\title{
Hydrology of Indiana Lakes
}

By J. I. PERREY and D. M. CORBETT

GEOLOGICAL SURVEY WATER-SUPPLY PAPER 1363

In cooperation with the Indiana

Department of Conservation, Division of Water Resources

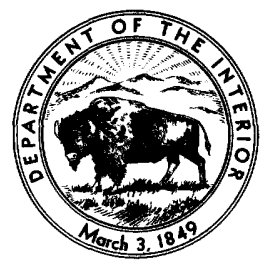




\section{UNITED STATES DEPARTMENT OF THE INTERIOR}

Fred A. Seaton, Secretary

\section{GEOLOGICAL SURVEY}

Thomas B. Nolan, Director 


\section{PREFACE}

This report was prepared by the U.S. Geological Survey, Water Resources Division, C. G. Paulsen, chief, under the general direction of J. V. B. Wells, chief, Surface Water Branch.

The field work and the collection and tabulation of basic information is part of a continuous cooperative program with the Division of Water Resources of the Indiana Department of Conservation, and the preparation of the report was the culmination of this program.

The data presented in this report were collected and prepared for publication under the supervision of Don M. Corbett, district engineer, Indianapolis, Ind. The sections dealing with the origin and extinction of lakes, and with ice condition, temperature and evaporation were prepared by J. I. Perrey. The introduction and the sections dealing with basic data on lake levels and stabilization of lakes were prepared jointly by J. I. Perrey and Don M. Corbett. Acknowledgement is made to Charles H. Bechert, Director, Division of Water Resources, Indiana Department of Conservation, for furnishing the table for the section on Legal lake levels, and the gage-height hydrographs and for reviewing the report. 

Page

Abstract $\ldots \ldots \ldots \ldots \ldots \ldots \ldots \ldots \ldots \ldots \ldots \ldots \ldots \ldots \ldots \ldots \ldots \ldots \ldots \ldots \ldots \ldots$

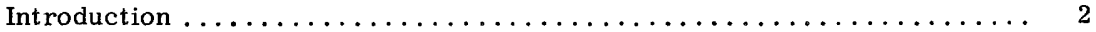

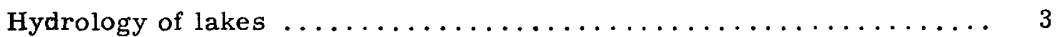

Indiana laws for preservation of lakes $\ldots \ldots \ldots \ldots \ldots \ldots \ldots$

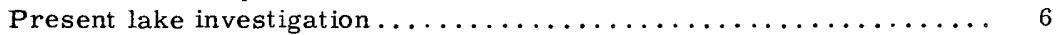

Creative and destructive forces affecting lakes .............. 7

Origin and distribution of Indiana lakes $\ldots \ldots \ldots \ldots \ldots \ldots \ldots$

Gradual extinction of northern Indiana lakes . . . . . . . . . . 8

Filling by organic material and marl deposits............. 9

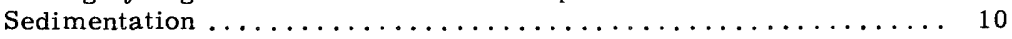

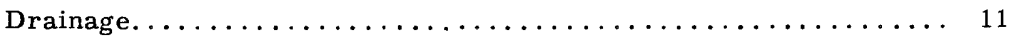

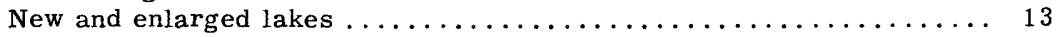

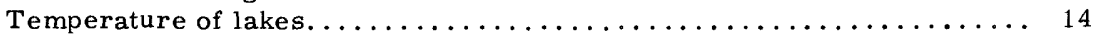

Scope of studies $\ldots \ldots \ldots \ldots \ldots \ldots \ldots \ldots \ldots \ldots \ldots \ldots \ldots \ldots \ldots \ldots$

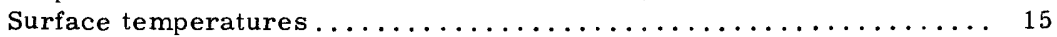

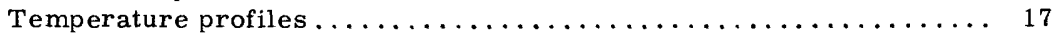

Theory of temperature changes below the surface $\ldots \ldots \ldots \ldots \ldots$

Comparison of temperature profiles in ten Indiana lakes . . . . . . 31

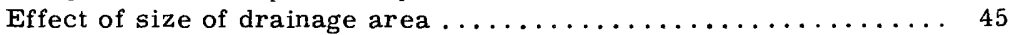

Effect of size of surface area $\ldots \ldots \ldots \ldots \ldots \ldots \ldots \ldots \ldots \ldots \ldots$

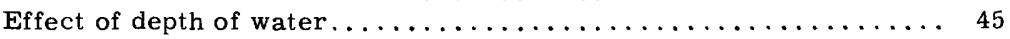

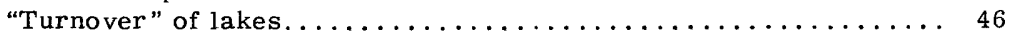

Implications of temperature information. . . . . . . . . . 47

Evaporation from lakes ............................ 49

Measurement of evaporation from water surfaces . . . . . . . . . 50

Collection of evaporation data in Indiana $\ldots \ldots \ldots \ldots \ldots \ldots \ldots \ldots \ldots \ldots$

Variation in evaporation in Indiana . . . . . . . . . . . . . . 52

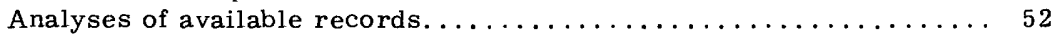

Ice conditions on northern Indiana lakes. . . . . . . . . . . . . 59

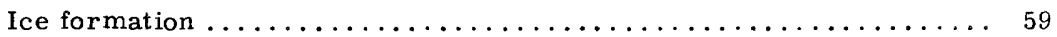

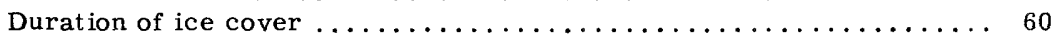

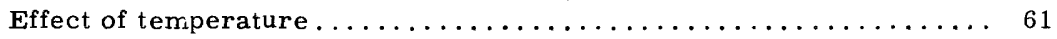

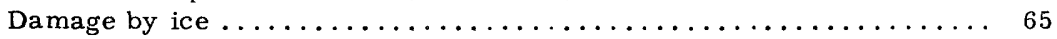

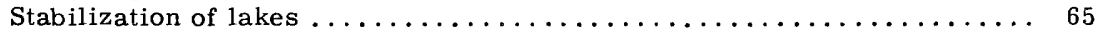

Improved transportation and its effect on use of lakes. . . . . . . . 65

Agricultural and recreational use of lakes. . . . . . . . . . . 66

Requirements for maintaining constant lake levels . . . . . . . . 67

Problems of lake-level control on some Indiana lakes . . . . . . . . . 68

Effect of inflow and outflow on planning control dams . . . . . . . . 69

Effect of storage in lakes on streamflow ............... 70

Effect of control dam on Ridinger Lake . . . . . . . . . . . . . 72

Basic data on lake levels for selected lakes $\ldots \ldots \ldots \ldots \ldots \ldots \ldots \ldots \ldots \ldots$

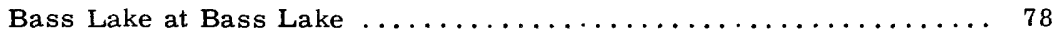

Bear Lake at Wolflake . . . . . . . . . . . . . . . . . . . 84

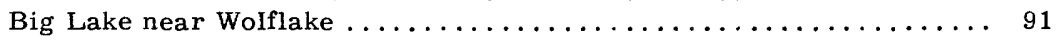

Bixler Lake at Kendallville ... . . . . . . . . . . . . . . . . 98

Bruce Lake at Lake Bruce. . . . . . . . . . . . . . . . . . . . 104

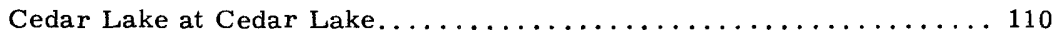

Cedar Lake near Waterloo. . . . . . . . . . . . . . . . . 117

Crooked Lake near Wolflake . . . . . . . . . . . . . . . . . 124

Flint Lake near Valparaiso . . . . . . . . . . . . . . . . . . . 130

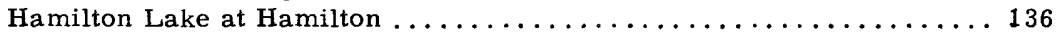

Jimerson Lake at Nevada Mills . . . . . . . . . . . . . . . . . 143

Koontz Lake at Koontz Lake . . . . . . . . . . . . . . . . . . . 152

Lake James at Lake James . . . . . . . . . . . . . . . . . . 159

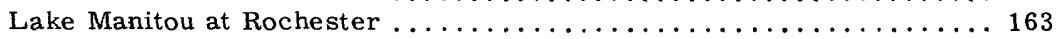

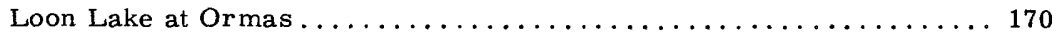

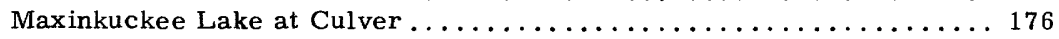


Basic data on lake levels for selected lakes--Continued

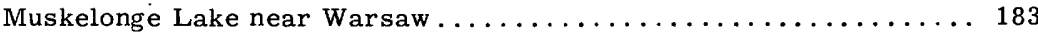

Ridinger Lake near Pierceton . . . . . . . . . . . . . . . . . 189

Round Lake and Cedar Lake at Tri-Lakes . . . . . . . . . . . . 197

Round Lake and Clear Lake at Clear Lake . . . . . . . . . . . . 205

Shriner Lake at Tri-Lakes. . . . . . . . . . . . . . . . . . . . . 212

Smalley Lake near Washington Center.................. 218

Sylvan Lake at Rome City. . . . . . . . . . . . . . . . . . . . . . 224

Syracuse Lake at Syracuse. . . . . . . . . . . . . . . . . 231

Tippecanoe Lake at Oswego ...................... 238

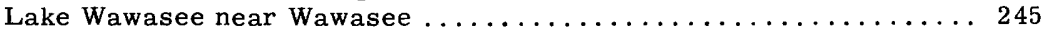

Webster Lake at North Webster. . . . . . . . . . . . . . . . . 252

Winona Lake at Warsaw ........................ 258

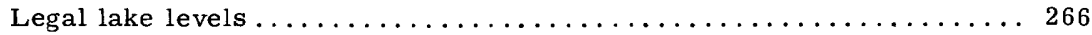

Temperatures for selected lakes . . . . . . . . . . . . . . . 273

Bass Lake at Bass Lake . . . . . . . . . . . . . . . . . . 274

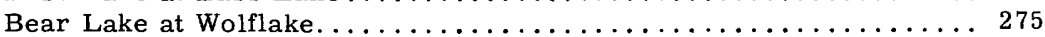

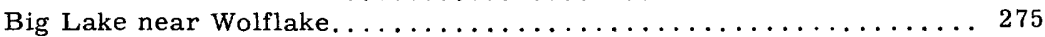

Bixler Lake at Kendallville . . . . . . . . . . . . . . . 276

Bruce Lake at Lake Bruce . . . . . . . . . . . . . . . . . . 278

Cedar Lake at Cedar Lake . . . . . . . . . . . . . . . . . . . . . . 279

Cedar Lake near Waterloo ......................... 280

Crooked Lake near wolflake $\ldots \ldots \ldots \ldots \ldots \ldots \ldots \ldots \ldots \ldots \ldots \ldots \ldots \ldots \ldots$

Flint Lake near Valparaiso . . . . . . . . . . . . . . . . . . 281

Hamilton Lake at Hamilton . . . . . . . . . . . . . . . . . 283

Jimerson Lake at Nevada Mills. . . . . . . . . . . . . . . . 283

Koontz Lake at Koontz Lake. . . . . . . . . . . . . . . . . . . . . . 283

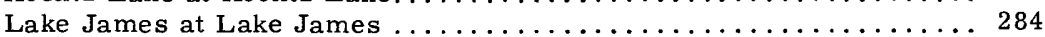

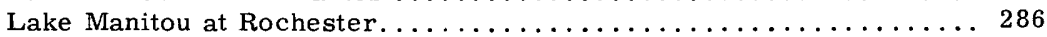

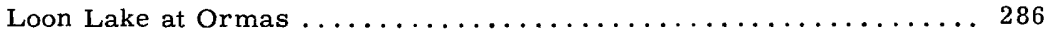

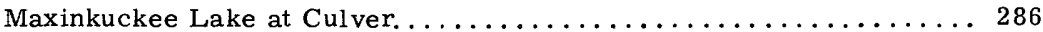

Muskelonge Lake near Warsaw ...................... 310

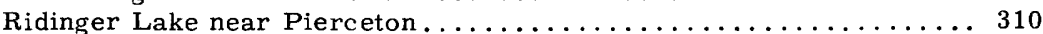

Round Lake and Cedar Lake at Tri-Lakes . . . . . . . . . . . . . 310

Round Lake and Clear Lake at Clear Lake . . . . . . . . . . . . . 311

Shriner Lake at Tri-Lakes. . . . . . . . . . . . . . . . . . 315

Smalley Lake near washington Center. . . . . . . . . . . . . . 315

Sylvan Lake at Rome City. . . . . . . . . . . . . . . . . . . 315

Syracuse Lake at Syracuse. . . . . . . . . . . . . . . . . . 316

Tippecanoe Lake at Oswego . . . . . . . . . . . . . . . . . 316

Lake Wawasee near Wawasee ..................... 318

Webster Lake at North Webster. . . . . . . . . . . . . . . . . . . 319

Winona Lake at Warsaw ........................ 320

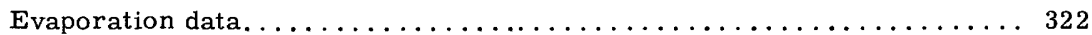

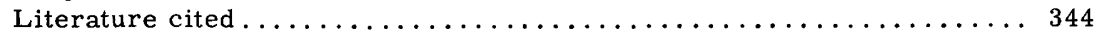

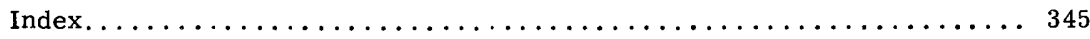

\section{ILLUSTRATIONS}

1. Sediment collector used by Scott and Miner............. Page

2. Water temperature-density relationship $\ldots \ldots \ldots \ldots \ldots \ldots \ldots$

3. Surface water and mean air temperatures, Maxinkuckee Lake ............................. 20

4. Typical temperature profile graph with temperature zones indicated.......................... 23 
Figure 5. Temperature profiles, Maxinkuckee Lake, at intervals

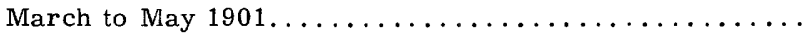

6. Temperature profiles, Maxinkuckee Lake, at intervals

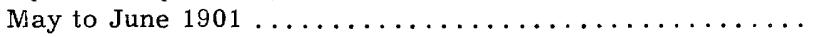

7. Temperature profiles, Maxinkuckee Lake, at intervals

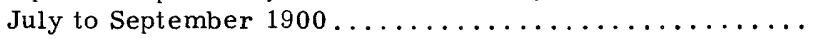

8. Temperature profiles, Maxinkuckee Lake, at intervals October to December $1900 \ldots \ldots \ldots \ldots \ldots \ldots \ldots \ldots \ldots \ldots \ldots \ldots \ldots \ldots$.

9. Temperature profiles, Maxinkuckee Lake, at intervals

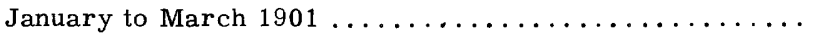

10. Temperature profiles, Tippecanoe Lake, at intervals January to November $1947 \ldots \ldots \ldots \ldots \ldots \ldots \ldots \ldots$. . . . . . . . . . . . . . .

11. Temperature profiles, Maxinkuckee Lake, at intervals

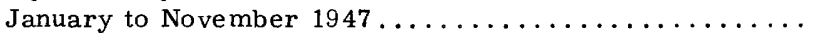

12. Temperature profiles, Cedar Lake (Lake County) at intervals February to November $1947 \ldots \ldots \ldots \ldots \ldots$

13. Temperature profiles, Flint Lake, at intervals February to

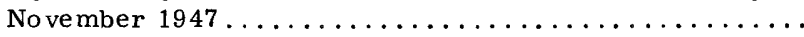

14. Temperature profiles, Bass Lake, at intervals May to

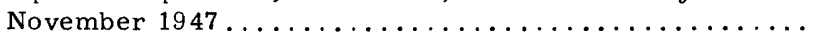

15. Temperature profiles, Winona Lake, at intervals April to

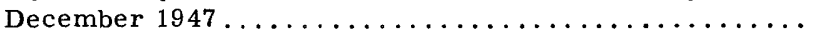

16. Temperature profiles, Lake Wawasee, at intervals February to November $1947 \ldots \ldots \ldots \ldots \ldots \ldots \ldots \ldots \ldots \ldots$

17. Temperature profiles, Bixler Lake, at intervals March

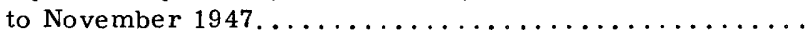

18. Temperature profiles, Lake James, at intervals April to

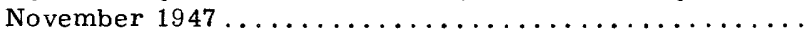

19. Temperature profiles, Clear Lake, in Steuben County, at intervals February to December $1947 \ldots \ldots \ldots \ldots \ldots \ldots$

20. Class A evaporation station, Kendallville .............

21. Graph of average daily evaporation, Geist Reservoir,

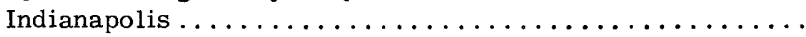

22. Comparison of evaporation loss at Kendallville with water loss at Bixler Lake .....................

23. Comparison of evaporation loss at Kendallville with water

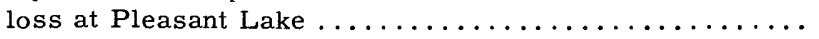

24. Comparison of evaporation loss at Valparaiso with water

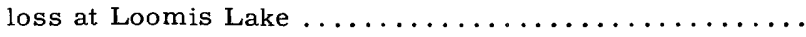

25. Comparison of evaporation loss at Valparaiso with water loss at Clear Lake .......................

26. Time required to freeze ice of various thicknesses . . . . . .

27. Graphs showing possible control of Ridinger Lake through operation of a control dam, $1944 \ldots \ldots \ldots \ldots \ldots \ldots \ldots$

28. Graphs showing actual control of Ridinger Lake through operation of control dam, $1948 \ldots \ldots \ldots \ldots \ldots \ldots \ldots$

29. Lake-level hydrographs for Bass Lake at Bass Lake, for

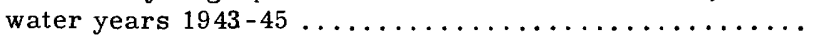

30. Lake-level hydrographs for Bass Lake for water years

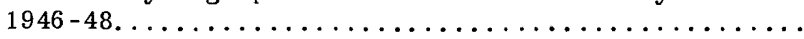

31. Lake-level hydrographs for Bass Lake for water years 1947-51............................

32. Lake-level hydrographs for Bass Lake for water years

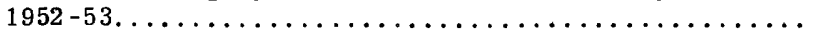

33. Lake-level hydrographs for Bear Lake at Wolflake, for water years $1943-45 \ldots \ldots \ldots \ldots \ldots \ldots \ldots \ldots \ldots$

34. Lake-level hydrographs for Bear Lake for water years 1946-48........................... 
Figure 35. Lake-level hydrographs for Bear Lake for water years

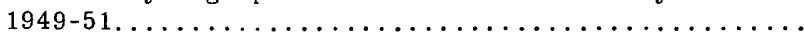

36. Lake-level hydrographs for Bear Lake for water years 1952-53.

37. Lake-level hydrographs for Big Lake at Wolflake, for

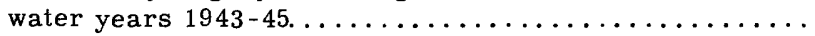

38. Lake-level hydrographs for Big Lake for water years 1946-48.............................

39. Lake-level hydrographs for Big Lake for water years $1949-51$.

40. Lake-level hydrographs for Big Lake for water years $1952-53$.

41. Lake-ıevel hydrographs for Bixler Lake at Kendallville, for

42. Lake-level hydrographs for Bixler Lake for water years

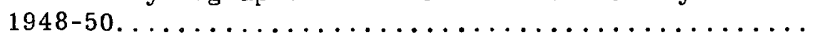

43. Lake-level hydrographs for Bixler Lake for water years 1951-53.

44. Lake-level hydrographs for Bruce Lake at Lake Bruce, for water years $1943-45 \ldots \ldots \ldots \ldots \ldots \ldots \ldots \ldots$. . . . . .

45. Lake-level hydrographs for Bruce Lake for water years

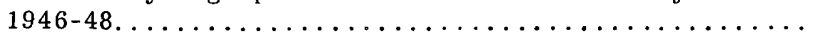

46. Lake-level hydrographs for Bruce Lake for water years

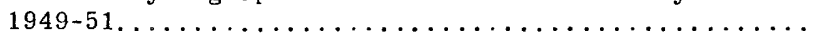

47. Lake-level hydrographs for Bruce Lake for water year 1952.

48. Lake-level hydrographs for Cedar Lake at Cedar Lake for water years $1943-45 \ldots \ldots \ldots \ldots \ldots \ldots \ldots \ldots$

49. Lake-level hydrographs for Cedar Lake at Cedar Lake, for

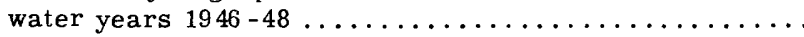

50. Lake-level hydrographs for Cedar Lake at Cedar Lake, for

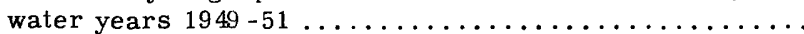

51. Lake-level hydrographs for Cedar Lake at Cedar Lake, for

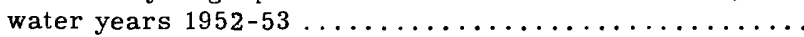

52. Lake-level hydrographs for Cedar Lake near Waterloo, for water years $1943-45 \ldots \ldots \ldots \ldots \ldots \ldots \ldots \ldots \ldots$

53. Lake-level hydrographs for Cedar Lake near Waterloo, for

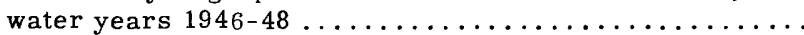

54. Lake-level hydrographs for Cedar Lake near Waterloo, for

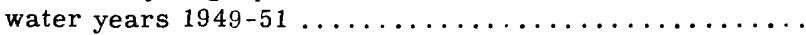

55. Lake-level hydrographs for Cedar Lake near Waterloo, for

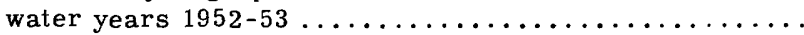

56. Lake-level hydrographs for Crooked Lake near Wolflake, for water years $1943-45 \ldots \ldots \ldots \ldots \ldots \ldots \ldots \ldots$

57. Lake-level hydrographs for Crooked Lake for water years $1946-48$

58. Lake-level hydrographs for Crooked Lake for water years 1949-51

59. Lake-level hydrographs for Crooked Lake for water year

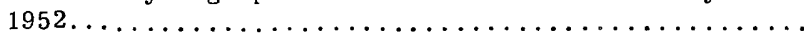

60. Lake-level hydrographs for Flint Lake near Valparaiso, for

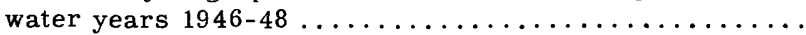

61. Lake-level hydrographs for Flint Lake for water years 1949-51.

62. Lake-level hydrographs for Flint Lake for water years

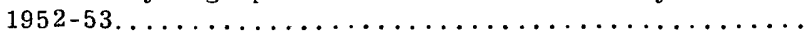

63. Lake-level hydrographs for Hamilton Lake at Hamilton, for

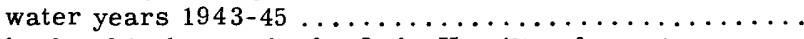

64. Lake-level hydrographs for Lake Hamilton for water years 1946-48. 
Figure 65. Lake-level hydrographs for Hamilton Lake for water years

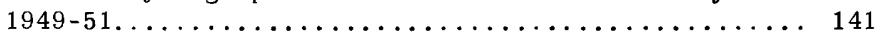

66. Lake-level hydrographs for Hamilton Lake for water years 1952-53.

67. Lake-level hydrog raphs for Jimerson Lake at Nevada Mills, for water years 1937-39 .................

68. Lake-level hydrographs for Jimerson Lake for water years 1940-42............................

69. Lake-level hydrographs for Jimerson Lake for water years $1943-44$ and 1944.

70. Lake-level hydrographs for Jimerson Lake for water years

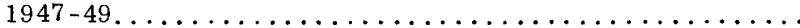

71. Lake-level hydrographs for Jimerson Lake for water years

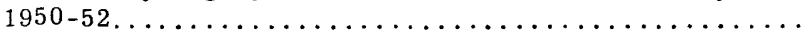

72. Lake-level hydrograph for Jimerson Lake for water year 1953.

73. Lake-level hydrographs for Koontz Lake at Koontz Lake, for water years $194-45 \ldots \ldots \ldots \ldots \ldots \ldots \ldots \ldots$

74. Lake-level hydrographs for Koontz Lake for water years

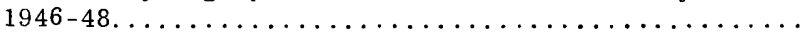

75. Lake-level hydrographs for Koontz Lake for water years

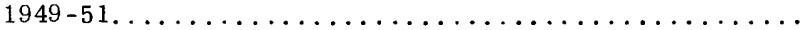

76. Lake-level hydrographs for Koontz Lake for water years

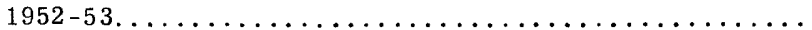

77. Lake-level hydrographs for Lake James at Lake James, for water years $1943-45 \ldots \ldots \ldots \ldots \ldots \ldots$

78. Lake-level hydrographs for Lake James for water years

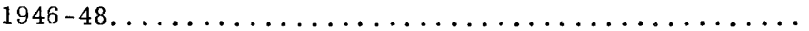

79. Lake-level hydrographs for Lake James for water year

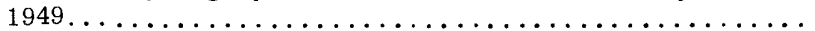

80. Lake-level hydrographs for Lake Manitou at Rochester, for water years $1943-45 . \ldots \ldots \ldots \ldots \ldots \ldots \ldots \ldots$

81. Lake-level hydrographs for Lake Manitou for water years

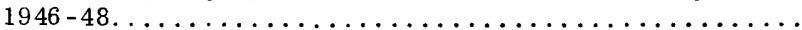

82. Lake-level hydrographs for Lake Manitou for water years

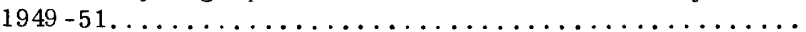

83. Lake-level hydrographs for Lake Manitou for water years $1952-53$.

84. Lake-level hydrographs for Loon Lake at Ormas, for water

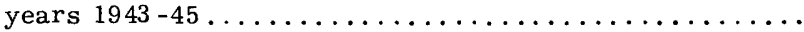

85. Lake-level hydrographs for Loon Lake for water years

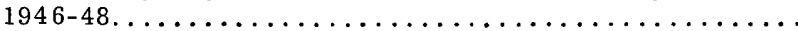

86. Lake-level hydrographs for Loon Lake for water years 1949-51.............................

87. Lake-level hydrographs for Loon Lake for water years 1952-53............................

88. Lake-level hydrographs for Maxinkuckee Lake at Culver, for water years $1943-45 \ldots \ldots \ldots \ldots \ldots \ldots \ldots \ldots \ldots$

89. Lake-level hydrographs for Maxinkuckee Lake for water

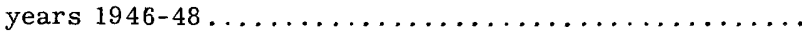

90. Lake-level hydrographs for Maxinkuckee Lake for water

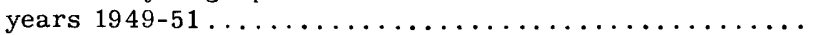

91. Lake-level hydrographs for Maxinkuckee Lake for water

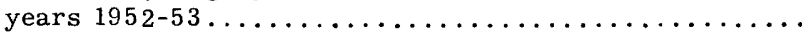

92. Lake-level hydrographs for Muskelonge Lake near Warsaw, for water years $1943-45 \ldots \ldots \ldots \ldots \ldots \ldots \ldots$

93. Lake-level hydrographs for Muskelonge Lake for water

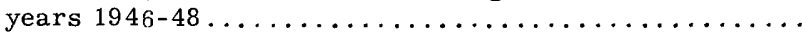

94. Lake-level hydrographs for Muskelonge Lake for water

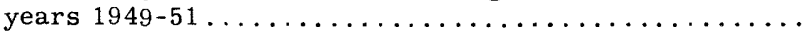


Figure 95. Lake-level hydrographs for Muskelonge Lake for water Page

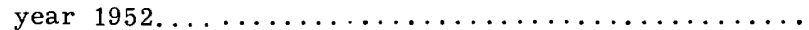

96. Lake-level hydrographs for Ridinger Lake near Pierceton,

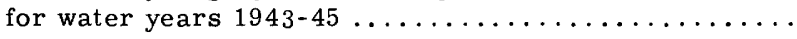

97. Lake-level hydrog raphs for Ridinger Lake for water years

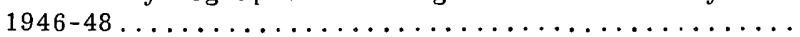

98. Lake-level hydrographs for Ridinger Lake for water years

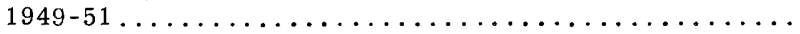

99. Lake-level hydrographs for Ridinger Lake for water years

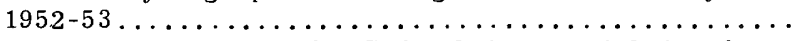

100. Lake-level hydrographs for Cedar Lake at Tri-Lakes for

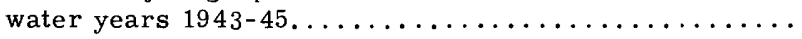

101. Lake-level hydrographs for Cedar Lake for water years

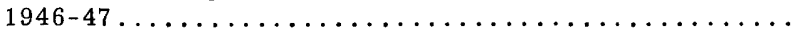

102. Lake-level hydrographs for Round and Cedar Lakes at Tri-Lakes for water years $1948-50 \ldots \ldots \ldots \ldots \ldots$

103. Lake-level hydrographs for Round and Cedar Lakes for

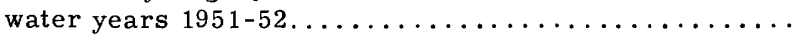

104. Lake-level hydrographs for Round Lake and Clear Lake at Clear Lake, for water years $1943-45 \ldots \ldots \ldots \ldots \ldots \ldots$. . . . .

105. Lake-level hydrographs for Round Lake and Clear Lake for

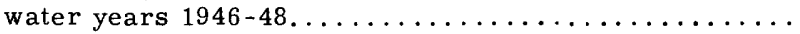

106. Lake-level hydrographs for Round Lake and Clear Lake for

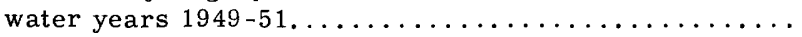

107. Lake-level hydrographs for Round Lake and Clear Lake for

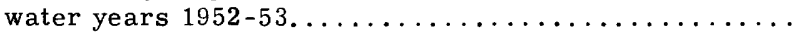

108. Lake-level hydrographs for Shriner Lake at Tri-Lakes for water years $1943-45 \ldots \ldots \ldots \ldots \ldots \ldots \ldots \ldots \ldots \ldots \ldots \ldots \ldots \ldots \ldots \ldots$

109. Lake-level hydrographs for Shriner Lake for water years

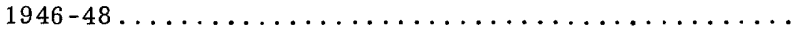

110. Lake-level hydrographs for Shriner Lake for water years 1949-51 ..........................

111. Lake-level hydrographs for Shriner Lake for water years

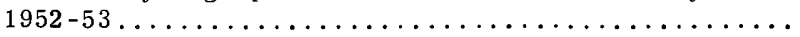

112. Lake-level hydrographs for Smalley Lake near Washington Center, for water years $1943-45 \ldots \ldots \ldots \ldots \ldots \ldots$

113. Lake-level hydrographs for Smalley Lake for water years

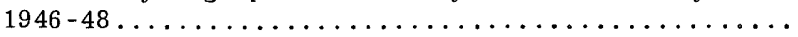

114. Lake-level hydrographs for Smalley Lake for water years

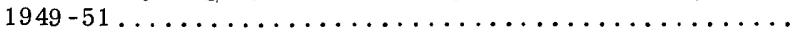

115. Lake-level hydrographs for Smalley Lake for water years

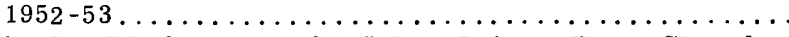

116. Lake-level hydrographs for Sylvan Lake at Rome City, for

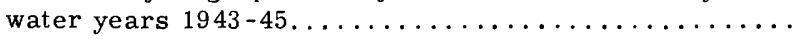

117. Lake-level hydrographs for Sylvan Lake for water years

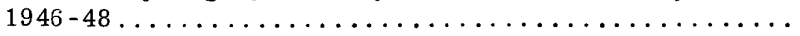

118. Lake-level hydrographs for Sylvan Lake for water years

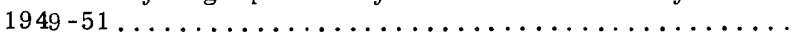

119. Lake-level hydrographs for Sylvan Lake for water years

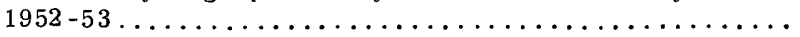

120. Lake-level hydrog raphs for Syracuse Lake at Syracuse,

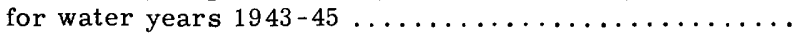

121. Lake-level hydrographs for Syracuse Lake for water years

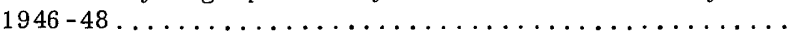

122. Lake-level hydrographs for Syracuse Lake for water years

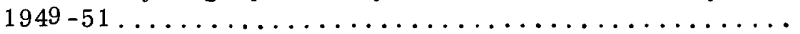

123. Lake-level hydrographs for Syracuse Lake for water years

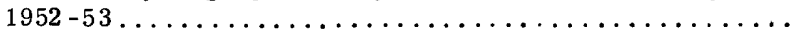


Figure 124. Lake-level hydrographs for Tippecanoe Lake at Oswego,

Page

for water years $1943-45 \ldots \ldots \ldots \ldots \ldots \ldots \ldots \ldots$

125. Lake-level hydrographs for Tippecanoe Lake for water

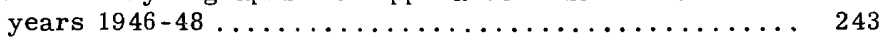

126. Lake-level hydrographs for Tippecanoe Lake for water

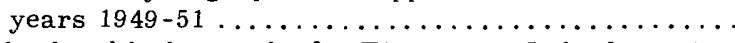

127. Lake-level hydrographs for Tippecanoe Lake for water

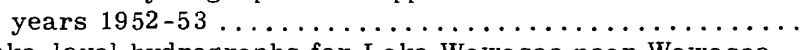

128. Lake-level hydrographs for Lake Wawasee near Wawasee, for water years $1943-45 \ldots \ldots \ldots \ldots \ldots \ldots \ldots \ldots$

129. Lake-level hydrographs for Lake Wawasee for water years

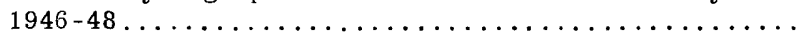

130. Lake-level hydrographs for Lake Wawasee for water years

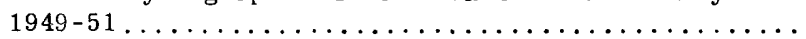

131. Lake-level hydrographs for Lake Wawasee for water years

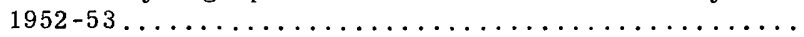

132. Lake-level hydrographs for Webster Lake at North Webster, for water years $1943-45 \ldots \ldots \ldots \ldots \ldots \ldots \ldots \ldots$

133. Lake-level hydrographs for Webster Lake for water years

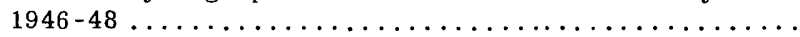

134. Lake-level hydrographs for Webster Lake for water years $1949-51$

243

135. Lake-level hydrographs for Webster Lake for water years

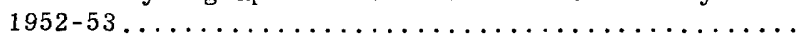

136. Lake-level hydrographs for Winona Lake at Warsaw, for

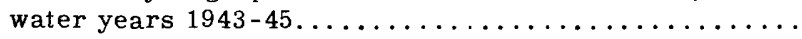

137. Lake-level hydrographs for Winona Lake for water years $1946-48$

138. Lake-level hydrographs for Winona Lake for water years

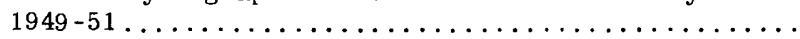

139. Lake-level hydrographs for Winona Lake for water years $1952-53$

\section{TABLES}

Table 1. Summary of legal lake levels 



\title{
HYDROLOGY OF INDIANA LAKES
}

\author{
By J. I. Perrey and D. M. Corbett
}

\section{A BSTRACT}

Indiana's lakes are a valuable resource for both recreational use and their industrial potential. Some lakes are used for water supply.

The natural lakes are glacial in origin and are most concentrated in northeastern Indiana. Many of the lakes were drained by the early settlers. The natural processes of sedimentation and accumulation of organic deposits tend also to reduce the number and size of lakes. The trend toward fewer lakes has been reversed in recent years by the construction of artificial lakes; the number of lakes has increased from a little over 500 in 1800 to over 1,000 at the present time.

The recreational value of the lakes and the desirability of maintaining constant lake levels led to legislation relative to the establishment of legal lake levels and to a program of lake-level observations, supplemented by the collection of hydrologic data affecting lake levels.

Observations of both surface temperature and temperature profile were made by investigators as early as 1875, The earlier data and data collected since 1946 have been analyzed to show the fluctuation of temperature with season, depth of water, size of lake, and other influencing factors. Surface temperatures, which may vary several degrees within 24-hour period, fluctuate with air temperature and reach highs of about $85^{\circ} \mathrm{F}$ in August. The bottom temperatures of lakes more than 100 feet deep seldom rise above $45^{\circ} \mathrm{F}$. Subsurface water temperatures below ice cover reach a uniform value of about $39^{\circ} \mathrm{F}$ except that just below the ice which remains closely to $32^{\circ} \mathrm{F}$. During summer, temperature stratification is established as the lake warms. Shallow lakes reach temperature extremes about $5^{\circ} \mathrm{F}$ above and below those of the deep lakes. Size of the contributing drainage area, surface area of the lake, and flow through the lake have little effect on water temperatures below a depth of about 20 feet. Little aquatic life exists below depths of 30 feet from mid April to mid September owing to lack of oxygen in the water. Large amounts of cool water, 5 to 10 degrees cooler than ground water which averages $50^{\circ}$ to $55^{\circ} \mathrm{F}$ in northern Indiana, are available in the lower portions of the deep lakes.

Evapotranspiration accounts for the disposal of about 70 percent of the precipitation in Indiana. During a year's time evaporation from the lake surface about equals the precipitation falling on the lake. During dry periods evaporation from a lake may exceed the inflow from small drainage areas and cause a lowering of lake levels. For the period April through October, evaporation as measured in a class A land pan of the U. S. Weather Bureau has averaged 44 inches at Evansville, 34 inches at Indianapolis, and 31 inches at Valpariso. A coefficient of about 0.7 is usually applied to yearly evaporation data from Class A land pans to obtain equivalent evaporation from lake surfaces.

Ice cover on the lakes extends from about December 15 to March 15 and reaches thickness of 24 to 30 inches during the colder winters. Ice can be damaging to lakeside installations by thrust action or by the wind action on ice cakes.

The stabilization of lake levels often requires the construction of outlet control structures. A detailed study of past lake-level elevations and other hydrologic data is necessary to establish a level that can be maintained and to determine the means necessary for maintaining the established level. Detailed lake-level records for 28 lakes are included in the report, and records for over 100 other lakes are available in the U. S. Geological Survey office, Indianapolis, Ind. Evaporation data from the four Class A evaporation stations of the U. S. Weather Bureau have been compiled in this report. A table showing the established legal lake level and related data is included. 


\section{INTRODUCTION}

To the casual observer a lake is a relatively stable body of water, nearly always of the same area and level. To the more discerning person, who may be concerned about property or facilities located on the shores, it is soon apparent that a lake is a variable thing whose surface expands and contracts, and whose level rises and falls. Closer observation will reveal that these oscillations have seasonal trends, and operate between variable extremes.

With the intensive use of lakes and their shores, it is common for variable surface levels to interfere with the use of lake property. This problem may be solved by either accommodating the use of the property to the conditions of the lake, or by controlling the lake itself. The latter method is frequently selected because it will generally increase the utility of the lake.

The degree to which a lake may be controlled will depend largely upon physical conditions such as the elevation of surrounding land, the presence of an outlet, the size of contributing watershed, and the flow of water into the lake. The water flowing into the lake will determine largely the amounts of water that must be regulated to gain control.

Not all problems stem from a need for control or stabilization, some may arise from a desire to merely increase or decrease the surface area, or raise or lower the average level. Whatever the purpose, the solution will involve a change in some physical feature connected with the lake, and some manipulation that will tend to counteract the normal processes of nature: precipitation, evaporation, transpiration, change of temperature, surface runoff, and underground inflow and outflow. Physical features frequently may be changed, and the changes made quite permanent, but usually it is practical to obtain only a limited control over the natural processes.

In designing any type of control device it is necessary to have a knowledge of conditions that are undesirable and in need of control, factors that are causing the undesirable conditions, and quantities that must be controlled. The features of a lake most frequently in need of control are the level and surface areas. The surface area is a function of the level since it varies with the level, and may be treated as a problem in level control. In turn, all problems in levels are related to the volume of water in the lake, and become problems in volume control. It is also readily apparent that variations in volume are directly connected with the gain or loss of water in the lake. 
With a little imagination one can quickly visualize the complicated intertwining of natural processes that are constantly changing the volume of water. The rainfall on the surface, the water entering from inflowing streams, the contribution from springs, the evaporation from the surface, the water used by vegetation and returned to the air through the process of transpiration, the flow through the outlet, and seepage into the ground, are all continually adding to or subtracting from the water in the lake. With this multiplicity of processes the study of lakes becomes a study in hydro$\log$.

\section{Hydrology of Lakes}

Hydrology, as related to lakes, is the science that deals with the processes governing the depletion and replenishment of the waters of lakes. It takes into consideration precipitation, evaporation, transpiration, flow into and out of the lake, from both sur face and underground sources, temperature, ice formation, sedimentation, and the effects of artificial control. It is the tool by which the behavior of lakes may be analyzed. Once the causes for the behavior are recognized and evaluated, means for their control modification, or counteraction may be devised. The recognition of causes for given conditions may be relatively simple, for example, the relation between high lake levels and excessive precipitation is apparent to the most casual observer. The evaluation of the cause, that is, the determination of the amount of water involved, is not so simple.

The determination of all the hydrologic factors is dependent on some type of measurement: the amount of precipitation is determined from rainfall caught and measured in rain gages; the surface inflow or outflow are determined from measurements of streamflow; gains and losses in lake volume are determined from measurements of lake levels.

The measurements of the various hydrologic factors are the basis of any hydrologic study. Data from observations of hydrologic phenomena are therefore called basic data. Basic data peculiar to lakes are information on size of contributing drainage areas, size of surface area, lake temperatures, and lake levels. Precipitation, evaporation, and streamflow are types of basic information that are common to other fields of study as well as lakes. Of the various types of basic data, lake levels are perhaps the most easily understood by the layman, the easiest to apply to many problems, and the easiest to observe and measure. 
Although many types of basic data may be determined by direct measurements, some must be obtained by indirect means. Losses due to evaporation generally cannot be measured with accuracy by direct observations of lake surfaces; they are usually estimated by comparisons with evaporation losses from standard evaporation pans. However, other methods are being developed that will eventually improve the technique of determining such losses (anonymous 1952). Water gained by inflow from the ground or lost by seepage into the ground cannot be measured directly, and must be determined by indirect methods.

Nearly all water problems involve problems of volume or quality. The solutions to the problems are dependent upon the evaluation of basic factors affecting the volume to be controlled. In other words, the solutions are dependent to a large degree upon basic data.

If a problem involves lake levels, as most lake problems do, it is desirable to have a knowledge of the levels that might be considered normal during the various seasons, the maximum and minimum levels, and the frequency and duration of extreme levels. Reliable information can be obtained only from frequent measurements or observations of the levels. Such observations must be made as events occur. We cannot go out today to observe last year's levels, nor even the one that occurred yesterday. Problems must be anticipated far in the future, and the collection of basic data started years before it is needed so that the range of conditions may be as wide as possible.

The importance of lake hydrology will grow with the increasing importance of lakes in our economy. Year by year the lakes of Indiana are increasing in value.

The importance of lakes for recreation and utility is easily understood when one considers that the number of lakes in Indiana of 5 acres or more in size has grown from a little over 500 in 1800 to more than 1,000 natural and artificial lakes today. The increase has been due to the construction of artificial lakes, mainly in those portions of the State where few or no lakes existed for merly.

Not all the construction of lakes has been done in recent years. Some of the largest artificial lakes were constructed during the canal-building days, over a hundred years ago. The old Wabash and Erie Canal was fed by two great reservoirs, no longer in existence. The Splunge Creek reservoir, along the Clay-Vigo County line, is said to have covered 4,000 acres, and the nearby Birch Creek reservoir in Clay County covered 1,400 acres. The former, 
if the area was correct, was the largest body of water in the State, excluding Indiana's portion of Lake Michigan and the old Kankakee swamp areas.

Although the building of artificial lakes was started early in the history of Indiana, the greatest activity is now taking place. Many of the recently constructed lakes are used for water supply. These lakes required hydrologic studies to determine the size of spillways required to discharge floodwaters without overtopping their dams, studies of the amount of storage required to meet anticipated demands for water, and studies of the ability of the watershed to replenish the water used by evaporation and for water supply.

Some lakes now under construction for recreational purposes will be among the deepest in the State when they are completed and so large in relation to their drainage areas that they will require 6 to 10 years to fill. Determination of the length of the filling period required studies of hydrologic data.

\section{Indiana Laws for Preservation of Lakes}

In 1905 the Indiana Legislature passed the first law "to preserve the fresh water lakes of the State of Indiana at their established level and protect them from danger of being injuriously affected or destroyed by the lowering of waters thereof by any drains or ditches." This law recognized the growing importance of lakes as centers for recreation, and tended to stop some of the land reclamation practices of former years, which had lowered many of the natural lakes and greatly reduced their areas.

As the use of lakes increased through the years new laws were passed to amend, strengthen or supplement the 1905 law. By 1935 it was realized that some definite elevation should be used in establishing a level for a lake. Consequently, a law was enacted which formulated a procedure to be followed in establishing a lake level, and provided for recording the level in the county recorder's office.

In 1947 another law was enacted that made records of lake-level observations essential to the administration of laws establishing levels for lakes. The 1947 law stated in part:

The Indiana Department of Conservation of the State of Indiana is hereby authorized and empowered to establish, by appropriate monuments, the average normal water level of all natural and artificial lakes of the State of 
Indiana and to construct or sponsor and supervise the construction of dams, spillways and control works necessary to maintain the average normal lake level. Such water level monuments shall be as permanent in character as practicable and shall fix the average normal level, and indicate the elevation of the highest point to which the water of such lake or lakes shall have risen within the past ten years prior to the passage of this act.

In 1951 the Legislature repealed the 1935 lake-level law and enacted a new statute designed to accomplish more effectively the same purpose as the 1935 law.

\section{Present Lake Investigation}

Sometime after the passage of the 1935 lake-level law, it became apparent that to accomplish the purpose of the law more knowledge of lake levels and their variations was needed. Consequently, in the fall of 1942, the Geological Survey, in cooperation with the Water Resources Division of the Indiana Department of Conservation, established 11 gaging stations on lakes in northern Indiana. In succeeding years, a definite program of lake-level observations was established; by November 1946 gages had been installed on 109 lakes, and the collection of records, on a daily basis wherever practicable, had begun.

About that time it was also realized that data on lake levels a lone were not sufficient to provide a full understanding of the various factors that affect lakes and account for differences in their behavior. To provide additional data for the lake study, the number of precipitation stations in the northern lake section were increased and two evaporation stations were established in cooperation with the U. S. Weather Bureau. Several lakes were selected for intensive studies that involved the recording of temperatures of lake-water, measuring inflow and outflow, mapping of the topography of lake beds to permit determination of volume of water, and collecting of other pertinent hydrologic data.

During the period 1947 to 1951 , additional lakes were included in the program and some of less importance were omitted from the study. The number of lake gages in operation reached a peak of 122 in 1949, but has averaged about 110 in recent years.

The study of the hydrology of Indiana lakes was carried on simultaneously with the collection of basic data, and has now reached the point where its results may be presented to the public together with basic data for selected lakes. 
This report describes the development of Indiana lakes, the factors that tend eventually to obliterate them, the annual cycle of water temperature, the theory of temperature changes within the body of a lake, evaporation from lakes, ice conditions on northern Indiana lakes, and problems involved in attempting to stabilize lake levels. It presents also the basic data for selected lakes.

The report is intended to provide the layman as well as the engineer an understanding of the natural processes that affect the behavior of all lakes. It should provide the basic information necessary to analyze many of the problems connected with lakes, and aid in reaching sound decisions regarding the solutions to these problems.

\section{CREATIVE AND DESTRUCTIVE FORCES AFFECTING LAKES}

\section{Origin and Distribution of Indiana Lakes}

Some thousands of years ago the region that is now Indiana was covered by great glaciers or ice sheets, hundreds of feet thick, that moved down from the north over a large part of the State. As these ice sheets advanced they changed every square mile of land surface they touched. They planed off the existing hilltops and filled in the former valleys in the manner of a gigantic bulldozer. Their final retreat produced the land surface upon which the lakes of Indiana were formed.

Two stages of glaciation have been recognized in Indiana. During the older, the Illinoian, glaciers covered about 82 percent of the State, leaving only a rough triangular area within the State, from Evansville to Martinsville to Jeffersonville, unglaciated. The younger, the Wisconsin, occurred many thousands of years after the Illinoian. During this stage the ice advanced as far south as a line roughly defined by Terre Haute, Rockville, Greencastle, Mooresville, North Vernon, Connersville, and Brookville. This advance of ice overrode the surface deposits of the older Illinoian glaciation and materially reduced or removed them entirely. The younger Wisconsin glaciation is responsible for the hundreds of natural lakes in northern Indiana.

The advancing ice transported large quantities of rock material; some was pushed before the ice mass and some was contained within it. As the ice retreated to the north it left a covering of glacial debris called drift or till. Along the margins of the ice, piles of glacial debris, liberated by the retreating ice front, were dumped, thus forming irregular and lobate ridges known as moraines. Between moraines, the ice dropped its load as a sheet of till of variable thickness called ground moraine. Melt waters from the ice left stratified deposits known as outwash within and on top 
of the till sheets, along the margins, and behind and beyond the moraines.

The moraines were hilly ridges in which mammoth blocks of ice were buried. The buried ice melted slowly and eventually left water-filled holes, which became the deeper lakes in Indiana. On the rolling surface of the drift shallow depressions were filled with water where there were no outlets to permit the water to drain away. These depressions formed shallow lakes, and eventually many became swamps and marshes.

After the disappearance of the glaciers, those lakes without sufficient inflow of surface water and ground water to satisfy the demands of evaporation, transpiration, and seepage losses gradually dried up. Others with large contributing watersheds overflowed at outlets formed at the lowest point in their brims, and as water flowed from one lake to another, drainage systems were formed involving a chain of lakes. Such chains are now found in the upper reaches of the Tippecanoe and Pigeon Rivers. Others, without surface outlets, existed as long as there was a balance between inflow from surface and underground sources and water losses from the lake.

The greatest concentration of glacial lakes in Indiana is along the Mississinewa and Packerton morainic systems which extend from Steuben County in the northeast corner of the State diagonally southwestward for about 75 miles. Hundreds of lakes are located on and behind these moraines and their various arms in Steuben, Noble, Kosciusko, and Whitley Counties and northern Wabash County. The grouping of the lakes becomes less dense away from the main moraines in the direction of Fort Wayne or South Bend.

Other groups of lakes, although more scattered, are found in other morainic systems. The Valparaiso moraine follows a general northeasterly course from the Illinois boundary across Lake, Porter, and LaPorte Counties, and the Maxinkuckee moraine, a course along the Pulaski-Fulton and Starke-Marshall County lines.

\section{Gradual Extinction of Northern Indiana Lakes}

Once created, lakes are doomed to gradual extinction by natural causes. Hundreds had disappeared in the thousands of years that elapsed between the Ice Age and the first settlement in the area by white men.

With the advent of our civilization and the growth and spread of populations, more tillable land was brought into production. This demand led to more land drainage which hastened the extinction of many lakes. 


\section{Filling by Organic Material and Marl Deposits}

After the glaciers, vegetation took root in the soil and spread to the shallower portions of lakes. The growth of aquatic plants produced deposits of organic muck along the shores and on the bottoms of many lakes and slowly filled the depressions that were once occupied by water. The shallower lakes, in which vegetation covered the entire bottom, gradually deterioratedinto swamps and then marshes with comparative rapidity. The deeper lakes with organic deposits around the edges show only retreating shorelines.

The multitude of muck beds scattered over the northern part of the State are evidence of the lakes and swamps that once existed, at elevations much above present lake levels, and indicating that many of the lakes were much larger in the past. The muck deposits have considerable range in depth averaging 3 to 4 feet in many lakes, but some extinct lakes and ponds have organic deposits more than 30 feet deep.

Marly soil, found extensively in and around northern Indiana lakes, has helped to decrease the size and depth of these lakes. It is a fresh-water deposit of high calcium carbonate content, mixed with clay, silt, and small amounts of other minerals and organic matter. It is a soft, granular, earthy material, ranging in color from milky white to bluish gray or yellowish brown, depending on the amounts of mineral and organic matter mixed with it.

The calcium carbonate of the marlis precipitated from the water in the lake or pond, in part through the action of aquatic organisms. Where the lake or pond is fed at a relatively high rate by hard spring water of high calcium bicarbonate content, the precipitation of marl is especially vigorous.

Marl beds range in thickness from a few inches to more than 45 feet. Deposits of 6 or 7 feet are common and many between 10 and 20 feet are found in Indiana. Blatchley and Ashley (1900) surveyed the marl deposits of many Indiana lakes in 1899 and 1900 and reported that "in some cases the accumulation of marl has almost or quite filled up the lake."

Fresh-water marl deposits are now used commercially to some extent as a source of agricultural lime. During the period 1899 to 1940 , the deposits were used also in a number of places as a source of lime in the production of Portland cement. The largest and longest used deposit, for this purpose, is located on Big and Little Turkey Lakes in LaGrange County. The Wabash Portland Cement Co. erected a plant at Elmira on Big Turkey Lake and began the manufacture of cement in August 1900. The plant, the last of its kind in Indiana, has not been operated since 1945. 


\section{Sedimentation}

From their very beginning, the lakes of Indiana have been decreasing in both area and depth owing in part to the quantities of sediment carried into them by their inflowing streams. However, the process of filling these lakes by sediments has not been as rapid as that caused by the organic deposits, as the sediment load in most northern Indiana streams does not appear to be heavy.

The largest deposits of sediment are found near the mouths of the tributary streams. In some lakes, a combination of sediment and organic deposits have built up large swamp and marsh areas around the mouths of the inlets resulting in corresponding decreases in the areas of the lakes. Although a large part of the sediment, particularly the larger and heavier particles, settles as soon as the inflowing water reaches the still water of the lake, a certain amount moves out into the main body of water, where the smaller and lighter particles settle at a much slower rate. Thus, deposition of sediment over the entire bottom of the lake gradually makes the lake shallower.

Extensive investigations of the amount and distribution of the accumulated sediment in Winona and Tippecanoe Lakes were conducted by Wilson (1935, p. 302-303; 1937, p. 248) of Heidelberg College. These studies indicate that the maximum depth of Winona Lake decreased from an original 127 feet to 79 feet, and the original area and volume were reduced 37.4 and 43.6 percent, respectively. In Tippecanoe Lake the maximum depth decreased from 164 feet to 123 feet, and the original area and volume were reduced 20.8 and 31.9 percent respectively. The original depth, area, and volume are based on geologic evidence and it is believed that they indicate conditions at the time the lakes were formed after the ice age.

Although the depth of sediment deposits may be determined by simple measurement of samples of bottom materials taken from the beds of lakes, it is more difficult to determine the rates at which the materials were deposited. Rates may be determined indirectly by sampling the bottom at periodic intervals, but the samples must be taken in the same locations each time to avoid errors resulting from irregularities in the bed and varying distances from the point at which the sediment enters the lake.

Direct measurements of sediment depositions were made with collectors installed by Scott and Miner (1935) at various locations on Winona and Tippecanoe Lakes in July 1930. The collector (fig. 1) consisted of a wire cable with a float attached at the upper end, an anchor at the lower end, and cylindrical glass collecting 
jars wired to the cable at various intervals. These collectors were allowed to remain in position in the lakes for periods ranging from 10.8 to 60 months. As a result of this study the sediment

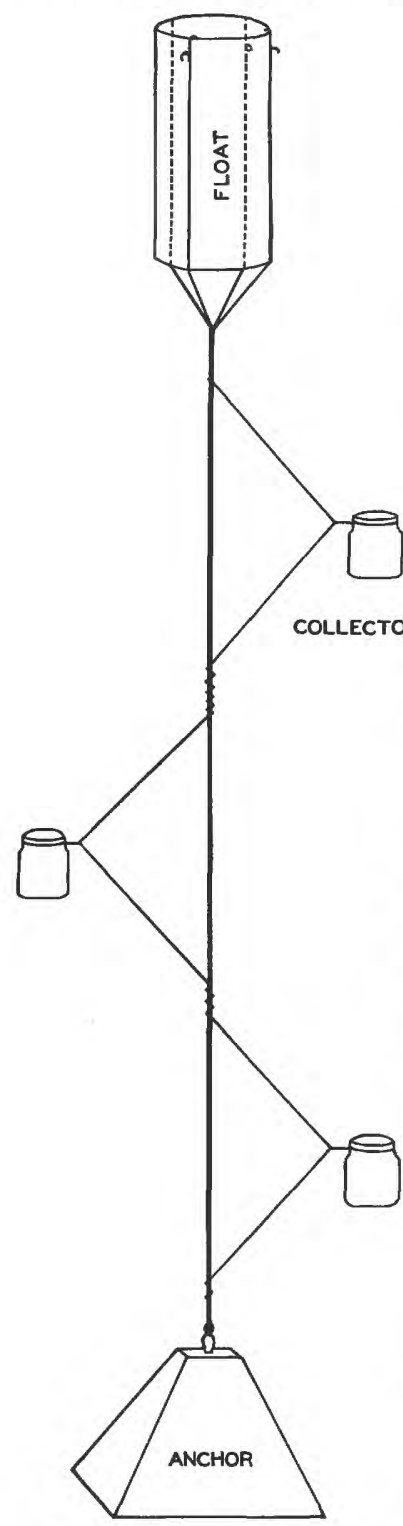

Figure 1. --Sediment collector used by Scott and Miner. in the deepest parts was found to be increasing at the rate of about one-third inch per year in Winona Lake, and at the rate of about one-fifth inch per year in Tippecanoe Lake.

After taking the observations in 1935 , Mr. Miner set out four strings of collectors in the deep water of Winona Lake for longer periods of observation. In the summer of 1949 he removed the collectors from Winona Lake after they had been submerged for 14 years. "The minimum rate of sedimentation near the bottom in deep water was about half an inch per year, or at the rate of a foot every 24 years." (Miner, 1949) This rate appears to be somewhat greater than that determined in the summer of 1935.

Because of the long existence of the lakes it is apparent that the rate of sedimentation is either variable or has not always been as rapid as these recent short period observations indicate. At the rate of one half inch per year, it would have taken only 1,150 years for the 48 feet of sediment in the deep part of Winona Lake to be deposited. Geologic evidence indicates that the period of accumulation must have been much longer.

\section{Drainage}

The most rapid changes in lake levels are believed to have been manmade and to have taken place since the settlement of the State. The demand for agricultural land brought about the draining of swamps and, consequently, the lowering of many lake levels as areas of rich muck lands were reclaimed. This process brought about the complete disappearance of some of the shallower lakes. 
For example, Cedar Lake in Steuben County has disappeared as a result of drainage. An examination of aerial photographs of this area reveals evidence that the lake once had a surface area of 557 acres. However, it has not existed as an open body of water for many years. In 1899 Ashley wrote (Blatchley and Ashley, 1900) "It [Cedar Lake] has now been partly drained so that with the exception of a few lagoons its former area is a marsh." Topographic maps made as recent as 1938 show a marsh area of 392 acres remaining at that time. Today the lake is completely drained and its former bottom is being cultivated. Cedar Lake was always shallow and probably was not more than 15 feet deep when originally formed. At the time of the first drainage project, the water had a maximum depth of perhaps not more than about 5 feet.

Many other lakes that have not been completely drained have decreased in area as a result of artificial drainage. Although some of these lakes still are sizable bodies of water, their present water-surface areas are by no means an indication of their original size.

According to early reports, Cedar Lake in Lake County was lowered 8 to 12 feet prior to 1900 by dredging an outlet to Cedar Creek.

Eagle Lake in Starke County was reported, in May 1900, to have been reduced in size by half or more, to an area of about 70 acres as a result of dredging. The area of the same lake was found to be only 24 acres in 1939 .

In 1897, a ditch was dredged into Bruce Lake in Fulton County and the lake lowered considerably. The lower level was found unsatisfactory, and a dam was built later to restore the lake to approximately its former level.

Chapman Lake, in Kosciusko County, was lowered twice prior to 1900 , the total amounting to about 6 feet. The water area before the lake was lowered was estimated to have included about 850 acres. Since 1900 the lake level has been raised slightly by the construction of a small dam and the combined area of Big and Little Chapman Lakes is now 526 acres. Chapman Lake appears to have had an original area of about 1, 370 acres as indicated by the muck deposits around the lake. The level of the lake at that time was at an elevation of about 836 feet above mean sea level, or about 8 feet higher than the present level. The decrease in size, prior to dredging of the outlet channel, came about through natural causes. 
Ridinger Lake, in Koscuisko County, was lowered 4 feet in 1900, but in 1947 a control dam was built to maintain a higher and more desirable level.

Many other lakes have been lowered sizable amounts in the past. Heaton Lake in Elkhart County was lowered about 5 feet in 1885; Troy Cedar Lake in Whitley County was lowered about 10 feet in 1900; Loon Lake in Whitley County was lowered about 12 feet in the spring of 1900, and in Noble County, Bear Lake was lowered about 6 feet and High Lake about 8 feet in 1899. Many other lakes have been lowered smaller amounts by drainage.

\section{New and Enlarged Lakes}

The creation of new lakes began during the epoch of canal building when lakes were needed as feeders for the canals. Sylvan Lake in Noble County is perhaps the first notable example of such work in Indiana. Entirely artificial, it was created in 1837 as a feeder lake for the proposed Michigan and Erie Canal.

In the early days the levels of some natural lakes were raised and their areas enlarged to furnish sources of power for grist mills. A dam for a mill was built at Webster Lake in Kosciusko County in 1830 and raised the lake level 7 feet. This mill continued to operate until 1951. Dams were erected and the water level raised in many other lakes. The level of Lake Manitou was raised about 7 feet in 1830; Lake Wawasee, 5 feet in 1833; Lake George in Steuben County, 4 feet in 1836; and Hamilton Lake, 9 feet in 1837.

In recent years, the needs of an increasing population brought about a great demand for more lakes for water supply, flood control, and recreation. A dam was constructed on Fall Creek, a tributary of White River, in 1942 by the Indianapolis Water Co. to increase the water supply of Indianapolis, thus creating Geist Reservoir, a lake of 1,800 acres, the largest artificial body of water in Indiana. Bloomington Reservoir was constructed on Beanblossom Creek, also a tributary of White River, in 1953 by the city of Bloomington to increase its source of water supply, creating an artificial lake of 1,650 acres at spillway level. The acute shortage of water at the Muscatatuck State School and for the city of North Vernon resulted in the creation of an 185-acre artificial lake in 1953 on Brush Creek in the vicinity of Butlerville. The towns of Batesville, Salem, Scottsburg, Huntingburg, and Oakland City also have created lakes for water supply.

The Cagle's Mill flood-control dam, completed in December 1951, has behind it a permanent lake area of 1,400 acres for 
recreational purposes. This is the first large flood control reservoir created in Indiana.

More and more lakes are being created for recreation purposes, particularly in the southern part of the State where natural lakes are very rare. Greenwood Lake in Martin County, with an area of 800 acres, is perhaps the largest of such lakes. However, this lake is now within the boundaries of the Crane Naval Ordnance Depot and not open to the public.

Brown County has severalartificial lakes of which Yellowwood, with a water surface of 147 acres, is the largest. Martin, Morgan and Monroe Counties have a scattering of artificial lakes. Clay, Vigo, Sullivan, and Knox Counties have several lakes formed from surface drainage into abandoned strip coal mines. As evidenced by the activities cited herein, it is apparent that man realizes the value of lakes and the multiple purpose they serve, and it is rather obvious that more new lakes will be planned and constructed in future years.

\section{TEMPERATURE OF LAKES}

\section{Scope of Studies}

The first account of observations of temperatures of Indiana lakes was prepared by Levette (1876). His report tabulates the surface and bottom temperatures and temperature profiles (temperatures at various depths from the surface to the bottom of the lake,formerly called serial temperatures) of 12 lakes in northern Indiana taken in the fall of 1875. Unfortunately, the dates on which the observations were made are not given; consequently it is difficult to compare these data with more recent observations of similar type as temperatures vary appreciably with the time of year. In 1896, temperature studies of Lake Wawasee were made by Dolan (1896).

The first extensive study of temperatures of lake waters was made at Maxinkuckee Lake between July 1899 and June 1901 by Evermann and Clark (1920, p. 152-216), in connection with a physical and biological survey of the lake by the United States Fish Commission (now the U.S. Fish and Wildlife Service). This study includes tabulated observations of both surface and temperature profiles of Lake Maxinkuckee.

Scott (1915) in his report on the lakes of the Tippecanoe basin, records temperature profiles taken in 13 lakes during the summers of 1912 and 1914. 
Beginning with the summer of 1946 and continuing through December 1948, the U. S. Geological Survey in cooperation with the Division of Water Resources, State Department of Conservation, recorded lake temperatures of a representative group of lakes of various sized and depths. The objective was to obtain comparative data. The 10 lakes selected for this study are scattered over northern Indiana and include Cedar Lake in Lake County, Flint Lake in Porter County, Bass Lake in Starke County, Maxinkuckee Lake in Marshall County, Winona and Tippecanoe Lakes and Lake Wawasee in Kosciusko County, Bixler Lake in Noble County, and Lake James and Clear Lake in Steuben County. Weekly observations of the temperature of the water near the surface of these lakes were made by local gage observers. Temperature profiles were taken in the deepest parts of the lakes about once a month. During the colder months, it was not always possible to obtain the profile readings because unsafe ice conditions prevented reaching observation points.

\section{Surface Temperatures}

A study of the available data clearly establishes these observed results: that, except when the lake is under ice cover, the temperature of the lake water at the surface fluctuates with the temperature of the air with which it is in contact; that these fluctuations follow seasonal trends of air temperature; and that the temperature of the water will vary several degrees within a 24-hour period if the air temperatures vary widely during the same period. Ice cover provides a blanket of insulation which keeps the water underneath at a nearly constant temperature. Prolonged spells of below $32^{\circ} \mathrm{F}$ temperatures merely increase the thickness of the ice blanket without lowering the temperature of the unfrozen water.

The work of Evermann and Clark (1920) at Maxinkuckee Lake affords an excellent source of information on daily temperature variations. Daily surface temperatures were taken at $6 \mathrm{a} . \mathrm{m}$. , $12 \mathrm{~m}$. , and $6 \mathrm{p} . \mathrm{m}$., for the period July 1, 1899, to June 30, 1901, and infrequent observations were taken during some of the following years until 1913 .

Observations during each winter period, December 15 to March 31 , generally gave variations in water-surface temperatures of less than $1^{\circ} \mathrm{F}$ in any one day. These small variations in water temperature may be accounted for, even on days of wide variations in air temperatures, by the fact that the temperatures of open surface water are generally below $39^{\circ} \mathrm{F}$ during this period and for the greater part of the time near the freezing point of $32^{\circ}$, and a continued drop in air temperature results in formation of ice. The 
temperature of surface water immediately below ice cover usually will be between $32^{\circ} \mathrm{F}$ and $34^{\circ} \mathrm{F}$, with that in intimate contact with the ice remaining close to $32^{\circ} \mathrm{F}$.

When lakes are open and water temperatures are between $32^{\circ} \mathrm{F}$ and $39.2^{\circ} \mathrm{F}$ any tendency for the water to become warmer will increase its density, as fresh water reaches its maximum density at $39.2^{\circ} \mathrm{F}$; consequently, the heavier water will move downward and be replaced by colder and lighter water from below, thus maintaining a nearly constant temperature at the water surface. Of course, if this process continues long enough, the whole body of water will eventually become warmed to $39.2^{\circ} \mathrm{F}$ and the temperature at the water surface will gradually increase.

When the air temperature drops rapidly and the surface temperature of the water is between $32^{\circ}$ and $39.2^{\circ} \mathrm{F}$, the cooling water at the surface becomes lighter and remains at the surface, and results in a significant decrease in surface temperatures or the formation of ice. Such a change is more likely to take place between evening and the following morning, as air temperatures usually rise during the day and fall at night.

At Maxinkuckee Lake very few observations show more than $1^{\circ} \mathrm{F}$ increase in daytime surface temperatures during the winter months, but several instances of temperature drops of $3^{\circ}$ to $4^{\circ} \mathrm{F}$ overnight have been recorded, particularly during mid-December when rapid cooling of the lake occurred as a result of below-freezing air temperatures.

During the spring and summer months when the water temperature of the lake surface is above $39.2^{\circ} \mathrm{F}$, daily variations of $6^{\circ}$, $8^{\circ}$, and $10^{\circ} \mathrm{F}$ in the water at the surface are not uncommon. These variations are possible because of a greater differential existing between surface temperatures and those a short distance below the surface. The top few inches of water may be warmed very rapidly under the hot summer sun, and because the water becomes lighter as its temperature increases it will remain on top. Consequently there will be very little mixing with the water underneath, particularly on calm days. On cool days this same top layer will be cooled until it reaches the temperature and density of the water of the next lower stratum after which any further cooling and increase in its density will cause it to decend and the lower, less dense water to rise to the top. After this action has started the movement of the lower and more uniform temperatured water to the top will maintain a rather constant or very slowly cooling lake surface temperature throughout the day. 
The general pattern of the annualcycle of lake surface temperatures for northern Indiana lakes is as follows. Temperatures for the months of January and February range between $32^{\circ}$ and $34^{\circ} \mathrm{F}$. March temperatures continue in this range until the ice cover leaves, usually about March 15th, then the temperatures begin to rise. The rapidity of this rise depends on the solar radiation absorbed by the water, the air temperature, the inflow of large quantities of relatively warm water from spring rains, and other factors. By April 1st the water temperature at the surface is up to $40^{\circ}$ or $45^{\circ} \mathrm{F}$. It will continue to rise in the following months, reaching about $55^{\circ}$ by May $1 \mathrm{st}$, about $65^{\circ} \mathrm{F}$ by June $1 \mathrm{st}$, and about $75^{\circ} \mathrm{F}$ by July 1st. The term "about" is used as the temperatures may vary $5^{\circ}$ in either direction, depending upon the weather conditions. During July and August the rate of increase diminishes, but temperatures continue to rise until about the third or fourth week of August when a maximum is reached. During July and the early part of August the water temperatures at the surface are about $80^{\circ} \mathrm{F}$ and then rise to a maximum of about $85^{\circ} \mathrm{F}$ during the latter part of August. Once the peak is passed, the water begins to cool rapidly, dropping to about $65^{\circ} \mathrm{F}$ by October 1st, about $55^{\circ} \mathrm{F}$ by November $1 \mathrm{st}$, about $40^{\circ} \mathrm{F}$ by December $1 \mathrm{st}$, and about $33^{\circ} \mathrm{F}$ by December 15 th. The lakes usually freeze over during mid-December each year and there is little change from that time until the ice leaves the next spring.

\section{Temperature Profiles}

Although lake temperature profiles were made as early as 1875 it was not until the exhaustive studies of Evermann and Clark (1920) that data in sufficient detail were collected to trace the temperature changes that take place from day to day in the depths of a lake.

On July 18,1899 , a regular observation station for temperature profiles was established at the deepest place in Maxinkuckee Lake, known as the Deep Hole, where the water had a maximum depth of 89 feet at the ordinary stage of the lake. Temperature profile readings were taken daily from July 18 to September 30, 1899, and from two to three times a week from July 17, 1900 to June 28,1901 . Readings were taken at the lake surface and usually at 5-foot intervals from the surface to the bottom.

The equipment used to obtain these observations is described by Evermann and Clark (1920, p. 197) as follows: "From July 18 to August 14, 1899, a self-registering Negretti-Zâmba deep-sea thermometer was used. From August 15 to September 21, a Ritchie Thermophone was used, with not wholly satisfactory results, after which the deep-sea thermometer was again used." 
Although these observations were made more than 50 year ago they still furnish one of the most complete sets of data upon which a theory for lake-temperature phenomena may be based.

Starting with the basic fact that water reaches its maximum density at a temperature of $39.2^{\circ} \mathrm{F}$ and that at lower or higher temperatures it will be less dense and therefore weigh less per cubic foot, it may be concluded that when waters of different densities come in contact with each other the heavier and more dense water will descend and the lighter or less dense water will rise.

There is only a small range of temperature between $32^{\circ}$ and $39.2^{\circ} \mathrm{F}$ where colder unfrozen water will rise above warmer water. Above $39.2^{\circ} \mathrm{F}$ there is a range of about $50^{\circ} \mathrm{F}$ in temperature through which the water becomes progressively lighter in Indiana lakes. The density-temperature graph (fig. 2) shows that through

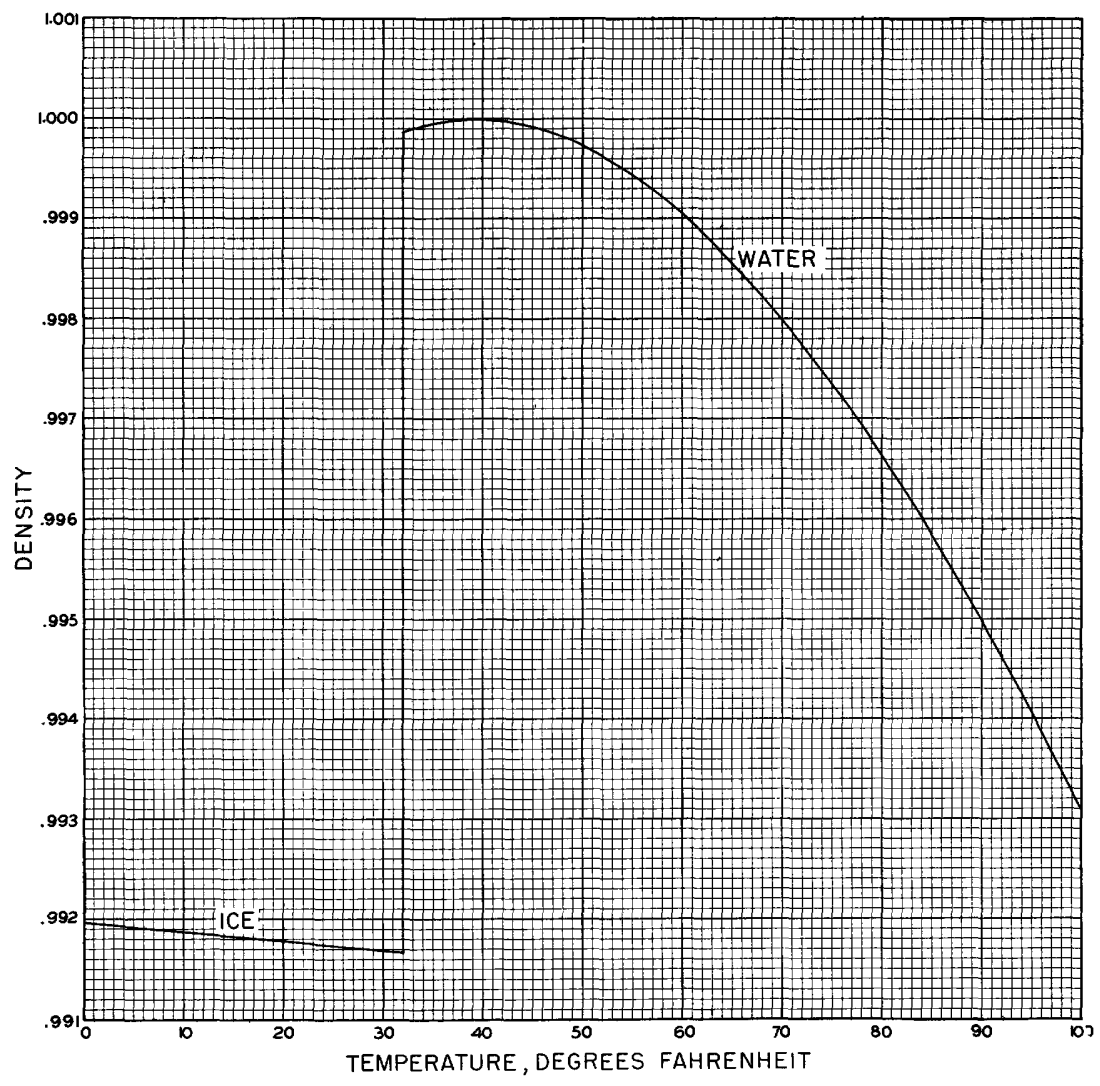

Figure 2. --Water temperature-density relationship. 
the range of $37^{\circ}$ to $42^{\circ} \mathrm{F}$ the variation from the maximum density is very slight. Beyond this range the density decreases with increasing rapidity. For example, the difference in the densities of water at $80^{\circ}$ and $85^{\circ} \mathrm{F}$ is more than four times as great as at $45^{\circ} \mathrm{F}$ and at $50^{\circ} \mathrm{F}$, and more than six times as great as at $42^{\circ} \mathrm{F}$ and at $47^{\circ} \mathrm{F}$. The practical implication of this phenomenon is that there will be over six times as much resistance for water at $85^{\circ} \mathrm{F}$ to mix with water at $80^{\circ} \mathrm{F}$ as there will be for water at $47^{\circ} \mathrm{F}$ to $\mathrm{mix}$ with water at $42^{\circ} \mathrm{F}$. It also means that at the higher temperatures there will be more stratification of water, or in other words, water of different temperatures existing in separate layers.

Water is a poor conductor of heat; so poor in fact, that so far as lake temperatures are concerned the amount of heat that one layer of water will absorb from another layer with which it is in contact is negligible. Most of the heat in the water is transferred by convection - that is, by water of one temperature moving through water of another temperature.

The principal gain or loss in heat of the whole body of water in a lake is largely due to absorption of solar radiation or radiation from the lake surface and to the energy used for evaporation. There is also some gain or loss of heat by contact of the water with the bed of the lake, and by the sensible heat carried into or away from the lake by the inflowing and outflowing water.

\section{Theory of Temperature Changes Below the Surface}

With the foregoing principles in mind, it is possible to analyze the processes that take place in changing water temperatures within the body of a lake. Starting at a time when the entire lake is at a temperature of $39.2^{\circ} \mathrm{F}$, the point of maximum density, which occurs shortly after the ice leaves, progressive changes that occur in the spring, summer, fall and winter months may be followed and analyzed in a logical manner.

From figure 3 it is evident that the temperature of the air in contact with the surface of the lake varies considerably from day to day and that the temperature of the water at the surface of the lake follows the trend of the air temperatures. In the spring months the general tendency is for the air temperatures to gradually increase. As the surface of the lake warms in following this trend, the warm water remains at the surface provided the weather is calm, but if the weather is windy the warm surface water mixes with the water immediately below, thus deepening the layer of warmer water to the depth of wave disturbance. 


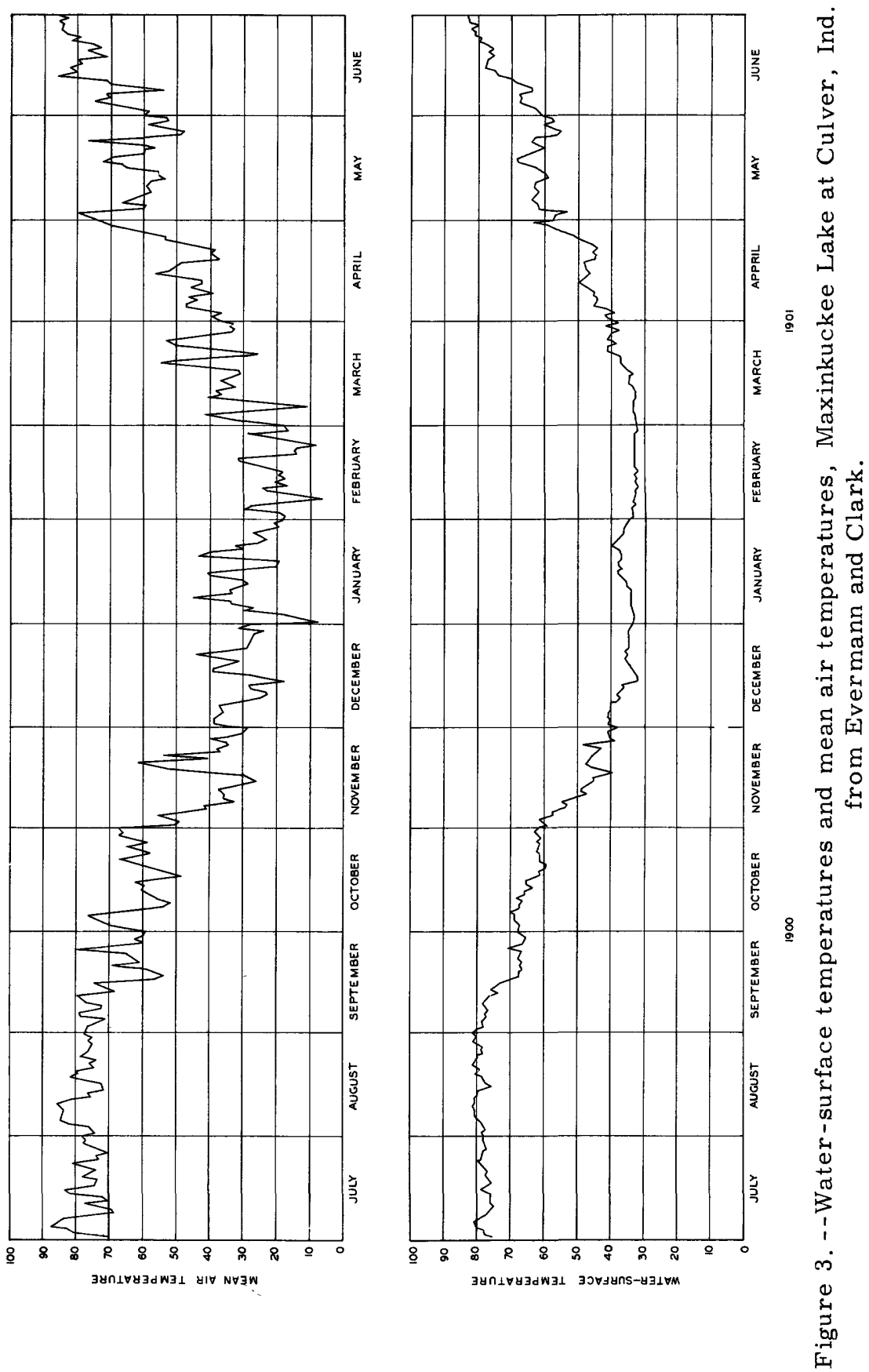


Wind, undoubtedly, plays an important part in creating a uniform temperature in the upper layers of lake water. This may be particularly true of the larger lakes where the wind has sufficient sweep to create sizable waves. The depth to which wind action affects water temperatures in relatively small lakes is still uncertain, but the data available on air and water temperatures tend to indicate that such effects are far less significant than those produced by the convection currents in the water.

There is very little variation in the density of water in the temperature range $37^{\circ}$ to $42^{\circ} \mathrm{F}$. Consequently, as the surface temper atures increase slightly above $39.2^{\circ} \mathrm{F}$, there is very little resistance to water of different temperatures mixing and the least disturbance tends to mix the whole body of water and raise its temperature uniformly. As the water temperatures rise beyond $42^{\circ} \mathrm{F}$ more resistance to mixing develops and stratification begins to take place. At this point the resistance to mixing is still not too great and water at the lower depth will continue to warm slowly for another $2^{\circ}$ or $3^{\circ} \mathrm{F}$, but at the same time the surface temperature will increase at a more rapid rate and thereby increase the differential between surface temperature and temperatures at lower depths.

If a situation could exist in which the air temperature would continue to gradually increase without the usual daily fluctuations and the weather would continue calm, a layer of warm water of relatively shallow depth would form on the surface with a gradation to the $42^{\circ}$ to $45^{\circ} \mathrm{F}$ water below. But as the air temperature does fluctuate there will be periods when it will be cooling the water surface. As the water cools it becomes heavier and descends until it reaches its own temperature level displacing the lighter and warmer water. In this process the warmer water is cooled somewhat by the passage of the colder water through it and a vertical uniform temperature zone is developed in the upper layer of water. During the early stages of this process, while the surface temperatures are below $50^{\circ} \mathrm{F}$, solar radiation or insolation plays an important part in shaping the events that follow.

In clear water "the sun's rays penetrate to considerable depths so that their energy is distributed throughout a large mass, with a consequent slow rise in temperature. About one-tenth of the insolation incident at the water's surface reaches a depth of 30 feet, and small amounts penetrate much deeper. ***In quiet water daily temperature changes are felt at least 20 feet below the surface." (Trewartha, 1943). Consequently, during the early stages when the variation in density per degree of temperature is low and the resistance to mixing is slight a gradation in the temperatures $f$ :om $45^{\circ}$ to $50^{\circ} \mathrm{F}$ takes place through a depth of about 20 feet. 
The surface temperatures of northern Indiana lakes reach $50^{\circ}$ during the latter part of April or about the first of May. At that time of year the air temperatures do not drop much below $45^{\circ} \mathrm{F}$. Consequently, during the cooler spells the surface water is not cooled enough to descendbelow a depth of about 20 feet where the water remains at a temperature of $45^{\circ}$ to $46^{\circ} \mathrm{F}$. As the process of heating and cooling goes on three distinct temperature zones are developed. The first, a rather uniform warm zone, extends down from the surface to the second zone in which the temperature changes quite rapidly, and finally the third, a colder zone, that reaches to the bottom. These zones are called the epilimnion, thermocline, and hypolimnion respectively (see fig. 4).

When the epilimnion temperature graph is vertical, that is, when a uniform temperature exists throughout this stratum, water that has been cooled at the surface descends rapidly to the thermocline. As this water reaches its own temperature level it has sufficient momentum to slightly penetrate or dig into the stratum of colder water below. This disturbance mixes warmer water with colder water and raises the temperature of the next layer slightly and thus increases the depth of the epilimnion and gradually moves the thermocline to lower levels. During the warming stages of the lake the thermocline descends rather slowly and the epilimnion will not extend deeper than to about the 20 foot depth, because the portion of the day devoted to cooling the water is less than that devoted to warming the water and the descending cold water has not been cooled enough to penetrate deeper than the upper part of the thermocline.

After the water temperature peak has been passed, in the latter part of August, the portion of each day during which the water is cooled exceeds the warming period and the thermally induced cur rents in the water are working longer hours. The temperature of the epilimnion decreases rapidly and extends to deeper depths as the currents eat into the thermocline and push it toward the bottom. This process continues until the epilimnion extends from top to bottom in the lake. As cooling goes on the water remains at a uniform temperature throughout its depth until it has cooled to about $37^{\circ}$ or $38^{\circ} \mathrm{F}$. Any further cooling will lower the surface temperatures and produce a reversed thermocline in the upper layers.

At the time ice forms, the water below the surface will be at very nearly $35^{\circ}$ or $36^{\circ} \mathrm{F}$ throughout its depth except for the last few feet at the bottom which will approach $39.2^{\circ} \mathrm{F}$. This uniform temperature is possible because of the slight difference in density of water at $36^{\circ} \mathrm{F}$ and $39.2^{\circ} \mathrm{F}$ and the lack of resistence to mixing. After the ice cover has formed, the water in contact with the ice will be only slightly above $32^{\circ} \mathrm{F}$. The effect of air temperatures on the water is then very slight since the water is protected by the ice blanket. 


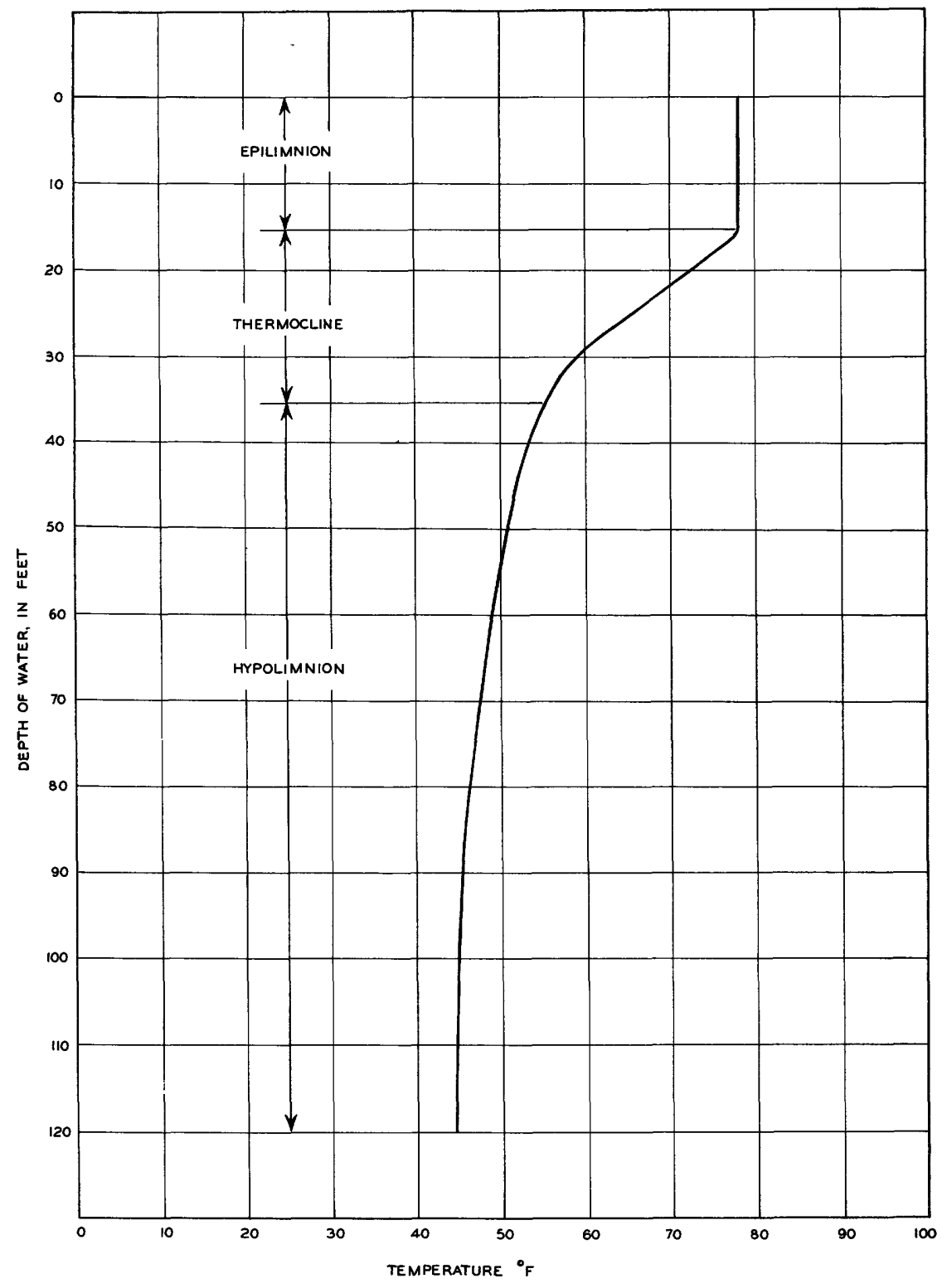

Figure 4. --Typical temperature profile graph with temperature zones indicated. 
During the period of ice cover, ground temperatures have more effect on the temperature of the water than air temperatures. Ground temperatures some distance below the ground surface remain at approximately $52^{\circ} \mathrm{F}$ in the lake region of northern Indiana. The ground in contact with the water is somewhat colder than $52^{\circ} \mathrm{F}$ during the winter months because it is cooled by the water. In cooling the ground, the water picks up heat which gradually raises the temperature of the lake through the winter until a temperature of about $39^{\circ} \mathrm{F}$ is reached. Heating above this point is prevented because the warm water rises to the top where it is immediately cooled by the ice and any excess temperature is taken up in melting the ice. From this point on there is practically no change in lake temperatures until the ice melts.

The temperature profiles taken in Maxinkuckee Lake during 1900 and 1901 (Evermann and Clark, 1920) offer a splendid oppor tunity to follow day to day changes in water temperature and to observe how the theory of temperature change, as describec' above, really works. From these observations, 30 graphs (figs. 5-9) have been prepared for selected days to show the progress of temperature changes throughout the year. The graphs are considered to be typical of those that might occur at approximately the same time in any year.

The normal temperature cycle extends from the breakup of ice, usually about March 15, in one year until the breakup the following year. Unfortunately, the temperature profiles of 1900 and 1901 do not coincide with the cycle as described, but begin at another point on the cycle. For illustrative purposes the sequence of observations have been changed to coincide with the pattern of the normal temperature cycle. The observations for the period March 26, 1901, (the day after the ice disappeared) to June 30, 1901 (figs. 5 and 6), have been arranged ahead of the observations for the period July 17, 1900 to March 14, 1901 (figs. 7-9).

Figure 5, A shows that a uniform temperature of $40^{\circ} \mathrm{F}$ existed from the surface to the bottom of the lake on March 26 the day after the ice disappeared. This follows the theory that the entire body of water will be very near the temperature of maximum density, $39.2^{\circ} \mathrm{F}$, at the time of the breakup of ice in the spring.

By April 19 (fig. 5, B) the entire body except for that in contact with the ground, has warmed up about $2^{\circ} \mathrm{F}$. This indicates that free circulation took place in the $40^{\circ}$ to $42^{\circ} \mathrm{F}$ range of water temperatures that existed during the period from March 26 to April 10. In this range the variation from maximum density is small and the resistance to mixing of water of different temperatures is slight. 


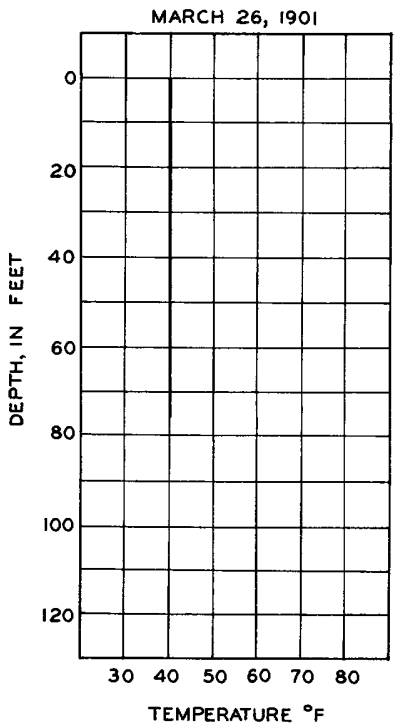

A

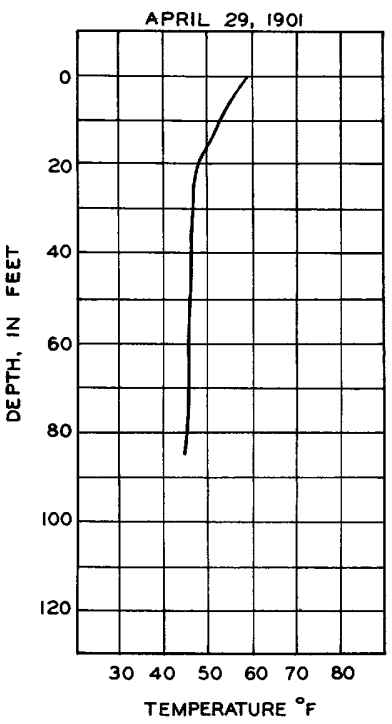

D

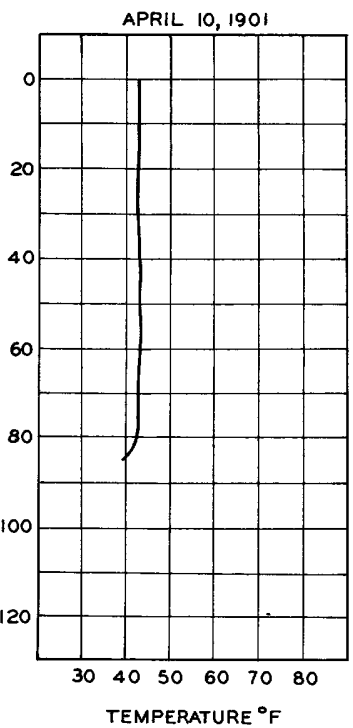

B

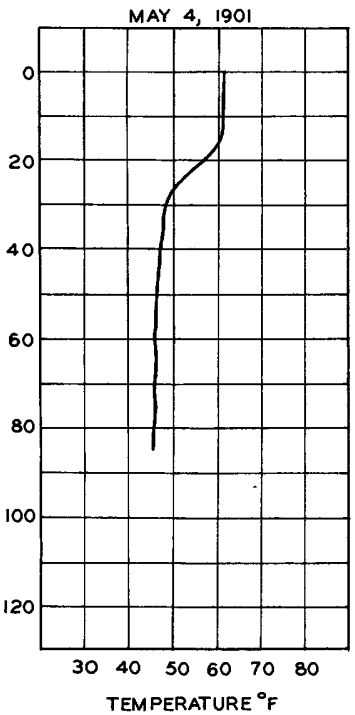

E

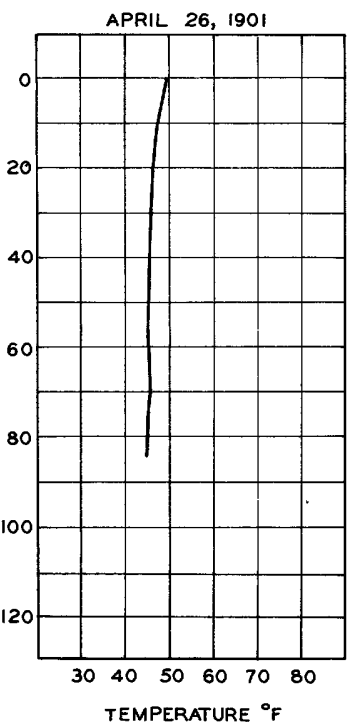

C

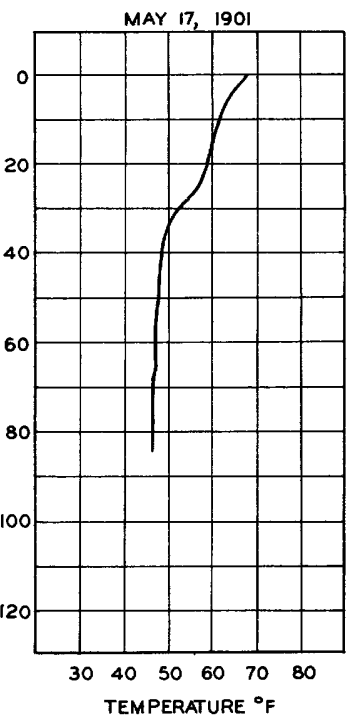

$\mathbf{F}$

Figure 5. --Temperature profiles for Maxinkuckee Lake at Culver, Ind., at intervals for March to May 1901. Data from Evermann and Clark. 
On April 26 (fig. 5, C) the water surface had warmed enough so that resistance to mixing of water of different temperatures was noticeable and a thermocline started to develop. By April $2 \Omega$ (fig. 5, D) this thermocline extended to a depth of 20 feet, a deptr significant in that for all the deeper lakes in northern Indiana, it has been found to be the depth to which the thermocline descends in the early stages of its development and the depth at which the epilimnion ends during the warming period. The depth is believed to be controlled by the depth to which the sun's rays are able to penetrate with sufficient energy to warm the water. The thermocline for April 29 also indicates that gradual warming of the water was continuing after April 26 with no cooling periods to develop an epilimnion. This is also borne out by the minimum and maximum daily recorded air temperatures which gradually increased from a minimum of $50^{\circ} \mathrm{F}$ on April 25 to a maximum of $85^{\circ} \mathrm{F}$ or April 30, 1901, without the minimum air temperatures ever falling below the minimum observed temperature of the water. On May 3 and 4,1901 , the minimum daily air temperature fell to $52^{\circ} \mathrm{F}$ and $49^{\circ} \mathrm{F}$ respectively. These air temperatures developed an epilimnion and caused the thermocline to descend as shown in figure. 5 E.

Air temperatures registered in the fifties from May 4 to 15, with a minimum of $43^{\circ} \mathrm{F}$ occurring on May 15. This period was followed by rapidly warming weather with the air temperature rising to $84^{\circ} \mathrm{F}$ on May 17,1901 . The water-temperature grapl for May 17 (fig. $5 \mathrm{~F}$ ) shows that the cold weather during the period May 4-17 forced the thermocline to descend deeper, and the warm weather at the end of the period caused a secondary thermocline to start forming in the upper layers. Temperature profiles for other dates during this period confirm the depressing of the thermocline during cooling periods.

Cooling weather between May 17 and 24 caused a vertical epilimnion (fig. $6 \mathrm{~A}$ ) to form again, the secondary thermocline to be deepened, and the primary thermocline to be shortened greatly.

Variable air temperatures, with minimums as low as $43^{\circ} \mathrm{F}$ between May 24 and June 6, caused the primary thermocline (fig. 6 B) to descend to nearly the 60-foot depth, with secondary and tertiary thermoclines slightly in evidence at about the 38-and 20foot depths. These thermoclines merged to form an essentially new elongate major thermocline.

Warm weather between June 6 and June 28 gradually increased temperatures at the surface and in the upper layers, but made onl" very slight changes in water temperatures below a depth of 30 feet. The graphs for this period are shown in figures $6 \underline{\mathrm{C}}-\underline{\mathrm{F}}$. 


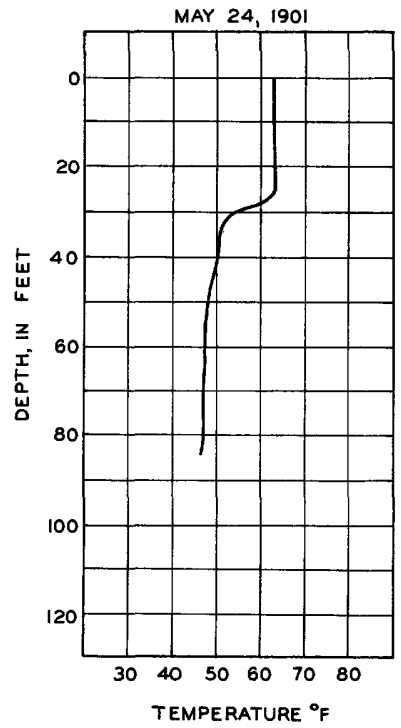

A

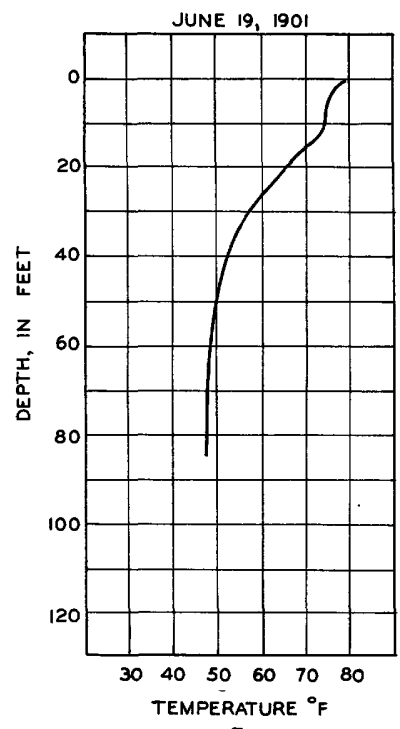

D

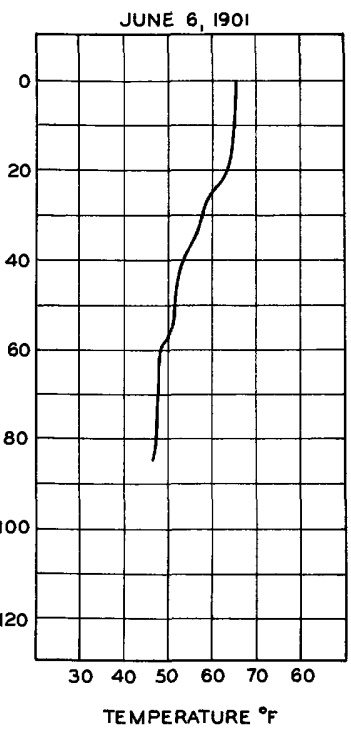

B

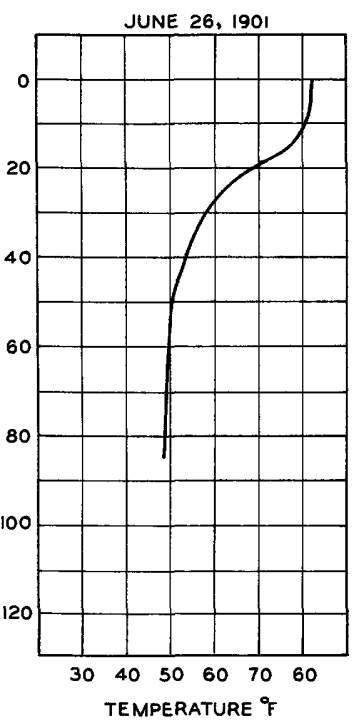

E.
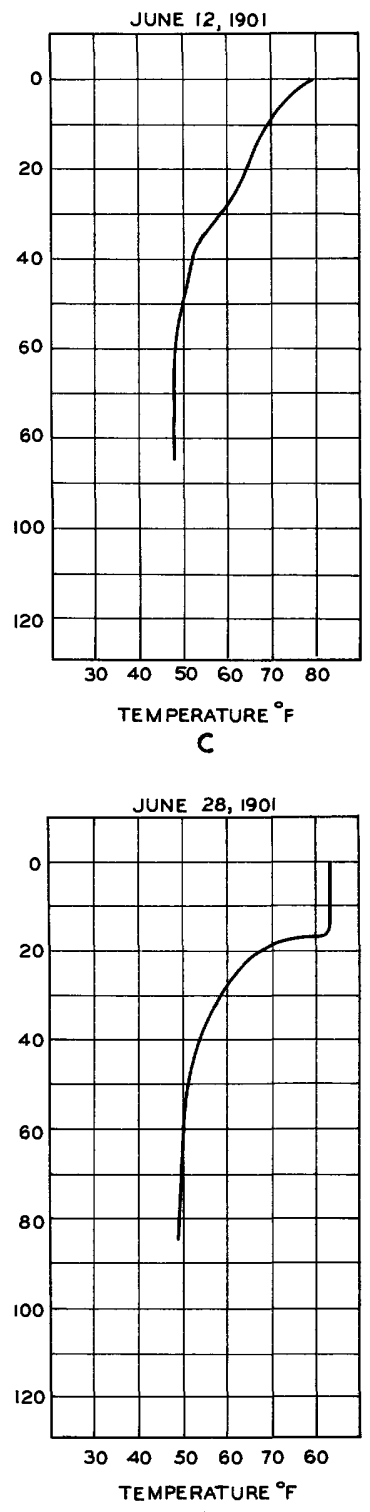

F

Figure 6. --Temperature profiles, for Maxinkuckee Lake at Culver, Ind., at intervals for May to June 1901. Data from Evermann and Clark. 
The graphs in figures $7 \mathrm{~A}-\mathrm{D}$ cover the period of gradual warming of the upper layers of the lake until the maximum amount of heat is reached about the third week of August. Until this time, the lake has been warming since the breakup of ice and all the temperature-profile graphs have been typical of the warming period.

The graph for September 5, 1900 (fig. 7 E), is representative of the first cooling period. The epilimnion has retreated and the upper part of the thermocline descended slightly.

By September 22 (fig. $7 \underline{\mathrm{F}}$ ), the epilimnion has cooled $11^{\circ}$ or $12^{\circ} \mathrm{F}$ and is vertical for a depth of 40 feet. The thermocline has descended to nearly the 50-foot depth and is much shortened. For the first time during the year, water of over $60^{\circ} \mathrm{F}$ temperature has penetrated below a depth of 40 feet.

By October 17 (fig. $8 \mathrm{~A}$ ), the thermocline is down to a depth of about 55 feet, $60^{\circ} \mathrm{F}$ wate $\bar{r}$ down to the 52 -foot depth, and the epilimnion cooled to $63^{\circ} \mathrm{F}$.

The thermocline dropped to a depth of 65 feet by November 2, (fig. $8 \mathrm{~B}$ ), and the epilimnion with water $60^{\circ} \mathrm{F}$ descended to 60 feet.

By November 9 (fig. $8 \mathrm{C}$ ), the thermocline had disappeared entirely and only the epilimnion remained from surface to bottom. a nearly uniform temperature existed throughout the entire body of water.

A point of particular interest is that the epilimnion did not penetrate below 25 feet from March 26, when the ice cover disappeared, until September 5 , a period of 5 months. Then during the cooling period September 6 to November 9, a period of slightly more than 2 months, the epilimnion dropped from a depth at 20 feet to the bottom at 85 feet. This phenomenon clearly demonstrates the greater rapidity of heat penetration to the lower depths during the cooling period than during the warming period.

Once the epilimnion reaches the bottom of the lake the whole body of water cools uniformly and the temperature-profile graph remains a straight line (figs. $8 \mathrm{D}$ and $8 \mathrm{E}$ ) until the water has reached a temperature of about $40^{\circ} \mathrm{F}$.

By December 17, 1900 (fig. $8 \mathrm{~F}$ ), the lake had been almost completely frozen over several times. Water temperatures lowered past the point of maximum density and a slight temperature inclination had formed with temperatures ranging between $34^{\circ} \mathrm{F}$ at the surface and $36^{\circ} \mathrm{F}$ at the bottom. 

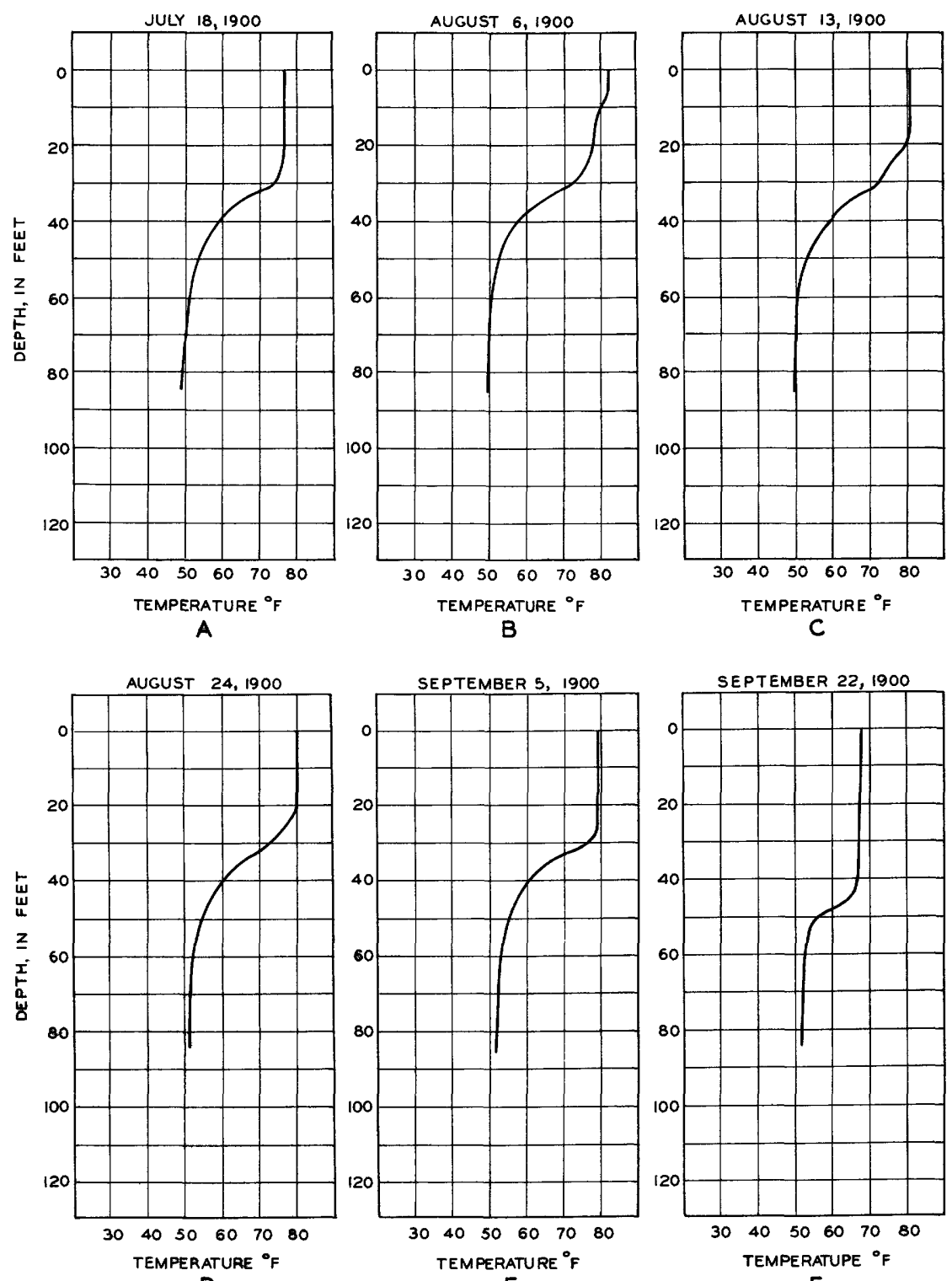

$E$

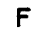

Figure 7.--Temperature profiles for Maxinkuckee Lake at Culver, Ind., at intervals for July to September 1900. Data from Evermann and Clark. 

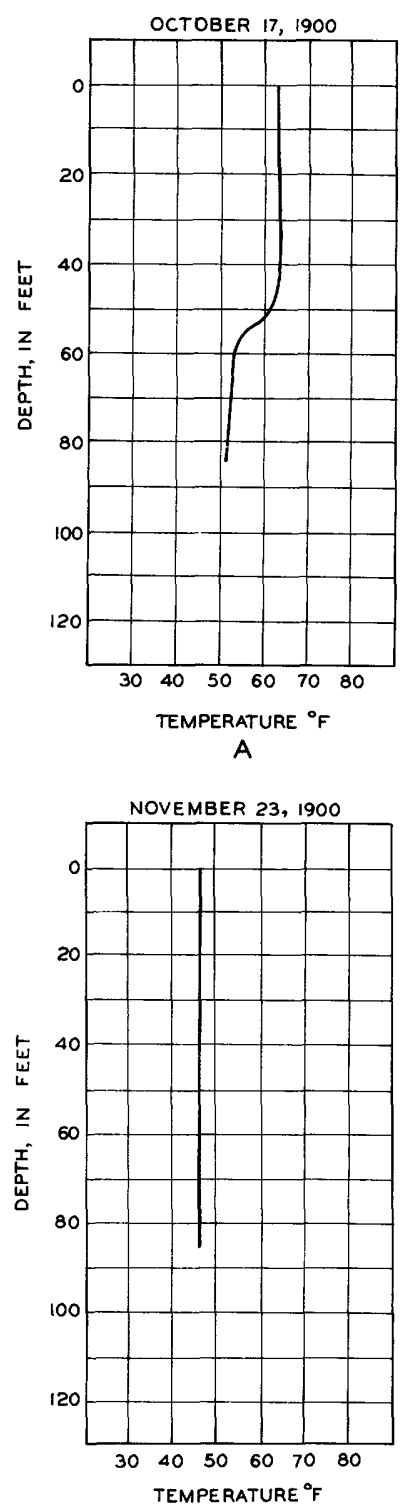

D

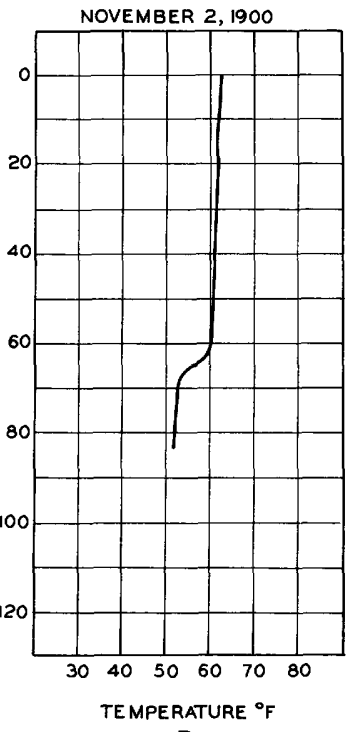

$B$

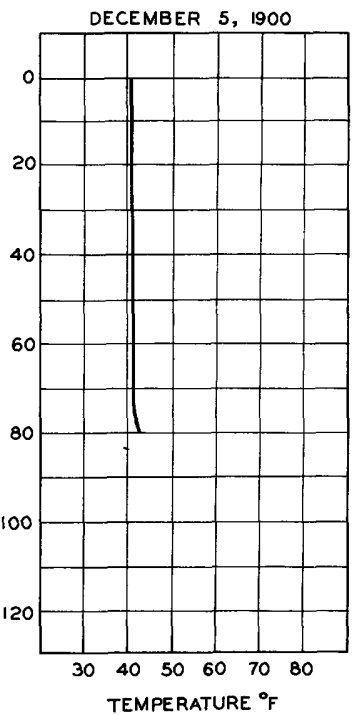

$E$

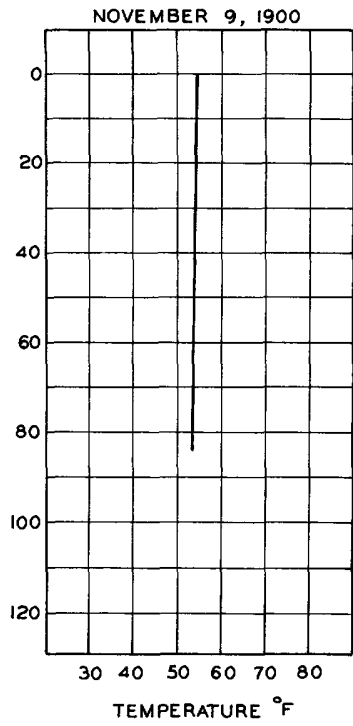

$C$

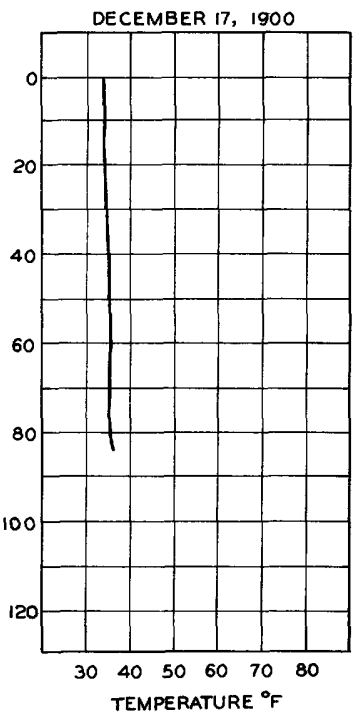

$F$

Figure 8. --Temperature profiles for Maxinkuckee Lake at Culver, Ind., at intervals for October to December 1900. Data from Evermann and Clark. 
The temperature graphs shown in figure 9 are all for the period of ice cover. During this period the lake was either completely or almost frozen over.

The graphs show practically no change in water temperatures during late December and early January as open water existed at the observation point although most of the lake was under ice cover.

By January 26, (fig. 9 B), under the influence of ice cover, a slight reversed thermocline existed, water temperature throughout most of the depth had increased slightly, and water in contact with the bottom was somewhat warmer. The graphs infigures $9 \mathrm{C}$ to $9 \mathrm{~F}$ are all typical of conditions under the ice cover. They show a gradual warming of the water through the winter with a tendency to approach a temperature of $39.2^{\circ} \mathrm{F}$.

The effect of ground temperatures upon the lower lake strata may be seen by examining some of the temperature-profile graphs. When the water temperature at the bottom of the lake is above $52^{\circ} \mathrm{F}$, the lower end of the temperature graph will incline in the direction of the $52^{\circ} \mathrm{F}$ temperature. When the water temperatures are between $39^{\circ} \mathrm{F}$ and $52^{\circ} \mathrm{F}$ there is no noticeable effect from the higher ground temperatures because any increase in water temperature will immediately cause the water so affected to rise to the surface (fig. $8 \mathrm{D}$ ). When the lake temperature is below $39^{\circ} \mathrm{F}$ the lower end of the $\overline{g r a p h}$ will again bend in the direction of $52^{\circ} \mathrm{F}$ (fig. 9 B).

In the system of heat transfer herein described it should be noted that heat is carried to the depths of the lake only when the surface is cooling and verticalcurrents move down into the cooler strata of the thermocline below. While water is being heated on the surface the vertical currents are nonexistent and heat cannot penetrate to any considerable depth. Frequently, when warming periods are rather protracted secondary thermoclines will be formed. When this happens the primary thermocline does not deepen until the secondary thermocline has descended and merged with the primary thermocline (figs. $7 \underline{B}$ to $7 \underline{E}$ ).

\section{Comparison of Temperature Profiles in Ten Indiana Lakes}

The temperature-profile observations since 1946 have been taken about once a month and therefore do not show the daily changes shown by the Evermann and Clark (1920) studies. However, as they have been made on a number of lakes they do show the effects of depth, size, and other characteristics. 

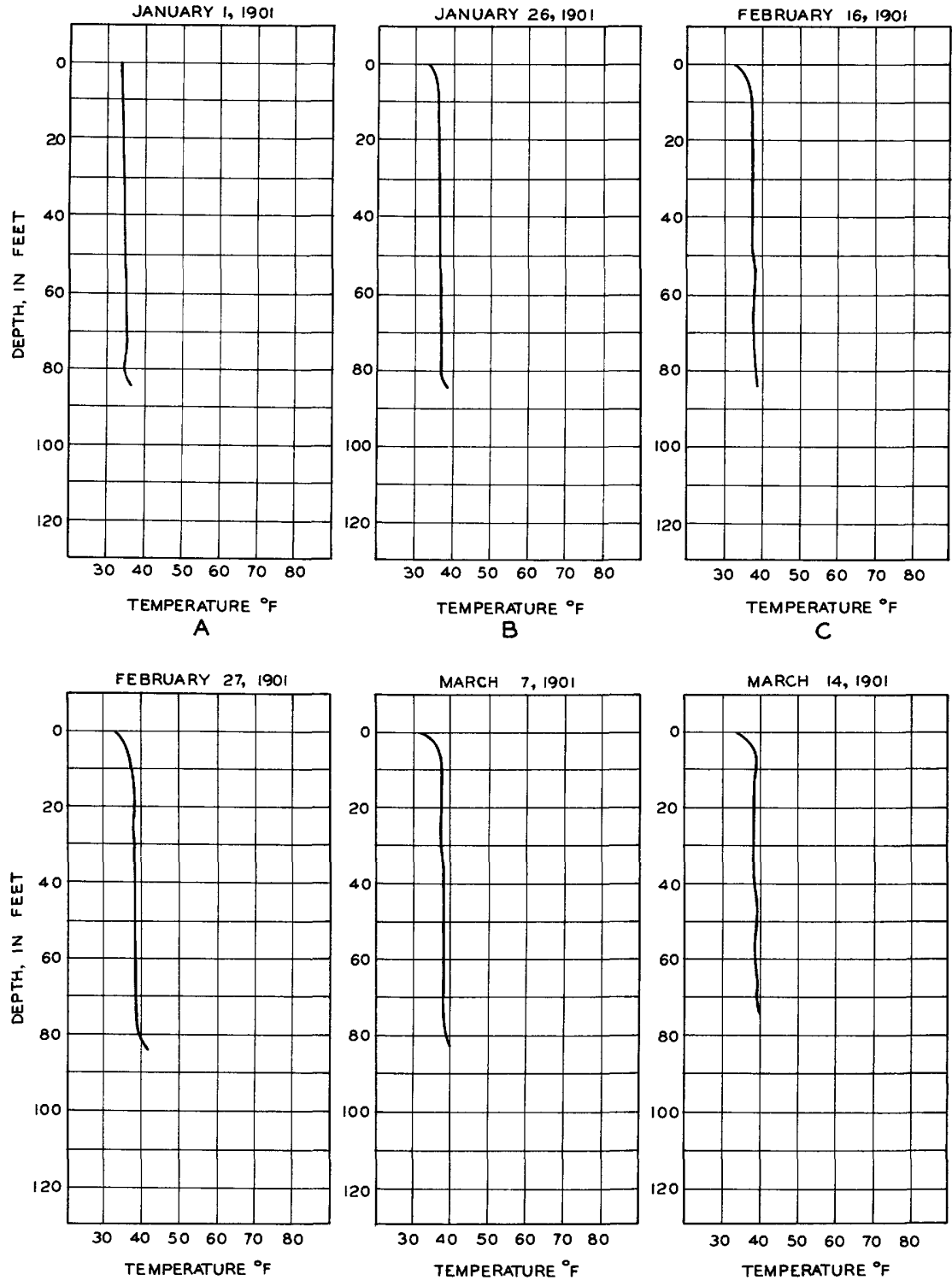

D

E

$F$

Figure 9. --Temperature profiles for Maxinkuckee Lake at Culver, Ind., at intervals for January to March 1901. Data from Evermann and Clark. 
The equipment used was a Leeds and Northrup Thermohm calibrated to give direct temperature readings. The temperature scale was graduated to half degrees, and readings to tenths of a degree were obtained by estimating between scale graduations. Surface temperatures were also read on a mercury thermometer or tube thermometer filled with red liquid, graduated in degrees from $20^{\circ}$ to $120^{\circ} \mathrm{F}$ for comparison with the Thermohm readings. Surface readings were made about 1 inch beneath the surface of the water.

Tippecanoe Lake, 117 feet deep, is the deepest lake in the State, and at the bottom has the coldest water recorded for any lake in the State. The temperature of the water at that depth does not rise above $45^{\circ} \mathrm{F}$. The upper part of the temperature graph follows the general pattern previously described. The epilimnion does not extend below 20 feet until after the peak temperatures have been reached in August (fig. 10); then it descends rapidly to about 100 feet and is obliterated because the temperatures at the surface have decreased to the same temperatures as those at the bottom.

In Maxinkuckee Lake (fig. 11) the bottom temperatures at 80 feet average about $10^{\circ} \mathrm{F}$ warmer in the summer and early fall than the temperatures at the same depth in Tippecanoe Lake. Temperatures at the surface and first 10 to 15 feet of depth are about the same as those of Tippecanoe Lake, but the thermocline is generally at a considerable lower depth.

Cedar Lake (Lake County), the shallowest lake studied, with a maximum depth of 15 feet, is $4^{\circ}$ to $5^{\circ} \mathrm{F}$ warmer in spring and $4^{\circ}$ to $5^{\circ} \mathrm{F}$ cooler in the fall than Tippecanoe and other deeper lakes. The temperature graphs for Cedar Lake (fig. 12) is about the same as the temperature graphs for the upper 15 feet of other lakes. The lake, being less than 20 feet deep, seldom develops more than one temperature zone and thus lacks a thermocline and hypolimnion. Because of the shallower depths and the smaller volume of water in proportion to surface area, the temperature changes are more rapid and pronounced than in the deeper lakes.

In the upper 10 feet, Flint Lake (fig. 13) has about the same temperature in summer as Tippecanoe Lake. However, it is much colder between the depths of 10 and 40 feet. The colder thermocline may result from the considerable quantities of ground water pumped into the lake in the summer months by the Valparaiso Water Department. At 70 feet it is $3^{\circ}$ to $4^{\circ} \mathrm{F}$ warmer than Tippecanoe at the same depth except in the winter months.

Bass Lake (fig. 14) has a large surface area and is very shallow, having an average depth of only 6 feet. Because of its shallowness it warms more rapidly in the spring and cools more 

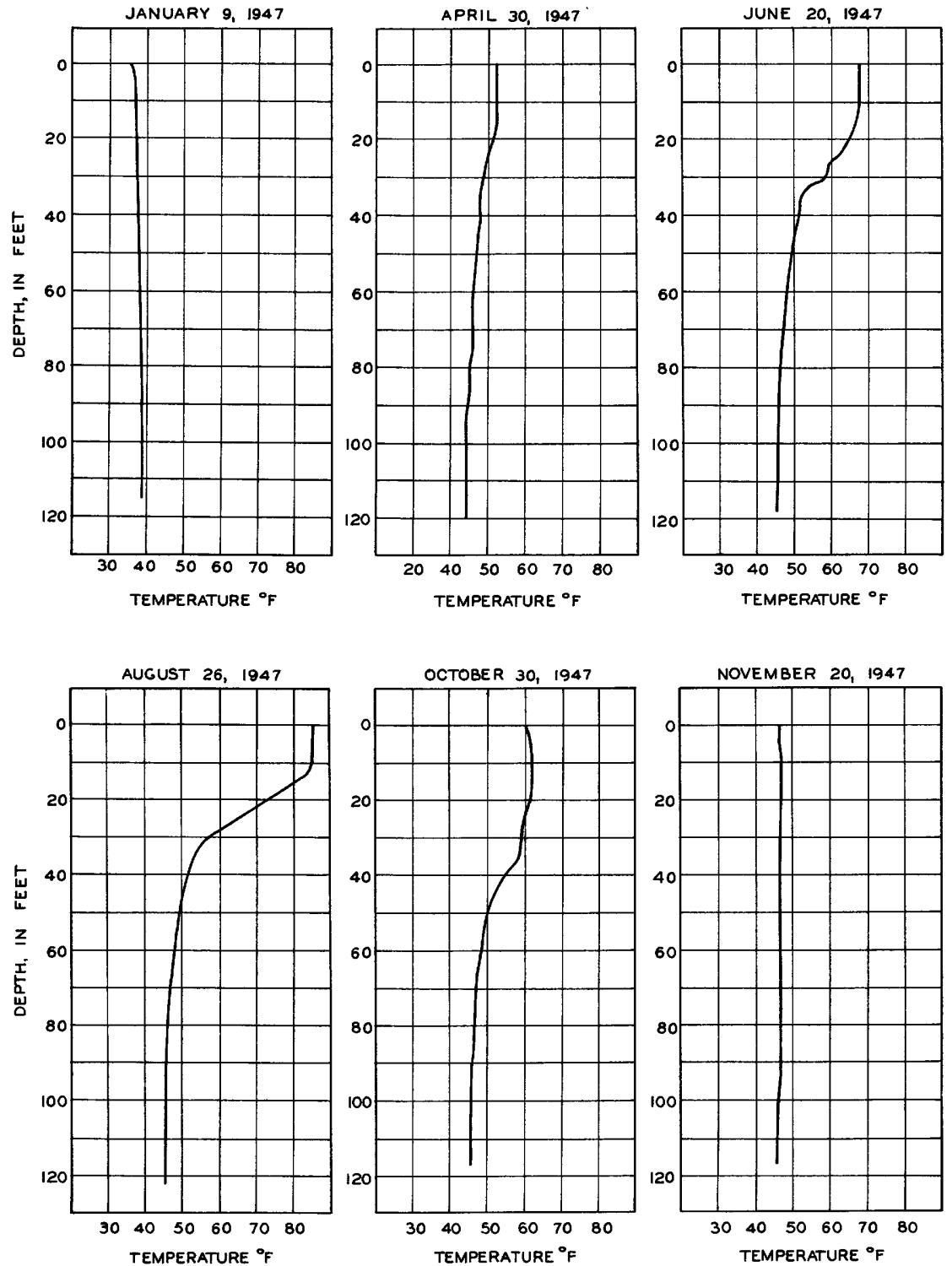

Figure 10. - Temperature profiles for Tippecanoe Lake at Oswego, Ind., at intervals for January to November 1947. 

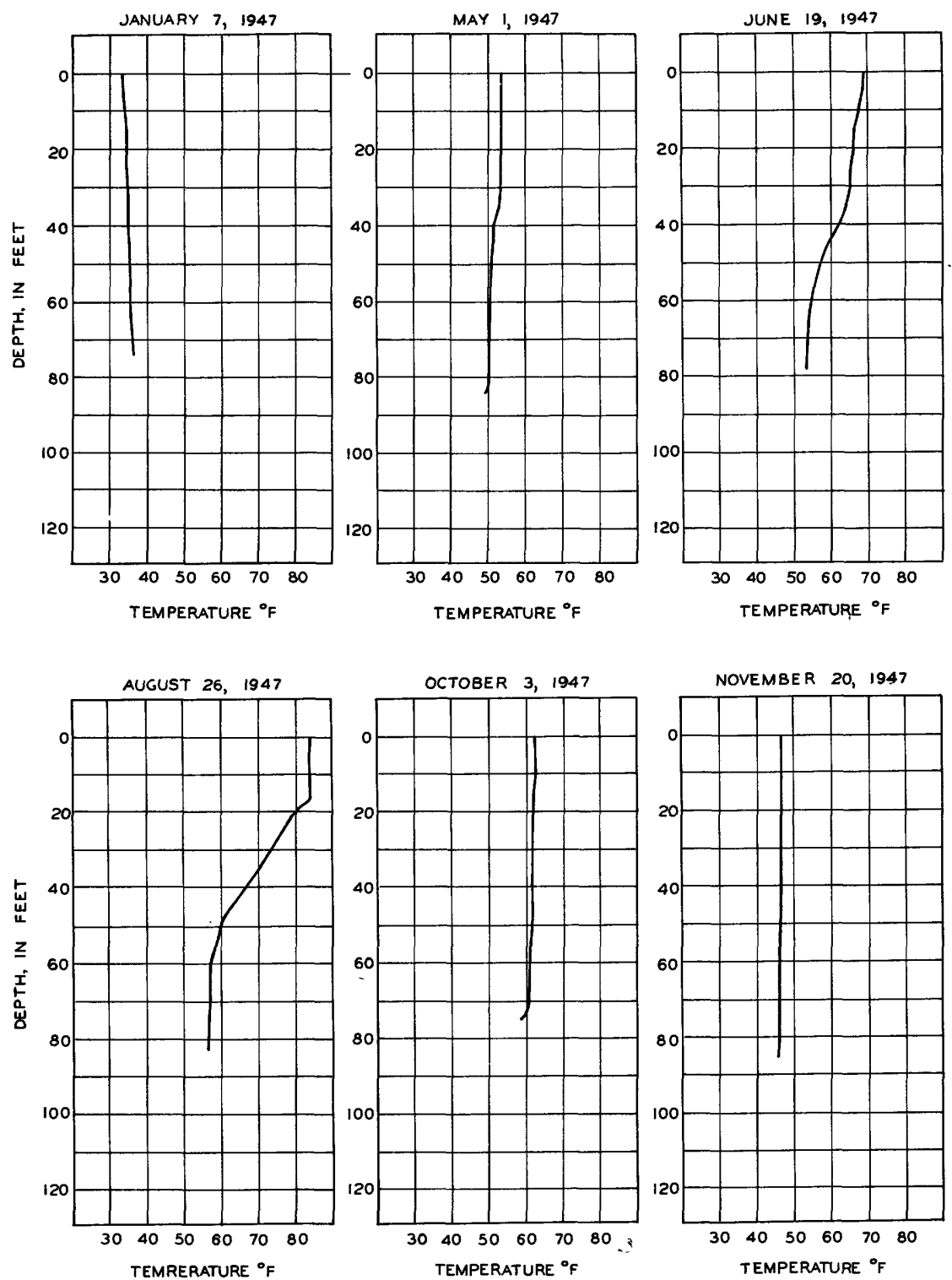

Figure 11. --Temperature profiles for Maxinkuckee Lake at Culver, Ind., at intervals for January to November 1947. 

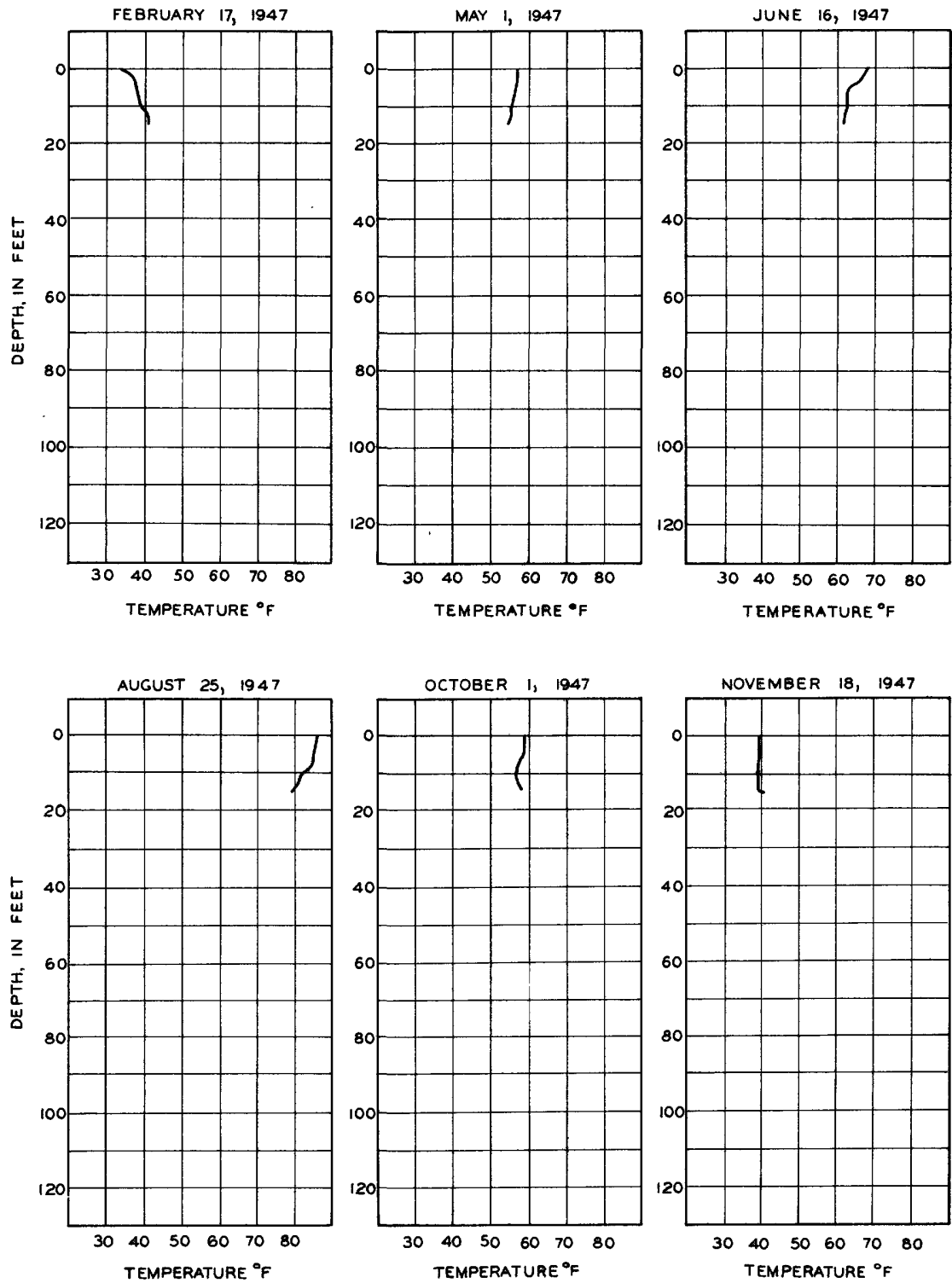

Figure 12. --Temperature profiles for Cedar Lake at Cedar Lake in Lake County, Ind. , at intervals for February to November 1947. 

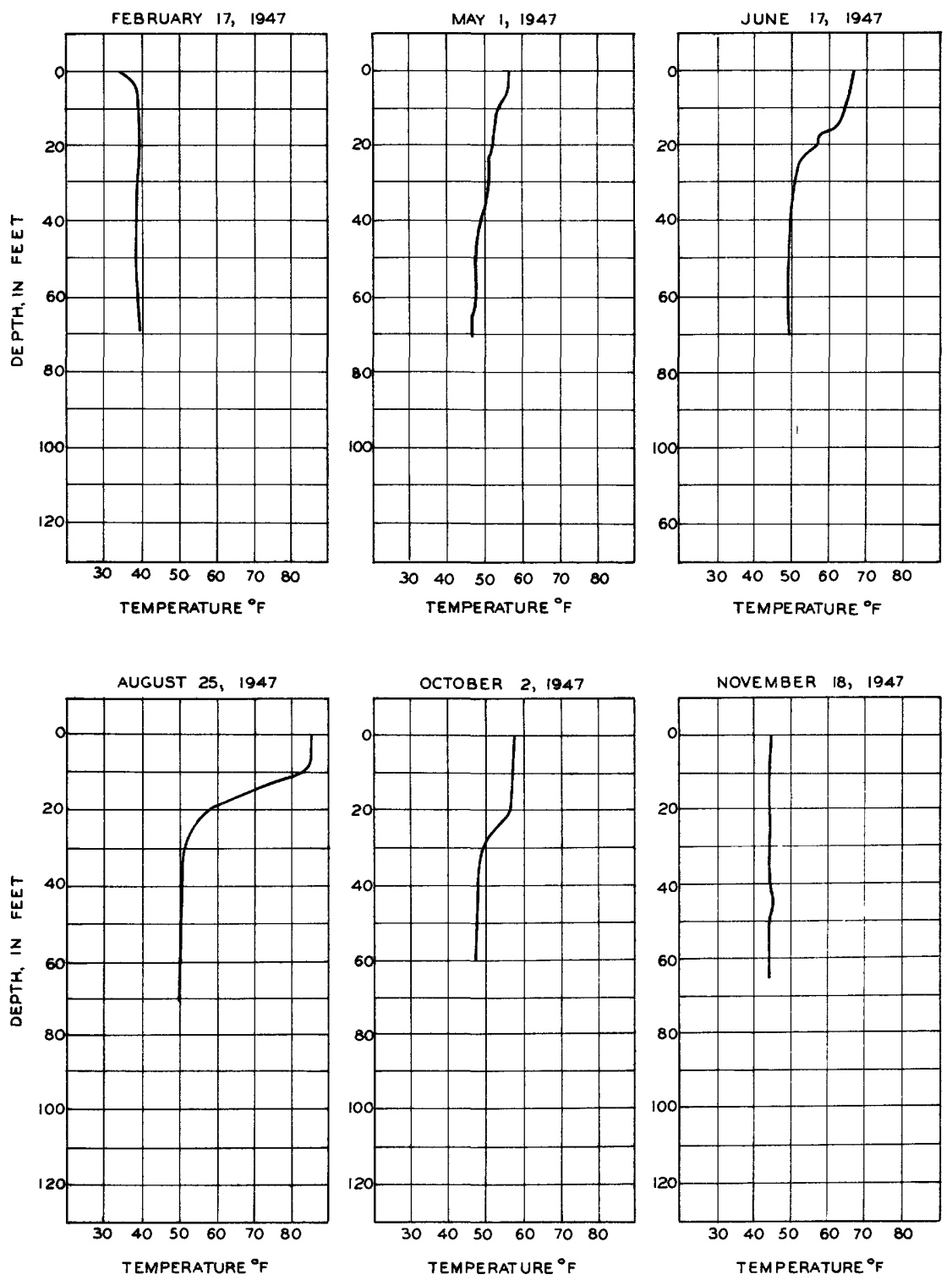

Figure 13. --Temperature profiles for Flint Lake near Valparaiso, Ind., at intervals for February to November 1947. 

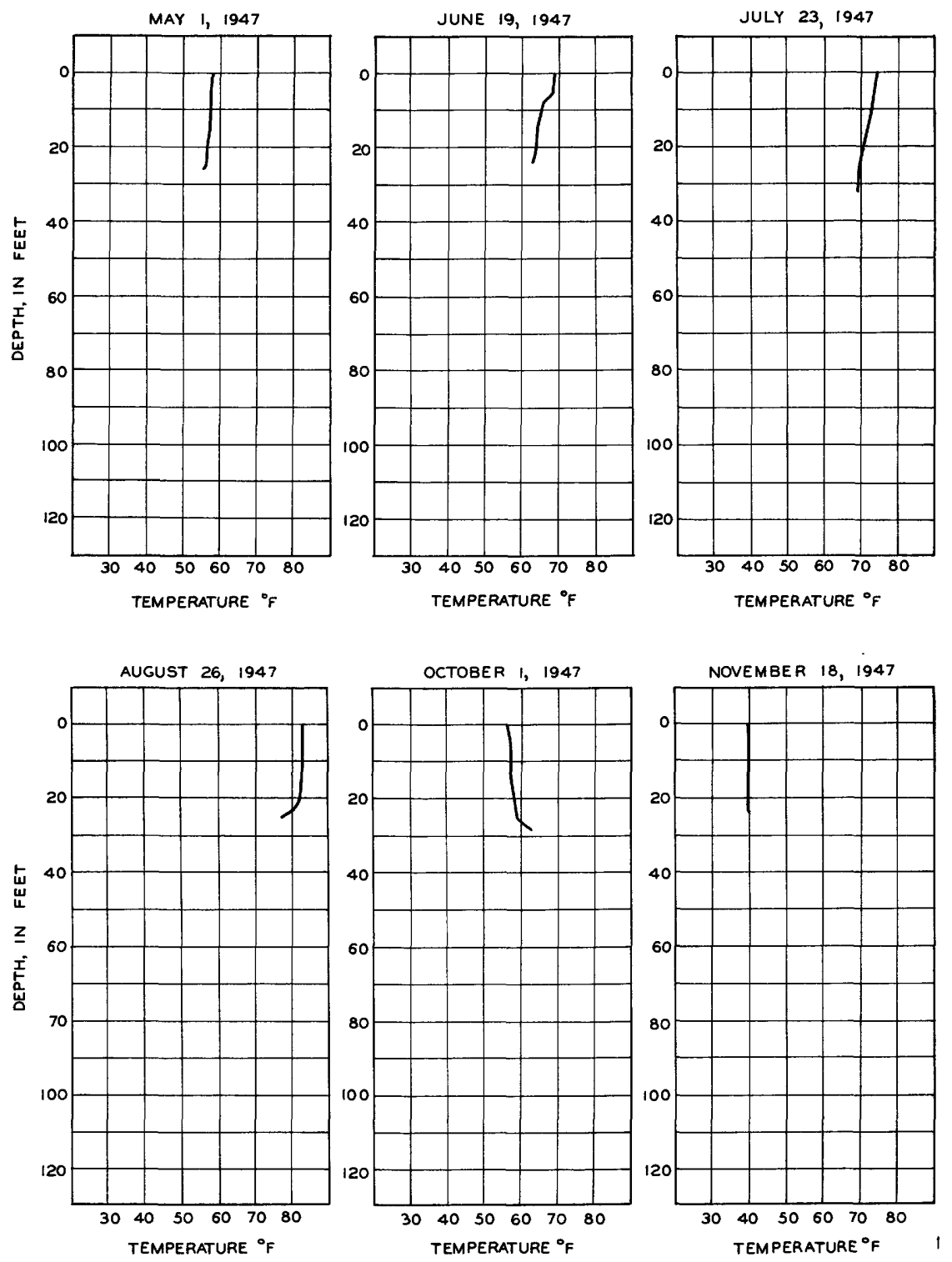

Figure 14. --Temperature profiles for Bass Lake at Bass Lake, Ind., at intervals for May to November 1947. 
quickly in the fall than the deeper lakes. The surface temperatures during the summer are about the same as for other lakes but the warm water extends to much greater depths. The absence of a thermocline during most of the year is a significant difference from most of the other lakes studies. A nearly uniform temperature from the surface to the bottom at 33 feet is common even in the summer months, possibly due to the small volume of water to be changed in temperature in proportion to surface area available to absorb or radiate heat.

The temperature graph for Winona Lake (fig. 15) closely follows that for Tippecanoe Lake to a depth of 40 feet and then shows gradually warmer temperatures as the bottom is approached. The bottom temperatures at 75 feet average about $3^{\circ} \mathrm{F}$ warmer than the temperatures at a corresponding depth in Tippecanoe Lake.

In Wawasee Lake (fig. 16) the temperatures for the first 10 to 15 feet of depth are about the same as those in Tippecanoe Lake, but lower depths are much warmer than those for corresponding depths in Tippecanoe Lake, being about $8^{\circ} \mathrm{F}$ warmer at a depth of 60 feet, the bottom. Another peculiarity is that the thermocline extends to a depth of about 55 feet during the summer months, which is much deeper than in other lakes.

The temperatures for Bixler Lake (fig. 17) are about the same as those in Tippecanoe Lake for the upper $10 \mathrm{feet}$ of depth and slightly cooler between 10 and 25 feet. At a depth of 37 feet, which is the bottom of Bixler Lake, the temperatures are about $3^{\circ} \mathrm{F}$ warmer than at the same depth in Tippecanoe Lake.

At Lake James (fig. 18) the temperatures are almost exactly the same as in Tippecanoe Lake except at the bottom, where the temperatures at 85 feet may be $1^{\circ}$ to $2^{\circ} \mathrm{F}$ colder than those at the same depth of Tippecanoe Lake. This lake is the same depth as Maxinkuckee Lake but the bottom temperatures average about $14^{\circ} \mathrm{F}$ colder than those of Maxinkuckee Lake.

Clear Lake, with a maximum depth of 105 feet, is the second deepest lake in Indiana. The temperature graph (fig. 19) generally follows that for Tippecanoe Lake, except near the surface the temperatures for Clear Lake are about $2^{\circ}$ to $3^{\circ} \mathrm{F}$ cooler and the bottom temperatures $2^{\circ}$ to $3^{\circ} \mathrm{F}$ warmer than those for corresponding depths in Tippecanoe Lake. The thermoclines for Clear Lake and Tippecanoe Lake coincide.

From this survey and study of the various lakes in northern Indiana, the author concludes that in general the lakes follow the same pattern of temperature changes with no significant differences except in the shallow lakes. 

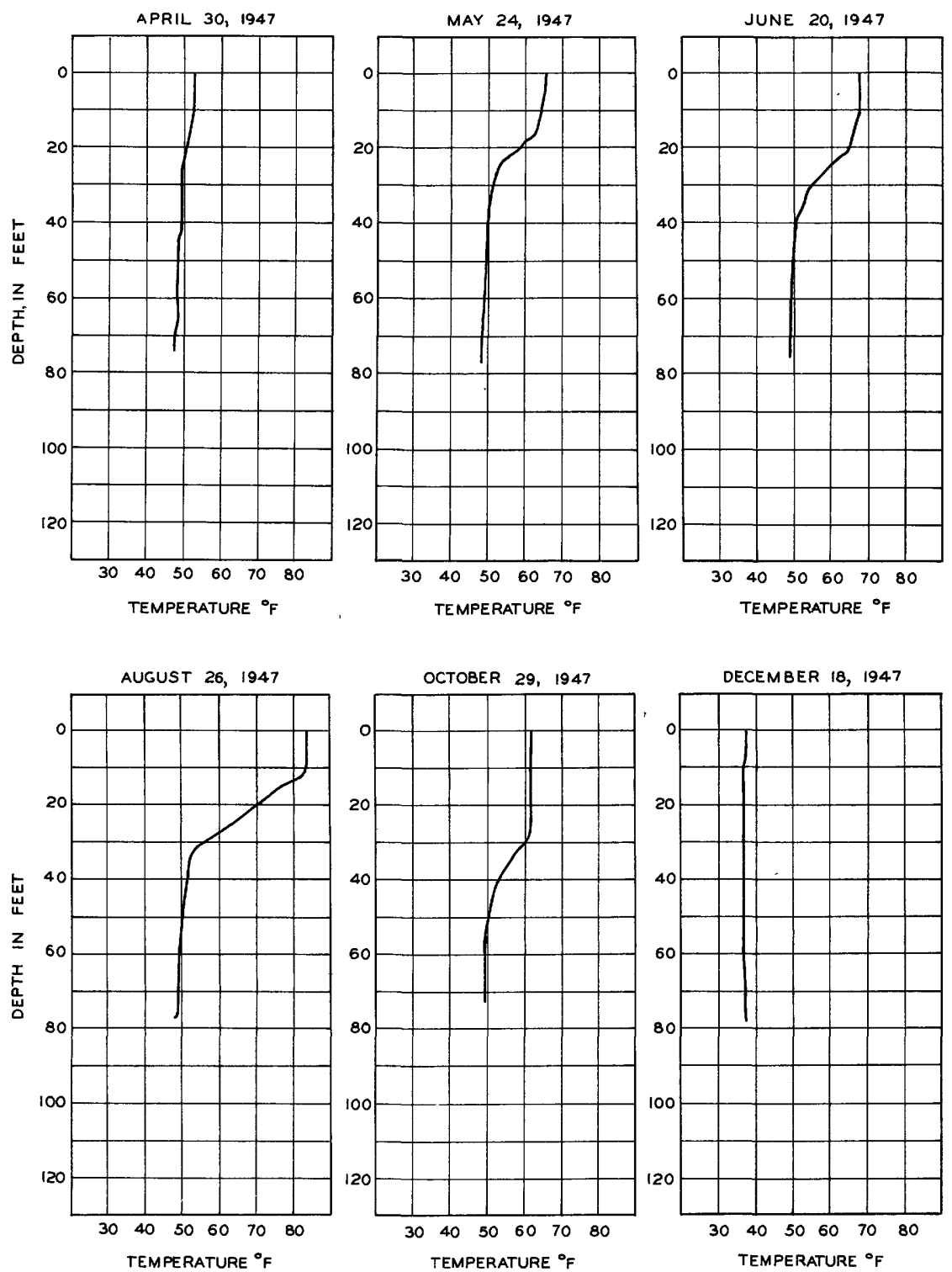

Figure 15. --Temperature profiles for Winona Lake at Warsaw, Ind., at intervals for April to December 1947. 

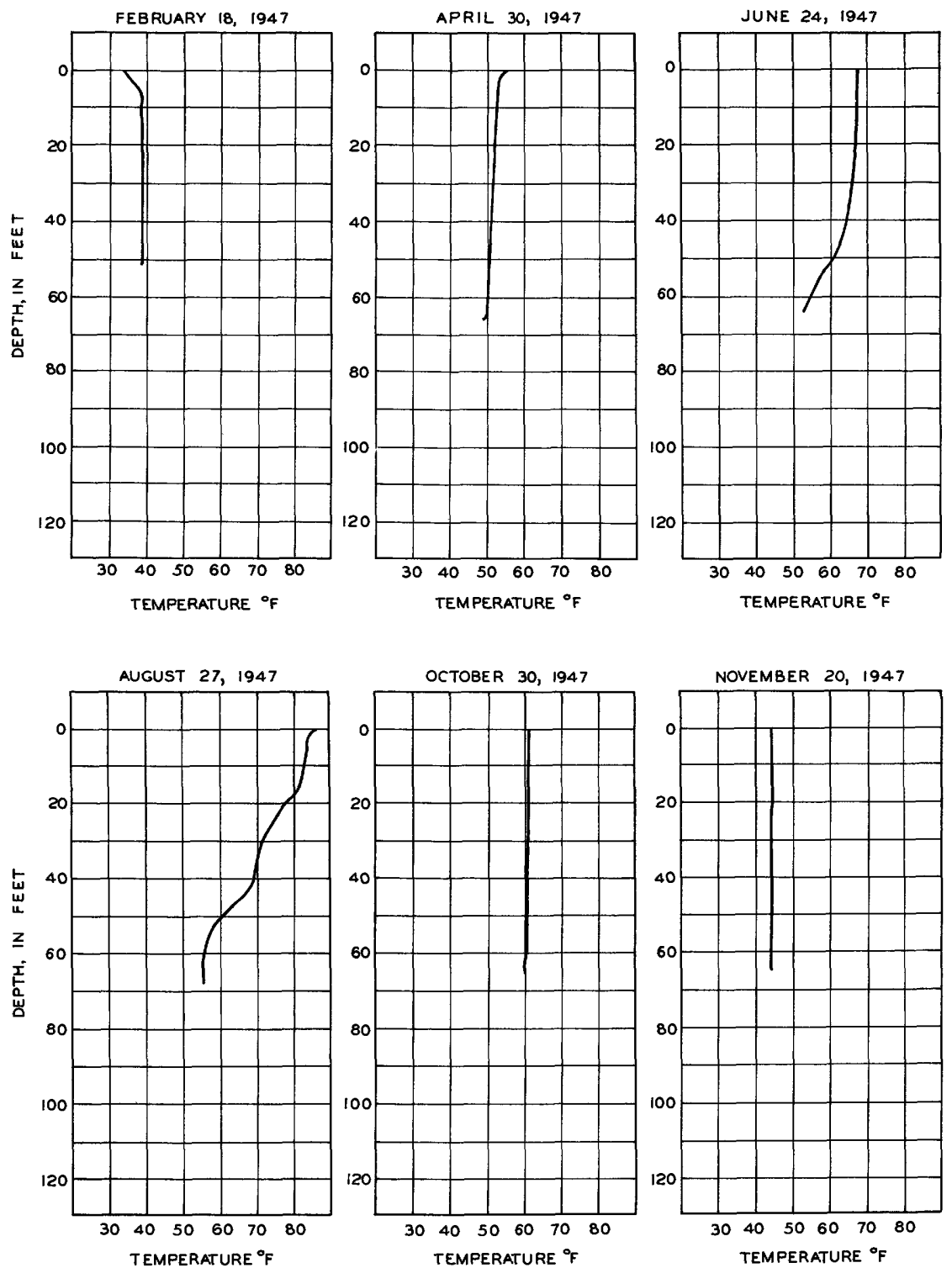

Figure 16. --Temperature profiles for Lake Wawasee near Wawasee, Ind., at intervals for February to November 1947. 

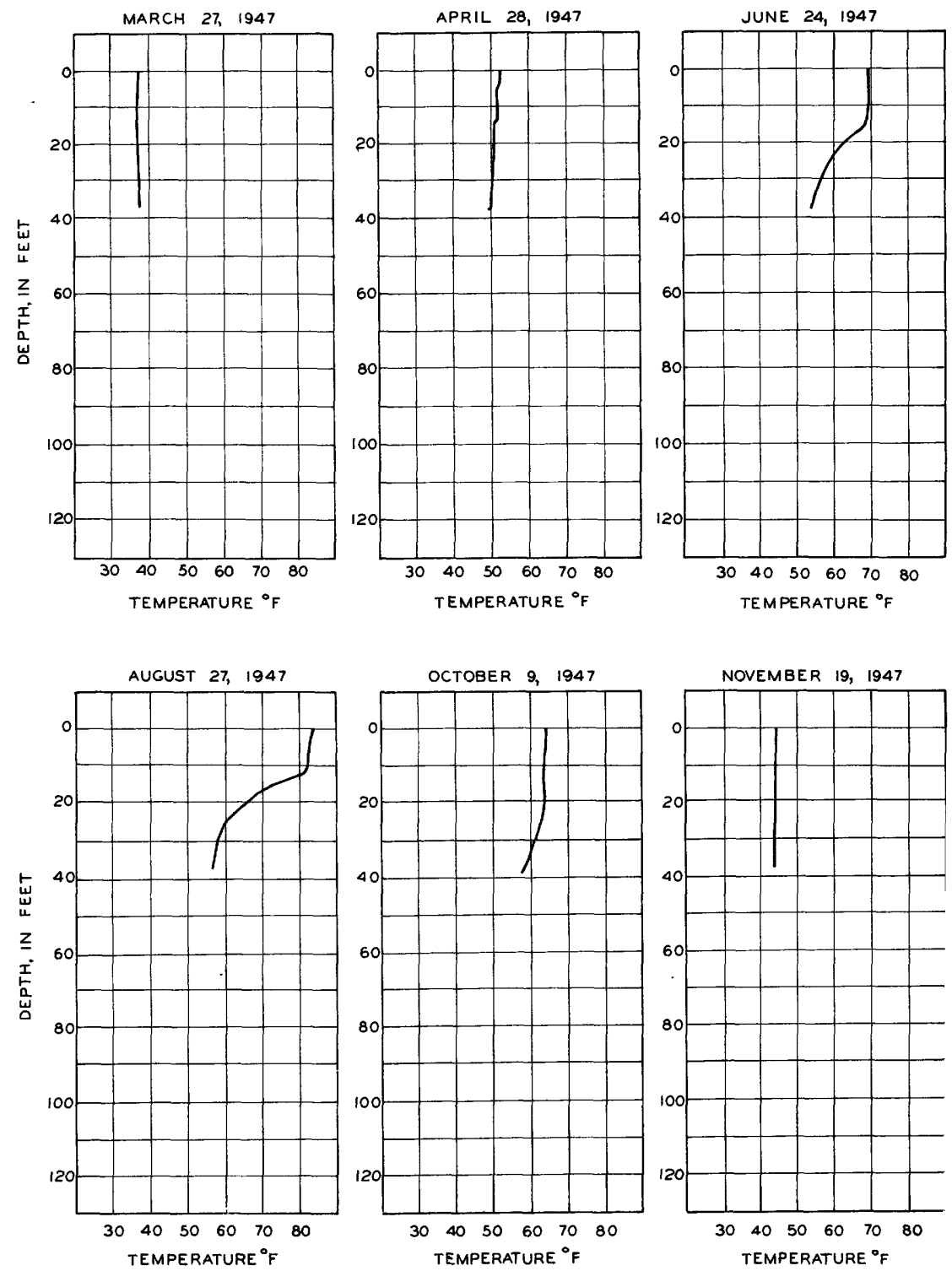

Figure 17. --Temperature profiles for Bixler Lake at Kendallville, Ind. , at intervals for March to November 1947. 

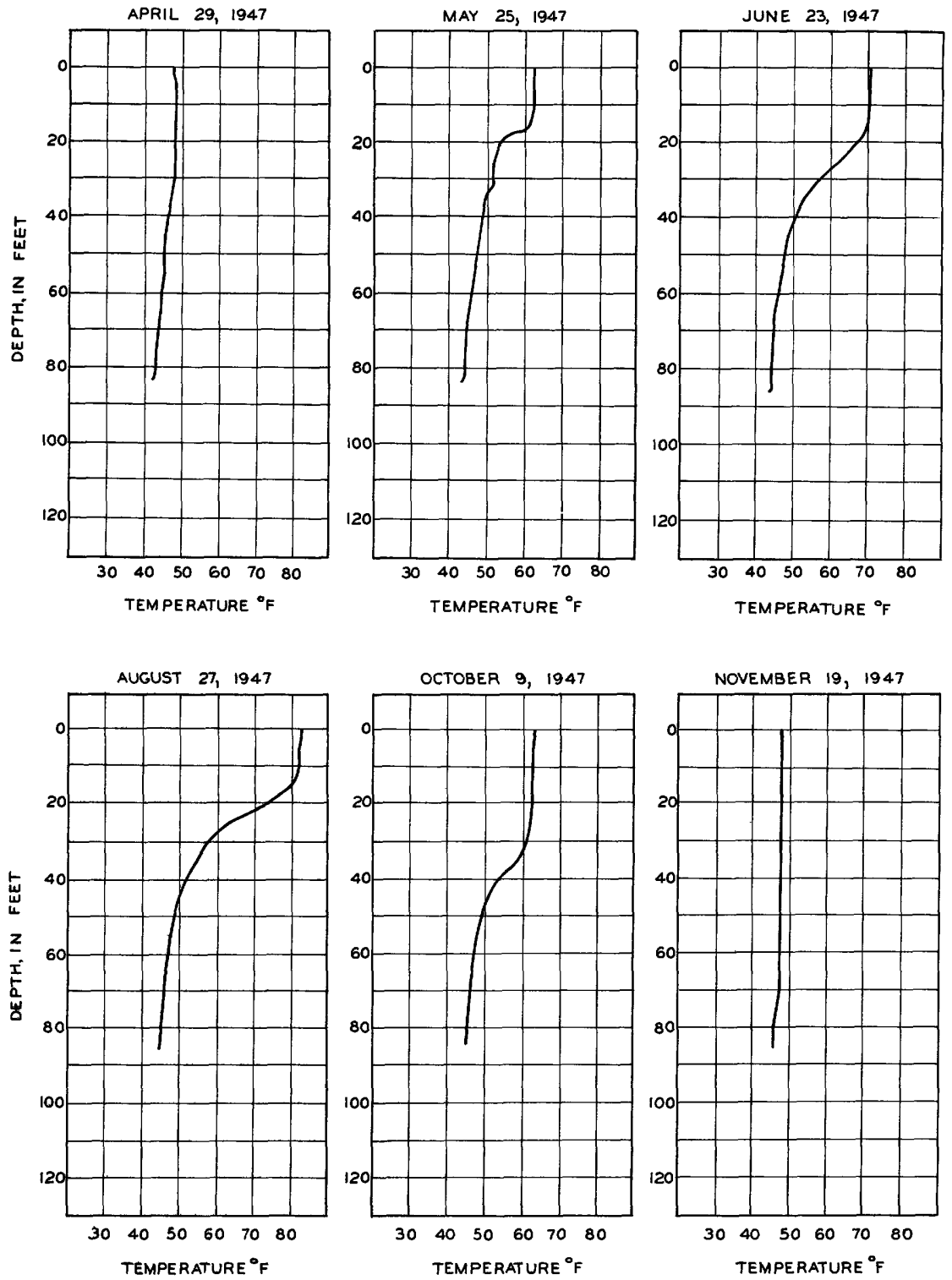

Figure 18. --Temperature profiles for Lake James at Lake James, Ind., at intervals for April to November 1947. 

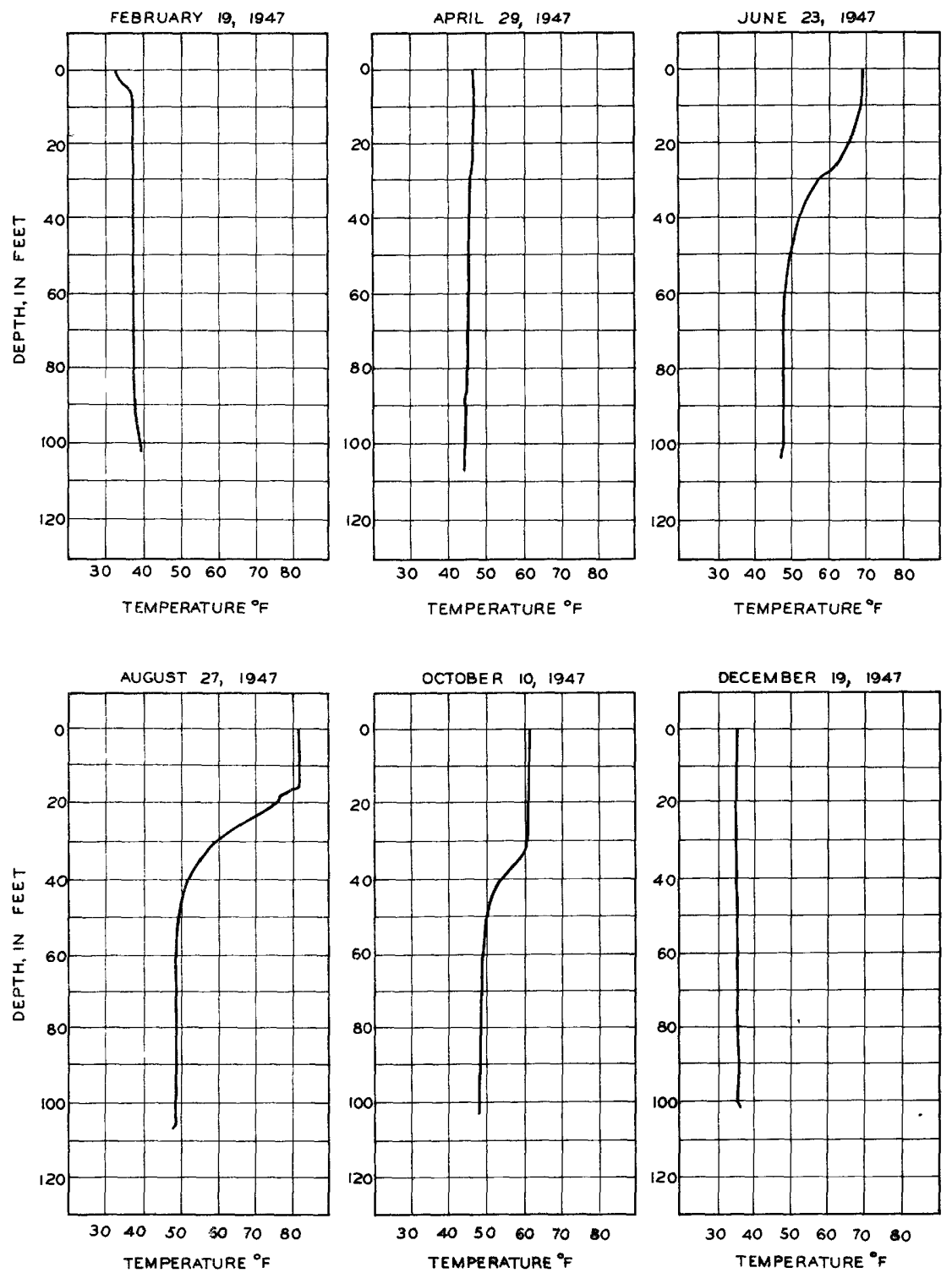

Figure 19. --Temperature profiles for Clear Lake at Clear Lake, Ind., at intervals for February to December 1947. 


\section{Effect of Size of Drainage Area}

The size of surface drainage area contributing water to a lake appears to have little effect upon water temperatures, particularly temperatures below a depth of 20 feet. This indicates that the inflowing water, which is generally of a higher temperature than the lake water, flows across the surface of the lake rather than penetrating into the lower depths. This point is illustrated by the records for Clear Lake and Tippecanoe Lake. Both are deep lakes, but the former has a drainage area of only 7.25 square miles whereas the latter has a drainage area of 118 square miles. Clear Lake has no outflow during the low-water season; whereas Tippecanoe Lake has a considerable outflow throughout the year. The temperature graphs for these two lakes show no significant difference that might be interpreted as being due to the size of the drainage area.

Another and perhaps more significant reason for the inflowing water not materially affecting the temperatures is that the inflow is not in sufficient quantities to make a complete change in the water in the lake as rapidly as ordinary temperature changes occur. The average inflow into Tippecanoe Lake is about $100 \mathrm{cfs}$; the maximum daily flow is perhaps about five times as much. The total volume of the lake is $1,472,152,000$ cubic feet. At a rate of $100 \mathrm{cfs}$ inflow it would take 170 days to change the water in the lake completely, or if the maximum daily rate could be maintained for an extended period it would require about 35 days. These periods are too long for the inflow that actually occurs to have any appreciable effect upon the entire body of water in Tippecanoe Lake.

\section{Effect of Size of Surface Area}

The surface area of the lake does not appear to influence temperatures greatly. A comparison of the temperature graphs for Bixler Lake, area 112 acres, and Tippecanoe Lake, area 1, 037 acres, shows great similarity. The slight difference in the two lakes are probably due to factors other than surface area.

\section{Effect of Depth of Water}

The depth of the water is the most important factor, other than climatological conditions, in determining the temperature characteristics of a lake. The deeper the lake the more water there is to be warmed or cooled per square foot of surface area. Lakes more than 20 feet deep have the same general characteristics for comparable depths. Those lakes less than 20 feet deep are likely to warm faster during the spring months and coolfaster in the fall 
months because the heat absorbed or lost per square foot of water surface will be confined to smaller volumes of water in the shallow lakes.

For those lakes studied that have depths of more than 20 feet, the thermocline, whose upper end usually was in the vicinity of the 20 foot depth through the summer months, appears to act as an artificial bottom to limit vertical circulation of the water and in effect makes all the deeper lakes react as though they were of the same depth.

Many of the small variations in temperature previously described for various lakes are probably due to variations in local weather condition. It should also be recognized that lakes located in somewhat warmer or colder climates would have characteris tics peculiar to those climates, but the method of heat transfer within the bodies of the lakes would still be the same as those for the lakes of northern Indiana.

\section{"Turnover" of Lakes}

The spring and autumn "turnover" of lakes to which reference is frequently made is a phenomenon in which the water on the bottom of the lake rises to the top rather suddenly and the water at the surface descends to the bottom. A "turnover" on Maxinkuckee Lake in 1900 is described (Evermann and Clark, 1920) as follows:

So long as the colder, heavier water is at the bottom, it will so remain, but let the upper end of the column (of water) become colder and, consequently, the heavier, it will go down, and the lighter bottom water will come up. This is precisely what does happen. If the series of temperatures from July 24 onward be examined, it will be observed that the difference between surface and bottom temperatures becomes successively less. By the 23rd of November, 1900 , the difference was only 0.5 of a degree, the surface being $46.7^{\circ}$ and the bottom $46.2^{\circ}$. Sometime between the $23 \mathrm{rd}$ and the $26 \mathrm{th}$, probably on the night of the 24 th, the temperature throughout the upper column fell below $46^{\circ}$, or lower than that of the lower end of the column. The upper half of the column then being the heavier, it of necessity went to the bottom, the bottom half came up, and the temperature of the lake became uniform throughout. This is shown by the serial temperature readings of November 26, which show that the temperature at all depths from top to bottom was $44^{\circ}$, a condition which practically continued until the ice went off. It can therefore be safely assumed that, in 1900, Lake Maxinkuckee "turned over" 
some time between the evening of November 23 and the morning of the 26th; and what occurred then, in all probability occurs every November or December.

On the basis of studies and analyses of changing temperatures in Indiana lakes as contained in the preceding pages, the change from top to bottom does not take place suddenly but is a prolonged process extending over several months. Consequently, there is no "turnover" as described by Clark.

In the fall of the year there is no circulation throughout the entire depth of water until a uniform temperature has been established from top to bottom. Previous to such a time the circulation is only from the top to the thermocline. With the establishment of uniform temperatures throughout, the depth circulation continues until stratification again takes place at temperatures below $39^{\circ} \mathrm{F}$.

During periods of ice cover, circulation occurs as a result of warming of the water from the bottom. This takes place probably at a rather slow rate, as it requires about 2 months for the water to rise from the $35^{\circ} \mathrm{F}$ temperature at the time the ice cover forms, to the $38^{\circ} \mathrm{F}$ temperature that is acquired later in the winter.

As soon as the ice cover disappears the convection currents are again set up from the top to the bottom until stratification again is established, after which the circulation is only between the surface and the thermocline.

As there is no circulation in the water below the thermocline from about mid-April to mid-October, the water becomes stagnant in depths below about 30 feet. This has been proven by profile chemical analysis of lake waters collected by various observers (Birge and Juday, 1911; and Ricker, 1937). During the late summer the epilimnion is usually well supplied with oxygen, while through the thermocline there is a gradually diminishing supply, and the hypolimnion is deficient in oxygen. The entire volume of water does not again become recharged with oxygen until the water temperatures have become uniform from top to bottom in the fall.

Implications of Temperature Information

In order to live, aquatic life requires that the water contain enough absorbed oxygen to support that life. Most lakes become stagnant and deficient in oxygen below a depth of 30 feet for approximately 5 months from mid-April to mid-September. Therefore, aquatic life accustomed to living in depths of 30 feet or more 
which cannot readily adapt itself to shallower depths through a considerable portion of the year, cannot exist in most Indiana lakes. Also, as there is an increasing deficiency in oxygen in depths from 20 to 30 feet during mid-summer, it is probable that certain types of aquatic life will not be found in depths of more than 20 feet, Maxinkuckee Lake and Lake Wawasee are possible exceptions to this rule because the thermoclines in those lakes are located somewhat deeper than in other Indiana lakes. The water might be suitable for aquatic life 10 feet deeper in these lakes than in most other lakes. Conversely, Flint Lake has a summer thermocline at a higher level than other lakes and depths over 10 feet might be found unsuitable for some aquatic life.

One of the mostinteresting facts revealed by the studies of lake temperature in northern Indiana lakes is the large volume of cold water stored in the deeper lakes. During the late summer months the water from the deeper parts of these lakes is from $5^{\circ}$ to $10^{\circ} \mathrm{F}$ colder than the nearby ground water. This cold water, because of its greater density, has been trapped near the bottom of the deep lakes since the previous winter and has warmed very slowly as the summer progressed.

For example, in Tippecanoe Lake, the deepest known lake in Indiana, it is common during late summer to find water $80^{\circ} \mathrm{F}$ in the upper layers and $45^{\circ} \mathrm{F}$ in the bottom 50 feet, and all water from 50 to 117 feet below the surface less than $50^{\circ} \mathrm{F}$ in temperature.

The following table gives the volume in gallons at different levels of Tippecanoe Lake:

Capacity of Tippecanoe Lake

\begin{tabular}{c|c||c|c}
\hline $\begin{array}{c}\text { Depth } \\
\text { (feet) }\end{array}$ & $\begin{array}{c}\text { Volume } \\
\text { (gallons) }\end{array}$ & $\begin{array}{c}\text { Depth } \\
\text { (feet) }\end{array}$ & $\begin{array}{c}\text { Volume } \\
\text { (gallons) }\end{array}$ \\
\hline $\begin{array}{c}\text { Surface } \\
5\end{array}$ & $11,011,697,000$ & 60 & $1,932,383,000$ \\
10 & $9,600,812,000$ & 65 & $1,665,998,000$ \\
15 & $8,472,559,000$ & 70 & $1,420,385,000$ \\
20 & $7,452,922,000$ & 75 & $1,192,910,000$ \\
25 & $6,495,774,000$ & 80 & $981,675,000$ \\
30 & $5,612,805,000$ & 85 & $786,806,000$ \\
35 & $4,811,360,000$ & 90 & $606,815,000$ \\
40 & $4,113,671,000$ & 95 & $442,008,000$ \\
45 & $3,536,073,000$ & 100 & $292,543,000$ \\
50 & $3,057,891,000$ & 105 & $165,084,000$ \\
55 & $2,640,836,000$ & 110 & $73,267,000$ \\
\hline
\end{tabular}


The above table shows that the capacity of Tippecanoe Lake below the 50 -foot level, where water $50^{\circ} \mathrm{F}$ or colder is found throughout the year, is $2,640,836,000$ gallons. This enormous volume would provide $7,235,000$ gallons of water $50^{\circ} \mathrm{F}$ every day in the year without giving consideration to the 4-month period December 1 to March 31 each year when the temperature of the entire lake is quite likely to be below $50^{\circ} \mathrm{F}$. By distributing the total volume of water below the 50 -foot level over the period April 1 to November 30 , it would provide an average of $10,820,000$ gallons for each day of that 8-month period.

The same table shows that the capacity of Tippecanoe Lake below the 90 -foot level, where water $45^{\circ}$ or colder is found throughout the year, is $606,815,000$ gallons. This volume would provide $1,662,500$ gallons of $45^{\circ} \mathrm{F}$ water every day in the year if no other cold water was available. However, the whole body of the lake is at a temperature of $45^{\circ} \mathrm{F}$ or less from about December 1 to April 1 , after which the level below which $45^{\circ}$ water would be available gradually declines through the spring months so that withdrawal below the 90 -foot level would be limited to the months of August, September, and October. Therefore, the 606, 815, 000 gallons of water below the 90 -foot level would all be available for use in these 3 months, which would raise the rate at which water could be used to $6,650,000 \mathrm{gpd}$. By distributing the total volume of water below the 65-foot level over the period April 1 to November 30 it would provide an average of $6,828,000$ gallons for each day of the 8month period.

\section{EVAPORATION FROM LAKES}

Evapotranspiration is nature's most thirsty consumer of water. It alone consumes about 70 percent of the water that falls on Indiana as precipitation. The remaining 30 percent is drained away by streams.

Between precipitation and evaporation, water may pass through many processes and uses by nature. It may be temporarily stored in the ground, used by vegetation and later transpired from the leaves, collected in lakes and streams to be evaporated over a period of time, or passed on by streams to the ocean where it is evaporated into the atmosphere. In any case, water is almost continuously passing into the atmosphere wherever moisture is in contact with the air. Even during periods of precipitation some water is being evaporated into the atmosphere.

The rate of evaporation from water surfaces is dependent upon such conditions as temperature of water, area of water surface 
in contact with the air, temperature and relative humidity of air, and movement of air. As these conditions change from place to place and season to season, the rate of evaporation changes also.

In Indiana evaporation usually reaches its highest rate in July and is lowest in January. Because of high evaporation rates in late spring, summer, and early fall, water losses from lakes due to evaporation are likely to exceed precipitation on the lake during those periods. The opposite is true during late fall, winter, and early spring.

Lake levels tend to rise during periods of low evaporation rates, when water accumulations are in excess of water losses, and tend to decline during the remainder of the year. The combination of a high precipitation and moderate evaporation in spring usually results in high lake levels in late April or early May. Minimum levels usually occur in October.

Evaporation is of particular importance to lakes that have no outlets. It is usually the principal means of water loss for such lakes, although in some instances transpiration by vegetation around the borders of the lake and underground outflow may account for sizable water losses.

The levels of lakes with outlets, particularly if there is constant inflow and outflow, are likely to be affected to a lesser degree by direct evaporation from the lake surface than lakes without outlets, even though the quantities of water evaporated are equivalent for equal areas. In such cases the inflow and outflow are the predominant factors.

\section{Measurement of Evaporation from Water Surfaces}

So many factors affect the amount of water in a lake that it is generally impractical or even impossible to separate and make direct measurements of the amount of water lost by evaporation. Factors such as inflow, outflow, precipitation, seepage, and changes in level must all be determined with a high degree of precision if the residual is to be considered evaporation. More precise methods for determining lake evaporation have been recently developed (Anonymous, 1952).

The usual method of determining amounts of evaporation is to measure the evaporation loss from a large pan. The loss from the pan is then corrected by a factor to reduce it to equivalent loss from a lake or pond. Many types of evaporation pans, both floating and land, have been used. The standard pan used by the U. S. Weather Bureau for collecting the data used in this report is the class A land pan (see fig. 20). This pan is 4 feet in diameter and 


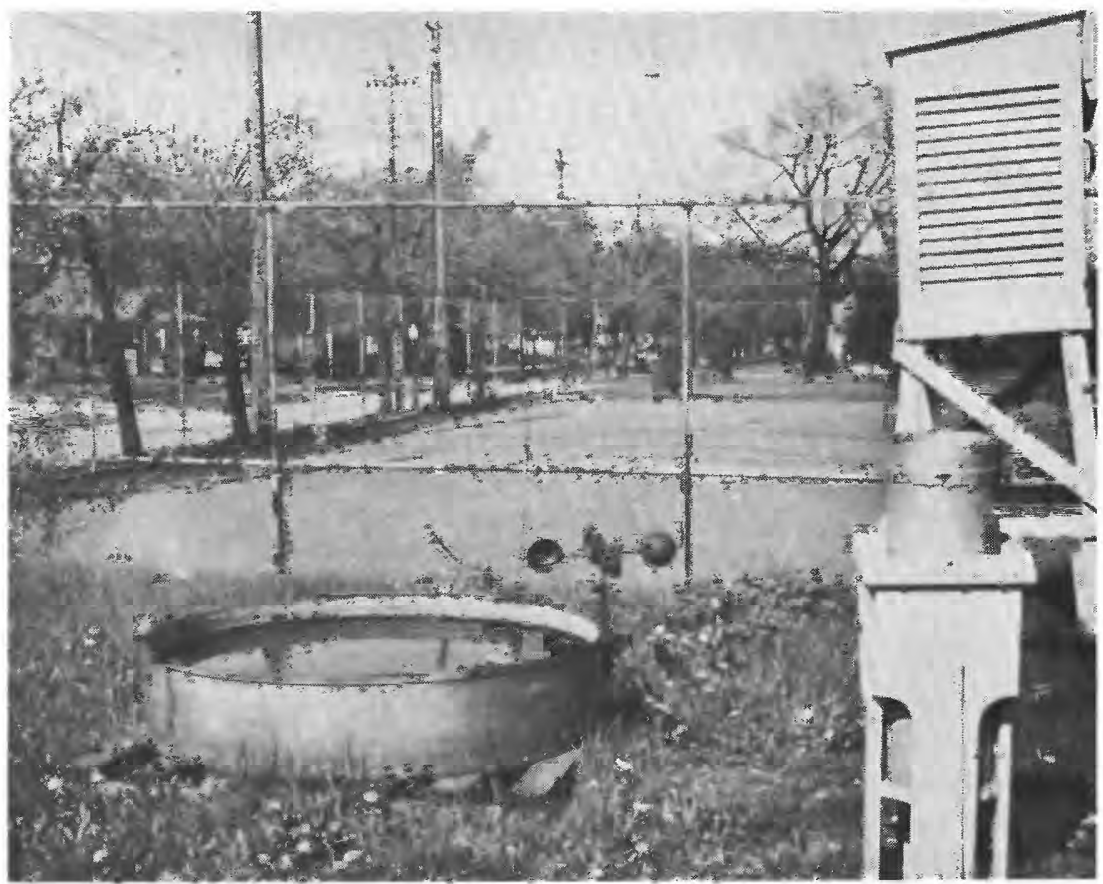

Figure 20. --Class A evaporation station, Kendallville, Ind.

and 10 inches deep, and a depth of water between 7 and 8 inches is maintained. The unpainted, galvanized iron pan is placed on timbers so that the bottom of the pan is about 6 inches above the ground. The pan is placed on a level, open spot exposed to the maximum possible sunshine. The depth of water is measured with a hook gage in a stilling well.

The total annual evaporation from the surfaces of lakes and streams is usually considered to be about 0.7 of the evaporation from a class $A$ pan. The daily and monthly rates, however, may vary widely from this ratio. Pan evaporation is likely to be higher than lake evaporation in the spring months and lower in the fall months because the temperature of the shallow water in the evaporation pan changes much more rapidly than that of large bodies of water.

\section{Collection of Evaporation Data in Indiana}

Realizing that evaporation is an important factor affecting the levels of Indiana lakes, two evaporation stations were established in cooperation with the U. S. Weather Bureau in the lake region of northern Indiana on April 1, 1947. One station was located at 
the Kendallville water works (see fig. 20) on the shore of Bixler Lake and the other at the Valparaiso water plant on the shore of Flint Lake. Both are class A evaporation stations where daily observations are made of relative humidity, maximum and minimum temperatures, wind movement, precipitation, and evaporation.

In addition to the two stations in northern Indiana an evaporation station has been operated in Indianapolis since May 1, 1937, and at Evansville since May 1, 1946.

Evaporation observations in Indiana are generally made only during the months of April through October. This period was selected because it is not practicable to make observations during freezing weather when ice formation prevents reading of the hook gage used in measuring the amounts of water evaporated.

\section{Variation in Evaporation in Indiana}

Temperature is the principal factor affecting the average rate of evaporation. It is, therefore, logical to expect evaporation rates to be higher in the southern part of the State with its higher temperatures than in the northern part. This assumption is borne out by records obtained at the various evaporation stations. For the period April to October, the average evaporation from a class $A$ evaporation pan totals about 44 inches at Evansville, about $\overrightarrow{34}$ inches near Indianapolis, and about 31 inches at Valparaiso. When pan evaporation is converted to evaporation from a free water sur face by using the average coefficient of 0.7 and when allowances are made for evaporation during the months of November to March, it will be found that the average annual evaporation from open water surfaces is about equal to the average annual precipitation in Indiana. However, during periods of drought the evaporation rates are generally higher than average and during wet periods the reverse is likely to be true.

\section{Analyses of Available Records}

For purposes of design, studies of water losses, and other water utilization problems it is frequently desirable to know the amount of water losses that may normally be expected for particular days, periods, or seasons; the time of greatest or least evaporation; and the variations from the normal values. For such purposes, average or "normal" values are usually determined by averaging observations taken over a long period of time. To determine normal values of daily evaporation with sufficient accuracy to preclude appreciable changes in the averages by one or two future extreme observations, at least 25 years of record, and preferably more, should be used in computing the normals. 
None of the evaporation records being collected in Indiana are as much as 25 years long. The Indianapolis record, which is the longest, covers a period of 17 years, except for the months of April and July, which are 16 years in length; June, which is 15 years in length; and October, which is 9 years in length. Although this record is not enough for determining good averages, it can be used as a guide to indicate the amount of evaporation that may be expected on any day.

In figure 21, the average daily evaporation for various dates has been plotted for Geist Reservoir station near Indianapolis to demonstrate how the evaporation varies in the period April to October. A curve of normal evaporation drawn through these points shows that it reaches its maximum about July 10. The minimum point is not shown because of the lack of winter evaporation observations.

The evaporation from a lake or pond may vary considerably from that observed in a land evaporation pan. The temperatures of the two bodies of water will have a decided effect upon their relative rates of evaporation. The water in the evaporation pan will follow the temperature of the air closely and will seldom vary more than $3^{\circ} \mathrm{F}$ from the air temperature. Lake temperatures, on the other hand, may vary considerably from air temperatures. During the spring months, the lake temperatures will average several degrees colder than the air temperatures, and during the fall months will be several degrees warmer. Consequently, the rate of evaporation from lakes will be lower in the spring and higher in the fall for corresponding air temperatures.

Evaporation from a lake surface is generally less than that from an evaporation pan. A coefficient of 0.7 is generally used to convert annual class $A$ pan evaporation to equivalent lake evaporation. However, the evaporation from lakes for individual days or months may vary considerably from 70 percent of pan evaporation and may even exceed the pan evaporation at times. Furthermore, pan coefficients ranging from 0.6 to 0.8 or greater have been reported.

Even though there are differences in evaporation from lakes and evaporation pans, the data observed in the latter can be very useful in the study of lakes and reservoirs. For example, comparisons have been made of the observed evaporation at Kendallville and Valparaiso for the months of August and September, 1948, and the losses from several lakes during the same period.

To simplify the computations, lakes having no surface outflow (and presumably negligible or balancing underground inflow and 


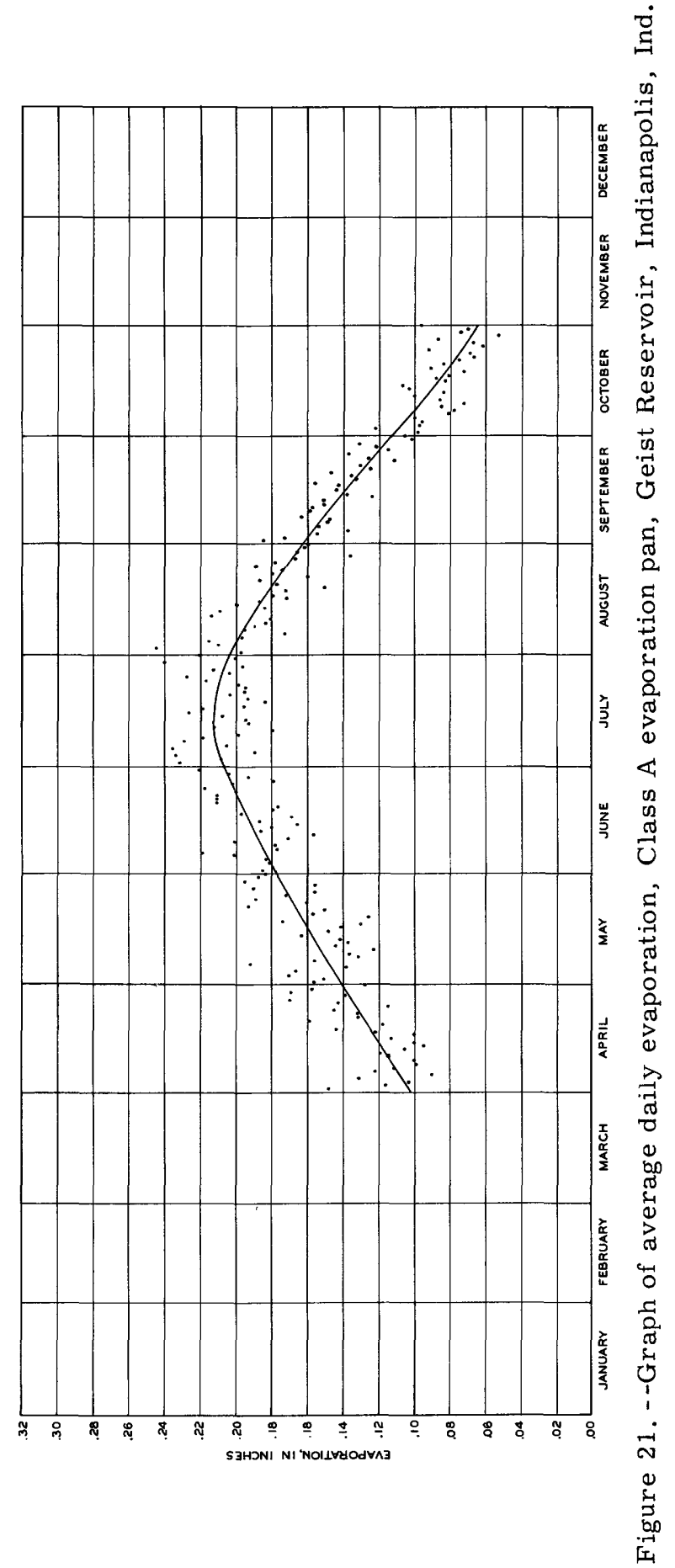


outflow) during this period and for which rainfall data for nearby areas is available have been used. Lakes having small drainage areas are best suited for such studies because both surface inflow and surface outflow generally stop during the drier months and the only factors left to affect the lakes are evaporation, transpiration, precipitation, and seepage. The rainfall data used should be obtained near the lake because summer storms are likely to be quite variable in intensity over relatively small areas.

Bixler Lake with a drainage area of 3.63 square miles has no surface inflow nor surface outflow during the drier periods of the year. During the months of August and September 1948 there was no outflow but there may have been a small amount of inflow for a few days after the heavy rain on August 10, 1948. During the remainder of the period there was no rainfall heavy enough to cause an appreciable amount of inflow other than the rain that fell upon the lake itself.

Figure 22 shows a progressive graph of the daily net water loss from Bixler Lake as determined from the daily water-level readings. This graph, in general, shows the decline in lake level through this period. The effect of rain which fell on August 4, 5, $11,17,30$, and September 7, 9, 18, 20,21, 29, and 30 can be seen in the rise in the lake level as indicated by decreases in net water loss. Consequently, if the rainfall amounted to more than the evaporation and other losses, the lake would rise and show a

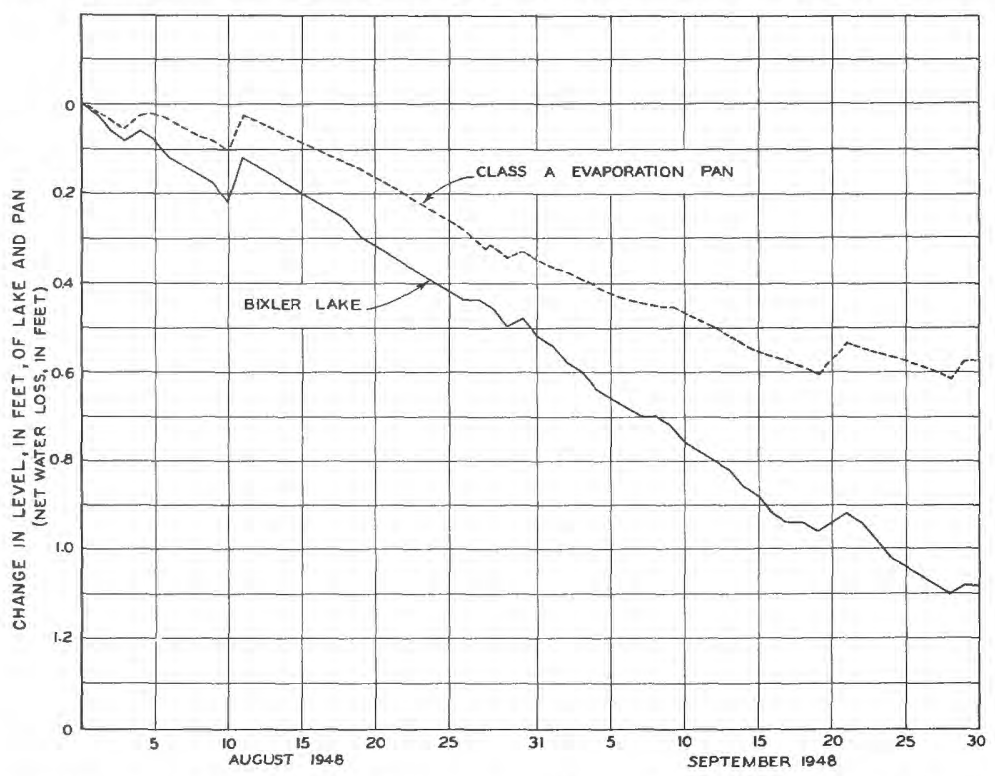

Figure 22. --Comparison of measured evaporation loss at Kendallville with water loss at Bixler Lake, evaporation adjusted for rainfall at Kendallville. 
net gain rather than loss. If the rainfall was just equal to the losses, there would be no change in level.

On the same chart is shown the accumulative daily net loss from the evaporation pan at the evaporation station on the shore of Bixler Lake. The two graphs are very similar in shape, but the lake graph descends more rapidly and indicates greater losses than those observed in the evaporation pan. Probably the excess losses, are due to differences in rates of evaporation, transpiration, and seepage into the ground.

During the month of September, when surface runoff into the lake is believed to have been negligible, the water in the evaporation pan declined only 0.22 foot while the lake level fell 0.56 foot, or 0.34 foot more than the water in the pan. A study of the daily records shows that the rate of divergence of the two curves was constant throughout the month and that the loss in excess of pan evaporation was, therefore, constant.

A similar study of Pleasant Lake at Pleasant Lake (fig. 23) was made by using the evaporation data from Kendallville and precipitation data at Angola. It is recognized that the evaporation and rainfall data in other areas are not strictly applicable at Pleasant Lake, but they are believed to be generally near enough to the conditions at the lake to furnish a reasonable comparison. The comparison of losses through the period is quite good except during

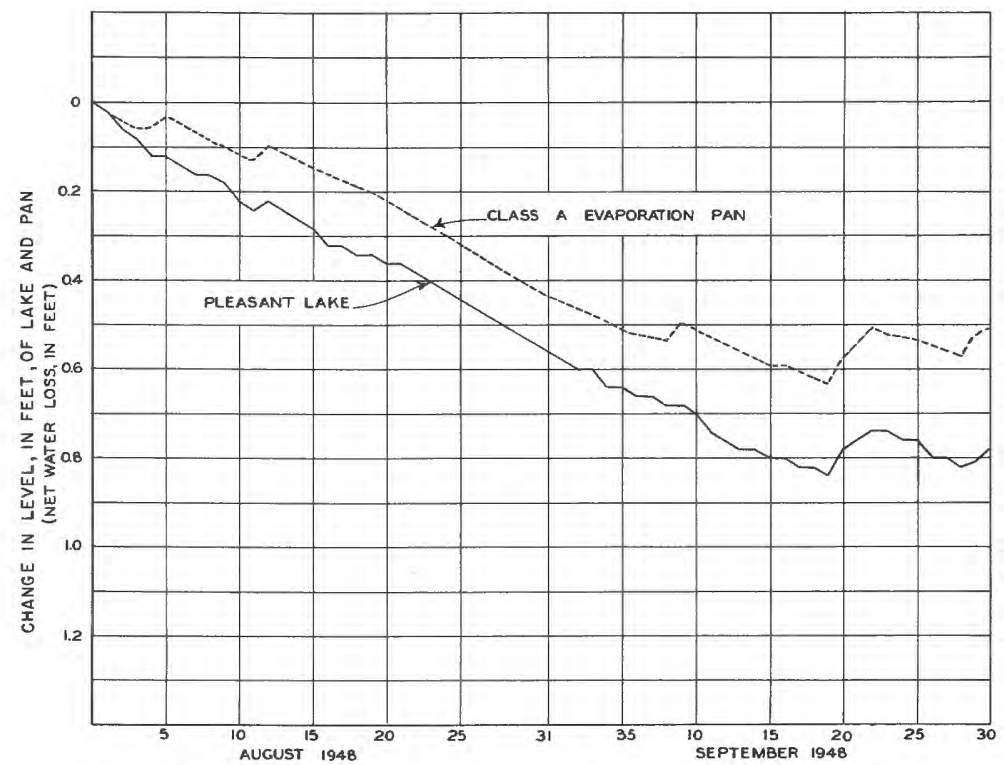

Figure 23. --Comparison of measured evaporation loss at Kendall ville with water loss at Pleasant Lake, evaporation adjusted for rainfall at Angola. 
September 1948, when the rainfall at Angola apparently was greater than at Pleasant Lake. The difference in rainfall is particularly noticeable on September 9, when it made a noticeable hump in the graph for the evaporation pan but caused only a flattening of the lake graph.

The period from August 13 to September 5 is best for study pur poses as there was very little rainfall during that time. The two graphs are nearly parallel through the period indicating that the rates of loss from the pan and the lake were the same and that there was no appreciable loss in excess of evaporation.

Similar studies were made for Loomis Lake (fig. 24) which is quite near the evaporation station on the shore of Flint Lake near Valparaiso, and for Clear Lake (fig. 25) at LaPorte. Flint Lake could not be studied because it is affected by pumpage of water into and out of the lake by the Valparaiso Water Department. Loomis Lake, however, is close enough for the evaporation data observed at the water works to apply directly. For comparison with Clear Lake, the Valparaiso evaporation data and LaPorte rainfall data were used.

The comparison for Loomis Lake shows that the graph of water loss from the evaporation pan is generally parallel to that for the lake. The same is true for the comparison with Clear Lake. Both of these graphs indicate only small losses from the lakes in excess of losses from evaporation.

The data for Pleasant, Loomis, and Clear lakes shows tilat water losses from the lakes during the rain-free periods are very nearly equal to and very nearly the same rate as the losses from the evaporation pans. This indicates that the evaporation from the lake surfaces during these periods was very nearly equal to that from the pans. It seems unlikely that other gains (considered negligible for these lakes) or losses should so nearly equal any difference in the evaporation rates. Much of the divergence in the graphs at the end of the period probably can be attributed more to the rainfall data used than to actual seepage losses.

The Bixler Lake graph, because of its steady divergence from the pan evaporation graph, certainly can be considered as indicating a rather steady loss other than evaporation. The Kendallville water plant is located on the shore of Bixler Lake and operates a number of ground water wells near the lake. It is quite possible that a sizable amount of water infiltrates into the ground in the vicinity of the wells. 


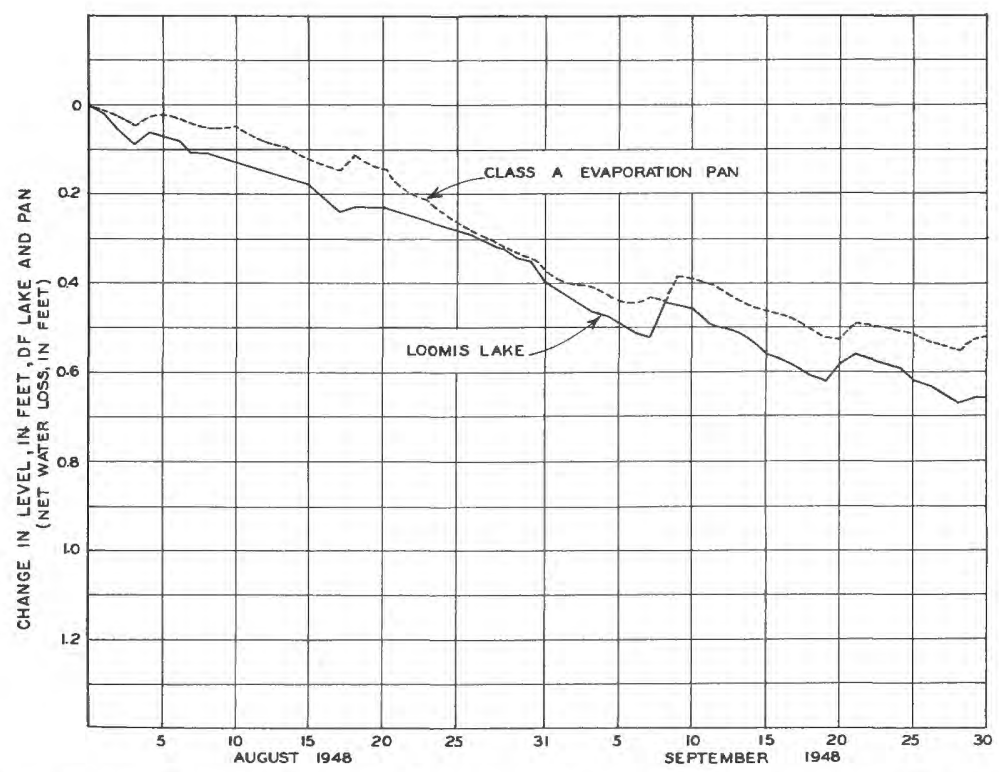

Figure 24. --Comparison of measured evaporation loss at Valparaiso with water loss at Loomis Lake, evaporation adjusted for rainfall at Valparaiso.

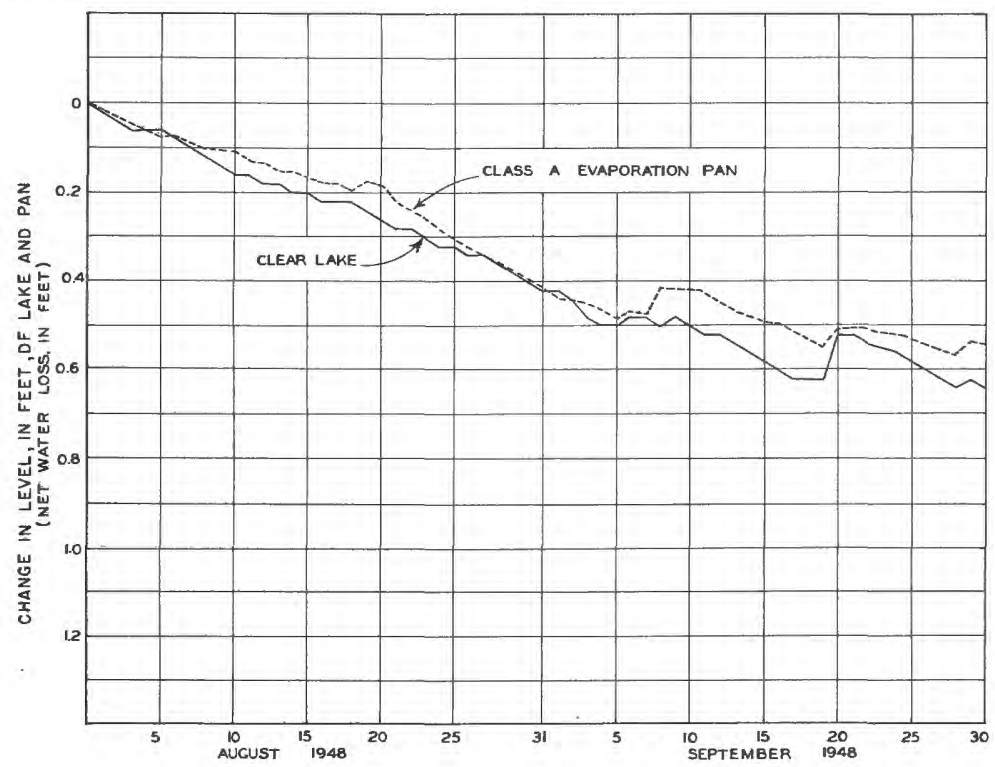

Figure 25. --Comparison of measured evaporation loss at Valparaiso, Ind., with water loss at Clear Lake, evaporation adjusted for rainfall at La Porte, Ind. 
Although all the illustrations of water losses from lakes given here have indicated some loss in excess of that due to evaporation, it should not be inferred that all lakes are subject to losses in excess of the evaporation. There are a number of lakes that are known to have definite accretions during the dry season when surface runoff is at a minimum. Wabee Lake is an example. It is fed by springs and the minimum outflow observed on October 17, 1946, at the end of a long dry period, was 0.65 cfs or 420,000 gpd. This represented water left after water losses of all types had been satisfied." Lakes of this type would have been included in the illustrations but lack of rainfall data in their vicinity or other deficiencies in the records prevented their use.

\section{ICE CONDITIONS ON NORTHERN INDIANA LAKES}

The freezing of a lake is an interesting phenomenon which varies considerably from winter to winter and with the size and depth of the lake. The general pattern is nearly the same each year: small shallow lakes and ponds freeze first, then the large shallow lakes, and finally the larger deep lakes. The length of time from the first appearance of ice along the shores to the complete freezing of an ice cover on a lake, and the dates on which ice is first noticed and on which complete cover is achieved, vary widely from year to year. Likewise, the dates on which the last ice disappears may vary by 6 weeks or more.

\section{Ice Formation}

The small and shallow lakes cool rapidly during the fall months and readily freeze over during the first severe cold spell after the whole mass of water has been chilled to near the freezing point. They are usually more protected from the winds than the large lakes and freeze with a smooth, clear sheet of ice over the surface rather early in the winter.

The large and deep lakes, with their greater volumes of water, cool more slowly and are likely to have ice only around the edges when small lakes have become completely frozen over. If the weather continues calm and cold, these lakes freeze over rather quickly, although it usually takes several days even in the most favorable freezing weather for them to become completely covered withice as the surface is always somewhat disturbed by winds. However, if during the early freezing weather there are protracted periods of wind, the freezing occurs later and proceeds at a slower pace. 
Evermann and Clark, (1920, p. 223) describe the freezing process as follows:

The ice first forms in sheltered calm places and any object that breaks the wind or tends to calm the water tends to the formation of a sheet of ice. Thus ice appears not only along the shore in sheltered bays, but also around any projecting body in the water - a stake, or a patch of bulrushes or pondweeds. Usually the formation of ice proceeds from day to day in a more or less orderly manner from these nuclei to the middle of the lake until the whole is frozen, ---.

After a lake has frozen over, continued cold increases the thickness of the ice. The maximum thickness reached during the winter depends upon the degree of coldness and the length of time the cold weather persists. Ice 7 to 8 inches thick is common for winters in northern Indiana, but during colder winters it may attain thicknesses of 24 to 30 inches.

\section{Duration of Ice Cover}

In the northern portion of the State the average duration of complete ice cover is approximately 90 days, extending from December 15 to March 15. The date on which the lakes freeze over in any particular year may range over a period of a month, while the date of disappearance of ice may range over a period of six weeks. The table below lists the average periods of ice cover for several seasons.

\begin{tabular}{|c|c|c|}
\hline Season & Average period of ice cover & $\begin{array}{c}\text { Average duration } \\
\text { (Days) }\end{array}$ \\
\hline $1942-43$ & December 5 to March 15 & 100 \\
\hline \multirow[t]{2}{*}{$1943-44$} & December 15 to February 28- & \\
\hline & March 11 & $76-87$ \\
\hline $1944-45$ & December 15 to March 12 & 87 \\
\hline $1945-46$ & December 15 to March 5 & 80 \\
\hline \multirow{2}{*}{$1946-47$} & December 19-January 4 to & \\
\hline & March 25-April 6 & $81-109$ \\
\hline $1947-48$ & December 17 to March 20 & 95 \\
\hline $1948-49$ & December 22 to February 17 & 59 \\
\hline $1950-51$ & December 6 to March 3 & 86 \\
\hline
\end{tabular}

Where one beginning and one ending date are given in the above table, most lakes froze over or thawed out within a few days of the given dates. When more than one date is given, freezing over or thawing out was so variable and uncertain on different lakes that an average period of beginning or ending is shown. 
The 1946-47 season was particularly variable. A number of the smaller lakes froze over around December 2, with the ice disappearing around December 7 th or 8 th. For example, Loomis Lake, a small lake near Valparaiso, froze over on December 13, and then was followed by a succession of other lakes freezing over. Of 87 lakes, for which ice reports are available, 2 froze over on the $14 \mathrm{th}, 3$ on the $15 \mathrm{th}, 44$ between the 16 th and $19 \mathrm{th}, 6$ between the 20 th and $24 \mathrm{th}, 14$ between the $25 \mathrm{th}$ and $31 \mathrm{st}$, and 17 between January 1 and 9, 1947.

During the 1946-47 year the disappearance of the ice from the lakes was nearly as variable as its formation. Between March 16 th and 31 st, 15 lakes were reported free of ice, and between April 1 and 6, 39 lakes were reported ice free. Other reports of ice conditions on lakes did not definitely indicate the day on which the ice disappeared.

\section{Effect of Temperature}

A decrease in temperature below $39.2^{\circ} \mathrm{F}$ causes water to expand slightly until the freezing point is reached. As water changes into ice its volume suddenly increases. It is needless to say that the sudden increase in volume does not take place through a great thickness of ice, but starts with a very thin skim of ice. The ice then grows in thickness at a rate which is dependent principally on the air temperature.

The water at the surface of a lake is usually very close to $32^{\circ} \mathrm{F}$ at the time of freezing. As long as the unfrozen water remains at $32^{\circ} \mathrm{F}$, the rate at which it freezes will be dependent upon air temperature. However, if the water should become supercooled, as it may on rare occasions, the formation of ice throughout the body of supercooled water will take place very rapidly. In this case, the rate at which the ice is formed will be influenced also by the amount that the water is cooled below the normal freezing point of $32^{\circ} \mathrm{F}$.

The length of time required to transform water at $32^{\circ} \mathrm{F}$ into ice of various thicknesses is shown in figure 26. The graphs in this illustration show that an infinite amount of time is required to freeze ice to any appreciable thickness if the air temperature remains at $32^{\circ} \mathrm{F}$. However, if the air temperature drops to $30^{\circ} \mathrm{F}$ and remains constant, ice 1 inch thick will freeze in 18.5 hours, and at $0^{\circ} \mathrm{F}$ an inch can be frozen in 1 hour and 10 minutes. Three days of continuous zero weather would result in the freezing of 10 inches of ice. 


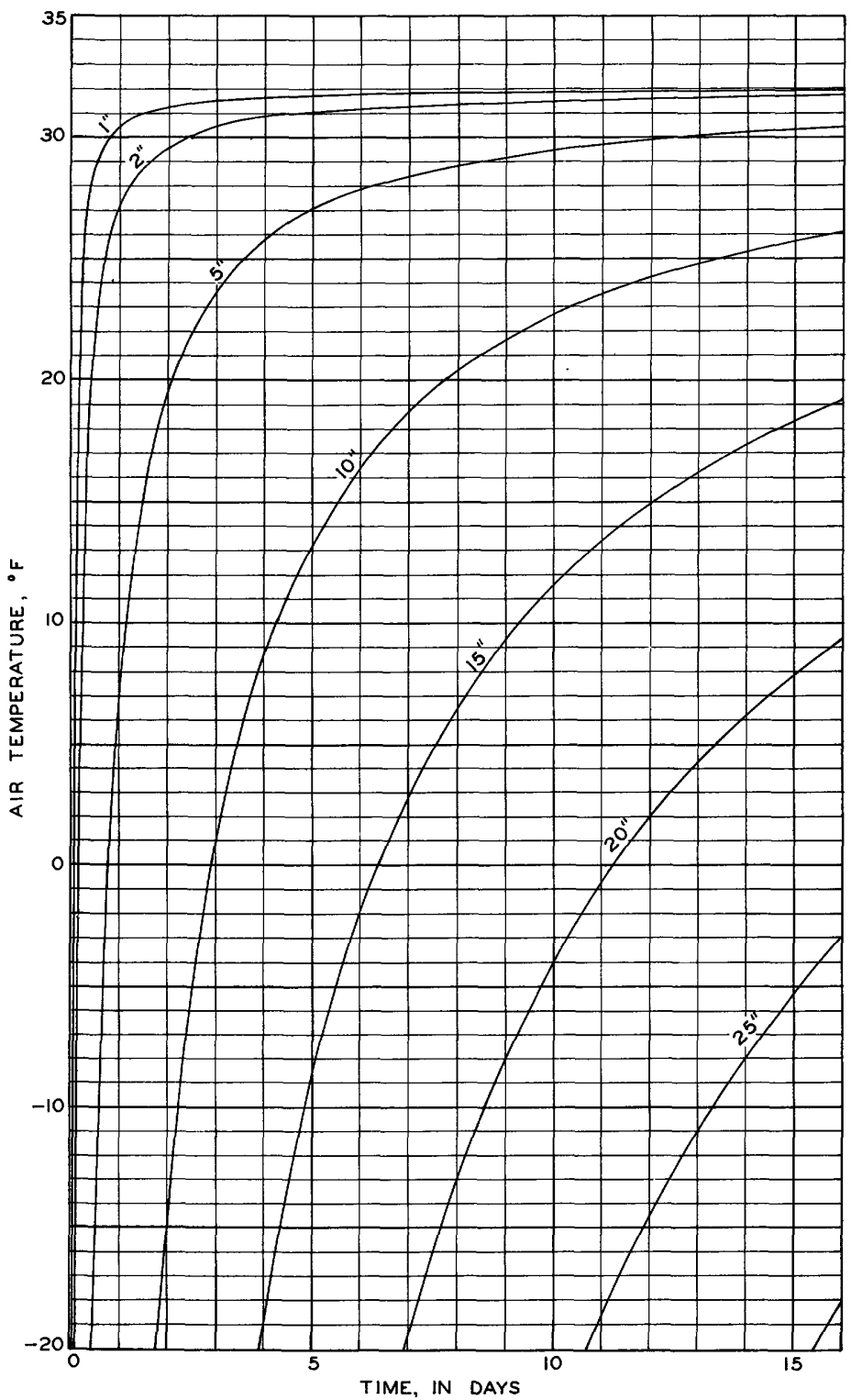

Figure 26. --Graph showing time required to freeze ice of various thicknesses. 
The graphs of ice thickness are based on constant temperatures, and, because of their curvature, cannot be used directly with average temperatures. In general, during a period of variable temperatures, more ice will be formed than the chart indicates for the average temperature. The greater the spread between the maximum and minimum temperatures, the greater will be the divergence from the chart.

As long as the air temperatures remain below $32^{\circ} \mathrm{F}$ the only ef fect of air-temperature variations on ice thickness is to vary the rate at which the ice sheet is growing in thickness. Air at temperatures above $32^{\circ} \mathrm{F}$, of course, melts the ice and tends to decrease its thickness. In addition to the air, solar radiation is another source from which ice may gain heat. If the ice surface is free from snow, solar radiation may heat the ice considerably and cause thawing, even though the air temperatures are well below freezing. On sunny days solar radiation rather than air temperatures may be responsible for the predominating temperature effect on ice, but on cloudy or overcast days the air temperatures will provide the overriding influence.

Although considerable expansion takes place at the moment of freezing, ice, once formed, reacts to temperature changes in the same manner as other solids. As temperatures decrease the ice contracts, and as they increase it expands.

The coefficient of linear expansion of ice varies from 0.00002928 per degree Fahrenheit at $32^{\circ}$ to 0.00002806 at $0^{\circ} \mathrm{F}$, with an average value of 0.00002867 per degree Fahrenheit in this range. Using the average coefficient of linear expansion it is found that for a drop in temperature from $32^{\circ} \mathrm{F}$ to $0^{\circ} \mathrm{F}$ ice will contract 11.0 inches per thousand feet. If the ice completely covers the lake and is firmly attached to the shores, the contraction will introduce strains in the ice and cause cracks to form. When temperature changes slowly little cracking will occur, because the ice, which behaves in a somewhat plastic manner, will flow sufficiently to relieve the contraction strains. But when temperature changes rapidly the ice is unable to relieve the strains in this manner and wide cracks will appear.

If the weather remains cold, a state of equilibrium is reached in which the strains have been relieved by movement and cracking of the ice. The cracks fill with water and freeze, and the ice again becomes a continuous mass.

As the temperature rises after a cold spell the ice will expand, and if held firmly at the shores, the expansion will cause the ice to bulge in the center or plow up the earth at the shore line. Great pressure is exerted by the expanding ice and is capable of moving boat piers and stout seawalls. 
If the ice bulges in the center, owing to expansion, the bending will introduce strains and cause it to crack. At times the ice does not crack in an exactly vertical plane, and this permits one side of a cracked ice sheet to ride up the inclined face of the crack and overlap the other side, thus relieving the pressure. At other times the pressure is relieved by the ice merely buckling and cracking in many places.

On a mild sunny day following cold weather one can hear for considerable distance the booming and rumbling as new cracks are formed to relieve the stresses in the ice cover. In fact, these sounds can be followed as the cracks progress from one side of the lake to the other.

During periods of thick ice cover (10 inches or more) rapidly rising air temperatures may not greatly raise the temperature of the ice through its entire thickness. At such times the surfaceice will expand as the temperature rises; consequently the lower por tion of the ice cover will not expand nearly so much because it will be maintained at a nearly constant temperature by the water underneath. The expanding surface ice will then cause the ice underneath to crack, and the cracks will penetrate only part way to the surface. In clear ice, mosaic patterns made by these partial cracks can frequently be seen.

The quality of the ice cover varies with the temperature of the air. As the air temperatures fall below $32^{\circ} \mathrm{F}$ the ice becomes colder and also increases in thickness. With increasing coldness the ice becomes harder and more brittle. At temperatures below $0^{\circ} \mathrm{F}$ it is very hard and brittle. In very cold weather a decrease in hardness and brittleness is noticed in a thick ice cover as cuts are made into the lower and warmer parts of the ice. The temperatures in thick ice will be graded from near the air temperature on the surface to near $32^{\circ} \mathrm{F}$ at the bottom where it is in contact with water.

During thawing periods ice generally does not become gradually thinner and thinner until it finally disappears, but becomes gradually warmer throughout its thickness and begins to melt internally as well as on the top and bottom surfaces. Solar radiation is very effective in heating and melting ice internally and if the ice surface is free of snow can greatly increase the melting rate over that caused by air temperatures. The internal melting reduces the ice to a porous mushy state in which the ice becomes softer and softer until it loses most of its strength. It is then said to be rotten. Once it has reached the mushy state a little wind will cause it to mix with the water, and it will break up, melt, and disappear very rapidly. 


\section{Damage by Ice}

Alternating warm and cold weather will cause ice to go through a series of expansions and contractions. The cracks produced by this process fill with water and freeze, and each successive period of expansion causes the ice boundaries, if not firmly held in place, to push out more and more. Such movements can be very destructive to shore installations.

Another type of destructive movement of ice is the movement of ice sheets by wind. As long as the ice cover is firmly attached to the shores it is not affected much by wind. But after rainstorms, when the lake has risen and the ice cover has been separated from the shores, it becomes a large freely floating body, and is easily moved by wind. Once the whole sheet of ice has begun to move, it is of such great mass, especially if very thick, that it possesses great inertia and will push up on the shore in great cakes. In severe windstorms it has been known to plow up great mounds of earth, move trees, and crush houses.

\section{STABILIZATION OF LAKES}

In the early days of the settlement of Indiana, the fluctuations of lakes were of little concern to people. Land was plentiful and that affected by flooding or marshy conditions was disregarded in favor of other lands more favorable for settlement. As the State became more densely populated, land became more valuable and farmers, aware of the potentialities of the rich muck lands in the swamps and marshes bordering the lakes, constructed drainage ditches to drain the swamps and even to lower the lakes to provide more tillable acreage. Very little objection was raised to this practice, for, before the days of easy travel, the city dwellers were not using the lakes on a large scale for recreational pur poses.

\section{Improved Transportation and its Effect on Use of Lakes}

At about the beginning of the present century, the era of the electric interurban transportation came into existence, providing quick transportation from city to city and points in between. Weekend travel to many areas that were formerly beyond the practical reach of the horse and buggy was now possible. Those lakes within easy reach of the interurban lines were invaded by people who bought lake front lots for summer cottages. Streetcar lines were built from some cities and towns to nearby lakes to facilitate the new traffic. The automobile gave additional impetus to the increasing use of lakes for recreational purposes, and as automobile 
transportation became common, the population around the lakes increased by leaps and bounds.

The change in use of the lakes and their shorelines from agricultural activities to recreational and habitational functions brought new problems. No longer could one make changes in lakes by draining or damming them without affecting the rights of others whose interests in the lakes were different.

\section{Agricultural and Recreational Use of Lakes}

The increasing demand for agricultural products has made it profitable for farmers to drain and reclaim low lands in the vicinity of lakes. These low lands are not always adjacent to the lakes, but, as in the case of lands adjacent to lakes, they cannot be drained without affecting lake levels. In other cases, the lands to be drained are downstream from the lakes, and in deepening the ditches to obtain adequate drainage the lake levels also are affected. The cottage owners, boat livery operators, fishermen, and resort proprietors generally are interested in maintaining lake levels at the elevations to which they have been accustomed. This is particularly important during low-water periods to preserve fish spawning and feeding beds and provide better boating and fishing. However, the conflicting interests are in general agreement that unusually high levels, as are common on many lakes during the spring months, are undesirable and limit the use of the lakes. High levels submerge piers, flood low lands and lake cottages, render septic tanks inoperative, and create many other difficulties.

From the standpoint of the cottage owner and recreationalist, it is generally desirable to keep the lakes at about the same level the year around. It is recognized that there may be disagreement on which level is most desirable by parties with different interests. For example, the cottage owner who built on low land during a dry year may desire a lower lake level than another owner who is located on higher land and who has trouble getting his boat through the shallow water to the shore. Some farmers may want low lake levels for better drainage, while others may want higher lake levels to make the water more accessible to them. In spite of the many conflicting interests, it is frequently possible to agree on a lake level that will, in general, be of some benefit to all concerned even though not all the objectionable features are eliminated entirely.

When a given lake level with limited variations in elevation has been decided upon, a problem of obtaining it and its maintenance 
arises. With the problem recognized the degree of satisfaction obtained in the solution will depend largely on the type of lake involved and the extent to which those who are interested in stabilizing the lake level will cooperate.

\section{Requirements for Maintaining Constant Lake Levels}

To maintain a lake at a constant level requires an outlet structure that will permit the discharge of excess quantities of water as fast as they flow into the lake as well as hold the water in the lake during periods of $\mathrm{dry}$ weather. In addition to this requirement the outlet channel or ditch must have sufficient capacity to carry away the maximum discharge from the lake without causing appreciable backwater on the dam. If the level of the lake is to remain at or near the crest elevation of the dam during dry weather the inflow must be perennial and sufficient to offset losses from the lake due to seepage into the ground, transpiration, and evaporation.

To maintain an absolutely constant level on a lake at all times is seldom practical because of the necessity of providing constant regulation in keeping with the variation in inflow. Usually lakelevel control is limited to maintaining levels within certain limits that will keep the regulation of the outlet gates to a minimum without producing objectionable levels. In general, the narrower the limits within which the lake will be permitted to vary, the more difficult and expensive will be the problem of control.

The range in level of an uncontrolled lake depends upon the maximum quantity of water flowing into the lake, the storage capacity of the lake, and the capacity of the outlet to discharge excess water. Thus it is apparent that a small lake with a large inflow will have a much greater range in level than a large lake with the same or a smaller inflow, provided the outflow channel capacities are the same.

By proper design and regulation of outlet control works, lakes that normally fluctuate through a wide range in levels may frequently be controlled within much narrower limits. Likewise, those lakes that normally fluctuate through small ranges may have the range reduced to narrower limits if the normal fluctuation is induced largely by factors other than seepage, transpiration, and evaporation, which are generally impractical to control.

For lakes with small drainage areas the problem of maintaining a sutiable level during the dry periods of the year is frequently more difficult than that of trying to dispose of the excess water 
during wet periods. Sometimes it is possible to improve the lowwater situation by increasing the size of the drainage area and the inflow through the construction of new drainage-ditch feeders. This remedy may also create a high-water problem if the lake has no outlet, such as in the case of Pine and Stone Lakes in LaPorte County. In 1924 the Kabelin Ditch was constructed to intercept the drainage of a larger area to relieve the unusual low-water conditions of Pine and Stone Lakes. Since that time the levels of these lakes have risen to such heights that damage to realestate on low lands around the lake shores has become a problem, previously given little or no consideration, and the construction of an outlet is being considered as a means of alleviating this condition. The problem of regulating this condition will probably require careful study and analysis of the factors producing it, particularly the weather factors.

\section{Problems of Lake-Level Control on Some Indiana Lakes}

Bass Lake in Starke County is an example of a lake with insufficient drainage area to maintain the level through the summer months. The lake has a surface area of 1,405 acres and occupies 60 percent of its drainage area, thus 60 percent of the rain falling in this basin falls directly on the surface of the lake. A dam at the outlet helps store water in the lake through the winter and spring months for the purpose of maintaining higher levels during the dry summer months. Very little of the water escapes from the lake by way of the outlet, yet the annual variation in level averages about 1.53 feet. To help relieve the low-water conditions during the summer months, when the lake is used most for recreation, the property owners have considered the installation of pumps to pump water into the lake from other drainage basins or from wells. Maintaining the lake level during the dry periods would require the pumping of approximately 5 million gallons of water per day.

The easiest solution for the low-water problem, although it is not a complete solution, is to store as much water in the lake as is possible during the high-water season for tiding over the lowwater season. This method does not help reduce the range in lake levels on the lake nor decrease high-water levels but it helps preserve a higher low-water level.

For the lakes with year around inflow the elimination of high water is usually the most serious problem. Webster, Sylvan, and Ridinger Lakes are good examples of this type of lake. Excess water is controlled by discharging water from the lake nearly as fast as it is received, usually by operation of a dam equipped with gates that may be operated as required. If the range in stage is 
not too great, a fixed dam without gates may be used to keep the lake within limits of a desired level.

Webster Lake is controlled by two outlet structures. The main structure is a concrete dam, 35 feet long by 8 feet high, with seven 5 -foot wooden, adjustable sluice gates mounted on the crest. The auxiliary structure is a 20 -foot concrete dam with five 4 -foot wooden, adjustable sluice gates mounted on the crest (this structure is the old mill dam). During periods of low runoff the gates are left in place to maintain the lake at nearly constant level. During the periods of high runoff the gates on both structures may be opened manually to discharge excess water.

Sylvan Lake at Rome City is controlled by a concrete dam with a spillway 50 feet long surmounted with 12 gates which are manually operated as required. This dam has sufficient height to provide complete regulation without danger of submergence from the channel downstream.

Ridinger Lake has a concrete control dam with a 10-foot fixed concrete spillway and a 6 -foot sluiceway equipped with stop logs. The fixed spillway maintains the lake at a desirable level during the low-water season. The high-water conditions are relieved by removing stop logs from the sluiceway.

Tippecanoe Lake is controlled by placing boards between upright $\mathrm{H}$-beams set in concrete in the bed of Tippecanoe River. The boards are installed during the low-water months to raise the lake level and removed during the high-water months to provide a completely opened channel.

These controldams are all located on lakes where the low-water flow is in excess of the requirements for seepage, transpiration, and evaporation.

\section{Effect of Inflow and Outflow on Planning Control Dams}

In planning for the design and construction of control dams, consideration must be given to factors other than the lake. A preliminary step is the study of the inflow and outflow of the lake to determine the effect of storage upon the outlet discharge. As the lake level rises, part of the water entering the lake is withheld in storage and for a short period of time the outflow will be less than the inflow. When the flow is through a chain of lakes, a flood peak passing downstream becomes progressively smaller as it passes through each lake because of the water retained in storage. During falling stages, the outflow is greater than the inflow because water is coming out of storage as the lake levels recede. 
Studies of inflow and outflow have been made on several lakes; the difference between the rate of inflow and outflow is surprisingly large for some lakes, and the larger the lake-surface area, the larger this difference will be. These conditions are not unusual but common to any unregulated lake.

At Cedar Lake in Lake County, during the storm of March 13 and 14, 1944, the peak inflow was $328 \mathrm{cfs}$ and the outflow at the same time was only $42 \mathrm{cfs}$, or one-eighth of the inflow. The maximum outflow of $75 \mathrm{cfs}$, which was the capacity of the outlet, was reached on March 16, two days after the peak inflow. Cedar Lake, with a surface area of 805 acres (1.25 square miles), has considerable storage capacity to accommodate runoff from the 8.05 square miles of its drainage area.

Bixler Lake, during the storm of June 12, 1946, had a maximum inflow of $183 \mathrm{cfs}$ and an outflow of less than $2 \mathrm{cfs}$ at the same time. A high rate of inflow was sustained for several hours and by the time the inflow had dropped to $115 \mathrm{cfs}$, the outflow had increased to $10 \mathrm{cfs}$. The outflow finally reached a maximum of 13 cfs on June 15 when the inflow had also dropped to $13 \mathrm{cfs}$. In this case, the maximum outflow was delayed 3 days after the maximum inflow. Bixler Lake has a surface area of 112 acres $(0.175$ square miles), with a contributing drainage area of 3.63 square miles.

The high water of April 12, 1944 at Ridinger Lake showed a peak inflow of $316 \mathrm{cfs}$ with an outflow of $170 \mathrm{cfs}$ at the same time. The peak outflow of 216 cfs came 24 hours later. Ridinger Lake with a surface area of 115 acres ( 0.179 square miles) is about the same size as Bixler Lake, but its drainage area of 32.5 square miles is nearly ten times that of Bixler Lake. Its storage capacity is, therefore, less in proportion to the drainage area and a smaller ratio between inflow and outflow exists.

\section{Effect of Storage in Lakes on Streamflow}

Because of the dampening effect of lakes on floods, consideration must be given to the effect downstream if the flood storage capacity of a lake is eliminated and the discharge passed on downstream as fast as it enters the lake. First, the typical outlet channel probably is only large enough to accommodate the discharges that have customarily come from the lake in the past. If the peak discharges should be increased 5 to 10 times, as would be necessary to hold constant levels on Cedar Lake or Bixler Lake, then the outlet channels would become inadequate, resulting in water overflowing to adjacent lands. This condition could be prevented by dredging the outlet to provide a channel with sufficient capacity to carry the increased flow. If the outlets are 
enlarged to carry larger flood flows, the water will arrive downstream sooner and in larger volumes. In the examples cited above, the peak discharges were delayed 1 to 3 days in passing through the lakes. A more rapid concentration of flows will increase the high water in lakes and streams downstream unless they also are prepared to carry more water. If a stream should pass through a chain of lakes and each lake in succession should be regulated to discharge flood waters rapidly, the flood hazard on the lower reaches of the stream would be increased many times.

An idea of the flood-retention properties of a group of lakes may be obtained by comparing the peak discharges during the high water caused by several days of heavy rainfall shown in the following table. The storm during the period May 14-17, 1945, was selected because of its nearly equal intensity over the northern part of the State.

Comparison of peak discharge for the storm of May 14-17, 1945

[*Adjusted to equivalent discharge for drainage area (126 sq mi) equal to that for Tippecanoe River

\begin{tabular}{|c|c|c|c|c|c|c|}
\hline Stream & $\begin{array}{c}\text { Drainage } \\
\text { area } \\
\text { (sq } \mathrm{mi} \text { ) }\end{array}$ & $\begin{array}{c}\text { Lake } \\
\text { area } \\
\text { (acres) }\end{array}$ & $\begin{array}{c}\text { Total } \\
\text { precipi- } \\
\text { tation } \\
\text { (in) }\end{array}$ & Date & $\begin{array}{c}\text { Peak } \\
\text { discharge } \\
\text { (cfs) }\end{array}$ & $\begin{array}{c}\text { *Adjusted } \\
\text { discharge } \\
\text { (cfs) }\end{array}$ \\
\hline $\begin{array}{l}\text { Tippecanoe River, } 15 \text { miles } \\
\text { north of Warsaw }\end{array}$ & 126 & 4822 & 3.00 & May 20 & 320 & 320 \\
\hline Hart Ditch, at Munster & 57 & 0 & 2.63 & May 15 & 918 & 1600 \\
\hline Wildcat Creek, at Greentown--- & 166 & 0 & 2. 75 & May 17 & 1680 & 1400 \\
\hline Little River, at Huntington & 259 & 0 & 3.00 & May $17-18$ & 2750 & 1720 \\
\hline Eel River, at North Manchester- & 423 & 804 & 3. 50 & May 17 & 3990 & 1750 \\
\hline
\end{tabular}

The Tippecanoe River drainage basin above Warsaw, contains 39 lakes, with a combined surface area of approximately 4, 822 acres, which constitute 6.0 percent of the drainage area. The drainage basins for the other streams, excepting Eel River, listed in the table above, contain no lakes or ponds of consequence. The Eel River drainage basin contains 14 small lakes with a combined surface area of 804 acres, or only 0.3 percent of the drainage area. The storage capacity of these lakes, which are all in the upper headwater areas, is considered as having a very minor effect on Eel River discharge.

In comparing the peak discharges one should bear in mind that this storm traveled from west to east and that Hart Ditch, which is near the Illinois State line, received its heaviest rainfall on May 14, and the other streams, which are on the eastern side of the State, received their rainfall on May 15 and 16. The time factor in the eastward movement of the storm across the State accounts for the progressively later dates of the peaks for ali streams except the Tippecanoe River, where the peak was retarded approximately 3 days longer than can be accounted for by the eastward movement of the storm. This phenomenon is attributed to the storage of water in the 39 lakes contained in the Tippecanoe River drainage basin. 
If the peak discharges for the other streams are adjusted (on basis of a plot of peak discharge against drainage area) to the Tippecanoe River drainage area and rainfall, they will average about $1,600 \mathrm{cfs}$. This discharge may be taken as the equivalent of what would have occurred in the Tippecanoe River if it had not been for the storage and retarding effect of the lakes. In other words, the lakes in the Tippecanoe drainage basin reduced the peak flow of the river 79 percent.

As a result of the natural flood control properties of the upper Tippecanoe drainage basin, the stream channel is much smaller than other streams of equal drainage area, the flow is more uniform throughout the year, and no serious flood problems have developed.

The foregoing examples have indicated the effect upon streamflow that might be expected with lake storage and without lake storage. In considering the effect of lake stabilization upon the downstream reaches of a stream it is not usually necessary to consider such extreme changes as might be brought about by the totalelimination of lake storage. Usually it is impractical to achieve such conditions, and some intermediate plan that will permit a certain amount of fluctuation in lake levels is adopted.

\section{Effect of Control Dam on Ridinger Lake}

To illustrate what can be accomplished in a practical way by the construction of a control dam, the case of Ridinger Lake is cited. In June 1946 a petition to clean out Grassy Creek or Elder Ditch, the outlet of Ridinger Lake, wes approved by the Circuit Court of Kosciusko County upon condition that a control dam be constructed to maintain the lake level at 843.12 feet, mean-sealevel datum. The ditch was cleaned in June 1947 and the control dam was built in October of the same year. The dam consists of a fixed spillway of a concrete gravity-type section 3.25 feet high with a 10 -foot horizontal crest, and a 6 -foot sluiceway equipped with stop logs. These stop logs eventually will be replaced with a vertical lift gate.

By using the discharge rating curves determined before and since the construction of the dam, it is possible to determine how the cleaning of the outlet and operation of the dam has affected the level of the lake and discharges in the outlet. Two high-water periods have been selected to demonstrate the effect of the dam. The first is the period April 8-21, 1944, when the highest level of the period of record occurred and before the ditch was cleaned. The second is the period February 26 to March 10, 1948, after the dam was built. 
The period April 8-21, 1944, includes three separate rainstorms. The first covered a period of four consecutive days, April 9-12, during which an average of about 3.60 inches of rain fell over the drainage basin. The next rainfall averaged about 0.85 inch on April 15 and 16. The last rainfall, reported on April 20 , amounted to about 0.20 inch over the area. These three storms resulted in three crests instead of one on the lake during the period considered.

In figure 27 discharge graphs show the inflow and the outflow of the lake that occurred from April 8 to 21, 1944, and the possible outflow in the same period had the control dam been installed at that time. In making the graph to show the effect of operating the dam, an assumed condition of all but two stop logs removed from the sluiceway was used to start the period in order to have a lake level nearly equal to that which actually existed during the first 2 days of the record. It was then assumed that the observer, on the morning of April 10, 1944, having witnessed the rain of the day before and the continuing rain, would remove all the stop logs at $10 \mathrm{o}^{\prime} \mathrm{clock}$, and leave the sluiceway entirely open. The stop logs would not be replaced during the remainder of the period.

With the assumed removal of the stop logs, the discharge would immediately increase by about 24 cfs and would continue to be somewhat larger than actually occurred until on April 14 when it would drop somewhat below the actual discharge for most of the remaining portion of the period. The effect of the increased dis charge during April 10-13 would be to permit less water to go into storage in the lake and thereby reduce lake levels. The outlet channel, on the assumption that it would be cleaned out, would permit passing a peak discharge $28 \mathrm{cfs}$ larger at a stage 2.0 feet lower than under conditions before the outlet was cleaned out.

The gage-height graph of the actual lake levels is shown un figure 27 and also the computed gage-height graph showing the levels that would have existed if the control dam had been in operation in April 1944. The computed graph shows that the maximum level would have been 2.0 feet lower and that the levels would have remained approximately 1.5 to 2.0 feet lower than actual conditions throughout the period.

During the period February 26 to March 10, 1948, after the construction of the control dam, two rainstorms took place. The first storm on February 27 and 28 averaged about 1.50 inches over the basin, and the second on March 1, averaged about 0.12 inch.

The discharge graphs for the storm of February 26 to March 10,1948 are shown in figure 28. The observed outflow curve is computed from gage readings and a rating for the dam. The period started with two stop logs in place in the sluiceway. One stop 


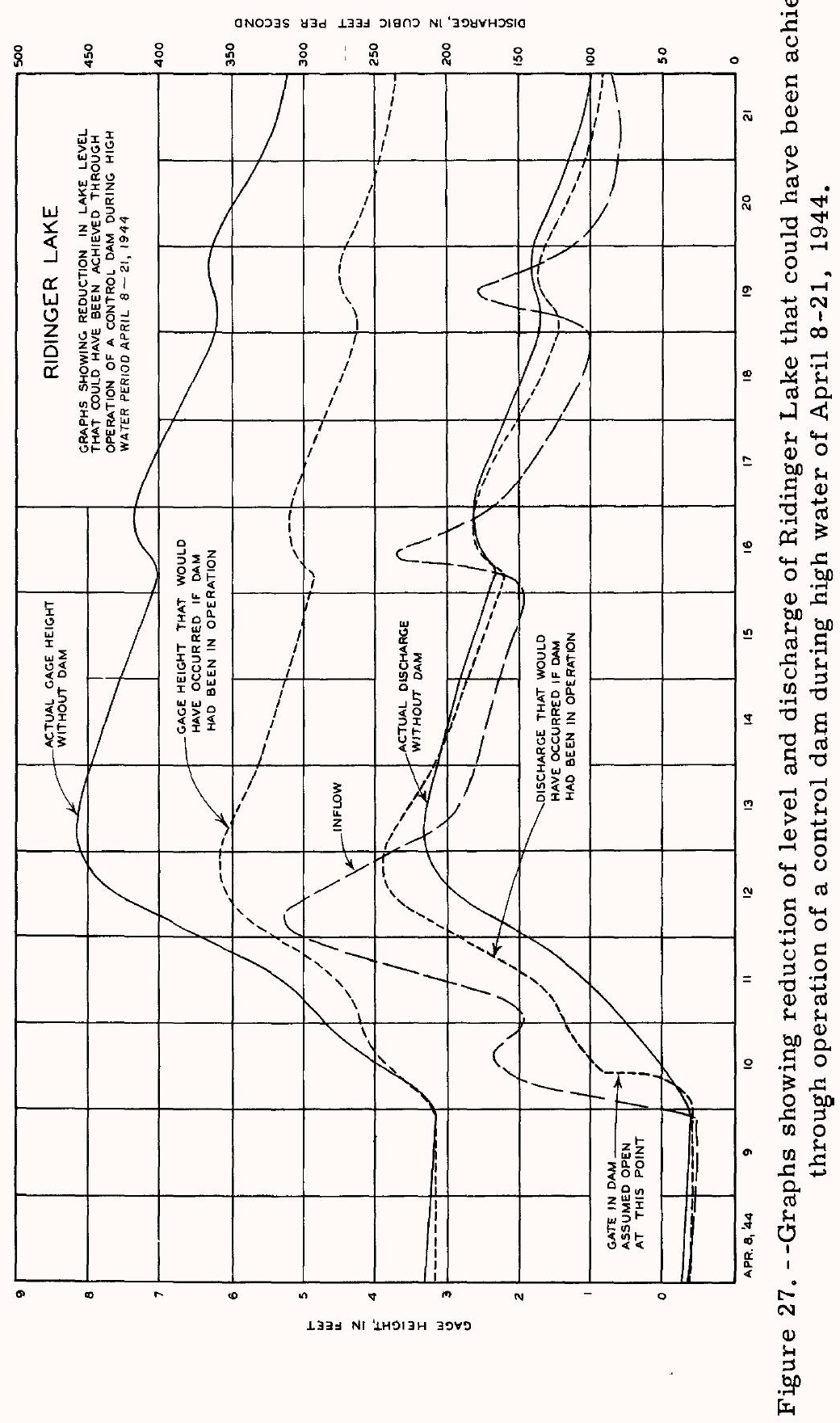




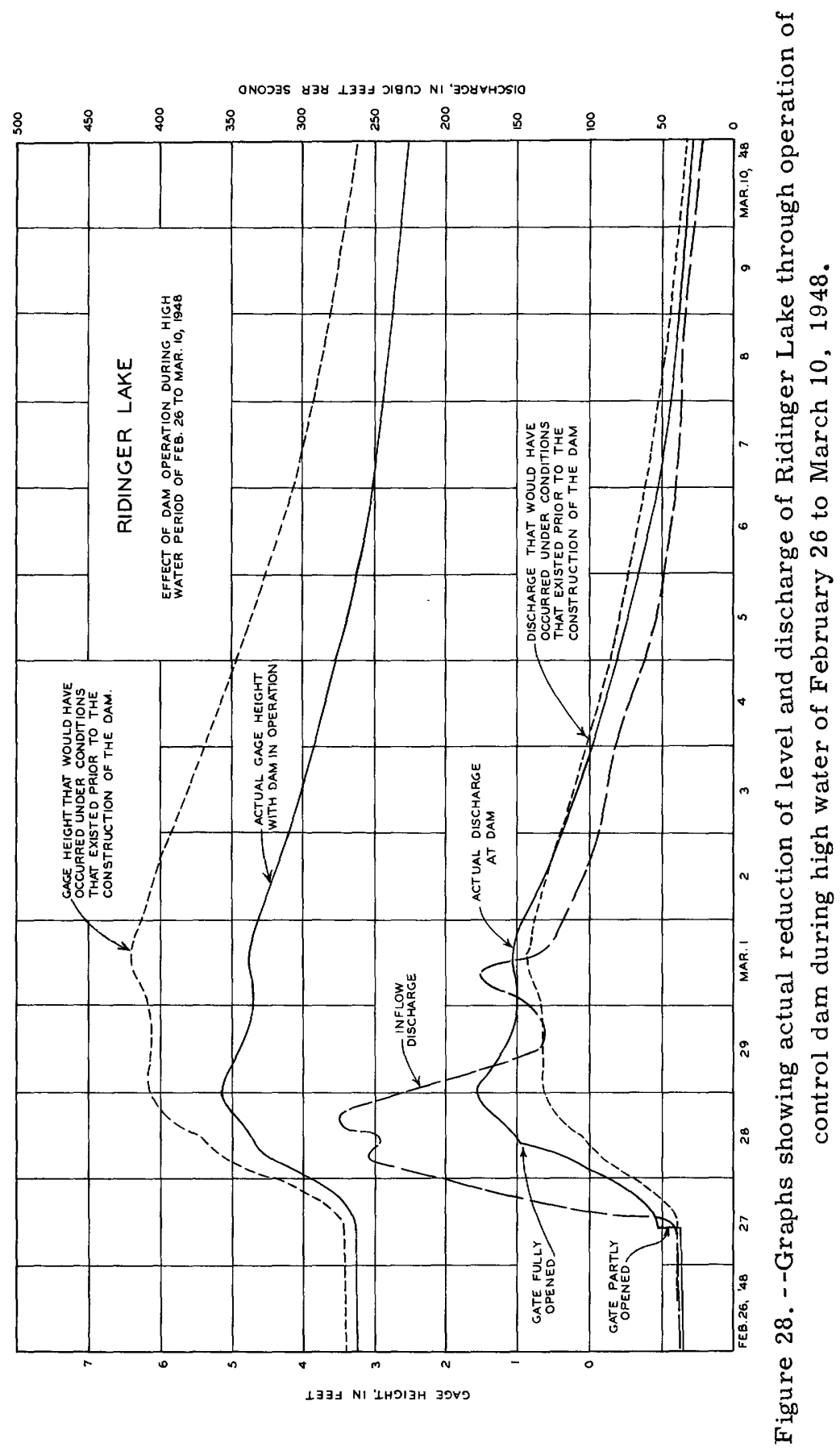


$\log$ was removed at 10 a.m. on February 27 and the other at 10 a. $m$. on February 28. No stop logs were in the sluiceway for the remainder of the period. The increase in discharge caused by the removal of the second stop log was not nearly as great as for the the removal of the first, because the second stop log was under a high degree of submergence at the stage at which it was removed. The inflow curve was computed from the outflow and rate of change in storage in the lake.

To determine the discharges that would have existed if the ditch had rot been cleaned and the dam built, the rating curve that applied before the cleaning was used to compute the new outflow and lake levels. The discharge curve obtained shows a smaller outflow during the first part of the storm period and later a greater outflow than existed with the dam. The peak discharge would be about $35 \mathrm{cfs}$ less than the $178 \mathrm{cfs}$ that occurred with the dam, and would occur a day and a half later. More water would go into storage during the rising stages and would be gradually released later.

The gage-height graphs in figure 28 show that the peak lake level without the dam would be 1.25 feet higher and would occur about a day and a half later. The lake levels on March 1, 2, and 3 would be about 1.5 feet higher than occurred on those days with the dam.

The lake levels could be lowered still more if the outlet had greater capacity. During the high water of February 29 and March 1,1948 , the outlet was filled to bank full and there was only about 3 inches fall over the dam. This caused the fixed spillway to operate under 83 percent submergence and thereby reduced its efficiency. The amount of water that could pass through the sluiceway also was reduced by the low head under which it operated; However, even under these adverse conditions, the peak lake level was 1.25 feet lower than it would have been if the outlet had not been cleaned.

Prior to the building of the dam, Ridinger Lake usually receded to a level of alout 1.5 feet on the gage during the lowest water period of the year, and during some years remained below 2.0 feet during the whole summer. These low levels were unsatisfactory from a recreational standpoint and the established level of 3.12 feet was much more desirable. During the summer of 1948, the first summer after the dam was built, the level remained between 3.00 and 3.30 feet from May 20 until November 16 and averaged about 3.15 feet for the period. Thus, a more desirable level was maintained throughout the whole low-water season. 


\section{BASIC DATA ON LAKE LEVELS FOR SELECTED LAKES}

The basic data collected at lake-level stations consists of readings of lake elevation, usually taken daily to the nearest hundreath of a foot, by a local observer. The records consist of daily, monthly, and yearly mean water-surface elevations and graphs showing the fluctuation in elevation. Discharge measurements made at the outflow are also available in some instances.

The lake records, except those contained herein, have not been published but are available in the files of the district office of the U. S. Geological Survey in Indianapolis, Ind. A list of stations where lake levels have been recorded may be found in the section Legal Lake Levels on page 266.

Records for 28 lakes have been selected for publication on the basis of completeness of record and general importance. The data for each station consists of a description of the station; tables listing the maximum and minimum lake level for each water year, the maximum recorded ice thickness and period of ice cover for each water year, discharge measurements made at the lake outlet, and average lake levels; and lake level hydrographs (figs. 29-139). The lake-level hydrographs were furnished by C. H. Bechert, director, Division of Water Resources, Department of Conservation, and show the variations in lake levels in each water year of record. A water year is the 12-month period ending September 30 of the indicated year.

The description of the station gives the location, surface area, drainage area, records available, type and history of gages, average level, the established legallevel, and the lake-level control. The location of the lake is obtained from the most accurate maps available. The surface area (when the lake is at the established legal level) and the drainage area, unless otherwise noted, are obtained from aerial photographs taken in 1938 by the Agricultural Adjustment Administration, Department of Agriculture (photographs are now in the custody of the Commodity Stabilization Service). Under "gage" are given the type, location, and datum of the present gage, the frequency of readings, and a history of past gages. In order to work with small numbers the gages have been set at datums differing from mean sea level by multiples of 10 feet. The elevation of the water surface above mean sea level may be determined by adding the gage reading to the gage datum. Under "Average level" is given the average lake level in gage height and in elevation above sea level for the number of years indicated. The established legal level is that elevation set by the courts to which the average level of the lake is to be held. It is normally set at about the average level that has prevailed for a number of years prior to the establishment of the legallevel. The means of maintaining the established level are described in the "Lake level control" paragraph. 
Bass Lake at Bass Lake, Ind.

Location. --Secs. 7 and 18, T. 32 N., R. 1 W. , and secs. 12, 13, 14,23 , and 24, T. 32 N., R. 2 W. , Starke County.

Surface area. $--1,405$ acres.

Drainage area. $-3.66 \mathrm{sq} \mathrm{mi}$.

Records available. --October 1942 to September 1953.

Gage. --Staff gage at south shore of lake at Fishburn's Store at Junction of State Highways 35 and 10. Gage read to hundredths once daily. Datum of gage is $700.00 \mathrm{ft}$ above mean sea level, datum of 1929 .

Average lake level. --11 years;gage height, $13.52 \mathrm{ft}$; elevation, $713.52 \mathrm{ft}$.

Established legal level. --Established Aug. 10, 1948, at gage height 13.65 ft; elevation, 713. $65 \mathrm{ft}$ above mean sea level.

Lake-level control. - - Lake level maintained by sheet-piling dam with crest $6.4 \mathrm{ft}$ long at gage height $14.08 \mathrm{ft}$. Prior to fall of 1946, control was an old concrete dam.

Extreme levels for Bass Lake, 1943-53

\begin{tabular}{|c|c|c|c|c|}
\hline \multicolumn{3}{|c|}{ Maximum } & \multicolumn{2}{|c|}{ Minimum } \\
\hline $\begin{array}{c}\text { Water } \\
\text { year }\end{array}$ & Date & $\begin{array}{c}\text { Gage } \\
\text { height } \\
\text { (feet) }\end{array}$ & Date & $\begin{array}{c}\text { Gage } \\
\text { height } \\
\text { (feet) }\end{array}$ \\
\hline 1943 & May $20,1943 \ldots$ & 14.04 & Oct. $27,1942 \ldots$ & 12.29 \\
\hline 1944 & Apr. $13,1944 \ldots$ & $* 14.15$ & Sept. 27, 1944 -- & 12.51 \\
\hline 1945 & June $22,23,1945-$ & 13.65 & Nov. $5,6,1944--$ & 12.33 \\
\hline 1946 & Mar. 19, $1946 \ldots$ & 14.01 & Sept. 30,1946 -- & 12.53 \\
\hline 1947 & June $19,1947 \ldots$ & 13.97 & Oct. $16,1946 \ldots$ & 12.23 \\
\hline 1948 & May 15, $1948 \ldots$ & 14.44 & Sept. 29, 30, 1948- & 13.06 \\
\hline 1949 & June $16,17,1949-$ & 14.14 & Oct. $29,30,1948-$ & 12.81 \\
\hline 1950 & Apr. 4, $1950 \ldots$ & 14.62 & Oct. 2, $1949 \ldots$ & 12.89 \\
\hline 1951 & May $11,1951 \ldots$ & 14.50 & Nov. $7-15,1950-$ & 13. 30 \\
\hline 1952 & Mar. 18, $1952 \ldots$ & 14.59 & Sept. 30, $1952 \ldots$ & 13. 19 \\
\hline 1953 & June $6-9,1953 \ldots$ & 13.63 & Sept. $30,1953-\cdots$ & 12.42 \\
\hline
\end{tabular}

* Estimated 
Maximum recorded thickness and periods of ice cover 1943-52

\begin{tabular}{|c|c|c|c|}
\hline $\begin{array}{l}\text { Water } \\
\text { year }\end{array}$ & $\begin{array}{l}\text { Maximum } \\
\text { thickness } \\
\text { (inches) }\end{array}$ & $\begin{array}{l}\text { Total } \\
\text { days of } \\
\text { cover }\end{array}$ & Period \\
\hline 1943 & ----- & 101 & Nov. 27, 1942, to Mar. 7, 1943. \\
\hline 1944 & 12 & 72 & Dec. 12,1943 , to Feb. 22, 1944. \\
\hline 1945 & 2 & $-\ldots$ & Dec. 5,1944 , to $-?$ \\
\hline 1946 & 10 & 50 & Jan. 10, 1946, to Feb. 28, 1946. \\
\hline 1947 & $-\cdots--$ & 73 & Dec. 18,1946 , to Feb. 28,1947 . \\
\hline 1948 & 24 & 131 & $\begin{array}{l}\text { Nov. } 6,1947 \text {, to Dec. } 6,1947 . \\
\text { Dec. } 10,1947 \text {, to Mar. } 18,1948 .\end{array}$ \\
\hline 1949 & 3 & 79 & Dec. 10,1948 , to Feb. 26,1949 \\
\hline 1950 & 3 & 81 & Jan. 4, 1949, to Mar. 25, 1950. \\
\hline 1951 & ----- & 95 & Nov. 23,1950 , to Feb. 25, 1951. \\
\hline 1952 & ----- & ---- & Dec. 2,1951 , to $-?$ \\
\hline
\end{tabular}

Discharge measurements at outlet of Bass Lake

\begin{tabular}{|c|c|c|c|c|c|}
\hline Date & $\begin{array}{c}\text { Gage } \\
\text { height } \\
\text { (feet }\end{array}$ & $\begin{array}{c}\text { Discharge } \\
\text { (cfs) }\end{array}$ & Date & $\begin{array}{r}\text { Gage } \\
\text { height } \\
\text { (feet) }\end{array}$ & $\begin{array}{c}\text { Discharge } \\
\text { (cfs) }\end{array}$ \\
\hline Aug. 6,1943 & 13.72 & 0.77 & Jan. 7, 1946- & 12.86 & 0 \\
\hline Oct. $12, \cdots$ & 13.53 & .05 & Feb. $8-\ldots$ & 13.73 & 1.20 \\
\hline Jan. 18,1944 & $a_{13.58}$ & 0 & Apr. $5 \ldots$ & 13.79 & 1.42 \\
\hline Mar. 17, - - & 14.05 & 12.00 & June $5 \ldots$ & 13.55 & 0 \\
\hline Apr. $25, \ldots$ & 14.10 & 15.0 & July $18 \ldots$ & 13.29 & 0 \\
\hline May $9, \ldots$ & 13.94 & 12.5 & July $24-\ldots$ & 13.19 & 0 \\
\hline June $11, \ldots$ & 13.49 & 0 & Nov. $12 \ldots$ & 12.69 & 0 \\
\hline June $23, \ldots$ & 13.63 & .60 & Dec. $12 \ldots$ & 12.69 & 0 \\
\hline July $11, \ldots$ & 13.29 & 0 & Dec. $20 \ldots$ & 12.76 & 0 \\
\hline Aug. $14, \ldots$ & 12.85 & 0 & Feb. 17, 1947- & 13.03 & 0 \\
\hline Sept. $12, \ldots-$ & 12.63 & 0 & Feb. 10, 1948 - & 13.51 & 0 \\
\hline Apr. $12, \ldots$ & 12.93 & 0 & May 3-...- & 14.12 & 0 \\
\hline May $14, \ldots$ & 13.19 & 0 & May $11 \div \ldots$ & 14.34 & 5.44 \\
\hline Aug. $21, \ldots$ & 13.12 & 0 & May 18..... & 14.39 & 3.73 \\
\hline Nov, $16, \ldots$ & 13.35 & $* .05$ & & & \\
\hline
\end{tabular}

* Estimated.

a Ice cover. 
Average lake level for Bass Lake for water years 1943-53

\begin{tabular}{l|c|c|c|c|c|c|c}
\hline Year & Oct. & Nov. & Dec. & Jan. & Feb. & Mar. & Apr. \\
\hline 1943 & $* 12.33$ & 12.53 & 12.68 & 13.07 & 13.34 & 13.52 & 13.65 \\
1944 & 13.60 & 13.72 & 13.69 & 13.61 & 13.67 & 13.93 & 14.00 \\
1945 & 12.53 & 12.39 & 12.43 & 12.48 & 12.50 & 12.72 & 13.02 \\
1946 & 13.36 & 13.37 & 13.49 & 13.73 & 13.74 & 13.89 & 13.80 \\
1947 & 12.39 & 12.68 & 12.72 & 12.92 & 13.03 & 13.08 & 13.37 \\
1948 & 13.30 & 13.33 & 13.46 & 13.59 & 13.57 & 13.90 & 14.13 \\
1949 & 12.92 & 12.94 & 13.02 & 13.42 & 13.84 & 14.02 & 14.04 \\
1950 & 13.12 & 12.99 & 13.16 & 13.78 & 14.23 & 14.45 & 14.48 \\
1951 & 13.52 & 13.33 & 13.58 & 13.78 & 13.86 & 14.11 & 14.31 \\
1952 & 13.61 & 13.92 & 14.18 & 14.36 & 14.33 & 14.36 & 14.42 \\
1953 & 13.00 & 12.80 & 12.92 & 12.93 & 13.01 & 13.16 & 13.40 \\
\hline Average & 13.06 & 13.09 & 13.21 & 13.42 & 13.56 & 13.74 & 13.87 \\
\hline \hline Year & May & June & July & Aug. & Sept. & Annual & \\
\hline 1943 & 13.86 & 13.89 & 13.74 & 13.77 & 13.82 & $* 13.43$ & \\
1944 & 13.90 & 13.66 & 13.24 & 12.86 & 12.62 & 13.54 & \\
1945 & 13.35 & 13.57 & 13.37 & 13.12 & 12.90 & 12.87 & \\
1946 & 13.67 & 13.66 & 13.37 & 12.95 & 12.60 & 13.47 & \\
1947 & 13.61 & 13.90 & 13.60 & 13.24 & 13.44 & 13.16 & \\
1948 & 14.25 & 14.07 & 13.92 & 13.60 & 13.22 & 13.70 & \\
1949 & 13.98 & 14.04 & 13.92 & 13.59 & 13.14 & 13.57 & \\
1950 & 14.20 & 14.25 & 14.29 & 14.04 & 13.75 & 13.89 & \\
1951 & 14.32 & 14.20 & 14.05 & 13.89 & 13.62 & 13.88 & \\
1952 & 14.33 & 14.32 & 13.94 & 13.71 & 13.38 & 14.07 & \\
1953 & 13.50 & 13.49 & 13.36 & 13.09 & 12.61 & 13.11 & \\
\hline Average & 13.91 & 13.91 & 13.71 & 13.44 & 13.19 & 13.52 & \\
\hline
\end{tabular}

* Partial month or partial year. 

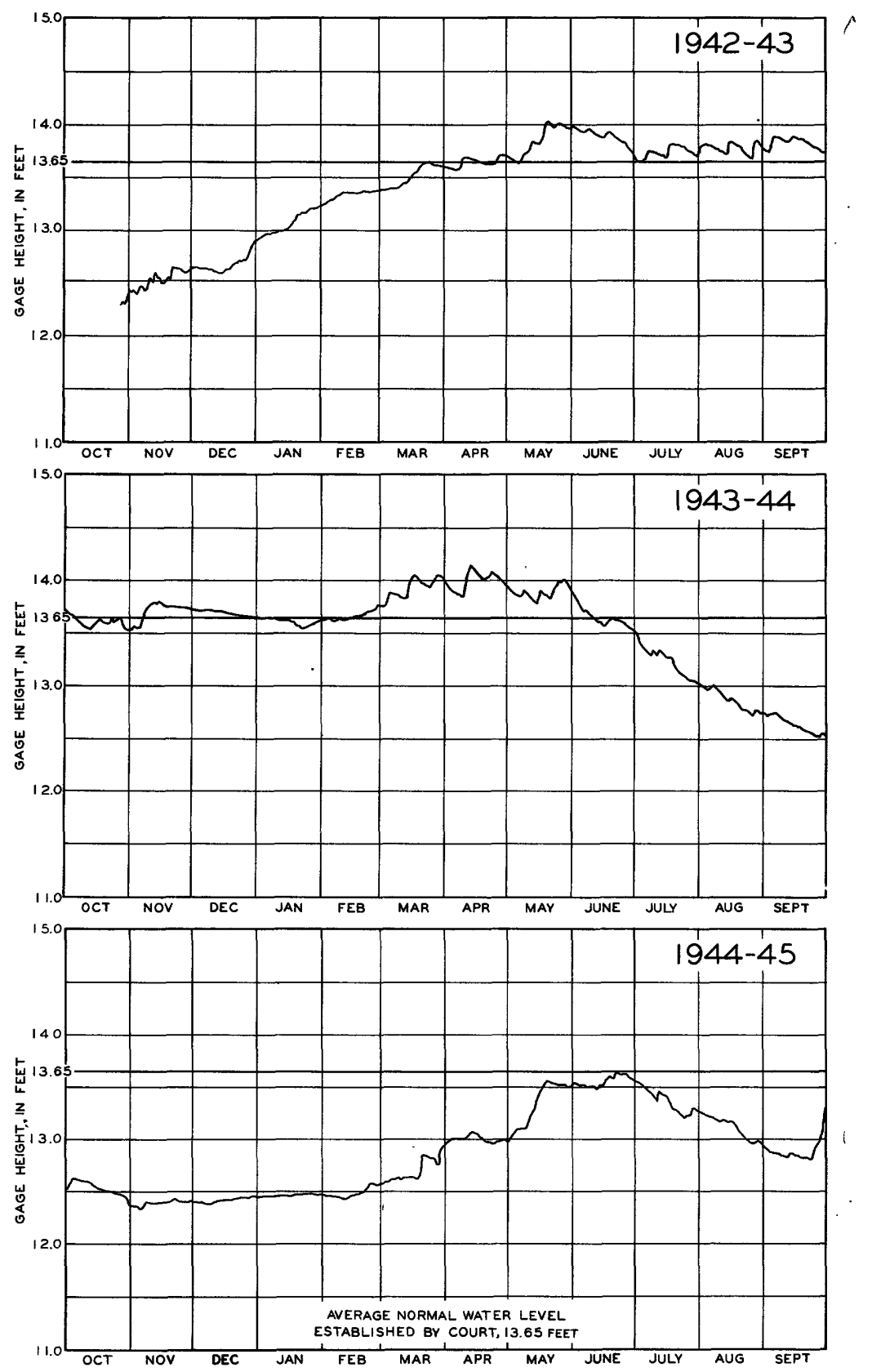

Figure 29. --Lake-level hydrographs for Bass Lake at Bass Lake, Ind., for water years 1943-45. 

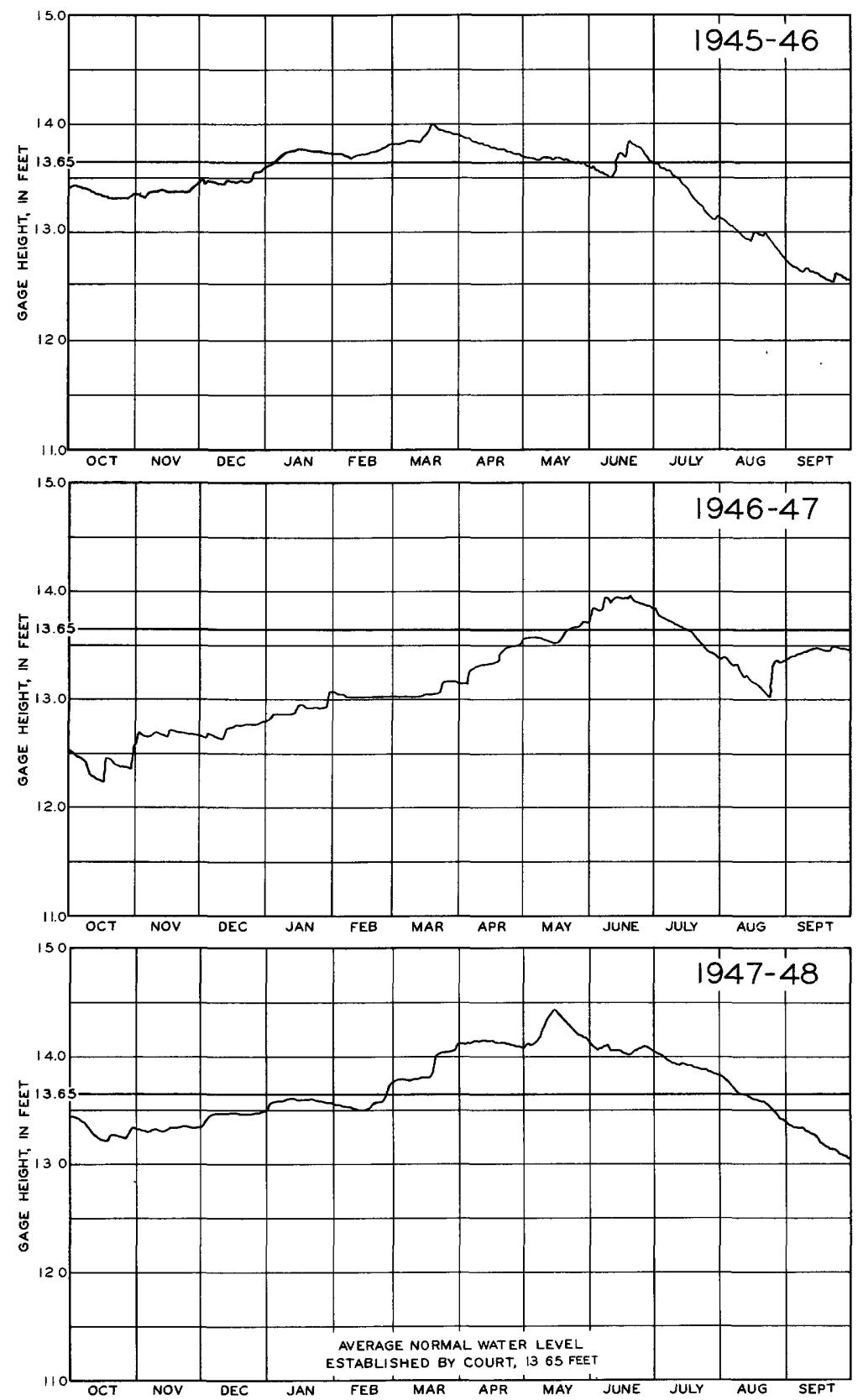

Figure 30. --Lake-level hydrographs for Bass Lake at Bass Lake, Ind., for water years 1946-48. 


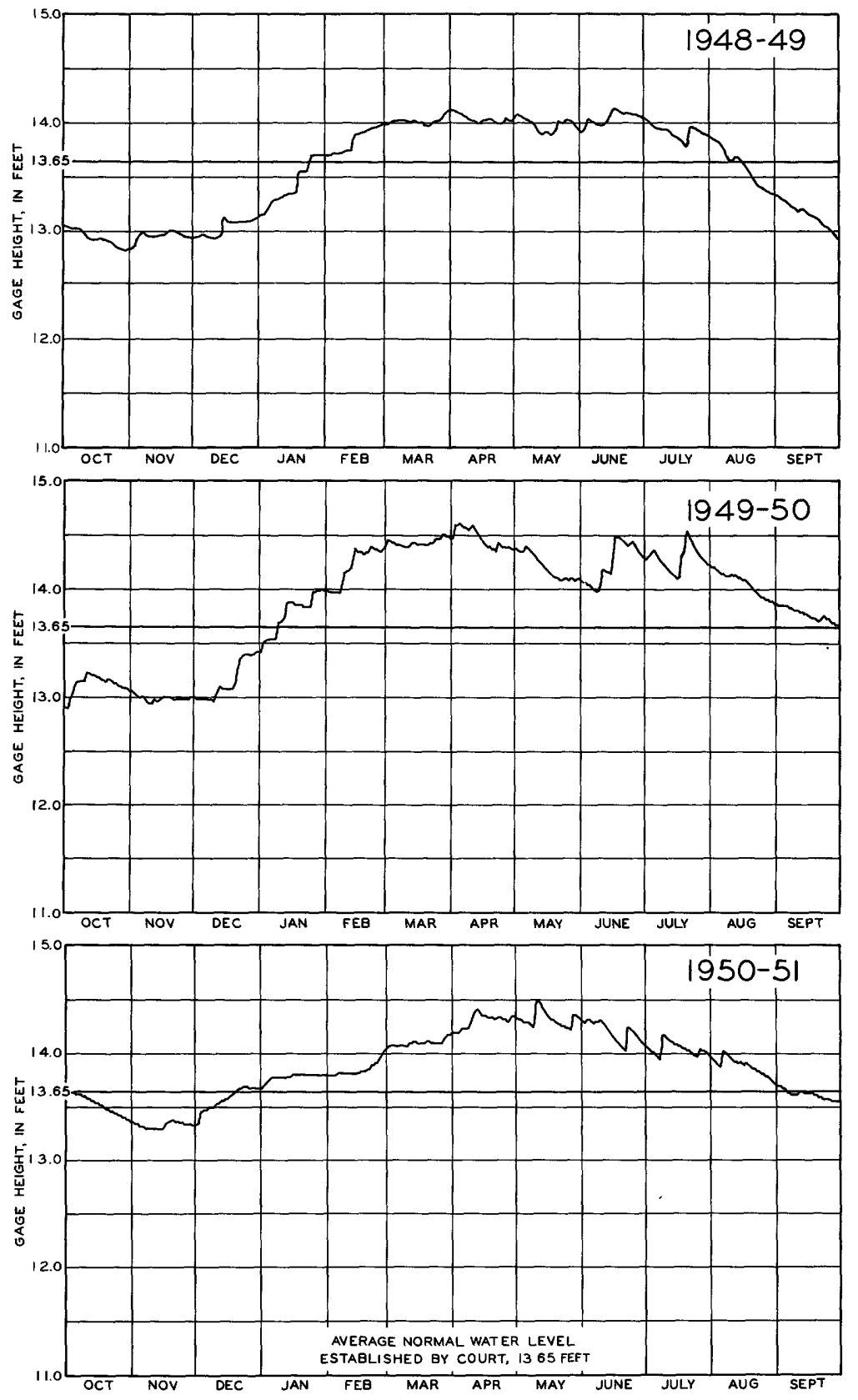

Figure 31. --Lake-level hydrographs for Bass Lake at Bass Lake, Ind., for water years 1949-51. 

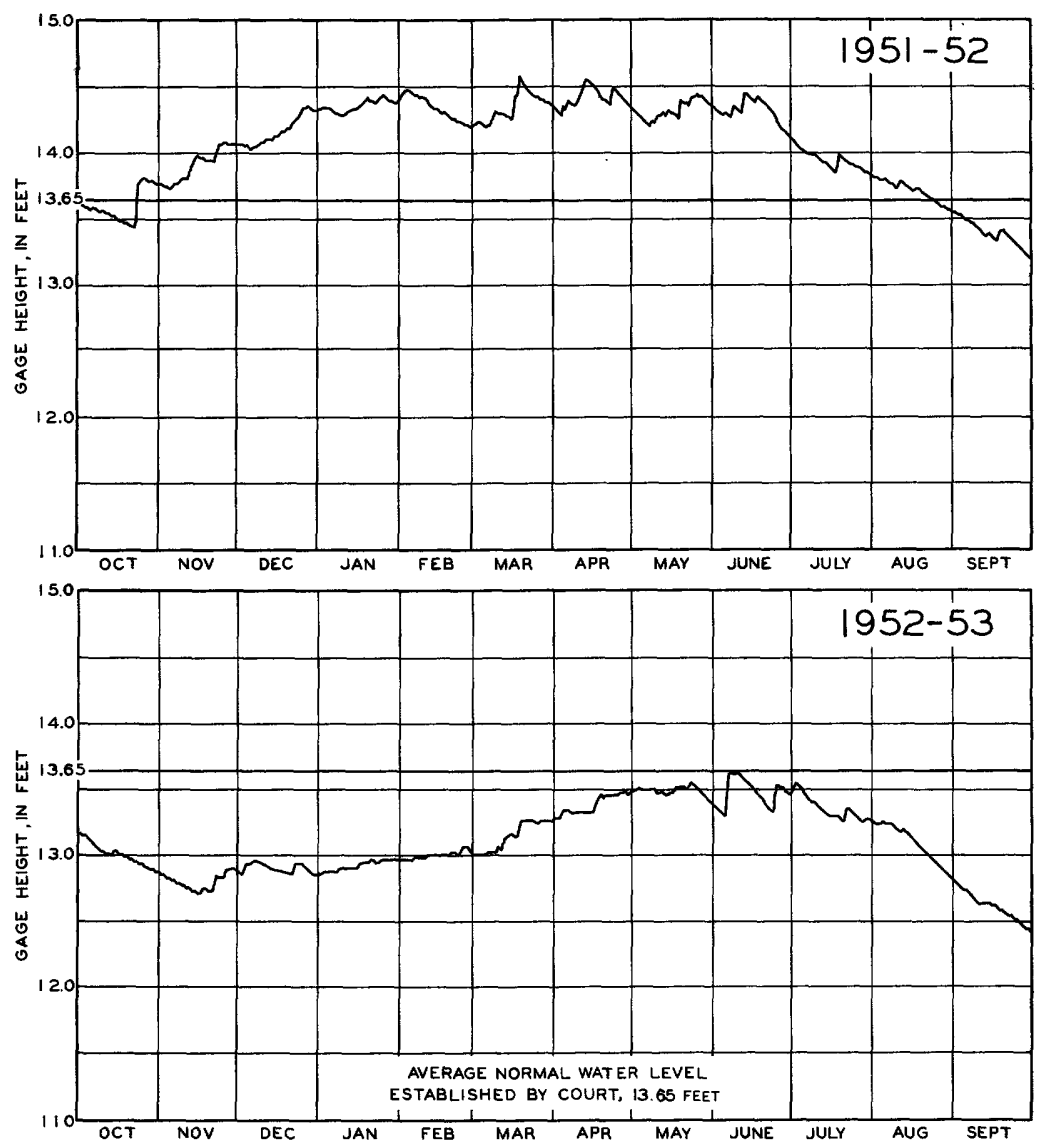

Figure 32. --Lake-level hydrographs for Bass Lake at Bass Lake, Ind., for water years 1952-53.

Bear Lake at Wolflake, Ind.

Location.--Secs. 7, 8, 17, and 18, T. 33 N., R. 9 E., Noble County; $1 \frac{1}{2}$ miles southwest of the town of Wolflake.

Surface area. -125 acres.

Drainage area. $-6.12 \mathrm{sq} \mathrm{mi}$.

Records available.. --November 1942 to September 1933, (no record Jan. 3, 1943, to Mar. 31, 1943).

Gage. - Staff gage on east shore of lake, $800 \mathrm{ft}$ north of outlet.

Gage read to hundredths once daily. Datum of gage is $890.00 \mathrm{ft}$ above mean sea level, datum of 1929. Prior to July 14, 1948, staff gage at north shore of lake at same datum.

Average lake level. --11 years; gage height, $4.81 \mathrm{ft}$; elevation, $894.81 \mathrm{ft}$.

Established legal level. - -None.

Lake-level control. --Lake level maintained by rock fill dam in outlet channel $380 \mathrm{ft}$ downstream from lake. 
Extreme levels for Bear Lake, 1943-53

\begin{tabular}{|c|c|c|c|c|}
\hline \multicolumn{3}{|c|}{ Maximum } & \multicolumn{2}{|l|}{ Minimum } \\
\hline $\begin{array}{c}\text { Water } \\
\text { year }\end{array}$ & Date & $\begin{array}{c}\text { Gage } \\
\text { height } \\
\text { (feet) }\end{array}$ & Date & $\begin{array}{c}\text { Gage } \\
\text { height } \\
\text { (feet) }\end{array}$ \\
\hline 1943 & Dec. $30,1942^{--}$ & 8.25 & July $3-6,1943 \ldots$ & $* 4.90$ \\
\hline 1944 & Apr. $12,1944^{--}$ & 6.61 & Sept. 26, 27, 1944- & 4.33 \\
\hline 1945 & Mar. $31,1945^{--}$ & $* 5.82$ & Nov. $1-3,1944 \ldots$ & 4.23 \\
\hline 1946 & Jan. $12,1946--$ & 5.90 & Sept. $29,30,1946$ & 3.90 \\
\hline 1947 & Apr. $22,1947^{--}$ & $* 5.29$ & Oct. $14-17,1946--$ & 3.74 \\
\hline 1948 & Mar. $6,1948^{--}$ & 5.50 & Sept. $28,1948 \ldots$ & 3.93 \\
\hline 1949 & Feb. $16,1949--$ & 5.50 & Oct. 30, Nov. 2,1948 & 3.78 \\
\hline 1950 & Apr. 5, $1950---$ & 6.06 & Oct. $2,1949 \ldots$ & 4.23 \\
\hline 1951 & Feb. 22, 1951-- & 5.70 & Sept. 30, $1951 \ldots$ & 4.08 \\
\hline 1952 & May 27, 1952--- & 5.36 & Sept. 29, 30, 1952- & 3.38 \\
\hline 1953 & Mar. 19, 1953-- & 5.48 & $\begin{array}{l}\text { Oct. } 31 \text { to Nov. } 3 \text {, } \\
\text { Nov. } 7-17,1952-\end{array}$ & $* 2.90$ \\
\hline
\end{tabular}

*Estimated.

Maximum recorded thickness and periods of ice cover for Bear Lake, 1943-53

\begin{tabular}{|c|c|c|c|}
\hline $\begin{array}{l}\text { Water } \\
\text { year }\end{array}$ & $\begin{array}{c}\text { Maximum } \\
\text { thickness } \\
\text { (inches) }\end{array}$ & $\begin{array}{l}\text { Total } \\
\text { days of } \\
\text { cover }\end{array}$ & Period \\
\hline 1943 & 6 & $--\cdots--$ & Dec. 3,1942, to-? \\
\hline 1944 & $-\cdots---$ & 98 & Dec. 5, 1943, to Mar. $12,1944$. \\
\hline 1945 & ------- & 81 & Dec. 14,1944 , to Mar. 4, 1945. \\
\hline 1946 & 8 & 87 & Dec. 12,1945 , to Mar. 8, 1946. \\
\hline 1947 & 11 & 101 & $\begin{array}{l}\text { Dec. } 19-24 ; \text { Dec. } 30,1946 \text {, to } \\
\text { Apr. } 3,1947 .\end{array}$ \\
\hline 1948 & 15 & 94 & Dec. 17,1947 , to Mar. $19,1948$. \\
\hline 1949 & 5 & 94 & Dec. 17, 1948, to Mar. 21, 1949. \\
\hline 1950 & 8 & 100 & Dec.. 6, 1949, to Mar. 24, 1950. \\
\hline 1951 & 20 & 81 & Dec. 11,1950 , to Mar. 1, 1951 \\
\hline 1952 & -- & 81 & Dec. 23, 1951, to Mar. 13, 1952. \\
\hline 1953 & 5 & 54 & $\begin{array}{l}\text { Dec. } 31,1952 \text {, to Feb. } 22,1953 \text {; } \\
\text { Mar. 8-12, } 1953 \text {. }\end{array}$ \\
\hline
\end{tabular}


Discharge measurements at outlet of Bear Lake

\begin{tabular}{l|c|c||c|c|c}
\hline \multicolumn{1}{c|}{ Date } & $\begin{array}{c}\text { Gage } \\
\text { height } \\
\text { (feet) }\end{array}$ & $\begin{array}{c}\text { Discharge } \\
\text { (cfs) }\end{array}$ & Date & $\begin{array}{c}\text { Gage } \\
\text { height } \\
\text { (feet) }\end{array}$ & $\begin{array}{c}\text { Discharge } \\
\text { (cfs) }\end{array}$ \\
\hline Aug. 12, 1943 & 5.17 & 0.35 & Jan. 20, 1947- & 2.74 & 0 \\
Oct. 8----- & 4.93 & .20 & Mar. 4, 1948- & 5.35 & 14.8 \\
Feb.16, 1944 & 5.05 & $: 95$ & Mar. 4, 1949- & 4.92 & 6.67 \\
Mar.31----- & 4.83 & $* 4$ & Sept. 7----- & 4.40 & 0 \\
Aug.11----- & 4.60 & 0 & Dec. 1----- & 4.60 & 1.03 \\
July 11, 1945 & 4.77 & .10 & Jan. 17, 1950 & 5.64 & 27.5 \\
Sept. 4---- & 5.09 & $* .10$ & Mar. 8---- & 5.32 & 20.7 \\
Dec.19----- & 5.55 & 5.57 & May 4----- & 4.95 & 8.39 \\
May 17, 1946 & 5.18 & .05 & Sept. 13----- & 4.86 & 6.69 \\
June 19---- & 5.24 & 10.4 & Nov. 21----- & 4.79 & 6.51 \\
July 3---- & 4.89 & .80 & Nay 15, 1951- & 4.83 & 8.75 \\
\hline
\end{tabular}

* Estimated.

Average lake level for Bear Lake for water years 1943-53

\begin{tabular}{|c|c|c|c|c|c|c|c|}
\hline Year & Oct. & Nov. & Dec. & Jan. & Feb. & Mar. & Apr. \\
\hline 1943 & -- & $* 7.94$ & 7.93 & 6.82 & 5.45 & 5.29 & 5.48 \\
\hline 1944 & 4.91 & 4.92 & 5. 14 & 5.06 & 5.07 & 5.35 & 5.79 \\
\hline 1945 & 4. 36 & 4. 33 & 4. 44 & 4.66 & 4.94 & 5.59 & 4.73 \\
\hline 1946 & 5.49 & 5.44 & 5.41 & 5.48 & 5.39 & 5.39 & 5.17 \\
\hline 1947 & 3.80 & 4.04 & 4.09 & 4.42 & 4.96 & 4.89 & 5.10 \\
\hline 1948 & 4.74 & 4.83 & 5.04 & 5.09 & 5.11 & 5.24 & 4.92 \\
\hline 1949 & 3.86 & 4.00 & 4.25 & 4.98 & 5.09 & 4.84 & 4.78 \\
\hline 1950 & 4.54 & 4.53 & 4. 74 & 5. 51 & 5.24 & 5.23 & 5.44 \\
\hline 1951 & 4.55 & 4.63 & 4.99 & 4.86 & 5.03 & 4.87 & 4.90 \\
\hline 1952 & 4.20 & 4.50 & 4.56 & 4.92 & 4.78 & 4.72 & 4.74 \\
\hline 1953 & 3.14 & 3.05 & 3.73 & 4.62 & 5.18 & 5.34 & 5.14 \\
\hline Average & 4.36 & 4.75 & 4.94 & 4.96 & 5.08 & 5.15 & 5.11 \\
\hline Year & May & June & July & Aug. & Sept. & Annual & \\
\hline 1943 & 5.89 & 5.17 & 5.11 & 5.23 & 5.16 & $* 5.84$ & \\
\hline 1944 & 5.16 & 4.96 & 4. 96 & 4. 56 & 4.42 & 5.02 & \\
\hline 1945 & 4.83 & 4.88 & 4.75 & 5.07 & 5.07 & 4.80 & \\
\hline 1946 & 5.27 & 5.22 & 4.68 & 4.35 & 4.04 & 5.11 & \\
\hline 1947 & 5.06 & 4.98 & 4.78 & 4.64 & 4.72 & 5.62 & \\
\hline 1948 & 4.99 & 4.80 & 4.68 & 4.43 & 4.08 & 4.83 & \\
\hline 1949 & 4.77 & 4.96 & 4.64 & 4. 44 & 4.33 & 4.58 & \\
\hline 1950 & 4.80 & 4.94 & 4.84 & 4.65 & 4.86 & 4.94 & \\
\hline 1951 & 4. 69 & 4.56 & 4.96 & 4.52 & 4.24 & 4.73 & \\
\hline 1952 & 4. 60 & 4. 60 & 4.24 & 3.86 & 3.51 & 4.44 & \\
\hline 1953 & 5.10 & 4. 81 & 4.52 & 4.41 & 3.86 & 4.41 & \\
\hline Average & 5.01 & 4.90 & 4.74 & 4.56 & 4.39 & 4.81 & \\
\hline
\end{tabular}

* Partial month or partial year. 

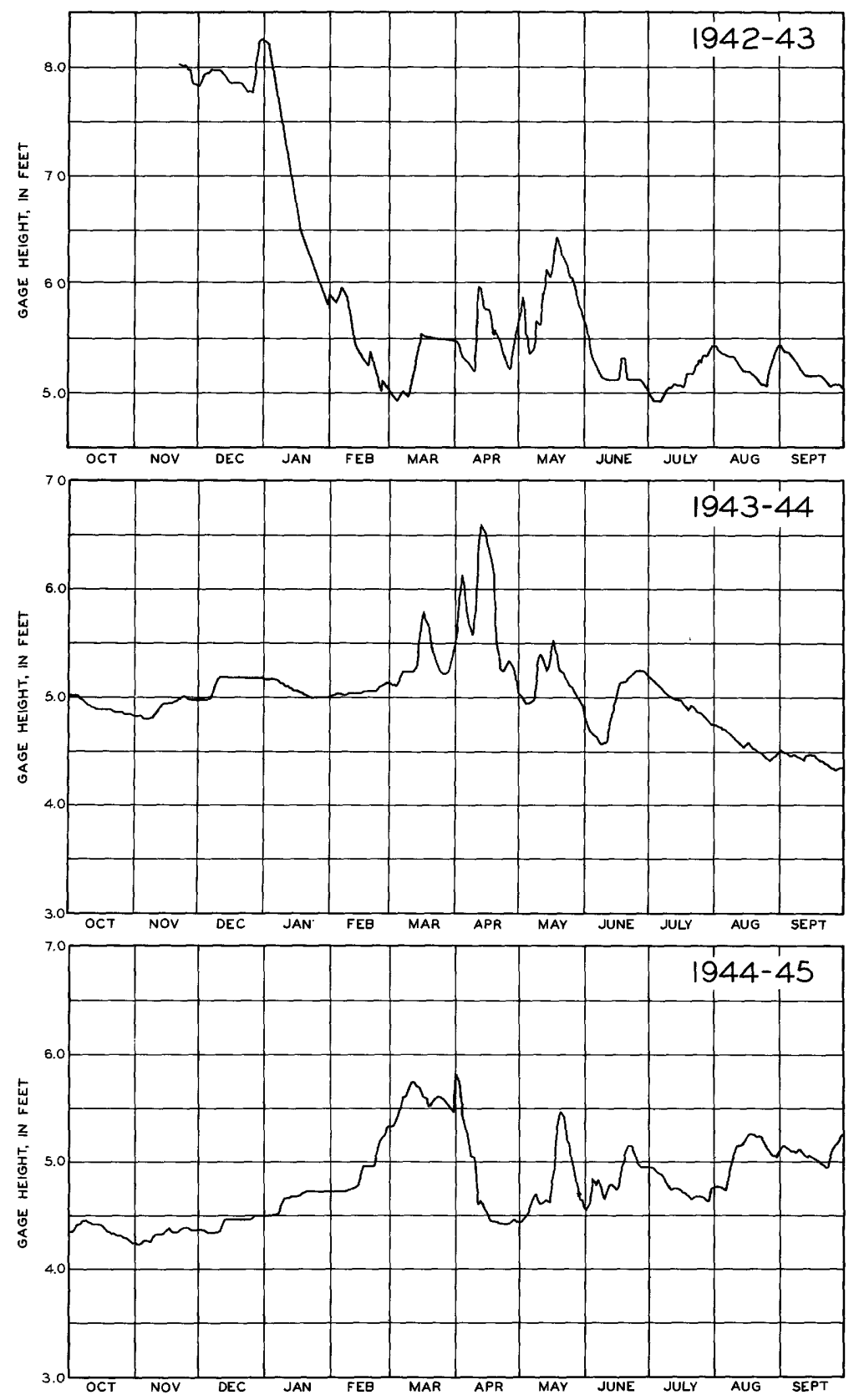

Figure 33. --Lake-level hydrographs for Bear Lake at Wolflake, Ind., for water years 1943-45. 

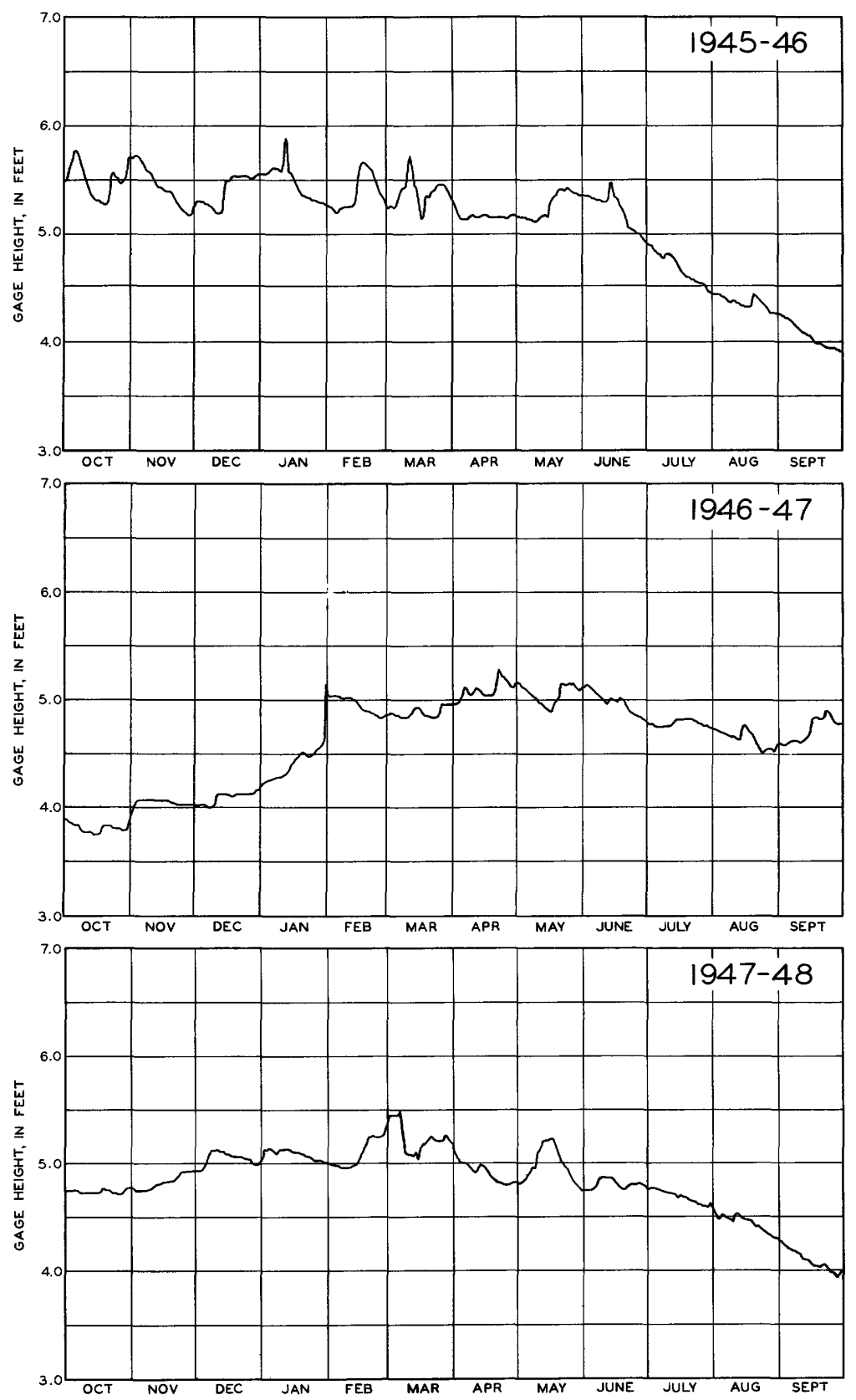

Figure 34. --Lake-level hydrographs for Bear Lake at Wolflake, Ind., for water years 1946-48. 

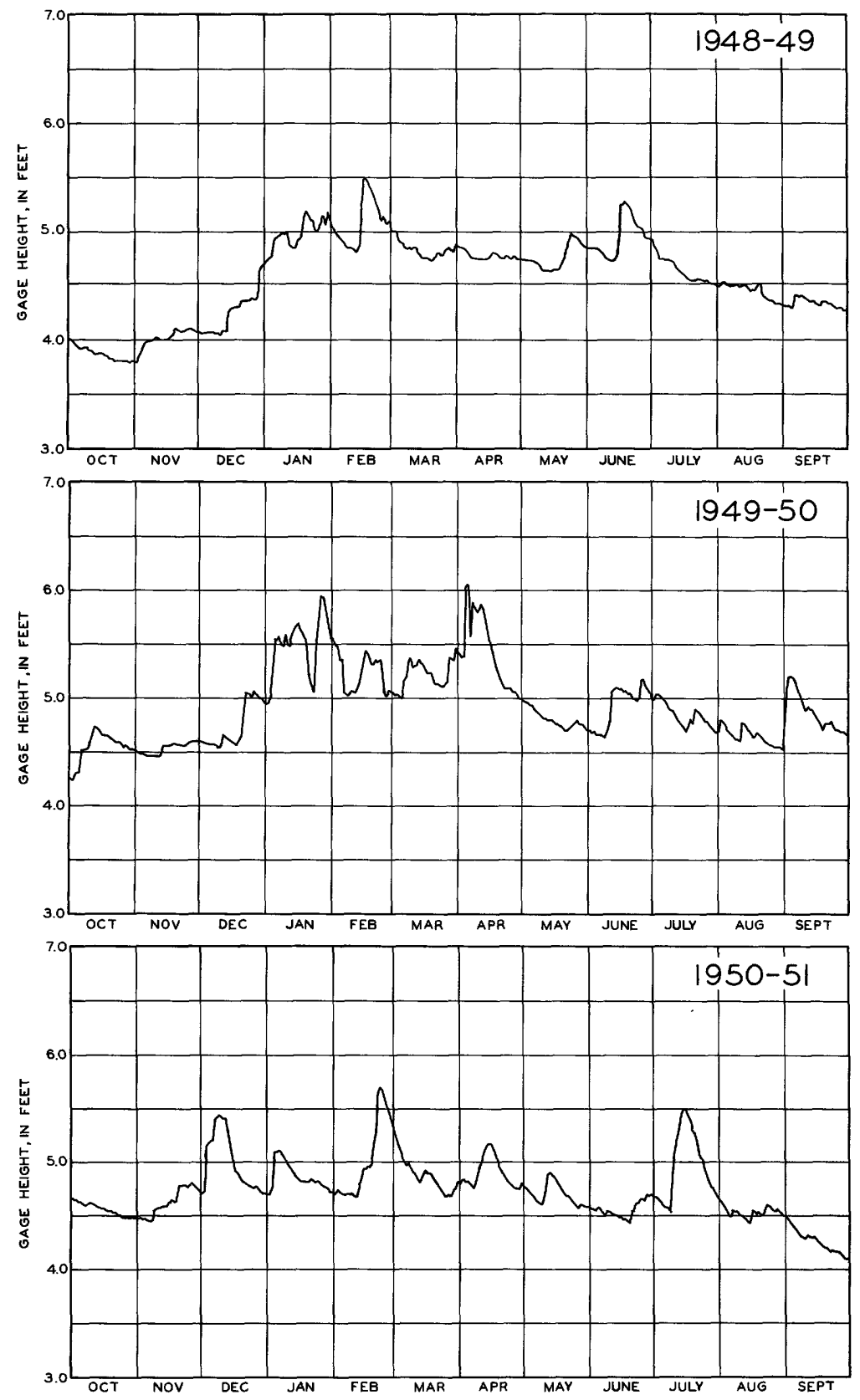

Figure 35. --Lake-level hydrographs for Bear Lake at Wolflake, Ind., for water years 1949-51. 

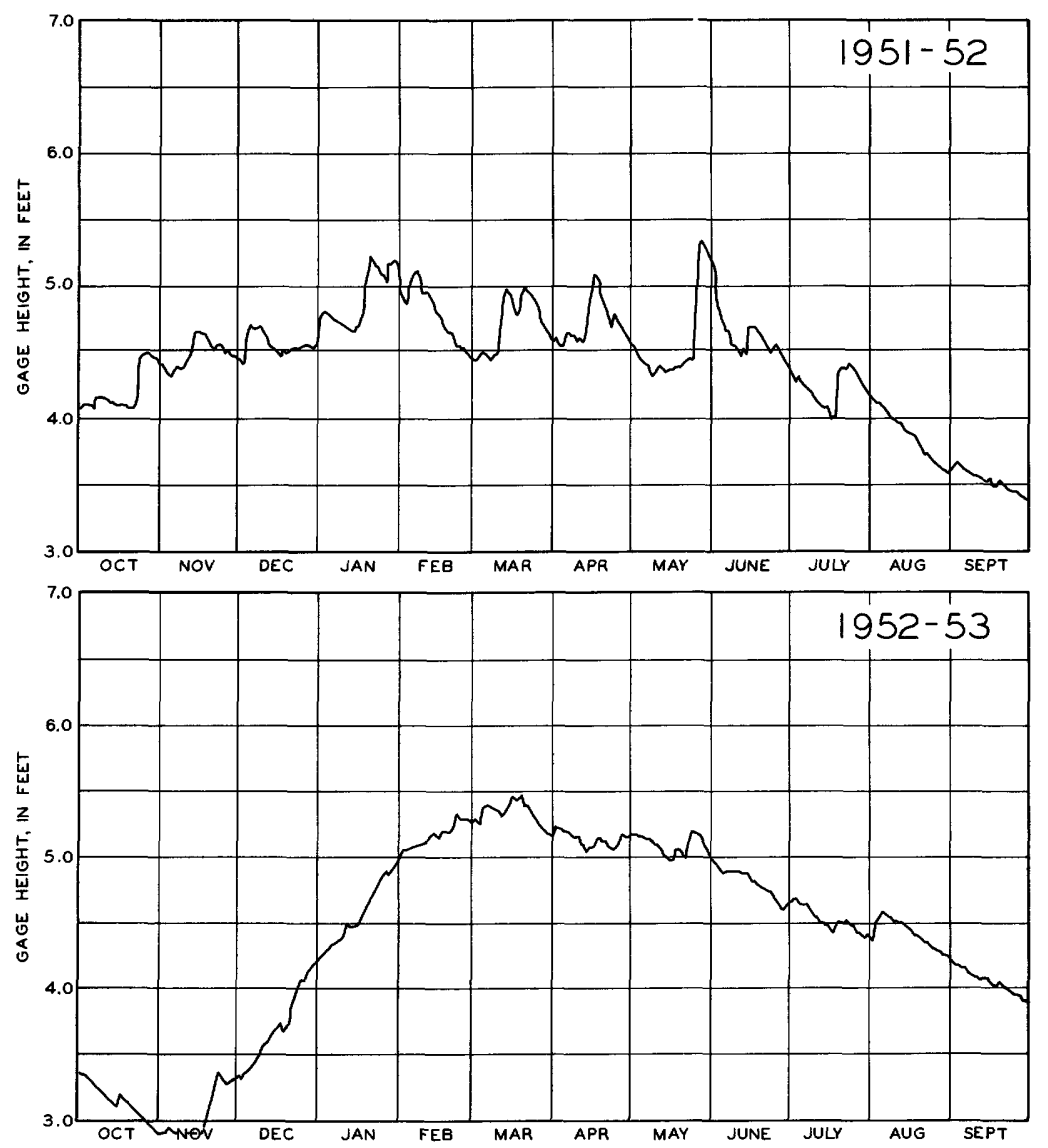

Figure 36. --Lake-level hydrographs for Bear Lake at Wolflake, Ind., for water years 1952-53. 
Big Lake near Wolflake, Ind.

Location.--Secs. 32 , and 33, T. 33 N., R. 9 E., Noble County, 3.5 miles south and 0.3 mile southwest of the town of Wolflake.

Surface area. --204 acres.

Drainage area. $--6.77 \mathrm{sq} \mathrm{mi}$.

Records available. --August 1943 to September 1953 (no record, Jan. 1, 1944, to Feb. 15, 1944).

Gage. - Staff gage on piling in lake at northwest shore. Gage read to hundredths once daily. Datum of gage is $890.00 \mathrm{ft}$ above mean sea level, datum of 1929 .

Average lake level. - - 10 years; gage height, $9.22 \mathrm{ft}$; elevation, $899.22 \mathrm{ft}$.

Established legal level. --None.

Lake-level control. - - Lake level maintained by the bed of outlet channel.

Extreme levels for Big Lake, 1944-53

\begin{tabular}{|c|c|c|c|c|}
\hline \multicolumn{3}{|c|}{ Maximum } & \multicolumn{2}{|l|}{ Minimum } \\
\hline $\begin{array}{c}\text { Water } \\
\text { year }\end{array}$ & Date & $\begin{array}{c}\text { Gage } \\
\text { height } \\
\text { (feet) }\end{array}$ & Date & $\begin{array}{c}\text { Gage } \\
\text { height } \\
\text { (feet) }\end{array}$ \\
\hline $1943^{\bullet}$ & Aug. $4,5,1943-$ & $* 9.20$ & Aug. 24, $1943 \ldots$ & 8.79 \\
\hline $1944 b$ & Apr. 16, 1944-- & 11.65 & Sept. $29,30,1944 \ldots$ & 8.51 \\
\hline 1945 & May $19,20,1945$ & 11.29 & Oct. 31 to Nov. 2,1944 & 8.47 \\
\hline 1946 & Oct. $7,1945 \ldots$ & 9.95 & Sept. $30,1946 \ldots$ & 8.02 \\
\hline 1947 & Apr. $23,1947 \ldots$ & 11.18 & Oct. $17,1946 \ldots$ & 7.82 \\
\hline 1948 & Mar. 29, 1948-... & 11.01 & Sept. 28, $1948 \ldots$ & 8.70 \\
\hline 1949 & Feb. $18,19,1949 \ldots$ & 11.36 & Oct. 29 to Nov. 2,1948 & 8.59 \\
\hline 1950 & Apr. 4, 1950- & $* 12.76$ & Oct. $1-3,1949 \ldots$ & 9.02 \\
\hline 1951 & Oct. $1,1950 \ldots$ & 9.96 & Sept. 29, 30, $1951 \ldots$ & 7.80 \\
\hline 1952 & Jan. $28,1952 \ldots$ & 9.88 & Sept. 17, $1952 \ldots$ & $* 7.66$ \\
\hline 1953 & May 24-26, $1953 \ldots$ & 8.60 & Sept. 22-30, $1953 \ldots$ & 7.32 \\
\hline
\end{tabular}

* Estimated.

a August and September.

b Jan. 15, 1944, to Feb. 15, 1944, missing record. 
Maximum recorded thickness and periods of ice cover for Big Lake, 1944-52

\begin{tabular}{|c|c|c|c|}
\hline $\begin{array}{l}\text { Water } \\
\text { year }\end{array}$ & $\begin{array}{l}\text { Maximum } \\
\text { thickness } \\
\text { (inches) }\end{array}$ & $\begin{array}{l}\text { Total } \\
\text { days of } \\
\text { cover }\end{array}$ & Period \\
\hline 1944 & $1 \frac{1}{2}$ & $-\cdots--$ & \\
\hline 1945 & ----- & $---1-$ & \\
\hline 1946 & 1 & 79 & Dec. 17,1945 , to Mar. 5, 1946. \\
\hline 1947 & $9 \frac{1}{2}$ & 101 & $\begin{array}{c}\text { Dec. } 15-26 ; \text { Dec. } 31,1946 \text {, to } \\
\text { Mar. 29, } 1947 .\end{array}$ \\
\hline 1948 & 15 & 70 & Dec. 17, 1947, to Feb. 24, 1948. \\
\hline 1949 & 3 & $-\ldots-$. & Dec. 25,1948 , to $-?$ \\
\hline 1950 & 6 & 100 & Dec. 15, 1949, to Mar. 24, 1950. \\
\hline 1951 & $---\cdots$ & ----- & Nov. 27,1950 to $-?$ \\
\hline 1952 & $-\cdots---$ & $-\cdots--$ & Dec. 14,1951 , to $-?$ \\
\hline
\end{tabular}

Record for period from Jan. 15, 1944, to Feb. 15, 1944 missing.

Discharge measurements at outlet of Big Lake

\begin{tabular}{|c|c|c|c|c|c|}
\hline Date & $\begin{array}{l}\text { Gage } \\
\text { height } \\
\text { (feet) }\end{array}$ & $\begin{array}{c}\text { Discharge } \\
\text { (cfs) }\end{array}$ & Date & $\begin{array}{l}\text { Gage } \\
\text { height } \\
\text { (feet) }\end{array}$ & $\begin{array}{c}\text { Discharge } \\
\text { (cfs) }\end{array}$ \\
\hline Aug. $3,1943-$ & 17 & 2.04 & Jan. 15,1950 & 12.08 & 57.2 \\
\hline Oct. $\quad 8-\cdots$ & 8.70 & .44 & Jan, $20 \cdots-\cdots$ & 11.88 & 47.6 \\
\hline Feb. $16,1944-$ & 8.71 & .46 & Jan. 27-... & 12.26 & 57.9 \\
\hline Aug. $11 \ldots$ & 8.77 & .00 & Feb. $7 \ldots$ & 11.02 & 30.1 \\
\hline Apr. $10,1945-$ & 10.43 & 29.3 & Feb. 22- - - & 11.34 & 35.0 \\
\hline July $11 \ldots$ & 9.14 & 2.53 & Mar 24-... & 11.09 & 28.0 \\
\hline Sept. $6 \ldots-\ldots$ & 8.97 & 1.03 & Apr. 8-..- & 12.52 & 60.3 \\
\hline Nov. $28 \ldots$ & 8.87 & 1.89 & May 3-..- & 10.43 & 19.2 \\
\hline Dec. $17 \ldots$ & 8.77 & 1.04 & May 24-.-.- & 9.74 & 4.99 \\
\hline May $15,1946-$ & 8.76 & 1.84 & Sept.13-...- & 9.92 & 1.52 \\
\hline Oct. $\quad 1 \ldots$ & 8.00 & .00 & Oct. $17-\ldots$ & 9.63 & 2.08 \\
\hline Mar. $9,1949-$ & 10.22 & 17.0 & Nov. $1-\cdots$ & 9.19 & 14.7 \\
\hline Apr. $27 \ldots \ldots$ & 9.48 & 6.24 & Nov. 21---- & 8.66 & 9.11 \\
\hline Sept. $6 \ldots$ & 9.22 & .58 & Feb. 20, 1951 & 9.27 & 37.6 \\
\hline Dec. $1 \ldots$ & 9.28 & .90 & May 15-...- & 8.64 & 15.5 \\
\hline Dec. $19 \ldots \ldots$ & 9.40 & 2.43 & & & \\
\hline
\end{tabular}


Average lake level for Big Lake for water years 1943-53

\begin{tabular}{|c|c|c|c|c|c|c|c|}
\hline Year & Oct. & Nov. & Dec. & Jan. & Feb. & Mar. & Apr. \\
\hline 1944 & 8.65 & 8.73 & 8.65 & $* 8.60$ & $* 8.76$ & 9.78 & 10.75 \\
\hline 1945 & 8.57 & 8.59 & 8.66 & 8.74 & 8.97 & 9.50 & 9.98 \\
\hline 1946 & 9.41 & 8. 90 & 8.84 & 9.46 & 9.41 & 9.60 & 9.04 \\
\hline 1947 & 7.93 & 8.18 & 8.32 & 8.80 & 9.43 & 9.46 & 10.53 \\
\hline 1948 & 9.01 & 9.06 & 9.33 & 9.51 & 9.56 & 10.52 & 10.28 \\
\hline 1949 & 8.67 & 8.80 & 9.01 & 10.17 & 10.72 & 9.99 & 9.49 \\
\hline 1950 & 9.47 & 9.34 & 9.79 & 11.71 & 11.30 & 11.25 & 11.76 \\
\hline 1951 & 9.68 & 8.69 & 8.97 & 9.14 & 9.10 & 8.63 & 8.69 \\
\hline 1952 & 7.92 & 8.25 & 8.40 & 9.15 & 8.87 & 8.52 & 8.41 \\
\hline 1953 & 7.64 & 7.50 & 7.87 & 8. 22 & 8. 20 & 8.38 & 8.26 \\
\hline Average & 8.70 & 8. 60 & 8.78 & 9.35 & 9.43 & 9.56 & 9.72 \\
\hline Year & May & June & July & Aug. & Sept. & Annual & \\
\hline 1943 & & --- &.-- & $* 8.98$ & 8.90 & $* 8.94$ & \\
\hline 1944 & 10.39 & 9.81 & 9.17 & 8.75 & 8.59 & 9.22 & \\
\hline 1945 & 9.99 & 9.72 & 9.15 & 9.33 & 8.95 & 9.18 & \\
\hline 1946 & 8.80 & 9.09 & 9.01 & 8.51 & 8.18 & 9.02 & \\
\hline 1947 & 10.18 & 9.86 & 9.50 & 9.12 & 9.11 & 9.20 & \\
\hline 1948 & 10.11 & 9.90 & 9.69 & 9.32 & 8.86 & 9.60 & \\
\hline 1949 & 9.53 & 10.09 & 9.82 & 9.26 & 9.12 & 9.55 & \\
\hline 1950 & 9.97 & 10.11 & 10.23 & 9.98 & 9.90 & 10.40 & \\
\hline 1951 & 8.40 & 8.16 & 8.47 & 8.20 & 7.97 & 8.67 & \\
\hline 1952 & 8.26 & 8. 35 & 8.44 & 8.11 & 7.86 & 8.37 & \\
\hline 1953 & 8.39 & 8. 15 & 7.73 & 7.68 & 7. 37 & 7.95 & \\
\hline Average & 9.40 & 9.32 & 9.12 & 8.84 & 8.62 & 9.22 & \\
\hline
\end{tabular}

* Partial month or partial year. 

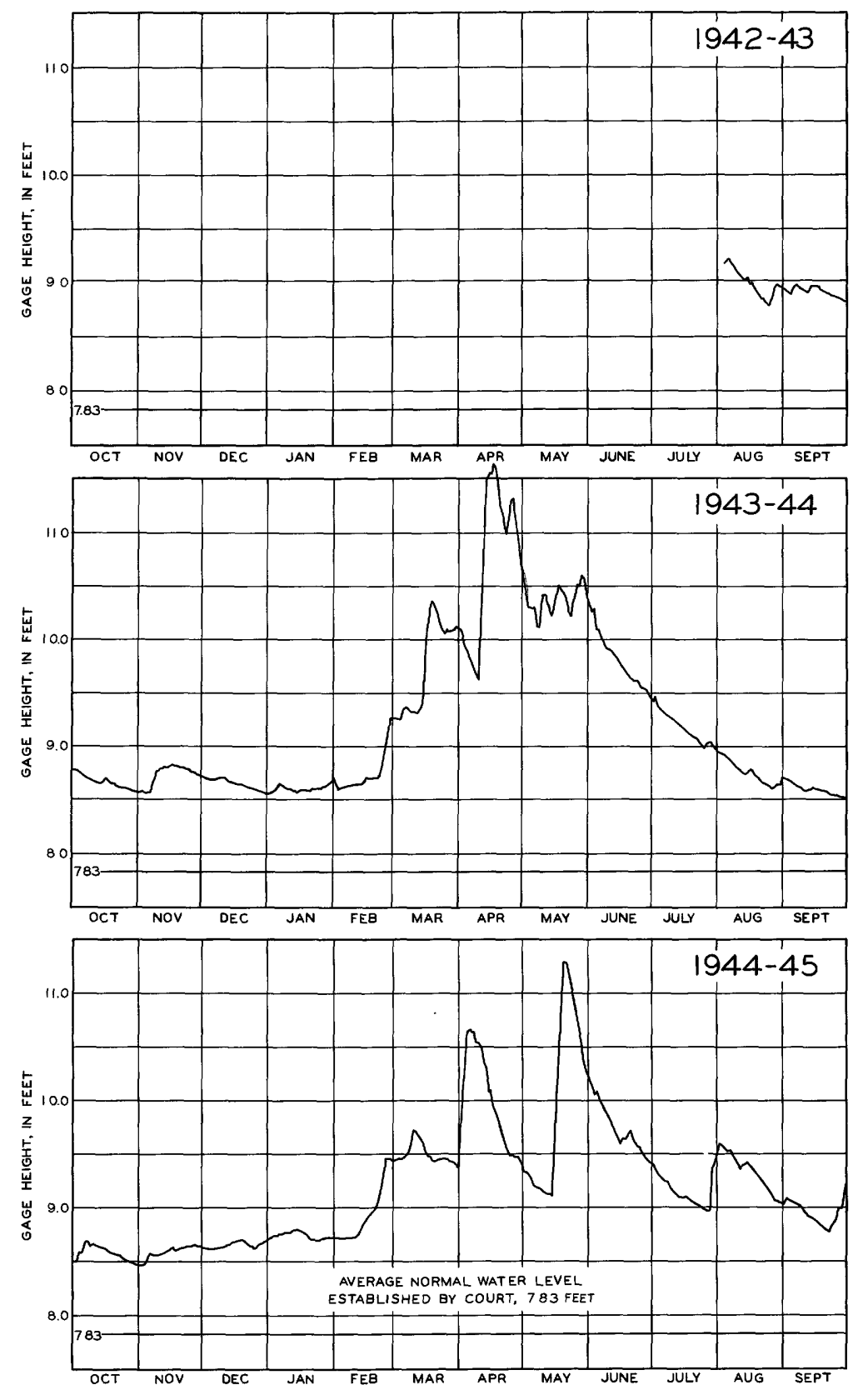

Figure 37. --Lake-level hydrographs for Big Lake at Wolflake, Ind., for water years 1943-45. 

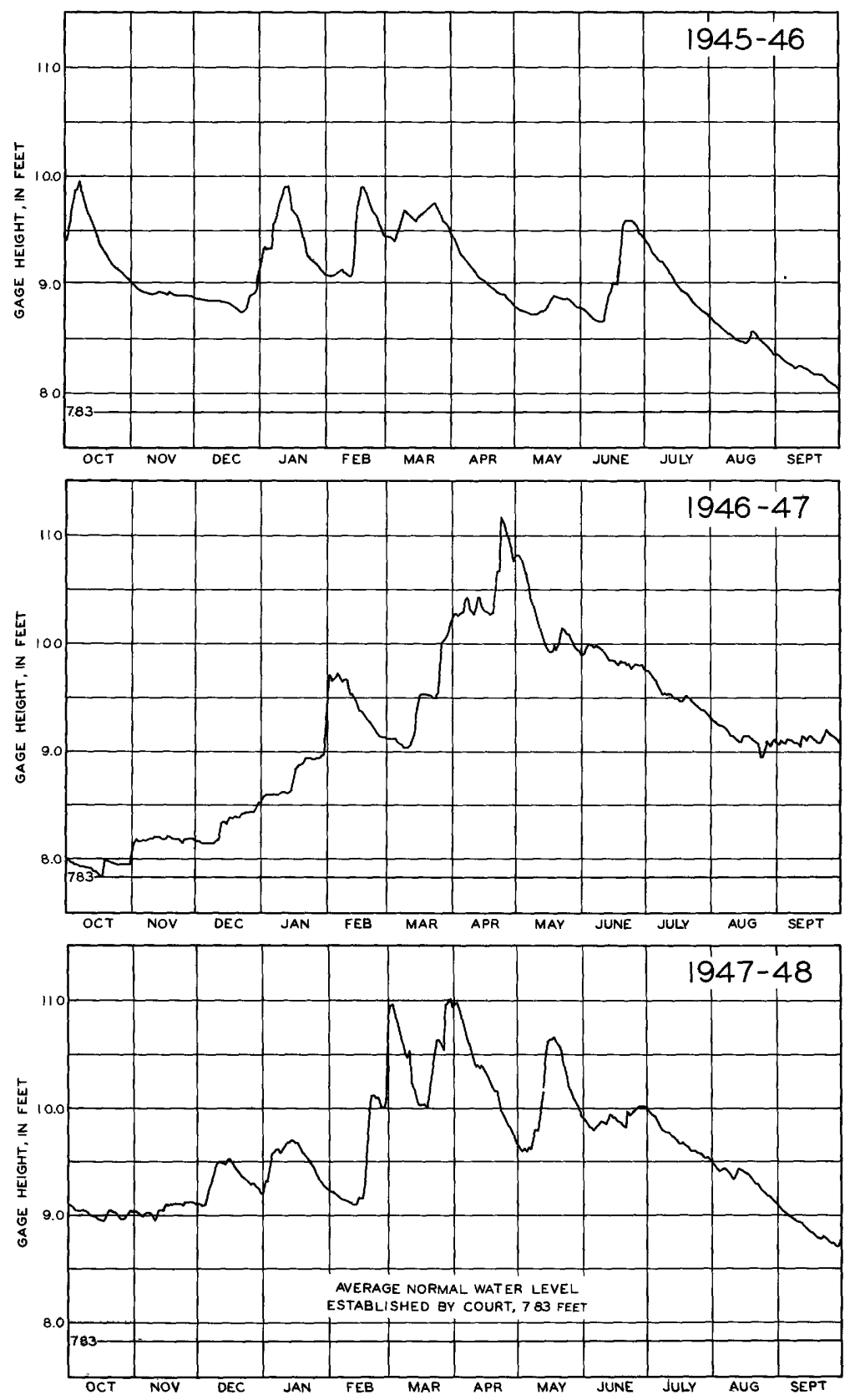

Figure 38. --Lake-level hydrographs for Big Lake at Wolflake, Ind., for water years 1946-48. 

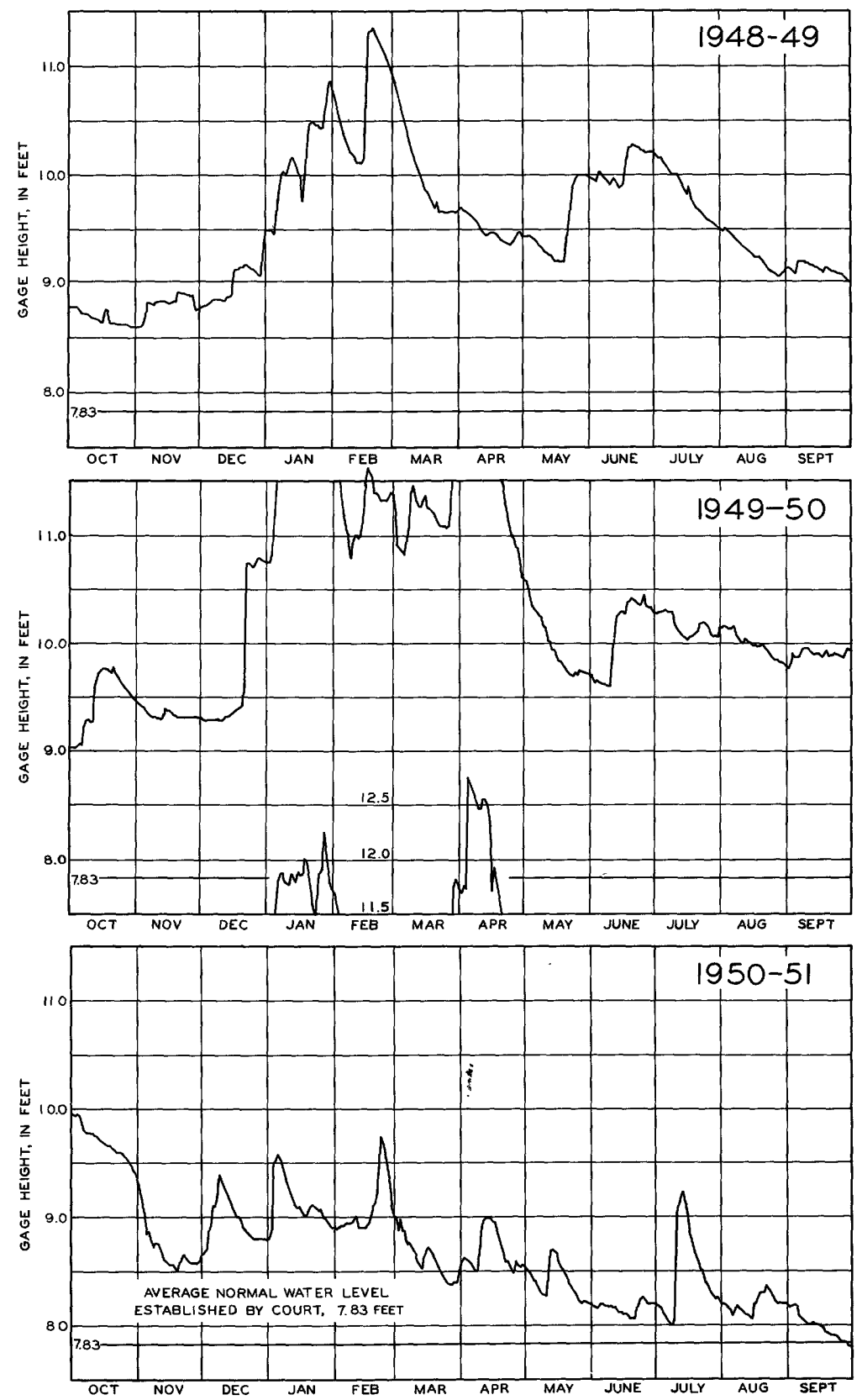

Figure 39. --Lake-level hydrographs for Big Lake at Wolflake Ind., for water years 1949-51. 

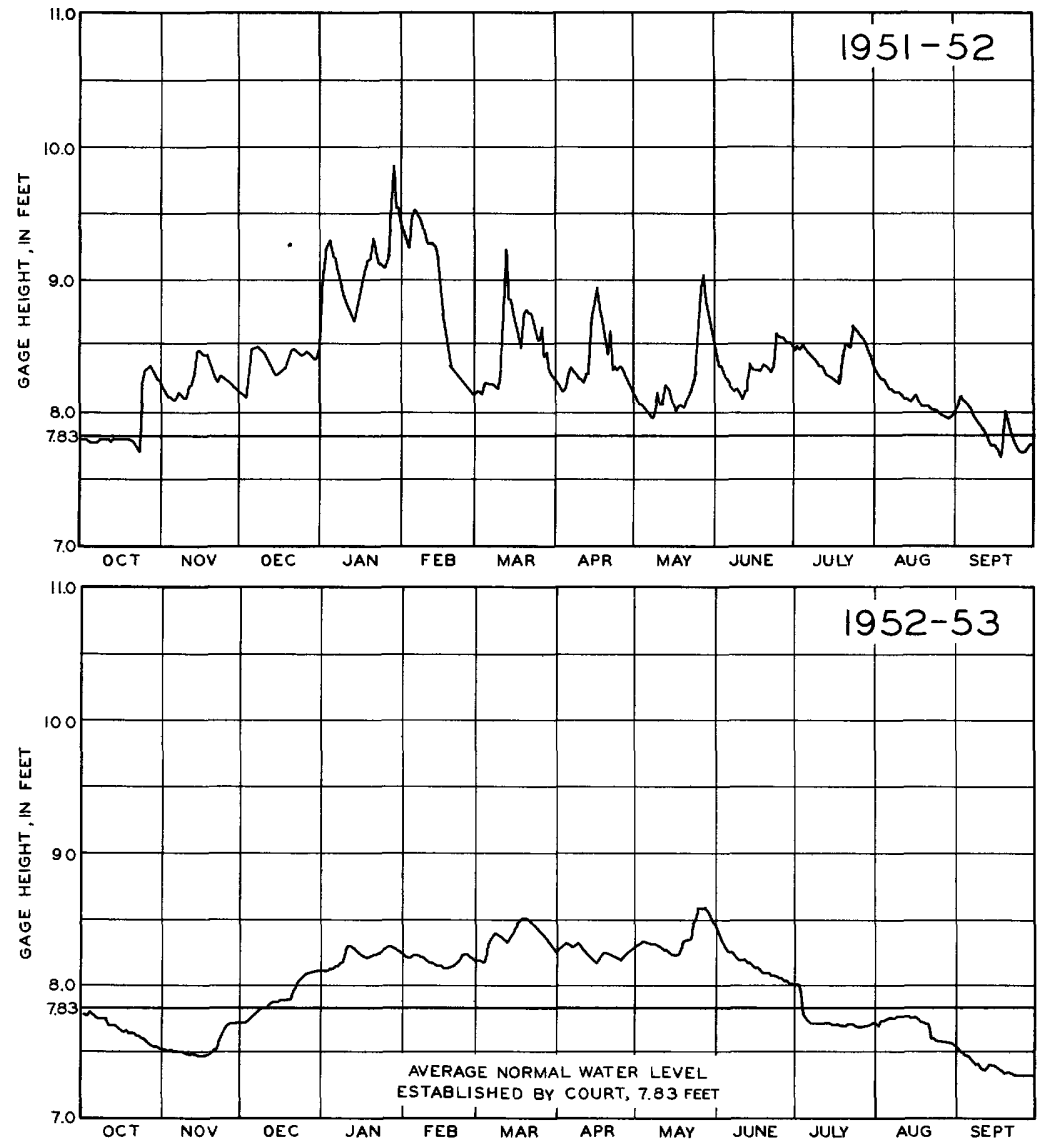

Figure 40. --Lake-level hydrographs for Big Lake at Wolflake Ind., for water years 1952-53. 
Bixler Lake at Kendallville, Ind.

Location. --Secs. 3, 4, T. 34 N. , R. 11 E., secs. 33, 34, T. 35 N., R. 11 E., Noble County.

Surface area. - -112 acres (determined from Bixler Lake contour map).

Drainage area. $--3.63 \mathrm{sq} \mathrm{mi}$.

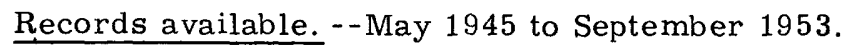

Gage. - -Staff gage on concrete pier $20 \mathrm{ft}$ upstream from control dam. Gage read to hundredths once daily. Datum of gage is $960.00 \mathrm{ft}$ above mean sea level, unadjusted.

Average lake level. --8 years; gage height, $2.87 \mathrm{ft}$; elevation, $962.87 \mathrm{ft}$ above mean sea level.

Established legal level. --Established Apr. 25, 1952, at gage height $3.65 \mathrm{ft}$, elevation, $963.65 \mathrm{ft}$ above mean sea level.

Lake-level control. - - Lake level maintained by Columbus deepnotch-type concrete control dam built in October 1946. A flood gate 18 inches in diameter in each abutment provides relief from extremely high water.

Extreme levels for Bixler Lake, 1946-53

\begin{tabular}{|c|c|c|c|c|}
\hline \multicolumn{3}{|c|}{ Maximum } & \multicolumn{2}{|l|}{ Minimum } \\
\hline $\begin{array}{c}\text { Water } \\
\text { year }\end{array}$ & Date & $\begin{array}{c}\text { Gage } \\
\text { height } \\
\text { (feet) }\end{array}$ & Date & $\begin{array}{c}\text { Gage } \\
\text { height } \\
\text { (feet) }\end{array}$ \\
\hline 1946 & June 20,1946 & 5.10 & Sept. $30,1946 \ldots$ & 2.64 \\
\hline 1947 & Apr. 2, $1947 \ldots$ & 4.89 & Oct. $17,1946 \ldots$ & 2.36 \\
\hline 1948 & Feb. 28, $1948 \ldots$ & 5.15 & Sept. 28, 1948--- & 2.51 \\
\hline 1949 & Feb. $15,1949 \ldots$ & 5.23 & Oct. $30,1948 \ldots$ & 2.09 \\
\hline 1950 & Apr. 4, $1950 \ldots$ & 5.75 & Oct. 2,1949 & 3.12 \\
\hline 1951 & Dec. $3,4,1950$ & 5.08 & Feb. 11, $1951 \ldots$ & 2.58 \\
\hline 1952 & Jan. $20,1952 \ldots$ & 4.75 & Sept. $30,1952 \ldots$ & $* 2.34$ \\
\hline 1953 & May $23,1953^{-\cdots}$ & 4.36 & Nov. $18,1952 \ldots$ & 1.71 \\
\hline
\end{tabular}

* Estimated. 
Maximum recorded thickness and periods of ice cover for Bixler Lake, 1946-53.

\begin{tabular}{|c|c|c|c|}
\hline $\begin{array}{l}\text { Water } \\
\text { year }\end{array}$ & $\begin{array}{l}\text { Maximum } \\
\text { thickness } \\
\text { (inches) }\end{array}$ & $\begin{array}{l}\text { Total } \\
\text { days of } \\
\text { cover }\end{array}$ & Period \\
\hline 1946 & 9 & 71 & Dec. 16, 1945, to Feb. 24, 1946. \\
\hline 1947 & 4 & 62 & Dec. 18,1946 , to Feb. $17,1947$. \\
\hline 1948 & $127 / 8$ & --- & Dec. 16,1947 , to $-?$ \\
\hline 1949 & $5 \frac{1}{2}$ & 62 & Dec. 26, 1948, to Feb. 25, 1949 \\
\hline 1950 & $6 \frac{1}{2}$ & 54 & $\begin{array}{l}\text { Dec. } 15-21,1949 ; \text { Jan. } 8-10,17-25 \\
\text { 1950; Feb. 5, 6; Feb. } 20 \text { to Mar. } \\
\text { 24, } 1950 .\end{array}$ \\
\hline 1951 & 8 & 89 & Dec. 1, 1950, to Feb. 27, 1951 \\
\hline 1952 & $7 \frac{1}{2}$ & 78 & $\begin{array}{l}\text { Dec. } 14,1951 \text {, to Jan. } 19,1952 \text {; } \\
\text { Jan. 24, to Mar. 4, } 1952 \text {. }\end{array}$ \\
\hline 1953 & ----- & 31 & $\begin{array}{l}\text { Dec. 28, } 1952 \text {, to Jan. 15, 1953; } \\
\text { Jan. 26, to Feb. 6, 1953. }\end{array}$ \\
\hline
\end{tabular}

Discharge measurements at outlet of Bixler Lake

\begin{tabular}{|c|c|c|c|c|c|}
\hline Date & $\begin{array}{c}\text { Gage } \\
\text { height } \\
\text { (feet) }\end{array}$ & $\begin{array}{c}\text { Discharge } \\
\text { (cfs) }\end{array}$ & Date & $\begin{array}{c}\text { Gage } \\
\text { height } \\
\text { (feet) }\end{array}$ & $\begin{array}{c}\text { Discharge } \\
\text { (cfs) }\end{array}$ \\
\hline Oct. 1,1945 & 3.53 & 1.16 & July 1,1946 & 4.46 & 6.67 \\
\hline Dec. $29-\cdots$ & 3.41 & 1.04 & July $16 \ldots$ & 3.82 & .12 \\
\hline Jan. $11,1946-$ & 4. 48 & 9.93 & Aug. 20-... & 3.34 & .02 \\
\hline Jan. $29 \cdots-\cdots$ & 3.60 & 2.91 & Aug. 28-... & 3.16 & 0 \\
\hline Feb. $5 \cdots$ & 3.50 & 1.32 & Sept. 9-..- & 2.92 & 0 \\
\hline Feb. 14- - & 4.31 & 8. 41 & Oct. $1---$ & 2.61 & 0 \\
\hline Feb. $14 \ldots$ & 4.47 & 8.53 & Oct. $10 \ldots$ & 2.47 & 0 \\
\hline Feb. 15- & 4.53 & 9.84 & Oct. $16-\cdots$ & 2.36 & 0 \\
\hline Feb. 18- & 4.58 & 12.2 & Nov. 21-..- & 2.62 & 0 \\
\hline Feb. 23- & 4.28 & 8.74 & Dec. 17 ... & 2.86 & 0 \\
\hline Feb. 25- - & 4.16 & 7.71 & Jan. 8,1947 & 3.02 & 0 \\
\hline Mar. 2-... & 4.14 & 6.79 & Jan. 28-.-- & $3 . \dot{9} 3$ & .42 \\
\hline Apr. $11 \cdots$ & 3.62 & 2.21 & Feb. 6-- & 4.55 & 6.14 \\
\hline May 15- & 3.20 & .06 & Feb. 13- & 4.24 & 1.74 \\
\hline May 27-...- & 3.32 & .45 & Feb. 20- - & 4.26 & 1.60 \\
\hline June $13 \ldots$ & 4.86 & 11.2 & Mar.27.... & 4.69 & 9.64 \\
\hline June $14-\cdots$ & 5.00 & 12.8 & Apr. 12- & 4.28 & 17.8 \\
\hline June 15 & 5.03 & 12.9 & Apr. 19-.. & 3.71 & $a_{12.4}$ \\
\hline June $16 \cdots$ & 5.02 & 12.6 & July 29-... & 3.43 & 0 \\
\hline June $17 \cdots$ & 5.00 & 12.5 & July $31 \ldots$ & 3.35 & 0 \\
\hline June $20 \ldots$ & 5.10 & 12.7 & Oct. $31-\cdots$ & 2.28 & 0 \\
\hline June $22 \ldots$ & 4.98 & 12.0 & Mar. 1, 1948 & 5.03 & $a_{26.4}$ \\
\hline June $26 \cdots-\cdots$ & 4.63 & 8.91 & Mar. 1-..- & 5.02 & 19.2 \\
\hline
\end{tabular}


Discharge measurements at outlet of Bixler Lake--Con.

\begin{tabular}{|c|c|c|c|c|c|}
\hline Date & $\begin{array}{l}\text { Gage } \\
\text { height } \\
\text { (feet) }\end{array}$ & $\left|\begin{array}{c}\text { Discharge } \\
\text { (cfs) }\end{array}\right|$ & Date & $\begin{array}{c}\text { Gage } \\
\text { height } \\
\text { (feet) }\end{array}$ & $\begin{array}{c}\text { Discharge } \\
\text { (cfs) }\end{array}$ \\
\hline Mar. 3, 1948- & 4.68 & a 22.4 & Aug. 30, 1951 & 3.56 & 0.01 \\
\hline Mar. 3-... & 4.73 & 9.87 & Oct. $8 \ldots$ & 3.28 & 0 \\
\hline June $10-\ldots$ & 4.17 & 1.08 & Nov. $20-\cdots$ & 3.84 & .01 \\
\hline June $10 \ldots$ & 4.13 & a 9.98 & Dec. $20 \ldots$ & 4.01 & .14 \\
\hline Nov. $5-\cdots$ & 2.24 & 0 & Feb. 7,1952 & 4.42 & 16.6 \\
\hline Feb. 2, 1949- & 4.28 & 1.72 & Mar. 25 .... & 4. 12 & $a_{13.1}$ \\
\hline May $5 \ldots \ldots$ & 4. 31 & 1.95 & July $17 \ldots$ & 3.34 & 0 \\
\hline Sept. $9 \ldots \ldots$ & 3.45 & 0 & Dec. $10 \ldots$ & 1.75 & 0 \\
\hline Mar. 15, 1950- & 4.41 & $a_{17.0}$ & Jan. 13,1953 & 1. 70 & 0 \\
\hline Nov. $9 \ldots$ & 3.95 & 0 & Feb. $6 \ldots-\cdots$ & 2.09 & 0 \\
\hline Nov. 15-... & 4.03 & .81 & Mar. $27 \ldots$ & 4.25 & 1. 76 \\
\hline Jan. $31,1951-$ & 2.76 & a 3.05 & Apr. $16 \ldots$ & 3.61 & $a_{13.7}$ \\
\hline Apr. $10 \ldots$ & 4.50 & 4.94 & June $4 \cdots--$ & 3.84 & 0 \\
\hline May $1-\cdots--$ & 3.81 & .21 & July $\quad 6 \cdots---$ & 3.30 & 0 \\
\hline June $6 \cdots-\cdots$ & 3.83 & .06 & July $28 \ldots$ & 2.81 & 0 \\
\hline July $10 \ldots \ldots$ & 4.86 & a 29.5 & Aug. $26 \ldots$ & 2.59 & 0 \\
\hline Aug. $1 \ldots$ & 3.89 & .14 & Sept. $23 \ldots$ & 2.13 & 0 \\
\hline
\end{tabular}

a Gates on dam open.

Average lake level for Bixler Lake for water years 1946-53

\begin{tabular}{c|c|c|c|c|c|c|c}
\hline Year & Oct. & Nov. & Dec. & Jan. & Feb. & Mar. & Apr. \\
\hline 1946 & 3.70 & 3.33 & 3.38 & 4.02 & 4.01 & 4.28 & 3.54 \\
1947 & 2.47 & 2.60 & 2.75 & 3.47 & 4.32 & 4.36 & 4.36 \\
1948 & 2.90 & 3.05 & 3.84 & 4.36 & 4.30 & 4.51 & 4.43 \\
1949 & 2.27 & 2.26 & 2.38 & 4.26 & 4.43 & 3.71 & 4.31 \\
1950 & 3.63 & 3.65 & 4.01 & 4.87 & 4.13 & 4.34 & 4.49 \\
1951 & 4.04 & 4.12 & 3.89 & 3.52 & 3.59 & 3.79 & 4.07 \\
1952 & 3.38 & 3.95 & 3.98 & 4.38 & 4.08 & 4.17 & 3.97 \\
1953 & 2.08 & 1.78 & 1.88 & 2.28 & 2.73 & 3.87 & 4.16 \\
\hline Average & 3.06 & 3.09 & 3.26 & 3.90 & 3.95 & 4.13 & 4.17 \\
\hline \hline & & & & & & & \\
Year & May & June & July & Aug. & Sept. & Annual & \\
\hline 1946 & 3.26 & 4.14 & 3.92 & 3.34 & 2.82 & 3.64 & \\
1947 & 3.87 & 4.32 & 3.65 & 3.02 & 3.07 & 3.52 & \\
1948 & 4.56 & 4.20 & 3.86 & 3.35 & 2.75 & 3.84 & \\
1949 & 4.33 & 4.21 & 3.97 & 3.73 & 3.34 & 3.59 & \\
1950 & 3.71 & 3.92 & 4.28 & 4.04 & 4.22 & 4.11 & \\
1951 & 3.87 & 3.96 & 4.19 & 3.91 & 3.36 & 3.86 & \\
1952 & 3.97 & 3.86 & 3.41 & 2.9 .1 & 2.54 & 3.73 & \\
1953 & 4.09 & 3.67 & 3.07 & 2.79 & 2.22 & 2.88 & \\
\hline Average & 3.96 & 4.04 & 3.79 & 3.39 & 3.04 & 2.87 & \\
\hline
\end{tabular}



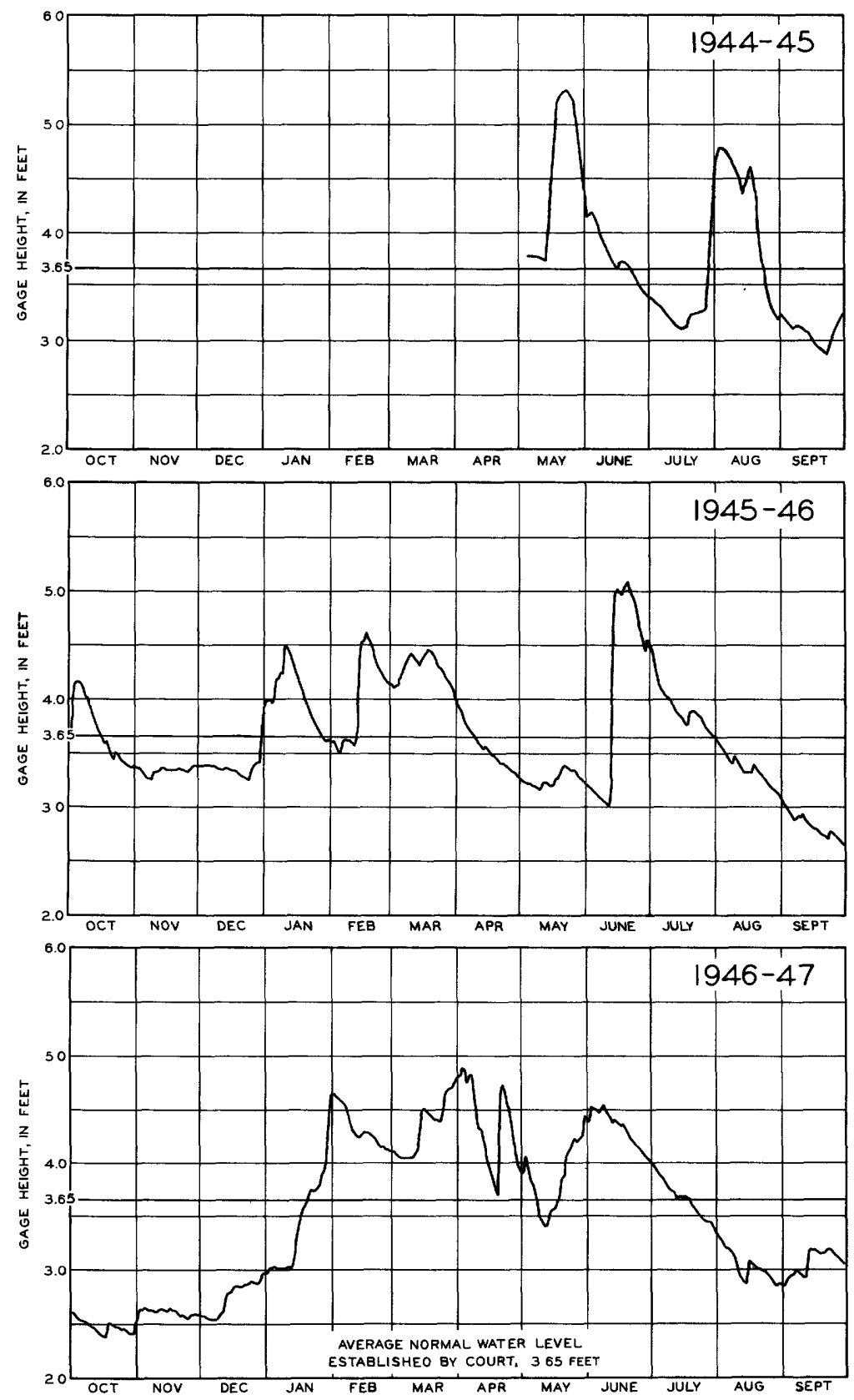

Figure 41. --Lake-level hydrographs for Bixler Lake at Kendallville, Ind., for water years 1945-47. 


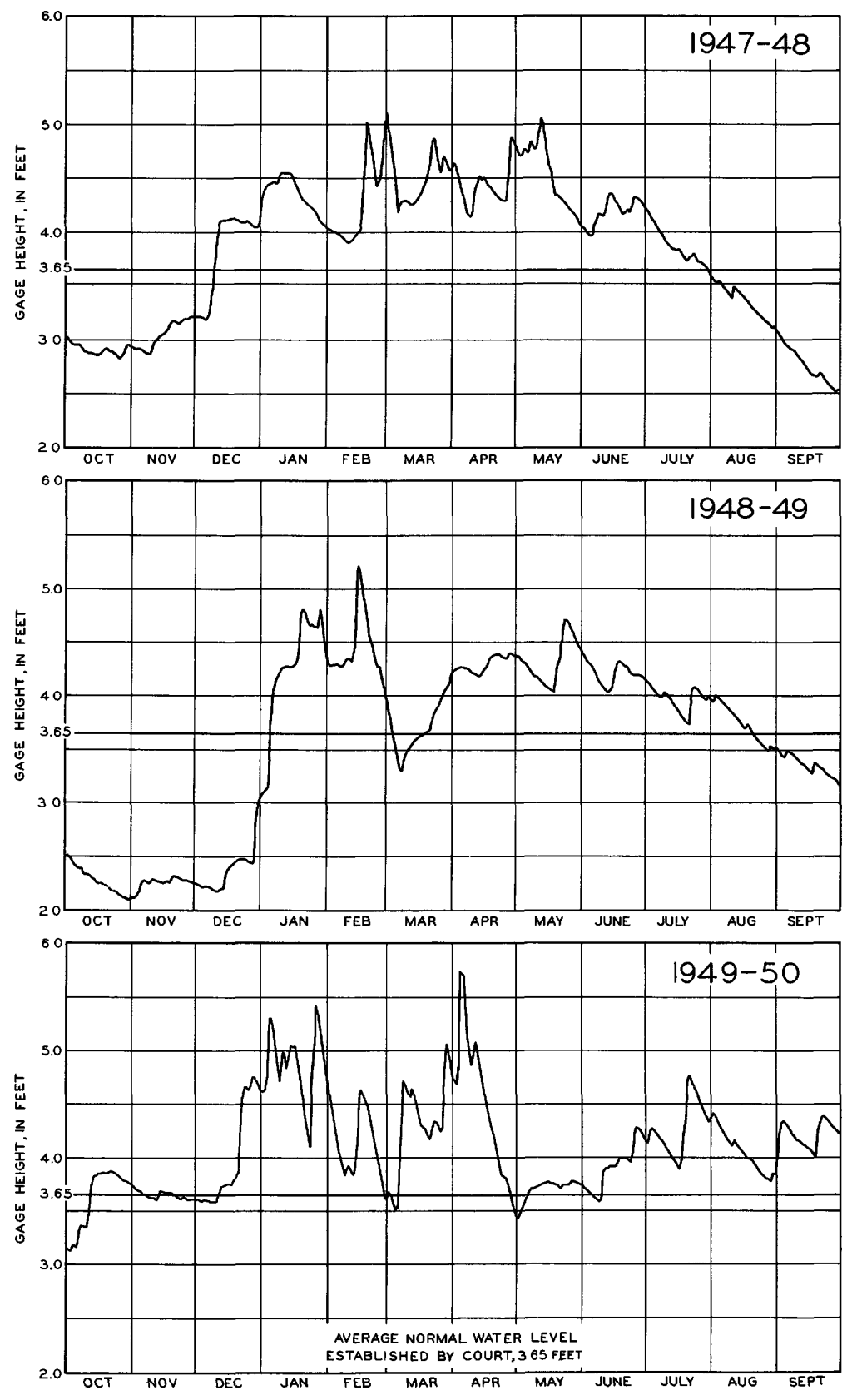

Figure 42. --Lake-level hydrographs for Bixler Lake at Kendallville, Ind., for water years 1948-50. 

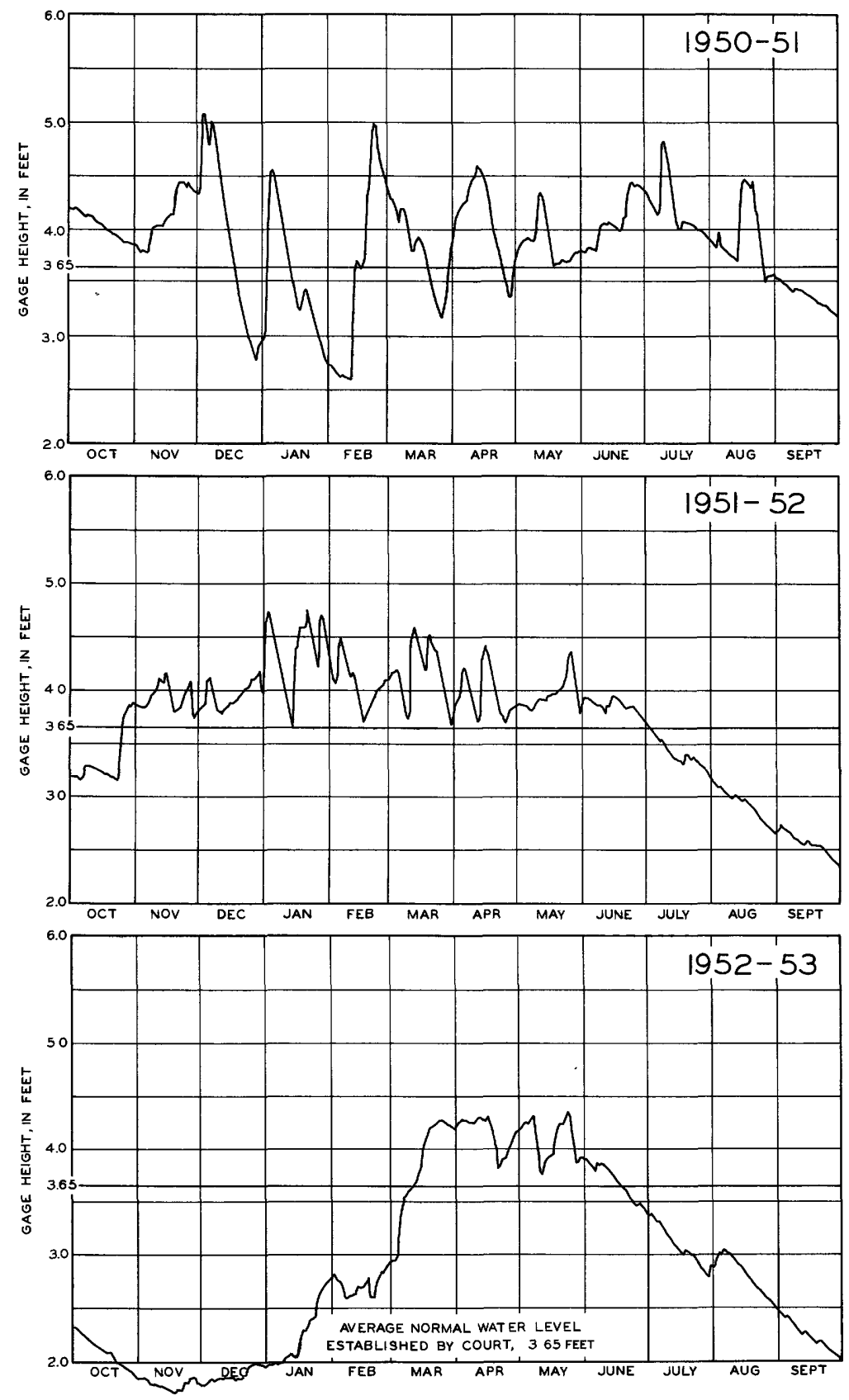

Figure 43. --Lake-level hydrographs for Bixler Lake at Kendallville, Ind., for water years 1951-53. 
Bruce Lake at Lake Bruce, Ind.

Location. --Sec. 1, T. 30 N., R. 1 W., Pulaski County and secs. 6 and 7, T. 30 N. , R. 1 E., Fulton County.

Surface area. --202 acres.

Drainage area. $--5.19 \mathrm{sq} \mathrm{mi}$.

Records available. --August 1943 to November 1952.

Gage. - - Staff gage at south shore of the western tip of lake at Egleston Inn. Gage read to hundredths once daily. Datum of gage is $720.00 \mathrm{ft}$ above mean sea level, datum of 1929 .

Average lake level. --9 years; gage height, $3.72 \mathrm{ft}$; elevation, $723.72 \mathrm{ft}$.

Established legal level. --Established June 20, 1950, at gage height $3.69 \mathrm{ft}$, elevation, $723.69 \mathrm{ft}$ above mean sea level.

Lake-level control. - - Lake level maintained by concrete dam with rounded crest, about $18 \mathrm{in}$ wide and $27 \mathrm{ft}$ long. Crest at gage height $3.55 \mathrm{ft}$.

Extreme levels for Bruce Lake, 1944-52

\begin{tabular}{|c|c|c|c|c|}
\hline \multicolumn{3}{|c|}{ Maximum } & \multicolumn{2}{|l|}{ Minimum } \\
\hline $\begin{array}{r}\text { Water } \\
\text { year }\end{array}$ & Date & $\begin{array}{c}\text { Gage } \\
\text { height } \\
\text { (feet) }\end{array}$ & Date & $\begin{array}{l}\text { Gage } \\
\text { height } \\
\text { (feet) }\end{array}$ \\
\hline 1944 & Apr. $12,1944 \ldots \ldots$ & 4.90 & Sept. 26, 1944-..- & $* 3.04$ \\
\hline 1945 & May $17,18,1945 \ldots$ & 4.80 & Nov. 2, $1944 \ldots-\ldots$ & $* 3.06$ \\
\hline 1946 & June $15,1946 \ldots$ & 4.52 & Sept. $28-30,1946-$ & 3.06 \\
\hline 1947 & Apr. $21,1947 \ldots$ & 4.71 & Oct. $14-17,1946$ - & 2. 90 \\
\hline 1948 & Feb. 28, 29, 1948 $\ldots$ & 4. 58 & Oct. $12-17,1947 \ldots$ & 3.18 \\
\hline 1949 & Jan. $19,1949 \ldots$ & 4.64 & Oct. $29,1948 \ldots$ & $* 3.16$ \\
\hline 1950 & Apr. $4,1950 \ldots$ & 4.64 & Oct. $1,2,1949 \ldots$ & 3.36 \\
\hline 1951 & July $10,1951 \ldots$ & 4.42 & Nov. $6-8,1950 \ldots$ & 3.46 \\
\hline 1952 & June $15,1952 \ldots$ & 4.28 & Aug. 7, 8, $1952 \ldots$ & 3.42 \\
\hline
\end{tabular}

* Estimated 
BASIC DATA ON LAKE LEVELS FOR SELECTED LAKES 105

Maximum recorded thickness and periods of ice cover 1944-52

\begin{tabular}{|c|c|c|c|}
\hline $\begin{array}{l}\text { Water } \\
\text { year }\end{array}$ & $\begin{array}{l}\text { Maximum } \\
\text { thickness } \\
\text { (inches) }\end{array}$ & $\begin{array}{l}\text { Total } \\
\text { days of } \\
\text { cover }\end{array}$ & Period \\
\hline 1944 & 10 & 72 & Dec. 14, 1943, to Feb. 23, 1944. \\
\hline 1945 & $---n$ & --- & \\
\hline 1946 & 12 & 81 & Dec. 13,1945 , to Mar. 3, 1946. \\
\hline 1947 & 8 & 95 & Dec. 20, 1946, to Mar. 24, 1947. \\
\hline 1948 & 15 & 106 & $\begin{array}{l}\text { Nov. } 30 \text { to Dec. } 5 \text {; Dec. } 9,1947 \text {, } \\
\text { to Mar. } 18,1948 .\end{array}$ \\
\hline 1949 & 6 & 62 & Dec. 26, 1948, to Feb. 25, 1949. \\
\hline 1950 & 5 & 43 & $\begin{array}{l}\text { Dec. 9, 15-17, 1949; Jan. 17-21, } \\
\text { 1950; Feb. 1-11; Feb. 20, to } \\
\text { Mar. 9, 1950; Mar. 14-18, 1950. }\end{array}$ \\
\hline 1951 & 14 & 101 & Nov. 24, 1950, to Mar. 4, 1951 \\
\hline 1952 & 8 & 62 & Nov. 20,1951 , to Jan. $20,1952$. \\
\hline
\end{tabular}

Discharge measurements at outlet of Bruce Lake

\begin{tabular}{|c|c|c|c|c|c|}
\hline Date & $\begin{array}{l}\text { Cage } \\
\text { height } \\
\text { (feet) }\end{array}$ & $\begin{array}{c}\text { Discharge } \\
\text { (cfs) }\end{array}$ & Date & $\begin{array}{l}\text { Gage } \\
\text { height } \\
\text { (feet) }\end{array}$ & $\begin{array}{c}\text { Discharge } \\
\text { (cfs) }\end{array}$ \\
\hline Aug. $\quad 5,1943$ & 4.39 & 29.0 & Feb. 11,1947 & 4.03 & 4.11 \\
\hline Oct. $12 \ldots$ & 3.67 & 1.06 & Feb. $21-\ldots$ & 3.97 & 2. 76 \\
\hline Jan. 18,1944 & 3.63 & .75 & Nov. $19 \ldots$ & 3.66 & 1. 39 \\
\hline $9-\cdots$ & 4.08 & 11.8 & Apr. 8,1948 & 4. 31 & 19.8 \\
\hline $11 \ldots \ldots$ & 3.49 & .01 & May $13 \ldots$ & 4.45 & 60.2 \\
\hline June 12,1945 & 3.72 & 2.21 & May $18 \ldots$ & 3.95 & 19.1 \\
\hline Aug. $20 \ldots$ & 3.56 & .20 & June $24 \ldots$ & 3.78 & 9.07 \\
\hline $27 \ldots \ldots$ & 3.79 & 3.20 & Nov. $2 \cdots$ & 3.35 & 0 \\
\hline 3,1946 & 4.01 & 6.59 & Jan. 12, 1949 & 3. 78 & 11.4 \\
\hline $5 \ldots \ldots$ & 3.67 & .86 & Feb. $22 \cdots \cdots$ & 3.89 & 18. 2 \\
\hline June & 4. 16 & 9.65 & Mar. $21 \ldots$ & 3.63 & 4.15 \\
\hline July & 3.76 & 1.04 & Apr. $25 \cdots$ & 3.63 & 3.60 \\
\hline July & 3.58 & .07 & Mar. 16, 1950 & 3.82 & 12.8 \\
\hline July & 3.42 & 0 & Nov. $24 \ldots$ & 3.67 & 4.87 \\
\hline
\end{tabular}


Average lake level for Bruce Lake for water years 1943-52

\begin{tabular}{c|c|c|c|c|c|c|c}
\hline Year & Oct. & Nov. & Dec. & Jan. & Feb. & Mar. & Apr \\
\hline 1944 & 3.71 & 3.85 & 3.70 & 3.65 & 3.81 & 4.13 & 4.28 \\
1945 & 3.15 & 3.18 & 3.29 & 3.40 & 3.59 & 3.91 & 4.04 \\
1946 & 3.83 & 3.77 & 3.73 & 4.05 & 4.00 & 4.23 & 3.88 \\
1947 & 3.02 & 3.67 & 3.79 & 4.02 & 4.08 & 3.95 & 4.36 \\
1948 & 3.27 & 3.58 & 3.78 & 3.94 & 3.97 & 4.22 & 4.03 \\
1949 & 3.26 & 3.58 & 3.66 & 3.93 & 3.87 & 3.69 & 3.66 \\
1950 & 3.62 & 3.58 & 3.73 & 4.10 & 3.95 & 3.88 & 4.00 \\
1951 & 3.54 & 3.59 & 3.65 & 3.78 & 3.79 & 3.76 & 3.85 \\
1952 & 3.66 & 3.77 & 3.73 & 3.91 & 3.82 & 3.79 & 3.82 \\
\hline Average & 3.45 & 3.62 & 3.67 & 3.86 & 3.88 & 3.95 & 3.99 \\
\hline \hline & & & & & & & \\
Year & May & June & July & Aug. & Sept. & Annual & \\
\hline 1943 & ---- & ---- & ---- & $* 4.06$ & 3.95 & $* 4.01$ & \\
1944 & 4.08 & 3.84 & 3.46 & 3.18 & 3.12 & 3.73 & \\
1945 & 4.13 & 3.77 & 3.59 & 3.58 & 3.46 & 3.59 & \\
1946 & 3.80 & 4.01 & 3.64 & 3.30 & 3.12 & 3.78 & \\
1947 & 4.10 & 4.09 & 3.57 & 3.25 & 3.20 & 3.76 & \\
1948 & 4.00 & 3.63 & 3.63 & 3.52 & 3.36 & 3.74 & \\
1949 & 3.70 & 3.67 & 3.59 & 3.51 & 3.42 & 3.63 & \\
1950 & 3.62 & 3.70 & 3.79 & 3.52 & 3.55 & 3.75 & \\
1951 & 3.70 & 3.64 & 3.86 & 3.65 & 3.56 & 3.70 & \\
1952 & 3.74 & 3.78 & 3.56 & 3.68 & 3.57 & 3.74 & \\
\hline Average & 3.87 & 3.79 & 3.63 & 3.52 & 3.43 & 3.72 & \\
\hline
\end{tabular}

* Partial month or partial year. 

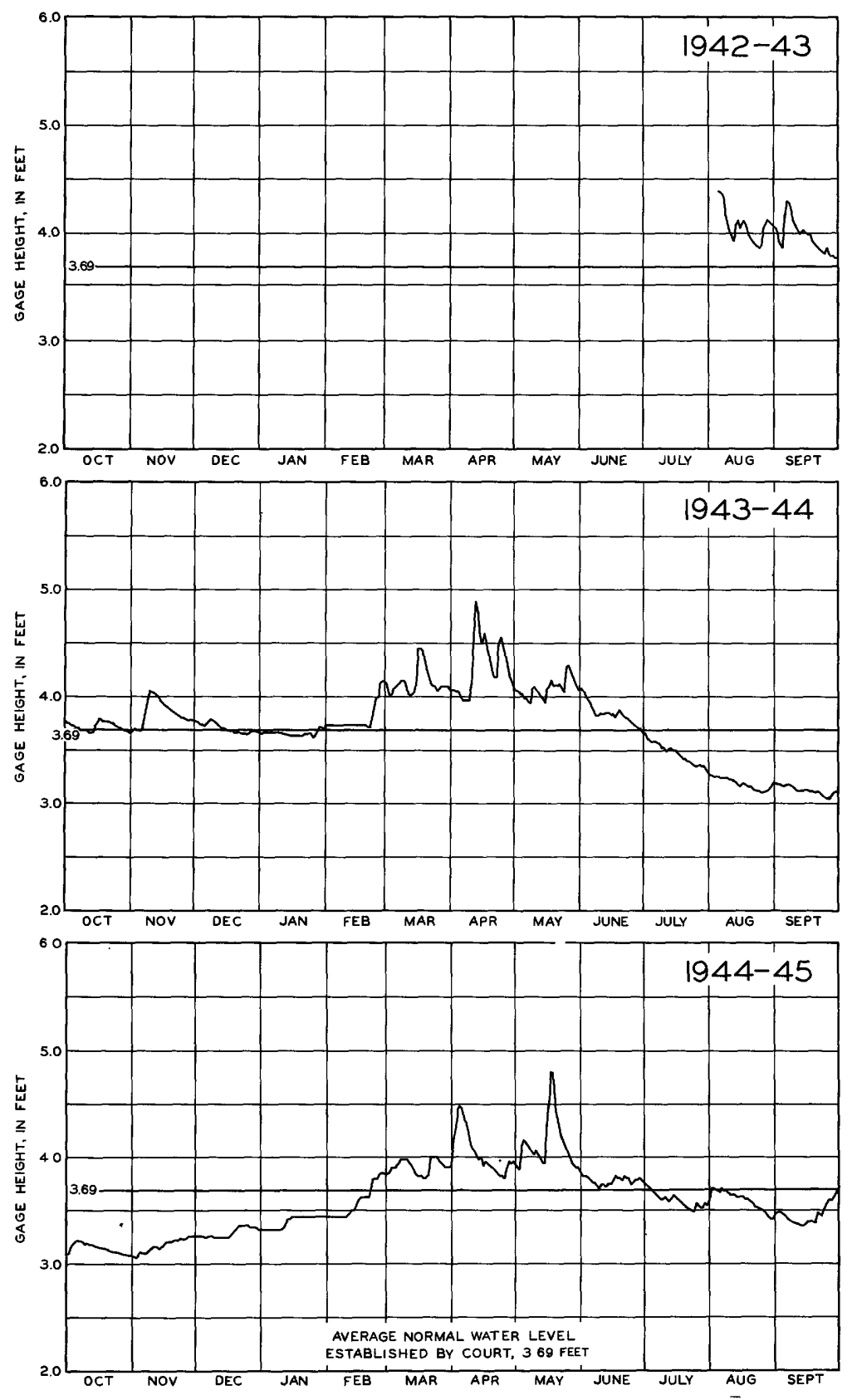

Figure 44. --Lake-level hydrographs for Bruce Lake at Lake, Bruce, Ind., for water years 1943-45. 

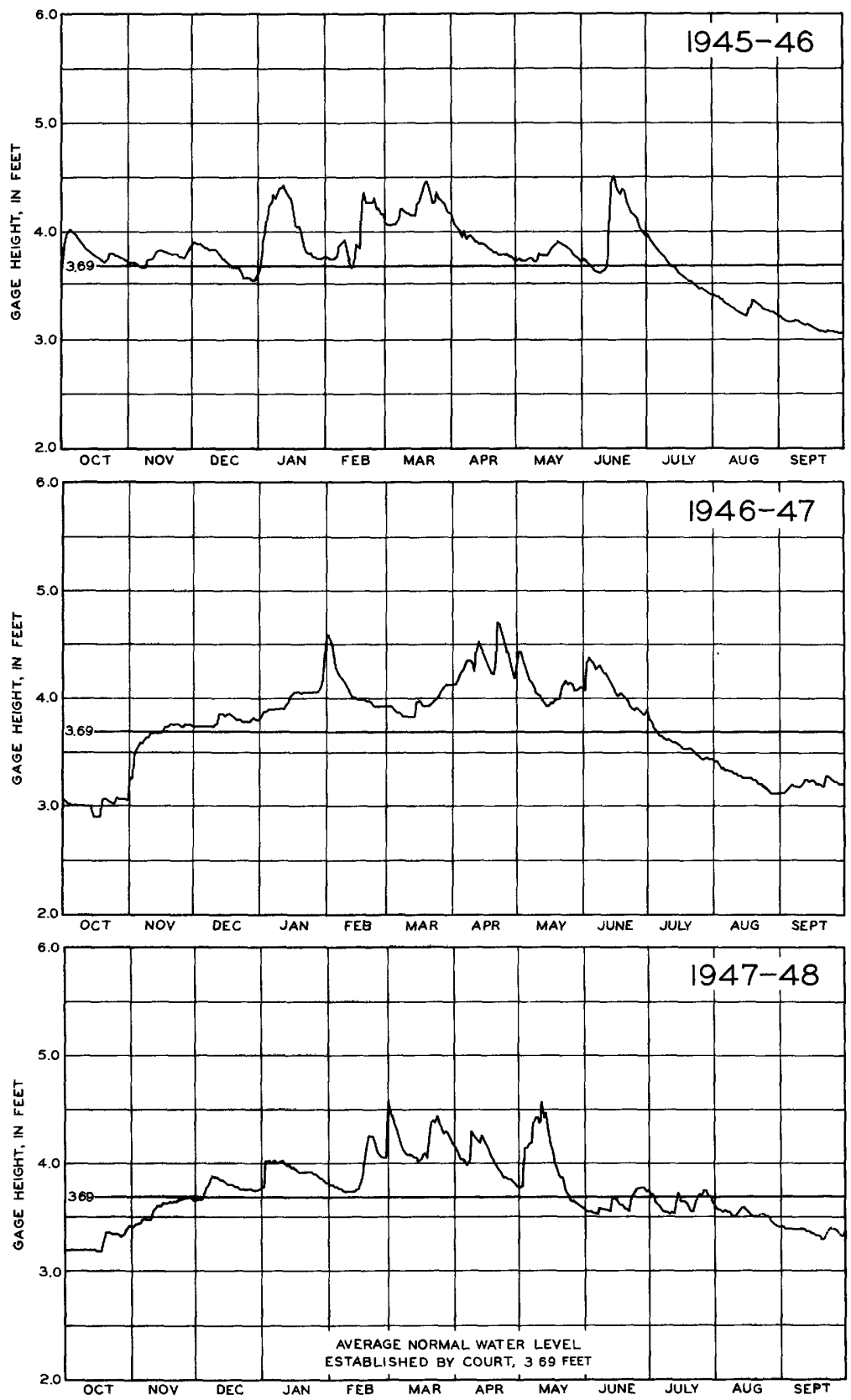

Figure 45. --Lake-level hydrographs for Bruce Lake at Lake Bruce. Ind. , for water years 1946-48. 

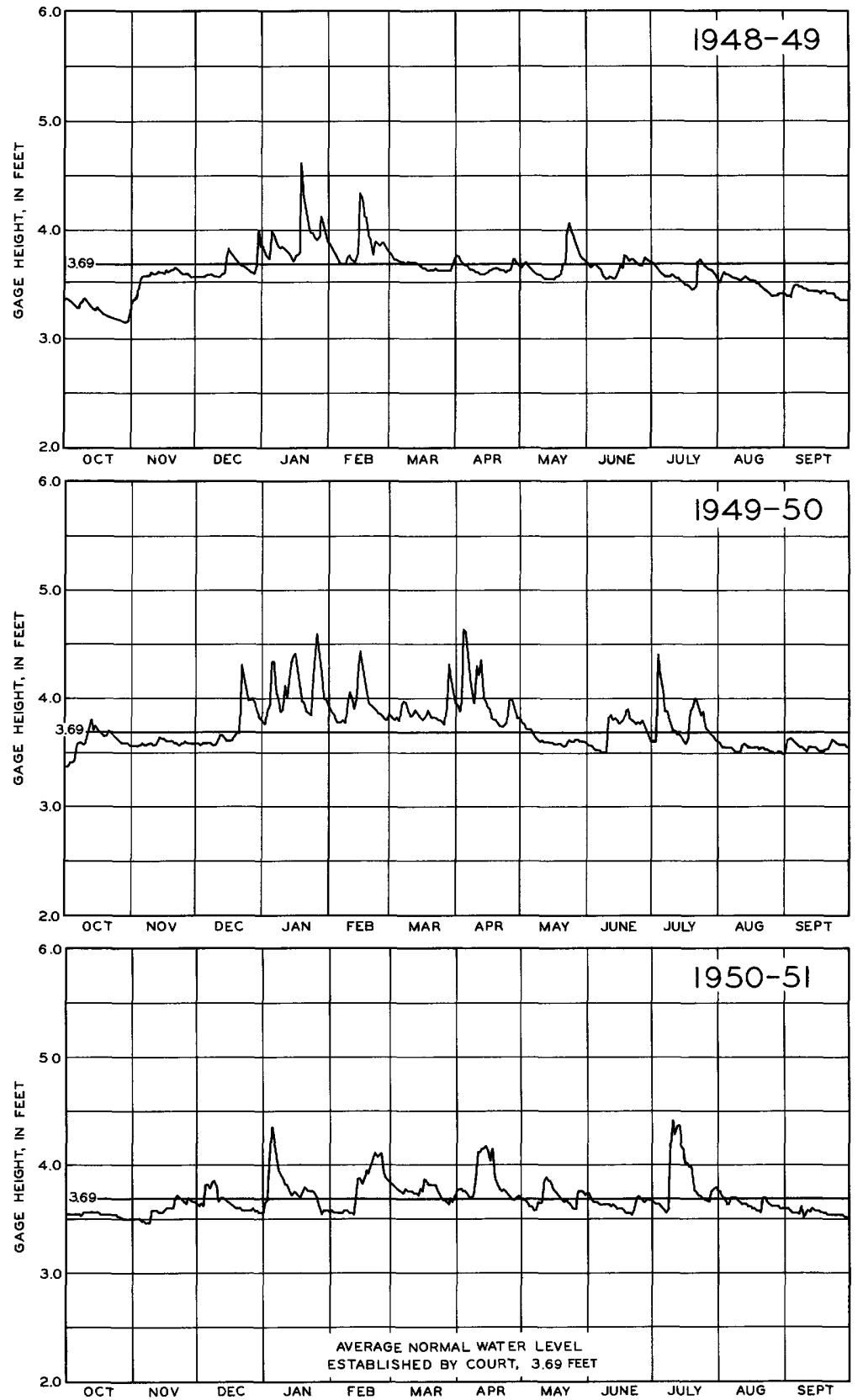

Figure 46. --Lake-level hydrographs for Bruce Lake at Lake Brı Ind., for water years 1949-51. 


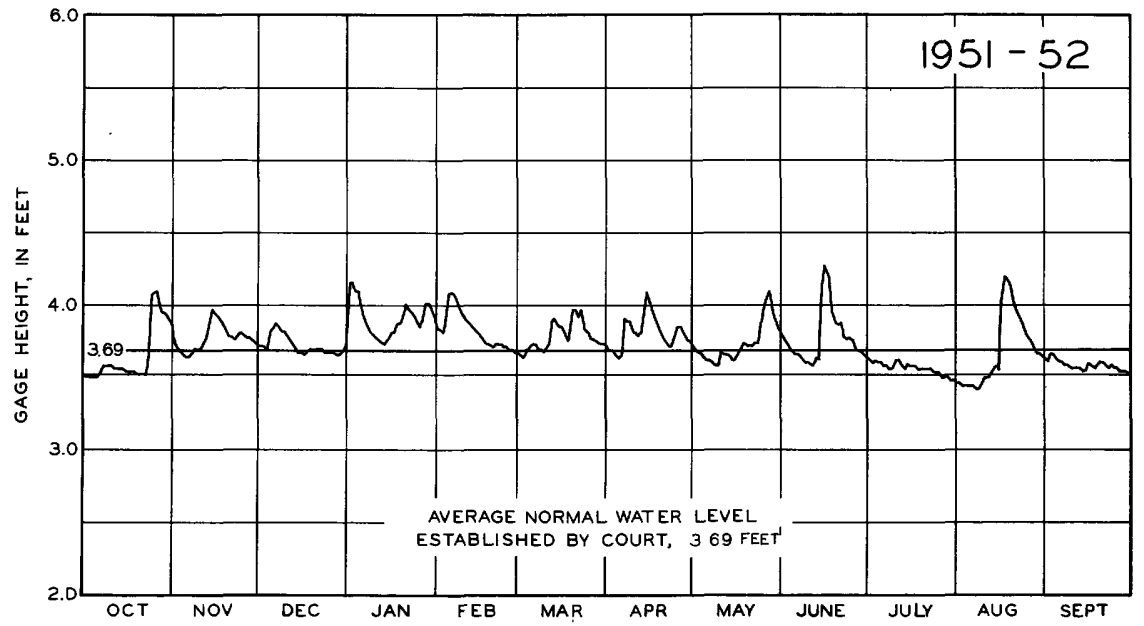

Figure 47. --Lake-level hydrographs for Bruce Lake at Lake Bruce, Ind., for water year 1952.

Cedar Lake at Cedar Lake, Ind.

Location.--Secs. 22, 23, 26, 27, 34, and 35, T. 34 N., R. 9 W. , Lake County.

Surface area. --805 acres.

Drainage area. $--8.05 \mathrm{sq} \mathrm{mi}$.

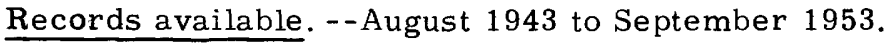

Gage. --Staff gage at middle of east shore, on boat crane post of Lawson's Marine Service Building. Gage read to hundredths once daily. Datum of gage is $690.00 \mathrm{ft}$ above mean sea level, datum of 1929 .

Average level. --10 years (1944-53); gage height, $2.94 \mathrm{ft}$; elevation, $692.94 \mathrm{ft}$.

Established legal level. --None.

Lake-level control. --Lake level maintained by concrete dam, $25 \mathrm{ft}$ long. Crest at gage height, 2.90 ft. 
Extreme levels for Cedar Lake, 1944-53

\begin{tabular}{|c|c|c|c|c|}
\hline \multicolumn{3}{|c|}{ Maximum } & \multicolumn{2}{|l|}{ Minimum } \\
\hline $\begin{array}{l}\text { Water } \\
\text { year }\end{array}$ & Date & $\begin{array}{l}\text { Gage } \\
\text { height } \\
\text { (feet) }\end{array}$ & Date & $\begin{array}{l}\text { Gage } \\
\text { height } \\
\text { (feet) }\end{array}$ \\
\hline $1943^{a}$ & Aug. $16,1943 \ldots$ & 3.45 & Sept. $27-30,1943^{-}$ & a2.97 \\
\hline 1944 & Mar. 16, 1944_... & 4.23 & 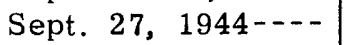 & 2.31 \\
\hline 1945 & May $19,1945 \ldots$ & 3.87 & Dec. $3=5,1944 \cdots$ & 2.27 \\
\hline 1946 & Jan. 12, 1946- & 3.73 & Sept. 29, 30, 1946 - & 2.35 \\
\hline 1947 & May 1, 1947 & 4. 11 & Oct. $16,17,1946^{--}$ & 2.21 \\
\hline 1948 & May $11,1948 \ldots$ & 4.02 & Sept. 28, 1948--- & 2.07 \\
\hline 1949 & Feb. 15, 22, 1949 & 3.36 & Oct. $29,30,1948--$ & 1.93 \\
\hline 1950 & Dec. 23,1949 & 3.90 & Aug. 27, $1950 \cdots$ & 2. 40 \\
\hline 1951 & Feb. $20,21,1951 \ldots$ & 3.52 & Nov. $6,1950 \ldots$ & 2.45 \\
\hline 1952 & June 14, $1952 \ldots$ & 4.02 & Sept. $30,1952 \cdots--$ & 2.32 \\
\hline 1953 & Mar. $18,19,1953$ & 3.39 & Nov. $7-9,16-17,1952$ & 1.96 \\
\hline
\end{tabular}

$a$ August and September.

Maximum recorded thickness and periods of ice cover 1944-52

\begin{tabular}{|c|c|c|c|}
\hline $\begin{array}{c}\text { Water } \\
\text { year }\end{array}$ & $\begin{array}{c}\text { Maximum } \\
\text { thickness } \\
\text { (inches) }\end{array}$ & $\begin{array}{l}\text { Total } \\
\text { days of } \\
\text { cover }\end{array}$ & Period \\
\hline 1944 & $-\cdots-\cdots$ & 110 & $\begin{array}{l}\text { Nov, } 14,1943, \text { to Feb. } 6,1944 ; \\
\text { Feb. } 11 \text { to Feb. 27; Mar. 8-15, } \\
\text { 1944. }\end{array}$ \\
\hline 1945 & 14 & 100 & Dec. 3, 1944, to Mar. 12, 1945 \\
\hline 1946 & 10 & 88 & Dec. 6,1945, to Mar. $3,1946$. \\
\hline 1947 & 7 & 102 & Dec. 19,1946 , to Mar. $30,1947$. \\
\hline 1948 & 16 & 111 & Nov. 27, 1947, to Mar. 16, 1948. \\
\hline 1949 & 9 & 80 & Dec. 11,1948 , to Feb. 28, 1949. \\
\hline 1950 & 5 & 100 & $\begin{array}{l}\text { Nov. 26-30; Dec. 9, 1949, to Jan. 1, } \\
\text { 1950; Jan. 6-25; Jan. 27, to } \\
\text { Mar. 18, 1950. }\end{array}$ \\
\hline 1951 & 6 & ---- & \\
\hline 1952 & ---- & ---- & Nov. 8, 1951 to - ? \\
\hline
\end{tabular}

Discharge measurements at outlet of Cedar Lake

\begin{tabular}{|c|c|c|c|c|c|}
\hline Date & $\begin{array}{l}\text { Gage } \\
\text { height } \\
\text { (feet) }\end{array}$ & $\begin{array}{c}\text { Discharge } \\
\text { (cfs) }\end{array}$ & Date & $\begin{array}{c}\text { Gage } \\
\text { height } \\
\text { (feet) }\end{array}$ & $\begin{array}{c}\text { Discharge } \\
\text { (cfs) }\end{array}$ \\
\hline Aug. $\quad 9,1943-$ & 3.19 & 7. 48 & Aug. 25,1945 & 2.67 & 0 \\
\hline Oct. $14 \ldots-\cdots$ & 2.91 & 0 & Oct. $26 \cdots$ & 2.85 & 0 \\
\hline Jan. 21, 1944- & 2.95 & .25 & Apr. 10,1946 & 3.18 & 4.78 \\
\hline $11 \cdots-\cdots$ & 3.37 & 15.6 & July 24----- & 2.93 & a. .04 \\
\hline $12-\cdots-$ & 2.99 & .73 & Aug. 26- & 2.62 & 0 \\
\hline June $7,1945-$ & 3.25 & 2.95 & 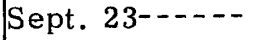 & 2.44 & 0 \\
\hline
\end{tabular}


Discharge measurements at outlet of Cedar Lake--Continued

\begin{tabular}{|c|c|c|c|c|c|}
\hline Date & $\begin{array}{l}\text { Gage } \\
\text { height } \\
\text { (feet) }\end{array}$ & $\begin{array}{c}\text { Discharg } \\
\text { (cfs) }\end{array}$ & Date & $\begin{array}{l}\text { Gage } \\
\text { height } \\
\text { (feet) }\end{array}$ & $\begin{array}{c}\text { Discharge } \\
\text { (cfs) }\end{array}$ \\
\hline Oct. $21,1946-$ & 2.25 & 0 & Apr. $19,1948-$ & 3.09 & 4. 43 \\
\hline Nov. $18 \cdots$ & 2.53 & 0 & May $11 \ldots$ & 4.06 & 72.7 \\
\hline Dec. $20 \cdots$ & 2. 79 & 0 & May $13 \ldots \ldots$ & 3.91 & 65.6 \\
\hline Jan. $\quad 6,1947-$ & 2.95 & 0 & May $18 \ldots \ldots$ & 3.58 & 35.8 \\
\hline Feb. $17 \ldots$ & 3.25 & 2.09 & Aug. $2 \ldots$ & 4. 35 & 0 \\
\hline Mar. $5 \ldots$. & 3.23 & .40 & Nov, $2 \ldots$ & 1.97 & 0 \\
\hline $7 \ldots-$ & 3.85 & 43.5 & Feb. 21, 1949- & 3.28 & 12.3 \\
\hline $1-\cdots$ & 4.03 & 61.1 & Oct. $17-\ldots$ & 3.00 & .82 \\
\hline Jure $16 \ldots$ & 3. 37 & 10.6 & Mar. 7, 1951- & 3.22 & 13.5 \\
\hline Dec. $16 \ldots$ & 3.05 & 2.01 & May $21 \ldots \ldots$ & 3.17 & 8.03 \\
\hline Feb. 28, 1948- & 3.49 & 31.8 & Jan. $20,1953-$ & 2.52 & 0 \\
\hline
\end{tabular}

$a$ Twigs and brush on dam crest restricting outflow.

Average lake level for Cedar Lake for water years 1943-53

\begin{tabular}{c|l|l|l|l|l|l|l}
\hline Year & Oct. & Nov. & Dec. & Jan. & Feb. & Mar. & Apr. \\
\hline 1944 & 2.94 & 3.05 & 3.00 & 2.97 & 3.06 & 3.59 & 3.66 \\
1945 & 2.40 & 2.34 & 2.32 & 2.39 & 2.54 & 3.06 & 3.34 \\
1946 & 2.88 & 2.84 & 2.94 & 3.45 & 3.38 & 3.46 & 3.15 \\
1947 & 2.26 & 2.50 & 2.66 & 3.04 & 3.29 & 3.31 & 3.70 \\
1948 & 2.65 & 2.75 & 2.99 & 3.04 & 3.08 & 3.36 & 3.17 \\
1949 & 2.03 & 2.07 & 2.19 & 2.65 & 3.18 & 3.18 & 3.18 \\
1950 & 2.97 & 2.87 & 3.19 & 3.45 & 3.36 & 3.36 & 3.49 \\
1951 & 2.58 & 2.51 & 2.74 & 3.03 & 3.21 & 3.22 & 3.27 \\
1952 & 2.84 & 3.06 & 3.11 & 3.22 & 3.12 & 3.23 & 3.29 \\
1953 & 2.13 & 2.02 & 2.24 & 2.44 & 2.65 & 3.13 & 3.04 \\
\hline Average & 2.57 & 2.60 & 2.74 & 2.97 & 3.09 & 3.29 & 3.33 \\
\hline Year & May & June & July & Aug. & Sept. & Annual & \\
\hline 1943 & --- & --- &.--- & $* 3.29$ & 3.08 & $* 3.17$ & \\
1944 & 3.37 & 3.19 & 2.90 & 2.54 & 2.41 & 3.06 & \\
1945 & 3.60 & 3.28 & 2.98 & 2.73 & 2.62 & 2.80 & \\
1946 & 3.12 & 3.32 & 3.09 & 2.66 & 2.46 & 3.06 & \\
1947 & 3.64 & 3.44 & 3.00 & 2.75 & 2.70 & 3.02 & \\
1948 & 3.40 & 2.95 & 2.72 & 2.49 & 2.19 & 2.90 & \\
1949 & 3.08 & 2.99 & 3.16 & 2.99 & 2.87 & 2.80 & \\
1950 & 3.26 & 3.06 & 2.93 & 2.67 & 2.56 & 3.09 & \\
1951 & 3.21 & 2.88 & 2.99 & 3.85 & 2.77 & 2.95 & \\
1952 & 3.12 & 3.36 & 2.97 & 2.74 & 2.50 & 3.05 & \\
1953 & 3.03 & 2.98 & 2.92 & 2.61 & 2.22 & 2.62 & \\
\hline Average & 3.28 & 3.14 & 2.97 & 2.85 & 2.58 & 2.94 & \\
\hline
\end{tabular}

* Partial month or partial year. 

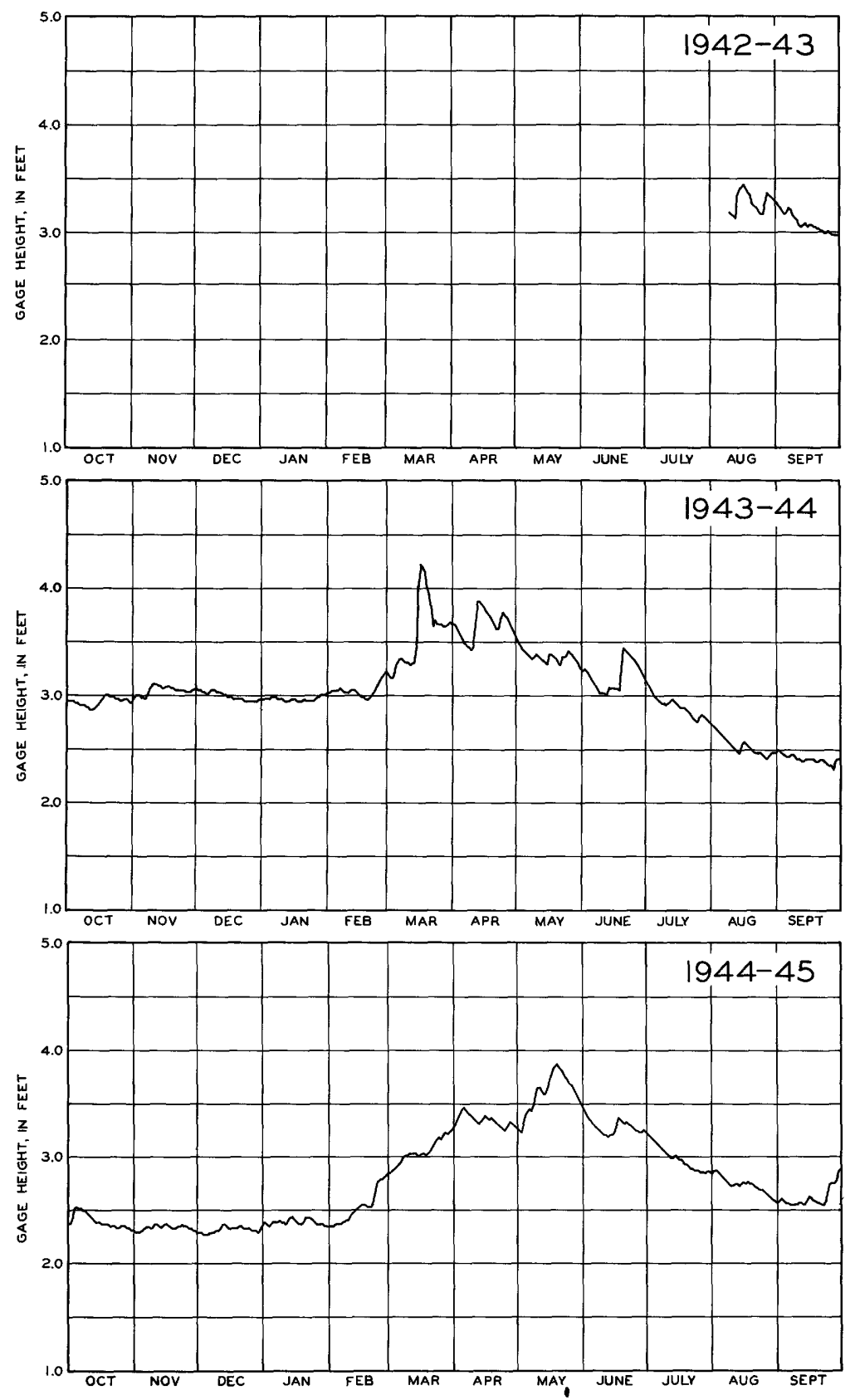

Figure 48. --Lake-level hydrographs for Cedar Lake at Cedar Lake, Ind., for water years 1943-45. 

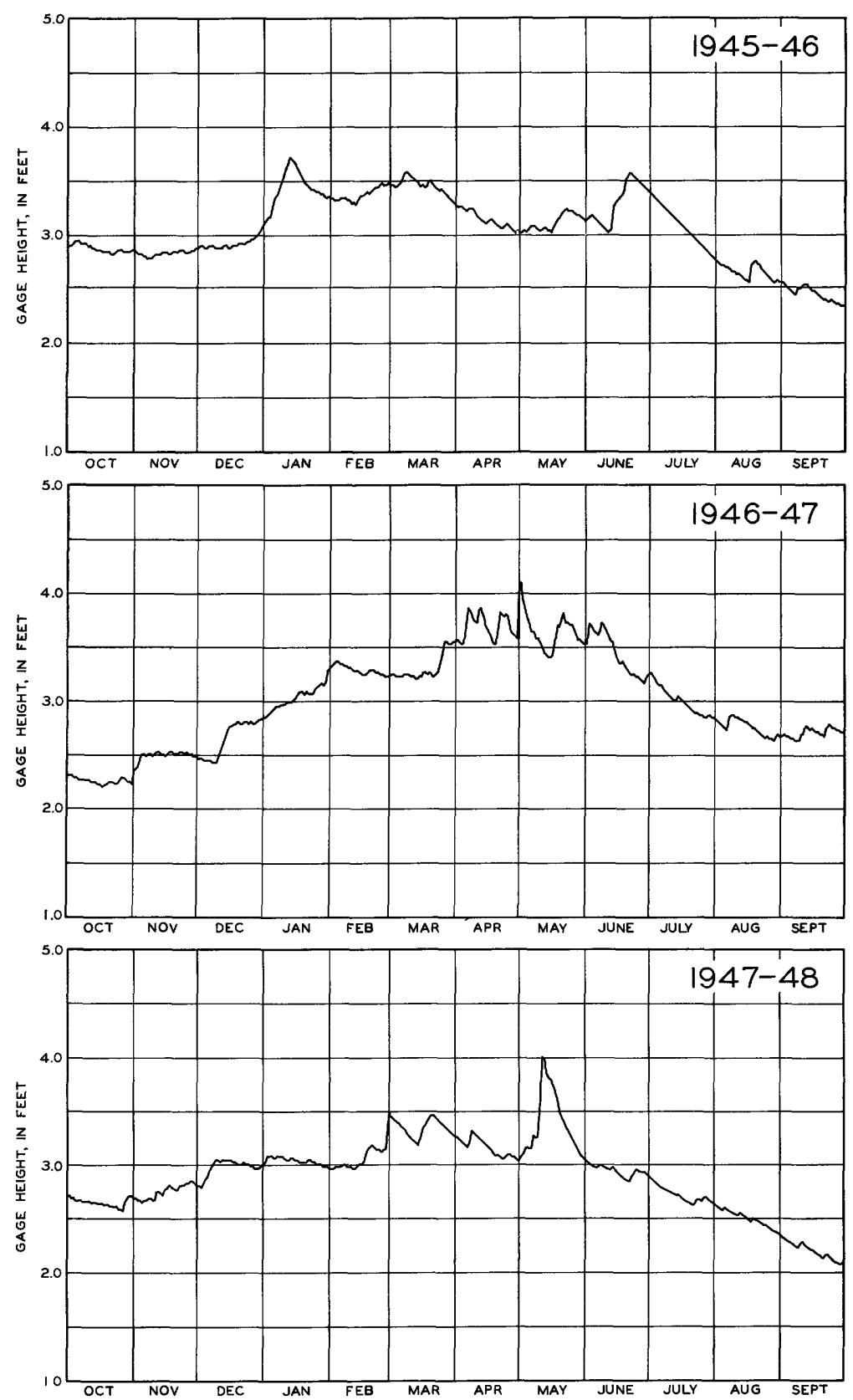

Figure 49. --Lake-level hydrographs for Cedar Lake at Cedar Lake, Ind., for water years 1946-48. 

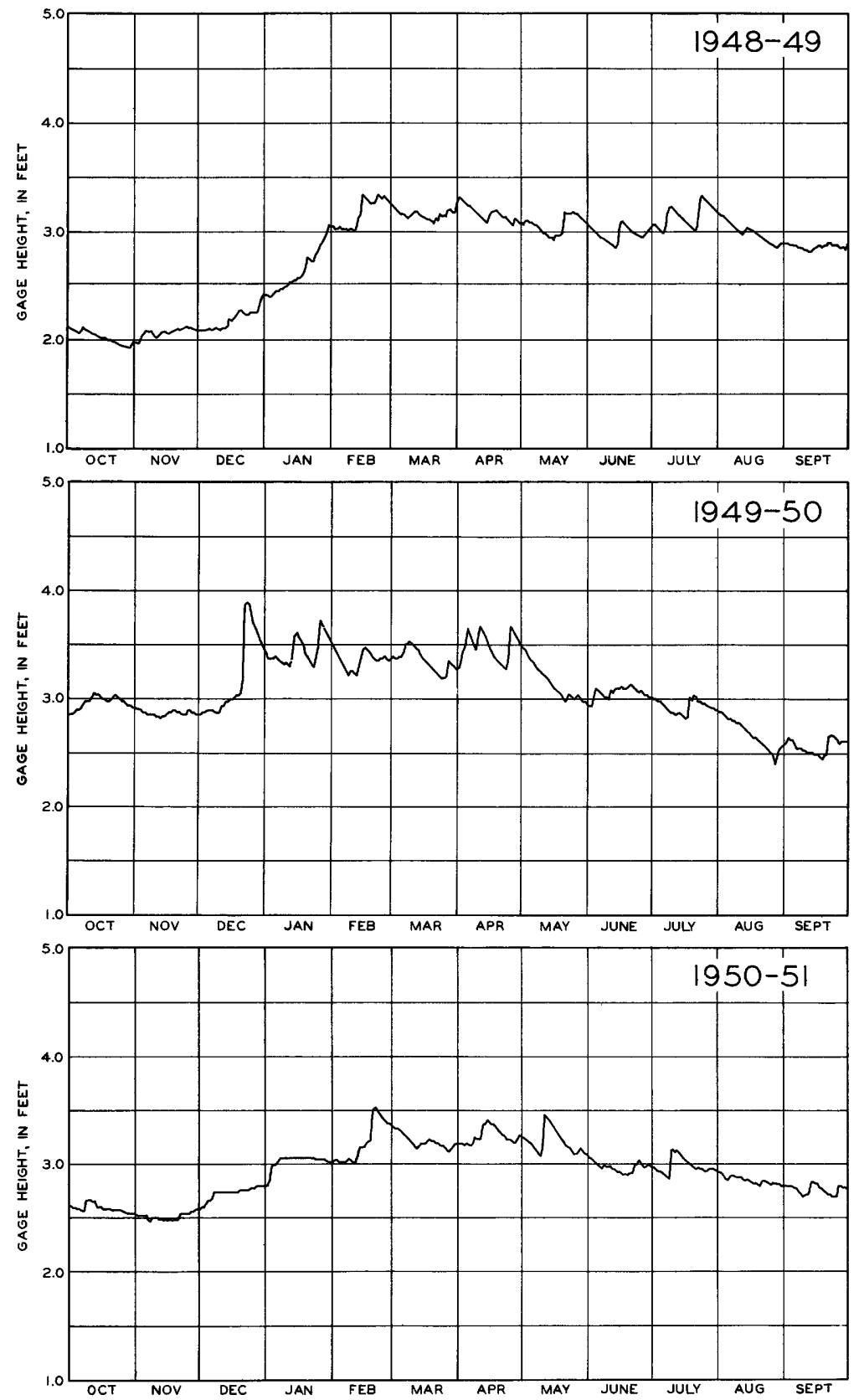

Figure 50.--Lake-level hydrographs for Cedar Lake at Cedar Lake, Ind. , for water years 1949-51. 


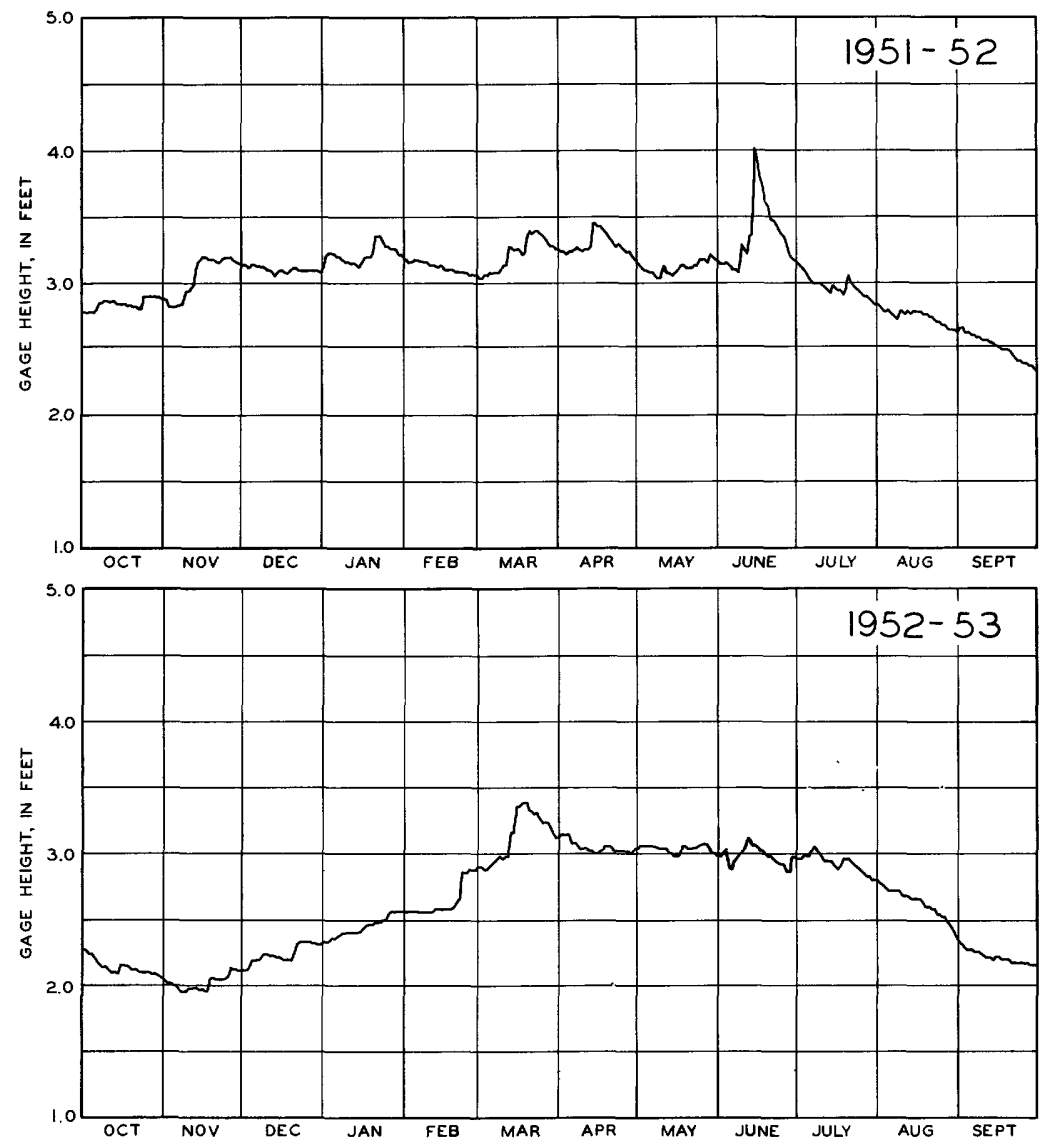

Figure 51. --Lake-level hydrographs for Cedar Lake at Cedar Lake, Ind., for water years 1952-53, 
Cedar Lake near Waterloo, Ind.

Location. --Sec. 30, T. 35 N., R. 13 E., DeKalb County, 3.5 miles northwest of Waterloo.

Surface area. -27 acres.

Drainage area. $--21.8 \mathrm{sq} \mathrm{mi}$.

Records available.--August 1943 to September 1953.

Gage. - -Staff gage attached to outlet control dam. Gage read to hundredths once daily. Datum of gage is $890.00 \mathrm{ft}$ above mean sea level, unadjusted.

Average lake level. --10 years; gage height, $6.79 \mathrm{ft}$; elevation, $896.79 \mathrm{ft}$.

Established legal level. --Level established Apr. 10, 1953, at gage height $6.76 \mathrm{ft}$; elevation, $896.76 \mathrm{ft}$ above mean sea level.

Lake-level control. - - Lake level controlled by a concrete dam with sill elevation at gage height $4.65 \mathrm{ft}$. Stop logs can be placed on sill to raise lake level.

Extreme levels for Cedar Lake near Waterloo, 1944-53

\begin{tabular}{|c|c|c|c|c|}
\hline \multicolumn{3}{|c|}{ Maximum } & \multicolumn{2}{|l|}{ Minimum } \\
\hline $\begin{array}{c}\text { Water } \\
\text { year }\end{array}$ & Date & $\begin{array}{l}\text { Gage } \\
\text { height } \\
\text { (feet) }\end{array}$ & Date & $\begin{array}{l}\text { Gage } \\
\text { height } \\
\text { (feet) }\end{array}$ \\
\hline 1944 & Apr. $12,1944 \cdots$ & 10.58 & Aug. $1,1944 \ldots$ & 6.41 \\
\hline 1945 & May $18,1945 \ldots \ldots$ & 9.80 & $\begin{array}{l}\text { Oct. } 2,21-31 \text {, } \\
\text { Nov. } 1-4,1944\end{array}$ & 6.40 \\
\hline 1946 & June $13,1946 \ldots$ & 10.03 & Aug. 2 to Sept. 21, & 6.38 \\
\hline 1947 & Apr. $21,1947 \ldots$ & 10.78 & Aug. $25,1947 \ldots$ & 6.40 \\
\hline 1948 & Feb. $28,1948 \ldots$ & 10.60 & $\begin{array}{l}\text { Sept. } 16-20,25- \\
28,1948 \ldots\end{array}$ & 6.14 \\
\hline 1949 & Feb. 16, 1949 & 11.90 & Aug. 20, $1949 \ldots$ & 6.10 \\
\hline 1950 & Apr. $5,1950 \ldots$ & 12.84 & Aug. $24,1950 \ldots$ & 5.50 \\
\hline 1951 & Feb. $20,1951 \ldots$ & 10.62 & $\begin{array}{l}\text { July } 7 \text {, Sept. } 18- \\
20,1951-\end{array}$ & 5.96 \\
\hline 1952 & Mar. $13,1952 \ldots$ & 9.72 & July $10-14,1952-$ & 5. 78 \\
\hline 1953 & Mar. 4, 1953 & 8.46 & Oct. $8,9,1952 \ldots$ & 5.62 \\
\hline
\end{tabular}




\begin{tabular}{|c|c|c|c|}
\hline $\begin{array}{l}\text { Water } \\
\text { year }\end{array}$ & $\begin{array}{r}\text { Maximum } \\
\text { thickness } \\
\text { (inches) }\end{array}$ & $\begin{array}{l}\text { Total } \\
\text { days of } \\
\text { cover }\end{array}$ & Period \\
\hline 1945 & 8 & ---- & \\
\hline 1946 & $-\ldots-$. & ---- & Unknown to Mar. $6,1946$. \\
\hline 1947 & ----- & $--\cdot$ & $\begin{array}{l}\text { Dec. 2-9, 1946; unknown to Apr. 4, } \\
\text { 1947. }\end{array}$ \\
\hline 1948 & 10 & 104 & $\begin{array}{l}\text { Nov. } 28 \text { to Dec. } 8 \text {; Dec. } 19,1947 \text {, } \\
\text { to Mar. 20, } 1948 .\end{array}$ \\
\hline 1949 & $--\cdots$ & 71 & Dec. 27,1948 , to Mar. 7,1949 \\
\hline 1950 & $-\cdots--$ & 78 & $\begin{array}{l}\text { Dec. } 7-12,14-19,1949 ; \text { Jan. } 7-13 \text {, } \\
\text { 19-29; Feb. 3-14; Feb. } 19 \text { to } \\
\text { Mar. 26, } 1950\end{array}$ \\
\hline 1951 & 4 & 98 & Nov. 28,1950 , to Mar. 4,1951 \\
\hline 1952 & $--\cdots$ & 96 & $\begin{array}{l}\text { Nov. } 20-26 \text {; Dec. } 14,1951 \text {, to } \\
\text { Mar. } 11,1952 .\end{array}$ \\
\hline 1953 & ----- & 106 & Nov. 30,1952 , to Mar. $15,1953$. \\
\hline
\end{tabular}

Discharge measurements at outlet of Cedar Lake near Waterloo

\begin{tabular}{|c|c|c|c|c|c|}
\hline Date & $\begin{array}{l}\text { Gage } \\
\text { height } \\
\text { (feet) }\end{array}$ & $\begin{array}{c}\text { Discharge } \\
\text { (cfs) }\end{array}$ & Date & $\begin{array}{l}\text { Gage } \\
\text { height } \\
\text { (feet) }\end{array}$ & $\begin{array}{c}\text { Discharge } \\
\text { (cfs) }\end{array}$ \\
\hline Aug. 2, 1943 & 6.61 & 6.47 & June 15,1946 & 68.53 & 98.6 \\
\hline Oct. $\quad 4----$ & 6.45 & 2.37 & June 17 & -7.64 & 47.5 \\
\hline Feb. 11, 1944 & 6.49 & 2.61 & June $18 \ldots$ & -7.44 & 41.1 \\
\hline Apr. $24 \cdots--$ & 8.68 & 170 & June 20 & -7.88 & 64.5 \\
\hline Aug. $\quad 1 \ldots-\cdots$ & 6.41 & *. 15 & July $24 \ldots \ldots$ & -6.42 & 0 \\
\hline July $\quad 2,1945$ & 6.54 & 3.59 & Feb. 13,1947 & 76.70 & 23.8 \\
\hline Sept. $\quad 4-\cdots$ & 6.46 & 1. 58 & Oct. $23 \ldots$ & -6.51 & 3.04 \\
\hline Dec. $12 \ldots-$ & 6.54 & 4.23 & Feb. 29, 1948 & 89.78 & 189 \\
\hline Apr. 11,1946 & 6.61 & 6.83 & Mar. $22 \cdots$ & -8.68 & 122 \\
\hline May $24 \ldots---$ & 6.62 & 8. 19 & Sept. $29 \ldots$ & -6.18 & 0 \\
\hline
\end{tabular}

*Estimated 
Average lake level for Cedar Lake near Waterloo for water years 1943-53

\begin{tabular}{c|c|c|c|c|c|c|c}
\hline Year & Oct. & Nov. & Dec. & Jan. & Feb. & Mar. & Apr. \\
\hline 1944 & 6.52 & 6.64 & 6.51 & 6.69 & 6.78 & 7.37 & 7.77 \\
1945 & 6.44 & 6.46 & 6.47 & 6.45 & 6.73 & 7.09 & 7.21 \\
1946 & 6.87 & 6.60 & 6.62 & 6.89 & 7.06 & 6.90 & 6.61 \\
1947 & 6.46 & 6.53 & 6.63 & 6.78 & 6.80 & 7.13 & 8.04 \\
1948 & 6.52 & 6.65 & 6.92 & 6.93 & 7.48 & 7.51 & 7.09 \\
1949 & 6.20 & 6.32 & 6.53 & 7.57 & 7.54 & 6.66 & 6.73 \\
1950 & 6.35 & 6.12 & 6.90 & 9.04 & 7.73 & 8.59 & 8.63 \\
1951 & 6.64 & 7.07 & 7.30 & 7.07 & 7.55 & 6.87 & 6.83 \\
1952 & 6.82 & 7.17 & 7.06 & 8.05 & 7.43 & 7.51 & 7.23 \\
1953 & 6.67 & 7.00 & 7.04 & 6.71 & 6.48 & 6.85 & 6.67 \\
\hline Average & 6.55 & 6.66 & 6.80 & 7.22 & 7.16 & 7.25 & 7.28 \\
\hline \hline Year & May & June & July & Aug. & Sept. & Annual & \\
\hline 1943 &.--- & --- &.-- & 6.61 & 6.61 & $* 6.61$ & \\
1944 & 7.03 & 6.71 & 6.46 & 6.43 & 6.43 & 6.76 & \\
1945 & 7.26 & 6.70 & 6.50 & 6.56 & 6.51 & 6.70 & \\
1946 & 6.58 & 7.23 & 6.54 & 6.38 & 6.39 & 6.72 & \\
1947 & 7.31 & 6.94 & 6.50 & 6.43 & 6.47 & 6.83 & \\
1948 & 7.44 & 6.65 & 6.29 & 6.19 & 6.16 & 6.82 & \\
1949 & 6.57 & 6.47 & 6.34 & 6.31 & 6.97 & 6.68 & \\
1950 & 6.66 & 6.51 & 6.57 & 5.92 & 6.68 & 7.14 & \\
1951 & 6.40 & 6.61 & 6.52 & 6.44 & 6.05 & 6.77 & \\
1952 & 6.58 & 6.05 & 6.65 & 7.43 & 7.67 & 7.14 & \\
1953 & 6.71 & 6.59 & 6.44 & 6.50 & 6.92 & 6.70 & \\
\hline Average & 6.85 & 6.65 & 6.48 & 6.47 & 6.62 & 6.74 & \\
\hline
\end{tabular}

* Partial month or partial year. 

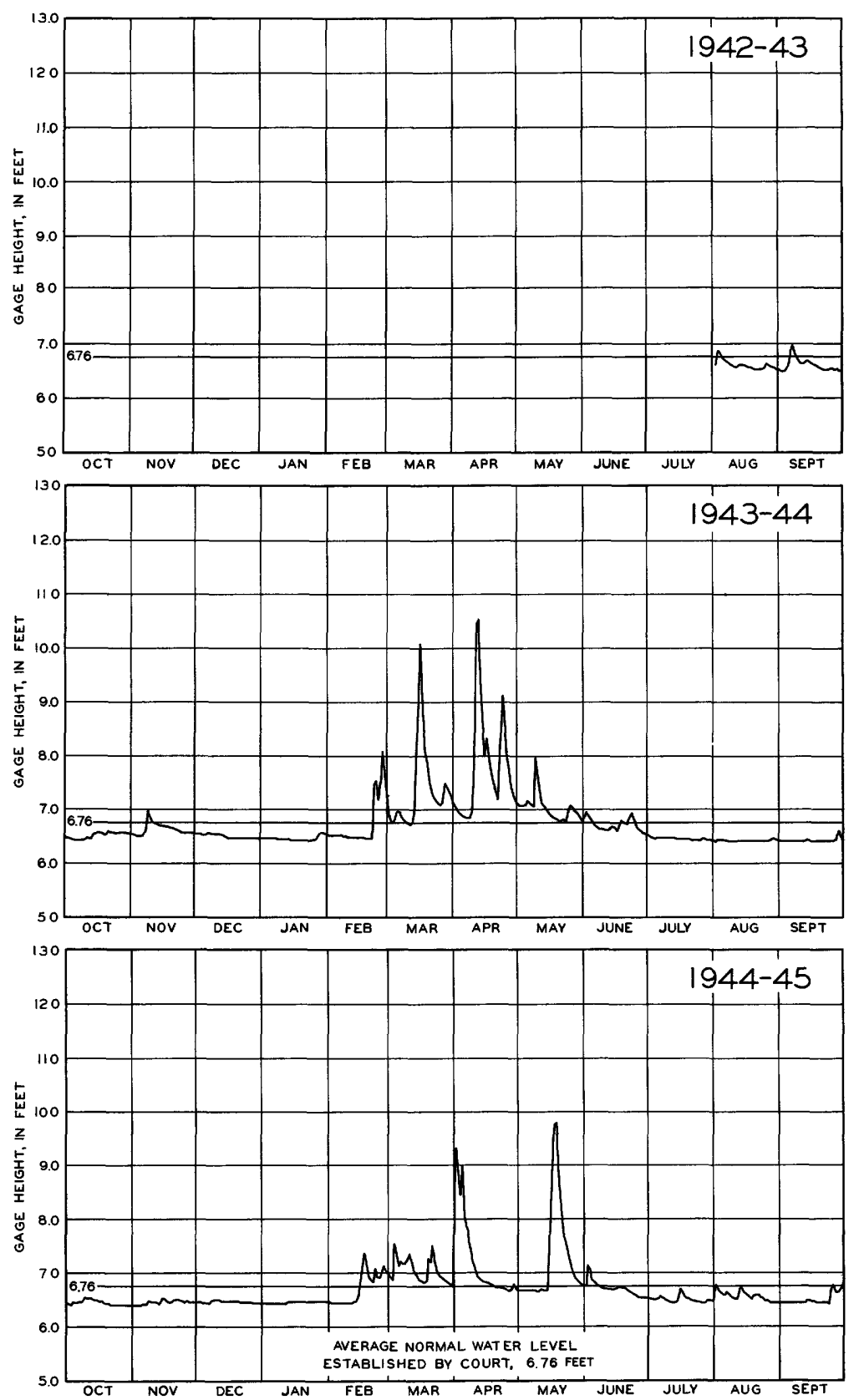

Figure 52. --Lake-level hydrographs for Cedar Lake near Waterloo, Ind., for water years 1943-45. 

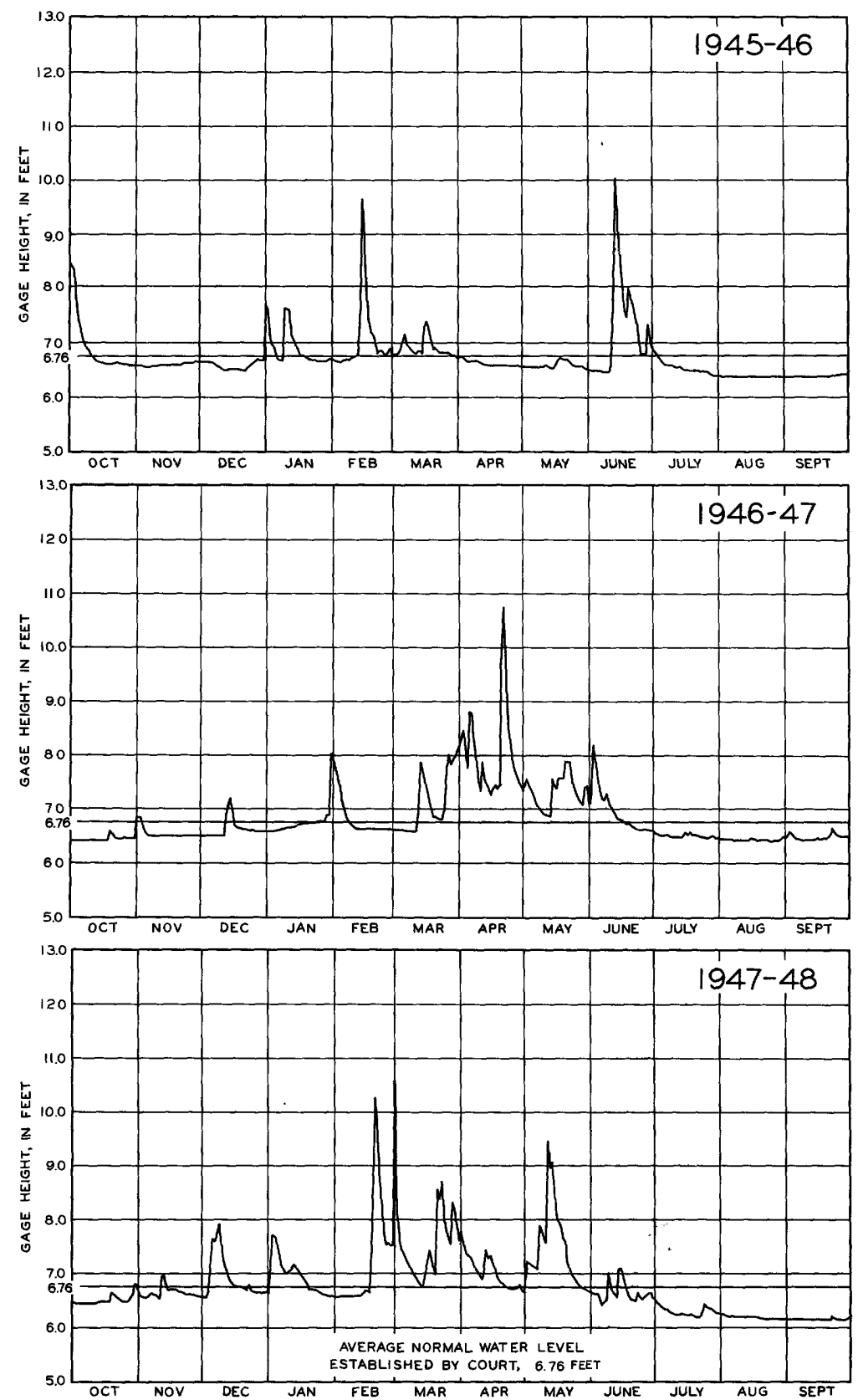

Figure 53. --Lake-level hydrographs for Cedar Lake near Water100 , Ind., for water years 1946-48. 

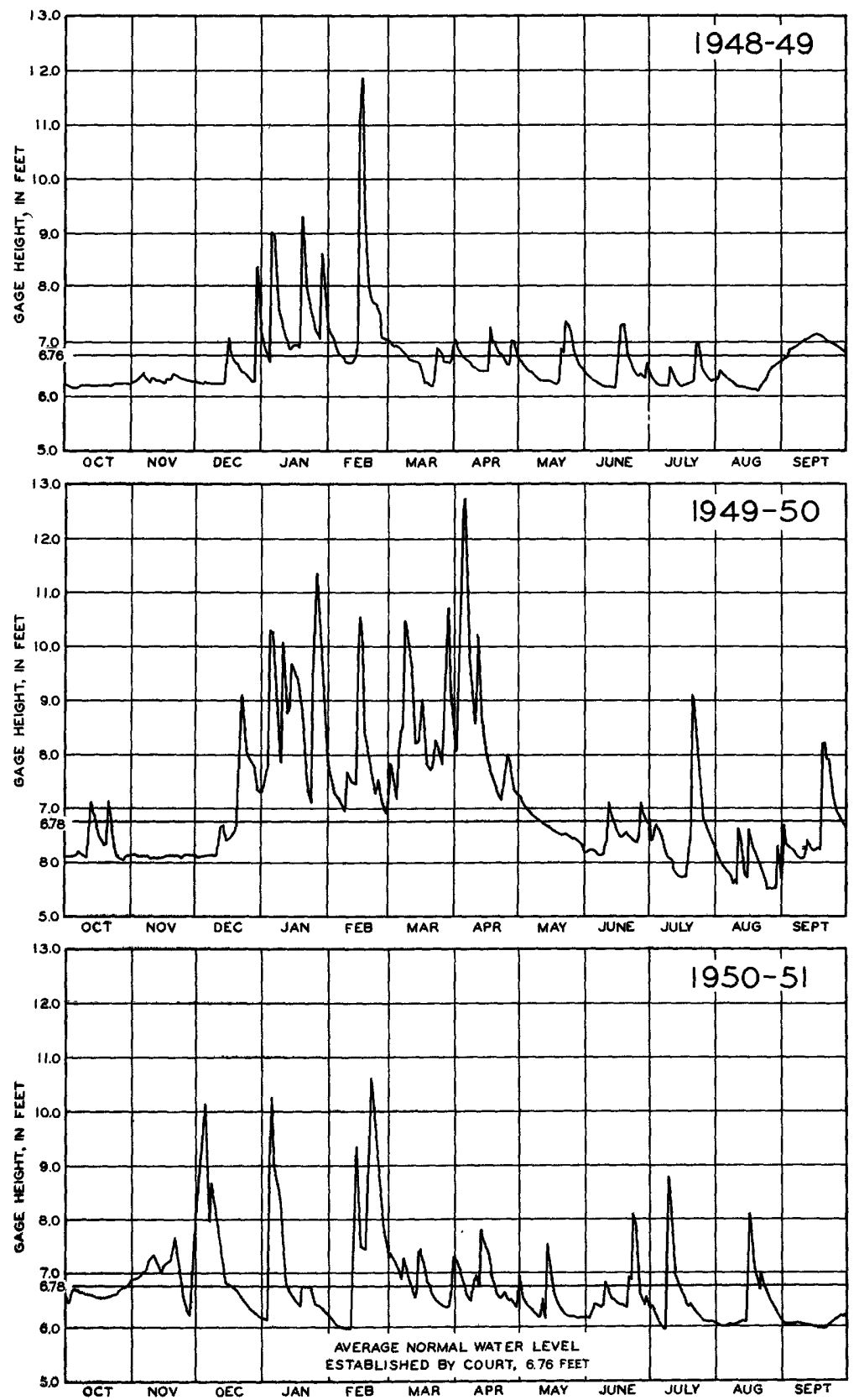

Figure 54. - Lake-level hydrographs for Cedar Lake near Water100, Ind., for water years 1949-51. 


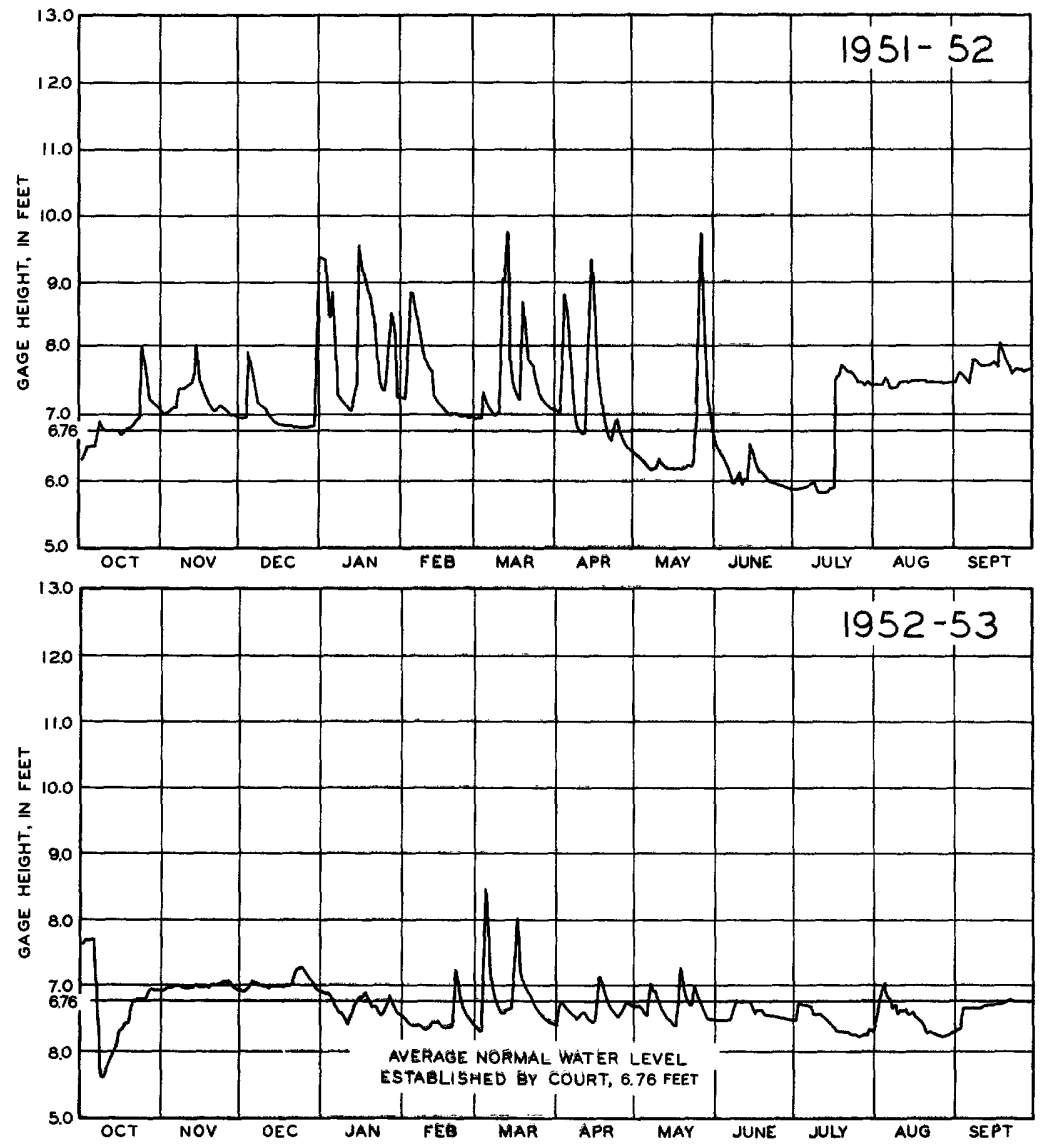

Figure 55. --Lake-level hydrographs for Cedar Lake near Waterlo o, Ind., for water years $1952-53$. 
Crooked Lake near Wolflake, Ind.

Location. --Secs. 33 and 34, T. 33 N., R. 9., E. , Noble County, and sections 3 and 4, T. 32 N., R. 9 E., Whitley County.

Surface area. --172 acres.

Drainage area. $-1.32 \mathrm{sq} \mathrm{mi}$.

Records available. - -August 1943 to November 1952 (Intermittent period to Mar. 27, 1947).

Gage. - -Staff gage at extreme west end of lake attached to a 12-inc maple tree, $250 \mathrm{ft}$ east of road. Gage read to hundredths onc daily. Datum of gage is $900.00 \mathrm{ft}$ above mean sea level, datun of 1929 .

Average lake level. --8 years; gage height, $5.68 \mathrm{ft}$; elevation, $905.68 \mathrm{ft}$

Established legal level. - -Established June 29, 1948 at gage heigh1 $5.69 \mathrm{ft}$, elevation, $905.69 \mathrm{ft}$ above mean sea level.

Lake-level control. - - Lake level maintained by bed of outlet channel.

Extreme levels for Crooked Lake, 1944-52

\begin{tabular}{|c|c|c|c|c|}
\hline \multicolumn{3}{|c|}{ Maximum } & \multicolumn{2}{|l|}{ Minimum } \\
\hline $\begin{array}{c}\text { Water } \\
\text { year }\end{array}$ & Date & $\begin{array}{l}\text { Gage } \\
\text { height } \\
\text { (feet) }\end{array}$ & Date & $\begin{array}{l}\text { Gage } \\
\text { height } \\
\text { (feet) }\end{array}$ \\
\hline $1945^{a}$ & May $17,18,1945 \ldots$ & 6.56 & Sept. 21, 22, 1945- & $a 5.38$ \\
\hline $1946^{a}$ & Mar. 9, 19-23, 1946-- & 6.14 & Sept. $28-30,1946-$ & $a_{4.85}$ \\
\hline $1947^{a}$ & Apr. 21-23, $1947 \ldots$ & 6.27 & Oct. $15-17,1946--$ & $a_{4.71}$ \\
\hline 1948 & Mar. 27, $1948 \ldots$ & 6.08 & Sept. 28, $1948 \ldots$ & 4.73 \\
\hline 1949 & Feb. $15,1949 \ldots$ & 6.48 & Oct. 20-22, 1948-- & 4. 66 \\
\hline 1950 & Apr. $4,1950 \ldots$ & 6.91 & Oct. $1,2,1949 \cdots$ & 5.13 \\
\hline 1951 & Feb. 22, $1951 \ldots$ & 6.47 & Nov. $7,1950 \ldots$ & 5.35 \\
\hline 1952 & Jan. 27, $1952 \ldots$ & 6.17 & Sept. 30, $1952 \ldots$ & 5. 20 \\
\hline
\end{tabular}

a Partial record only. 
Maximum recorded thickness and periods of ice cover 1944-51

\begin{tabular}{|c|c|c|c|}
\hline $\begin{array}{l}\text { Water } \\
\text { year }\end{array}$ & $\begin{array}{r}\text { Maximum } \\
\text { thickness } \\
\text { (inches) }\end{array}$ & $\begin{array}{l}\text { Total } \\
\text { days of } \\
\text { cover }\end{array}$ & Period \\
\hline 1944 & 5 & 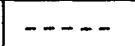 & \\
\hline 1945 & 11 & $\ldots-$ & \\
\hline 1946 & 8 & $-\ldots$ & \\
\hline 1947 & 5 & 91 & Jan. 5 to Apr. 5, 1947 \\
\hline 1948 & 15 & 107 & $\begin{array}{l}\text { Nov. } 30 \text { to Dec. } 4 \text {; Dec. } 10,1947 \text {, } \\
\text { to Mar. } 20,1948 .\end{array}$ \\
\hline 1949 & 4 & --- & $\begin{array}{l}\text { Dec. } 26,1948 \text {, to Jan. } 22,1949 \text {, } \\
\text { to-? }\end{array}$ \\
\hline 1950 & 5 & --- & \\
\hline 1951 & 11 & 83 & Dec. 12,1950 , to Mar. $4,1951$. \\
\hline
\end{tabular}

Discharge measurements at outlet of Crooked Lake

\begin{tabular}{|c|c|c|c|c|c|}
\hline Date & $\begin{array}{l}\text { Gage } \\
\text { height } \\
\text { (feet) }\end{array}$ & $\begin{array}{c}\text { Discharge } \\
\text { (cfs) }\end{array}$ & Date & $\begin{array}{l}\text { Gage } \\
\text { height } \\
\text { (feet) }\end{array}$ & $\begin{array}{c}\text { Discharge } \\
\text { (cfs) }\end{array}$ \\
\hline Aug. 12, 1943 & 5. 74 & 0.43 & Aug. 21, 1946 & 5.28 & 0 \\
\hline Oct. $\quad 8$ & 5.60 & *. 18 & Feb. 17, 1947 & 5.60 & .54 \\
\hline Feb. 16, 1944- & 5.32 & $* 10$ & Apr. $23 \cdots-$ & 6.22 & 4.77 \\
\hline May $15 \ldots$ & 6.30 & $* 4.00$ & Mar. 9, 1949 & 6.04 & 2.42 \\
\hline Apr. 10,1945 & 6.25 & 3.82 & Sept. $6 \ldots$ & 5.28 & .80 \\
\hline June 14 & 5. 96 & 1.47 & Dec. $\quad 1 \ldots-$ & 5.42 & .07 \\
\hline Sept. $\quad 7 \ldots$ & 5.51 & *. 18 & Jan. 17,1950 & 6.55 & 9.21 \\
\hline Dec. $\quad 6$ & 5.60 & $* .05$ & Apr. $13 \ldots-$ & 6.56 & 9.35 \\
\hline Mar. 11, 1946 & 6.12 & 2. 14 & Nov, $21 \ldots \ldots$ & 5.58 & .13 \\
\hline May $27 \ldots$ & 5.74 & $* .55$ & Feb. 20, 1951 & 6.24 & 5.31 \\
\hline June 19 & 5.91 & 1. 52 & May $15 \ldots$ & 6.10 & 2.38 \\
\hline
\end{tabular}

*Estimated. 
Average lake level for Crooked Lake for water years 1943-52

\begin{tabular}{c|l|l|r|r|r|r|l}
\hline Year & Oct. & Nov. & Dec. & Jan. & Feb. & Mar. & \multicolumn{1}{|c}{ Apr. } \\
1946 & 5.80 & --- & --- & --- & --- & 6.09 & 5.90 \\
1947 & 4.82 & --- & --- & $* 5.20$ & $* 5.56$ & $* 5.85$ & 6.03 \\
1948 & 5.32 & 5.36 & 5.53 & 5.66 & 5.87 & 5.93 & 5.84 \\
1949 & 4.92 & 5.08 & 5.26 & 5.93 & 6.14 & 5.90 & 5.86 \\
1950 & 5.47 & 5.44 & 5.62 & 6.44 & 6.34 & 6.33 & 6.34 \\
1951 & 5.44 & 5.49 & 6.01 & 6.08 & 6.13 & 6.13 & 6.17 \\
1952 & 5.45 & 5.68 & 5.75 & 5.96 & 5.89 & 5.84 & 5.85 \\
\hline Average & 5.32 & 5.41 & 5.63 & 5.88 & 5.99 & 6.01 & 6.00 \\
\hline \hline & & & & & & & \\
Year & May & June & July & Aug. & Sept. & Annual & \\
\hline 1943 & ---- & --- & --- & $* 5.73$ & ---- & $* 5.73$ & \\
1945 & 6.13 & 6.00 & 5.76 & 5.77 & 5.50 & $* 5.84$ & \\
1946 & 5.75 & 5.73 & 5.59 & 5.22 & 4.96 & $* 5.63$ & \\
1947 & 5.98 & 5.83 & 5.55 & 5.32 & 5.37 & $* 5.55$ & \\
1948 & 5.78 & 5.52 & 5.31 & 5.12 & 4.84 & 5.49 & \\
1949 & 5.77 & 5.70 & 5.45 & 5.23 & 5.21 & 5.52 & \\
1950 & 5.92 & 5.88 & 5.72 & 5.53 & 5.53 & 5.88 & \\
1951 & 6.00 & 5.83 & 6.06 & 5.86 & 5.54 & 5.89 & \\
1952 & 5.75 & 5.72 & 5.59 & 5.48 & 5.30 & 5.69 & \\
\hline Average & 5.88 & 5.78 & 5.63 & 5.47 & 5.28 & 5.68 & \\
\hline
\end{tabular}

*Partial month or partial year. 


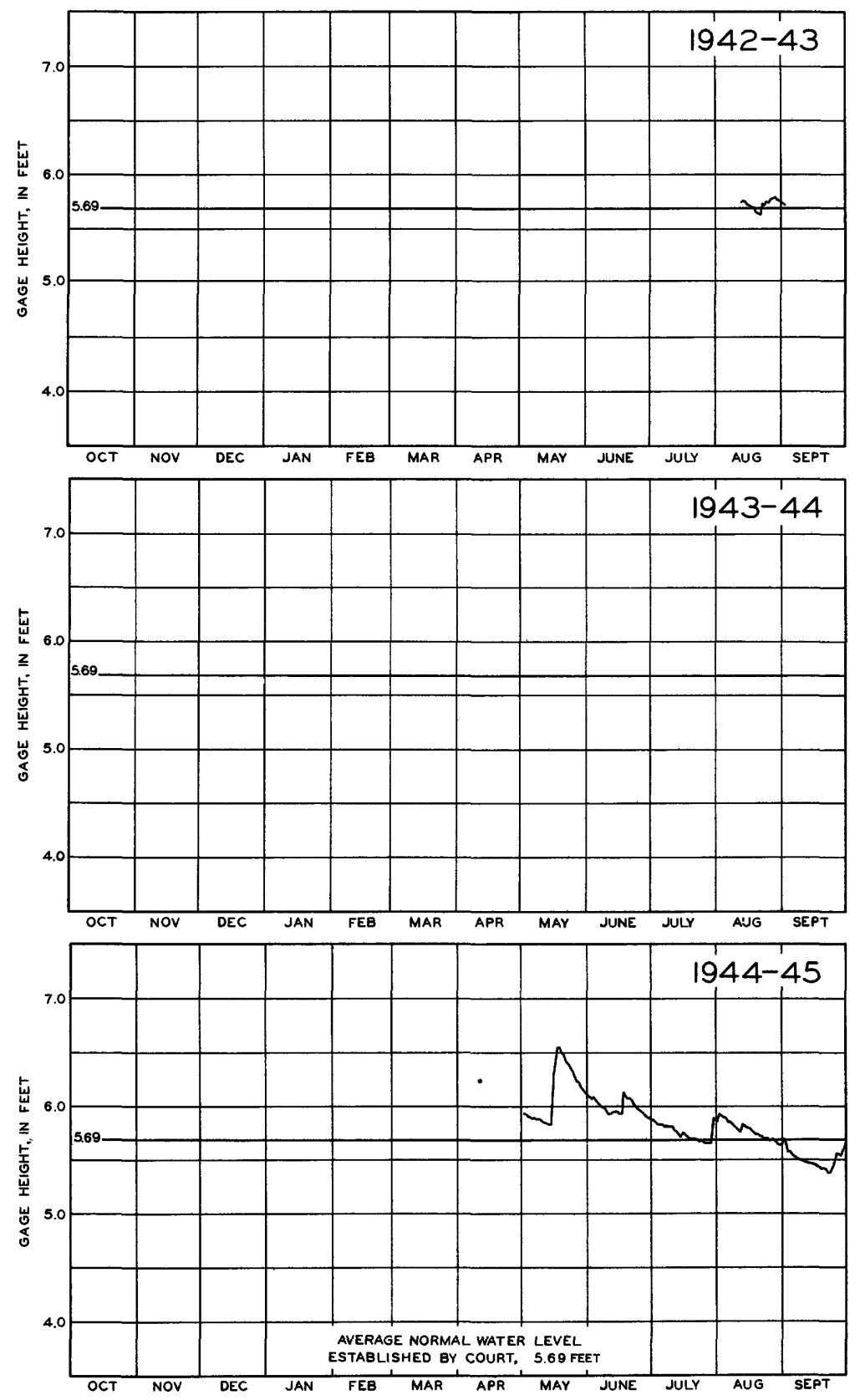

Figure 56. --Lake-level hydrographs for Crooked Lake near Wolflake, Ind., for water years 1943-45. 

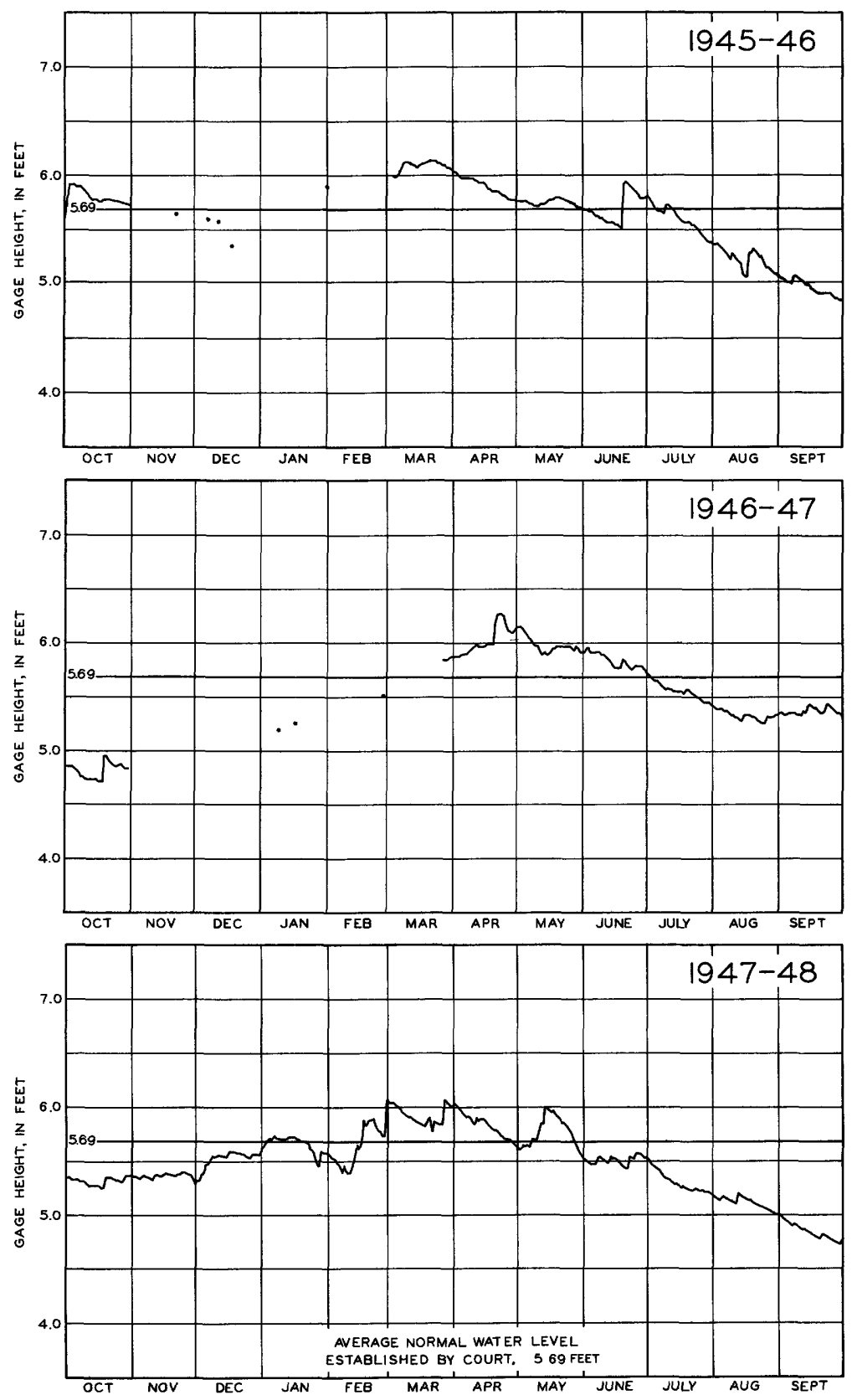

Figure 57. --Lake-level hydrographs for Crooked Lake near Wolflake, Ind., for water years 1946-48. 


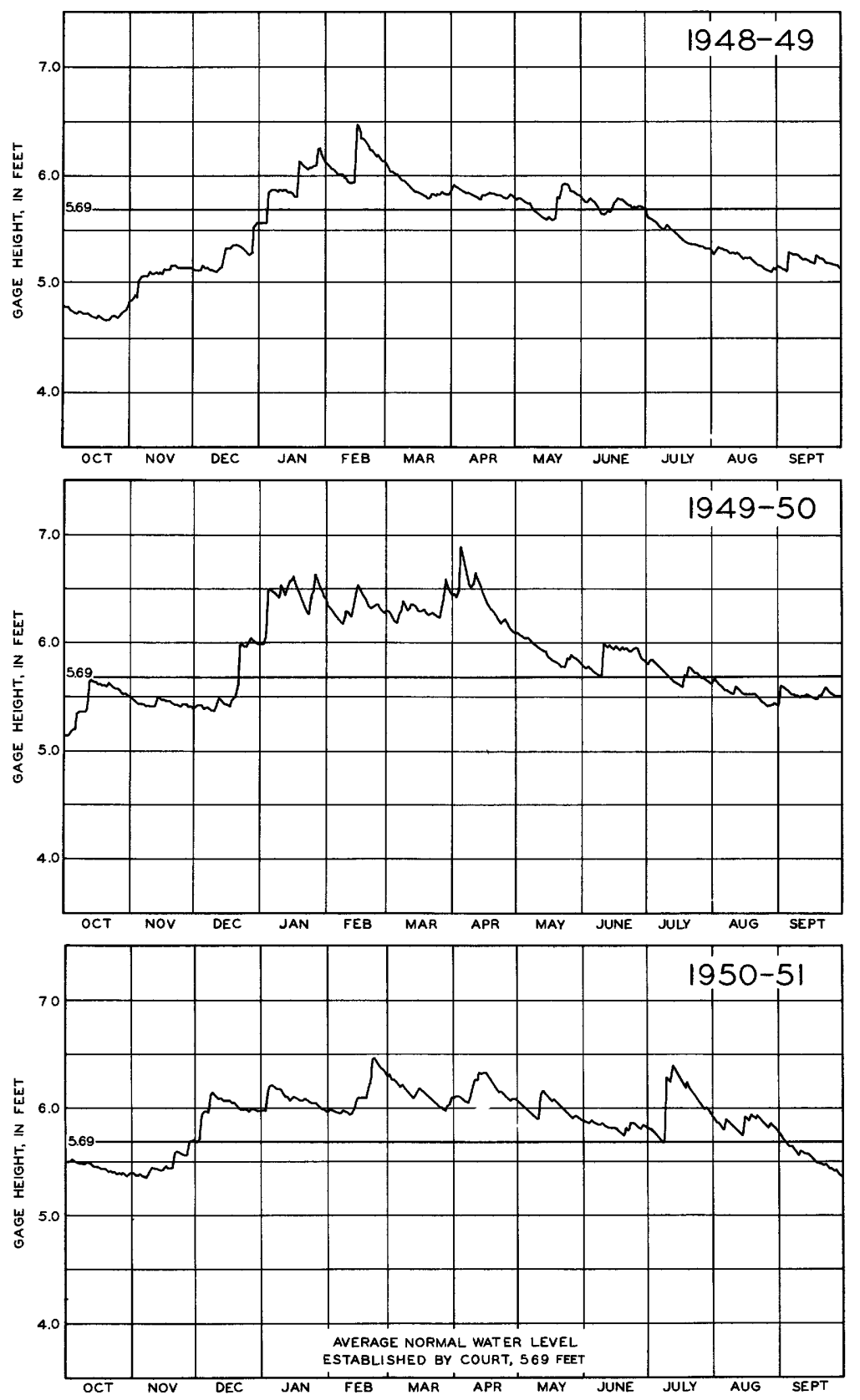

Figure 58. --Lake-level hydrographs for Crooked Lake near Wolflake, Ind., for water years 1949-51. 


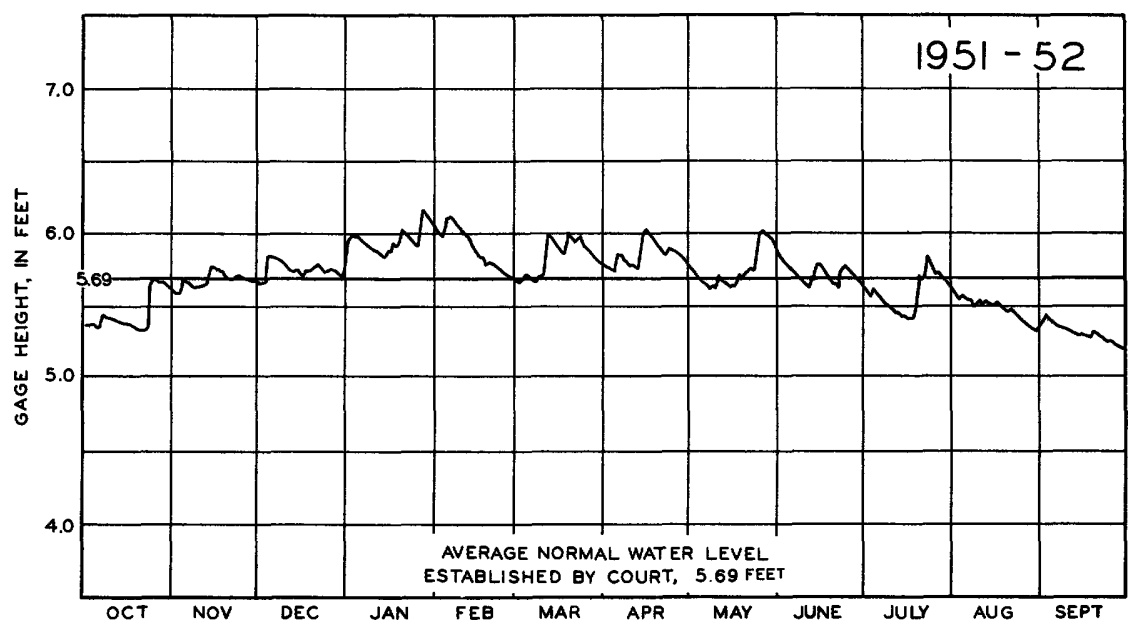

Figure 59. --Lake-level hydrographs for Crooked Lake near Wolflake, Ind., for water year 1952 .

Flint Lake near Valparaiso, Ind.

Location. --Sec. 6, T. 35 N., R. 5 W., and sec. 1, T. 35 N., R. 6 W., Porter County, 3.2 miles northeast of Valparaiso.

Surface area. --86.0 acres.

Drainage area. $--2.88 \mathrm{sq} \mathrm{mi}$.

Records available. --August 1946 to September 1953.

Gage.--Float type gage inside the water plant. Gage read to hundredths once daily. Datum of gage is $780.00 \mathrm{ft}$ above mean sea level, datum of 1929. Prior to Mar. 26, 1950, staff gage in front of waterworks at same datum.

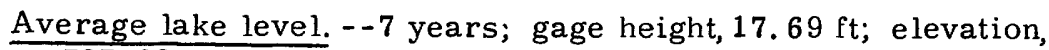
$797.69 \mathrm{ft}$.

Established legal level. --None.

Lake-level control. --Lake level maintained by bed of outlet channel ditch. 
Extreme levels for Flint Lake, 1947-53

\begin{tabular}{|c|c|c|c|c|}
\hline \multicolumn{3}{|c|}{ Maximum } & \multicolumn{2}{|c|}{ Minimum } \\
\hline $\begin{array}{l}\text { Water } \\
\text { year }\end{array}$ & Date & $\begin{array}{c}\text { Gage } \\
\text { height } \\
\text { (feet) }\end{array}$ & Date & $\begin{array}{c}\text { Gage } \\
\text { height } \\
\text { (feet) }\end{array}$ \\
\hline 1947 & June $3,1947 \ldots$ & 20.27 & Mar. 22, 23, 1947- & 15.24 \\
\hline 1948 & May $19,20,1948 \ldots$ & 19.58 & Feb. 15,1948 & $* 12.97$ \\
\hline 1949 & May 27, 1949 & 17.99 & Dec. $29,1948-\cdots$ & 12.59 \\
\hline 1950 & Apr. 5,1950 & 20.84 & Dec. $9,10,1949--$ & 15.75 \\
\hline 1951 & May $13-15,1951 \ldots$ & 20.03 & Dec. $1,2,1950 \cdots$ & 16.69 \\
\hline 1952 & Jan. $20,1952 \ldots$ & 20.40 & Sept. 30, $1952 \ldots$ & 17.40 \\
\hline 1953 & May 26, 1953 & 18.32 & Sept. $30,1953---$ & 15. 23 \\
\hline
\end{tabular}

* Established.

Maximum recorded thickness and periods of ice cover 1947-53

\begin{tabular}{c|c|c|c|c}
\hline $\begin{array}{c}\text { Water } \\
\text { year }\end{array}$ & $\begin{array}{c}\text { Maximum } \\
\text { thickness } \\
\text { (inches) }\end{array}$ & $\begin{array}{c}\text { Total } \\
\text { days of } \\
\text { cover }\end{array}$ & \multicolumn{2}{|c}{ Period } \\
\hline 1947 & $13 \frac{1}{2}$ & 106 & Dec. 19, 1946, to Apr. 3, 1947. \\
1948 & $14 \frac{1}{2}$ & 101 & Dec. 11, 1947, to Mar. 20, 1948. \\
1949 & $8 \frac{1}{2}$ & 81 & Dec. 20, 1948, to Mar. 10, 1949. \\
1950 & $6 \frac{1}{2}$ & --- & a Dec. 9, 1949, to Mar. 25, 1950. \\
1951 & 13 & 91 & Dec. 5, 1950, to Mar. 5, 1951. \\
1952 & 7 & 92 & Dec. 15, 1951, to Mar. 15, 1952. \\
1953 & -- & -- & Mar. 7, 1953 to-? \\
\hline
\end{tabular}

$a^{a}$ Lake open for intermittent periods.

Discharge measurements at outlet of Flint Lake

\begin{tabular}{|c|c|c|c|c|c|}
\hline Date & $\begin{array}{c}\text { Gage } \\
\text { height } \\
\text { (feet) }\end{array}$ & $\begin{array}{c}\text { Discharge } \\
\text { (cfs) }\end{array}$ & Date & $\begin{array}{c}\text { Gage } \\
\text { height } \\
\text { (feet) }\end{array}$ & $\begin{array}{c}\text { Discharge } \\
\text { (cfs) }\end{array}$ \\
\hline Sept. 23,1946 & 18.27 & 0 & Feb. 17, 1947- & 16.36 & 0 \\
\hline $21 \cdots-\cdots$ & 17.51 & 0 & Feb. 26- & 15.95 & 0 \\
\hline $18 \cdots \cdots$ & 17.27 & 0 & Apr. $\quad 7-\cdots$ & 18. 14 & 0 \\
\hline $20 \cdots-$ & 16.87 & 0 & May 1-n- & 16.93 & 1.44 \\
\hline $27 \cdots--$ & 16. 70 & 0 & Sept. 23---- & 18.27 & 0 \\
\hline $31 \ldots-$ & 16.65 & 0 & Dec. $16-\cdots$ & 15.07 & 0 \\
\hline Jan. $\quad 6,1947$ & 16.57 & 0 & Nov. $3,1948-$ & 14.28 & 0 \\
\hline
\end{tabular}


Average lake level for Flint Lake for water years 1946-53

\begin{tabular}{c|c|c|c|c|c|c|c}
\hline Year & Oct. & Nov. & Dec. & Jan. & Feb. & Mar. & Apr. \\
\hline 1947 & 17.62 & 17.26 & 16.77 & 16.42 & 16.49 & 15.65 & 18.42 \\
1948 & 16.59 & 15.67 & 14.87 & 14.42 & 13.56 & 16.39 & 17.98 \\
1949 & 14.82 & 13.73 & 12.73 & 12.94 & 14.09 & 15.43 & 17.33 \\
1950 & 16.59 & 16.06 & 16.41 & 18.65 & 19.64 & 20.24 & 20.61 \\
1951 & 17.58 & 16.86 & 16.90 & 17.31 & 17.40 & 18.10 & 19.08 \\
1952 & 19.39 & 19.73 & 19.99 & 20.27 & 20.09 & 19.95 & 20.06 \\
1953 & 16.89 & 16.42 & 16.33 & 16.95 & 17.35 & 17.94 & 18.08 \\
\hline Average & 17.07 & 16.53 & 16.29 & 16.71 & 16.95 & 17.67 & 18.79 \\
\hline \hline & & & & & & & \\
Year & May & June & July & Aug. & Sept. & Annual & \\
\hline 1946 & ---- & ---- & ---- & $* 18.73$ & 18.39 & $* 18.56$ & \\
1947 & 19.75 & 19.98 & 19.25 & 18.36 & 17.56 & 17.80 & \\
1948 & 18.99 & 19.03 & 18.40 & 17.44 & 16.06 & 16.63 & \\
1949 & 17.71 & 17.63 & 17.23 & 16.99 & 16.87 & 15.63 & \\
1950 & 19.99 & 19.95 & 19.50 & 18.91 & 18.26 & 18.73 & \\
1951 & 19.80 & 19.58 & 19.18 & 19.17 & 19.12 & 18.34 & \\
1952 & 19.86 & 19.67 & 19.11 & 18.45 & 17.77 & 19.53 & \\
1953 & 18.11 & 17.90 & 17.28 & 16.58 & 15.55 & 17.11 & \\
\hline Average & 19.17 & 19.11 & 18.56 & 18.08 & 17.45 & 17.69 & \\
\hline
\end{tabular}

*Partial month or partial year. 

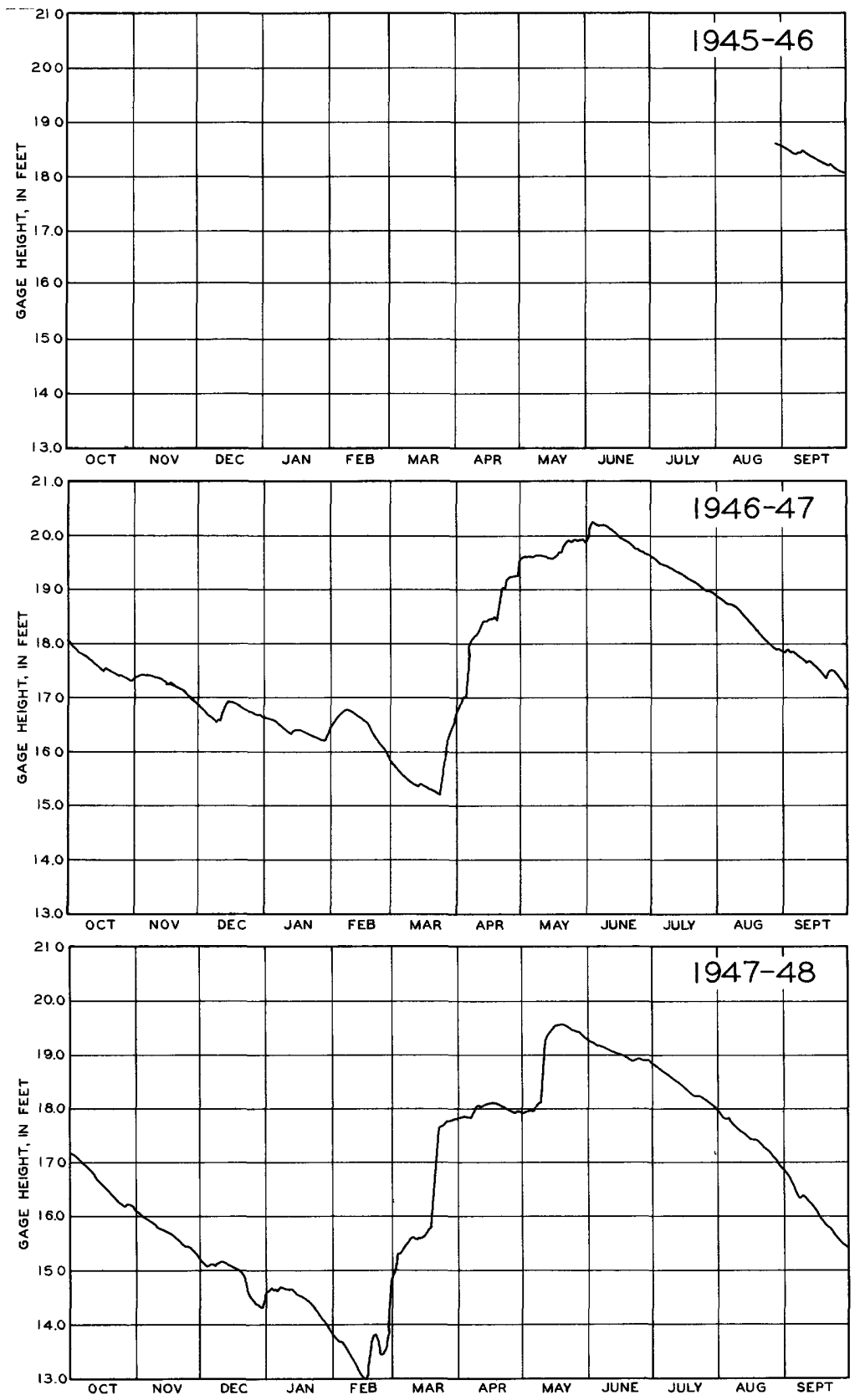

Figure 60. --Lake-level hydrographs for Flint Lake near Valparaiso, Ind., for water years 1946-48. 

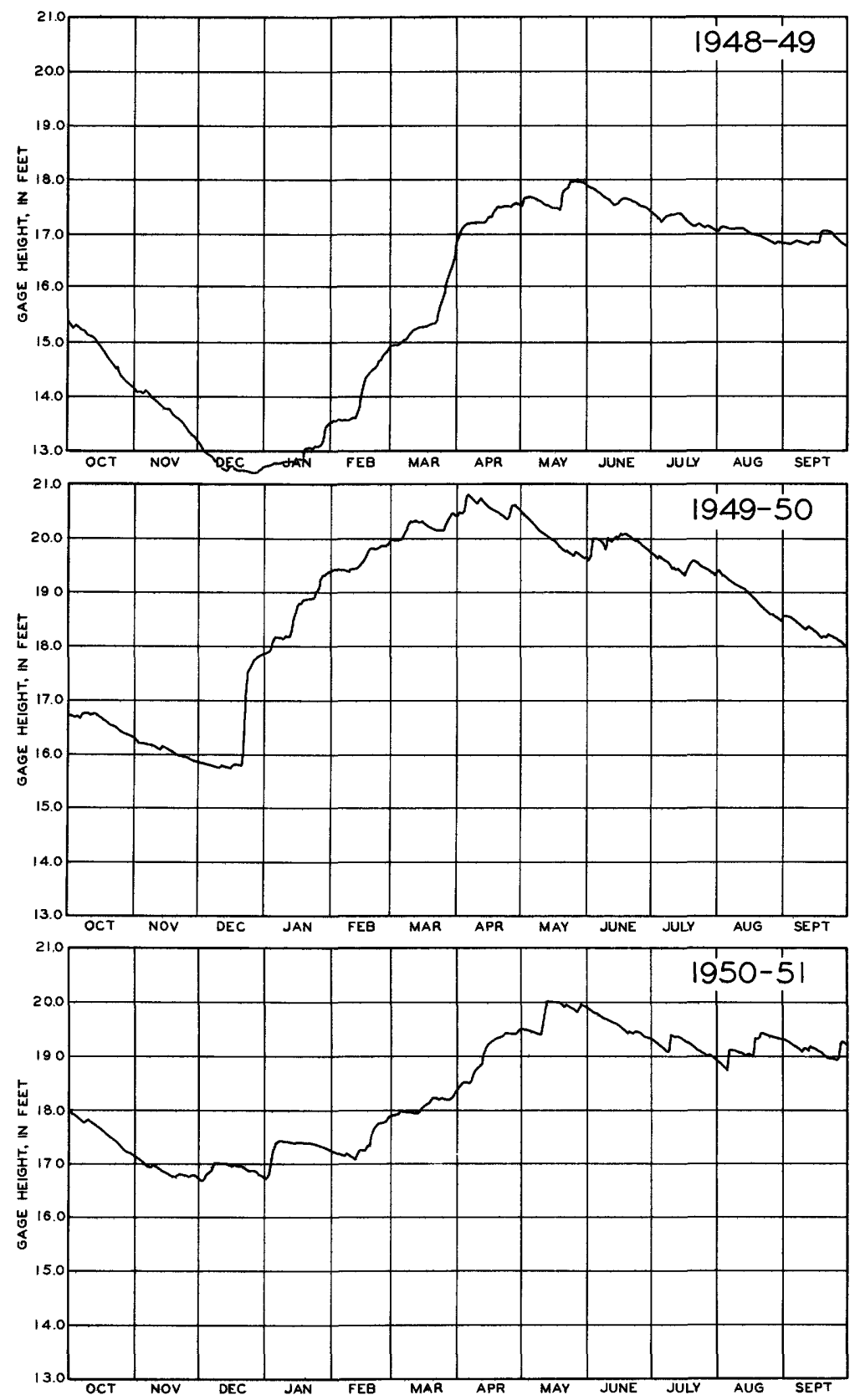

Figure 61. --Lake-level hydrographs for Flint Lake near Valparaiso, Ind., for water years 1949-51. 


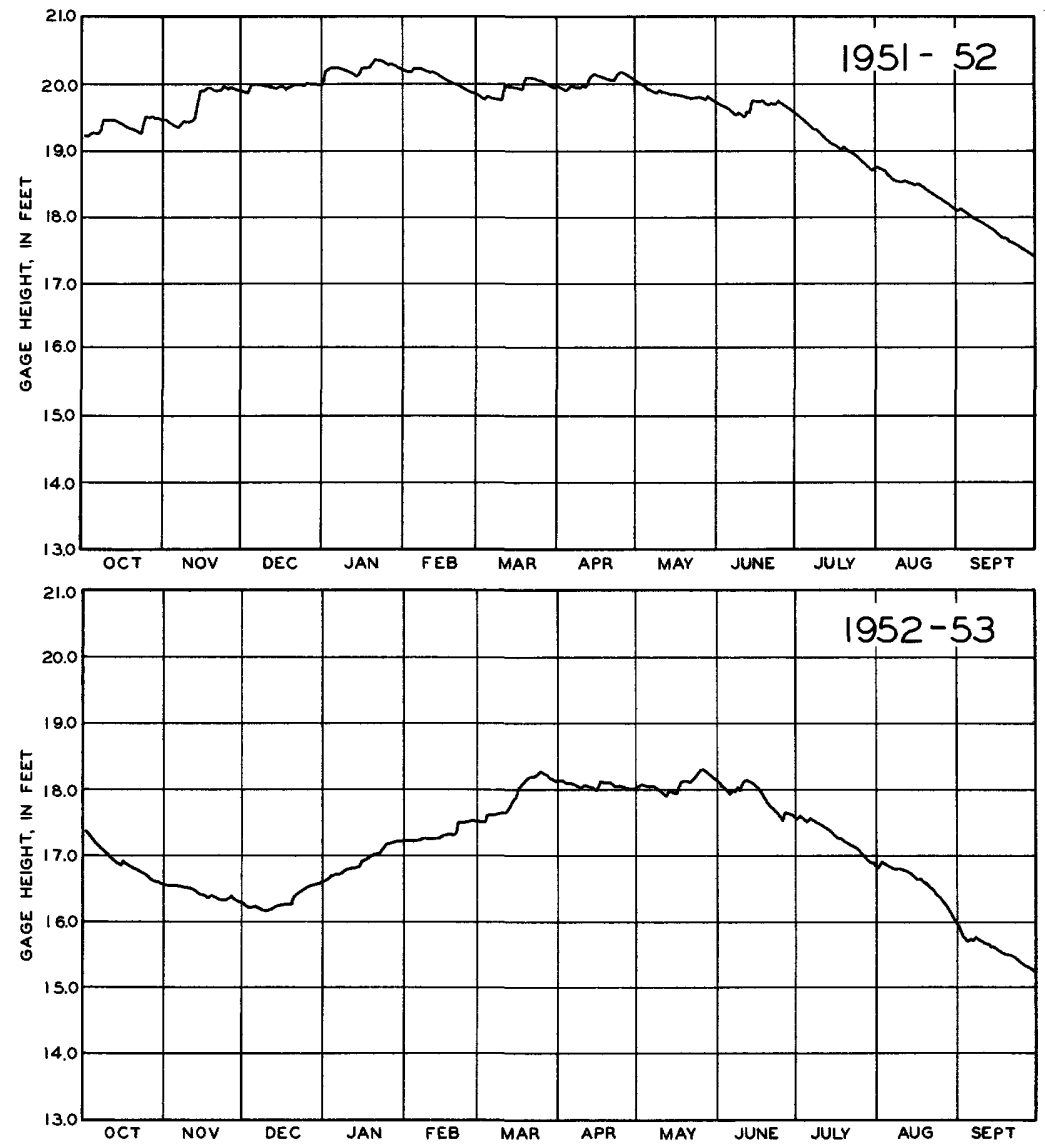

Figure 62. --Lake-level hydrog raphs for Flint Lake near Valparaiso, Ind., for water years 1952-53. 
Hamilton Lake at Hamilton, Ind.

Location.--Secs. 21, 22, 27, 28, 33 and 34, T. 36 N., R 14 E., Steuben County.

Surface area. - -765 acres.( USGS topographic maps, surveyed in 1938).

Drainage area. $--12.8 \mathrm{sq} \mathrm{mi}$ (determined from the same maps).

Records available. --July 1943 to September 1953.

Gage. - -Staff gage attached to concrete wing wall of south abutment of control dam on south out let at southeastern corner of lake. Gage read to hundredths once daily. Datum of gage is 890. $00 \mathrm{ft}$ above mean sea level, unadjusted.

Average level. --10 years; gage height, $8.37 \mathrm{ft}$; elevation, $898.37 \mathrm{ft}$.

Established legal level. --Established July 3, 1947, at gage height $8.38 \mathrm{ft}$, elevation, $898.83 \mathrm{ft}$ above mean sea level.

Lake-level control. - - Lake has two outlets about 800 it apart.

One outlet has concrete dam with fixed crest, the other a concrete dam with movable flood gates.

Extreme lake levels for Hamilton Lake, 1944-53

\begin{tabular}{|c|c|c|c|c|}
\hline \multicolumn{3}{|c|}{ Maximum } & \multicolumn{2}{|l|}{ Minimum } \\
\hline $\begin{array}{l}\text { Water } \\
\text { year }\end{array}$ & Date & $\begin{array}{l}\text { Gage } \\
\text { height } \\
\text { (feet) }\end{array}$ & Date & $\begin{array}{l}\text { Gage } \\
\text { height } \\
\text { (feet) }\end{array}$ \\
\hline 1944 & Apr. $13,1944 \ldots-\cdots$ & 9.35 & Sept. 22-27, 1944- & 7.61 \\
\hline 1945 & May $18,1945 \ldots$ & 9.55 & $\begin{array}{r}\text { Oct. } 22 \text { to Nov. } 3 \text {, } \\
1943\end{array}$ & 7.81 \\
\hline 1946 & Feb. 21, $1946 \ldots$ & 8.75 & Sept. 8, 9, 29, 1946 & 7.67 \\
\hline 1947 & Apr. $21,1947 \ldots$ & 9.51 & Oct. $14-16,1946--$ & 7.55 \\
\hline 1948 & Feb. 20, $1948 \ldots$ & 9.36 & Sept. 20, 1948-.- & 7. 78 \\
\hline 1949 & Feb. $15,1949 \ldots$ & 9.26 & Oct. $21-30,1949-$ & 7.88 \\
\hline 1950 & Apr. $4,1950 \ldots$ & 9.68 & Oct. $1,2,1950 \ldots$ & 8. 12 \\
\hline 1951 & Feb. 22, $1951 \ldots$ & 9.32 & Sept. 29, 30, $1951-$ & 8.22 \\
\hline 1952 & May 26, 1952- & 9.08 & Sept. $30,1952 \cdots$ & 7.97 \\
\hline 1953 & May 26, 1953 & $* 8.62$ & Jan. $4-9,1953 \cdots$ & 7.27 \\
\hline
\end{tabular}

*Estimated. 
BASIC DATA ON LAKE LEVELS FOR SELECTED LAKES 137

Maximum recorded thickness and periods of ice cover 1944-51

\begin{tabular}{|c|c|c|c|}
\hline $\begin{array}{l}\text { Water } \\
\text { year }\end{array}$ & $\begin{array}{l}\text { Maximum } \\
\text { thickness } \\
\text { (inches) }\end{array}$ & $\begin{array}{c}\text { Total } \\
\text { days of } \\
\text { cover }\end{array}$ & Period \\
\hline 1944 & 10 & 75 & Dec. 16, 1943, to Feb. 28, 1944 \\
\hline 1945 & --- & --- & Dec. 6,1944, to $-?$ \\
\hline 1946 & --- & 94 & Dec. 1,1945 , to Mar. 4, 1946. \\
\hline 1947 & $11 \frac{1}{2}$ & 129 & Nov. 29,1946 , to Apr. $6,1947$. \\
\hline 1948 & 25 & 97 & $\begin{array}{l}\text { Nov. } 30 \text {, to Dec. } 6 \text {; Dec. } 22,1947 \text {, } \\
\text { to Mar. } 21,1948 .\end{array}$ \\
\hline 1949 & 6 & ---- & \\
\hline 1950 & --- & --- & \\
\hline 1951 & 15 & --- & Dec. 9,1950 , to $-?$ \\
\hline
\end{tabular}

Discharge measurements at outlet of Hamilton Lake

\begin{tabular}{|c|c|c|c|c|c|c|c|}
\hline & Date & $\begin{array}{c}\text { Gage } \\
\text { height } \\
\text { (feet) }\end{array}$ & $\begin{array}{c}\text { Discharge } \\
\text { (cfs) }\end{array}$ & & Date & $\begin{array}{l}\text { Gage } \\
\text { height } \\
\text { (feet) }\end{array}$ & $\begin{array}{c}\text { Discharge } \\
\text { (cfs) }\end{array}$ \\
\hline Tuly & $30,1943-$ & 8.41 & 5.05 & July & $12,1951-$ & 8.83 & 38.4 \\
\hline Oct. & $4----$ & 8.31 & 1. 10 & Aug. & $9 \ldots$ & 8.38 & 1. 79 \\
\hline Feb. & $10,1944-$ & 8.39 & 3.08 & Sept. & $5 \ldots \ldots$ & 8.31 & .29 \\
\hline Apr. & $24 \ldots \ldots$ & 2.52 & $* 30.00$ & Oct. & $9 \ldots$ & 8.35 & 2.27 \\
\hline Aug. & $1-\cdots$ & 7.93 & *. 20 & Dec. & $14 \ldots$ & 8. 51 & 13.9 \\
\hline May & $1,1945^{-}$ & 8.45 & 8.12 & Feb. & $12,1952-$ & 8.68 & 31.9 \\
\hline June & $29-\cdots--$ & 8. 43 & 3.91 & Mar. & $26 \ldots-\cdots$ & 8.68 & 33.0 \\
\hline Sept. & $4-\cdots$ & 8.21 & .07 & Nov. & $13 \cdots-\cdots$ & 7.93 & 0 \\
\hline May & $24,1946-$ & 8.39 & 2.29 & Dec. & $10 \ldots$ & 8.28 & 1.07 \\
\hline July & $24-\cdots--$ & 8.13 & 0 & Jan. & $8,1953-$ & 8. 40 & 5.87 \\
\hline Dec. & $5-\cdots$ & 7.93 & 0 & Jan. & $30-\cdots-$ & 8.44 & 15. 7 \\
\hline Feb. & $14,1947^{-}$ & 8. 46 & 5.87 & Mar. & $10 \cdots \cdots-\cdots$ & 8.51 & 29.0 \\
\hline Nov. & $2,1950^{-}$ & 8.37 & 6.98 & Apr. & $2 \cdots--$ & 8.51 & 28.6 \\
\hline Nov. & $16-\cdots--$ & 8.53 & 26.6 & Apr. & $23-\ldots-$ & 8.47 & 18.9 \\
\hline Jan. & $24,1951-$ & 8.58 & 20.1 & May & $19 \ldots-$ & 8.57 & 32.4 \\
\hline Apr. & $12 \cdots-\cdots$ & 8.80 & 58.9 & June & $17 \cdots$ & 8.29 & *. 3 \\
\hline May & $8-\cdots--$ & 8.58 & 13.5 & Aug. & $5-\cdots$ & 8.33 & 8.53 \\
\hline June & $6-\cdots--$ & 8. 46 & 3.56 & Sept. & $1-\cdots--$ & 8.05 & 0 \\
\hline
\end{tabular}

*Estimated. 
Average lake level for Hamilton Lake for water years 1943-53

\begin{tabular}{c|l|l|l|l|l|l|l}
\hline Year & Oct. & Nov. & Dec. & Jan. & Feb. & Mar. & A pr. \\
\hline 1944 & 8.32 & 8.42 & 8.36 & 8.35 & 8.50 & 8.64 & 8.77 \\
1945 & 7.86 & 7.97 & 8.16 & 8.34 & 8.45 & 8.64 & 8.68 \\
1946 & 8.25 & 8.33 & 8.38 & 8.48 & 8.49 & 8.50 & 8.36 \\
1947 & 7.65 & 7.89 & 8.07 & 8.42 & 8.47 & 8.56 & 8.86 \\
1948 & 8.25 & 8.38 & 8.50 & 8.50 & 8.60 & 8.75 & 8.62 \\
1949 & 7.90 & 8.18 & 8.36 & 8.65 & 8.70 & 8.49 & 8.48 \\
1950 & 8.33 & 8.30 & 8.52 & 8.89 & 8.69 & 8.85 & 8.91 \\
1951 & 8.42 & 8.54 & 8.70 & 8.64 & 8.75 & 8.70 & 8.68 \\
1952 & 8.35 & 8.51 & 8.65 & 8.78 & 8.61 & 8.68 & 8.68 \\
1953 & 7.73 & 7.44 & 7.45 & 7.32 & 7.49 & 7.93 & 8.49 \\
\hline Average & 8.11 & 8.20 & 8.32 & 8.44 & 8.48 & 8.57 & 8.65 \\
\hline \hline Year & May & June & July & Aug. & Sept. & Annual & \\
\hline 1943 & ---- & ---- & $* 8.40$ & 8.33 & 8.35 & $* 8.34$ & \\
1944 & 8.65 & 8.38 & 8.09 & 7.82 & 7.67 & 8.33 & \\
1945 & 8.80 & 8.53 & 8.37 & 8.27 & 8.17 & 8.35 & \\
1946 & 8.34 & 8.35 & 8.21 & 7.90 & 7.73 & 8.28 & \\
1947 & 8.69 & 8.68 & 8.30 & 8.14 & 8.27 & 8.33 & \\
1948 & 8.62 & 8.41 & 8.32 & 8.18 & 7.89 & 8.42 & \\
1949 & 8.43 & 8.47 & 8.39 & 8.26 & 8.15 & 8.37 & \\
1950 & 8.51 & 8.47 & 8.48 & 8.33 & 8.49 & 8.56 & \\
1951 & 8.66 & 8.55 & 8.55 & 8.41 & 8.30 & 8.58 & \\
1952 & 8.56 & 8.52 & 8.33 & 8.24 & 8.15 & 8.50 & \\
1953 & 8.54 & 8.37 & 8.27 & 8.20 & 7.93 & 7.93 & \\
\hline Average & 8.58 & 8.47 & 8.34 & 8.19 & 8.10 & 8.37 & \\
\hline
\end{tabular}

* Partial month or partial year. 


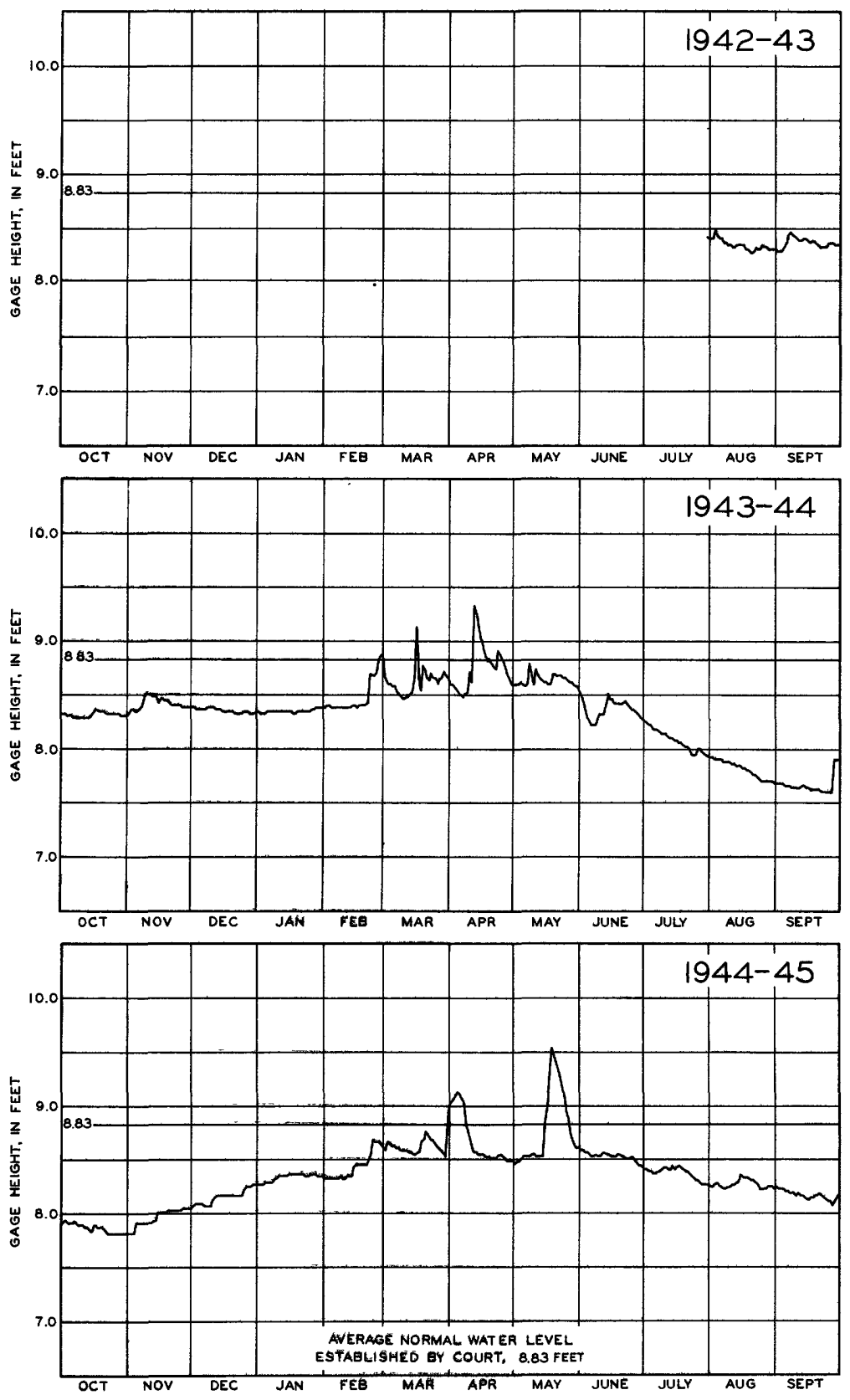

Figure 63. --Lake-level hydrographs for Hamilton Lake at Hamilton, Ind., for water years 1943-45. 

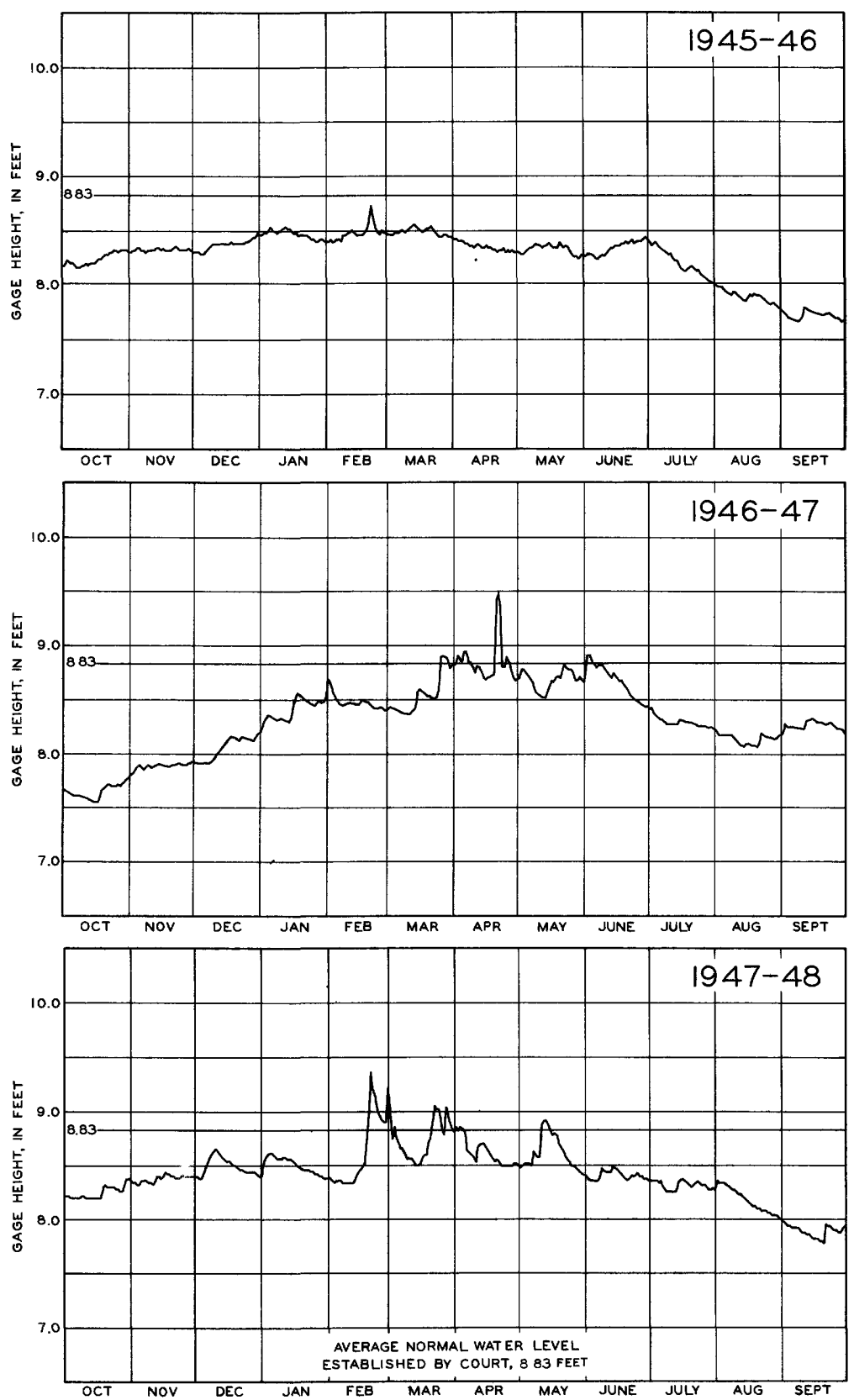

Figure 64. --Lake-level hydrographs for Hamilton Lake at Hamilton, Ind., for water years 1946-48. 

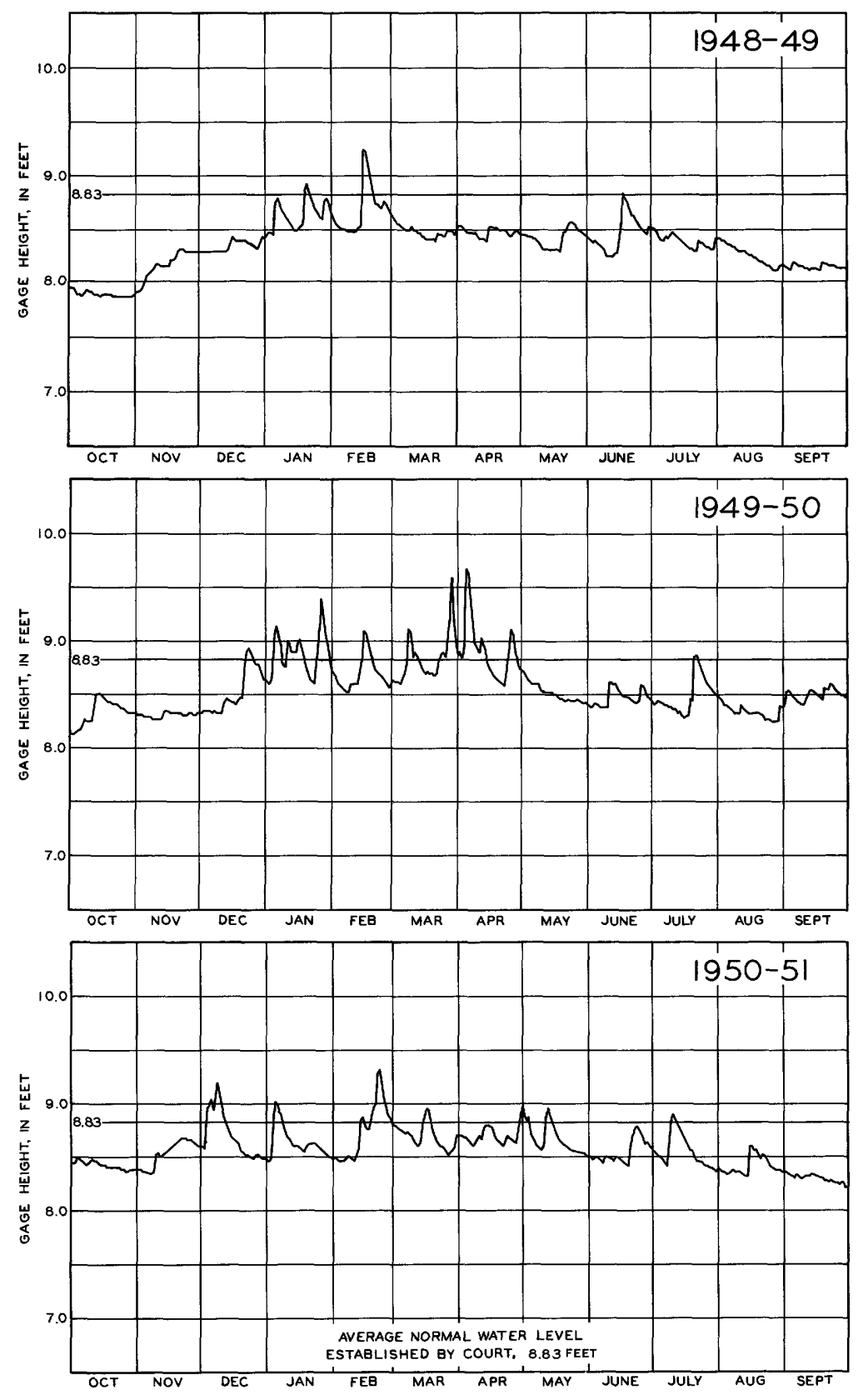

Figure 65. --Lake-level hydrographs for Hamilton Lake at HamiIton, Ind., for water years 1949-51. 

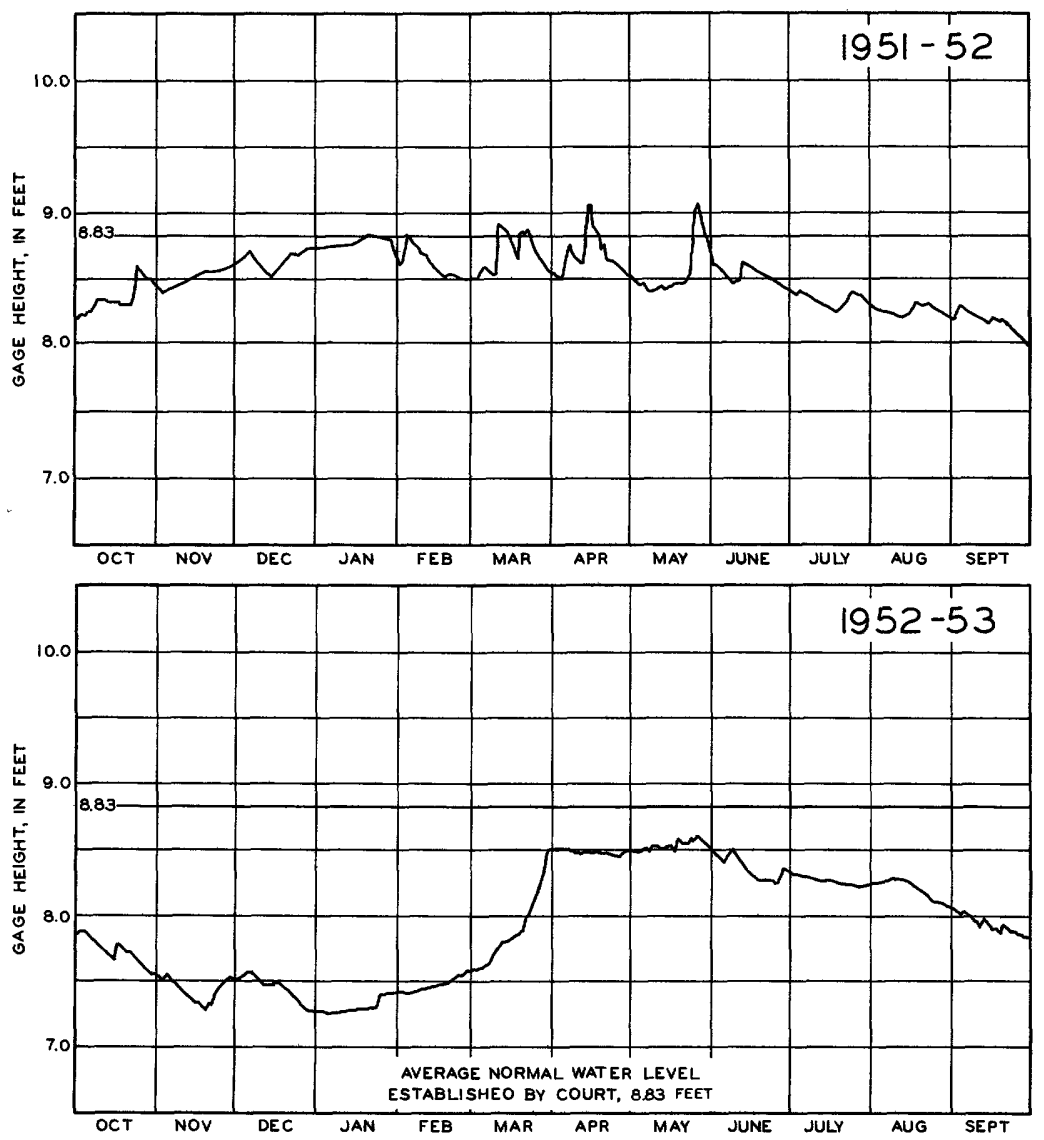

Figure 66. --Lake-level hydrographs for Hamilton Lake at Hamilton, Ind., for water years 1952-53. 
Jimerson Lake at Nevada Mills, Ind.

Location. --Secs. 29, 30, 31 and 32 , T. 38 N., R. 13 E., and sec. 5, T. 37 N., R. 13 E., Steuben County.

Surface area. --346 acres (determined from USGS topographic maps, surveyed in 1939).

Drainage area. $-470 \mathrm{sq} \mathrm{mi}$ (determined from the same maps).

Records available. --October 1945 to September 1953. January 1937 to December 1943, weekly readings by the Northern Indiana Public Service Company.

Gage. - Staff gage bolted to northwest wingwall of outlet dam at western end of lake. Gage read to hundredths once daily. Datum of gage is $960.00 \mathrm{ft}$ above mean sea level, datum of 1929. Prior to Oct. 9, 1945, reference point.

Average level. --8 years; gage height,4.73 ft; elevation, $964.73 \mathrm{ft}$.

Established legal level. --Established July 3, 1947, at gage height at $4.66 \mathrm{ft}$, elevation, $964.66 \mathrm{ft}$ above mean sea level.

Lake-level control. --Lake level controlled by concrete fixedcrest dam and culvert type sluice gate. The concrete dam is $23 \mathrm{ft} 1 \frac{1}{2}$ in long with a crest at gage height $4.67 \mathrm{ft}$. The adjustable metal sluice gate, width $3 \mathrm{ft} 9$ in and height $5 \mathrm{ft}$, is opened only during periods of high stages to release excess water. These structures control the levels of Jimerson Lake; Lake James, $2 \frac{1}{2}$ mi upstream; Snow Lake, $4 \mathrm{mi}$ upstream; and has a decided effect on the level of Big and Little Otter Lake. 
Extreme lake levels for Jimerson Lake, 1937-53

\begin{tabular}{|c|c|c|c|c|}
\hline \multicolumn{3}{|c|}{ Maximum } & \multicolumn{2}{|l|}{ Minimum } \\
\hline $\begin{array}{l}\text { Water } \\
\text { year }\end{array}$ & Date & $\begin{array}{l}\text { Gage } \\
\text { height } \\
\text { (feet) }\end{array}$ & Date & $\begin{array}{r}\text { Gage } \\
\text { height } \\
\text { (feet) }\end{array}$ \\
\hline $1937^{a}$ & Apr. 29, $1937-\cdots$ & 5.44 & Sept. 16, 1937- & $a 4.06$ \\
\hline $1938 b$ & Feb. 24, 1938- & 5.12 & Jan. $20,1938 \ldots$ & b3. 98 \\
\hline $1939 b$ & Mar. $2,18,1939--$ & 5. 31 & Nov. 17, Dec. 1,1938 & $b 4.06$ \\
\hline $1940^{b}$ & June $27,1940 \ldots$ & 5.18 & $\begin{array}{c}\text { Jan. } 11, \text { Feb. } 1-29, \\
1940-\end{array}$ & \\
\hline $1941^{a}$ & Feb. 12, 1941… & 5.06 & June $9,1941 \ldots$ & $a_{4} .19$ \\
\hline $1942 b$ & Mar. 19, $1942 \ldots$ & 5.62 & Jan. 22, 29, 1942-- & $b 4.29$ \\
\hline 1943 & May $21,27,1943 \ldots$ & c 6.48 & Sept. $2,1943 \ldots$ & $c 4.56$ \\
\hline 1944 & Mar. $17,18,19,1944$ & c 5.54 & Sept. $15,1944 \ldots$ & $c_{4} .17$ \\
\hline 1945 & May $23,1945 \ldots$ & $=5.86$ & Feb. $10,1945 \ldots$ & c3.69 \\
\hline 1946 & Mar. 12, $1946 \ldots$ & 5.17 & Sept. $16-22,1946-$ & 3.85 \\
\hline 1947 & Apr. $25,1947 \ldots$ & 5.55 & Oct. $13-17,1946$ & 3.81 \\
\hline 1948 & Dec. $12,1947 \ldots$ & 5.15 & Feb. $16,17,1948^{--}$ & 3.71 \\
\hline 1949 & Feb. $21,1949 \ldots$ & 5.32 & Oct. $4-7,1948$ & 4. 22 \\
\hline 1950 & Apr. $7,1950 \ldots$ & 6.00 & Sept. $30,1950 \ldots$ & 4.35 \\
\hline 1951 & July $14-16,1951---$ & 5.48 & Oct. $5-10,1950---$ & 4.08 \\
\hline 1952 & May $30,31,1952 \ldots$ & 5.20 & Apr. $4,1952 \cdots$ & 4. 54 \\
\hline 1953 & Apr. $30,1953 \ldots$ & 4. 98 & Sept. $14-16,1953--$ & 4.37 \\
\hline
\end{tabular}

a Weekly readings imcomplete year.

b Weekly readings.

c From James Lake at Lake James record.

Ice.--Ice, 6 inches thick, was reported on Jimerson Lake in 1949 and again in 1951. The ice cover in 1949 remained for a Feriod of 50 days, from Dec. 18, 1948, to Feb. 5, 1949. 
Discharge measurements at outlet of Jimerson Lake

\begin{tabular}{|c|c|c|c|c|c|}
\hline Date & $\begin{array}{l}\text { Gage } \\
\text { height } \\
\text { (feet) }\end{array}$ & $\begin{array}{c}\text { Discharge } \\
\text { (cfs) }\end{array}$ & Date & $\begin{array}{l}\text { Gage } \\
\text { height } \\
\text { (feet) }\end{array}$ & $\begin{array}{c}\text { Discharge } \\
\text { (cfs) }\end{array}$ \\
\hline $9,1945^{-}$ & 4.95 & 58.4 & Aug. $\quad 9,1951-$ & 4.44 & 56.4 \\
\hline Apr. $\quad 9,1946-$ & 4.63 & 38.5 & Sept. $5 \ldots$ & 4.55 & 37.5 \\
\hline May $21 \ldots-\cdots$ & 4.51 & 2.59 & Oct. $\quad 9 \ldots$ & 4.76 & 12. 3 \\
\hline June $14 \ldots$ & 4.57 & 2.42 & Nov, $17 \ldots$ & 5.00 & 59.1 \\
\hline July $24 \ldots-$ & 4.25 & 11.7 & Dec, $21 \ldots$ & 4.98 & 69.8 \\
\hline Nov. $23 \cdots$ & 4. 69 & 3.92 & Mar. 26, 1952 - & 4. 74 & 95.4 \\
\hline Feb. $14,1947-$ & 5.01 & 46.6 & Nov. $14 \ldots$ & 4.57 & 13.9 \\
\hline Jan. $\quad 6,1949-$ & 4. 76 & 39.7 & Dec. $10 \ldots$ & 4.75 & 24.6 \\
\hline Feb. $17 \ldots$ & 5.27 & 25.9 & Jan. $\quad 9,1953-$ & 4.83 & 31.0 \\
\hline Sept. 15 & 4. 60 & 5.20 & Jan. $30 \cdots$ & 4.94 & 39.5 \\
\hline Dec. 7 & 4.84 & 22.8 & Mar. $10-\ldots$ & 4.90 & 47.4 \\
\hline Oct. $19,1950-$ & 4. 50 & 17.9 & Apr. $3 \ldots-\ldots$ & 4.85 & 44.2 \\
\hline Nov. $15 \cdots$ & 4.60 & 50.5 & Apr. $24 \ldots$ & 4. 94 & 41.9 \\
\hline Jan. 24, 1951- & 4. 47 & 76.2 & May $19 \ldots$ & 4. 77 & 41.5 \\
\hline Apr. $11-\cdots--$ & 4.71 & 75.1 & June $15 \ldots$ & 4.61 & 10.5 \\
\hline May $2-\ldots-$ & 5. 13 & 137 & July $\quad 8 \ldots$ & 4. 51 & 5. 36 \\
\hline June $5 \ldots$ & 5.00 & 57.4 & $5 \ldots \ldots$ & 4.68 & 17.2 \\
\hline July $11 \ldots \ldots$ & 5.38 & 141 & $1 \ldots-\cdots$ & 4.45 & 10.6 \\
\hline
\end{tabular}


Average lake legal for Jimerson Lake for water years 1937-53

\begin{tabular}{|c|c|c|c|c|c|c|c|}
\hline Year & Oct. & Nov. & Dec. & Jan. & Feb. & Mar. & Apr. \\
\hline 1937 & --- & -.. & --- & $* 4.72$ & $* 5.08$ & $* 4.90$ & $* 5.09$ \\
\hline 1938 & $* 4.25$ & $* 4.18$ & $* 4.20$ & $* 4.12$ & $* 4.71$ & $* 4.89$ & $* 4.79$ \\
\hline 1939 & $* 4.48$ & $* 4.22$ & $* 4.21$ & $* 4.18$ & $* 4.48$ & $* 5.17$ & $* 5.00$ \\
\hline 1940 & $* 4.34$ & $* 4.14$ & $* 4.06$ & $* 3.87$ & $* 3.73$ & $* 3.98$ & $* 4.58$ \\
\hline 1941 & $* 4.35$ & $* 4.45$ & $* 4.60$ & $* 4.73$ & $* 4.97$ & $* 4.68$ & $* 4.57$ \\
\hline 1942 & $* 4.05$ & $* 4.30$ & $* 4.75$ & $* 4.46$ & $* 4.71$ & $* 5.22$ & $* 5.23$ \\
\hline 1943 & $* 4.66$ & $* 4.84$ & $* 4.79$ & $* 5.04$ & $* 4.98$ & $* 5.03$ & $* 5.00$ \\
\hline 1944 & $* 4.82$ & $* 4.94$ & $* 4.87$ & $-\cdots$ & $-\cdots$ & --- & -.. \\
\hline 1945 & --- & $\ldots$ & - - & ---- & --- & ---- & --- \\
\hline 1946 & 4.94 & 4.78 & 4.56 & 4.84 & 4.66 & 4.90 & 4. 53 \\
\hline 1947 & 3.92 & 4. 58 & 4.80 & 4.82 & 4.97 & 4.50 & 5. 22 \\
\hline 1948 & 4.75 & 4. 70 & 4. 94 & 4.63 & 3.96 & 4.55 & 4.55 \\
\hline 1949 & 4.25 & 4. 70 & 4.60 & 4.90 & 5. 14 & 5.04 & 5. 01 \\
\hline 1950 & 4.90 & 4.81 & 4.96 & 5.22 & 5. 26 & 5. 38 & 5. 79 \\
\hline 1951 & 4.31 & 4.55 & 4. 75 & 4.52 & 4.41 & 4. 68 & 4.74 \\
\hline 1952 & 4. 78 & 4.99 & 4.97 & 4.97 & 4.83 & 4.69 & 4.73 \\
\hline 1953 & 4.56 & 4.62 & 4.78 & 4.85 & 4.82 & 4.84 & 4.89 \\
\hline Average & 4.49 & 4.58 & 4.66 & 4.66 & 4.71 & 4.83 & 4.91 \\
\hline Year & May & June & July & Aug. & Sept. & Annual & \\
\hline 1937 & $* 5.15$ & $* 5.07$ & $* 4.86$ & $* 4.32$ & $* 4.21$ & $* 4.86$ & \\
\hline 1938 & $* 4.51$ & $* 4.79$ & $* 4.74$ & $* 4.70$ & $* 4.89$ & $* 4.56$ & \\
\hline 1939 & $* 5.00$ & $* 4.91$ & $* 4.94$ & $* 4.42$ & $* 4.30$ & $* 4.61$ & \\
\hline 1940 & $* 4.70$ & *4. 96 & $* 4.45$ & $* 4.40$ & $* 4.45$ & $* 4.29$ & \\
\hline 1941 & $* 4.31$ & $* 4.21$ & $* 4.10$ & $* 4.00$ & $* 3.95$ & $* 4.41$ & \\
\hline 1942 & $* 4.86$ & $* 4.66$ & $* 4.77$ & $* 4.96$ & $* 4.78$ & $* 4.73$ & \\
\hline 1943 & $* 5.64$ & *5. 16 & $* 4.71$ & $* 4.64$ & $* 4.81$ & $* 4.94$ & \\
\hline 1944 & $\ldots$ & $\ldots$ & - - & -.. & $-\cdots$ & $* 4.88$ & \\
\hline 1945 & $\cdots$ & $\cdots-$ & --- & --- & --- & --- & \\
\hline 1946 & 4. 36 & 4.59 & 4.56 & 4.06 & 3.89 & 4. 56 & \\
\hline 1947 & 5.01 & 4.93 & 4.39 & 4.40 & 4.76 & 4.69 & \\
\hline 1948 & 4. 76 & 4.57 & 4.29 & 4.40 & 4. 29 & 4. 54 & \\
\hline 1949 & 4.90 & 4.95 & 4.91 & 4. 74 & 4.66 & 4.82 & \\
\hline 1950 & 5. 31 & 5.03 & 5.01 & 4.93 & 4.66 & 5.10 & \\
\hline 1951 & 5.08 & 4.92 & 5.02 & 4.66 & 4. 56 & 4.68 & \\
\hline 1952 & 4.91 & 4.82 & 4.64 & 4.63 & 4.63 & 4.80 & \\
\hline 1953 & 4.78 & 4. 59 & 4.48 & 4. 61 & 4. 36 & 4.68 & \\
\hline Average & 4.89 & 4.81 & 4.66 & 4.52 & 4.48 & 4.73 & \\
\hline
\end{tabular}

*Partial month or partial year. 


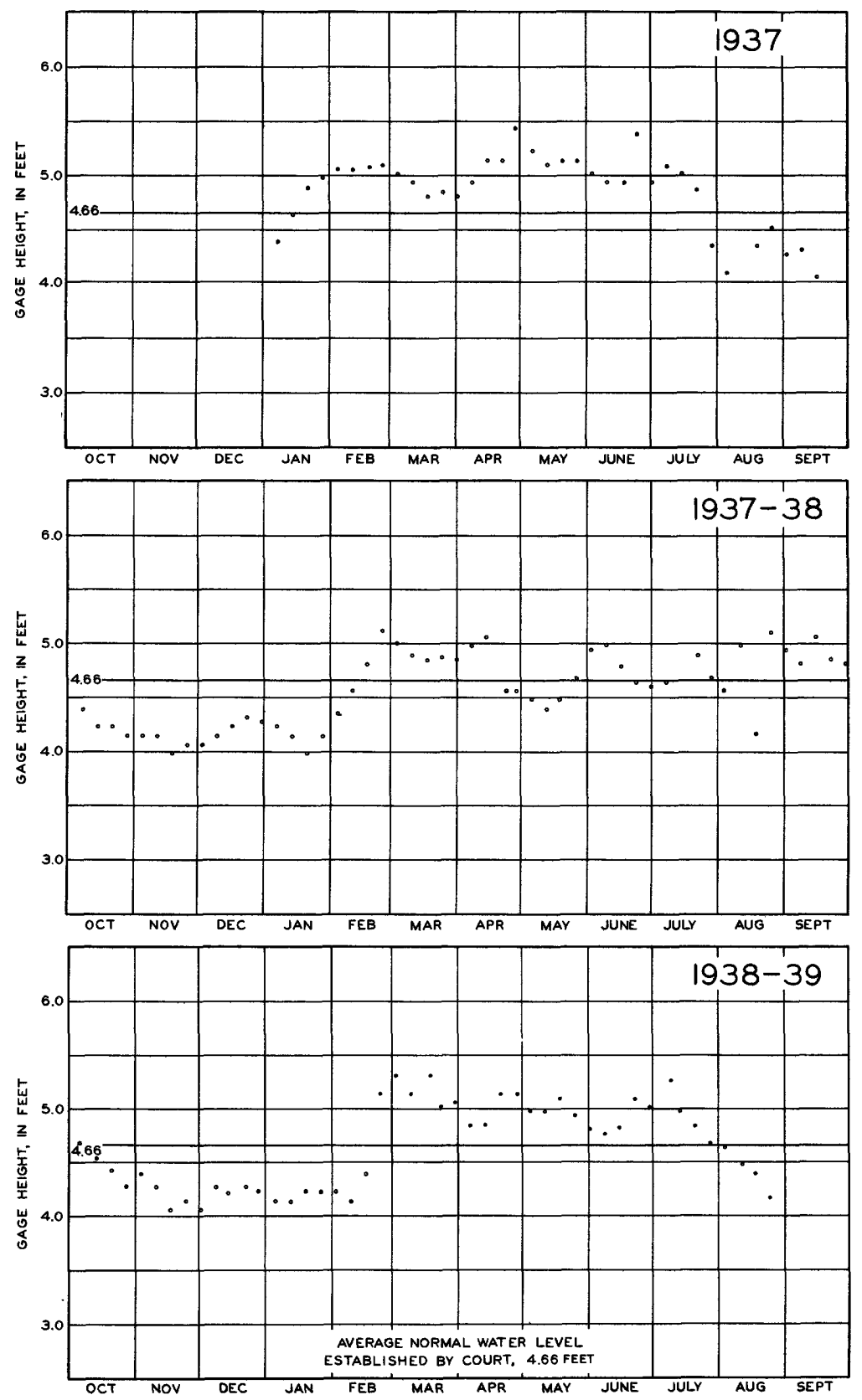

Figure 67. --Lake-level hydrographs for Jimerson Lake at Nevada Mills, Ind., for water years 1937-39. 

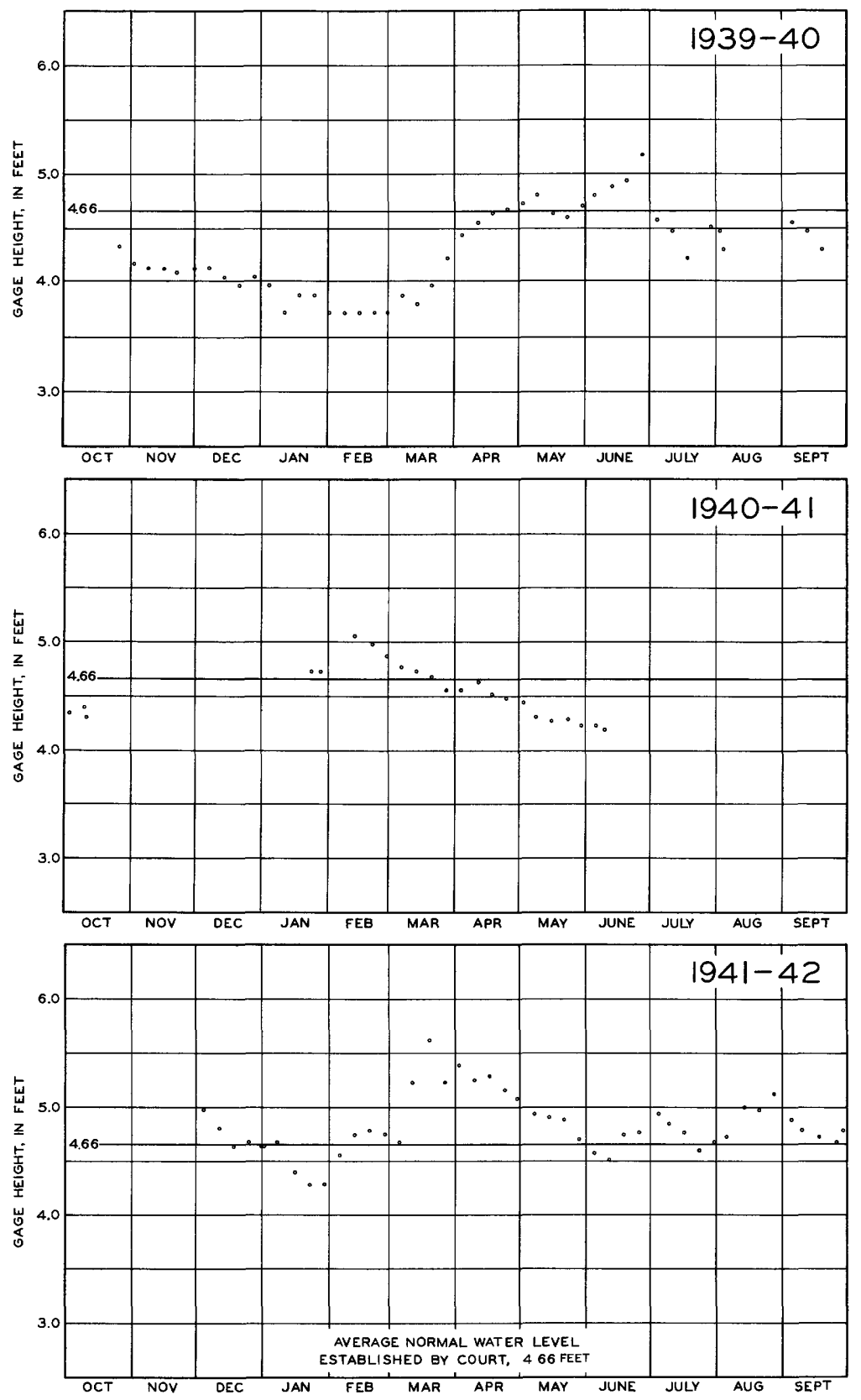

Figure 68. --Lake-level hydrographs for Jimerson Lake at Nevada Mills, Ind., for water years 1940-42. 

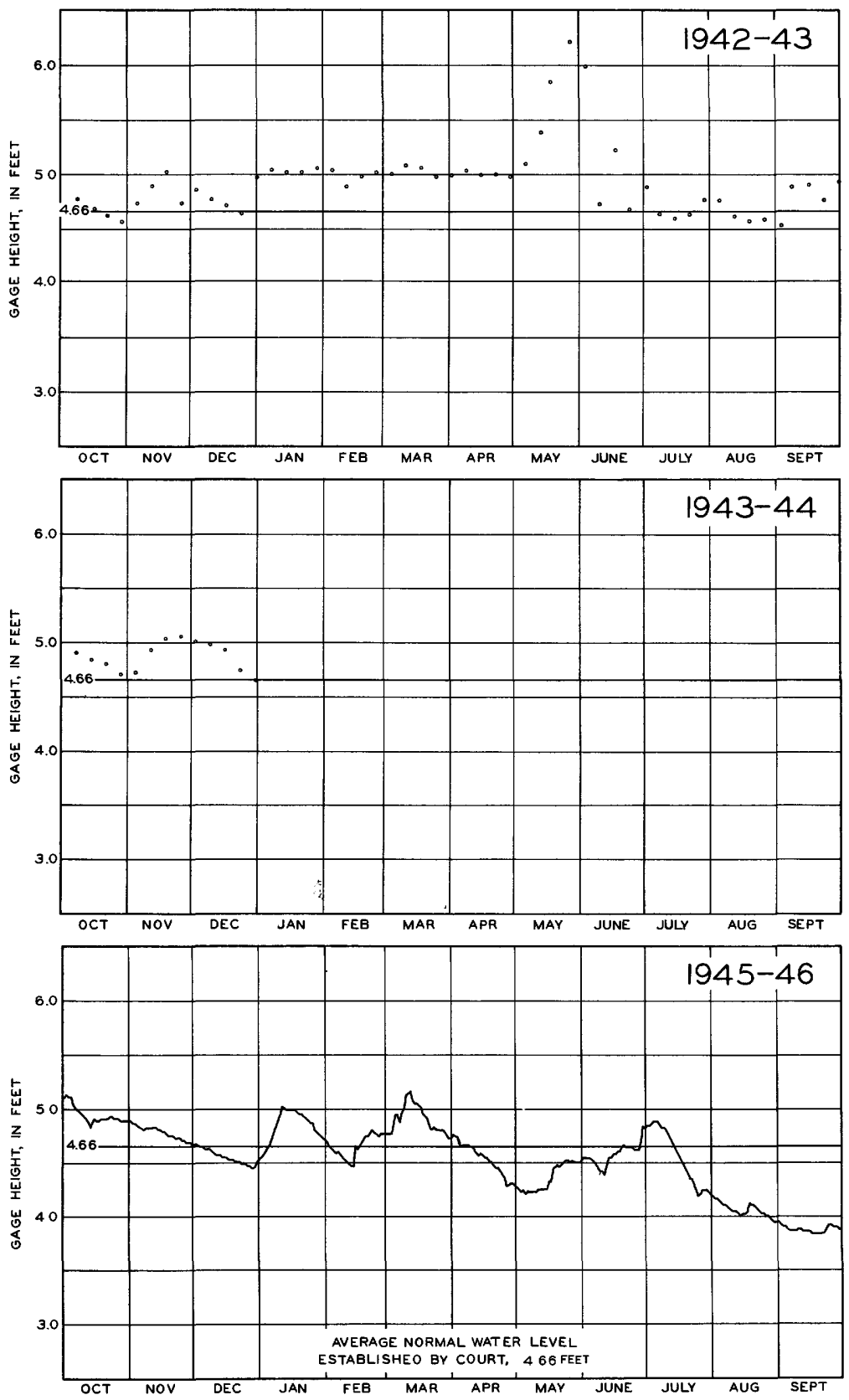

Figure 69. --Lake-level hydrographs for Jimerson Lake at Nevada Mills, Ind., for water years 1943-44 and 1946. 

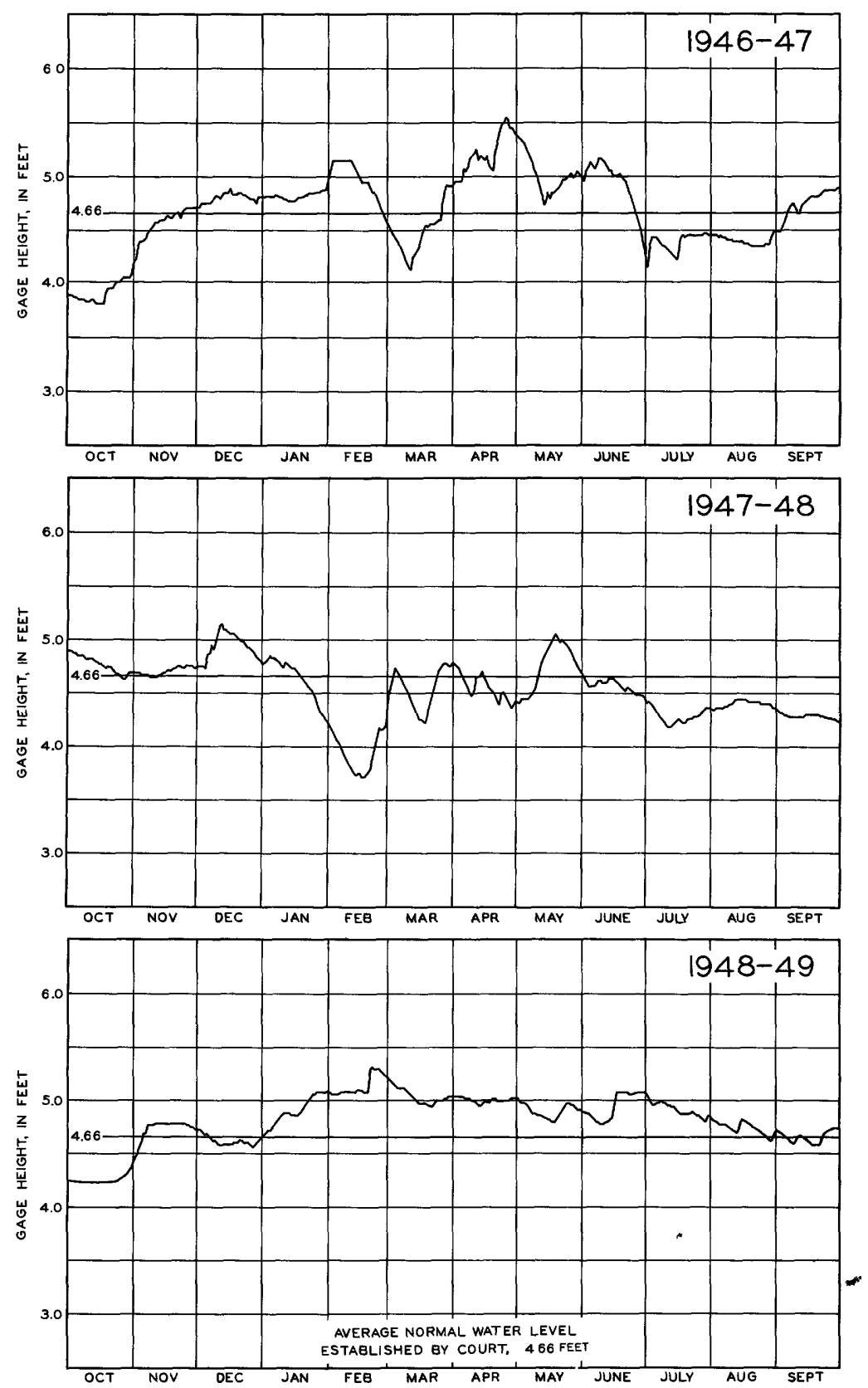

Figure 70. --Lake-level hydrog raphs for Jimerson Lake at Nevada Mills, Ind., for water years 1947-49. 

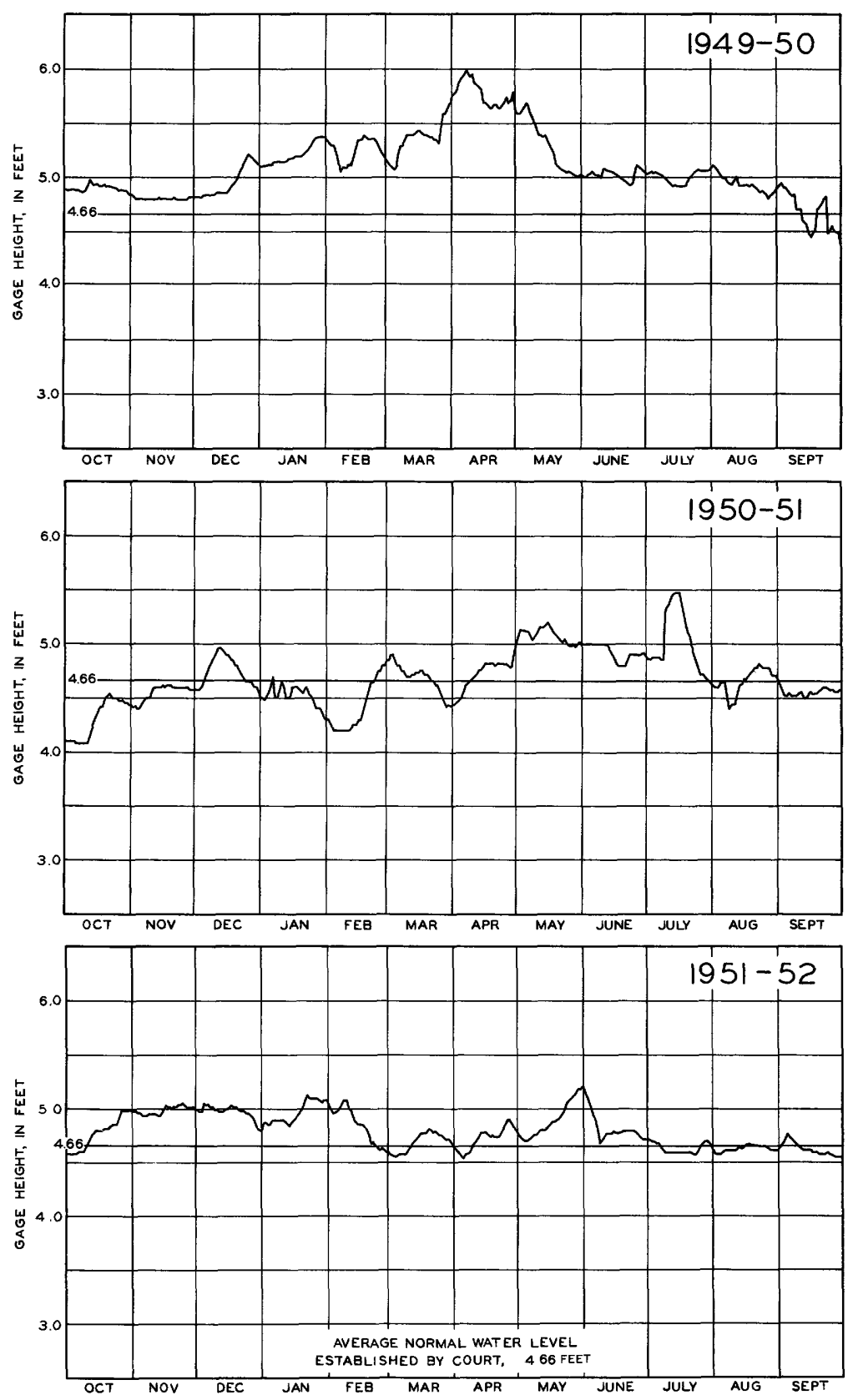

Figure 71. --Lake-level hydrographs for Jimerson Lake at Nevada Mills, Ind., for water years 1950-52. 


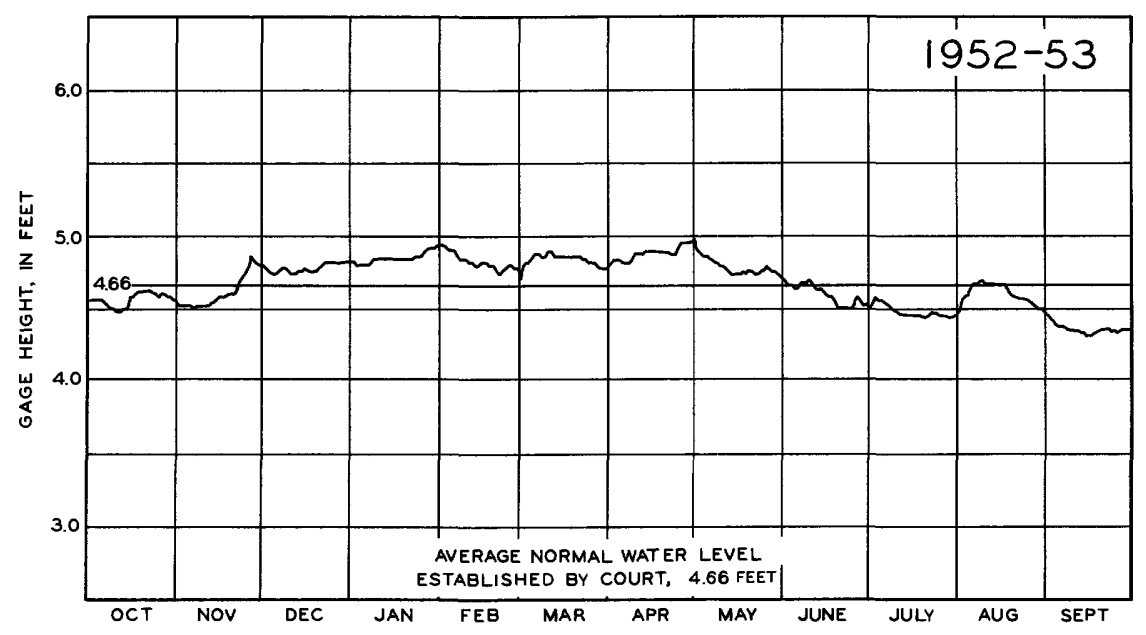

Figure 72. --Lake-level hydrograph for Jimerson Lake at Nevada Mills, Ind., for water year 1953.

Koontz Lake at Koontz Lake, Ind.

Location. --Secs. 1, 11, and 12, T. 34 N., R. 1 W. , Starke County, and sec. 7, T. 34 N., R. 1 E., Marshall County.

Surface area. -298 acres.

Drainage area. $--4.74 \mathrm{sq} \mathrm{mi}$.

Records available. --October 1942 to September 1953.

Gage. --Staff gage bolted to south side of concrete headwall of control gate at north outlet. Gage read to hundredths once daily. Datum of gage is $710.00 \mathrm{ft}$ above mean sea level, datum of 1929 .

Average lake level. --11 years; gage height, $4.59 \mathrm{ft}$; elevation, $714.59 \mathrm{ft}$.

Established legal level. --Established September 13, 1948, at gage height $4.56 \mathrm{ft}$; elevation, $714.56 \mathrm{ft}$ above mean sea level.

Lake-level control. --Lake controlled at two outlet channels which join about $500 \mathrm{ft}$ downstream from the lake. North outlet supplies water to two fish-rearing ponds and is controlled by two gates, each $38 \mathrm{in}$. wide and $40 \mathrm{in.} \mathrm{high,} \mathrm{located} \mathrm{at} \mathrm{the} \mathrm{head} \mathrm{of}$ two 30-in. pipes. The south outlet has a concrete control dam with sill $10 \mathrm{ft}$ long and $1.5 \mathrm{ft}$ wide at gage height $4.37 \mathrm{ft}$. Slots 4 in. wide are provided for placing stop logs on sill of dam. 
Extreme lake levels for Koontz Lake, 1943-53

\begin{tabular}{|c|c|c|c|c|}
\hline \multicolumn{3}{|c|}{ Maximum } & \multicolumn{2}{|l|}{ Minimum } \\
\hline $\begin{array}{c}\text { Water } \\
\text { year }\end{array}$ & Date & $\begin{array}{r}\text { Gage } \\
\text { height } \\
\text { (feet) }\end{array}$ & Date & $\begin{array}{l}\text { Gage } \\
\text { height } \\
\text { (feet) }\end{array}$ \\
\hline 1943 & May $19,1943^{\cdots} \cdots$ & 5.52 & Apr. $3,1943 \ldots$ & 4.14 \\
\hline 1944 & Mar. $16-18,1944--$ & 5.07 & $\begin{array}{r}\text { Aug. 29, 30, Sept. 10, } \\
1944 .\end{array}$ & 3.87 \\
\hline 1945 & June $27-30,1945 \cdots$ & 5.00 & Oct. $1,1944 \ldots-\ldots$ & 3.93 \\
\hline 1946 & June $22-24,1946 \ldots$ & 5.08 & $\begin{array}{c}\text { Sept. } 20-22,29,30 \\
1946 \\
\end{array}$ & 4.14 \\
\hline 1947 & May $21-23,1946 \ldots$ & 5. 16 & Oct. $16,17,1946 \ldots$ & 4.00 \\
\hline 1948 & May $10,1948 \ldots$ & 5.02 & Mar. 8, 1948 $\ldots$ & 4.04 \\
\hline 1949 & Dec. $19,1948 \ldots$ & 5. 10 & $\begin{array}{c}\text { Feb. 21, Apr. 13, } \\
1949\end{array}$ & 4.10 \\
\hline 1950 & Dec. $22,1949 \ldots$ & 5.20 & Oct. $1,2,1949 \ldots$ & 4.34 \\
\hline 1951 & July $10,1951 \ldots$ & 5.20 & Oct. $4,1950 \ldots$ & 4.14 \\
\hline 1952 & $\begin{array}{l}\text { Mar. 13, May } 28, \\
1952 .\end{array}$ & 5.10 & Nov. 17, 1951- & 4.20 \\
\hline 1953 & May 24, 1953 $\ldots$ & 5.08 & Sept. $30,1953 \ldots$ & 4.08 \\
\hline
\end{tabular}

Maximum recorded thickness and periods of ice cover 1943-53

\begin{tabular}{|c|c|c|c|}
\hline $\begin{array}{l}\text { Water } \\
\text { years }\end{array}$ & $\begin{array}{l}\text { Maximum } \\
\text { thickness } \\
\text { (inches) }\end{array}$ & $\begin{array}{l}\text { Total } \\
\text { days of } \\
\text { cover }\end{array}$ & Period \\
\hline 1943 & --- & --- & Dec. 3,1942 to-? \\
\hline 1944 & --- & --- & \\
\hline 1945 & --- & --- & Dec. 13,1944 to-? \\
\hline 1946 & 2 & --- & \\
\hline 1947 & 10 & 100 & Dec. 16,1946 , to Mar. 25, 1947. \\
\hline 1948 & 20 & 109 & $\begin{array}{c}\text { Nov. } 27 \text { to Dec. } 6,1947 \text {; Dec. } 12 \text {, } \\
1947 \text {, to Mar. } 19,1948 \text {. }\end{array}$ \\
\hline 1949 & 4 & 78 & Dec. 18,1948 , to Mar. 5,1949 \\
\hline 1950 & 5 & 50 & $\begin{array}{l}\text { Dec. } 15-19,1949 \text {; Jan. } 31,1950 \text {, to } \\
\text { Mar. 26, } 1950 .\end{array}$ \\
\hline 1951 & 10 & ---- & Nov. 25,1950 to $-?$ \\
\hline 1952 & 4 & 86 & Dec. 14,1951 , to Mar. 8,1952 , \\
\hline 1953 & 6 & 45 & $\begin{array}{l}\text { Nov. } 27 \text { to Dec. } 10,1952 \text {; Jan. } 1 \\
\text { to Jan. } 31,1953 .\end{array}$ \\
\hline
\end{tabular}


Discharge measurements at outlet of Koontz Lake

\begin{tabular}{|c|c|c|c|c|c|c|}
\hline & Date & $\begin{array}{l}\text { Gage } \\
\text { height } \\
\text { (feet) }\end{array}$ & $\begin{array}{c}\text { Discharge } \\
\text { (cfs) }\end{array}$ & Date & $\begin{array}{l}\text { Gage } \\
\text { height } \\
\text { (feet) }\end{array}$ & $\begin{array}{c}\text { Discharge } \\
\text { (cfs) }\end{array}$ \\
\hline Aug & $\overline{9}, 194 \overline{3-}$ & 4.40 & 1.09 & Apr. 16, 1946- & 4.82 & 2.80 \\
\hline Oct. & $13 \ldots$ & 4.72 & 1.53 & June $6 \ldots$ & 4. 73 & 4.71 \\
\hline Jan. & $19,1944-$ & 3.95 & 4.92 & July $19 \ldots \ldots$ & 4.52 & 1.33 \\
\hline May & $10 \ldots-$ & 4.49 & 14.2 & July $25 \ldots$ & 4.42 & .82 \\
\hline July & $13 \ldots \ldots$ & 4. 19 & .40 & Mar. 6, 1947- & 4.70 & 3.56 \\
\hline June & $11,1945-$ & 4.52 & .45 & May 21, 1948- & 4.26 & 23.9 \\
\hline Aug & $22 \ldots-\cdots$ & 4. 50 & .01 & Nov. $9,1949-$ & 4.59 & 0 \\
\hline Oct. & $23-\ldots--$ & 4.46 & .92 & Nov. 28 & 4.70 & 0 \\
\hline
\end{tabular}

Average lake level for Koontz Lake for water years 1943-53

\begin{tabular}{c|c|c|c|c|c|c|c}
\hline Year & Oct. & Nov. & Dec. & Jan. & Feb. & Mar. & Apr. \\
\hline 1943 & $* 4.68$ & 4.81 & 4.94 & 4.60 & 4.52 & 4.56 & 4.58 \\
1944 & 4.69 & 4.66 & 4.51 & 4.18 & 4.18 & 4.68 & 4.70 \\
1945 & 4.01 & 4.11 & 4.35 & 4.57 & 4.43 & 4.17 & 4.60 \\
1946 & 4.63 & 4.54 & 4.52 & 4.46 & 4.53 & 4.89 & 4.82 \\
1947 & 4.10 & 4.55 & 4.67 & 4.75 & 4.76 & 4.76 & 4.93 \\
1948 & 4.58 & 4.75 & 4.56 & 4.71 & 4.73 & 4.56 & 4.54 \\
1949 & 4.32 & 4.54 & 4.61 & 4.57 & 4.47 & 4.67 & 4.61 \\
1950 & 4.57 & 4.63 & 4.76 & 4.69 & 4.70 & 4.75 & 4.81 \\
1951 & 4.23 & 4.48 & 4.67 & 4.65 & 4.64 & 4.70 & 4.74 \\
1952 & 4.67 & 4.66 & 4.73 & 4.85 & 4.65 & 4.81 & 4.73 \\
1953 & 4.29 & 4.34 & 4.53 & 4.53 & 4.66 & 4.81 & 4.77 \\
\hline Average & 4.43 & 4.55 & 4.62 & 4.60 & 4.57 & 4.67 & 4.71 \\
\hline \hline Year & May & June & July & Aug. & Sept. & Annual & \\
\hline 1943 & 4.17 & 4.87 & 4.80 & 4.51 & 4.78 & 4.74 & \\
1944 & 4.54 & 4.26 & 4.14 & 4.05 & 3.89 & 4.38 & \\
1945 & 4.84 & 4.74 & 4.75 & 4.58 & 4.58 & 4.48 & \\
1946 & 4.88 & 4.92 & 4.62 & 4.32 & 4.19 & 4.61 & \\
1947 & 4.90 & 4.58 & 4.49 & 4.43 & 4.64 & 4.63 & \\
1948 & 4.59 & 4.59 & 4.68 & 4.55 & 4.39 & 4.60 & \\
1949 & 4.71 & 4.79 & 4.59 & 4.55 & 4.36 & 4.57 & \\
1950 & 4.70 & 4.85 & 4.78 & 4.48 & 4.55 & 4.69 & \\
1951 & 4.68 & 4.80 & 4.87 & 4.74 & 4.63 & 4.65 & \\
1952 & 4.81 & 4.85 & 4.64 & 4.52 & 4.38 & 4.69 & \\
1953 & 4.91 & 4.91 & 4.61 & 4.51 & 4.20 & 4.51 & \\
\hline Average & 4.70 & 4.74 & 4.63 & 4.48 & 4.42 & 4.59 & \\
\hline
\end{tabular}

* Partial month or partial year. 


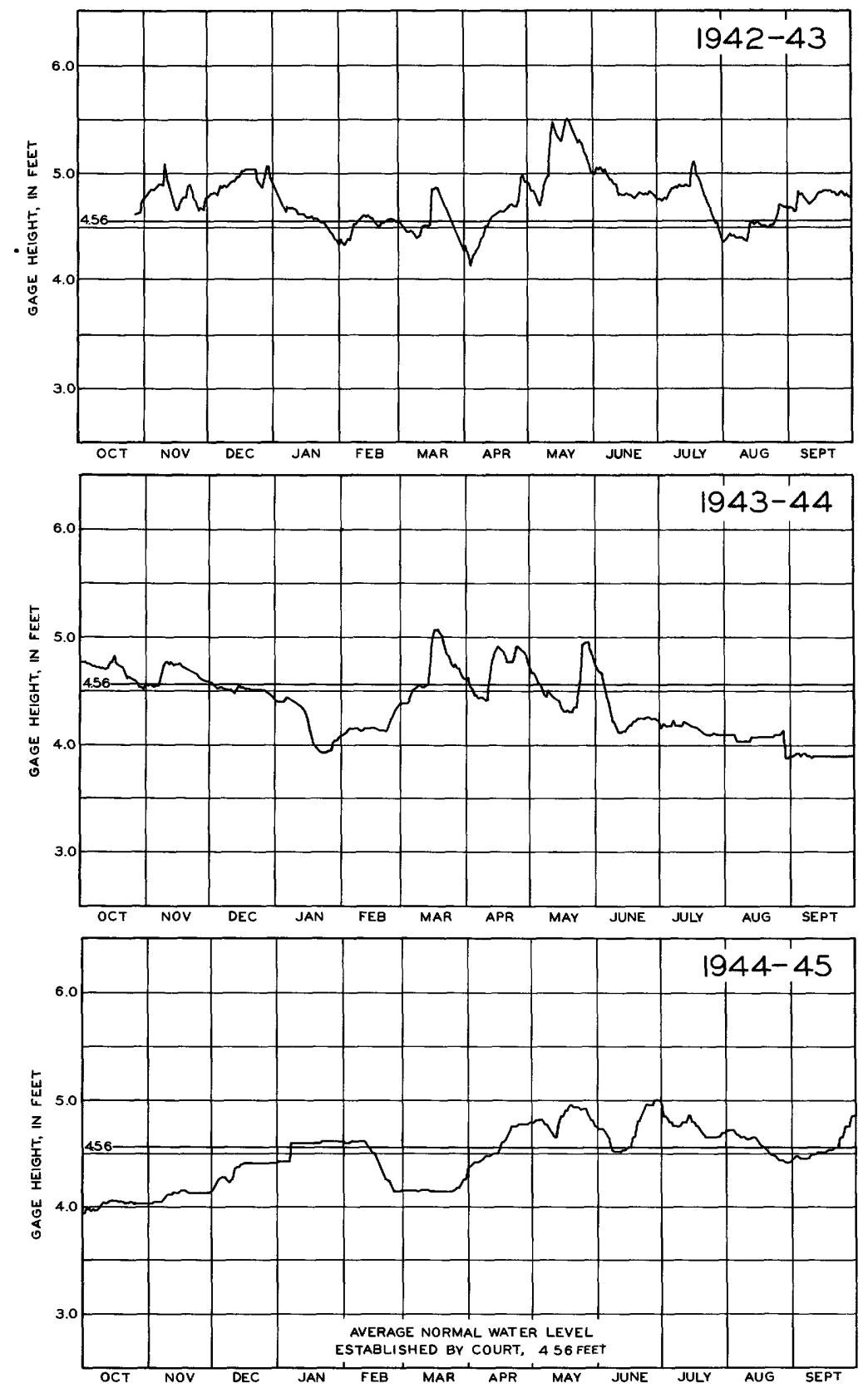

Figure 73. --Lake-level hydrographs for Koontz Lake at Koontz Lake, Ind., for water years 1943-45. 


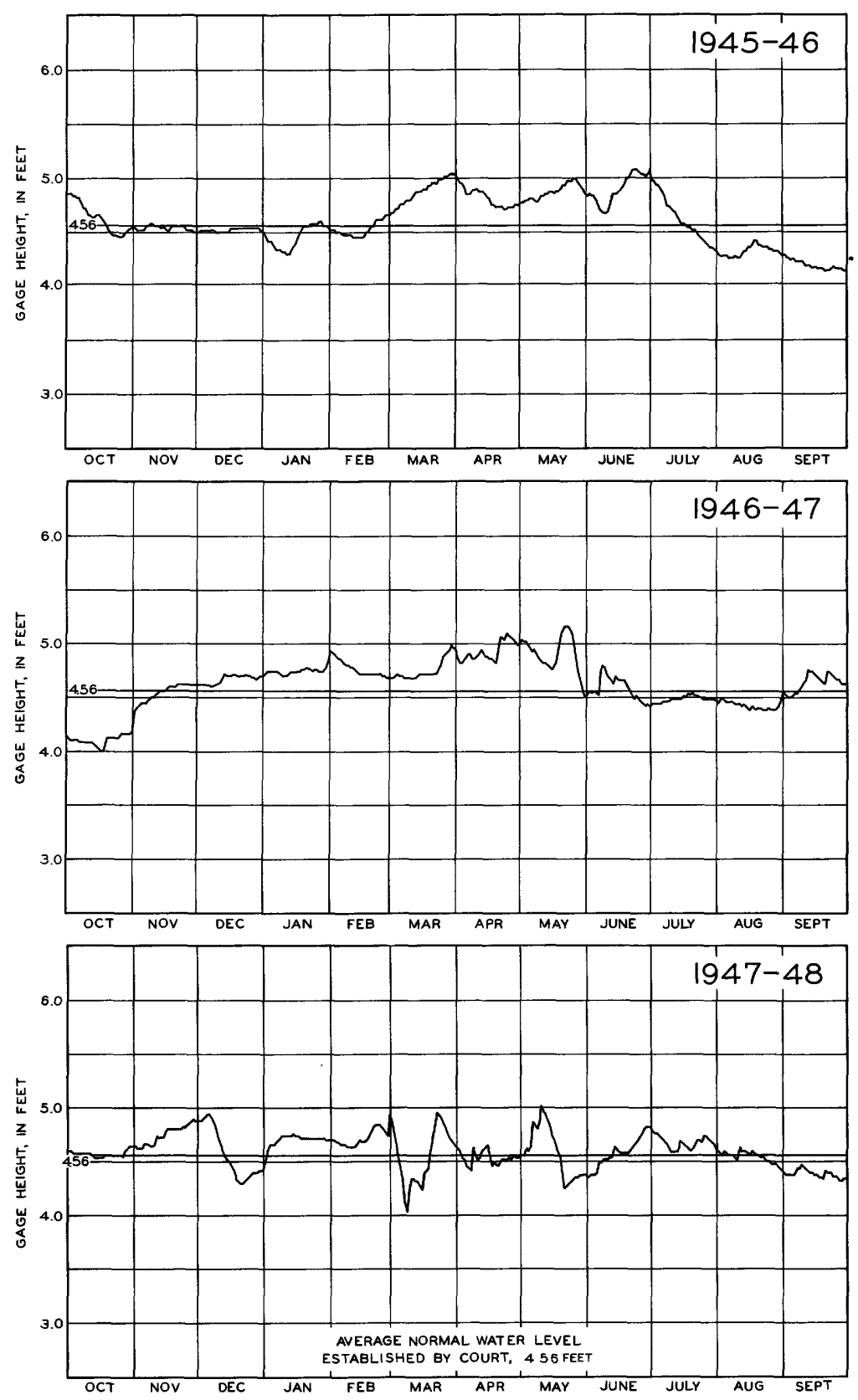

Figure 74. - Lake-level hydrographs for Koontz Lake at Koontz Lake, Ind., for water years 1946-48. 

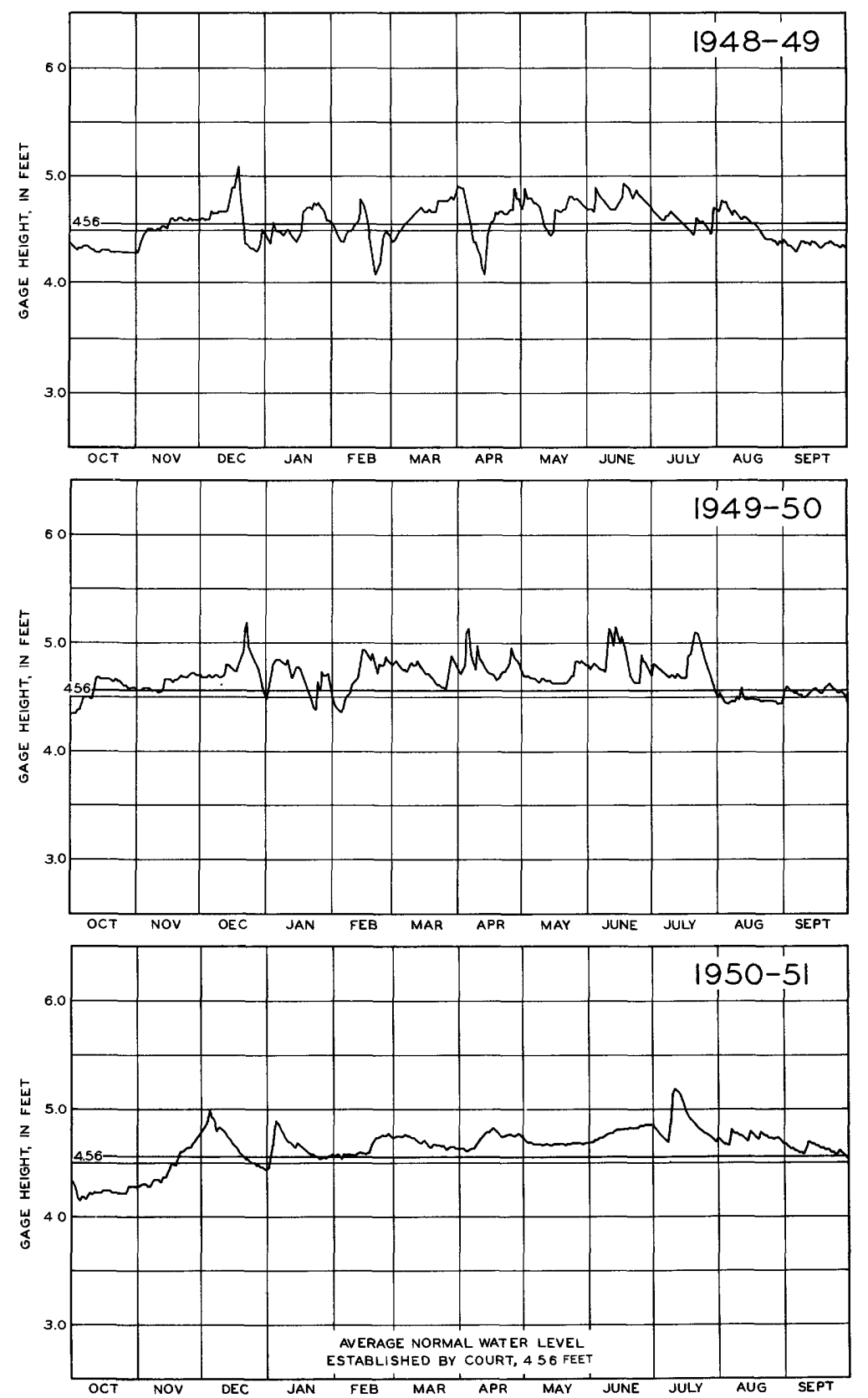

Figure 75. --Lake-level hydrographs for Koontz Lake at Koontz Lake, Ind., for water years 1949-51. 

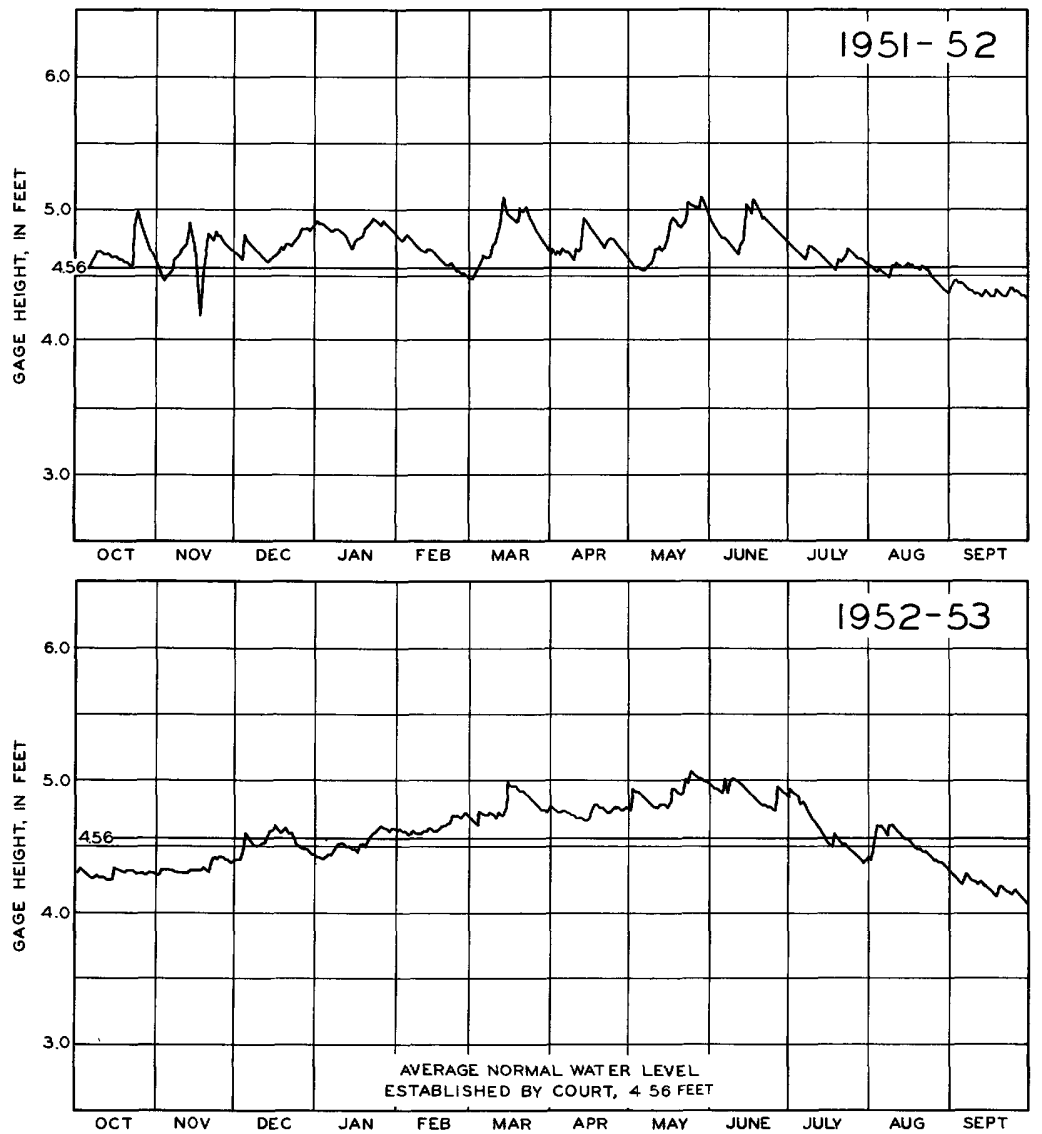

Figure 76. --Lake-level hydrographs for Koontz Lake at Koontz Lake, Ind., for water years 1952-53. 
Lake James at Lake James, Ind.

Location.--Secs. 21, 22, 27, 28, 33, and 34, T. 38 N., R. 13 E., and secs.3, 4, and 10, T. 37 N., R. 13 E., Steuben County.

Surface area. - -1, 034 acres for Lake James and 310 acres for Snow Lake which is at same level as Lake James (determined from USGS maps, surveyed in 1939).

Drainage area. $--43.0 \mathrm{sq} \mathrm{mi}$ including that of Snow Lake (determined from same maps).

Records available. --October 1942 to September 1949.

Gage. --Staff gage on County highway bridge over outlet, $500 \mathrm{ft}$ west of middle of second basin of lake. Gage read to hundredths once daily. Datum of gage is $960.00 \mathrm{ft}$ above mean sea level, datum of 1929.

Average level. --7 years; gage height, $4.94 \mathrm{ft}$; elevation, $964.94 \mathrm{ft}$.

Established legal level. --Established July 3, 1947, at gage height $4.96 \mathrm{ft}$; elevation, $964.96 \mathrm{ft}$ above mean sea level.

Lake-level control. --Lake level maintained by two dams at outlet of Jimerson Lake at Nevada Mills, $2 \frac{1}{2}$ miles downstream. The lake level of Lake James is generally $0.08 \mathrm{ft}$ above that of Jimerson Lake.

Extreme lake levels for Lake James, 1943-49

\begin{tabular}{|c|c|c|c|c|}
\hline \multicolumn{3}{|c|}{ Maximum } & \multicolumn{2}{|l|}{ Minimum } \\
\hline $\begin{array}{l}\text { Water } \\
\text { year }\end{array}$ & Date & $\begin{array}{l}\text { Gage } \\
\text { height } \\
\text { (feet) }\end{array}$ & Date & $\begin{array}{l}\text { Gage } \\
\text { height } \\
\text { (feet) }\end{array}$ \\
\hline $1943^{a}$ & May 21, 27, $1943 \ldots$ & 6.56 & Oct. $29,1942 \cdots$ & $a_{4.82}$ \\
\hline 1944 & Mar. 17-19, 1944-.-- & 5.62 & Sept. $15,1943 \cdots$ & 4.25 \\
\hline $1945^{a}$ & May 23, 1945 & 5.97 & Feb. 10,1945 & $a_{4} .04$ \\
\hline 1946 & $\begin{array}{r}\text { Oct. 2, 1945, Mar. 10, } \\
1946 .\end{array}$ & 5. 34 & Sept. $21,22,1946^{-}$ & 4.00 \\
\hline 1947 & Apr. 24, 1947 & 5.82 & Oct. $11-16,1946 \cdots$ & 3.98 \\
\hline 1948 & Dec. $10-12,1947 \ldots$ & 5. 30 & Feb. 12, 1948 & 4. 07 \\
\hline 1949 & June $21,1949 \ldots$ & 5.65 & Oct. $7-22,1948 \ldots$ & 4. 48 \\
\hline
\end{tabular}

a Partial year. 
Maximum recorded thickness and periods of ice cover 1946-48

\begin{tabular}{c|c|c|l}
\hline $\begin{array}{c}\text { Water } \\
\text { year }\end{array}$ & $\begin{array}{c}\text { Maximum } \\
\text { thickness } \\
\text { (inches) }\end{array}$ & $\begin{array}{c}\text { Total } \\
\text { days of } \\
\text { cover }\end{array}$ & \multicolumn{1}{|c}{ Period } \\
\hline 1946 & 8 & --- & Jan. 10, 1946, to-? \\
1947 & 12 & --- & Jan. 1, 1947, to-? \\
1948 & 16 & 92 & Dec. 20, 1947, to Mar. 21, 1948. \\
\hline
\end{tabular}

Discharge measurements at outlet of Lake James

\begin{tabular}{|c|c|c|c|c|c|}
\hline Date & $\begin{array}{c}\text { Gage } \\
\text { height } \\
\text { (feet) }\end{array}$ & $\begin{array}{c}\text { Discharge } \\
\text { (cfs) }\end{array}$ & Date & $\begin{array}{c}\text { Gage } \\
\text { height } \\
\text { (feet) }\end{array}$ & $\begin{array}{c}\text { Discharge } \\
\text { (cfs) }\end{array}$ \\
\hline Aug. 12, 1943- & 5.05 & 32.1 & Sept. 4, 1945- & 5.11 & 4.96 \\
\hline Oct. $\quad 4 \ldots \ldots$ & 5.22 & a 25.5 & Jan. 10, 1946- & 5. 17 & $a 23.4$ \\
\hline Feb. 10, 1944- & 4. 72 & 31.6 & May 21 $\ldots$ & 4. 70 & $a 14.5$ \\
\hline Apr. $25 \ldots$ & 5.24 & 113 & $24 \ldots \ldots$ & 4. 54 & $a_{26.3}$ \\
\hline July $3,1945-$ & 5.01 & $a 13.0$ & & & \\
\hline
\end{tabular}

$a$ Heavy growth in channel.

Average lake level for Lake James for water years 1943-49

\begin{tabular}{c|r|r|r|r|r|r|r}
\hline Year & Oct. & Nov. & Dec. & Jan. & Feb. & Mar. & Apr. \\
\hline 1943 & $* 4.90$ & $* 5.08$ & $* 5.06$ & 5.40 & $* 5.42$ & $* 5.30$ & 5.29 \\
1944 & 5.13 & 5.21 & 5.12 & 4.78 & 4.75 & 5.31 & 5.10 \\
1945 & 4.51 & 4.87 & 4.97 & 4.96 & 4.26 & 4.91 & 5.01 \\
1946 & 5.15 & 4.99 & 4.77 & 5.05 & 4.71 & 5.14 & 4.75 \\
1947 & 4.09 & 4.76 & 4.99 & 5.02 & 5.17 & 4.72 & 5.51 \\
1948 & 4.95 & 4.88 & 5.16 & 4.86 & 4.30 & 4.84 & 4.84 \\
1949 & 4.50 & 4.87 & 4.92 & 5.12 & 5.28 & 5.26 & 5.19 \\
\hline Average & 4.75 & 4.95 & 5.00 & 5.03 & 4.84 & 5.07 & 5.10 \\
\hline \hline Year & May & June & July & Aug. & Sept. & Annual & \\
\hline 1943 & 6.05 & $* 5.40$ & $* 5.00$ & $* 4.90$ & 5.16 & 5.25 & \\
1944 & 5.09 & 5.00 & 4.73 & 4.39 & 4.31 & 4.91 & \\
1945 & 5.40 & 5.09 & 4.98 & $* 5.00$ & $* 5.20$ & 4.93 & \\
1946 & 4.56 & 4.77 & 4.83 & 4.27 & 4.06 & 4.76 & \\
1947 & 5.28 & 5.29 & 4.62 & 4.56 & 4.96 & 4.91 & \\
1948 & 4.98 & 4.83 & 4.53 & 4.57 & 4.44 & 4.77 & \\
1949 & 5.19 & 5.37 & 5.26 & 4.94 & 4.85 & 5.06 & \\
\hline Average & 5.22 & 5.11 & 4.85 & 4.66 & 4.71 & 4.94 & \\
\hline
\end{tabular}

* Partial month or partial year. 

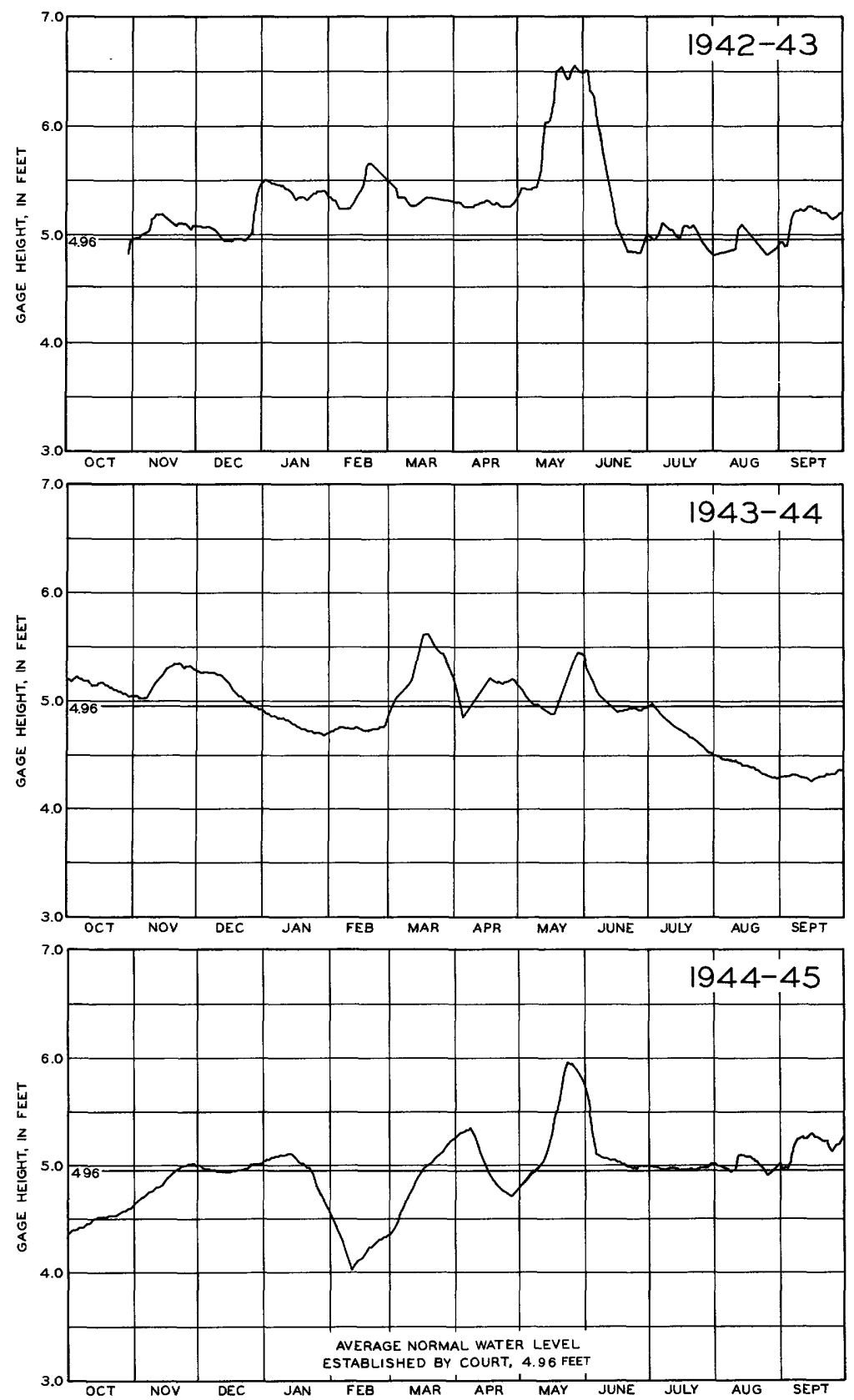

Figure 77. --Lake-level hydrographs for Lake James at Lake James, Ind., for water years 1943-45. 

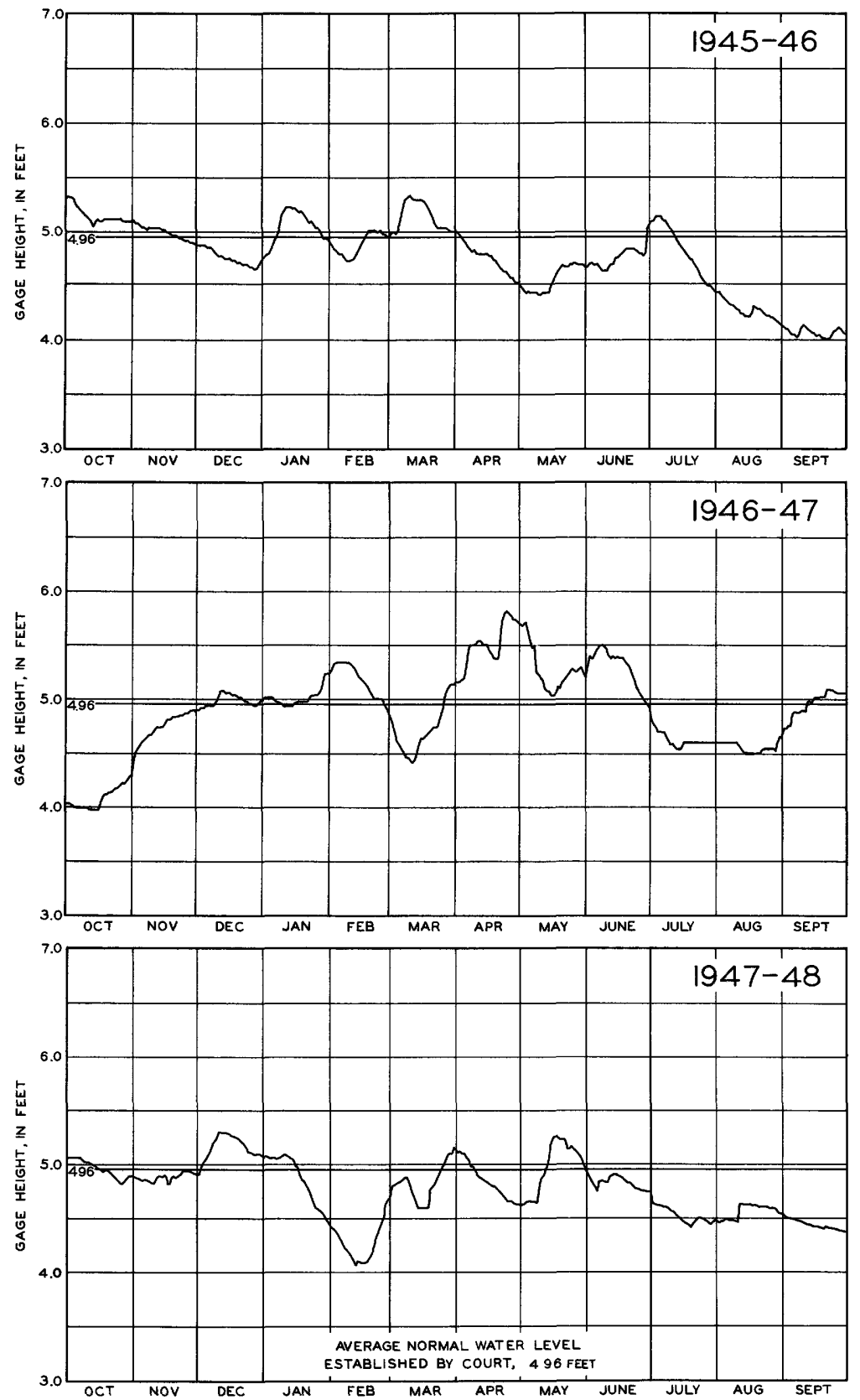

Figure 78. --Lake-level hydrographs for Lake James at Lake James, Ind., for water years 1946-48. 


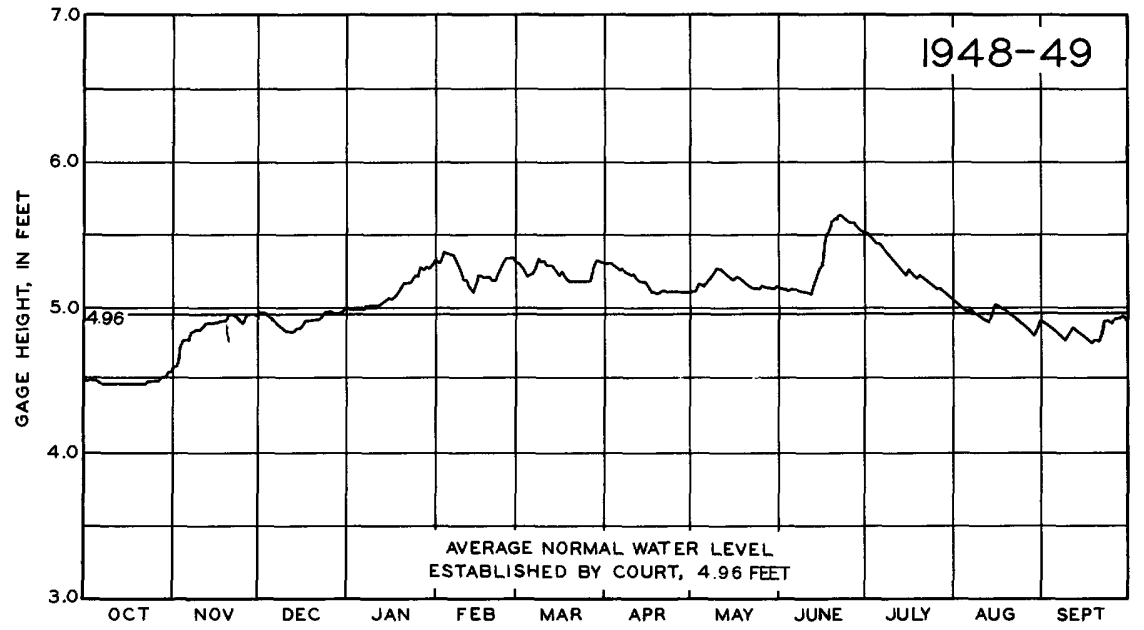

Figure 79. --Lake-level hydrograph for Lake James at Lake James, for water year 1947.

Lake Manitou at Rochester, Ind.

Location. --Secs. 9, 10,15,16, and 22, T. 30 N., R. 3 E., Fulton County.

Surface area. --631 acres.

Drainage area. $--38.1 \mathrm{sq} \mathrm{mi}$.

Records available.--October 1942 to September 1953.

Gage. - Staff gage attached to south wing wall of control gates on fish hatchery feeder canal. Gage read to hundredths once daily. Datum of gage is $770.00 \mathrm{ft}$ above mean sea level, datum of 1929 .

Average lake leve1. --11 years; gage height, $8.24 \mathrm{ft}$; elevation, $778.24 \mathrm{ft}$.

Established legal level. --Established September 27, 1948, at gage height $8.41 \mathrm{ft}$, elevation, $778.41 \mathrm{ft}$ above mean sea level.

Lake-level control. -- Lake level maintained by concrete dam, crest width $88 \mathrm{ft}$, at gage height $7.76 \mathrm{ft}$. A 24-in.culvert with movable steel gate controls amount of water going to the Federal Fish Hatchery fish ponds. 
Extreme lake levels for Lake Manitou, 1943-53

\begin{tabular}{|c|c|c|c|c|}
\hline \multicolumn{3}{|c|}{ Maximum } & \multicolumn{2}{|l|}{ Minimum } \\
\hline $\begin{array}{l}\text { Water } \\
\text { year }\end{array}$ & Date & $\begin{array}{l}\text { Gage } \\
\text { height } \\
\text { (feet) }\end{array}$ & Date & $\begin{array}{l}\text { Gage } \\
\text { height } \\
\text { (feet) }\end{array}$ \\
\hline 1943 & May $18,19,1943 \ldots$ & 9.25 & Sept. $28,29,1943 \ldots$ & 8.11 \\
\hline 1944 & Apr. $12,1944 \ldots \ldots$ & 9.13 & Sept. $26,1944 \ldots$ & 6.94 \\
\hline 1945 & May $17,1945 \ldots$ & 8.91 & Oct. $1,1944 \ldots$ & 7.07 \\
\hline 1946 & Feb. 14, $1946 \ldots$ & 8.84 & Sept. $30,1946 \ldots$ & 6.71 \\
\hline 1947 & Apr. $22,1947 \ldots$ & 8.91 & Oct. $16,1946 \ldots$ & 6.49 \\
\hline 1948 & Feb. 28, $1948 \ldots$ & 9.19 & Sept. $20,1948 \ldots$ & 7.67 \\
\hline 1949 & Feb. 16, $1949 \ldots$ & 9.24 & Oct. $28-31,1948-\ldots$ & 7.00 \\
\hline 1950 & Apr. $5,1950 \ldots$ & 9.38 & Oct. $2,1949 \ldots$ & 7.94 \\
\hline 1951 & Feb. 21, 1951-.... & 8.94 & Nov. $4,5,1950 \ldots$ & 8.00 \\
\hline 1952 & Feb. 6, 7, $1952 \ldots$ & 8.58 & $\begin{array}{l}\text { Nov. } 2-10,1951 \text {, } \\
\quad \text { Sept. } 3,5,6,12,14 \\
16,1952,\end{array}$ & - \\
\hline 1953 & Oct. $18,1952 \ldots$ & 8.48 & Sept. 30, $1953 \ldots$ & 7. 48 \\
\hline
\end{tabular}

Maximum recorded thickness and periods of ice cover 1943-51

\begin{tabular}{|c|c|c|c|}
\hline $\begin{array}{l}\text { Water } \\
\text { year }\end{array}$ & $\begin{array}{l}\text { Maximum } \\
\text { thickness d } \\
\text { (inches) }\end{array}$ & $\begin{array}{l}\text { Total } \\
\text { days of } \\
\text { cover }\end{array}$ & Period \\
\hline 1943 & $\ldots-$ & --- & Dec. 26,1942, to-? \\
\hline 1944 & --- & 89 & Dec. 14,1943 , to Mar. 11,1944 . \\
\hline 1945 & --- & --- & \\
\hline 1946 & 2 & --- & Dec. 17,1945 , to $-?$ \\
\hline 1947 & --- & 79 & Dec. 22,1946 , to Mar. $10,1947$. \\
\hline 1948 & 17 & 95 & Nov. 28, 1947, to Feb. 29, 1948. \\
\hline 1949 & 3 & --- & Dec. 9,1948 , to $-?$ \\
\hline 1950 & 3 & 58 & $\begin{array}{c}\text { Dec. } 13,1949 \text {, to Jan. } 24,1950, \\
\text { Jan. } 29 \text { to Feb. } 13,1950 .\end{array}$ \\
\hline 1951 & $-\cdots$ & 115 & $\begin{array}{c}\text { Nov. } 24,1950, \text { to Jan. } 20,1951 \text {, } \\
\text { Jan. } 28 \text { to Mar. 25, } 1951 .\end{array}$ \\
\hline
\end{tabular}


Discharge measurements at outlet of Lake Manitou

\begin{tabular}{|c|c|c|c|c|c|c|c|}
\hline & Date & $\begin{array}{l}\text { Gage } \\
\text { height } \\
\text { (feet) }\end{array}$ & $\begin{array}{c}\text { Discharge } \\
\text { (cfs) }\end{array}$ & & Date & $\begin{array}{c}\text { Gage } \\
\text { height } \\
\text { (feet) }\end{array}$ & $\begin{array}{c}\text { Discharge } \\
\text { (cfs) }\end{array}$ \\
\hline Aug. & $17,1943-$ & 8.43 & 75.5 & Apr. & $19,1946-$ & 8.23 & 0 \\
\hline Oct. & $11 \ldots$ & 8. 14 & 2.98 & June & $7 \ldots$ & 8.41 & 8.70 \\
\hline Jan. & $17,1944-$ & 7.93 & 5.74 & Aug. & $23 \ldots \ldots$ & 7.16 & 0 \\
\hline May & $8-\cdots$ & 8.23 & 58.1 & Dec. & $11 \ldots$ & 7. 36 & 0 \\
\hline July & $11 \cdots \cdots$ & 8.01 & 0 & July & $29,1947-$ & 8.05 & 0 \\
\hline Aug. & $20,1945-$ & 8.45 & $a_{11.8}$ & June & $24,1948-$ & 8. 61 & $a 47.8$ \\
\hline Sept. & $17 \cdots$ & 8.51 & a 1.00 & Oct. & $24,1950-$ & 8.02 & $a 8.37$ \\
\hline Nov. & $27 \ldots \ldots$ & 8. 09 & 7.53 & & & & \\
\hline
\end{tabular}

$a$ Restricted flow; debris in front of screens.

Average lake level for Lake Manitou for water years 1943-53

\begin{tabular}{c|c|c|c|c|c|c|c}
\hline Year & Oct. & Nov. & Dec. & Jan. & Feb. & Mar. & Apr. \\
\hline 1943 & $* 8.40$ & 8.31 & 8.28 & 8.35 & 8.33 & 8.36 & 8.42 \\
1944 & 8.19 & 8.21 & 8.08 & 7.98 & 8.15 & 8.31 & 8.58 \\
1945 & 7.21 & 7.40 & 7.69 & 7.81 & 7.91 & 8.23 & 8.40 \\
1946 & 8.27 & 8.14 & 8.21 & 8.51 & 8.54 & 8.47 & 8.29 \\
1947 & 6.67 & 7.22 & 7.44 & 7.91 & 8.59 & 8.59 & 8.66 \\
1948 & 8.19 & 8.49 & 8.41 & 8.30 & 8.50 & 8.53 & 8.39 \\
1949 & 7.56 & 8.09 & 8.41 & 8.59 & 8.54 & 8.32 & 8.39 \\
1950 & 8.30 & 8.23 & 8.35 & 8.70 & 8.53 & 8.53 & 8.71 \\
1951 & 8.06 & 8.14 & 8.32 & 8.36 & 8.41 & 8.38 & 8.40 \\
1952 & 8.27 & 8.31 & 8.31 & 8.31 & 8.42 & 8.42 & 8.38 \\
1953 & 8.42 & 8.32 & 8.28 & 8.27 & 8.27 & 8.29 & 8.28 \\
\hline Average & 7.96 & 8.08 & 8.16 & 8.28 & 8.38 & 8.40 & 8.44 \\
\hline \hline & & & & & & & \\
Year & May & June & July & Aug. & Sept. & Annual & \\
\hline 1943 & 8.72 & 8.59 & 8.51 & 8.60 & 8.26 & $* 8.43$ & \\
1944 & 8.47 & 8.48 & 7.94 & 7.28 & 7.04 & 8.06 & \\
1945 & 8.52 & 8.45 & 8.28 & 8.49 & 8.47 & 8.07 & \\
1946 & 8.54 & 8.45 & 8.08 & 7.30 & 6.87 & 8.14 & \\
1947 & 8.57 & 8.45 & 8.11 & 7.76 & 7.98 & 7.99 & \\
1948 & 8.48 & 8.51 & 8.32 & 8.08 & 7.76 & 8.33 & \\
1949 & 8.40 & 8.42 & 8.50 & 8.11 & 8.01 & 8.28 & \\
1950 & 8.33 & 8.35 & 8.46 & 8.35 & 8.27 & 8.42 & \\
1951 & 8.36 & 8.48 & 8.46 & 8.39 & 8.30 & 8.34 & \\
1952 & 8.39 & 8.37 & 8.32 & 8.32 & 8.29 & 8.34 & \\
1953 & 8.28 & 8.27 & 8.26 & 8.15 & 7.59 & 8.22 & \\
\hline Average & 8.46 & 8.44 & 8.29 & 8.08 & 7.89 & 8.24 & \\
\hline
\end{tabular}

*Partial month or partial year. 

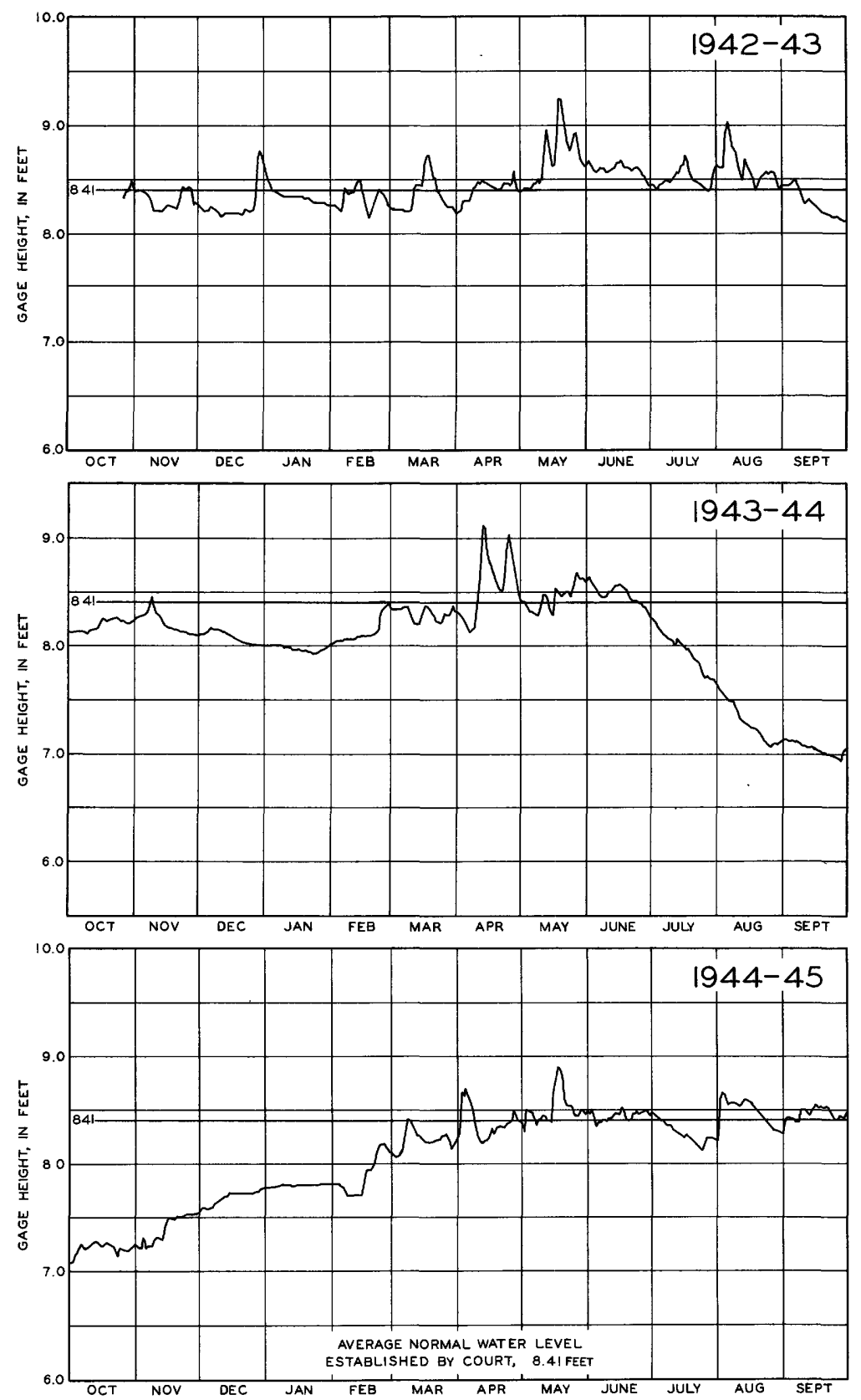

Figure 80. --Lake-level hydrographs for Lake Manitou at Rochester, Ind., for water years 1943-45. 

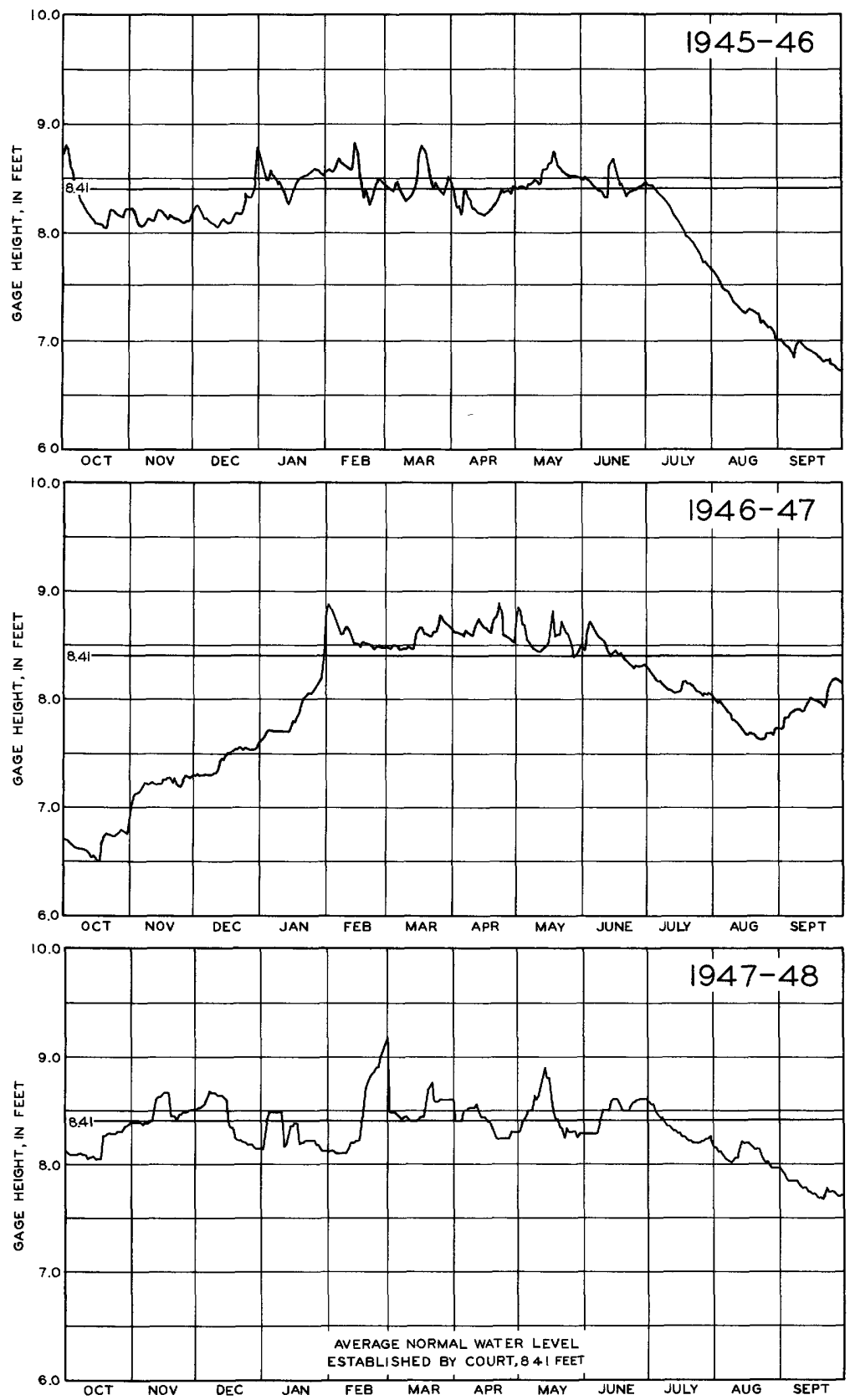

Figure 81. --Lake-level hydrographs for Lake Manitou at Roches ter, Ind., for water years 1946-48. 

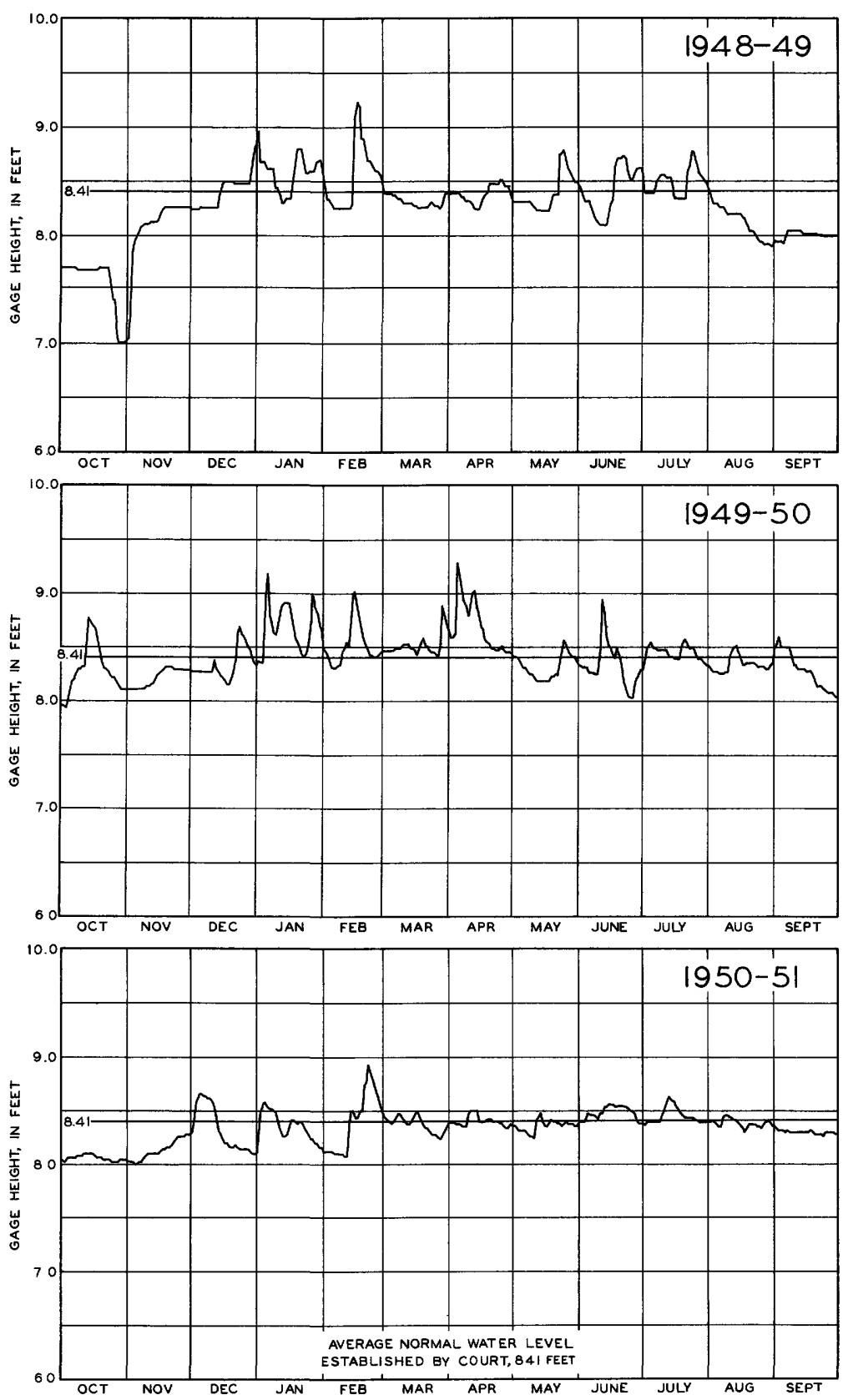

Figure 82. --Lake-level hydrographs for Lake Manitou at Rocheste $r$, Ind., for water years 1949-51. 
BASIC DATA ON LAKE LEVELS FOR SELECTED LAKES 169
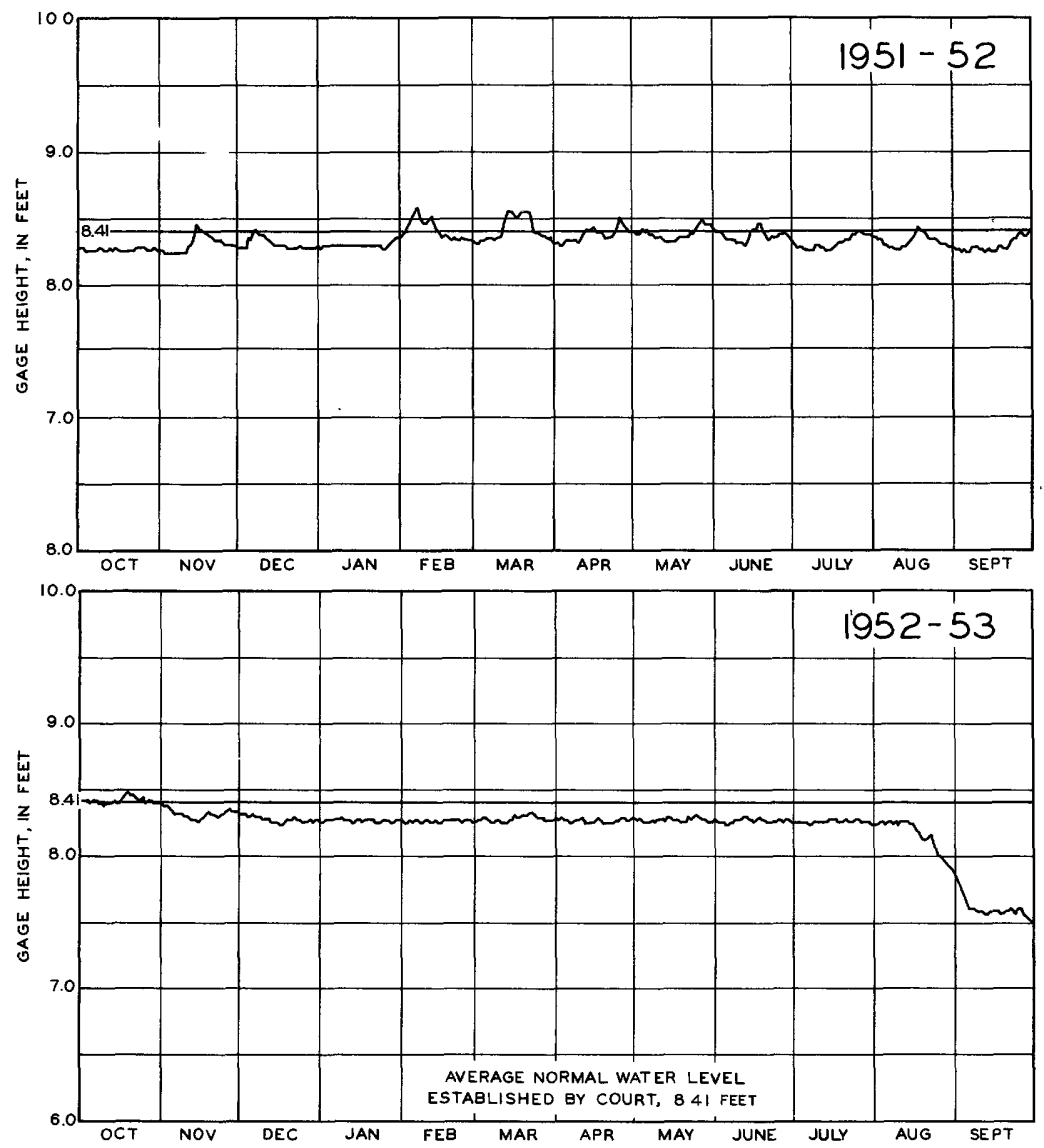

Figure 83. --Lake-level hydrographs for Lake Manitou at Rochester, Ind., for water years 1952-53. 
Loon Lake at Ormas, Ind.

Location. --Sec. 31, T. 33 N., R, 9 E. ,Noble County and secs. 25 and 36, T. 33 N., R. 8 E., sec.1, T. 32 N., R. 8 E., and sec. 6, T. 32 N., R. 9 E., Whitley County.

Surface area. --190 acres.

Drainage area. $--18.8 \mathrm{sq} \mathrm{mi}$.

Records available.--October 1942 to September 1953.

Gage. --Staff gage in two sections at outlet at extreme northeast corner of lake. Gage read to hundredths once daily. Datum of gage is $890.00 \mathrm{ft}$ above mean sea level, datum of 1929 .

Average lake level. --11 years; gage height, $5.34 \mathrm{ft}$; elevation, $895.34 \mathrm{ft}$.

Established legal leve1.--Established Dec. 8, 1953, at gage height $5.14 \mathrm{ft}$; elevation, 895. $14 \mathrm{ft}$ above mean sea level.

Lake-level control. - - Lake level maintained by bed of outlet channel. Court action now pending for recleaning outlet ditch and constructing control dam.

Extreme lake levels for Loon Lake, 1943-53

\begin{tabular}{|c|c|c|c|c|}
\hline \multicolumn{3}{|c|}{ Maximum } & \multicolumn{2}{|l|}{ Minimum } \\
\hline $\begin{array}{c}\text { Water } \\
\text { year }\end{array}$ & Date & $\begin{array}{l}\text { Gage } \\
\text { height } \\
\text { (feet) }\end{array}$ & Date & $\begin{array}{l}\text { Gage } \\
\text { heith } \\
\text { (feet) }\end{array}$ \\
\hline 1943 & May $20,21,1943 \ldots$ & 8.94 & Sept. $30,1943--$ & 4.66 \\
\hline 1944 & Apr. $17,1944 \ldots$ & 8.06 & Sept. $26-28,1944$ & 3.66 \\
\hline 1945 & May $20,21,1945 \ldots$ & 7.51 & $\begin{array}{r}\text { Oct. } 29 \text { to Nov. } 4 \text {, } \\
1944-\end{array}$ & 3.63 \\
\hline 1946 & Jan. $12,1946 \ldots$ & 6.16 & Sept. $30,1946 \cdots$ & 3.84 \\
\hline 1947 & Apr. $24,1947 \ldots$ & 7.18 & Oct. $16,17,1946$ & 3.66 \\
\hline 1948 & Mar. $2,3,1948 \ldots$ & 7. 16 & Sept. 27, 28, 1948 & 4.06 \\
\hline 1949 & Feb. 18, 1949 & 7.10 & Oct. $29,1948 \cdots$ & 3.96 \\
\hline 1950 & Apr. $5,1950 \ldots$ & 8.40 & Oct. $1,2,1949--$ & 4.38 \\
\hline 1951 & Feb. 23, 1951 $\ldots$ & 7.18 & Nov. $7,1950 \cdots$ & 4.76 \\
\hline 1952 & Jan. 28-31, 1952- - & 6.70 & Sept. $28-30,1952$ & 4.48 \\
\hline 1953 & Mar. $17,18,1953^{\cdots}$ & 5.84 & Sept. $30,1953--$ & $* 3.88$ \\
\hline
\end{tabular}

*Estimated. 
BASIC DATA ON LAKE LEVELS FOR SELECTED LAKES 171

Maximum recorded thickness and periods of ice cover 1943-52

\begin{tabular}{l|c|c|l}
\hline $\begin{array}{c}\text { Water } \\
\text { year }\end{array}$ & $\begin{array}{c}\text { Maximum } \\
\text { thickness } \\
\text { (inches) }\end{array}$ & $\begin{array}{c}\text { Total } \\
\text { days of } \\
\text { cover }\end{array}$ & \multicolumn{1}{|c}{ Period } \\
\hline 1943 & --- & 102 & Dec. 4, 1942, to Mar. 15, 1943. \\
1944 & $-\cdots-$ & --- & Dec. 15, 1943, to-? \\
1945 & --- & --- & Dec. 18, 1944, to-? \\
1946 & 11 & 84 & Dec. 12, 1945, to Mar. 5, 1946. \\
1947 & 10 & 91 & Jan. 4, 1947, to Apr. 4, 1947. \\
1948 & --- & 94 & Dec. 17, 1947, to Mar. 19, 1948. \\
1949 & 4 & --- & Jan. 30, 1949, to-? \\
1950 & 2 & --- & Dec. 15, 1949, to-? \\
1951 & --- & --- & Dec. 1, 1950, to-? \\
1952 & --- & --- & Dec. 13, 1951, to-? \\
\hline
\end{tabular}

Discharge measurements at outlet of Loon Lake

\begin{tabular}{|c|c|c|c|c|c|}
\hline Date & $\begin{array}{c}\text { Gage } \\
\text { height } \\
\text { (feet) }\end{array}$ & $\begin{array}{c}\text { Discharge } \\
\text { (cfs) }\end{array}$ & Date & $\begin{array}{c}\text { Gage } \\
\text { height } \\
\text { (feet) }\end{array}$ & $\begin{array}{c}\text { Discharge } \\
\text { (cfs) }\end{array}$ \\
\hline Aug. 13, 1943- & 5.13 & 3.67 & Feb. 16, 1949 - & 6.98 & 39.7 \\
\hline Oct. $\quad 8$ & 4.52 & 1.03 & Mar. $8 \ldots$ & 5.90 & 14.7 \\
\hline Jan. $\quad 7,1944-$ & 4.58 & .86 & Mar. $23 \ldots$ & 5.50 & 6.97 \\
\hline May 15 & 6.39 & 28.3 & Apr. $26 \ldots$ & 5.48 & 5.74 \\
\hline Apr. 10,1945 & 6.85 & 26.3 & Sept. $6 \ldots$ & 4.54 & .01 \\
\hline July 11 & 5. 36 & 3.64 & Nov. $30 \ldots$ & 5.03 & 1.01 \\
\hline Sept. $\quad 1 \ldots$ & 4.80 & 2.05 & Dec. 20 & 5.28 & 3.24 \\
\hline Oct. $\quad 5 \cdots-$ & 5.76 & 12.8 & Jan. $15,1950-$ & 7.91 & 69.1 \\
\hline Dec. 17 & 4.88 & 2.20 & Jan. $18 \ldots$ & 7.68 & 54.9 \\
\hline Mar. 12, $1946-$ & 5.94 & 13.4 & Jan. $27 \ldots$ & 7.85 & 64.4 \\
\hline May $14 \ldots$ & 4.78 & 1.70 & Feb. $\quad 7 \ldots$ & 6.43 & 24.8 \\
\hline July $25 \ldots$ & 4.58 & .51 & Feb. 22 & 6.88 & 32.6 \\
\hline Aug. $21 \ldots$ & 4.30 & .08 & Mar. 1 & 6.46 & 23.5 \\
\hline Oct. $\quad 1$ & 3.81 & 0 & May $\quad 3 \ldots$ & 6.06 & 16.4 \\
\hline Apr. $23,1947-$ & 7.16 & 24.1 & $24 \cdots---$ & 5.44 & 4.80 \\
\hline July $\quad 2$ & 5.42 & 8. 11 & Nov. $22 \ldots$ & 5.29 & 4. 34 \\
\hline Mar. 2, $1948-$ & 7.14 & 27.3 & May $9,1951-$ & 5.42 & 7.71 \\
\hline Sept. 28 & 4.06 & 0 & $7,1952-$ & 4. 76 & 0 \\
\hline Oct. 28 & 3.97 & 0 & $2,1953-$ & 4.76 & 1. 44 \\
\hline Dec. 29 & 5.20 & 4.90 & & & \\
\hline
\end{tabular}


Average lake level for Loon Lake for water years 1943-53

\begin{tabular}{r|c|l|l|l|l|l|l}
\hline Year & Oct. & Nov. & Dec. & Jan. & Feb. & Mar. & Apr. \\
\hline 1943 & $* 5.72$ & 6.36 & 6.29 & 6.85 & 6.44 & 6.66 & 6.19 \\
1944 & 4.51 & 4.72 & 4.67 & 4.52 & 4.80 & 5.85 & 7.13 \\
1945 & 3.72 & 3.74 & 3.92 & 4.17 & 4.79 & 5.85 & 6.35 \\
1946 & 5.48 & 5.20 & 4.99 & 5.72 & 5.69 & 5.96 & 5.28 \\
1947 & 3.75 & 4.01 & 4.20 & 4.84 & 5.57 & 5.48 & 6.59 \\
1948 & 4.64 & 4.84 & 5.19 & 5.40 & 5.51 & 6.69 & 6.30 \\
1949 & 4.04 & 4.26 & 4.72 & 6.11 & 6.41 & 5.75 & 5.54 \\
1950 & 5.04 & 5.05 & 5.55 & 7.42 & 6.80 & 6.87 & 7.31 \\
1951 & 4.85 & 5.03 & 5.89 & 5.83 & 6.01 & 5.99 & 6.08 \\
1952 & 4.98 & 5.50 & 5.60 & 6.28 & 6.08 & 5.89 & 5.94 \\
1953 & 4.35 & 4.37 & 4.87 & 5.18 & 5.24 & 5.56 & 5.33 \\
\hline Average & 4.64 & 4.83 & 5.08 & 5.67 & 5.76 & 6.05 & 6.19 \\
\hline \hline & & & & & & & \\
Year & May & June & July & Aug. & Sept. & Annual & \\
\hline 1943 & 7.49 & 6.43 & 5.59 & 5.12 & 4.83 & $* 6.20$ & \\
1944 & 6.88 & 5.43 & 4.40 & 3.90 & 3.77 & 5.05 & \\
1945 & 6.29 & 5.99 & 5.30 & 5.33 & 4.65 & 5.01 & \\
1946 & 4.97 & 5.18 & 4.87 & 4.29 & 4.00 & 5.13 & \\
1947 & 6.48 & 5.82 & 5.00 & 4.49 & 4.61 & 5.06 & \\
1948 & 6.09 & 5.55 & 4.95 & 4.55 & 4.21 & 5.33 & \\
1949 & 5.51 & 5.81 & 5.24 & 4.54 & 4.48 & 5.19 & \\
1950 & 5.66 & 5.64 & 5.38 & 4.91 & 5.14 & 5.89 & \\
1951 & 5.63 & 5.28 & 5.97 & 5.41 & 5.00 & 5.58 & \\
1952 & 5.62 & 5.97 & 5.58 & 4.99 & 4.61 & 5.59 & \\
1953 & 5.42 & 5.10 & 4.14 & 4.17 & 4.02 & 4.81 & \\
\hline Average & 6.00 & 5.65 & 5.13 & 4.70 & 4.48 & 5.34 & \\
\hline
\end{tabular}

* Partial year or partial month. 

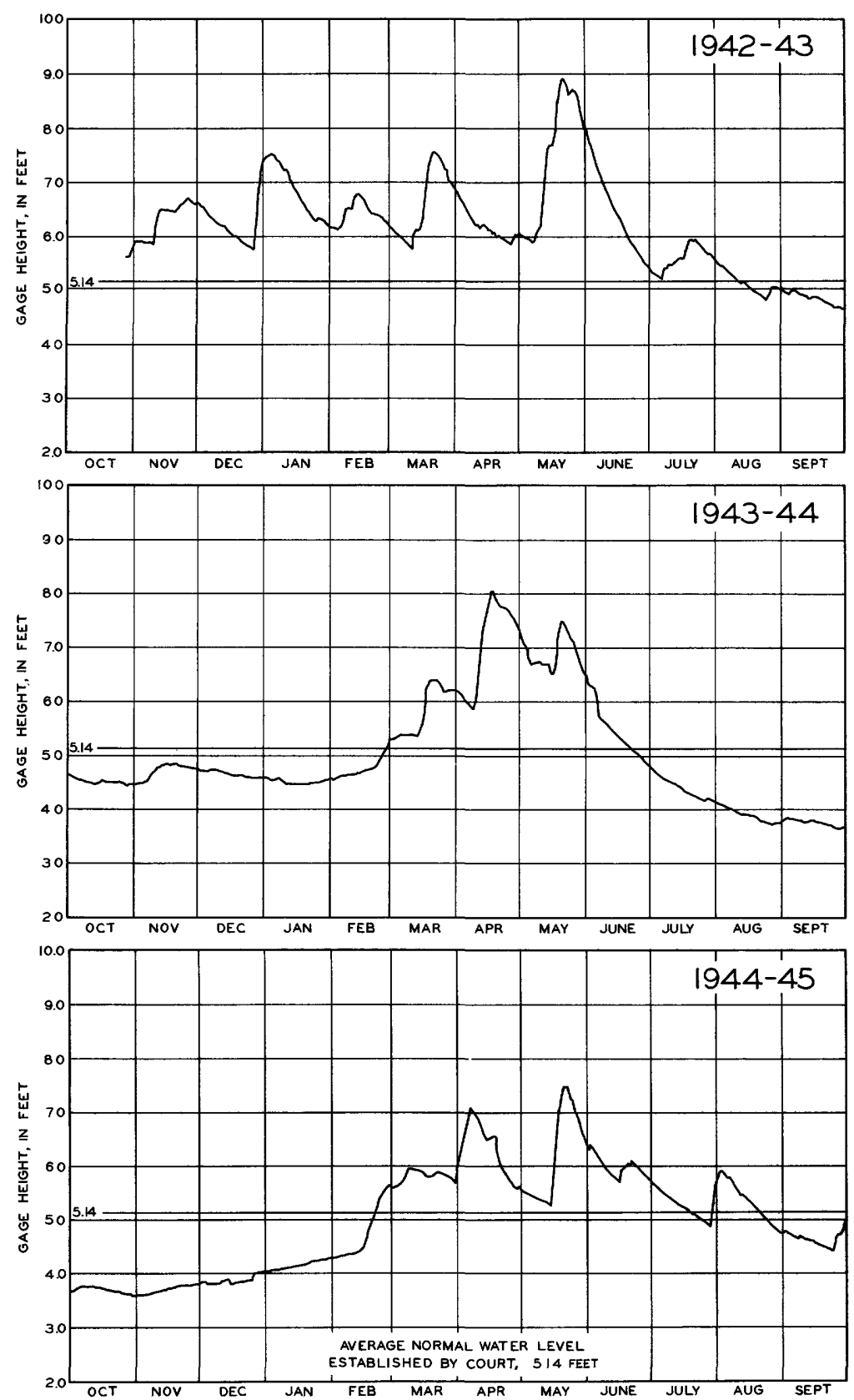

Figure 84. --Lake-level hydrographs for Loon Lake at Ormas Ind., for water years 1943-45. 

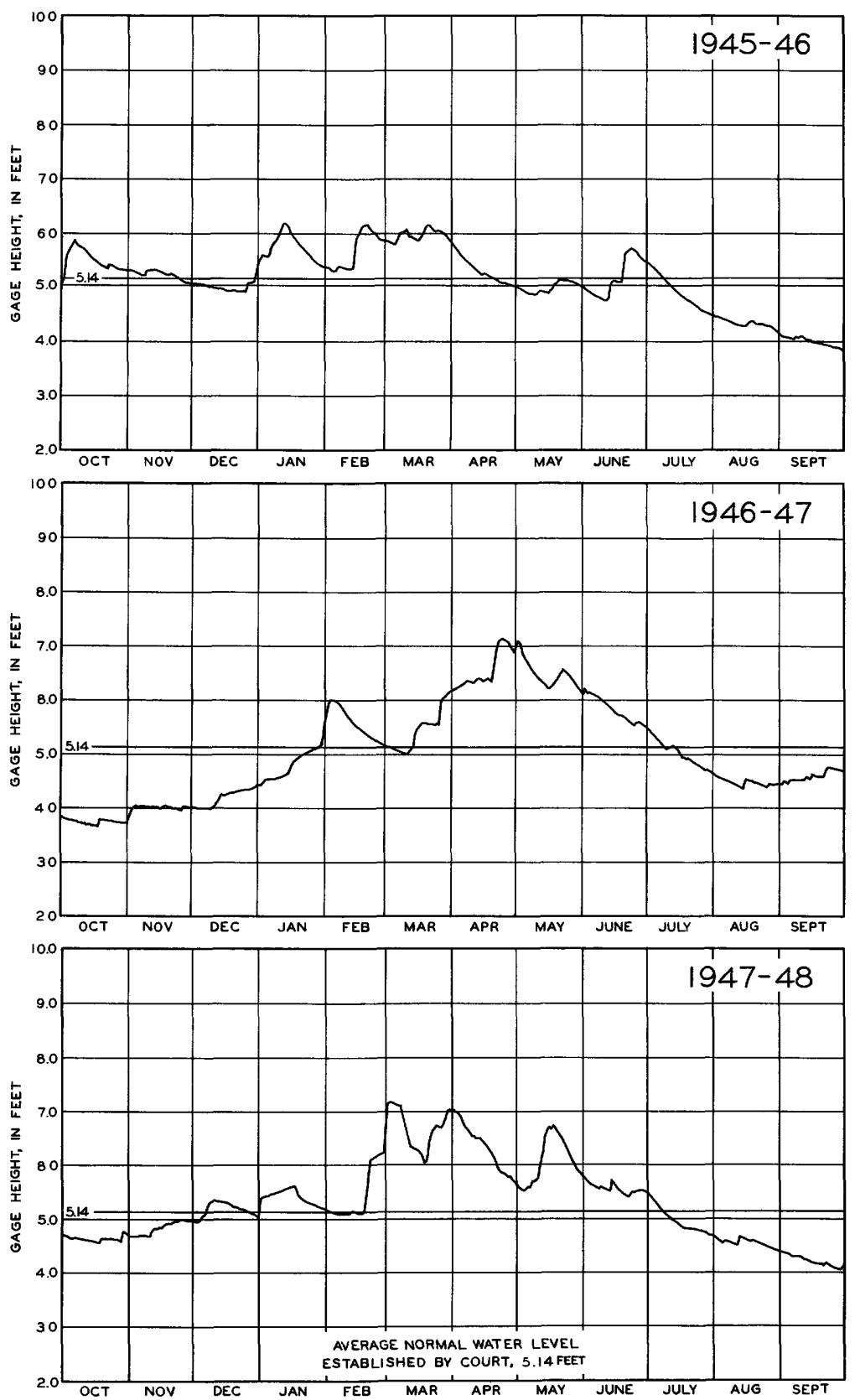

Figure 85. --Lake-level hydrographs for Loon Lake at Ormas, Ind., for water years 1946-48. 

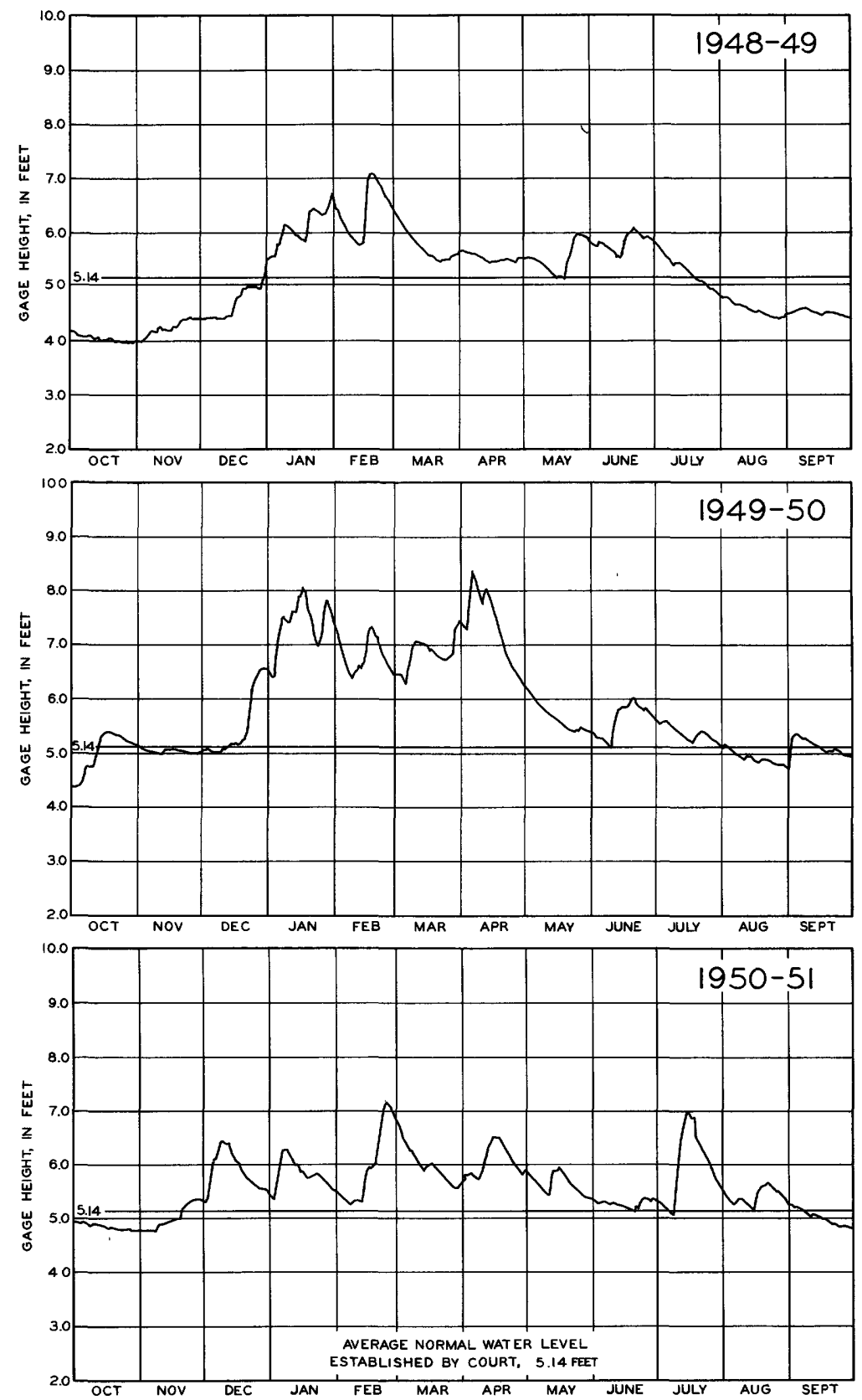

Figure 86. --Lake-level hydrographs for Loon Lake at Ormas, Ind., for water years 1949-51. 

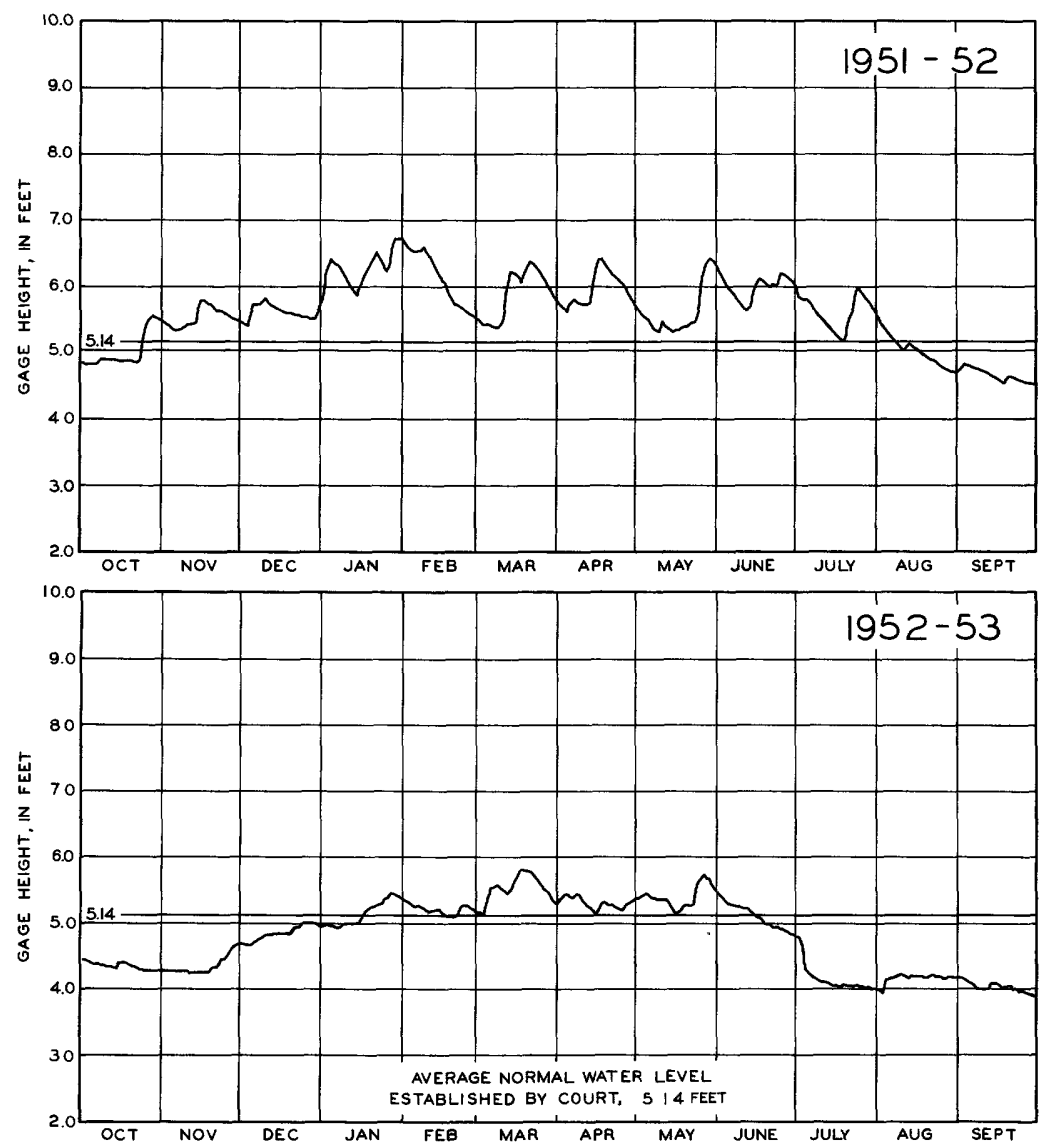

Figure 87. --Lake-level hydrographs for Loon Lake at Ormas, Ind., for water years 1952-53.

Maxinkuckee Lake at Culver, Ind.

Location. --Secs. 15, 16, 21, 22, 27, 28, 34, T. 32 N., R. 1 E. , Marshall County.

Surface area. $--1,650$ acres.

Drainage area. $--9.48 \mathrm{sq} \mathrm{mi}$.

Records available.--October 1942 to September 1953.

Gage.--Staff gage attached to east side of north abutment of out-

let dam. Gage read to hundredths once daily. Datum of gage

is $730.00 \mathrm{ft}$ above mean sea level, datum of 1929 .

Average level. --11 years; gage height $3.12 \mathrm{ft}$; elevation, $733.12 \mathrm{ft}$. Established legal level. --Established August 9, 1948, at gage height $3.12 \mathrm{ft}$; elevation, $733.12 \mathrm{ft}$ above mean sea level.

Lake-level control. - - Lake level maintained by 17-ft fixed-crest concrete dam, crest gage height, $2.9 \mathrm{ft}$. Dam was built in 1926 by Culver Military Academy. 
Extreme lake levels for Maxinkuckee Lake, 1943-53

\begin{tabular}{|c|c|c|c|c|}
\hline \multicolumn{3}{|c|}{ Maximum } & \multicolumn{2}{|l|}{ Minimum } \\
\hline $\begin{array}{l}\text { Water } \\
\text { year }\end{array}$ & Date & $\begin{array}{c}\text { Gage } \\
\text { height } \\
\text { (feet) }\end{array}$ & Date & $\begin{array}{l}\text { Gage } \\
\text { height } \\
\text { (feet) }\end{array}$ \\
\hline 1943 & May 20, 1943 $\ldots \ldots$ & 4. 58 & Oct. $27,28,1942--$ & 2.90 \\
\hline 1944 & Apr. 24, 1944 $\ldots$ & 3.92 & Sept, 25-27, 1944- & 2.54 \\
\hline 1945 & May $18,19,1945 \ldots$ & 3.72 & Oct. $30,21,1944-$ & 2.54 \\
\hline 1946 & Oct. 2, $1945 \ldots$ & 3.48 & Sept. $30,1946 \cdots$ & 2. 30 \\
\hline 1947 & June $8,1947 \ldots$ & 3.52 & Oct. $15,1946 \ldots$ & 2. 15 \\
\hline 1948 & May $14,1948 \ldots$ & 3.69 & Sept. $28,1948 \ldots$ & 2.62 \\
\hline 1949 & Feb. $16,17,1949$ & 3.65 & Sept. $30,1949 \ldots$ & 2.44 \\
\hline 1950 & Apr. 11. $1950 \ldots$ & 4. 38 & Oct. 2,1949 & 2. 42 \\
\hline 1951 & Apr. $15,1951 \ldots$ & 3.72 & Nov. $6,7,1950 \cdots$ & 2.75 \\
\hline 1952 & May 26-28, 1952- & 3.52 & Sept. $29,30,1952-$ & 2. 66 \\
\hline 1953 & May 23-25, 1953 $\ldots$ & 3.28 & Nov. $13-17,1952-$ & 2. 40 \\
\hline
\end{tabular}

Maximum recorded thickness and periods of ice cover 1943-52

\begin{tabular}{|c|c|c|c|}
\hline $\begin{array}{c}\text { Water } \\
\text { year }\end{array}$ & $\begin{array}{l}\text { Maximum } \\
\text { thickness } \\
\text { (inches) }\end{array}$ & $\begin{array}{c}\text { Total } \\
\text { days of } \\
\text { cover }\end{array}$ & Period \\
\hline 1943 & --- & 100 & Dec. 7,1942 , to Mar. $16,1943$. \\
\hline 1944 & --- & $-\ldots$ & Dec. 15,1943, to-? \\
\hline 1945 & --- & $-\cdots$ & Dec. 20,1944, to-? \\
\hline 1946 & --- & 80 & Dec. 18,1945 , to Mar. $7,1946$. \\
\hline 1947 & 5 & 89 & Jan. 4, 1947, to Apr. 2, 1947 \\
\hline 1948 & $165 / 8$ & 88 & Dec. 23,1947 , to Mar. $19,1948$. \\
\hline 1949 & 2 & 55 & $\begin{array}{l}\text { Jan. 1-9, 20-28; Jan. } 30 \text { to } \\
\text { Mar. } 7,1949 \text {. }\end{array}$ \\
\hline 1950 & $-\cdots$ & 25 & $\begin{array}{l}\text { Jan. 20-24; Feb. } 24 \text { to Mar. 15, } \\
\text { 1950. }\end{array}$ \\
\hline 1951 & $\ldots$ & 86 & Dec. 12,1950, to Mar. 7,1951 \\
\hline 1952 & --- & 57 & Dec. 17, 1951, to Feb. 11, 1952. \\
\hline
\end{tabular}


Discharge measurements at outlet of Maxinkuckee Lake

\begin{tabular}{|c|c|c|c|c|c|c|}
\hline & Date & $\begin{array}{c}\text { Gage } \\
\text { height } \\
\text { (feet) }\end{array}$ & $\begin{array}{c}\text { Discharge } \\
\text { (cfs) }\end{array}$ & Date & $\begin{array}{c}\text { Gage } \\
\text { height } \\
\text { (feet) }\end{array}$ & $\begin{array}{c}\text { Discharge } \\
\text { (cfs) }\end{array}$ \\
\hline Aug. & $6,1943-$ & 3.40 & 15.6 & Nov. $16,1950-$ & 2.80 & $a \quad 0.76$ \\
\hline Oct. & $12 \ldots-\cdots$ & 2.99 & 4.96 & Feb. 1, 1951 - & 3.19 & 17.1 \\
\hline Jan. & $21,1944-$ & 2.99 & 4.55 & Apr. $12 \ldots$ & 3.66 & 32.8 \\
\hline May & $11 \cdots-\cdots$ & 3.64 & 34.6 & May $16 \ldots$ & 3.47 & 28.1 \\
\hline July & $13 \ldots \cdots$ & 3.00 & 5.25 & June $21 \ldots$ & 3.10 & 11.5 \\
\hline Apr. & $12,1945-$ & 3.30 & 27.8 & July $18 \ldots$ & 3.28 & 21.5 \\
\hline June & $5 \cdots-$ & 3.64 & 30.6 & Aug. 28 & 3.01 & 6.01 \\
\hline Aug. & $21 \ldots \ldots$ & 3.07 & 8.18 & Sept. $19 \ldots$ & 2.89 & 2.29 \\
\hline Sept. & $17----$ & 2.86 & 1. 59 & Oct. $16 \ldots$ & 2.80 & 1.38 \\
\hline Nov. & $27 \cdots-\cdots$ & 3.06 & 8.26 & Nov. $19 \ldots \ldots$ & 3.23 & 21.5 \\
\hline Apr. & $3,1946-$ & 3.28 & 23.4 & Dec. $17 \ldots$ & 3.20 & 19.2 \\
\hline June & $5----$ & 3.02 & 7.04 & Jan. 14,1952 - & 3.29 & 23.2 \\
\hline Aug. & $26 \ldots---$ & 2.52 & 0 & Feb. $18 \ldots \ldots$ & 3.31 & 21.1 \\
\hline Oct. & $22-----$ & 2.26 & .04 & Mar. $19 \ldots$ & 3.35 & 27.7 \\
\hline Nov. & $19 \ldots$ & 2.68 & $a \quad .18$ & July $25 \ldots$ & 2.99 & $\begin{array}{ll}a & 5.78\end{array}$ \\
\hline Dec. & $20 \cdots-\cdots$ & 2. 74 & $* .042$ & Aug. $21 \ldots$ & 2.88 & 15.03 \\
\hline Jan. & $7,1947-$ & 2.89 & 2.02 & Sept. $19 \ldots$ & 2.78 & $a \quad .56$ \\
\hline Feb. & $17---$ & 3.12 & 10.0 & Oct. $14 \ldots \ldots$ & 2.56 & *. 10 \\
\hline Feb. & $20 \ldots-\cdots$ & 3.10 & 15.3 & Nov. $11 \ldots$ & 2.40 & 0 \\
\hline May & $1--$ & 3.49 & 33.0 & Dec. $12 \ldots$ & 2.70 & $* .20$ \\
\hline Dec. & $16 \cdots-\cdots$ & 3.11 & 8.26 & Jan. 22, 1953- & 3.04 & 7.60 \\
\hline Feb. & $23,1948-$ & 3.15 & 12.3 & Feb. $13 \ldots$ & 3.07 & 9.13 \\
\hline Feb. & $29 \cdots \cdots$ & 3.36 & 22.4 & Mar. 2 $2 \ldots$ & 3.10 & 10.2 \\
\hline May & $13 \ldots$ & 3.67 & 28.5 & Mar. 24-..- & 3.30 & 20.4 \\
\hline Aug. & $31 \ldots$ & 2.84 & a 1.40 & Apr. $13 \ldots$ & 3.19 & $a_{13.8}$ \\
\hline Feb. & $22,1949-$ & 3.61 & 35.1 & May $5 \ldots$ & 3.21 & 17.3 \\
\hline Apr. & $11 \cdots \cdots$ & 3. 17 & 16.5 & June $5 \ldots$ & 3.07 & 8. 16 \\
\hline Sept. & $2 \ldots-\cdots$ & 2.64 & $a \quad .61$ & June 29 & 3.06 & $a_{10.3}$ \\
\hline Nov. & $21 \cdots$ & 2. 70 & $a \quad .74$ & July $23-\ldots$ & 3.06 & a 9.76 \\
\hline Mar. & $16,1950-$ & 3.73 & 35.5 & Aug. $21 \ldots$ & 2.78 & a .49 \\
\hline May & $1----$ & 4.02 & 43.8 & Oct. $\quad 6 \cdots$ & 2.38 & 0 \\
\hline Oct. & $11 \ldots-\cdots$ & 2.90 & 2.54 & $9--\cdots$ & 2.18 & \\
\hline Nov. & $6----$ & 2.74 & a. .60 & & & \\
\hline
\end{tabular}

* Estimated.

${ }^{a}$ Leakage through dam. 
BASIC DATA ON LAKE LEVELS FOR SELECTED LAKES 179

Average lake level for Maxinkuckee Lake for water years 1943-53

\begin{tabular}{c|c|c|c|c|c|c|c}
\hline Year & Oct. & Nov. & Dec. & Jan. & Feb. & Mar. & Apr. \\
\hline 1943 & $* 2.93$ & 3.14 & 3.20 & 3.41 & 3.33 & 3.35 & 3.28 \\
1944 & 3.02 & 3.08 & 3.02 & 2.99 & 3.10 & 3.38 & 3.66 \\
1945 & 2.62 & 2.65 & 2.77 & 2.94 & 2.98 & 3.20 & 3.29 \\
1946 & 3.25 & 3.07 & 3.07 & 3.23 & 3.19 & 3.34 & 3.17 \\
1947 & 2.24 & 2.65 & 2.73 & 2.97 & 3.12 & 3.08 & 3.35 \\
1948 & 2.87 & 2.94 & 3.07 & 3.14 & 3.11 & 3.38 & 3.39 \\
1949 & 2.52 & 2.71 & 2.92 & 3.35 & 3.54 & 3.35 & 3.17 \\
1950 & 2.69 & 2.68 & 2.93 & 3.68 & 3.85 & 3.76 & 4.15 \\
1951 & 2.88 & 2.86 & 3.18 & 3.26 & 3.31 & 3.44 & 3.54 \\
1952 & 2.90 & 3.17 & 3.25 & 3.34 & 3.33 & 3.27 & 3.38 \\
1953 & 2.56 & 2.48 & 2.71 & 2.92 & 3.09 & 3.19 & 3.20 \\
\hline Average & 2.77 & 2.86 & 2.99 & 3.20 & 3.27 & 3.34 & 3.42 \\
\hline \hline & & & & & & & \\
Year & May & June & July & Aug. & Sept. & Annual & \\
\hline 1943 & 3.91 & 3.81 & 3.50 & 3.35 & 3.23 & $* 3.40$ & \\
1944 & 3.65 & 3.31 & 2.90 & 2.68 & 2.61 & 3.12 & \\
1945 & 3.47 & 3.54 & 3.16 & 3.09 & 2.94 & 3.05 & \\
1946 & 3.09 & 3.12 & 2.95 & 2.60 & 2.38 & 3.04 & \\
1947 & 3.36 & 3.39 & 2.98 & 2.75 & 2.94 & 2.96 & \\
1948 & 3.47 & 3.27 & 3.24 & 2.98 & 2.73 & 3.13 & \\
1949 & 3.20 & 3.21 & 3.01 & 2.85 & 2.56 & 3.03 & \\
1950 & 3.64 & 3.52 & 3.47 & 3.24 & 3.00 & 3.38 & \\
1951 & 3.44 & 3.18 & 3.20 & 2.98 & 2.88 & 3.18 & \\
1952 & 3.35 & 3.35 & 3.03 & 2.84 & 2.72 & 3.16 & \\
1953 & 3.21 & 3.11 & 3.08 & 2.86 & 2.55 & 2.91 & \\
\hline Average & 3.44 & 3.35 & 3.14 & 2.93 & 2.78 & 3.12 &. \\
\hline & & & & & & & \\
\hline
\end{tabular}

* Partial month or partial year. 

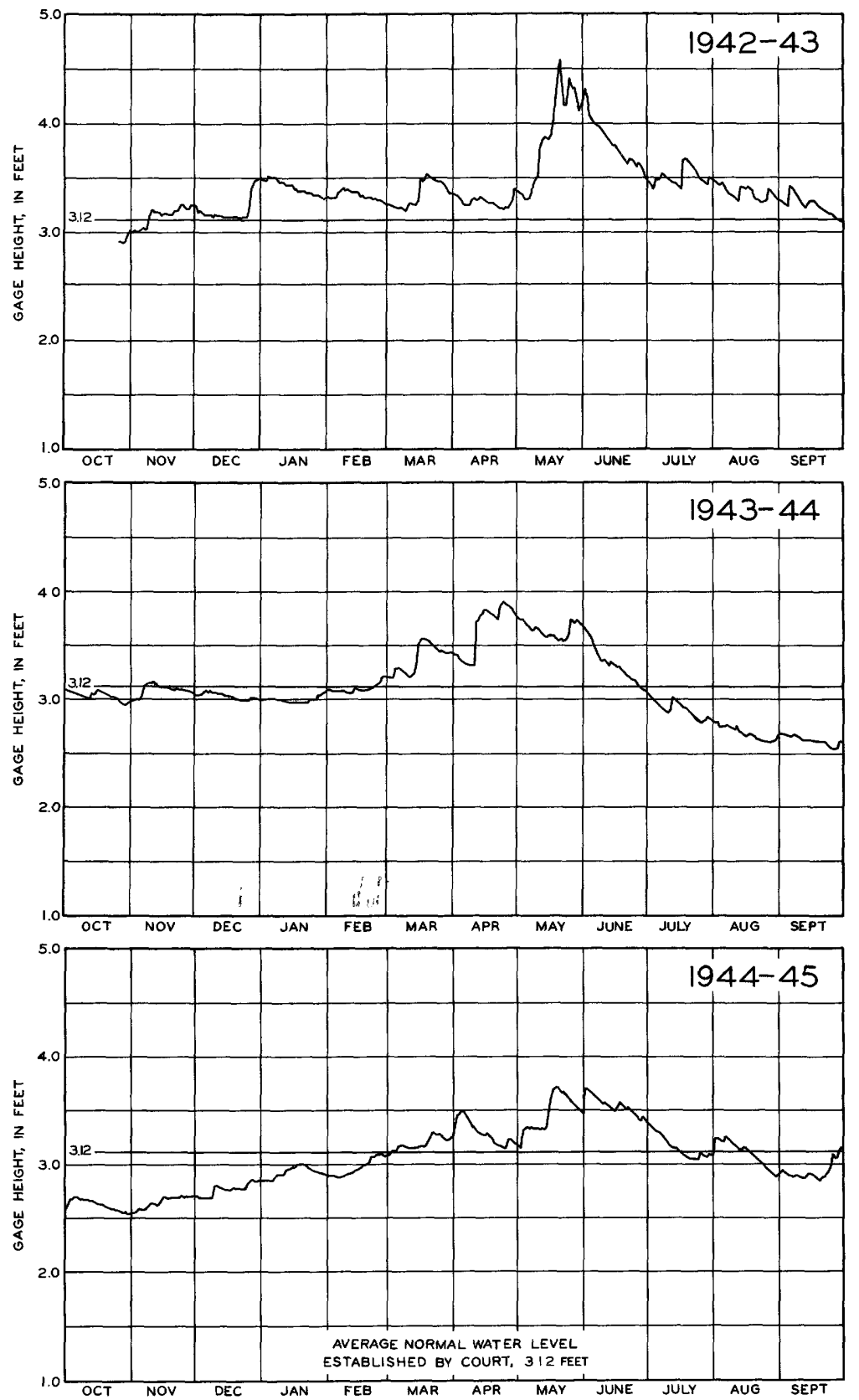

Figure 88. --Lake-level hydrographs for Maxinkuckee Lake at Culver, Ind., for water years 1943-45. 

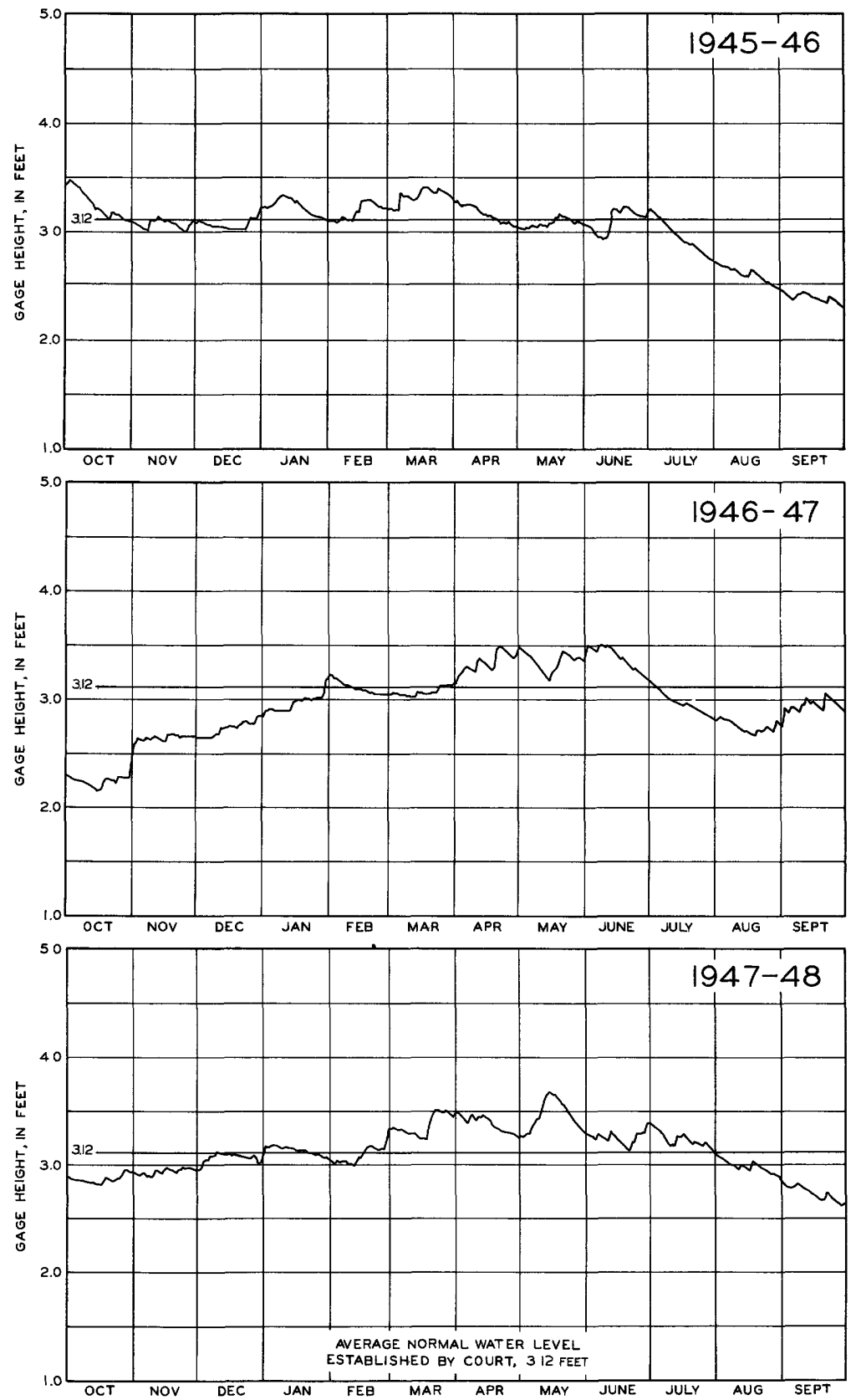

Figure 89. --Lake-level hydrographs for Maxinkuckee Lake at Culver, Ind., for water years 1946-48. 


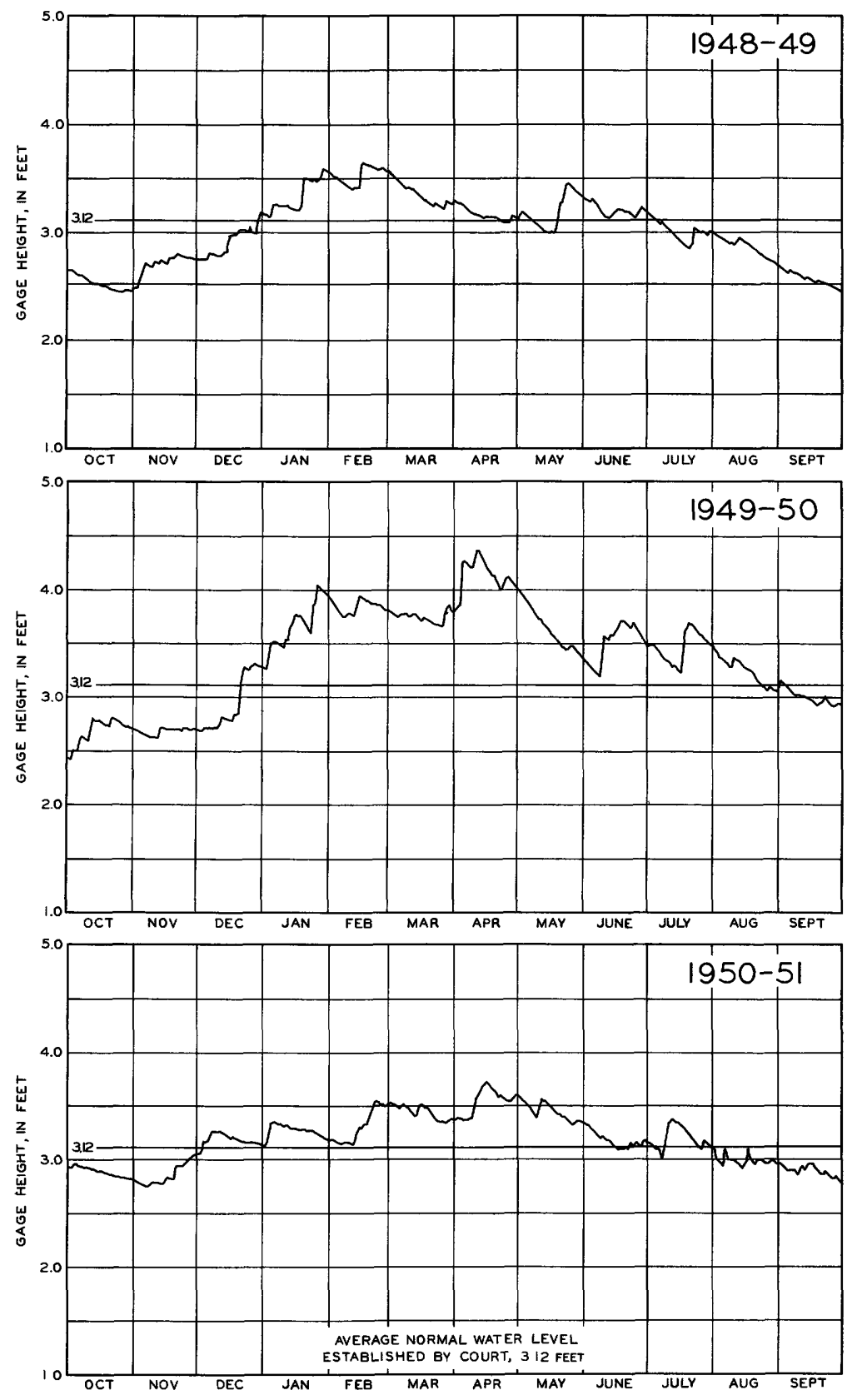

Figure 90. --Lake-level hydrographs for Maxinkuckee Lake at Culver, Ind., for water years 1949-51. 


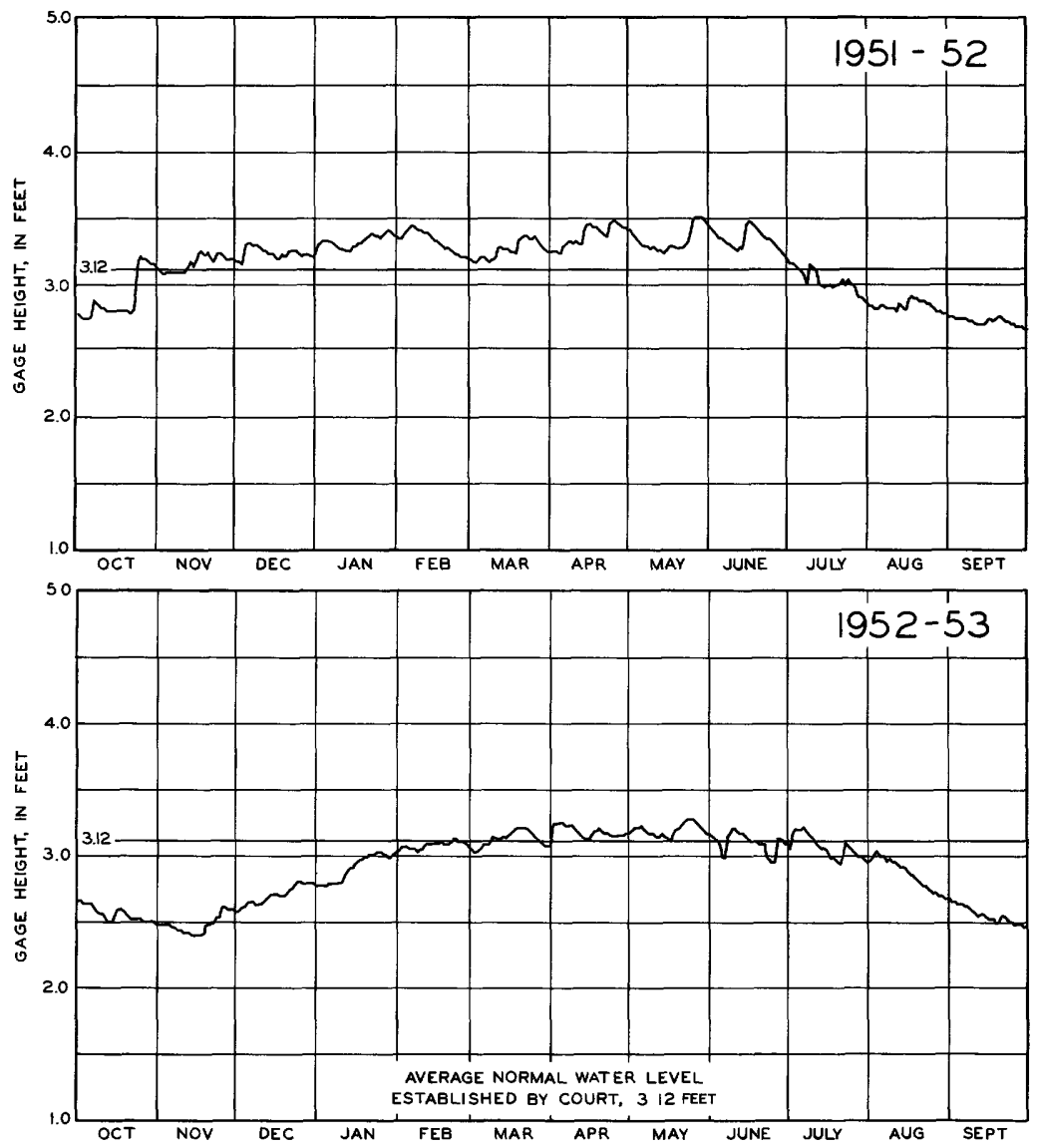

Figure 91. --Lake-level hydrographs for Maxinkuckee Lake at Culver, Ind., for water years 1952-53.

Muskelonge Lake near Warsaw, Ind.

Location.--Sec. 3, T. 31 N., R. 6 E., Kosciusko County, 4.9 miles southeast of Warsaw.

Surface area. -23.8 acres.

Drainage area. $--11.1 \mathrm{sq} \mathrm{mi}$.

Records available. --August 1943 to November 1952.

Gage. --Staff gage attached to willow tree on north bank of outlet,

$50 \mathrm{ft}$ downstream from lake. Gage read to hundredths once daily.

Datum of gage is $840.00 \mathrm{ft}$ above mean sea level, datum of 1929 . Average lake level. --9 years, gage height, $2.02 \mathrm{ft}$; elevation, $842.02 \mathrm{ft}$.

Established legal level. --Established September 20,1948, at gage height $2.67 \mathrm{ft}$; elevation, $842.67 \mathrm{ft}$ above mean sea level. Lake-level control. - - Lake level maintained by riffle about $0.5 \mathrm{mi}$ downstream from lake. 
Extreme lake levels for Muskelonge Lake, 1944-52

\begin{tabular}{|c|c|c|c|c|}
\hline \multicolumn{2}{|r|}{ Maximum } & \multicolumn{3}{|c|}{ Minimum } \\
\hline $\begin{array}{l}\text { Water } \\
\text { year }\end{array}$ & Date & $\begin{array}{l}\text { Gage } \\
\text { height } \\
\text { (feet) }\end{array}$ & Date & $\begin{array}{c}\text { Gage } \\
\text { height } \\
\text { (feet) }\end{array}$ \\
\hline $1943^{a}$ & Aug. 15, $1943-\cdots$ & $a 2.73$ & Sept. $3,1943 \ldots$ & $a_{1.66}$ \\
\hline 1944 & Apr. 13, 1944 & 5.22 & Aug. 21, 22, $1944-$ & $* 1.02$ \\
\hline 1945 & May $17,1945^{-\cdots--}$ & 4.76 & Oct. 1,1944 & 1.21 \\
\hline 1946 & Oct. $1,1945 \ldots$ & 4.98 & Sept. $27-30,1946-$ & 1.20 \\
\hline 1947 & Apr. $25,1947 \ldots$ & 3.44 & Oct. 5-16, 1946- - & 1.19 \\
\hline 1948 & Feb. 29, $1948 \ldots$ & 3.68 & Sept. 28, $1948 \ldots$ & 1.04 \\
\hline 1949 & Feb. 16, $1948 \ldots$ & 3.58 & $\begin{array}{l}\text { Oct. } 4-7,11-15, \\
1948\end{array}$ & 1. 10 \\
\hline 1950 & Apr. $5,1950 \cdots$ & 3.90 & Oct. 2, 1949- & 1. 36 \\
\hline 1951 & July $11,1951 \ldots$ & 3.50 & Sept. 28-30, $1951-$ & 1. 32 \\
\hline 1952 & May $25,1952 \ldots-$ & 3.46 & Oct. $1-6,1951 \cdots$ & 1. 32 \\
\hline
\end{tabular}

Maximum recorded thickness and periods of ice cover 1944-52

\begin{tabular}{|c|c|c|c|}
\hline $\begin{array}{l}\text { Water } \\
\text { year }\end{array}$ & $\begin{array}{c}\text { Maximum } \\
\text { thickness } \\
\text { (inches) }\end{array}$ & $\begin{array}{c}\text { Total } \\
\text { days of } \\
\text { cover }\end{array}$ & Period \\
\hline 1944 & 10 & --- & Unknown to Feb. $26,1944$. \\
\hline 1945 & --- & --- & \\
\hline 1946 & ---- & 83 & Dec. 16,1945 , to Mar. 8,1946 \\
\hline 1947 & 10 & 101 & Dec. 19,1946 , to Mar. 29, 1947. \\
\hline 1948 & 8 & -- & Unknown to Mar. 21,1948 \\
\hline 1949 & 6 & 52 & $\begin{array}{c}\text { Dec. } 25,1948 \text {, to Jan. } 15,1949 \text {; } \\
\text { Jan. } 31 \text { to Mar. 1, } 1949 .\end{array}$ \\
\hline 1950 & 4 & 43 & $\begin{array}{l}\text { Jan. } 15-28 ; \text { Feb. } 19 \text { to Mar. } 19 \text {, } \\
1950 .\end{array}$ \\
\hline 1951 & 8 & $-\ldots$ & Dec. 3,1950 , to-? \\
\hline 1952 & 6 & 20 & Jan. $1-20,1952$ \\
\hline
\end{tabular}


Discharge measurements at outlet of Muskelonge Lake

\begin{tabular}{|c|c|c|c|c|c|}
\hline Date & $\begin{array}{l}\text { Gage } \\
\text { height } \\
\text { (feet) }\end{array}$ & $\begin{array}{c}\text { Discharge } \\
\text { (cfs) }\end{array}$ & Date & $\begin{array}{l}\text { Gage } \\
\text { height } \\
\text { (feet) }\end{array}$ & $\begin{array}{c}\text { Discharge } \\
\text { (cfs) }\end{array}$ \\
\hline Aug. $\quad 5,1943$ & 2.58 & 17.8 & Jan. $\quad 4,1949-$ & 2.08 & 11.2 \\
\hline Oct. $\quad 6 \ldots$ & 1. 60 & 2.11 & Feb. $15 \ldots$ & 3.52 & 129 \\
\hline Jan. $\quad 6,1944$ & 1. 53 & 1. 56 & Mar. $2 \ldots$ & 2.37 & 25.1 \\
\hline Apr. $21 \cdots$ & 3.22 & 48.6 & Mar. $22 \ldots$ & 2.04 & 9.29 \\
\hline June 12,1945 & 2.05 & 7.55 & May $3 \ldots \ldots$ & 2.18 & 11.2 \\
\hline Sept. $7---$ & 1. 94 & 6.23 & Aug. $30 \ldots$ & 1.37 & .92 \\
\hline Nov. 26 & 1.98 & 7.59 & Nov. $1 \ldots$ & 1. 72 & 3.87 \\
\hline Mĩy 27, 1946 & 2.22 & 12.4 & Dec. $14 \ldots \ldots$ & 1. 92 & 8.35 \\
\hline June $21 \ldots$ & 2.59 & 19.2 & Jan. 12, 1950 - & 3.09 & 69.8 \\
\hline Dec. $\quad 3 \ldots$ & 1. 52 & 1.69 & Jan. $19 \ldots$ & 2.85 & 54.9 \\
\hline Apr. 22,1947 & 3.36 & 48.7 & Jan. $31 \ldots \ldots$ & 2.76 & 49.6 \\
\hline Jan. 27,1948 & 1. 73 & 4.00 & Mar. $14 \ldots$ & 2. 64 & 38.9 \\
\hline Mar. $1 \ldots$ & 3.51 & 49.3 & May $2 \ldots \ldots$ & 2. 42 & 21.6 \\
\hline Mar. $11 \ldots$ & 2.05 & 12. 6 & Sept. $12 \ldots$ & 1.59 & 2.97 \\
\hline Mar. $24 \ldots---$ & 3.11 & 37.6 & Oct. $31 \ldots \ldots$ & 1. 52 & 1.83 \\
\hline Sept. $28 \ldots$ & 1.05 & .21 & Nov. $20 \ldots$ & 2.09 & 14.5 \\
\hline Oct. $14 \ldots$ & 1.11 & .27 & May 8, 1951 _ & 2.19 & 9.99 \\
\hline Nov. $30 \ldots$ & 1. 57 & 2. 14 & Aug. 18, 1952 & 1.71 & 4.96 \\
\hline
\end{tabular}

Average lake level for Miuskelonge Lake for water years 1943-52

\begin{tabular}{c|l|l|l|l|l|l|l}
\hline Year & Oct. & Nov. & Dec. & Jan. & Feb. & Mar. & Apr. \\
\hline 1944 & 1.65 & 1.87 & 1.64 & 1.36 & 1.73 & 2.46 & 3.42 \\
1945 & 1.34 & 1.48 & 1.51 & 1.51 & 1.67 & 2.16 & 2.49 \\
1946 & 2.81 & 2.10 & 2.05 & 2.43 & 2.48 & 2.45 & 1.94 \\
1947 & 1.28 & 1.59 & 1.57 & 1.90 & 2.46 & 2.24 & 2.62 \\
1948 & 1.63 & 1.89 & 2.07 & 2.13 & 2.13 & 2.67 & 2.42 \\
1949 & 1.13 & 1.55 & 1.73 & 2.45 & 2.49 & 2.13 & 2.08 \\
1950 & 2.04 & 1.69 & 2.05 & 3.05 & 2.72 & 2.70 & 2.89 \\
1951 & 1.50 & 1.74 & 2.29 & 2.34 & 2.42 & 2.46 & 2.61 \\
1952 & 1.54 & 1.82 & 2.01 & 2.50 & 2.36 & 2.35 & 2.48 \\
\hline Average & 1.66 & 1.75 & 1.88 & 2.19 & 2.27 & 2.40 & 2.55 \\
\hline \hline Year & May & June & July & Aug. & Sept. & Annual & \\
\hline 1943 & --- & --- & --- & $* 2.12$ & 1.96 & $* 2.04$ & \\
1944 & 2.99 & 1.98 & 1.48 & 1.18 & 1.19 & 1.91 & \\
1945 & 2.79 & 2.20 & 1.68 & 2.51 & 2.19 & 1.96 & \\
1946 & 2.17 & 2.11 & 1.88 & 1.44 & 1.21 & 2.09 & \\
1947 & 2.43 & 2.28 & 1.73 & 1.49 & 1.79 & 1.94 & \\
1948 & 2.42 & 2.11 & 1.72 & 1.36 & 1.12 & 1.97 & \\
1949 & 2.14 & 2.32 & 1.96 & 1.49 & 1.49 & 1.91 & \\
1950 & 2.17 & 2.45 & 1.93 & 1.72 & 1.68 & 2.25 & \\
1951 & 2.35 & 2.16 & 2.38 & 1.64 & 1.42 & 2.08 & \\
1952 & 2.43 & 2.26 & 1.80 & 1.51 & 1.71 & 2.06 & \\
\hline Average & 2.43 & 2.21 & 1.84 & 1.65 & 1.58 & 2.02 & \\
\hline
\end{tabular}

* Partial month or partial year. 

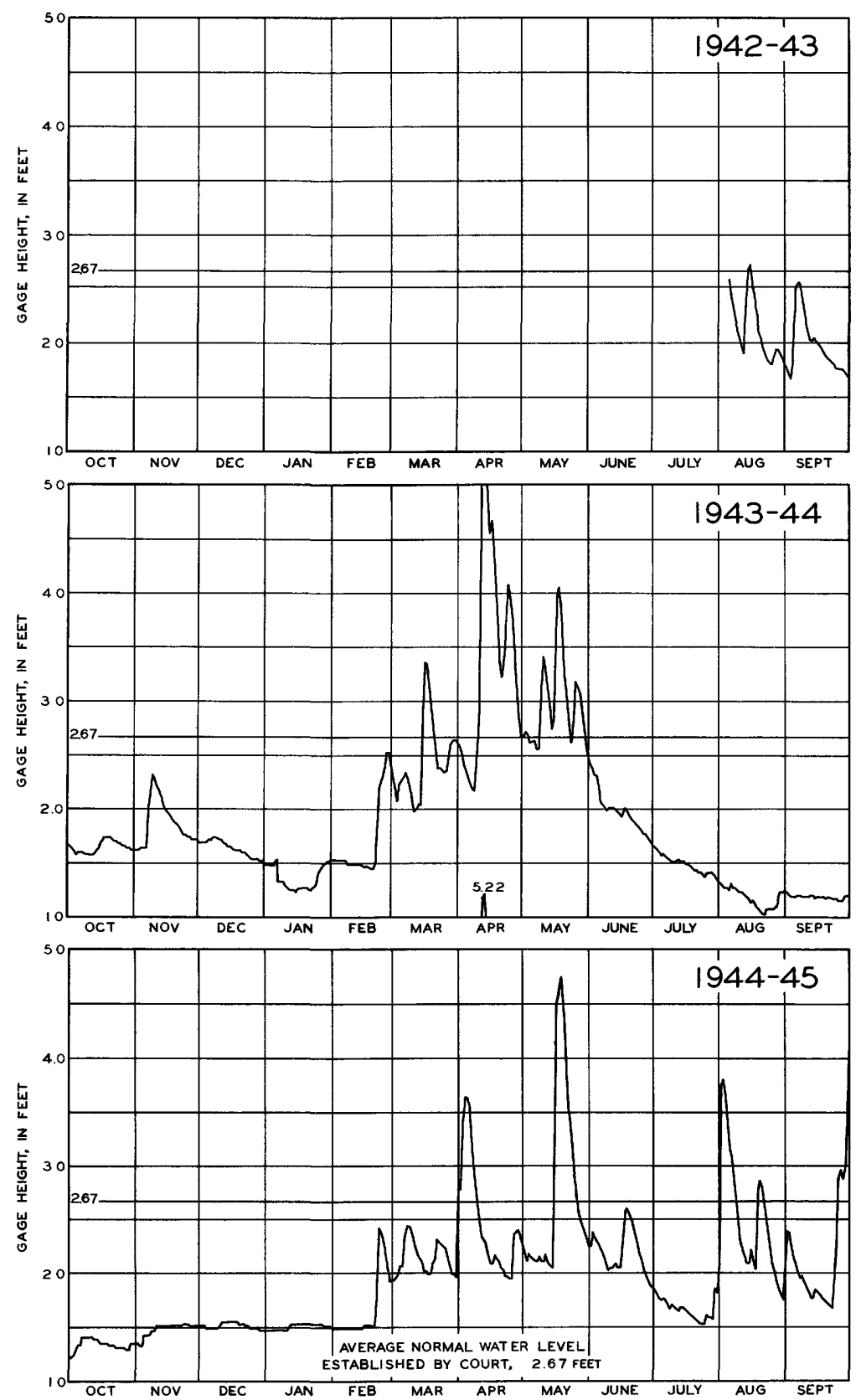

Figure 92. --Lake-level hydrographs for Muskelonge Lake near Warsaw, Ind., for water years 1943-45. 

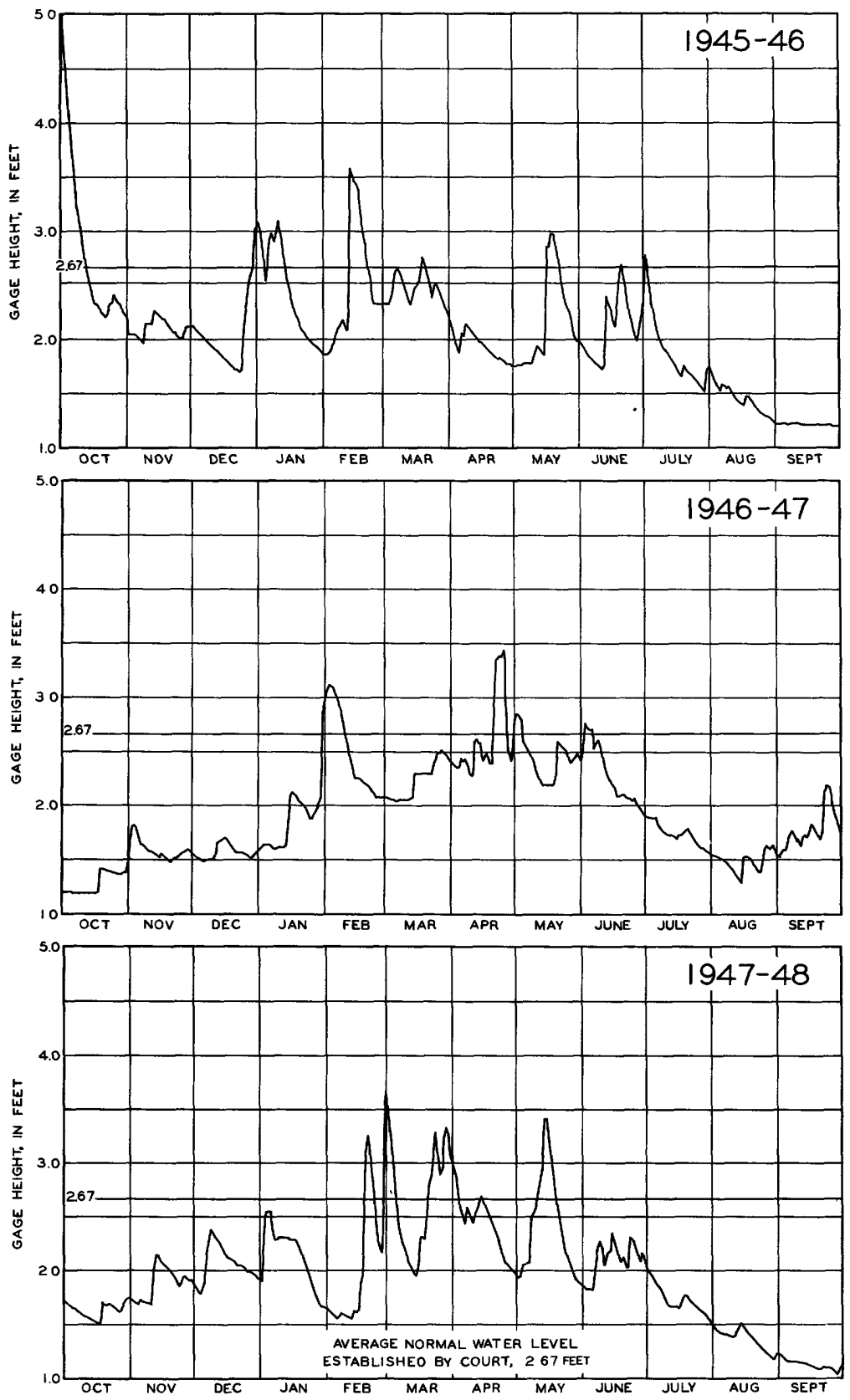

Figure 93. --Lake-level hydrographs for Muskelonge Lake near Warsaw, Ind., for water years 1946-48. 


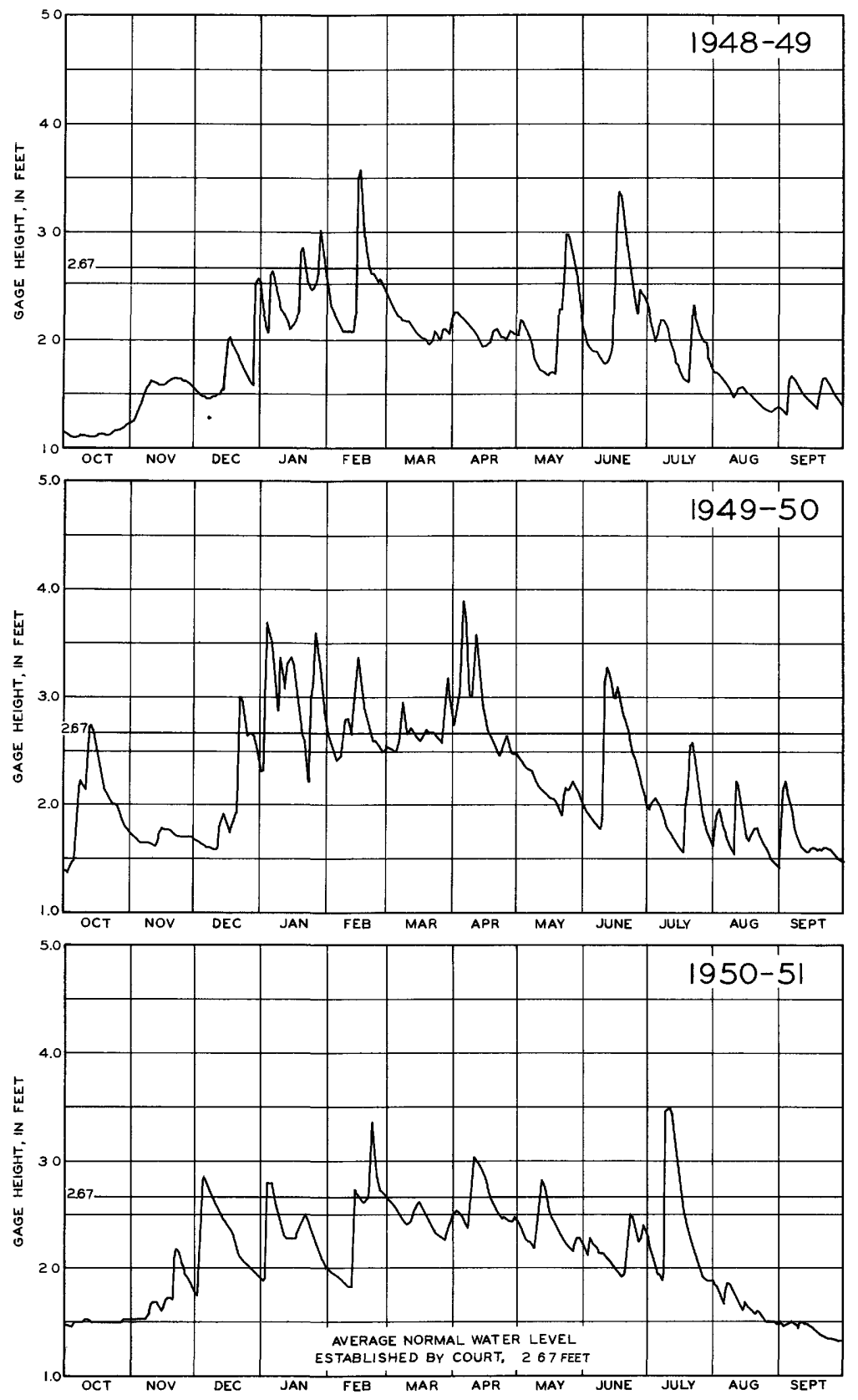

Figure 94. --Lake-level hydrographs for Muskelonge Lake near Warsaw, Ind., for water years 1949-51. 


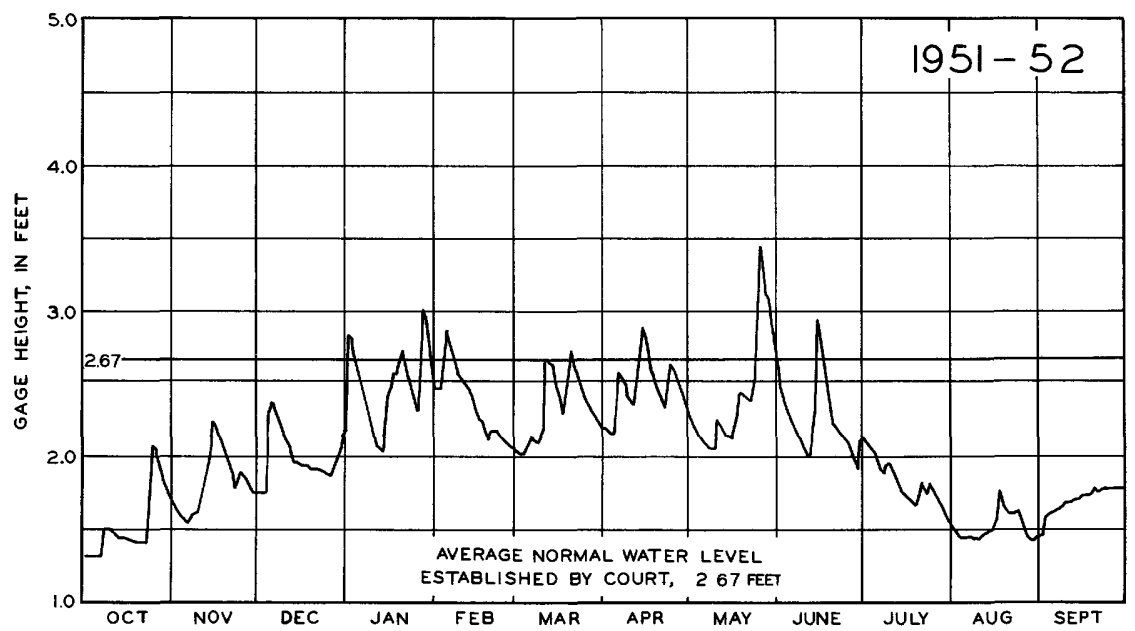

Figure 95. --Lake-level hydrographs for Muskelonge Lake near Warsaw, Ind, for water year 1952 .

Ridinger Lake near Pierceton, Ind.

Location. --Sec. 1, T. 32 N., R. 7 E., and sec. 36, T. 33 N., R. 7 E. , Kosciusko County, 4.6 miles northwest of Pierceton.

Surface area. --115 acres.

Drainage area. $--32.5 \mathrm{sq} \mathrm{mi}$.

Records available. --September 1943 to September 1953.

Gage. - Staff gage on north shore of east lobe of lake, at Riley resort. Gage read to hundredths once daily. Datum of gage is $840.00 \mathrm{ft}$ above mean sea level, datum of 1929 .

Average lake level. - -10 years; gage height, $3.05 \mathrm{ft}$; elevation, $843.09 \mathrm{ft}$.

Established legal-1evel. --Established June 17, 1949, at gage height $3.12 \mathrm{ft}$; elevation, $843.12 \mathrm{ft}$ above mean sea level.

Lake-level control. --Lake level controlled by concrete dam with Columbus type horizontal spillway, $10.0 \mathrm{ft}$ long, and sluiceway, $6.0 \mathrm{ft}$ wide. Crest of horizontal spillway at gage height $3.01 \mathrm{ft}$; base of sluiceway at gage height $0.10 \mathrm{ft}$. 
Extreme levels for Ridinger Lake, 1944-53

\begin{tabular}{|c|c|c|c|c|c|}
\hline \multicolumn{4}{|c|}{ Maximum } & \multicolumn{2}{|l|}{ Minimum } \\
\hline $\begin{array}{l}\text { Water } \\
\text { year }\end{array}$ & & Date & $\begin{array}{l}\text { Gage } \\
\text { height } \\
\text { (feet) }\end{array}$ & Date & $\begin{array}{l}\text { Gage } \\
\text { height } \\
\text { (feet) }\end{array}$ \\
\hline 1944 & Apr. 1 & $3,1944 \ldots$ & 8.06 & Jan. 17-19, 1944_-.-- & 1.35 \\
\hline 1945 & May 1 & $18,1945 \ldots$ & 7. 49 & Oct.23,31, Nov. 1,1944 & 1.45 \\
\hline 1946 & Oct. & $3,1945 \ldots$ & 6.03 & Sept. 6,7, 1946 & 1.44 \\
\hline 1947 & Apr. 2 & $23,1947 \ldots$ & 6.21 & Oct. $3-9,15-17,1946 \ldots$ & 1.56 \\
\hline 1948 & Feb. 2 & $29,1948 \ldots$ & 4.93 & Oct. $5,1947 \ldots$ & $* 2.00$ \\
\hline 1949 & Feb. 1 & $16,1949 \ldots$ & $* 5.55$ & Mar. $14-18,1949 \ldots$ & 2.80 \\
\hline 1950 & Apr. & $5,1950-\ldots$ & 7.44 & May 1,2 , July $15,1950-$ & 2.90 \\
\hline 1951 & Feb. 2 & $22,1951 \ldots$ & 6.28 & $\begin{array}{l}\text { Apr. } 29,30 \\
\quad \text { May } 1-8,1951 \ldots\end{array}$ & 2.80 \\
\hline 1952 & Jan. & $2,1952 \ldots$ & 5.45 & Feb. 21 to $\operatorname{Mar} .9,1952$ & 2.80 \\
\hline 1953 & Mar. & $5,1953 \ldots$ & 3.86 & Mar. 29 to Apr. 1, 1953- & 2.80 \\
\hline
\end{tabular}

* Estimated.

Maximum recorded thickness and periods of ice cover for Ridinger Lake, 1944-53

\begin{tabular}{|c|c|c|c|}
\hline $\begin{array}{l}\text { Water } \\
\text { year }\end{array}$ & $\begin{array}{l}\text { Maximurn } \\
\text { thickness } \\
\text { (inches) }\end{array}$ & $\begin{array}{c}\text { Total } \\
\text { days of } \\
\text { cover }\end{array}$ & Period \\
\hline 1944 & 2 & $--\cdots--$ & Dec. 19,1943 , to-? \\
\hline 1945 & ----- & $\cdots-\cdots$ & \\
\hline 1946 & 6 & 76 & Dec. 22, 1945, to Mar. 7, 1946. \\
\hline 1947 & 4 & 99 & $\begin{array}{l}\text { Dec. } 19-28,1946 \text {, and Jan. } 3 \text { to } \\
\text { Apr. 1, } 1947 \text {. }\end{array}$ \\
\hline 1948 & $15 \frac{1}{2}$ & 93 & Dec. 17,1947 , to Mar. 19, 1948. \\
\hline 1949 & 2 & 29 & Dec. 18,1948 , to Jan. 15,1949 . \\
\hline 1950 & ----- & $-\cdots--$ & Dec. 15,1949 , to $-?$ \\
\hline 1951 & 4 & 72 & Dec. 17,1950 , to Feb. $26,1951$. \\
\hline 1952 & 8 & $-\cdots---$ & \\
\hline 1953 & 4 & ----- & $\begin{array}{l}\text { Dec. } 18,1952 \text {, to Jan. 17, } 1953 \text {, } \\
\text { Feb. 21, to-? }\end{array}$ \\
\hline
\end{tabular}


Discharge measurements at outlet of Ridinger Lake

\begin{tabular}{|c|c|c|c|c|c|}
\hline Date & $\begin{array}{c}\text { Gage } \\
\text { height } \\
\text { (feet) }\end{array}$ & $\begin{array}{c}\text { Discharge } \\
\text { (cfs) }\end{array}$ & Date & $\begin{array}{l}\text { Gage } \\
\text { height } \\
\text { (feet) }\end{array}$ & $\begin{array}{c}\text { Discharge } \\
\text { (cfs) }\end{array}$ \\
\hline Aug. 4,1943 & 3.13 & 20.4 & Aug. 28, 1947- & 2. 48 & $* 4.50$ \\
\hline Oct. $\quad 6 \ldots-\cdots$ & 2.01 & 5.53 & Sept. $4 \ldots$ & 2.58 & 6.90 \\
\hline Jan. 7,1944 & 2.00 & 3.84 & Sept. $18 \cdots$ & 2.72 & 16.5 \\
\hline May $15 \ldots$ & 4. 31 & 68.8 & Oct. $25 \cdots-\cdots$ & 3.08 & .62 \\
\hline Mar. 2, 1945 & 3.45 & 39.5 & Oct. $30 \ldots$ & 3. 38 & 7.51 \\
\hline June $14 \ldots$ & 2.70 & 14.6 & Oct. $30 \cdots$ & 3.38 & 45.1 \\
\hline Sept. 7 & 2.60 & 13.6 & Jan. $\quad 4,1948$ & 3.87 & 65.9 \\
\hline Nov. $27 \ldots$ & 2.33 & 11.5 & Feb. 18-... & 4.52 & 127 \\
\hline Apr. 18,1946 & 2.39 & 12.7 & Feb. $18 \ldots$ & 4.59 & 143 \\
\hline May 22--- & 3.01 & 22.8 & Feb. $19 \ldots$ & 4.91 & 100 \\
\hline June 19 & 4. 24 & 50.7 & May $10 \ldots \ldots$ & 3.38 & 65.3 \\
\hline June $21 \ldots$ & 4.64 & 63.1 & May 13.... & 4.46 & 138 \\
\hline June $24 \ldots$ & 4.09 & 44.3 & May $14 \ldots \ldots$ & 4.50 & 139 \\
\hline June $26 \ldots$ & 3.60 & 32.0 & Aug. $19 \ldots$ & 3.18 & 1.95 \\
\hline June $28 \ldots$ & 3.25 & 24.0 & Dec. $23 \ldots-\ldots$ & 3.22 & 16.4 \\
\hline July $\quad 1 \ldots$ & 2.95 & 16.0 & Jan. $\quad 4,1949$ & 3.18 & 36.6 \\
\hline July & 2.61 & 8.43 & Mar. 2 & 2.96 & 51.4 \\
\hline July $\quad 6 \ldots$ & 2.60 & 8.71 & Mar. 22-..-- & 3.01 & 16.4 \\
\hline July $11 \ldots$ & 2.38 & 6.89 & May $\quad 4 \ldots \ldots$ & 3.12 & 18.3 \\
\hline July $26 \ldots$ & 2.08 & 2.95 & May $11 \ldots \ldots$ & 3.06 & 1.28 \\
\hline Aug. $22--$ & 1.72 & .94 & June $28 \ldots$ & 3.54 & 57.5 \\
\hline Oct. $11-$ & 1.60 & $* 1.25$ & Aug. $31 \ldots$ & 3.16 & 3.13 \\
\hline Dec. $4 \ldots$ & 1.83 & 3.83 & Nov, $29 \ldots$ & 3.04 & 7.59 \\
\hline Jan. 17,1947 & 2.95 & 24.3 & Jan. 14,1950 & 5.59 & 222 \\
\hline Feb, $19 \ldots$ & 2.64 & 18.2 & Nov. $22 \ldots$ & 3.64 & 38.6 \\
\hline Apr. $\quad 2--$ & 4. 15 & 53.2 & May $10,1951-$ & 3.00 & 12.3 \\
\hline Apr. $21 \ldots$ & 5.59 & 115 & Aug. 18, 1952- & 3.20 & 7.04 \\
\hline Apr. $27 \ldots$ & 6.17 & 135 & & & \\
\hline
\end{tabular}

*Estimated. 
Average lake level for Ridinger Lake for water years 1943-53

\begin{tabular}{c|c|c|c|c|c|c|c}
\hline Year & Oct. & Nov. & Dec. & Jan. & Feb. & Mar. & Apr. \\
\hline 1943 & -- & -- & -- & -- & -- & -- & - \\
1944 & 1.96 & 2.41 & 1.93 & 1.58 & 2.23 & 4.00 & 5.29 \\
1945 & 1.52 & 1.61 & 1.63 & 1.59 & 2.47 & 3.41 & 3.77 \\
1946 & 3.64 & 2.35 & 2.24 & 3.66 & 3.70 & 3.83 & 2.51 \\
1947 & 1.67 & 1.94 & 1.96 & 2.57 & 3.36 & 3.16 & 4.62 \\
1948 & 2.63 & 3.24 & 3.23 & 3.41 & 3.52 & 3.26 & 3.04 \\
1949 & 3.14 & 3.25 & 3.31 & 3.75 & 3.80 & 2.96 & 3.01 \\
1950 & 3.21 & 3.02 & 3.37 & 4.92 & 3.89 & 4.06 & 4.42 \\
1951 & 3.13 & 3.28 & 3.46 & 3.59 & 4.08 & 3.58 & 3.62 \\
1952 & 3.22 & 3.43 & 3.40 & 4.21 & 3.34 & 3.45 & 3.33 \\
1953 & 3.12 & 3.13 & 3.24 & 3.32 & 3.27 & 3.32 & 3.19 \\
\hline Average & 2.72 & 2.77 & 2.78 & 3.26 & 3.37 & 3.50 & 3.68 \\
\hline \hline & & & & & & & \\
Year & May & June & July & Aug. & Sept. & Annual & \\
\hline 1943 & -- & -- & -- & -- & 2.55 & $* 2.57$ & \\
1944 & 4.97 & 2.69 & 1.69 & 1.52 & 1.50 & 2.64 & \\
1945 & 4.04 & 3.28 & 2.51 & 2.84 & 2.57 & 2.60 & \\
1946 & 2.40 & 3.02 & 2.32 & 1.76 & 1.58 & 2.75 & \\
1947 & 4.03 & 3.59 & 2.28 & 2.70 & 2.75 & 2.88 & \\
1948 & 3.37 & 3.18 & 3.18 & 3.16 & 3.07 & 3.19 & \\
1949 & 3.40 & 3.61 & 3.18 & 3.15 & 3.15 & 3.31 & \\
1950 & 3.13 & 3.30 & 3.22 & 3.27 & 3.36 & 3.60 & \\
1951 & 3.15 & 3.08 & 3.70 & 3.26 & 3.12 & 3.42 & \\
1952 & 3.52 & 3.21 & 3.31 & 3.21 & 3.15 & 3.40 & \\
1953 & 3.25 & 3.07 & 3.08 & 3.19 & 3.10 & 3.19 & \\
\hline Average & 3.53 & 3.20 & 2.85 & 2.81 & 2.72 & 3.09 & \\
\hline & & & & & & & \\
\end{tabular}

* Partial month or partial year. 

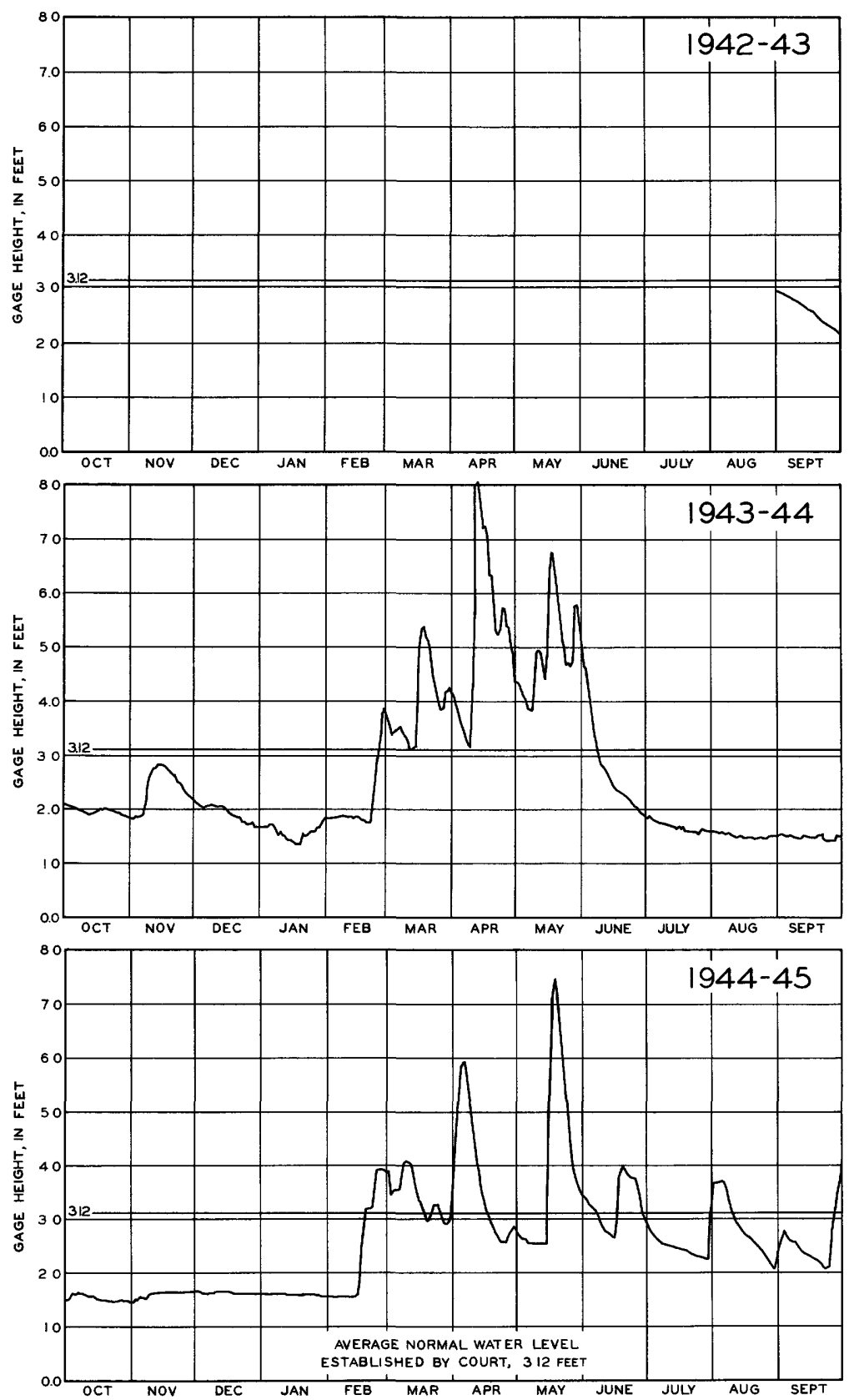

Figure 96. --Lake-level hydrographs for Ridinger Lake near Pierceton, Ind, , for water years 1943-45. 

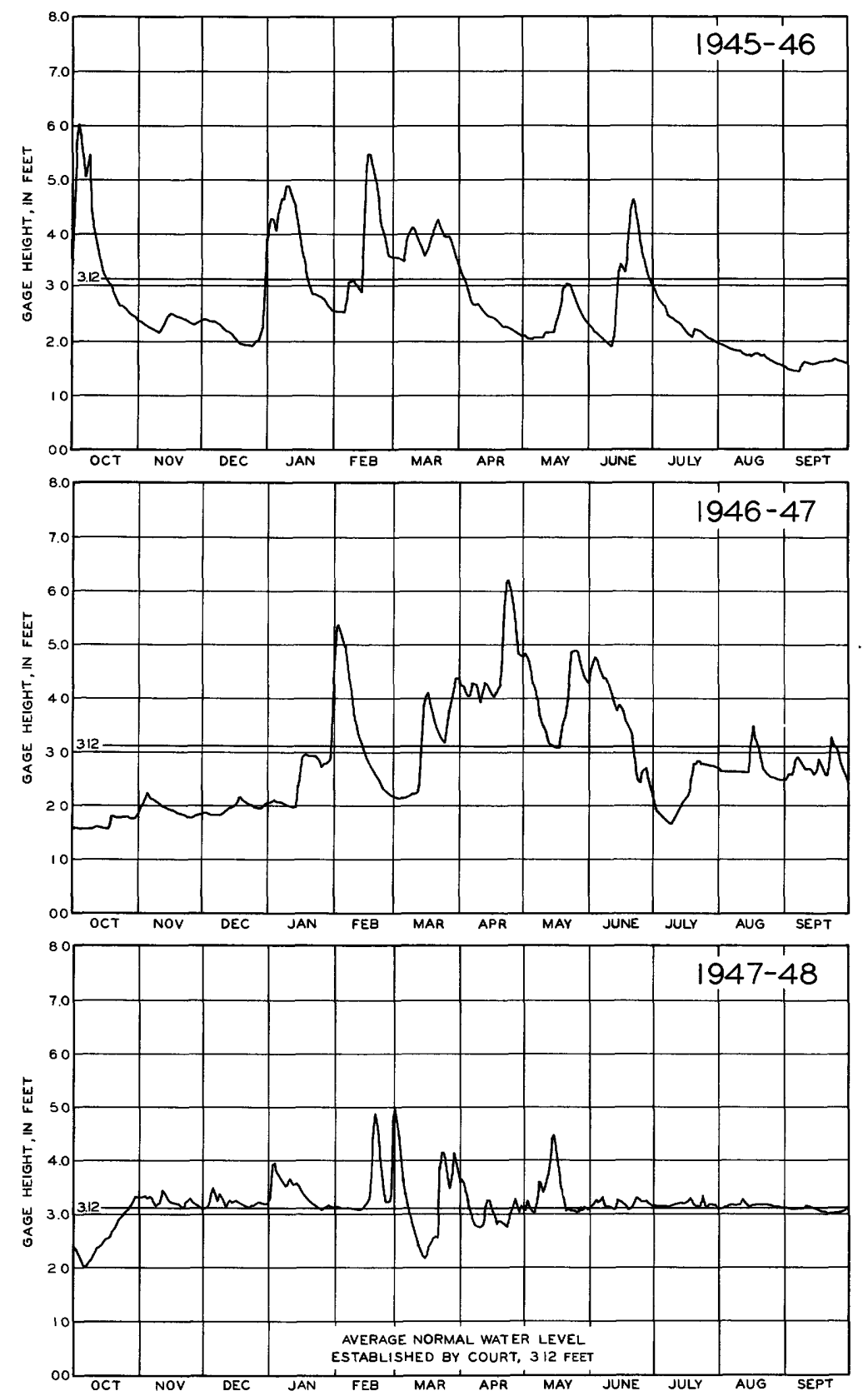

Figure 97. --Lake-level hydrographs for Ridinger Lake near Pierceton, Ind., for water years 1946-48. 

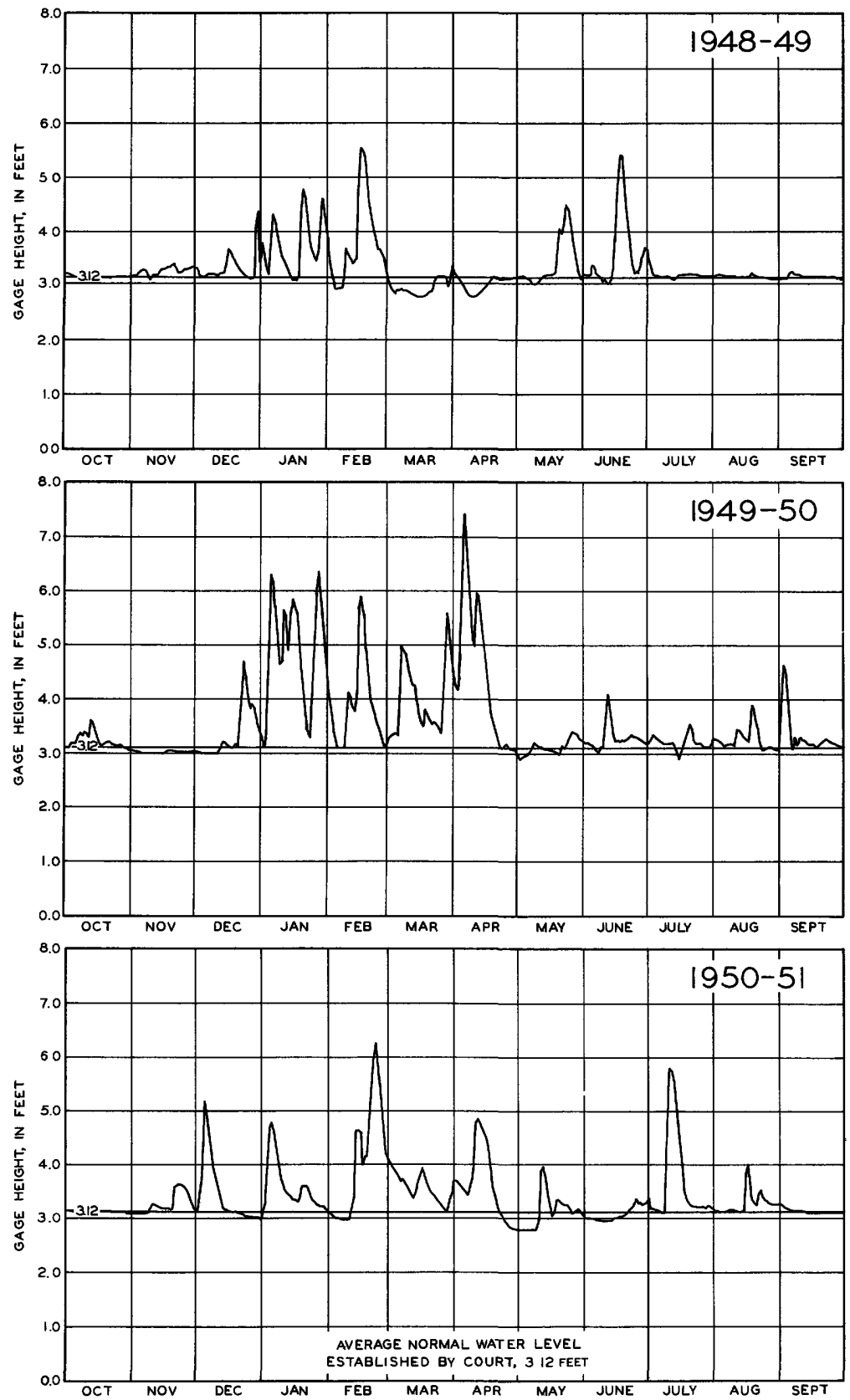

Figure 98. --Lake-level hydrographs for Ridinger Lake near Pierceton, Ind., for water years 1949-51. 

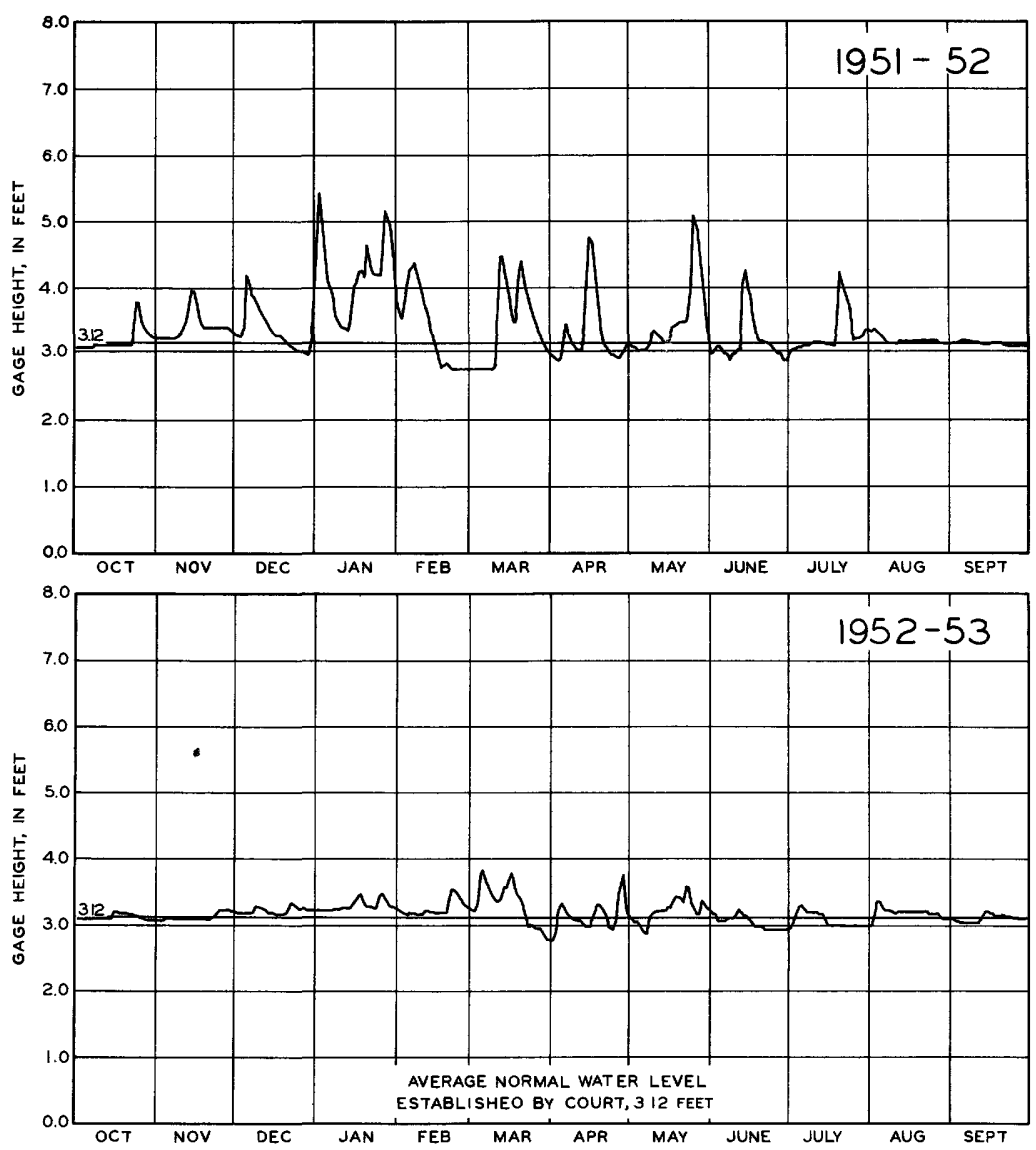

Figure 99. --Lake-level hydrographs for Ridinger Lake near Pierceton, Ind., for water years 1952-53. 
K ound Lake and Cedar Lake at Tri-Lakes, Ind.

Location.--Sec. 12, T. 32 N., R. 9 E., and sec. 7, T. 32 N., R. 10 E., Whitley County. Cedar Lake lies in sec. 2, 11, and 12, T. 32 N., R. 9 E., Whitley County.

Surface area. --Round Lake, 125 acres; Cedar Lake, 131 acres.

Drainage area. --Round Lake, $3.57 \mathrm{sq} \mathrm{mi}$; Cedar Lake, $1.62 \mathrm{sq} \mathrm{mi}$.

Records Available. - Cedar Lake, October 1942 to September 1947. Round Lake, January 1946 to September 1947. Round and Cedar Lake published as one record October 1947 to September 1952.

Gage. - Cedar Lake. Staff gage at east corner of north abutment of bridge over channel between Round and Cedar Lakes. Datum of gage is $900.00 \mathrm{ft}$ above mean sea level, datum of 1929 . Prior to July 11, 1946, staff gage on south shore, $900 \mathrm{ft}$ east of last used site.

Round Lake, staff gage bolted to east side of north dam abutment on outlet, 1,500 ft downstream from Round Lake. Datum of gage is $900.00 \mathrm{ft}$ above mean sea level, datum of 1929 .

Gages read to hundredths once daily.

Average level. - Cedar Lake (1942-47), gage height, $1.43 \mathrm{ft}$; elevation, $901.43 \mathrm{ft}$. Round Lake (1946-47), gage height, $1.25 \mathrm{ft}$; elevation, $901.25 \mathrm{ft}$. Round and Cedar Lakes (1947-52), gage height, $1.78 \mathrm{ft}$; elevation, $901.78 \mathrm{ft}$.

Established legal level. --Established June 29, 1948, at gage height, $1.90 \mathrm{ft}$; elevation, $901.90 \mathrm{ft}$ above mean sea level.

Lake-level control. - - Lake levels maintained by concrete dam at outlet of Round Lake. Dam constructed in August 1947, has a crest width of $25 \mathrm{ft}$ at gage height of $1.84 \mathrm{ft}$. 
Extreme levels for water years 1943-52

\begin{tabular}{c|c|c|c|c}
\hline \multicolumn{2}{c|}{ Maximum } & \multicolumn{2}{c}{ Minimum } \\
\hline $\begin{array}{c}\text { Water } \\
\text { year }\end{array}$ & Date & $\begin{array}{c}\text { Gage } \\
\text { height } \\
\text { (feet) }\end{array}$ & Date & $\begin{array}{c}\text { Gage } \\
\text { height } \\
\text { (feet) }\end{array}$ \\
\hline
\end{tabular}

Cedar Lake

\begin{tabular}{|c|c|c|c|c|}
\hline 1943 & May 17-21,25,26, 1943 & 2.42 & July $1-6,1943 \ldots$ & 1. 42 \\
\hline 1944 & Mar. 15-20, 1944-- & 2. 40 & Sept. 26-28, $1944 \ldots$ & .51 \\
\hline 1945 & May $17,1945 \ldots$ & 2.82 & Nov. $1-3,12,13,1944$ & .36 \\
\hline 1946 & Oct. $2,3,1945 \ldots$ & 1.99 & Sept. $30,1946 \ldots$ & .62 \\
\hline 1947 & Apr. $21,1947 \ldots$ & 2. 00 & Oct. $16,1946 \ldots$ & .42 \\
\hline
\end{tabular}

Round Lake

\begin{tabular}{|c|c|c|c|c|}
\hline $\begin{array}{l}1946 \\
1947\end{array}$ & $\begin{array}{l}\text { Feb. } 15-17,1946- \\
\text { Apr. } 21,1947 \ldots\end{array}$ & $\begin{array}{r}1.74 \\
* 1.92\end{array}$ & $\begin{array}{l}\text { June } 28,1946 \\
\text { Dec. } 4-9,1946\end{array}$ & $\begin{array}{l}a 1.41 \\
b \quad .67\end{array}$ \\
\hline
\end{tabular}

Round Lake and Cedar Lake

\begin{tabular}{|c|c|c|c|c|}
\hline 1948 & May $13,1948 \ldots$ & 2.20 & Oct. $15-17,1948$ & .68 \\
\hline 1949 & Feb, 15, $1949 \ldots$ & 2.52 & Oct. 27,1948 , to Nov. $1-$ & .96 \\
\hline 1950 & Apr. $4,1950 \cdots$ & 2.59 & Oct. $2,1949 \ldots$ & 1. 16 \\
\hline 1951 & Feb. 21, 1951 & 2. 38 & Nov. $6,7,1950$ & 1.68 \\
\hline 1952 & Jan. 27, 1952 $\cdots \cdots$ & 2.30 & Oct. $5,6,1951 \ldots$ & 1.59 \\
\hline
\end{tabular}

* Estimated.

$a$ Record for Jan. 23, 1946, to June 30, 1946.

$b$ Record for Dec. 1, 1946, to Sept. 30, 1947.

Maximum recorded thickness and periods of ice cover for the water years 1943-52

\begin{tabular}{c|c|c|c|}
\hline Water & $\begin{array}{c}\text { Maximum } \\
\text { year }\end{array}$ & $\begin{array}{c}\text { Total } \\
\text { dinchess } \\
\text { (inches of } \\
\text { cover }\end{array}$ & Period \\
\hline
\end{tabular}

\begin{tabular}{|c|c|c|c|}
\hline \multicolumn{4}{|c|}{ Cedar Lake } \\
\hline 1943 & $---1--1$ & $-\cdots--$ & Dec. 11,1942, to - ? \\
\hline 1944 & 5 & $-\cdots--$ & $\ldots-\cdots$ \\
\hline 1945 & $-\cdots-\cdots--\cdot$ & ---- & 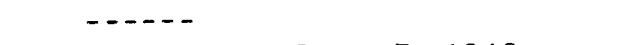 \\
\hline 1946 & 9 & 85 & Dec. 13,1945 , to Mar. 7,1946 \\
\hline 1947 & 12 & 69 & Dec. $19-23,1946 ; \operatorname{Jan} .3$ to Mar. 7,1947 \\
\hline \multicolumn{4}{|c|}{ Round Lake } \\
\hline 1946 & 12 & $-\cdots--$ & Unknown to Mar. 7,1946 \\
\hline 1947 & 13 & 95 & Dec. 30,1946 , to Apr. $3,1947$. \\
\hline \multicolumn{4}{|c|}{ Round Lake and Cedar Lake } \\
\hline 1948 & 16 & $-\cdots--$ & Dec. 17,1947, to $-?$ \\
\hline 1949 & 5 & 73 & $\begin{array}{l}\text { Dec. } 25,1948 \text {, to Jan. } 28,1949 \text {; Jan. } 31 \\
1949 \text {, to Mar. } 8,1949 .\end{array}$ \\
\hline 1950 & 3 & 44 & $\begin{array}{l}\text { Jan. } 8,9,17,19,25 ; \mathrm{Feb} .20 \text { to } \\
\text { Mar. 25, } 1950 .\end{array}$ \\
\hline 1951 & $-\ldots-n$ & ----- & Dec. 10,1950, to - ? \\
\hline
\end{tabular}


Discharge measurements at lake outlet'́ of Cedar Lake and Round Lake

\begin{tabular}{|c|c|c|c|c|c|}
\hline Date & $\begin{array}{c}\text { Gage } \\
\text { height } \\
\text { (feet) }\end{array}$ & $\begin{array}{c}\text { Discharge } \\
\text { (cfs) }\end{array}$ & Date & $\begin{array}{c}\text { Gage } \\
\text { height } \\
\text { (feet) }\end{array}$ & $\begin{array}{c}\text { Discharge } \\
\text { (cfs) }\end{array}$ \\
\hline \multicolumn{6}{|c|}{ Cedar Lake } \\
\hline June $14,1945-$ & 1.79 & 1.6 & May 22,1946 & 1.63 & 0 \\
\hline Sept. $6 \ldots-\ldots$ & 1. 40 & .02 & Aug. $21 \cdots--$ & 1.09 & 0 \\
\hline \multicolumn{6}{|c|}{ Round Lake } \\
\hline Jan. $17,1946-$ & 1.64 & 3.98 & July 24, 1946 & 1.36 & .38 \\
\hline Jan. $23 \ldots-\cdots$ & 1.60 & 3.50 & Sept. $9-\cdots$ & .67 & 0 \\
\hline Feb. $15 \ldots$ & 1.74 & 7.13 & Jan. 17,1947 & 1.12 & .14 \\
\hline Apr. $24 \ldots \ldots$ & 1.56 & 0 & Feb. 16- - - & 1.36 & 5.40 \\
\hline May $14 \ldots \ldots$ & 1.54 & .04 & Apr. 25--.- & 1.79 & 11.0 \\
\hline June $14 \ldots$ & 1.59 & 2.43 & July $29 \cdots--$ & 1.08 & 0 \\
\hline \multicolumn{6}{|c|}{ Round Lake and Cedar Lake } \\
\hline Mar. 4, 1948- & 2.06 & 8.21 & Apr. 24,1951 & 1.96 & 3.41 \\
\hline Mar. $16 \ldots$ & 2.00 & 4.85 & May 23-... & 1.93 & 1.30 \\
\hline May $26 \ldots$ & 1.87 & 0 & June $21 \ldots$ & 1.90 & 1.27 \\
\hline Nov. $4 \ldots$ & 1.05 & 0 & July 24----- & 2.00 & 6.05 \\
\hline Mar. 7, 1949- & 1.95 & 2.76 & Aug. 29-... & 1.93 & .81 \\
\hline Aug. $4 \ldots$ & 1.55 & 0 & Sept. 26---- & 1.70 & 0 \\
\hline Nov. $23 \ldots$ & 1.54 & 0 & Oct. 24---- & 1.92 & 1.43 \\
\hline Dec. $1 \ldots-\ldots$ & 1.54 & 0 & Nov. $28 \ldots-\cdots$ & 1.91 & 1.21 \\
\hline Mar. 23, 1950- & 2.10 & 10.9 & Dec. $20 \cdots--$ & 1.98 & 4. 30 \\
\hline Nov. $14-\cdots--$ & 1.75 & 0.0 & Feb. 7,1952 & 2.14 & 13.6 \\
\hline Jan. 23, $1951-$ & 2.00 & 4.96 & Mar.27-..- & 2.01 & 6.81 \\
\hline Feb. 20 & 2. 19 & 17.8 & July $17-\cdots$ & 1.76 & 0 \\
\hline Mar. $15 \ldots$ & 2.06 & 8.51 & & & \\
\hline
\end{tabular}

Average lake levels for water years 1943-52

\begin{tabular}{c|r|l|l|l|l|l|l}
\hline Year & Oct. & Nov. & Dec. & Jan. & Feb. & Mar. & Apr. \\
\hline \multicolumn{7}{c}{ Cedar Lake at Tri-Lakes, Ind. } \\
\hline 1943 & $* 1.64$ & 1.78 & 1.64 & 1.71 & 1.68 & 1.75 & 1.64 \\
1944 & 1.72 & 1.71 & 1.70 & 1.55 & 1.73 & 2.16 & 2.11 \\
1945 & 0.49 & 0.42 & 0.47 & 0.45 & 0.45 & 1.21 & 2.06 \\
1946 & 1.75 & 1.50 & 1.45 & 1.65 & 1.67 & 1.74 & 1.53 \\
1947 & 0.54 & 0.75 & 0.85 & 1.18 & 1.44 & 1.49 & 1.81 \\
\hline Average & 1.23 & 1.23 & 1.22 & 1.31 & 1.39 & 1.67 & 1.83 \\
\hline \hline
\end{tabular}


Average lake levels for water years 1943-52--Continued

\begin{tabular}{c|c|c|c|c|c|c|c}
\hline Year & May & June & July & Aug. & Sept. & Annual & \\
\hline \multicolumn{7}{c}{ Cedar Lake at Tri-Lakes, Ind. - Con. } \\
\hline 1943 & 2.12 & 1.77 & 1.69 & 1.73 & 1.81 & $* 1.76$ & \\
1944 & 2.08 & 1.70 & 1.17 & 0.80 & 0.57 & 1.58 & \\
1945 & 2.11 & 1.67 & 1.51 & 1.65 & 1.39 & 1.16 & \\
1946 & 1.55 & 1.61 & 1.46 & 1.08 & 0.77 & 1.48 & \\
1947 & 1.68 & 1.61 & 1.17 & 0.87 & 0.85 & 1.18 & \\
\hline Average & 1.91 & 1.67 & 1.40 & 1.23 & 1.08 & 1.43 & \\
\hline
\end{tabular}

Round Lake and Cedar Lake at Tri-Lakes, Ind.

\begin{tabular}{c|c|c|c|c|c|c|r}
\hline Year & Oct. & Nov. & Dec. & Jan. & Feb. & Mar. & Apr. \\
\hline 1946 & --- & --- & -- & $* 1.54$ & 1.61 & 1.62 & $a_{1.57}$ \\
1947 & $* 0.58$ & $* 0.76$ & 0.81 & 1.13 & 1.40 & 1.44 & $a_{1.77}$ \\
1948 & 0.74 & 0.78 & 0.93 & 1.34 & 1.65 & 2.06 & 1.97 \\
1949 & 1.02 & 1.22 & 1.40 & 2.02 & 2.08 & 1.95 & 1.92 \\
1950 & 1.55 & 1.56 & 1.73 & 2.18 & 2.10 & 2.11 & 2.14 \\
1951 & 1.80 & 1.83 & 1.99 & 2.02 & 2.06 & 2.00 & 2.05 \\
1952 & 1.72 & 1.91 & 1.97 & 2.11 & 2.03 & 2.03 & 2.01 \\
\hline Average & 1.24 & 1.34 & 1.47 & 1.76 & 1.85 & 1.89 & 1.92 \\
\hline \hline Year & May & June & July & Aug. & Sept. & Annual & \\
\hline 1946 & 1.55 & 1.47 & $* 1.42$ & $* 1.07$ & $* 0.81$ & $a_{*} 1.39$ & \\
1947 & 1.64 & 1.54 & 1.14 & 0.85 & 0.83 & \multicolumn{1}{|c|}{1.15} & \\
1948 & 1.97 & 1.85 & 1.71 & 1.53 & 1.23 & 1.48 & \\
1949 & 1.90 & 1.86 & 1.68 & 1.40 & 1.28 & 1.64 & \\
1950 & 1.91 & 1.95 & 1.90 & 1.82 & 1.93 & 1.91 & \\
1951 & 1.95 & 1.90 & 2.04 & 1.94 & 1.80 & 1.95 & \\
1952 & 1.96 & 1.94 & 1.90 & 1.83 & 1.77 & 1.93 & \\
\hline Average & 1.84 & 1.79 & 1.68 & 1.49 & 1.38 & 1.78 & \\
\hline
\end{tabular}

*Partial month or partial year.

${ }^{a}$ Data for Round Lake only. 

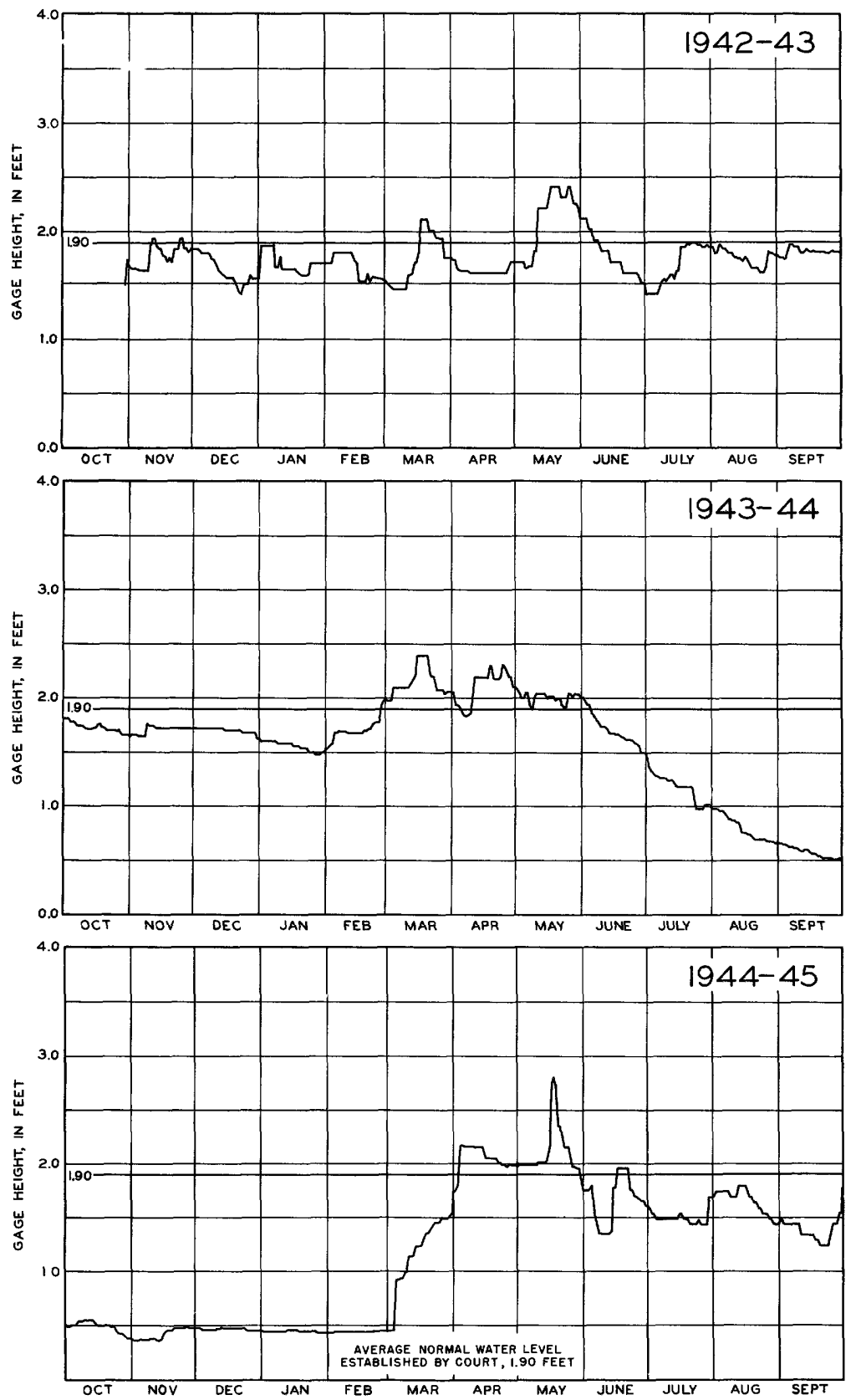

Figure 100. --Lake-level hydrographs for Cedar Lake at Tri-Lakes, Ind., for water years 1943-45. 

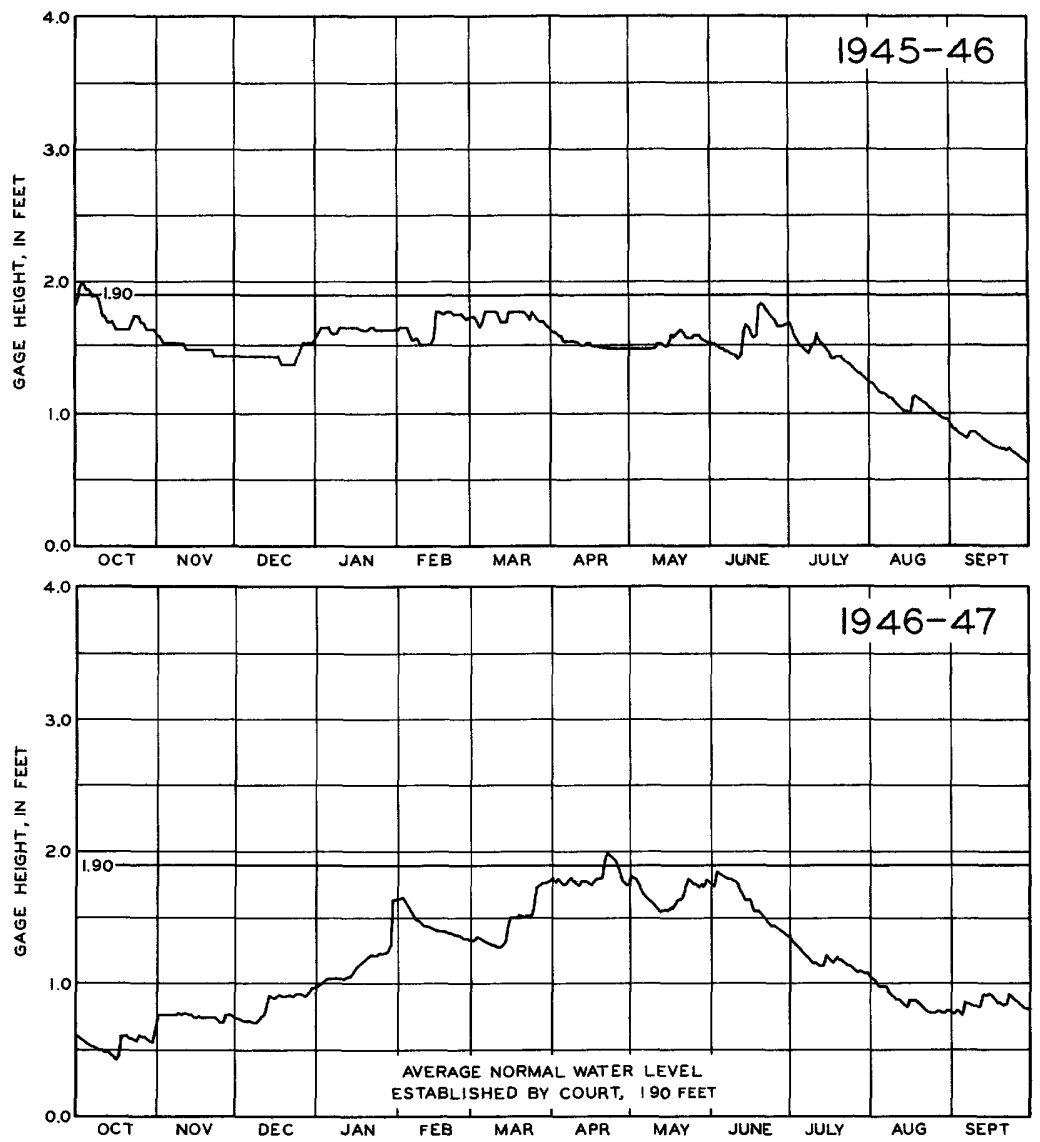

Figure 101. --Lake-level hydrographs for Cedar Lake at Tri-Lakes Ind. , for water years 1946-47. 

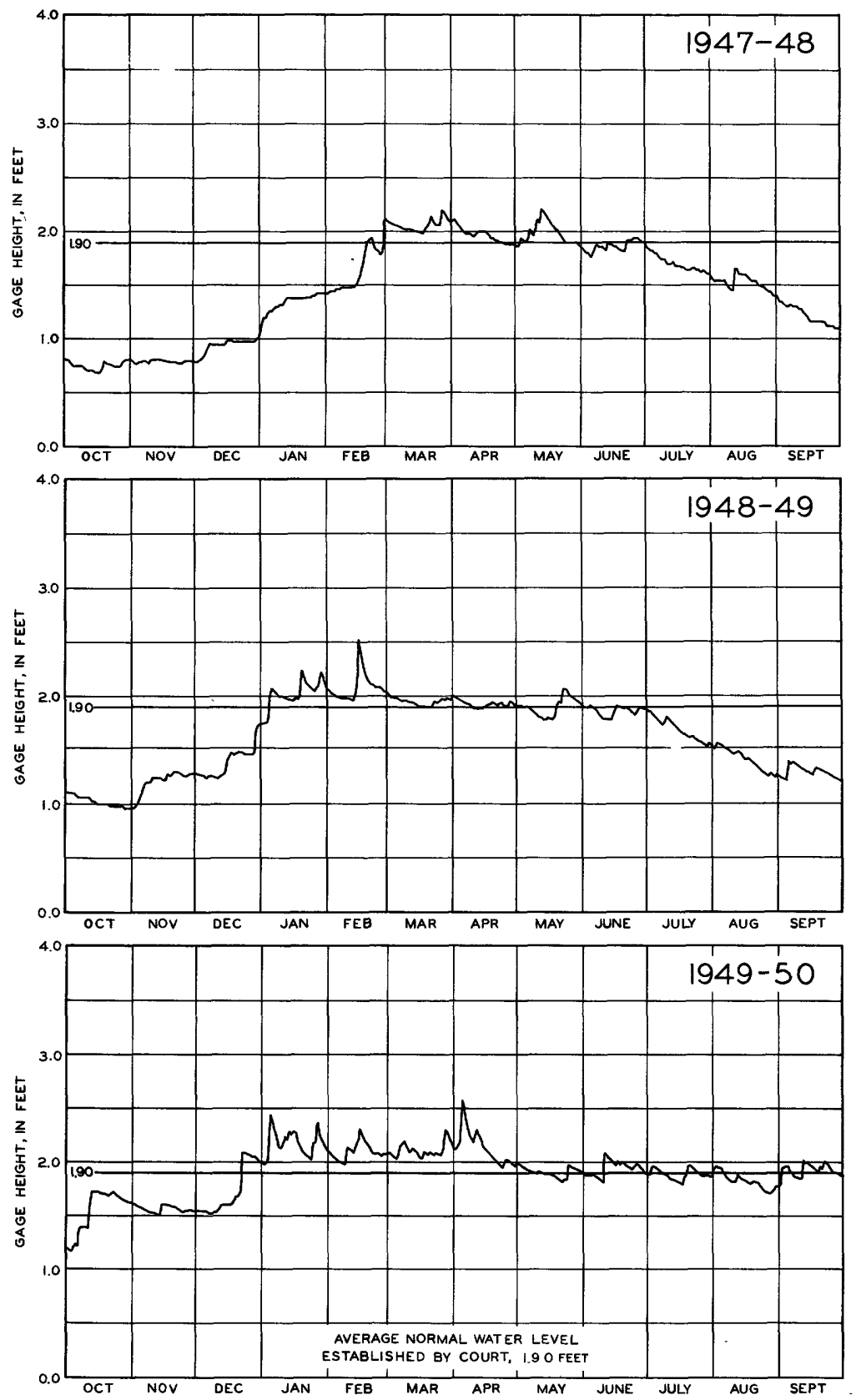

Figure 102. --Lake-level hydrographs for Round Lake and Cedar Lake at Tri-Lakes, Ind., for water years 1948-50. 

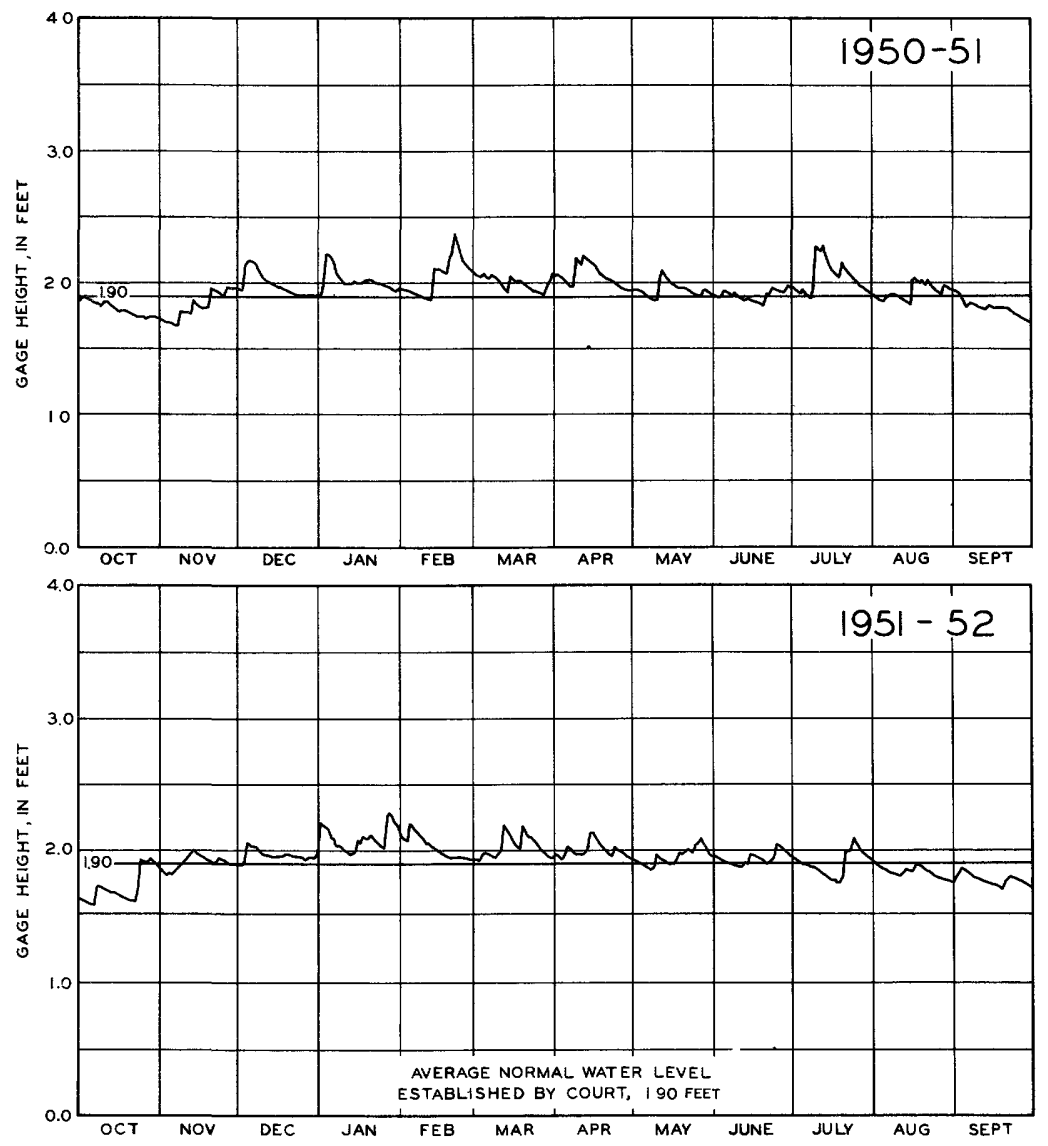

Figure 103. --Lake-level hydrographs for Round Lake and Cedar Lake at Tri-Lakes, Ind., for water years 1951-52. 
Round Lake and Clear Lake at Clear Lake, Ind.

Location. --Round Lake lies in sec. 17 and 18,T. 38 N., R. 15 E., and Clear Lake lies in secs. 17, 18, 19, 20, 29, and 30, T. 38 N., R. 15 E. , Steuben County.

Surface area. -Round Lake, 27.5 acres and Clear Lake, 765 acres from USGS topographic maps, surveyed 1938.

Drainage area. $--7.25 \mathrm{sq} \mathrm{mi}$. (determined from the same maps).

Records available.--August 1943 to September 1953.

Gage. - -Staff gage in outlet of Round Lake at extreme north end of Round Lake. Gage read to hundredths once daily. Datum of gage is $1,030.00 \mathrm{ft}$ above mean sea level, datum of 1929 .

Average lake level. --10 years; gage height, $7.11 \mathrm{ft}$; elevation, $1,037.11 \mathrm{ft}$.

Established legal level. --Established June 6, 1950, at gage height $7.38 \mathrm{ft}$; elevation, $1,037.38 \mathrm{ft}$ above mean sea level.

Lake-level control. - - Lake levels controlled by concrete dam with a movable 14 -inch high steel sluice gage on sill at gage height, $6.60 \mathrm{ft}$. Sill is 18 inches wide and $12.7 \mathrm{ft}$ long.

Extremes. - The maximum and minimum levels for the water years 1944-53 are contained in the following table:

Extreme levels for Round Lake and Clear Lake, 1944-53

\begin{tabular}{|c|c|c|c|c|}
\hline \multicolumn{3}{|c|}{ Maximum } & \multicolumn{2}{|c|}{ Minimum } \\
\hline $\begin{array}{l}\text { Water } \\
\text { year }\end{array}$ & Date & $\begin{array}{c}\text { Gage } \\
\text { height } \\
\text { (feet) }\end{array}$ & Date & $\begin{array}{c}\text { Gage } \\
\text { height } \\
\text { (feet) }\end{array}$ \\
\hline $1944^{a}$ & May 27, $1944 \ldots$ & 7.59 & Sept. 26, 1944 $\ldots$ & $* 6.36$ \\
\hline $1945^{a}$ & Apr. $4,1945 \ldots$ & 8.31 & Dec. $1,1944 \ldots$ & 6.33 \\
\hline 1946 & Mar. $10,1946 \ldots$ & 7.50 & Sept. $8,9,1946 \ldots$ & $* 6.63$ \\
\hline 1947 & Apr. $5,6,1947 \ldots$ & 7.94 & Oct. $13-16,1946 \ldots$ & 6.50 \\
\hline 1948 & May $11,1948 \ldots$ & 7.77 & Sept. $28,1948 \cdots$ & 6.79 \\
\hline 1949 & Jan. 20, 1949 & 7.65 & Sept. $30,1949 \ldots$ & 6.57 \\
\hline 1950 & Apr. $4,25,26,1950-$ & 7.66 & Oct. $1-3,5,6,1949--$ & 6.56 \\
\hline 1951 & July $9,1951 \ldots$ & 8.00 & Sept. $29,30,1951 \ldots$ & 6.96 \\
\hline 1952 & May $31,1952 \ldots--$ & 7.62 & Sept. $30,1952 \ldots$ & 6.84 \\
\hline 1953 & Apr. 17-23, $1953--$ & 7.39 & Sept. $30,1953 \ldots$ & 6.52 \\
\hline
\end{tabular}

* Estimated.

a Partial year record. 
Maximum recorded thickness and periods of ice cover for Round Lake and Clear Lake, 1946-53

\begin{tabular}{|c|c|c|c|}
\hline $\begin{array}{l}\text { Water } \\
\text { year }\end{array}$ & $\begin{array}{c}\text { Maximum } \\
\text { thickness } \\
\text { (inches) }\end{array}$ & $\begin{array}{l}\text { Total } \\
\text { days of } \\
\text { cover }\end{array}$ & Period \\
\hline 1945 & 6 & $---\cdots$ & Unknown to Feb. 20,1945 \\
\hline 1946 & 4 & 30 & Dec. 8,1945, to Jan. 6,1946 . \\
\hline 1947 & 15 & 113 & Dec. 14,1946 , to Apr. 5, 1947. \\
\hline 1948 & 21 & 116 & Nov. 30,1947 , to Mar. $24,1948$. \\
\hline 1949 & 9.5 & 93 & Dec.18, 1948, to Mar. 20,1949 \\
\hline 1950 & 8 & 118 & $\begin{array}{l}\text { Nov. } 26 \text { to Dec. } 12 \text {; Dec. } 14,1949 \text {, } \\
\text { to Jan. } 25,1950 ; \text { Jan. } 30 \text { to } \\
\text { Mar. } 28,1950 \text {. }\end{array}$ \\
\hline 1951 & 11 & 101 & Nov. 25, 1950, to Mar. 6, 1951. \\
\hline 1952 & 6 & ----- & Nov. 20,1951, to-? \\
\hline 1953 & 6 & 87 & $\begin{array}{l}\text { Nov. } 28 \text { to Dec. } 8 ; \text { Dec. } 15,1952 \text {, } \\
\text { to Feb. } 28,1953 \text {. }\end{array}$ \\
\hline
\end{tabular}

Discharge measurements at outlet of Round Lake and Clear Lake

\begin{tabular}{|c|c|c|c|c|c|}
\hline Date & $\begin{array}{c}\text { Gage } \\
\text { height } \\
\text { (feet) }\end{array}$ & $\begin{array}{c}\text { Discharge } \\
\text { (cfs) }\end{array}$ & Date & $\begin{array}{c}\text { Gage } \\
\text { height } \\
\text { (feet) }\end{array}$ & $\begin{array}{c}\text { Discharge } \\
\text { (cfs) }\end{array}$ \\
\hline Aug. 2, 1943 & 7.13 & 6.80 & Nov. 22, 1946 & 6.75 & 0 \\
\hline Oct. 4 & 6.93 & 0 & Dec. $18 \ldots$ & 6.81 & 0 \\
\hline Feb. 10,1944 & 6.85 & 2.25 & Feb. 6,1947 & 7.24 & 7.62 \\
\hline Apr. $25 \cdots$ & 7.30 & 18.9 & Feb. $19 \ldots$ & 7.18 & $* 1.77$ \\
\hline Dec. $\quad 1$ & 6.33 & 0 & Apr. $17 \ldots$ & 7.36 & 24.4 \\
\hline Apr. $\quad 8,1945$ & 7.38 & 28.1 & Apr. $23 \ldots$ & 7.56 & 35.0 \\
\hline $1----$ & 7.38 & 4.27 & Apr. $29 \ldots$ & 7.37 & 22.6 \\
\hline July $\quad 2 \ldots$ & 7.38 & 2.48 & Apr. 8, 1948 & 7.34 & 17.8 \\
\hline Sept. $4 \ldots$ & 7.06 & .16 & Jan. 16,1950 & 7.45 & 9.23 \\
\hline May 23, 1946 & 7.28 & $* .14$ & Mar. $9-\ldots$ & 7.20 & 16.4 \\
\hline July $23 \ldots$ & 7.06 & .05 & May 23, 1951 & 7.28 & 11.5 \\
\hline Sept. 26 & 6.67 & 0 & July 24, 1953 & 6.84 & .16 \\
\hline Oct. $24 \ldots \ldots$ & 6.54 & 0 & & & \\
\hline
\end{tabular}

* Leakage under gate. 
Average lake levels for Round Lake and Clear Lake for water years 1943-53

\begin{tabular}{c|r|r|r|r|r|r|r}
\hline Year & Oct. & Nov. & Dec. & Jan. & Feb. & Mar. & Apr. \\
\hline 1944 & 6.85 & 6.86 & 6.81 & 6.85 & 6.88 & 7.06 & 7.21 \\
1945 & $* 6.55$ & $* 6.65$ & $* 6.85$ & $* 7.00$ & $* 7.10$ & 7.28 & 7.53 \\
1946 & 7.13 & 7.19 & 7.24 & 7.33 & 7.31 & 7.34 & 7.29 \\
1947 & 6.55 & 6.75 & 6.80 & 6.93 & 7.20 & 7.33 & 7.55 \\
1948 & 7.02 & 6.90 & 6.92 & 6.93 & 6.97 & 7.32 & 7.35 \\
1949 & 6.71 & 6.88 & 6.84 & 7.10 & 7.27 & 7.11 & 7.11 \\
1950 & 6.69 & 6.63 & 6.76 & 7.20 & 7.26 & 7.24 & 7.49 \\
1951 & 7.18 & 7.24 & 7.30 & 7.24 & 7.27 & 7.32 & 7.30 \\
1952 & 7.09 & 7.31 & 7.26 & 7.27 & 7.21 & 7.16 & 7.24 \\
1953 & 6.74 & 6.70 & 6.76 & 6.73 & 6.75 & 7.17 & 7.26 \\
\hline Average & 6.85 & 6.91 & 6.95 & 7.06 & 7.12 & 7.23 & 7.33 \\
\hline \hline Year & May & June & July & Aug. & Sept. & Annual & \\
\hline 1943 & -- & -- & -- & $* 7.00$ & 6.92 & $* 6.96$ & \\
1944 & 7.30 & 7.16 & 6.79 & 6.55 & $* 6.35$ & 6.89 & \\
1945 & 7.55 & 7.53 & 7.30 & 7.14 & 7.01 & 7.12 & \\
1946 & 7.23 & 7.32 & 7.21 & 6.84 & 6.69 & 7.18 & \\
1947 & 7.44 & 7.36 & 7.04 & 6.79 & 7.09 & 7.07 & \\
1948 & 7.45 & 7.35 & 7.29 & 7.10 & 6.87 & 7.12 & \\
1949 & 7.26 & 7.24 & 7.06 & 6.81 & 6.64 & 7.00 & \\
1950 & 7.28 & 7.34 & 7.31 & 7.23 & 7.24 & 7.14 & \\
1951 & 7.38 & 7.34 & 7.53 & 7.31 & 7.06 & 7.29 & \\
1952 & 7.37 & 7.41 & 7.21 & 7.08 & 6.96 & 7.21 & \\
1953 & 7.23 & 7.19 & 7.13 & 6.98 & 6.65 & 6.94 & \\
\hline Average & 7.35 & 7.32 & 7.19 & 6.98 & 6.86 & 7.11 & \\
\hline
\end{tabular}

* Partial month or partial year. 

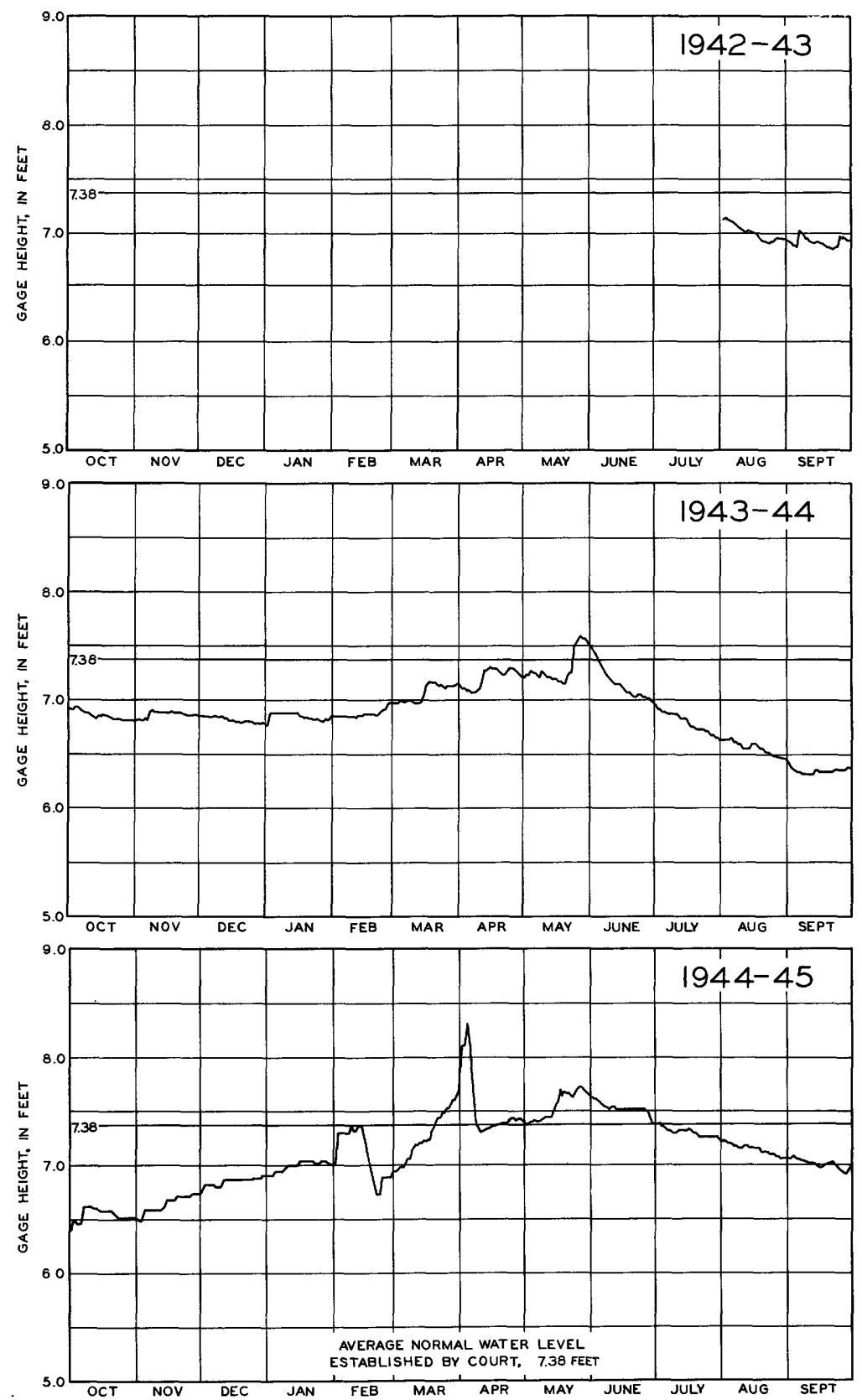

Figure 104. --Lake-level hydrographs for Round Lake and Clear Lake at Clear Lake, Ind., for water years 1943-45. 

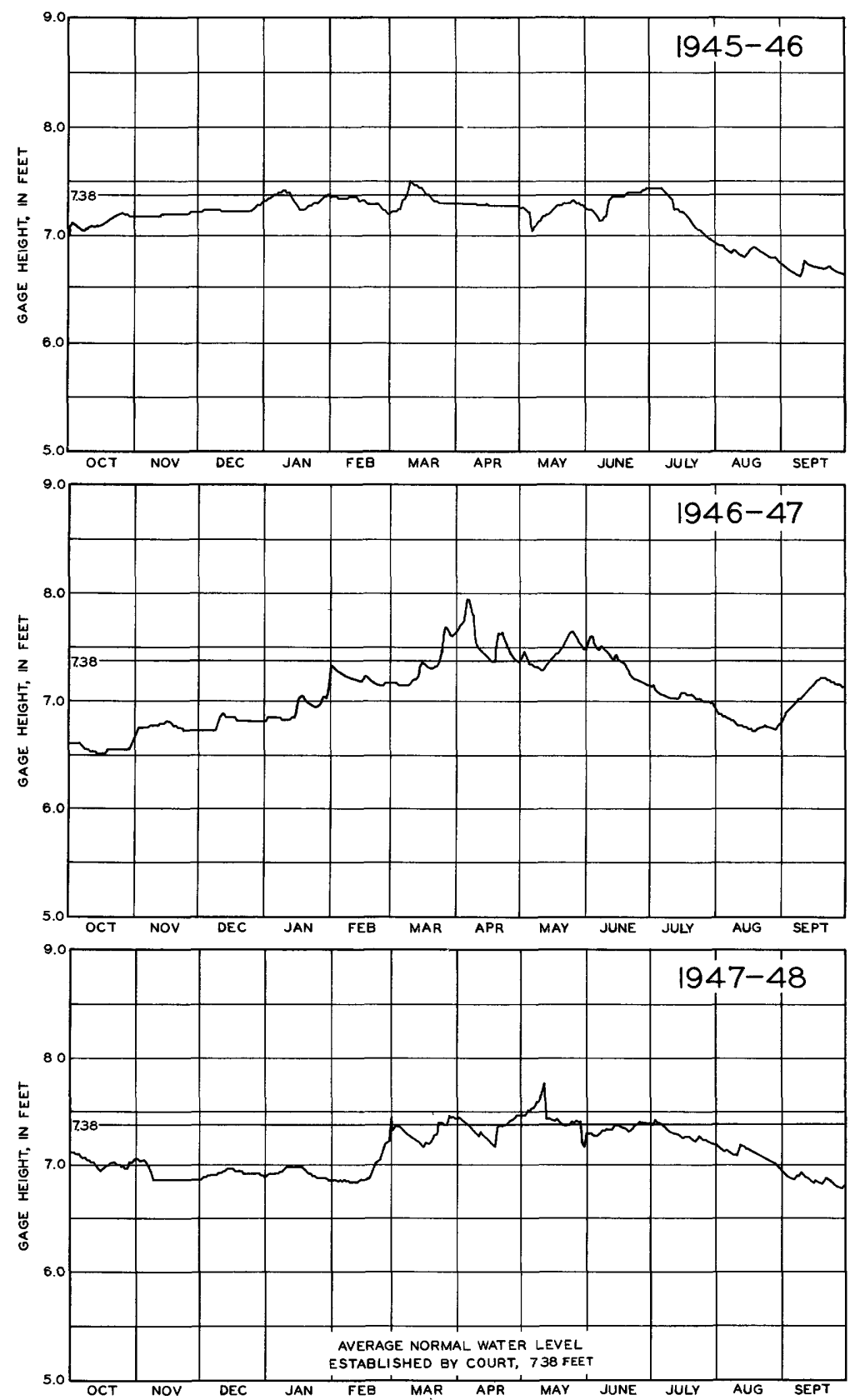

Figure 105. --Lake-level hydrographs for Round Lake and Clear Lake at Clear Lake, Ind., for water years 1946-48. 

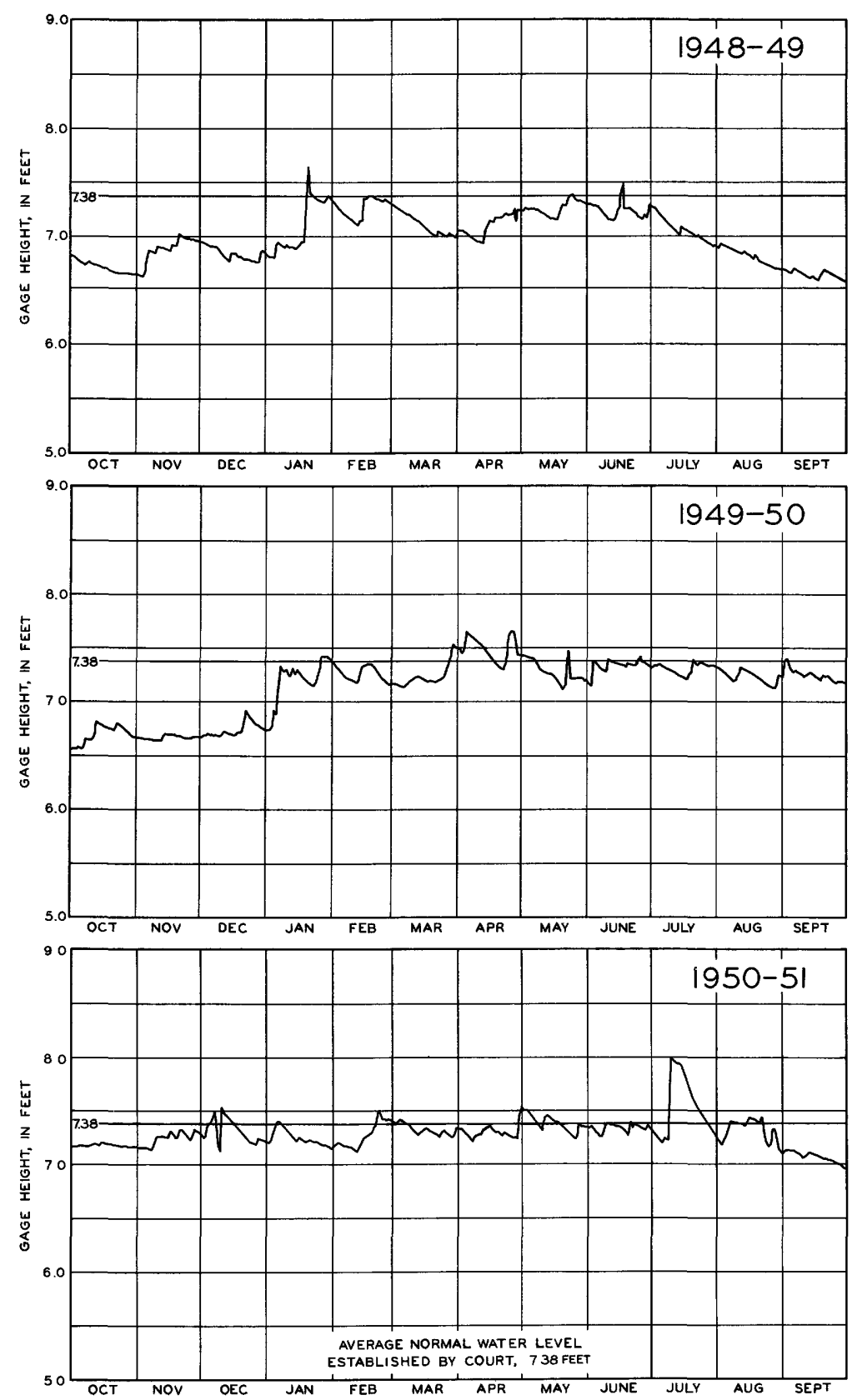

Figure 106. - -Lake-level hydrographs for Round Lake and Clear Lake at Clear Lake, Ind., for water years 1949-51. 


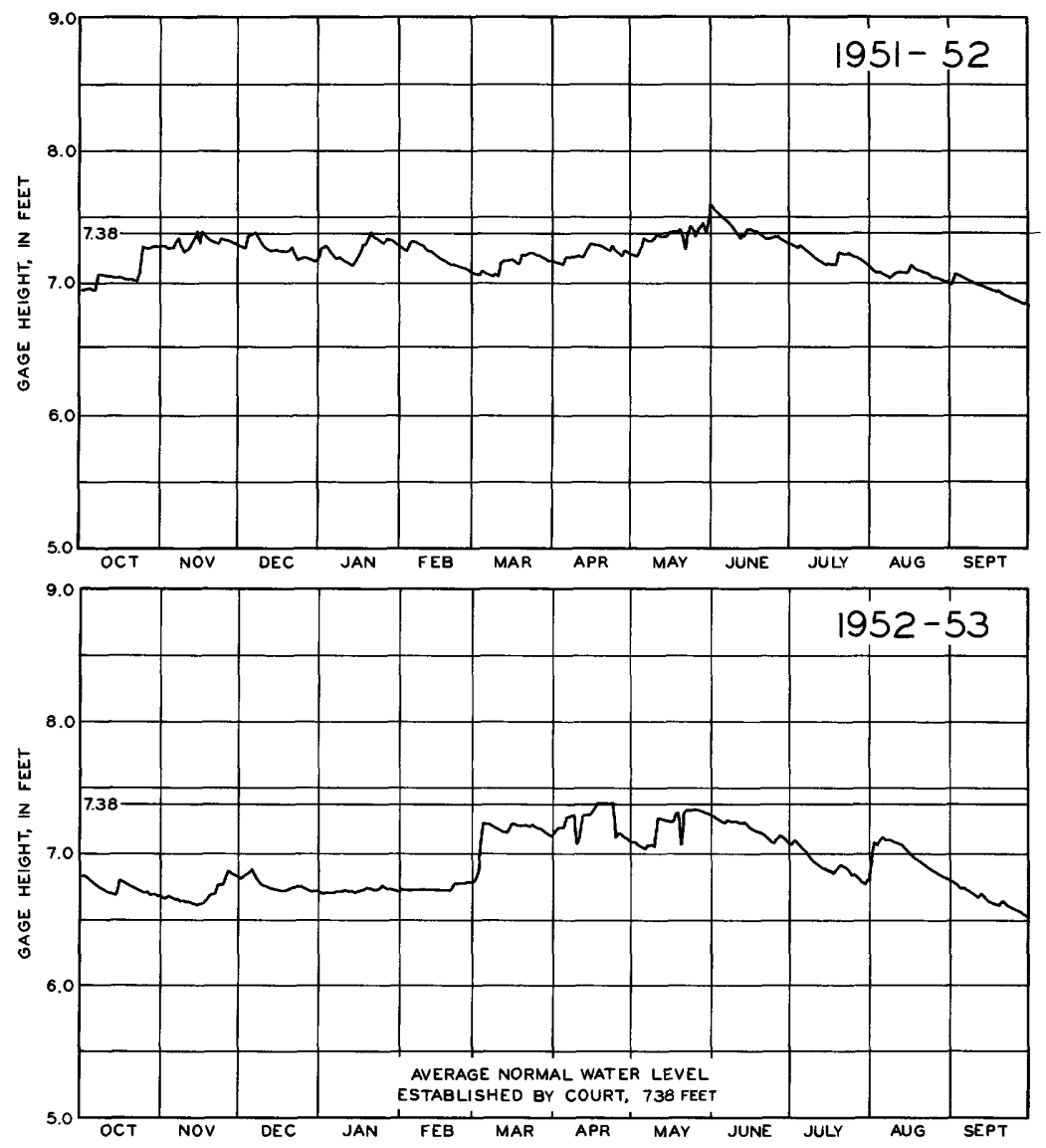

Figure 107. - -Lake-level hydrographs for Round Lake and Clear Lake at Clear Lake, Ind., for water years 1952-53. 


\section{Shriner Lake at Tri-Lakes, Ind.}

Location. --Secs. 2, 11, 12, T. 32 N., R. 9 E., Whitley County, 6.2 miles northeast of Columbia City.

Surface area. -111 acres.

Drainage area. $--1.12 \mathrm{sq} \mathrm{mi}$.

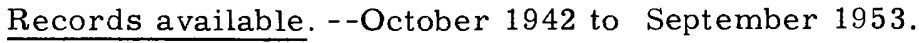

Gage. - Staff gage on extreme east shore, at outlet. Gage read to hundredths once daily. Datum of gage is $900.00 \mathrm{ft}$ above mean sea level, datum of 1929 .

Average lake level. --11 years; gage height, $6.72 \mathrm{ft}$; elevation, $906.72 \mathrm{ft}$.

Established legal level. --Established May 22, 1949, at gage height $7.04 \mathrm{ft}$; elevation, $907.04 \mathrm{ft}$ above mean sea level.

Lake-level control. --Lake level maintained by concrete dam in outlet channel, $300 \mathrm{ft}$ downstream from lake crest $4.8 \mathrm{ft}$ long at gage height $6.82 \mathrm{ft}$.

Extremes. - The maximum and minimum levels for water years $1943-53$ are contained in the following table:

Extreme levels of Shiner Lake, 1943-53

\begin{tabular}{|c|c|c|c|c|}
\hline \multicolumn{3}{|c|}{ Maximum } & \multicolumn{2}{|l|}{ Minimum } \\
\hline $\begin{array}{l}\text { Water } \\
\text { year }\end{array}$ & Date & $\begin{array}{l}\text { Gage } \\
\text { height } \\
\text { (feet) }\end{array}$ & Date & $\begin{array}{l}\text { Gage } \\
\text { height } \\
\text { (feet) }\end{array}$ \\
\hline 1943 & $\begin{array}{l}\text { Dec. } 28-30,1942, \\
\text { Mar. } 17-20, \\
\text { May } 17-20,25-26 \\
1943 .\end{array}$ & 7.54 & Sept. 28-30, 1943-.-- & 6.46 \\
\hline 1944 & Apr. $11-17,1944 \ldots$ & 7.56 & Sept. 28-30, 1944-.-- & 5.56 \\
\hline 1945 & May $17-19,1945 \ldots$ & 7. 44 & Dec. $9-11,23-30,1944-$ & 5.44 \\
\hline 1946 & Oct. $4,1945 \ldots$ & 7.18 & Sept. $30,1946 \ldots$ & 5.98 \\
\hline 1947 & Apr. $21,1947 \ldots$ & 7.40 & Oct. $15-17,1946 \ldots$ & 5.76 \\
\hline 1948 & Feb. 28, 1948 & 7.50 & Nov. $20,21,1947 \ldots$ & 6.06 \\
\hline 1949 & Feb. $17,18,1949 \ldots$ & 7.98 & Nov. $2,3,1948$ & 6.14 \\
\hline 1950 & Apr. $4,5,1950 \ldots$ & 8.16 & Oct. 2, $1949 \ldots$ & 6.12 \\
\hline 1951 & Feb. $21,22,1951 \ldots$ & 7.54 & Nov. $6,1950 \ldots$ & 6.24 \\
\hline 1952 & Jan. $27,1952 \ldots$ & 7.54 & Sept. 28-30, 1952-..- & 6.30 \\
\hline 1953 & May 23-25, 1953 & 7.40 & Nov. $12-18,1952 \ldots$ & 5.96 \\
\hline
\end{tabular}


Maximum recorded thickness and periods of ice cover for Shriner Lake, 1943-53

\begin{tabular}{|c|c|c|c|}
\hline $\begin{array}{l}\text { Water } \\
\text { year }\end{array}$ & $\begin{array}{c}\text { Maximum } \\
\text { thickness } \\
\text { (inches) }\end{array}$ & $\begin{array}{l}\text { Total } \\
\text { days of } \\
\text { cover }\end{array}$ & Period \\
\hline 1943 & 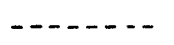 & $-\cdots--$ & Dec. 11,1942 , to-? \\
\hline 1944 & $-\ldots-n$ & --- & Dec. 15,1943 , to-? \\
\hline 1945 & $---1--$ & $-\cdots-$ & \\
\hline 1946 & 9 & 80 & Dec. 18, 1945, to Mar. 7, 1946. \\
\hline 1947 & 14 & 93 & Jan. 3, 1947, to Apr. 5, 1947 . \\
\hline 1948 & 14.5 & 102 & Dec. 10,1947 , to Mar. 20, 1948. \\
\hline 1949 & 4 & 25 & Dec. 18,1948 , to Jan. $11,1949$. \\
\hline 1950 & 4 & 43 & $\begin{array}{l}\text { Dec. } 16-18,1949 ; \text { Jan. } 19-29,1950 \text {; } \\
\text { Feb. } 23 \text { to Mar. 23, } 1950 .\end{array}$ \\
\hline 1951 & 14 & 69 & Dec. 16, 1950, to Feb. 22, 1951 \\
\hline 1952 & 4 & ----- & Dec. 17,1951, to-? \\
\hline 1953 & 4 & 19 & Jan. 5, 1953, to Jan. 23, 1953. \\
\hline
\end{tabular}

Discharge measurements. --None made; major part of outflow diverted to Tri-Lakes Fish Hatchery and cannot be related to gage height.

A verage lake level for Shriner Lake for water years 1943-53

\begin{tabular}{c|c|c|c|c|c|c|c}
\hline Year & Oct. & Nov. & Dec. & Jan. & Feb. & Mar. & Apr. \\
\hline 1943 & $* 6.82$ & 7.10 & 7.12 & 7.15 & 7.13 & 7.12 & 6.89 \\
1944 & 6.29 & 6.24 & 6.20 & 6.11 & 6.23 & 6.82 & 7.30 \\
1945 & 5.59 & 5.46 & 5.45 & 5.46 & 5.46 & 6.17 & 7.01 \\
1946 & 6.95 & 6.74 & 6.68 & 6.87 & 6.92 & 6.90 & 6.72 \\
1947 & 5.87 & 6.02 & 6.04 & 6.40 & 7.03 & 7.11 & 7.19 \\
1948 & 6.15 & 6.09 & 6.25 & 6.68 & 6.94 & 7.23 & 7.16 \\
1949 & 6.22 & 6.36 & 6.51 & 7.22 & 7.55 & 7.25 & 7.01 \\
1950 & 6.54 & 6.54 & 6.71 & 7.67 & 7.49 & 7.51 & 7.51 \\
1951 & 6.41 & 6.40 & 7.10 & 7.22 & 7.17 & 7.06 & 7.04 \\
1952 & 6.59 & 6.86 & 7.05 & 7.28 & 7.13 & 7.07 & 7.01 \\
1953 & 6.13 & 6.02 & 6.21 & 6.52 & 6.92 & 7.14 & 6.97 \\
\hline Average & 6.32 & 6.35 & 6.48 & 6.78 & 6.91 & 7.03 & 7.07 \\
\hline \hline Year & May & June & July & Aug. & Sept. & Annual & \\
\hline 1943 & 7.30 & 6.92 & 6.83 & 6.67 & 6.59 & 6.98 & \\
1944 & 7.17 & 6.87 & 6.38 & 5.92 & 5.62 & 6.43 & \\
1945 & 7.06 & 6.92 & 6.83 & 6.85 & 6.58 & 6.24 & \\
1946 & 6.64 & 6.76 & 6.91 & 6.50 & 6.13 & 6.73 & \\
1947 & 7.25 & 7.00 & 6.66 & 6.33 & 6.28 & 6.59 & \\
1948 & 7.21 & 7.07 & 6.98 & 6.78 & 6.44 & 6.75 & \\
1949 & 6.98 & 6.95 & 6.71 & 6.44 & 6.29 & 6.79 & \\
1950 & 6.79 & 6.86 & 6.69 & 6.44 & 6.49 & 6.94 & \\
1951 & 6.96 & 6.84 & 7.10 & 6.94 & 6.74 & 6.92 & \\
1952 & 6.95 & 6.96 & 6.81 & 6.62 & 6.43 & 6.89 & \\
1953 & 7.14 & 7.09 & 6.76 & 6.56 & 6.15 & 6.63 & \\
\hline Average & 7.04 & 6.93 & 6.79 & 6.55 & 6.34 & 6.72 & \\
\hline
\end{tabular}

* Partial month or partial year. 

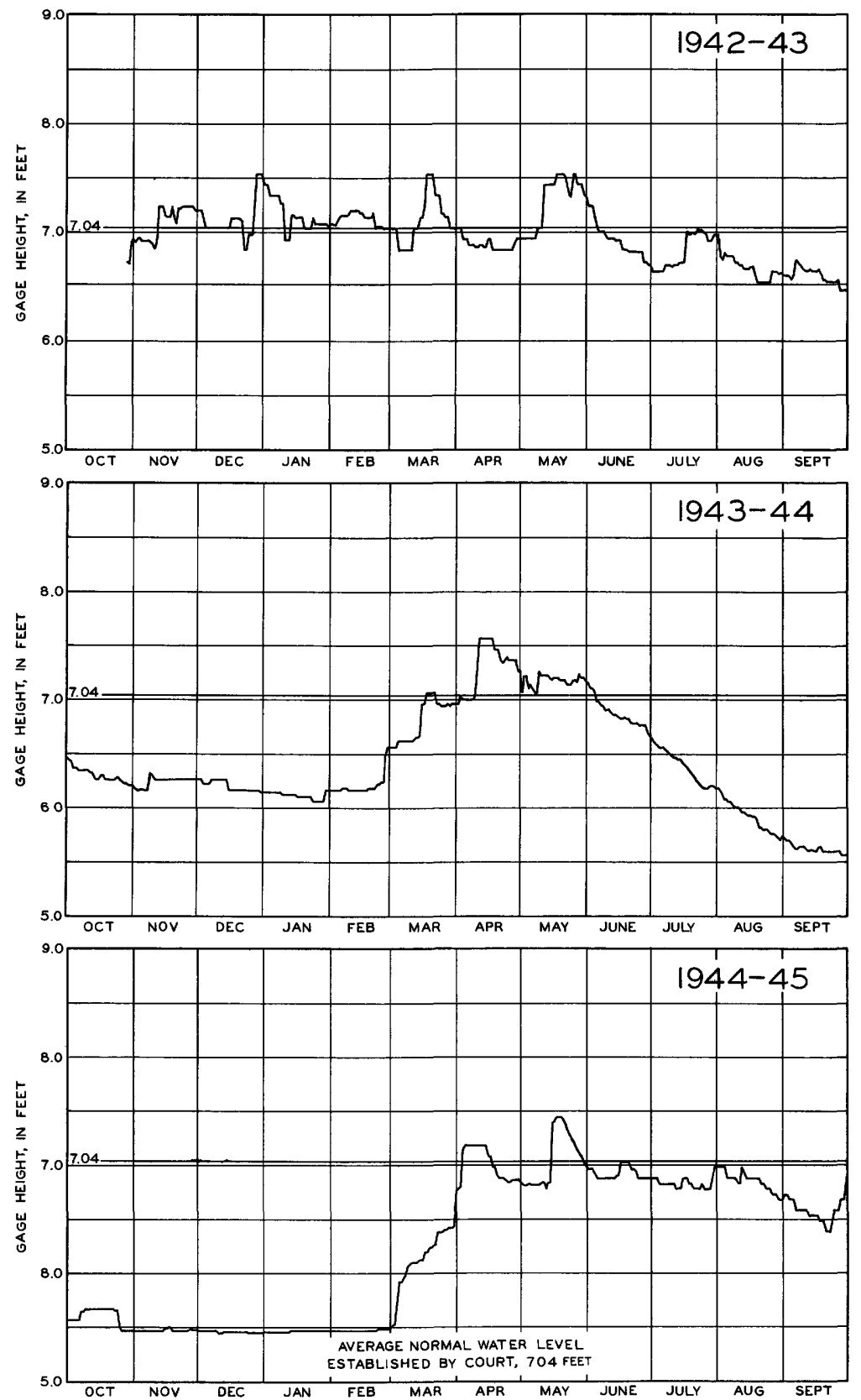

Figure 108. --Lake-level hydrographs for Shriner Lake at Tri Lakes, Ind., for water years 1943-45. 

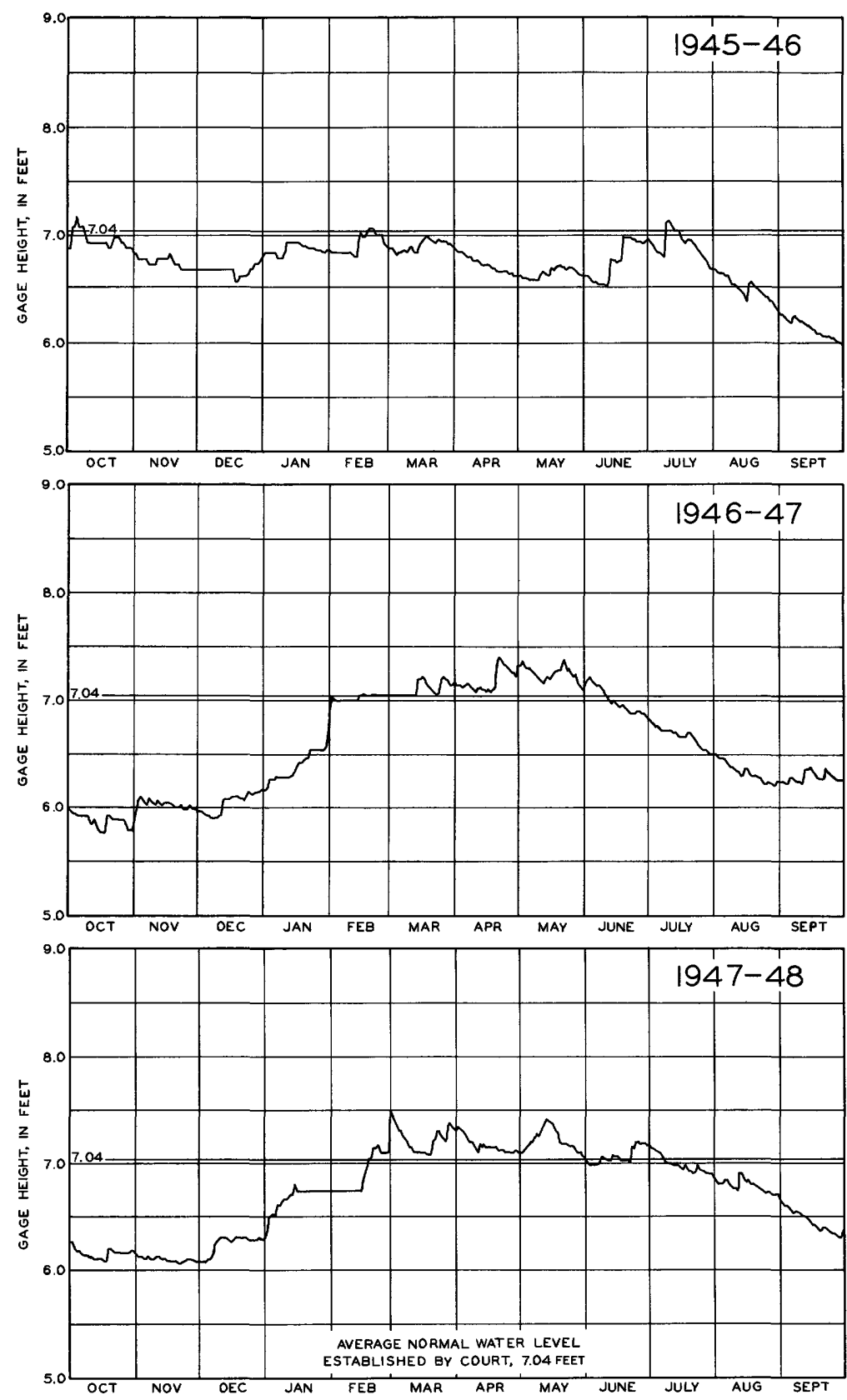

Figure 109. --Lake-level hydrographs for Shriner Lake at TriLakes, Ind., for water years 1946-48. 

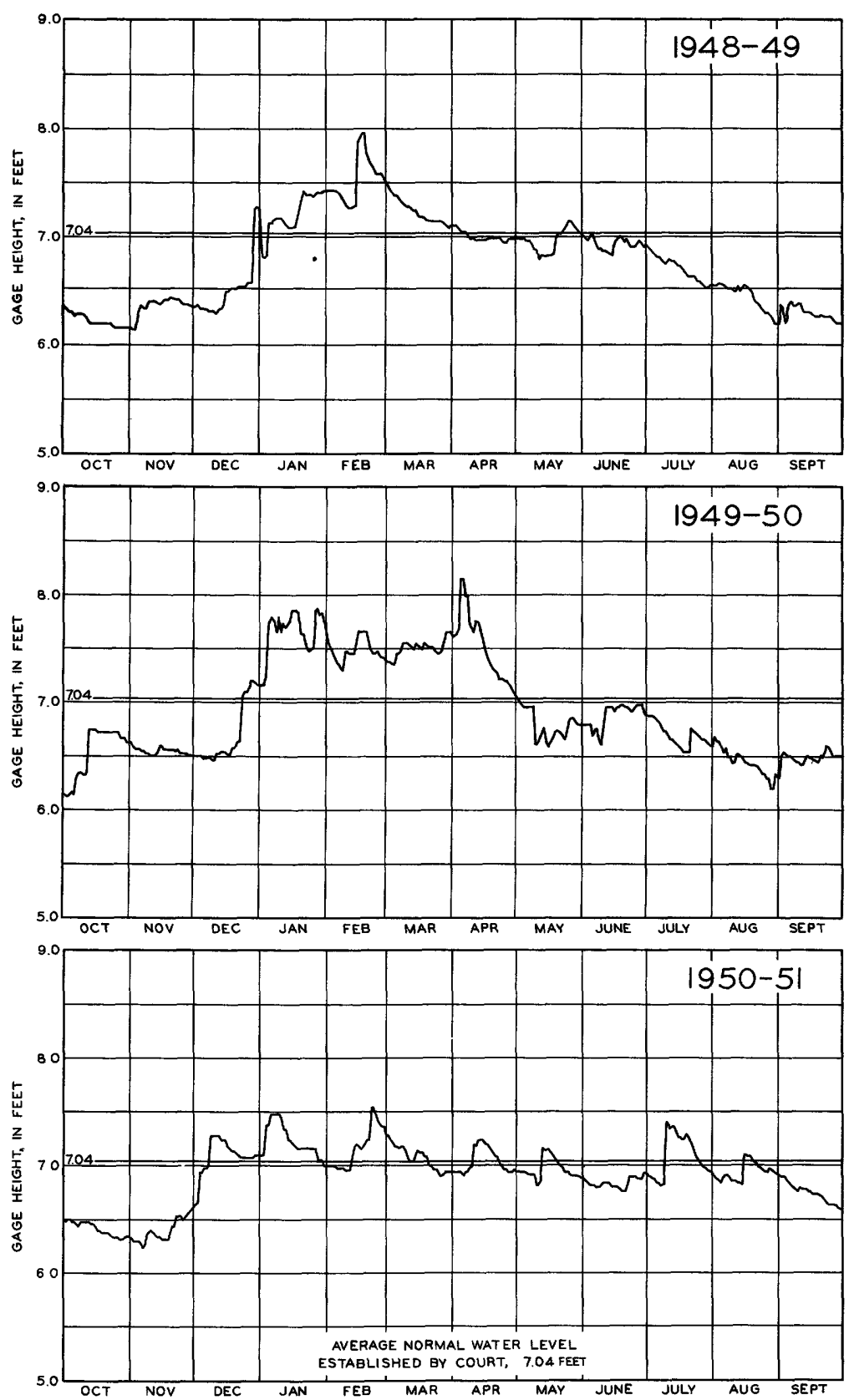

Figure. 110. --Lake-level hydrographs for Shriner Lake at TriLakes, Ind., for water years 1949-51. 

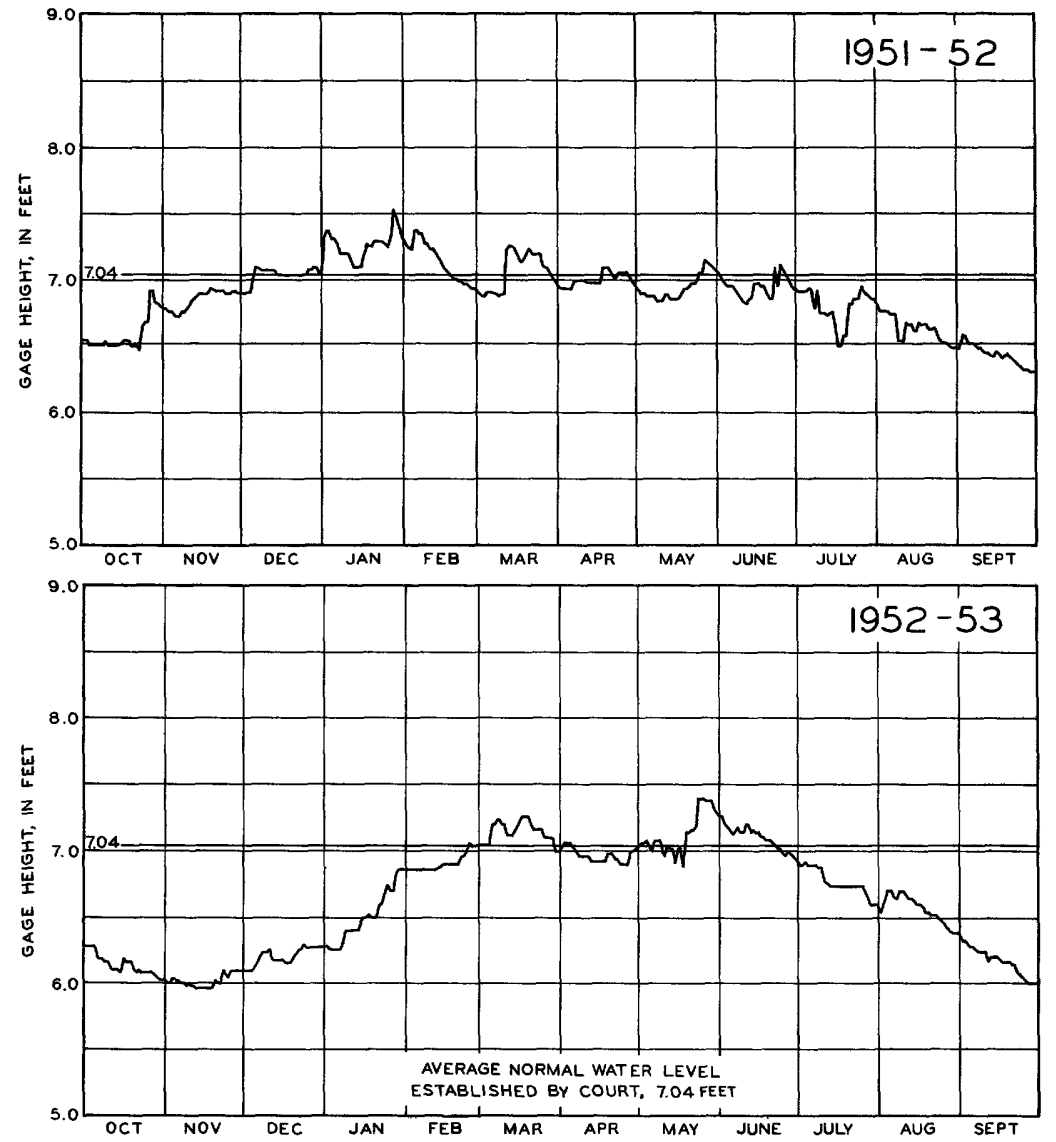

Figure 111. - Lake-level hydrographs for Shriner Lake at TriLakes, Ind., for water years 1952-53. 
Smalley Lake near Washington Center, Ind.

Location.--Secs. 15 and 22, T. 33 N., R. 8 E., Noble County, 0.9 mile southeast of Washington Center, and 4.7 miles soutl west of Wolflake.

Surface area. --61 acres.

Drainage area. $--32.6 \mathrm{sq} \mathrm{mi}$.

Records available.--August 1943 to September 1953.

Gage. - Chain gage on bridge over outlet $300 \mathrm{ft}$ below lake. Pric to Feb. 13, 1947, staff gage on the northeast shore. Datum ( gage is $880.00 \mathrm{ft}$ above mean sea level, datum of 1929. Gag read to hundredths once daily.

Average lake level. -10 years; gage height, $2.04 \mathrm{ft}$; elevation, $882.04 \mathrm{ft}$.

Established legal level. --None.

Lake-level control. --Lake level maintained at first bridge belov lake by gravel outlet channel.

Extreme levels for Smalley Lake, 1944-53

\begin{tabular}{|c|c|c|c|c|}
\hline \multicolumn{3}{|c|}{ Maximum } & \multicolumn{2}{|l|}{ Minimum } \\
\hline $\begin{array}{l}\text { Water } \\
\text { year }\end{array}$ & Date & $\begin{array}{c}\text { Gage } \\
\text { height } \\
\text { (feet) }\end{array}$ & Date & $\begin{array}{r}\text { Gag! } \\
\text { heigl } \\
\text { (feet } \\
\end{array}$ \\
\hline $1943 a$ & Aug. 4,1 & ${ }^{2} 2.17$ & Sept. $30,1943 \ldots$ & ${ }^{a} 1.7$ \\
\hline 1944 & May 16, 194 & 4.13 & Aug. 20,21, $1944 \ldots$ & 1.4 \\
\hline 1945 & May $17,1945 \ldots$ & 3.41 & Oct. 14 to Nov. 11,1944 & 1.4 \\
\hline 1946 & Oct. 2,194 & 2.98 & Sept. 3-9, 1946 & 1.3 \\
\hline 1947 & Apr. 21, 194 & 2.95 & Oct. $6-17,1946 \ldots$ & 1.3 \\
\hline 1948 & Feb. 29, $1948 \ldots$ & 2.95 & Sept. $18,19,1948 \ldots$ & 1.3 \\
\hline & Feb. 14, $1949 \ldots$ & 3.36 & Sept. 30, 1949 & 1.4 \\
\hline & Apr. $4,5,1950$ & 4.48 & Oct. 3,1949 & 1. 4 \\
\hline 1951 & Feb. 22, $1951 \ldots$ & $* 3.84$ & Sept. 30, 1951 $\ldots$ & 1.5 \\
\hline 195 & Jan. $27,1952 \ldots$ & 3.25 & Oct. $6,1951 \ldots$ & 1. 4 \\
\hline 1953 & Apr. $4,1953 \ldots$ & 3.20 & July $26,29,1953 \ldots$ & 1.3 \\
\hline
\end{tabular}

* Estimated.

a August and September. 
Maximum recorded thickness and periods of ice cover for Smalley Lake, 1944-51

\begin{tabular}{|c|c|c|c|}
\hline $\begin{array}{c}\text { Water } \\
\text { year }\end{array}$ & $\begin{array}{c}\text { Maximum } \\
\text { thickness } \\
\text { (inches) }\end{array}$ & $\begin{array}{c}\text { Total } \\
\text { days of } \\
\text { cover }\end{array}$ & Period \\
\hline 1944 & 3 & $-\cdots-1$ & Unknown to Mar. 28, 1944. \\
\hline 1945 & $---\cdots--$ & ------ & \\
\hline 1946 & $-\cdots-1-1$ & ------ & Dec. 13,1945 , to - ? \\
\hline 1947 & 3 & 43 & Dec. 30,1946 , to Feb. $13,1947$. \\
\hline 1948 & 10 & 98 & Dec. 13,1947 , to Mar. $19,1948$. \\
\hline 1949 & $-\ldots-.-$ & ----- & Dec. 18,1948 , to $-?$ \\
\hline 1950 & 6 & 62 & $\begin{array}{l}\text { Dec. } 15-20,1949 ; \text { Jan. } 4-10,19-25 ; \\
\text { Feb. 1-8; Feb. } 20 \text { to Mar. 25, } 1950\end{array}$ \\
\hline 1951 & $--\cdots--$ & 82 & $\begin{array}{l}\text { Dec. 1-3; Dec. 8, 1950, to Feb. } 27 \text {, } \\
\text { 1951. }\end{array}$ \\
\hline
\end{tabular}

Discharge measurements at outlet of Smalley Lake

\begin{tabular}{|c|c|c|c|c|c|}
\hline Date & $\begin{array}{c}\text { Gage } \\
\text { height } \\
\text { (feet) }\end{array}$ & $\begin{array}{c}\text { Discharge } \\
\text { (cfs) }\end{array}$ & Date & $\begin{array}{c}\text { Gage } \\
\text { height } \\
\text { (feet) }\end{array}$ & $\begin{array}{c}\text { Discharge } \\
\text { (cfs) }\end{array}$ \\
\hline Aug. $3,1943-$ & 2.13 & 11.1 & May 4,1950 & 2.50 & 43.6 \\
\hline Oct. $\quad 8 \ldots \ldots$ & 1.65 & 3.50 & Oct. $26 \cdots$ & 1. 64 & 4. 36 \\
\hline Jan. 7,1944 & 2.06 & 2.55 & Nov. $14 \ldots$ & 1.80 & 8.40 \\
\hline May 15-.... & 2.88 & 59.1 & Jan. 25,1951 & 2.31 & 30.4 \\
\hline Mar. 2, 1945- & 2.25 & 26.9 & Mar. 14-.-- & 2.50 & 45.5 \\
\hline Apr. 11- & 2.77 & 52.6 & Apr. 24 & 2. 44 & 40.7 \\
\hline July $11 \cdots$ & 1.89 & 8.82 & May 23----- & 2.18 & 19.0 \\
\hline Sept. $1 \ldots$ & 1.82 & 5.67 & June 20--.- & 1.89 & 13.6 \\
\hline Oct. 5 & 2.51 & 37.5 & July $25 \ldots$ & 2. 41 & 31.5 \\
\hline Mar. 13, $1946-$ & 2. 47 & 30.2 & Aug. $29 \ldots$ & 2.05 & 16.8 \\
\hline May 27. & 1.85 & 8.90 & Sept. 26 $\ldots$ & 1.63 & 3.57 \\
\hline June 19 & $2 . .28$ & 22.6 & Oct. 24- & 2.20 & 29.1 \\
\hline Aug. 21, $1946=$ & 1. 49 & 4.33 & Nov. 28-.-- & 2.06 & 21.8 \\
\hline Dec. 6 & 1.54 & a .53 & Dec. $19-\cdots$ & 2. 12 & a 23.1 \\
\hline Jan. 17, $1947-$ & 2.08 & 9.56 & Feb. 5, 1952 & 3.23 & 96.2 \\
\hline $\begin{array}{l}\text { Feb. } 13 \\
\text { Apr. } 23\end{array}$ & $\begin{array}{l}2.15 \\
2.87\end{array}$ & $\begin{array}{l}24.2 \\
64.9\end{array}$ & $\begin{array}{l}\text { Mar. 27 } \\
\text { July } 17 \ldots\end{array}$ & $\begin{array}{l}2.52 \\
1.89\end{array}$ & $\begin{array}{r}50.8 \\
9.30\end{array}$ \\
\hline July $2 \cdots-\cdots$ & 1.83 & 20.0 & Aug. 14 & 1.86 & 9.40 \\
\hline Oct. 30 & 1.67 & 2.81 & Nov. $18 \ldots$ & 1.61 & 2.37 \\
\hline Oct. $31 \ldots$ & 1.66 & 2.87 & Dec. $16 \ldots$ & 1.64 & 3.73 \\
\hline Mar. 4, 1948- & 2.84 & 70.8 & Jan. 15,1953 & 1.84 & 10.4 \\
\hline Apr. $1 \cdots$ & 2.87 & 56.2 & Feb. $3---$ & 1.96 & 15.9 \\
\hline Sept. 22- & 1.45 & 1.01 & Feb. 27-..-- & 2.09 & 19.8 \\
\hline Oct. $12 b$ & 1.48 & .66 & Mar. 25_...- & 2.37 & 35.0 \\
\hline Mar. 2, $1949-$ & 2.67 & 54.8 & Apr. 17.... & 2.20 & 26.6 \\
\hline Mar. 25 & 2. 07 & 20.9 & May 13-...- & 2.01 & 17.6 \\
\hline Sept. 7 $7-$ & 1.55 & 3.85 & June $8 \cdots$ & 1.94 & 11.2 \\
\hline Dec. 15- & 1.69 & 6.25 & July 2-..- & 1.67 & 3.52 \\
\hline Jan. 17,1950 & 3.92 & 136 & July 27---- & 1.53 & 1. 71 \\
\hline Feb. 8 & 2.78 & 65.1 & Aug. $25 b \ldots$ & 1.47 & b. 90 \\
\hline Mar. $1-\cdots$ & 2.83 & 65.7 & Sept. $18^{c} \ldots$ & 1.45 & c. 83 \\
\hline
\end{tabular}

a Leaves and debris restricting flow.

$b$ Point of zero flow determined 1. 17.

c Point of zero flow determined 1. 19. 
Average lake levels for Smalley Lake for water years 1943-53

\begin{tabular}{c|c|c|r|r|r|r|r}
\hline Year & Oct. & Nov. & Dec. & Jan. & Feb. & Mar. & Apr. \\
\hline 1944 & 1.65 & 1.77 & $* 1.68$ & $* 1.51$ & $* 1.58$ & 2.52 & 3.29 \\
1945 & 1.45 & 1.50 & $* 1.67$ & 1.70 & 1.88 & 2.48 & 2.68 \\
1946 & 2.18 & 1.84 & 1.80 & $* 2.29$ & $* 2.32$ & 2.49 & 2.01 \\
1947 & 1.39 & 1.56 & 1.72 & $* 2.02$ & $* 2.02$ & 2.01 & 2.59 \\
1948 & 1.56 & 1.71 & 1.96 & 2.06 & 2.10 & 2.70 & 2.50 \\
1949 & 1.50 & 1.64 & 1.74 & 2.49 & 2.84 & 2.29 & 2.00 \\
1950 & 1.78 & 1.64 & 1.93 & 3.48 & 3.05 & 3.00 & 3.42 \\
1951 & 1.70 & 1.80 & 2.36 & 2.50 & 2.72 & 2.59 & 2.64 \\
1952 & 1.78 & 2.21 & 2.17 & 2.85 & 2.69 & 2.57 & 2.53 \\
1953 & 1.55 & 1.59 & 1.65 & 1.94 & 2.04 & 2.41 & 2.57 \\
\hline Average & 1.65 & 1.73 & 1.87 & 2.28 & 2.32 & 2.51 & 2.62 \\
\hline \hline Year & May & June & July & Aug. & Sept. & Annual & \\
\hline 1943 & -- & -- & -- & $* 1.99$ & 1.86 & $* 1.92$ & \\
1944 & 3.21 & 2.21 & 1.62 & 1.46 & 1.46 & 1.99 & \\
1945 & 2.58 & 2.31 & 1.87 & 2.15 & 1.70 & 2.00 & \\
1946 & 1.80 & 1.96 & 1.75 & 1.44 & 1.34 & 1.93 & \\
1947 & 2.56 & 2.16 & 1.75 & 1.56 & 1.60 & 1.91 & \\
1948 & 2.37 & 1.94 & 1.78 & 1.57 & 1.44 & 1.97 & \\
1949 & 1.99 & 2.04 & 1.80 & 1.56 & 1.50 & 1.94 & \\
1950 & 2.18 & 2.05 & 1.91 & 1.83 & 1.92 & 2.34 & \\
1951 & 2.22 & 1.96 & 2.57 & 2.07 & 1.74 & 2.24 & \\
1952 & 2.34 & 2.19 & 2.08 & 1.87 & 1.62 & 2.25 & \\
1953 & 2.21 & 1.85 & 1.49 & 1.59 & 1.46 & 1.86 & \\
\hline Average & 2.35 & 2.07 & 1.86 & 1.74 & 1.60 & 2.04 & \\
\hline
\end{tabular}

* Partial year or partial month. 

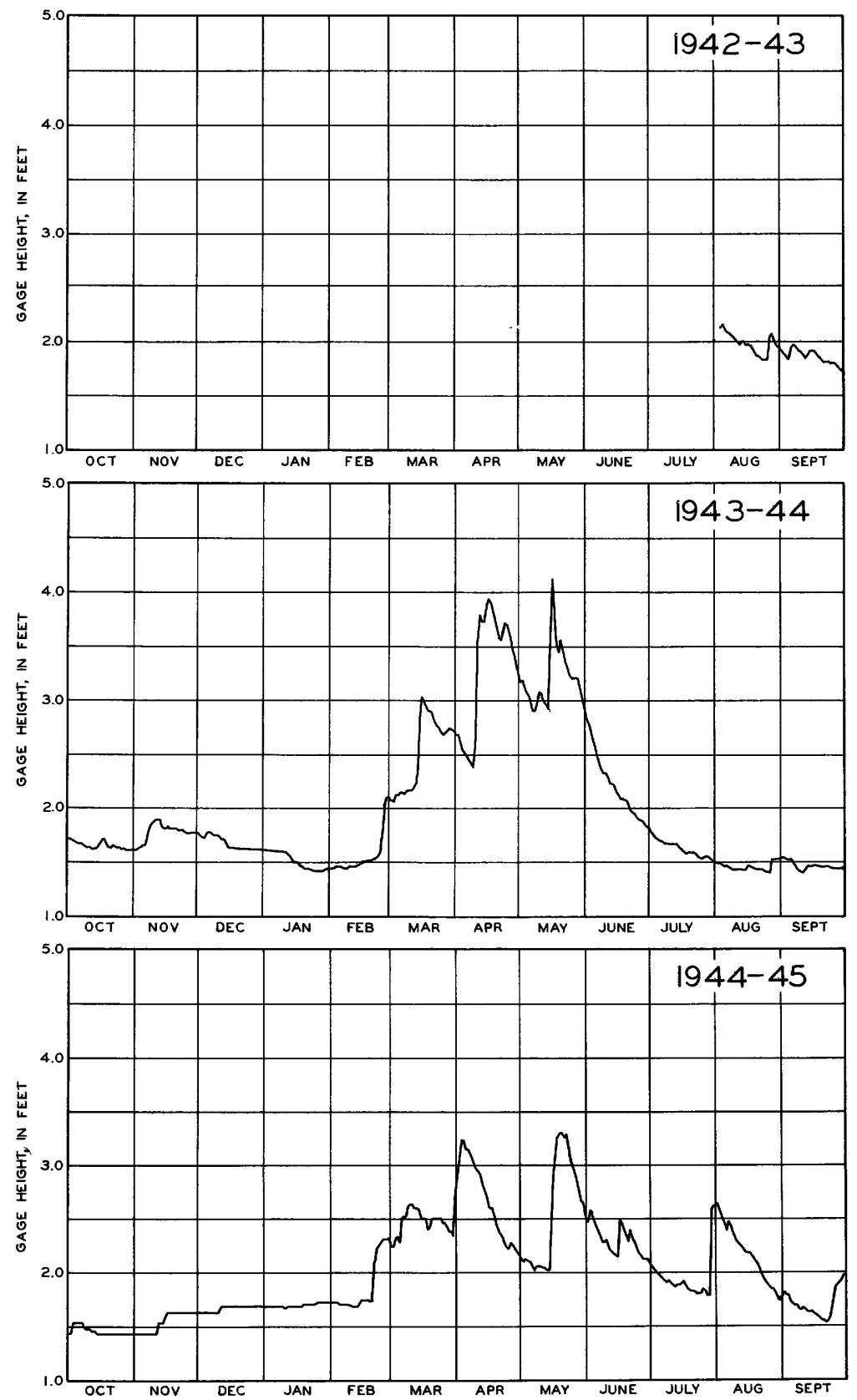

Figure 112.--Lake-level hydrographs for Smalley Lake near Washington Center, Ind., for water years 1946-48. 

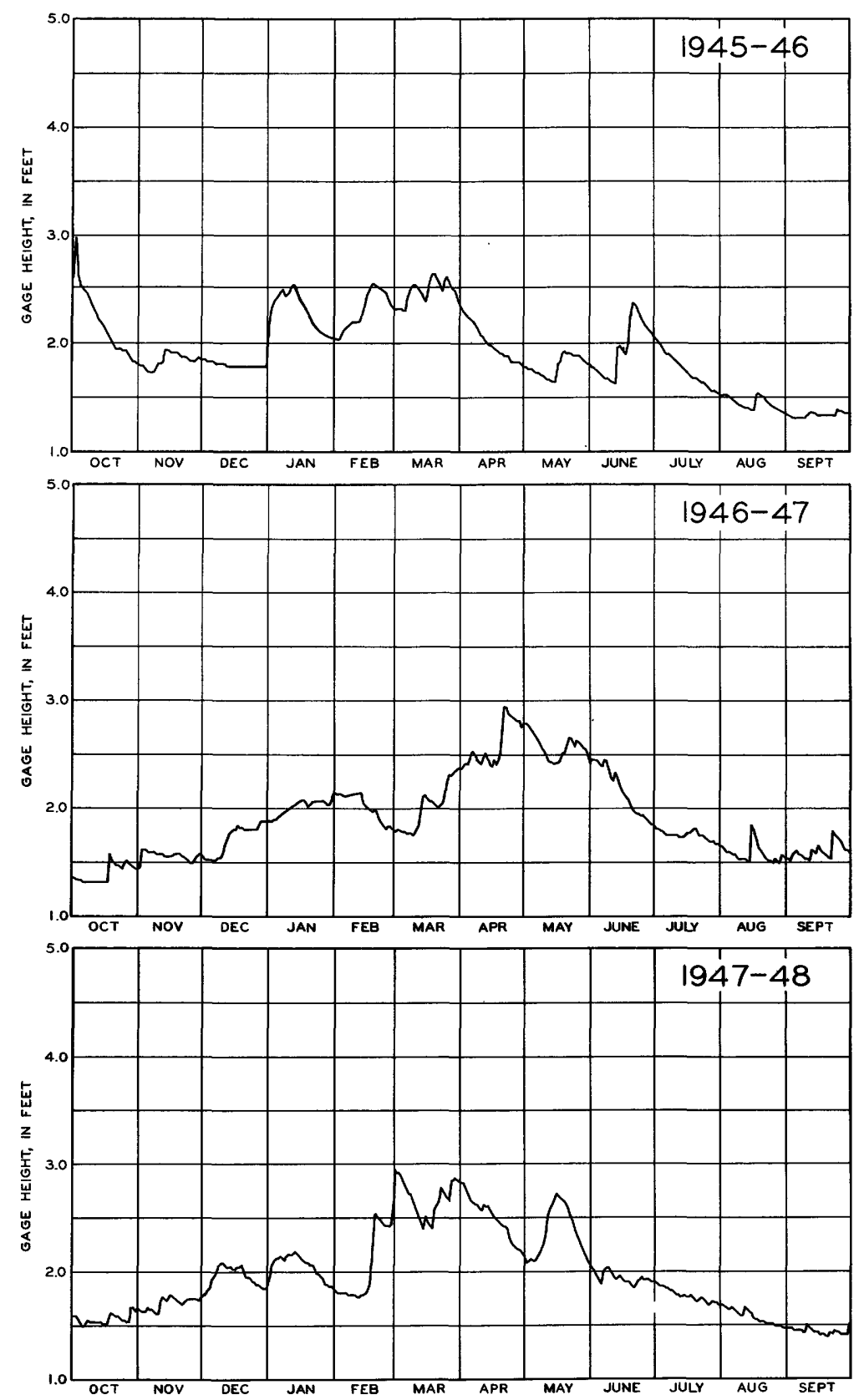

Figure 113. --Lake-level hydrographs for Smalley Lake near Washington Center, Ind., for water years 1946-48. 
BASIC DATA ON LAKE LEVELS FOR SELECTED LAKES 223
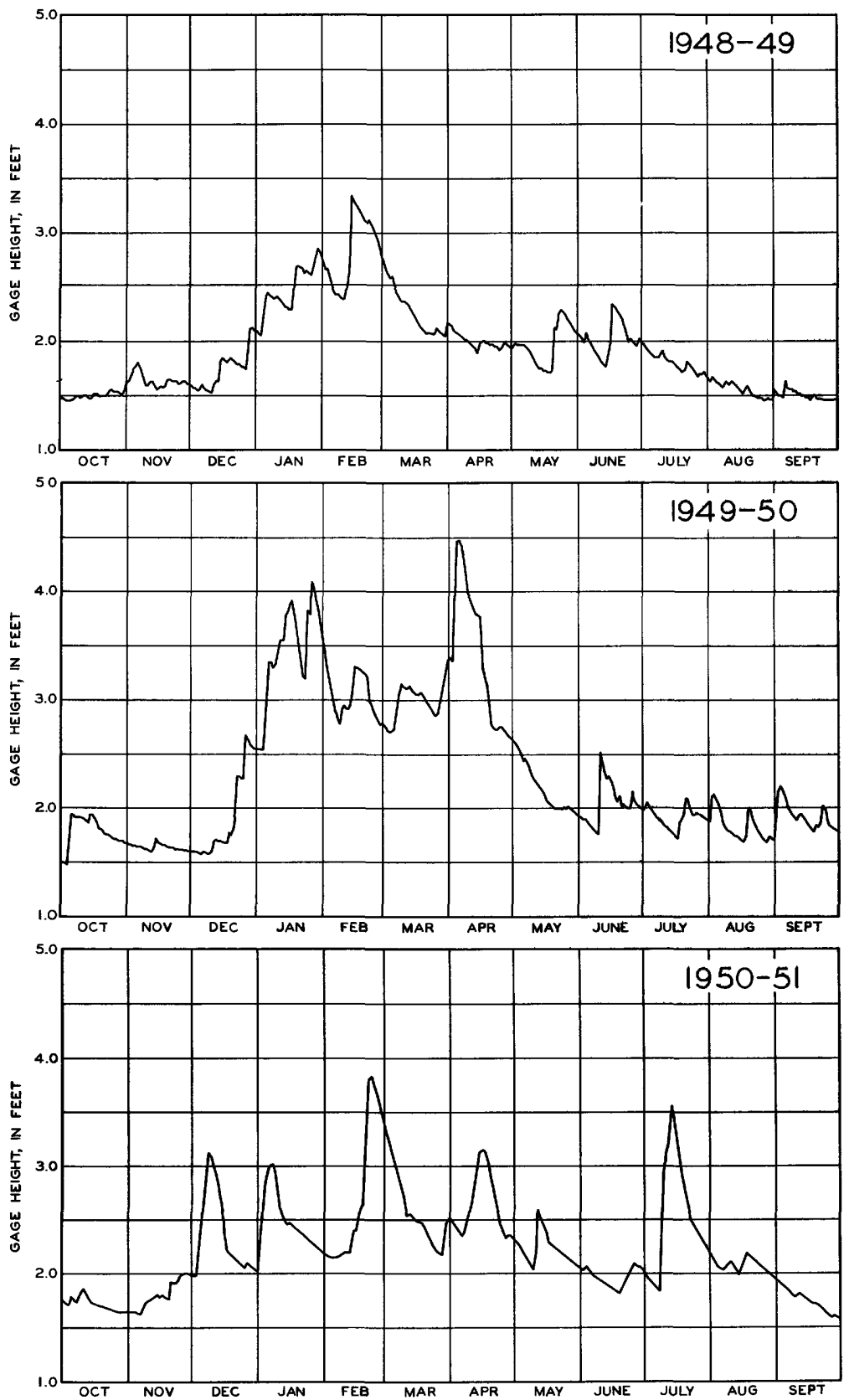

Figure 114. --Lake level hydrographs for Smalley. Lake near Washington Center, Ind., for water years 1949-51. 

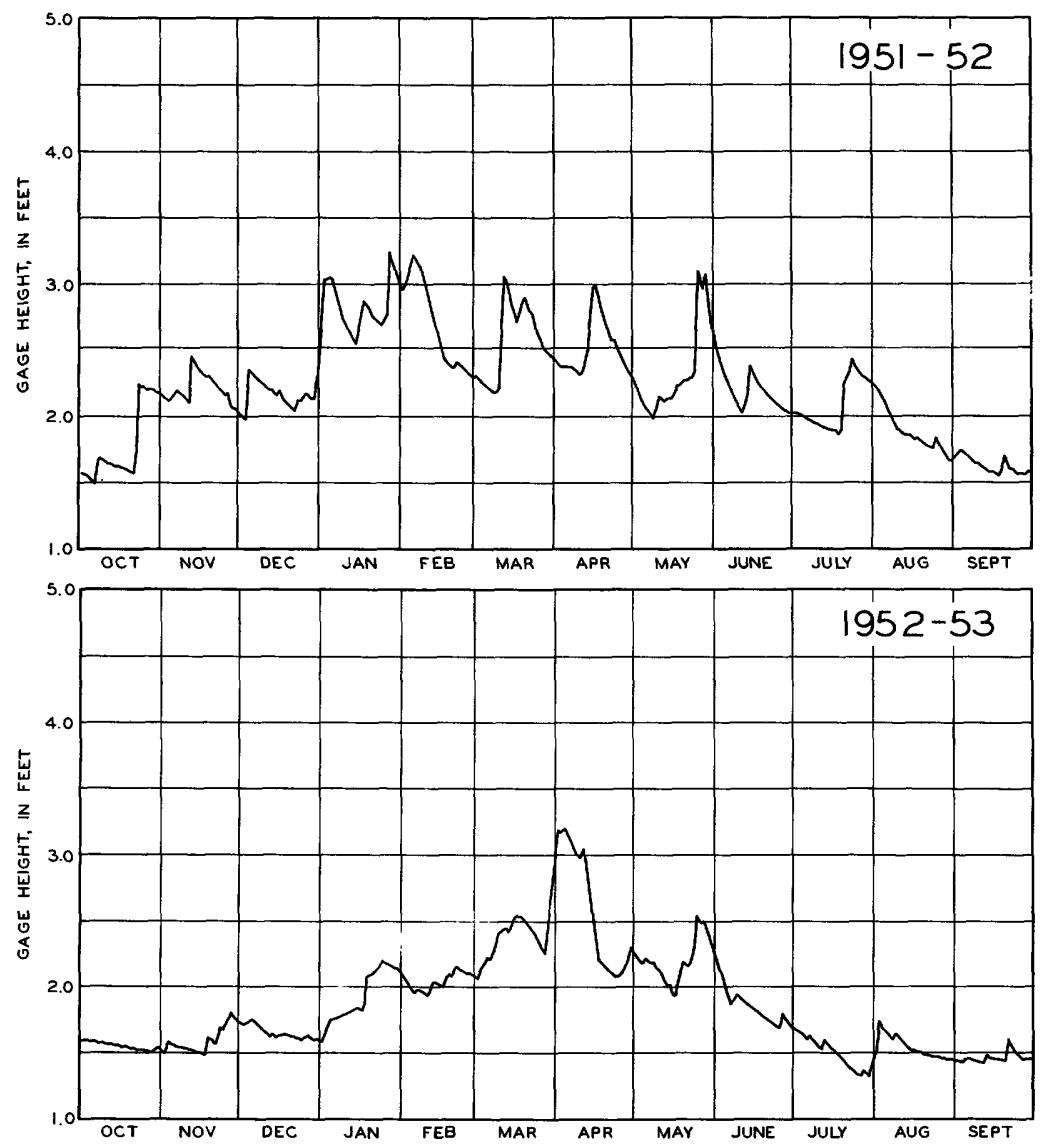

Figure 115. --Lake-level hydrographs for Smalley Lake near Washington Center, Ind. for water years 1952-53.

Sylvan Lake at Rome City, Ind.

Location. --Secs. 9, 13,14,15,16 and 23, T. 35 N., R. 10 E., Noble County.

Surface area. --575 acres.

Drainage area. $--31.5 \mathrm{sq} \mathrm{mi}$.

Records available. --October 1943 to September 1953.

Gage. --Staff gage on abutment of first bridge upstream from

control dam. Gage read to hundredths once daily. Datum of

gage is $910.00 \mathrm{ft}$ above mean sea level, datum of 1929 .

Average lake level. --11 years; gage height, $6.26 \mathrm{ft}$; elevation, $916.26 \mathrm{ft}$.

Established legal level. - Established June 14, 1951, at gage

height $6.20 \mathrm{ft}$; elevation, $916.20 \mathrm{ft}$ above mean sea level.

Lake-level control. - -Lake level controlled by concrete dam with

12 manually operated steel gates resting on the sill and spillway

$50 \mathrm{ft}$ long. 
Extreme levels for Sylvan Lake, 1943-53

\begin{tabular}{|c|c|c|c|c|}
\hline \multicolumn{3}{|c|}{ Maximum } & \multicolumn{2}{|l|}{ Minimum } \\
\hline $\begin{array}{c}\text { Water } \\
\text { year }\end{array}$ & Date & $\begin{array}{l}\text { Gage } \\
\text { height } \\
\text { (feet) }\end{array}$ & Date & $\begin{array}{l}\text { Gage } \\
\text { height } \\
\text { (feet) }\end{array}$ \\
\hline 1943 & May $18,1943 \ldots$ & 7.44 & Oct. $29,1942 \ldots$ & 6.21 \\
\hline 1944 & $\begin{array}{l}\text { Nov. } 9,1943, \text { Mar. } \\
\quad 16,17,1944 \ldots\end{array}$ & 6.44 & Apr. $3-5,1944-\ldots$ & 5.24 \\
\hline 1945 & May $18,1945 \ldots$ & 6.66 & Nov. $4,5,1944 \ldots$ & 6.00 \\
\hline 1946 & June $19,1946 \ldots$ & 6.97 & Sept. $28,1946 \ldots$ & 5.82 \\
\hline 1947 & Apr. 21, 1947 $\ldots$ & 6.80 & Oct. $16,17,1946 \ldots$ & 5.79 \\
\hline 1948 & Feb. 21, $1948 \ldots$ & 6.86 & $\begin{array}{c}\text { Mar. 8, Sept. 18, } \\
19,1948\end{array}$ & 5.90 \\
\hline 1949 & Feb. 15, $1949 \ldots$ & 6.74 & Mar. 14, 15, $1949-$ & 5.88 \\
\hline 1950 & Apr. 6, $1950 \ldots$ & 7. 56 & Oct. $1,2,1949 \ldots$ & 6.00 \\
\hline 1951 & Feb. 23, $1951 \ldots$ & 7. 14 & Sept. 23-30, $1951-$ & 6.10 \\
\hline 1952 & Dec. $18-20,1951 \ldots$ & 7. 10 & $\begin{array}{l}\text { Aug. } 3-9,29,30, \\
1952-\ldots\end{array}$ & 6.02 \\
\hline 1953 & Mar. 8, 9, 1953-... & 6.48 & Sept. $10,11,1953$ - & 5.80 \\
\hline
\end{tabular}

Maximum recorded thickness and periods of ice cover 1946-53

\begin{tabular}{|c|c|c|c|}
\hline $\begin{array}{l}\text { Water } \\
\text { year }\end{array}$ & $\begin{array}{c}\text { Maximum } \\
\text { thickness } \\
\text { (inches) }\end{array}$ & $\begin{array}{l}\text { Total } \\
\text { days of } \\
\text { cover }\end{array}$ & Period \\
\hline 1946 & $6 \frac{1}{2}$ & --- & Dec. 14,1945 , to - ? \\
\hline 1947 & 12 & 123 & Dec. 2,1946 , to Apr. 3, 1947. \\
\hline 1948 & 20 & 109 & $\begin{array}{c}\text { Nov. } 27 \text { to Dec. } 6,1947 \text {; Dec. } 10 \text {, } \\
1947 \text {, to Mar. } 19,1948 .\end{array}$ \\
\hline 1949 & 6 & 84 & $\begin{array}{l}\text { Dec. } 3-13 \text {; Dec. } 18,1948 \text {, to Jan. } \\
\text { 16; Jan. } 30 \text { to Feb. 19; Mar. 2- } \\
\text { 22, } 1949 \text {. }\end{array}$ \\
\hline 1950 & $-\cdots$ & 69 & $\begin{array}{c}\text { Dec. } 8-12,14-21,1949 ; \text { Jan. } 17- \\
\text { 25; Feb. } 3 \text { to Mar. } 26,1950 .\end{array}$ \\
\hline 1951 & 4 & 95 & Nov. 30,1950 , to Mar. 4,1951 \\
\hline 1952 & 6 & 87 & Dec. 16,1951 , to Mar. 11,1952 . \\
\hline 1953 & 5 & 89 & Dec. 14,1952 , to Mar. $12,1953$. \\
\hline
\end{tabular}


Discharge measurements at outlet of Sylvan Lake

\begin{tabular}{|c|c|c|c|c|c|}
\hline Date & $\begin{array}{l}\text { Gage } \\
\text { height } \\
\text { (feet) }\end{array}$ & $\begin{array}{c}\text { Discharge } \\
\text { (cfs) }\end{array}$ & Date & $\begin{array}{l}\text { Gage } \\
\text { height } \\
\text { (feet) }\end{array}$ & $\begin{array}{c}\text { Discharge } \\
\text { (cfs) }\end{array}$ \\
\hline Aug. $12,1943^{-}$ & 6.28 & 14.9 & Mar. 29, 1946- & 6.20 & 16.2 \\
\hline Oct. $\quad 5 \ldots$ & 6.16 & 5.79 & May $\quad 9-\ldots$ & 6.05 & 7. 90 \\
\hline Feb. 11, 1944- & 6.29 & 9.88 & $18-\cdots--$ & 6.09 & 6.32 \\
\hline Mar. 2, 1945- & 6.29 & 20.1 & Feb. 15, 1947- & 6.30 & 49.2 \\
\hline July $2 \ldots$ & 6. 16 & 12.8 & Oct. $25,1950-$ & 6.20 & 15. 7 \\
\hline Sept. $\quad 5 \ldots--$ & 6.08 & 7. 72 & $17,1951-$ & 6.54 & 55.2 \\
\hline Nov. 29 & 6.22 & 12.1 & & & \\
\hline
\end{tabular}

Average lake levels for Sylvan Lake for water years 1943-53

\begin{tabular}{c|c|c|c|c|c|c|c}
\hline Year & Oct. & Nov. & Dec. & Jan. & Feb. & Mar. & Apr. \\
\hline 1943 & $* 6.29$ & 6.40 & 6.45 & 6.40 & $* 6.44$ & 6.26 & 6.39 \\
1944 & 6.24 & 6.35 & 6.31 & 6.25 & 6.27 & 5.87 & 5.81 \\
1945 & 6.06 & 6.11 & 6.17 & 6.20 & 6.23 & 6.26 & 6.22 \\
1946 & 6.22 & 6.19 & 6.22 & 6.30 & 6.24 & 6.23 & 6.19 \\
1947 & 5.91 & 6.15 & 6.14 & 6.23 & 6.37 & 6.31 & 6.24 \\
1948 & 6.14 & 6.22 & 6.45 & 6.49 & 6.35 & 6.28 & 6.24 \\
1949 & 6.04 & 6.11 & 6.25 & 6.37 & 6.39 & 6.13 & 6.14 \\
1950 & 6.21 & 6.12 & 6.25 & 6.50 & 6.62 & 6.78 & 6.98 \\
1951 & 6.26 & 6.36 & 6.71 & 6.54 & 6.66 & 6.52 & 6.40 \\
1952 & 6.21 & 6.32 & 6.54 & 6.56 & 6.42 & 6.45 & 6.41 \\
1953 & 6.05 & 6.12 & 6.19 & 6.24 & 6.23 & 6.36 & 6.35 \\
\hline Average & 6.15 & 6.22 & 6.33 & 6.39 & 6.40 & 6.32 & 6.30 \\
\hline \hline & & & & & & & \\
Year & May & June & July & Aug. & Sept. & Annual & \\
\hline 1943 & $* 6.77$ & $* 6.59$ & 6.48 & 6.32 & 6.30 & $* 6.43$ & \\
1944 & 5.92 & 6.11 & 6.08 & 5.96 & 6.04 & 6.10 & \\
1945 & 6.25 & 6.33 & 6.11 & 6.29 & 6.13 & $* 6.21$ & \\
1946 & 6.10 & 6.20 & 6.18 & 5.98 & 5.87 & 6.16 & \\
1947 & 6.20 & 6.27 & 6.13 & 6.04 & 6.27 & 6.19 & \\
1948 & 6.28 & 6.38 & 6.25 & 6.11 & 5.98 & 6.26 & \\
1949 & 6.22 & 6.25 & 6.20 & 6.12 & 6.03 & 6.19 & \\
1950 & 6.39 & 6.36 & 6.36 & 6.27 & 6.46 & 6.44 & \\
1951 & 6.40 & 6.34 & 6.38 & 6.30 & 6.14 & 6.42 & \\
1952 & 6.38 & 6.30 & 6.13 & 6.07 & 6.10 & 6.34 & \\
1953 & 6.35 & 6.10 & 6.01 & 6.06 & 5.84 & 6.16 & \\
\hline Average & 6.30 & 6.29 & 6.21 & 6.14 & 6.10 & 6.26 & \\
\hline
\end{tabular}

*Partial year or partial month. 

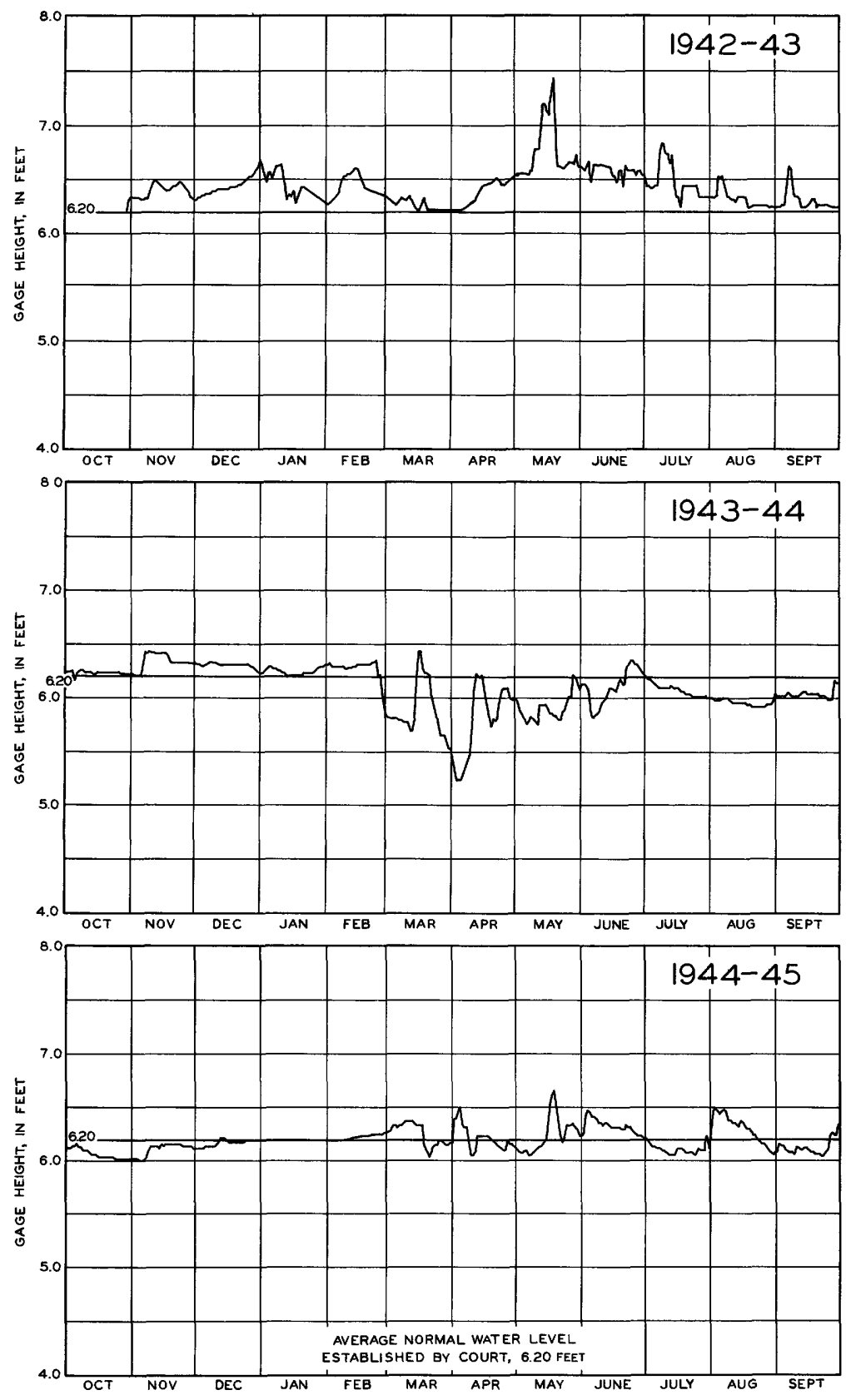

Figure 116. --Lake-level hydrographs for Sylvan Lake at Rome City, Ind., for water years 1943-45. 
228

HYDROLOGY OF INDIANA LAKES
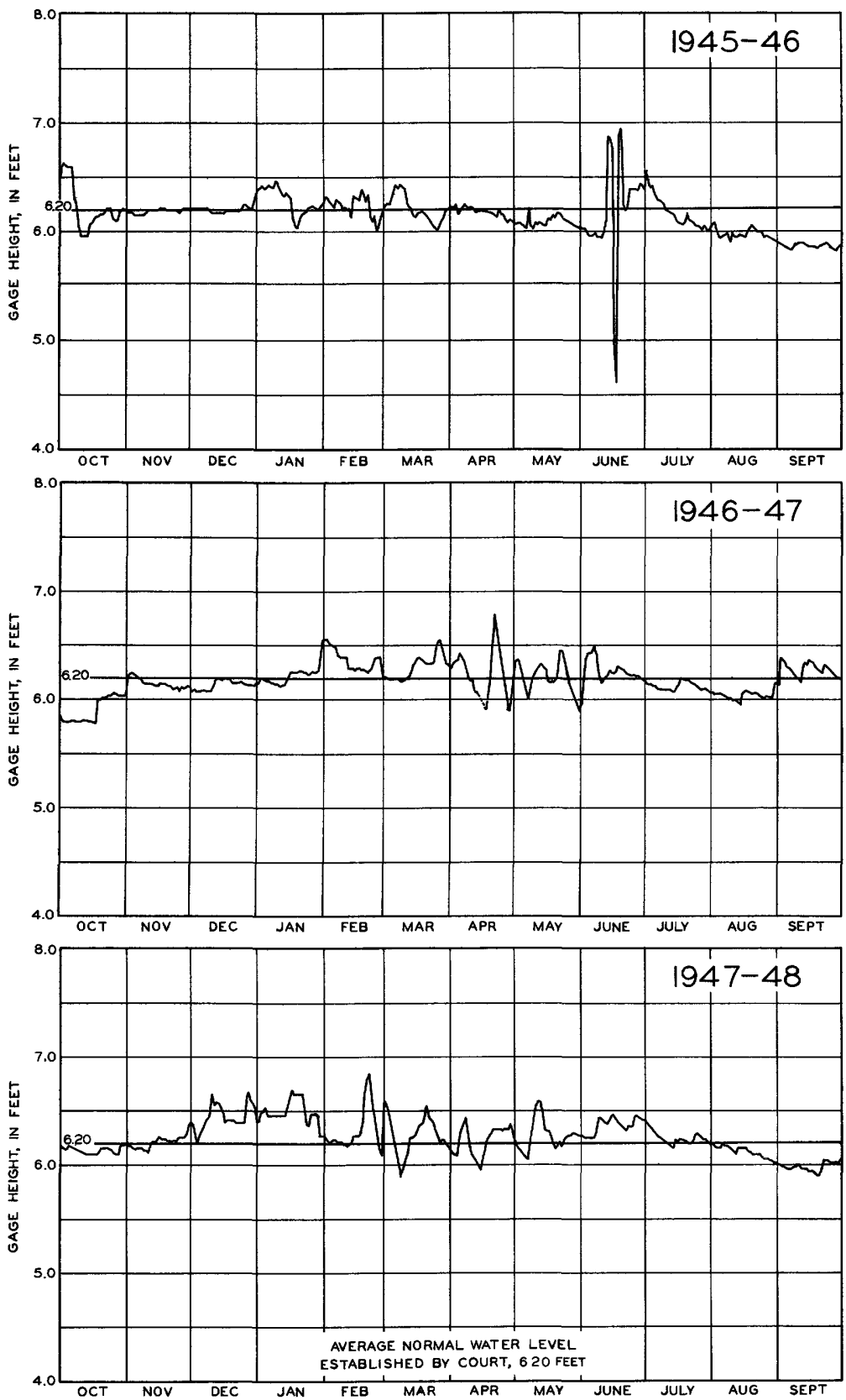

Figure 117. --Lake-level hydrograph for Sylvan Lake at Rome City, Ind., for water years 1946-48. 


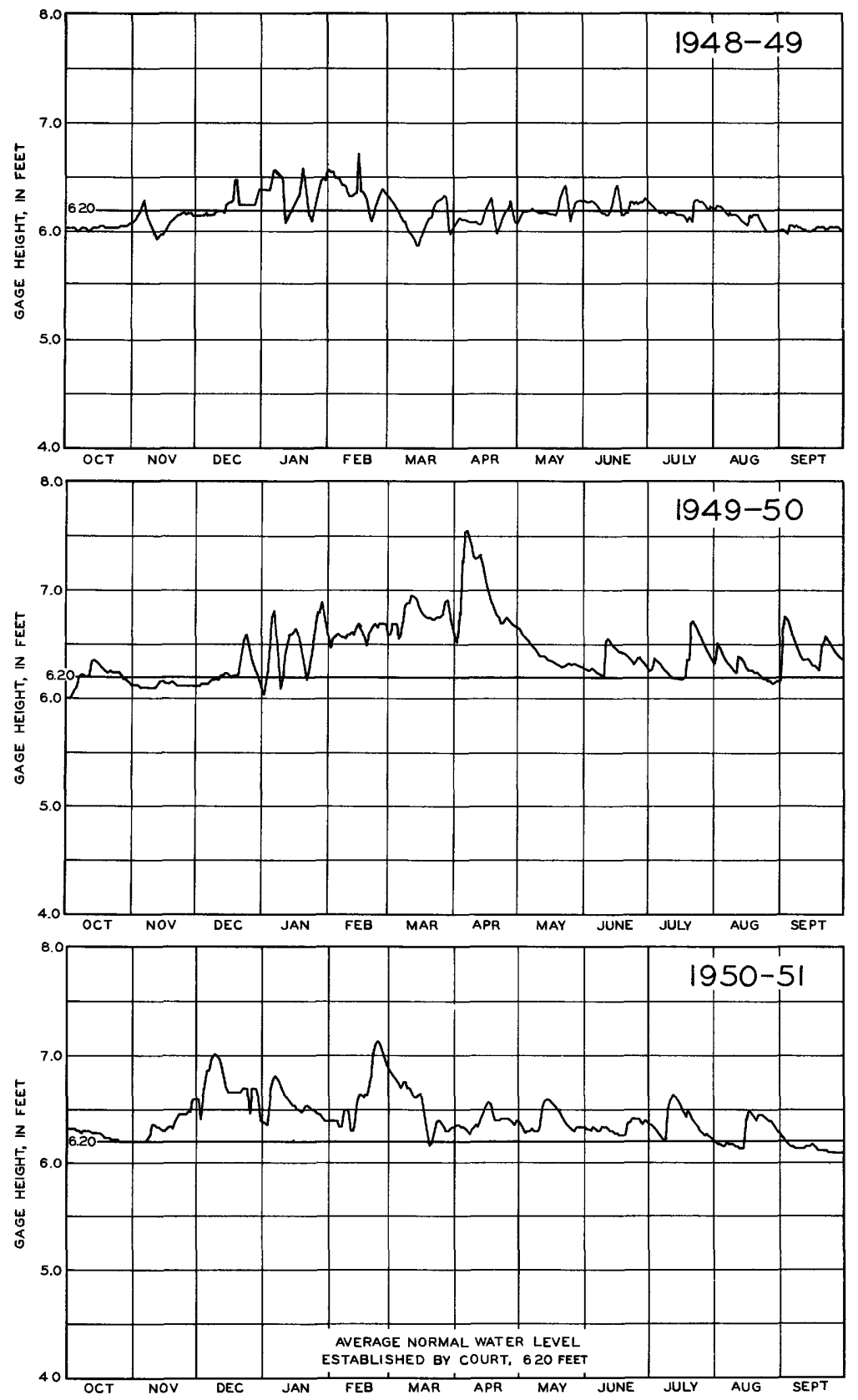

Figure 118. --Lake-level hydrographs for Sylvan Lake at Rome City, Ind., for water years 1949-51. 

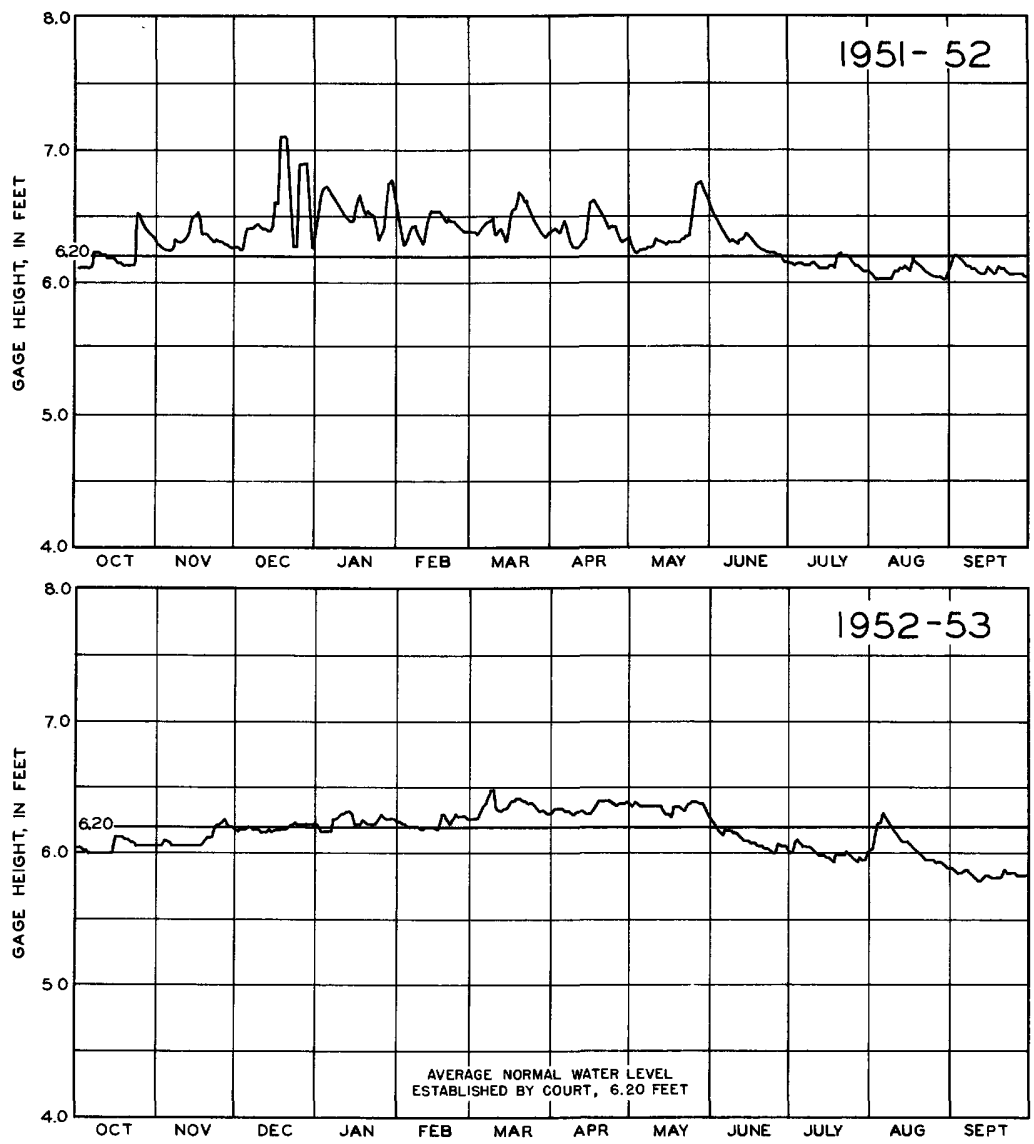

Figure 119. --Lake-level hydrographs for Sylvan Lake at Rome City, Ind., for water years 1952-53. 
Syracuse Lake at Syracuse, Ind.

Location. --Secs. 4, 5, 8, and 9, T. 34 N., R. 7 E., Kosciusko County.

Surface area. --367 acres.

Drainage area. $--37.3 \mathrm{sq} \mathrm{mi}$

Records available. --August 1943 to September 1953.

Gage. --Staff gage at second bridge upstream from control dam. Gage read to hundredths once daily. Datum of gage is $850.00 \mathrm{ft}$ above mean sea level, datum of 1929 .

Average level. --10 years; gage height, 8. $50 \mathrm{ft}$, elevation, $858.50 \mathrm{ft}$.

Established legal level. --Established September 20, 1948, at gage height $8.87 \mathrm{ft}$, elevation $858.87 \mathrm{ft}$ above mean sea level.

Lake-level control. - Lake level controlled by concrete dam with two steel sluice gates, each $6 \mathrm{ft}$ wide and $5 \mathrm{ft}$ high, with sill elevation at gage height $5.21 \mathrm{ft}$. A $14-\mathrm{ft}$ fixed crest spillway south of the gates has a crest at gage height, $8.87 \mathrm{ft}$.

Extreme levels of Syracuse Lake, 1944-53

\begin{tabular}{|c|c|c|c|c|}
\hline \multicolumn{3}{|c|}{ Maximum } & \multicolumn{2}{|l|}{ Minimum } \\
\hline $\begin{array}{l}\text { Water } \\
\text { year }\end{array}$ & Date & $\begin{array}{c}\text { Gage } \\
\text { height } \\
\text { (feet) }\end{array}$ & Date & $\begin{array}{r}\text { Gage } \\
\text { height } \\
\text { (feet) }\end{array}$ \\
\hline 1944 & Mar. 16-18, 1944-- & 9.34 & Sept. 27, $1944 \ldots$ & 7.48 \\
\hline 1945 & Sept. $30,1945 \ldots$ & 9.03 & Nov. $2,3,1944 \ldots$ & 7.28 \\
\hline 1946 & June $30,1946 \ldots$ & 9.11 & Sept. 30, $1946 \ldots$ & 7. 39 \\
\hline 1947 & Apr. $21-23,1947 \ldots$ & 9.36 & Oct. $17,1946 \ldots$ & 7.11 \\
\hline 1948 & Mar. 7, 1948 & 9.12 & Sept. 30, $1948 \ldots$ & 7.56 \\
\hline 1949 & July $9,1949 \ldots$ & 9.58 & Oct. $28,29,1948--$ & 7. 14 \\
\hline 1950 & Jan. $27,28,1950$ & $* 10.15$ & Dec. 10,1949 & $* 8.22$ \\
\hline 1951 & $\begin{array}{l}\text { Dec. } 10,11,12,1950 \\
\text { Apr. } 15,16,1951\end{array}$ & & Sept. 30, $1951 \ldots$ & 8.46 \\
\hline 1952 & Feb. 6-8, 1952 & 9.18 & $\begin{array}{r}\text { Sept. } 13,14,21,22, \\
1952\end{array}$ & 7.90 \\
\hline 1953 & May 25, 1953 & 8.82 & Sept. 27-30, $1953-$ & 7. 38 \\
\hline
\end{tabular}

*Estimated 
Maximum recorded thickness and periods of ice cover 1944-50

\begin{tabular}{c|c|c|l}
\hline $\begin{array}{c}\text { Water } \\
\text { year }\end{array}$ & $\begin{array}{c}\text { Maximum } \\
\text { thickness } \\
\text { (inches) }\end{array}$ & $\begin{array}{c}\text { Total } \\
\text { days of } \\
\text { cover }\end{array}$ & \multicolumn{1}{|c}{ Period } \\
\hline 1944 & 3 & 96 & Nov. 24, 1943, to Feb. 27, 1944. \\
1945 & --- & ---- & Dec. 10, 1944, to - ? \\
1946 & --- & 32 & Dec. 5, 1945, to Jan 5, 1946. \\
1947 & 4 & 94 & Dec. 21, 1946, to Mar. 24, 1947. \\
1948 & 16 & ---- & Dec. 15, 1947, to - ? \\
1949 & $6 \frac{1}{2}$ & ---- & Dec. 18, 1948, to - ? \\
1950 & 6 & ---- &
\end{tabular}

Discharge measurements at outlet of Syracuse Lake

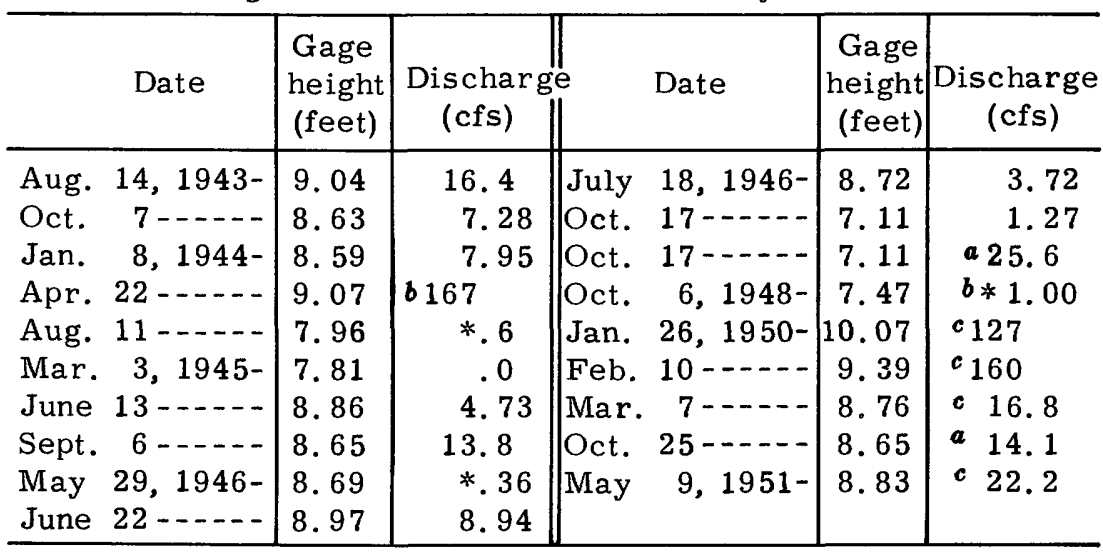

* Estimated because of leakage.

a Flood gates closed; power plant in operation.

$b$ Sluice gate partly open.

c Sluice gates partly open; power plant in operation. 
Average lake levels for Syracuse Lake for water years 1943-53

\begin{tabular}{c|l|l|l|l|l|l|l}
\hline Year & Oct. & Nov. & Dec. & Jan. & Feb. & Mar. & Apr. \\
\hline 1944 & ๖. & 8.66 & 8.62 & 8.59 & 8.74 & 9.08 & 8.98 \\
1945 & 7.46 & 7.36 & 7.40 & 7.49 & 7.54 & 8.13 & 8.77 \\
1946 & 8.78 & 8.70 & 8.67 & 8.73 & 8.71 & 8.61 & 8.58 \\
1947 & 7.23 & 7.35 & 7.34 & 7.48 & 7.88 & 8.11 & 9.01 \\
1948 & 8.02 & 7.88 & 8.07 & 8.39 & 8.56 & 9.02 & 8.98 \\
1949 & 7.32 & 7.37 & 7.43 & 7.98 & 8.91 & 9.12 & 8.87 \\
1950 & 8.50 & 8.36 & 8.46 & 9.67 & 9.36 & 8.88 & 9.50 \\
1951 & 8.74 & 8.70 & 8.98 & 8.86 & 8.81 & 8.88 & 8.94 \\
1952 & 8.46 & 8.75 & 8.90 & 8.96 & 9.02 & 8.92 & 8.99 \\
1953 & 7.76 & 7.66 & 7.53 & 7.47 & 7.61 & 8.13 & 8.57 \\
\hline Average & 8.09 & 8.08 & 8.14 & 8.36 & 8.51 & 8.69 & 8.92 \\
\hline \hline & & & & & & \\
Year & May & June & July & Aug. & Sept. & Annual & \\
\hline 1943 & ---- & ---- & ---- & $* 8.84$ & 8.80 & $* 8.81$ & \\
1944 & 8.94 & 8.77 & 8.37 & 7.88 & 7.63 & 8.57 & \\
1945 & 8.84 & 8.90 & 8.73 & 8.69 & 8.68 & 8.17 & \\
1946 & 8.60 & 8.81 & 8.76 & 8.13 & 7.65 & 8.56 & \\
1947 & 9.13 & 9.01 & 8.63 & 8.24 & 8.20 & 8.13 & \\
1948 & 8.90 & 8.90 & 8.82 & 8.42 & 7.82 & 8.48 & \\
1949 & 9.00 & 9.22 & 9.38 & 8.96 & 8.51 & 8.50 & \\
1950 & 8.83 & 8.87 & 9.07 & 8.94 & 8.86 & 8.94 & \\
1951 & 8.92 & 8.80 & 8.81 & 8.74 & 8.62 & 8.82 & \\
1952 & 8.92 & 8.88 & 8.61 & 8.42 & 8.05 & 8.74 & \\
1953 & 8.79 & 8.61 & 8.12 & 7.85 & 7.51 & 7.97 & \\
\hline Average & 8.89 & 8.88 & 8.73 & 8.46 & 8.21 & 8.50 & \\
\hline & & & & & & & \\
\hline
\end{tabular}

*Partial month or partial year. 

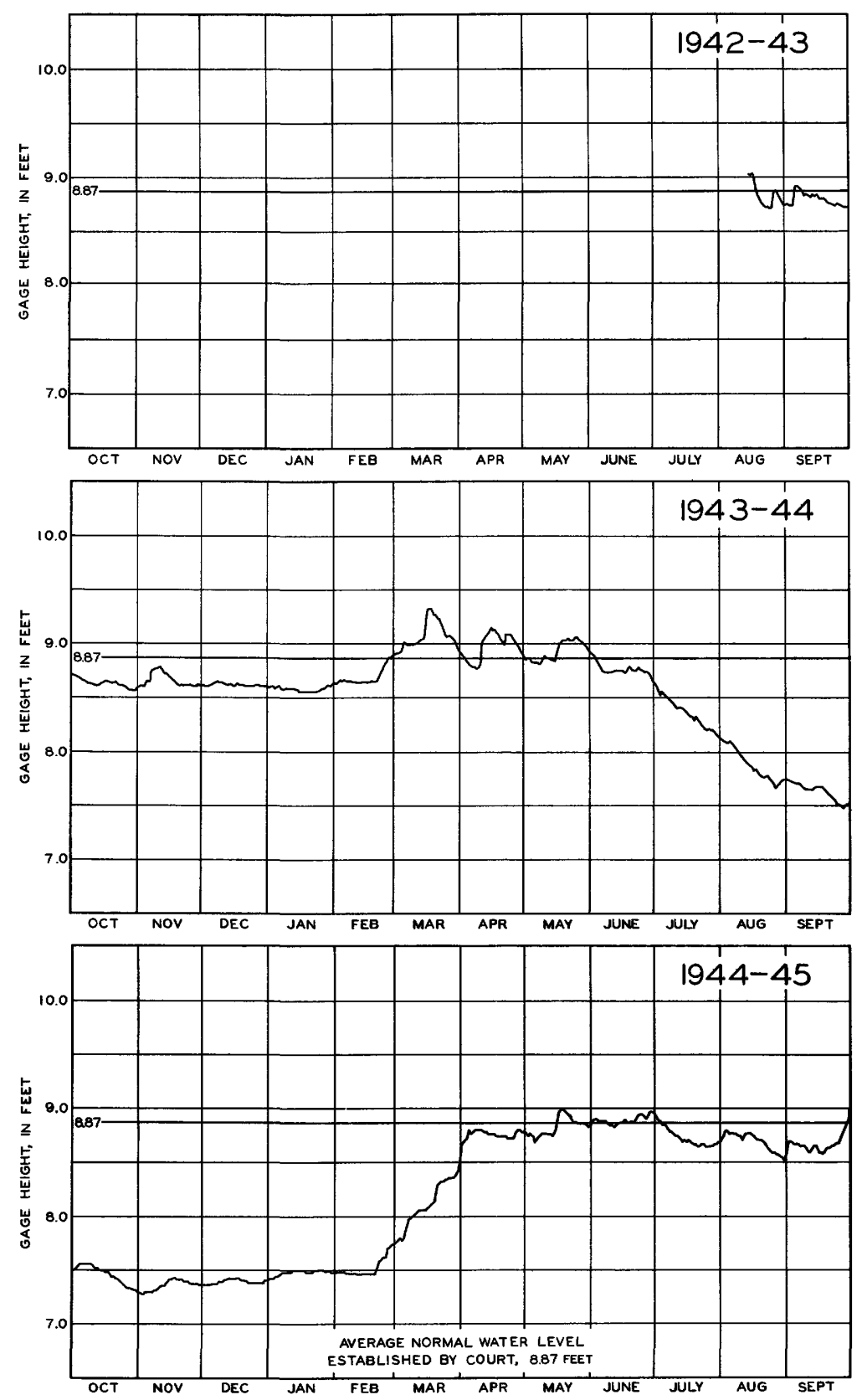

Figure 120. - - Lake-level hydrographs for Syracuse Lake at Syra cuse, Ind., for water years 1943-45. 

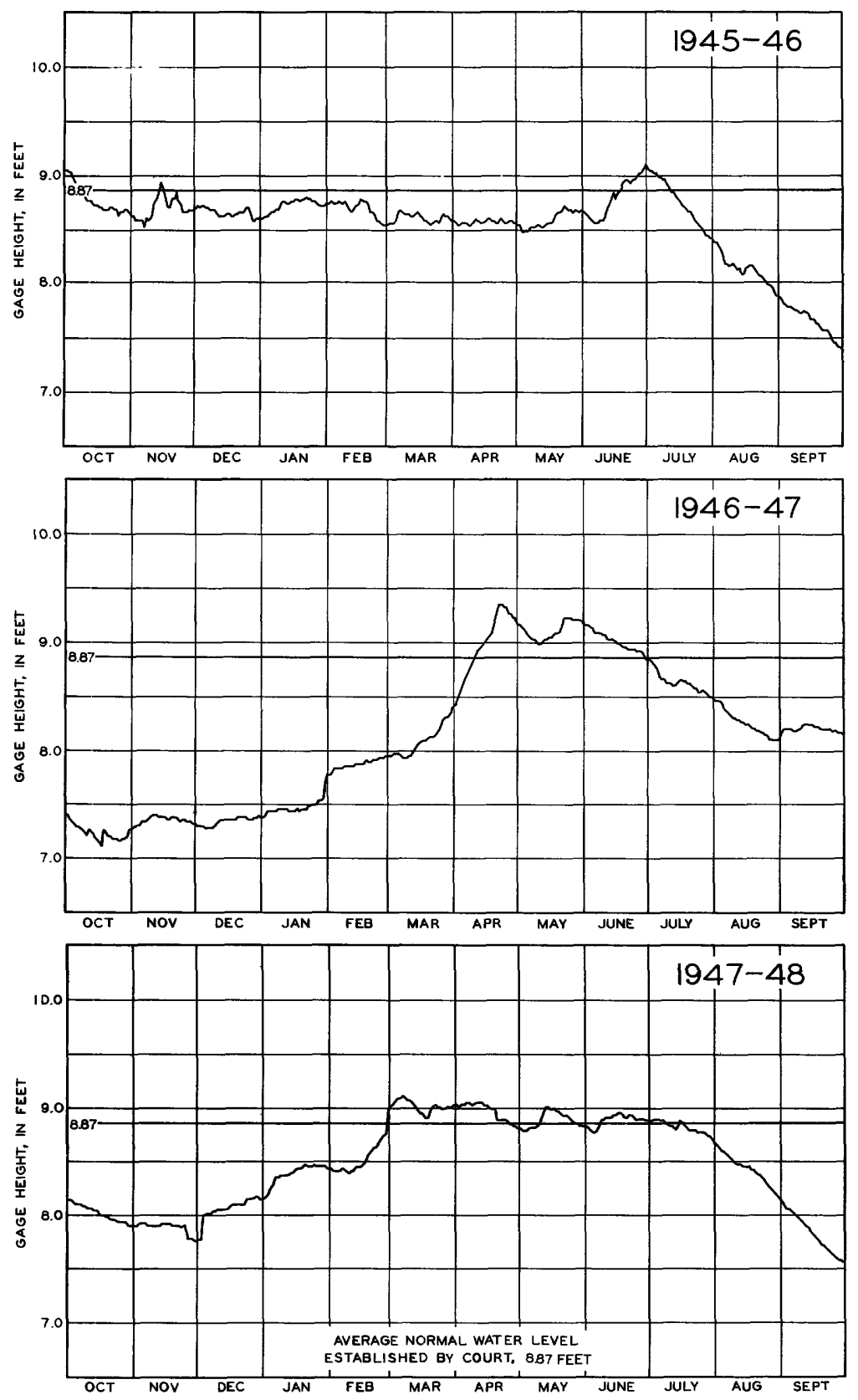

Figure 121. --Lake-level hydrographs for Syracuse Lake at Syracuse, Ind., for water years 1946-48. 

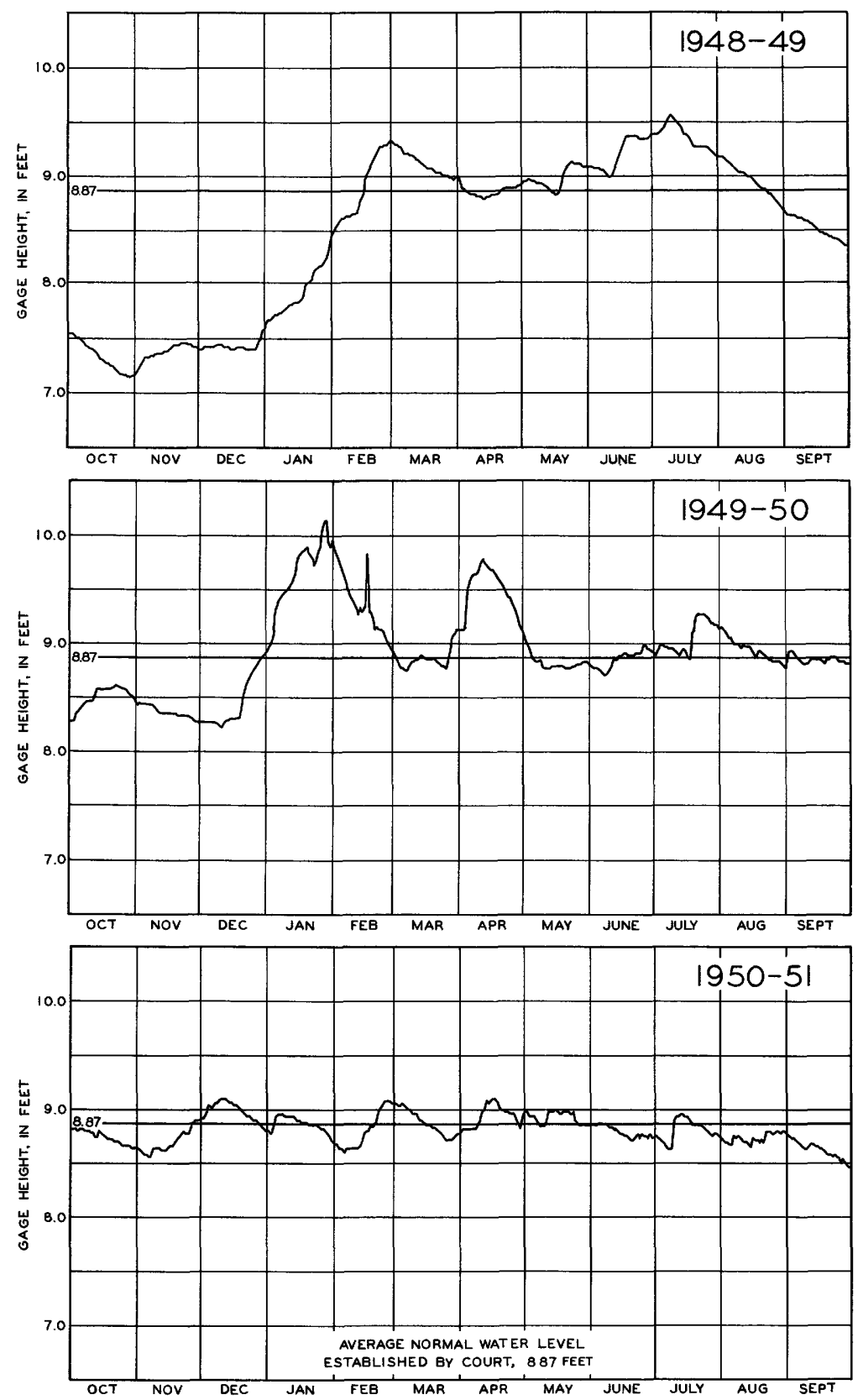

Figure 122. --Lake-level hydrographs for Syracuse Lake at Syracuse, Ind., for water years 1949-51. 


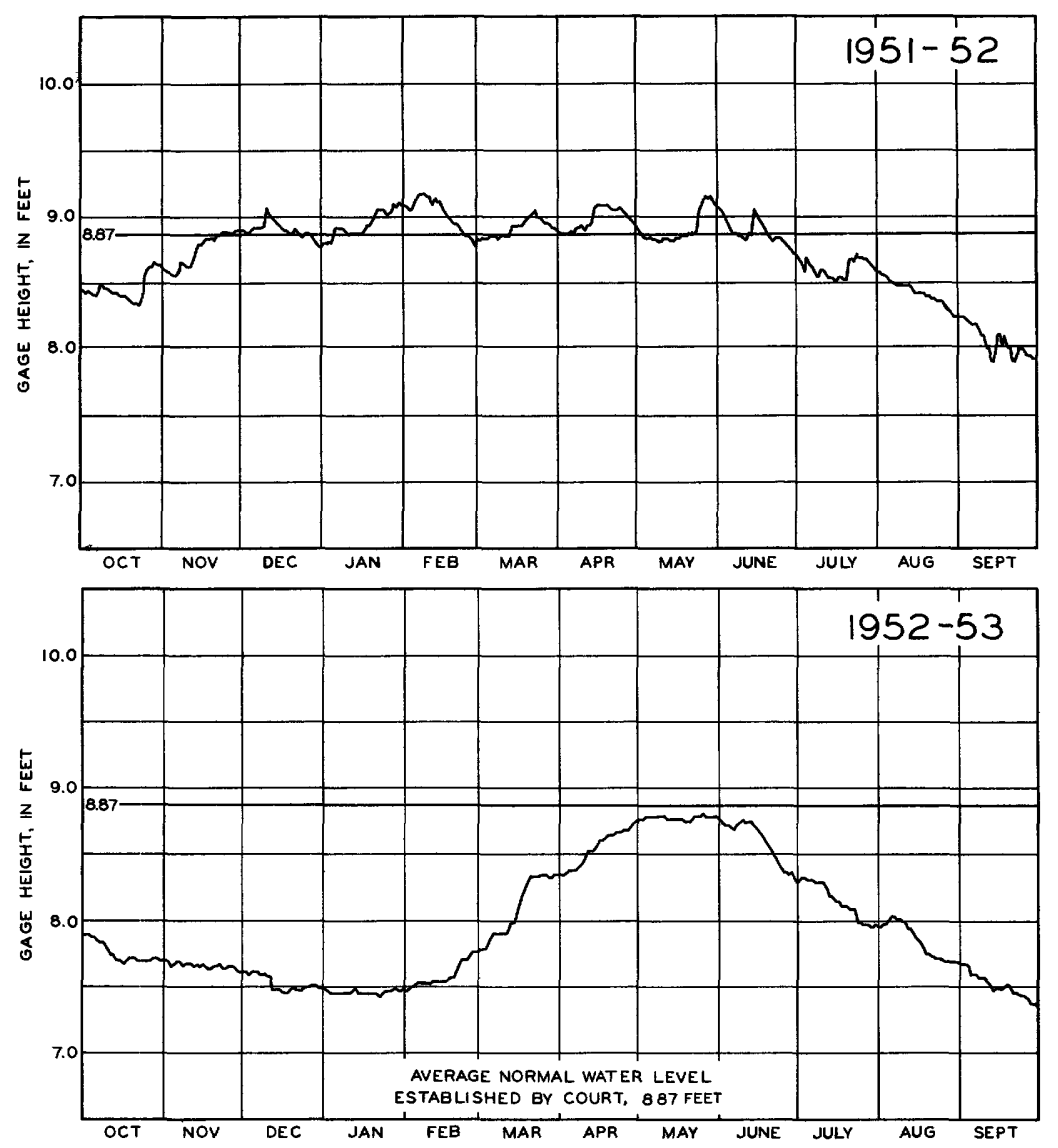

Figure 123. --Lake-level hydrographs for Syracuse Lake at Syracuse, Ind., for water years 1952-53. 
Tippecanoe Lake at Oswego, Ind.

Location.--Secs. 11, 12, and 14, T. 33 N., R. 6 E., and secs. 6, 7, $8,9,16,17$, and 18, T. 33 N. , R. 7 E. , Kosciusko, County.

Surface area. $--1,037$ acres, including area of Upper Tippecanoe or Lake James and Oswego Lake (determined from soil survey map of Kosciusko County).

Drainage area. $--118 \mathrm{sq} \mathrm{mi}$.

Records available. --October 1942 to September 1953.

Gage. - - Staff gage fastened to boat pier at Rookstool's home at west end of Oswego basin, at the head of outlet. Auxiliary headwater and tailwater gages are located at the outlet dam, about $800 \mathrm{ft}$ downstream from lake gage. Gages are read to hundredths once daily. Datum of gage is $830.00 \mathrm{ft}$ above mean sea level, datum of 1929 .

Average lake level. --11 years;gage height,6.50 ft; elevation, $836.50 \mathrm{ft}$.

Established legal level. --Established October 18, 1949, at gage height 6.40 ft; elevation, $836.40 \mathrm{ft}$ above mean sea level.

Lake-level control. - - Lake level controlled by a 56-ft concrete dam with 7-ft sluice ways. The average gage height of the dam sill is $4 \mathrm{ft}$. Lake level controlled by removable stop logs.

Extreme levels for Tippecanoe Lake, 1943-53

\begin{tabular}{|c|c|c|c|c|}
\hline \multicolumn{3}{|c|}{ Maximum } & \multicolumn{2}{|l|}{ Minimum } \\
\hline $\begin{array}{l}\text { Water } \\
\text { year }\end{array}$ & Date & $\begin{array}{l}\text { Gage } \\
\text { height } \\
\text { (feet) }\end{array}$ & Date & $\begin{array}{l}\text { Gage } \\
\text { height } \\
\text { (feet) }\end{array}$ \\
\hline 1943 & May 21, $1943 \ldots$ & 9.43 & Oct. 29, Nov. 5, 1942 & 6.32 \\
\hline 1944 & May $19,1944 \ldots$ & 8.64 & Aug. 27, $1944 \ldots$ & 5.36 \\
\hline 1945 & May 24, $1945 \ldots$ & 8.04 & $\begin{array}{l}\text { Oct. } 28 \text {, to Nov. 2, } \\
1944,\end{array}$ & 5.16 \\
\hline 1946 & Feb. 20, 1946 .... & 7.50 & Sept. $30,1946 \ldots$ & 5.20 \\
\hline 1947 & Apr. 26, 1947 & 7.92 & Oct. $16,1946 \ldots$ & 5.02 \\
\hline 1948 & Mar. 5, 1948 & 7.96 & Sjept. 27, $1948 \ldots-$ & 5.52 \\
\hline 1949 & Feb. 21, 1949- & 8.28 & $\begin{array}{l}\text { Oct. } 28 \text { to Nov. 2, } \\
1948-\end{array}$ & 5.46 \\
\hline 1950 & Apr. $10,1950 \cdots$ & 9.18 & Nov. 23, $1949 \ldots$ & 5.66 \\
\hline 1951 & Feb. 26, 27, 1951-- & 8.38 & Nov. $6,7,1950 \ldots$ & 5. 58 \\
\hline 1952 & Feb. $4,5,1952 \cdots$ & 8.02 & Oct. $21,22,1951 \cdots$ & 5. 58 \\
\hline 1953 & Mar. 23, 24, 1953-- & 7.02 & Nov. $14-18,1952--$ & 5.25 \\
\hline
\end{tabular}


BASIC DATA ON LAKE LEVELS FOR SELECTED LAKES 239

Maximum recorded thickness and periods of ice cover 1946-53

\begin{tabular}{|c|c|c|c|}
\hline $\begin{array}{l}\text { Water } \\
\text { year }\end{array}$ & $\begin{array}{l}\text { Maximum } \\
\text { thickness } \\
\text { (inches) }\end{array}$ & $\begin{array}{l}\text { Total } \\
\text { days of } \\
\text { cover }\end{array}$ & Period \\
\hline 1946 & ---- & ---- & Dec. 14,1945 , to $-?$ \\
\hline 1947 & 12.5 & 108 & Dec. 19,1946 , to Apr. 5, 1947. \\
\hline 1948 & 17 & 90 & $\begin{array}{l}\text { Nov. } 30 \text { to Dec. } 4 \text {; Dec. } 20,1947 \text {, } \\
\text { to Mar. } 19,1948 .\end{array}$ \\
\hline 1949 & --- & 76 & $\begin{array}{l}\text { Dec. } 26,1948 \text {, to Jan. } 16,1949 ; \\
\text { Jan. } 30 \text { to Mar. } 8 \text {; Mar. 16-21, } \\
\text { 1949. }\end{array}$ \\
\hline 1950 & 4 & 64 & $\begin{array}{l}\text { Dec. } 15-21,1949 \text {; Jan. } 19-25 \text {, } \\
\text { 1950; Feb. } 3 \text { to Mar. } 24,1950 .\end{array}$ \\
\hline 1951 & ---- & ---- & \\
\hline 1952 & ---- & $-\ldots-$ & Dec. 14,1951 , to $-?$ \\
\hline 1953 & ---- & ---- & $\begin{array}{l}\text { Dec. } 20,1952 \text {, to Jan. } 24,1953 \text {; } \\
\text { Jan. } 26,1953 \text {, to - ? }\end{array}$ \\
\hline
\end{tabular}

Discharge measurements at outlet of Tippecanoe Lake

\begin{tabular}{|c|c|c|c|c|c|c|}
\hline & Date & $\begin{array}{l}\text { Gage } \\
\text { height } \\
\text { (feet) }\end{array}$ & $\begin{array}{c}\text { Discharge } \\
\text { (cfs) }\end{array}$ & Date & $\begin{array}{l}\text { Gage } \\
\text { height } \\
\text { (feet) }\end{array}$ & $\begin{array}{c}\text { Discharge } \\
\text { (cfs) }\end{array}$ \\
\hline Aug. & $15,1943-$ & 6.80 & 89.0 & Aug. 25, 1948- & 5. 94 & 18.9 \\
\hline Oct. & $7 \ldots$ & 6.17 & 56.8 & Sept. 28-.... & 5. 54 & 14.6 \\
\hline Jan. & $8,1944-$ & 5. 56 & 41.2 & Nov. $30 \ldots$ & 5.64 & 33.0 \\
\hline Apr. & $21 \cdots \cdots$ & 8.50 & 455 & Dec. $23 \ldots-\ldots$ & 5.80 & 38.9 \\
\hline June & $13,1945-$ & 6.90 & 136 & Jan. $\quad 4,1949-$ & 6.26 & 74.7 \\
\hline Sept. & $8 \cdots-\cdots$ & 6.19 & 66.2 & $8---$ & 7.47 & 216 \\
\hline May & $22,1946-$ & 5. 78 & 51.2 & Mar. 23-.... & 6.70 & 124 \\
\hline June & $21 \ldots \ldots$ & 6.50 & 85.1 & May $3 \ldots \ldots$ & 6.15 & 76.3 \\
\hline July & $19 \cdots$ & 6.10 & 47. 3 & June 22-... & 7.44 & 170 \\
\hline Oct. & $23 \ldots \ldots$ & 5. 22 & 15.8 & Sept. $14 \ldots-\ldots$ & 5.92 & 16. 3 \\
\hline Nov. & $20 \cdots-\cdots$ & 5. 58 & 37.9 & Oct. $\quad 6 \ldots$ & 5.88 & 40.8 \\
\hline Dec. & $18 \cdots-$ & 5.47 & 33.1 & $7 \cdots-\cdots$ & 5.80 & 47.0 \\
\hline Jan. & $9,1947-$ & 5.70 & 41.7 & $7 \ldots$ & 5. 80 & 48.0 \\
\hline Feb. & $12 \ldots \ldots$ & 6.64 & 143 & $14,1950-$ & 8. 58 & 501 \\
\hline Feb. & $18 \cdots--$ & 6.44 & 85.9 & $15 \ldots \ldots$ & 8. 72 & 531 \\
\hline Apr. & $21 \ldots$ & 7.70 & 242 & $19 \ldots$ & 8.71 & 516 \\
\hline Apr. & $22 \ldots \ldots$ & 7. 86 & 262 & $25 \cdots \cdots$ & 8.50 & 445 \\
\hline Apr. & $29 \ldots$ & 7.78 & 251 & $1 \ldots$ & 8.74 & 533 \\
\hline July & $1 \ldots$ & 6.58 & 82.0 & $7 \ldots-\cdots$ & 7.97 & 305 \\
\hline Aug. & $28 \cdots \cdots$ & 5.60 & 26.4 & $6 \ldots-\cdots$ & 9.08 & 632 \\
\hline Nov. & $20 \ldots-\cdots$ & 5. 96 & 43.1 & $10-\ldots$ & 9.18 & 677 \\
\hline Mar. & $2,1948-$ & 7. 68 & 234 & $4 \ldots \ldots$ & 7.38 & 214 \\
\hline June & $9 \cdots$ & 6.74 & 84.5 & $8=----$ & 6.04 & 57.4 \\
\hline July & $2-\cdots--$ & 6.57 & 53.0 & $7-\cdots--$ & 6.70 & 98.8 \\
\hline
\end{tabular}


Discharge measurements at outlet of Tippecanoe Lake--Con.

\begin{tabular}{|c|c|c|c|c|c|}
\hline Date & $\begin{array}{l}\text { Gage } \\
\text { height } \\
\text { (feet) }\end{array}$ & Discharge & Date & $\begin{array}{l}\text { Gage } \\
\text { height } \\
\text { (feet) }\end{array}$ & $\begin{array}{c}\text { Discharge } \\
\text { (cfs) }\end{array}$ \\
\hline Aug. 22, $1950-$ & 6.42 & 76.0 & Apr. 17, 1952- & 7.52 & 259 \\
\hline Sept. $13 \ldots$ & 6.68 & 102 & May $8 \ldots \ldots$ & 6.64 & 130 \\
\hline Oct. $\quad 4 \ldots--$ & 6.18 & 67.5 & June $17 \ldots$ & 7.10 & 157 \\
\hline Oct. $31 \ldots$ & 5.78 & 46.2 & July $23 \ldots \ldots$ & 7.12 & 159 \\
\hline Nov. $29-\ldots$ & 6.28 & 84.2 & Aug. 14 & 6.30 & 67.5 \\
\hline Dec. $28 \ldots$ & 6.76 & 138 & Sept. $17 \ldots$ & 5.82 & 41.1 \\
\hline Jan. 31,1951 - & 6.66 & 130 & Oct. $\quad 7 \ldots$ & 6.01 & 59.5 \\
\hline Mar. $14 \ldots \ldots$ & 7.44 & 242 & Nov. $18 \ldots$ & 5.25 & 19.1 \\
\hline Apr. $24 \ldots-$ & 7.58 & 274 & Dec. $16 \ldots$ & 5.47 & 30.5 \\
\hline May $23 \ldots \ldots$ & 6.80 & 153 & Jan. 15, 1953- & 5.64 & 43.0 \\
\hline June $20 \ldots$ & 6.10 & 51.8 & Feb. $3 \ldots$ & 6.33 & 97.2 \\
\hline July $25 \ldots$ & 7.22 & 184 & Feb. $27 \ldots \ldots$ & 6.15 & 78.3 \\
\hline Aug. $29 \ldots$ & 6.66 & 106 & Mar. $26 \ldots$ & 6.98 & 181 \\
\hline Sept. $20-\cdots-$ & 5.96 & 53.3 & Apr. $17 \ldots$ & 6.54 & 114 \\
\hline Oct. $24 \ldots$ & 5. 86 & 61.9 & May $13 \ldots . .$. & 6.50 & 98.5 \\
\hline Nov. 28 & 6.58 & 126 & June $8 \ldots$ & 6.45 & 87.9 \\
\hline Dec. $18-\cdots-$ & 6.65 & 144 & $3----$ & 5.85 & 15.8 \\
\hline Feb. $\quad 4,1952-$ & 8.02 & 337 & July $27 \ldots \ldots$ & 5.73 & 8.40 \\
\hline Mar. $4 \ldots \ldots$ & 6.76 & 164 & Aug. $25 \ldots$ & 5. 76 & 8.11 \\
\hline Mar. 17 & 7.30 & 225 & Sept. 15 & 5.47 & 6.95 \\
\hline
\end{tabular}


Average lake levels for Tippecanoe Lake for water years 1943-53

\begin{tabular}{c|l|l|l|l|l|l|l}
\hline Year & Oct. & Nov. & Dec. & Jan. & Feb. & Mar. & Apr. \\
\hline 1943 & 6.38 & 6.72 & 6.76 & 7.43 & 7.17 & 7.36 & 7.14 \\
1944 & 6.09 & 6.00 & 5.82 & 5.48 & 5.57 & 6.68 & 8.02 \\
1945 & 5.32 & 5.32 & 5.39 & 5.41 & 5.49 & 6.51 & 7.10 \\
1946 & 6.85 & 6.13 & 5.76 & 6.77 & 6.75 & 7.08 & 6.32 \\
1947 & 5.17 & 5.54 & 5.43 & 5.75 & 6.45 & 6.32 & 7.46 \\
1948 & 6.00 & 5.88 & 6.32 & 6.56 & 6.43 & 7.57 & 7.40 \\
1949 & 5.56 & 5.63 & 5.74 & 7.02 & 7.77 & 7.10 & 6.38 \\
1950 & 6.06 & 5.79 & 6.14 & 8.35 & 8.21 & 8.09 & 8.64 \\
1951 & 6.08 & 5.86 & 7.04 & 6.98 & 7.04 & 7.42 & 7.34 \\
1952 & 5.81 & 6.40 & 6.68 & 7.49 & 7.59 & 7.10 & 7.29 \\
1953 & 5.71 & 5.34 & 5.51 & 5.84 & 6.21 & 6.61 & 6.53 \\
\hline Average & 5.91 & 5.87 & 6.05 & 6.64 & 6.79 & 7.08 & 7.24 \\
\hline \hline & & & & & & & \\
Year & May & June & July & Aug. & Sept. & Annual & \\
\hline 1943 & 8.33 & 7.50 & 7.04 & 6.79 & 6.55 & 7.14 & \\
1944 & 8.05 & 6.94 & 5.80 & 5.55 & 5.44 & 6.30 & \\
1945 & 7.22 & 6.98 & 6.22 & 6.48 & 6.10 & 6.18 & \\
1946 & 5.75 & 6.03 & 6.22 & 5.65 & 5.34 & 6.20 & \\
1947 & 7.36 & 6.95 & 6.28 & 5.71 & 5.88 & 6.19 & \\
1948 & 7.28 & 6.72 & 6.43 & 6.04 & 5.69 & 6.53 & \\
1949 & 6.30 & 6.97 & 6.69 & 6.00 & 5.92 & 6.41 & \\
1950 & 6.83 & 6.64 & 6.59 & 6.48 & 6.52 & 7.02 & \\
1951 & 6.79 & 6.24 & 6.96 & 6.58 & 6.09 & 6.70 & \\
1952 & 6.84 & 7.02 & 6.59 & 6.27 & 5.95 & 6.75 & \\
1953 & 6.56 & 6.15 & 5.76 & 5.86 & 5.47 & 5.97 & \\
\hline Average & 7.03 & 6.74 & 6.41 & 6.13 & 5.90 & 6.50 & \\
\hline & & & & & & & \\
\hline
\end{tabular}



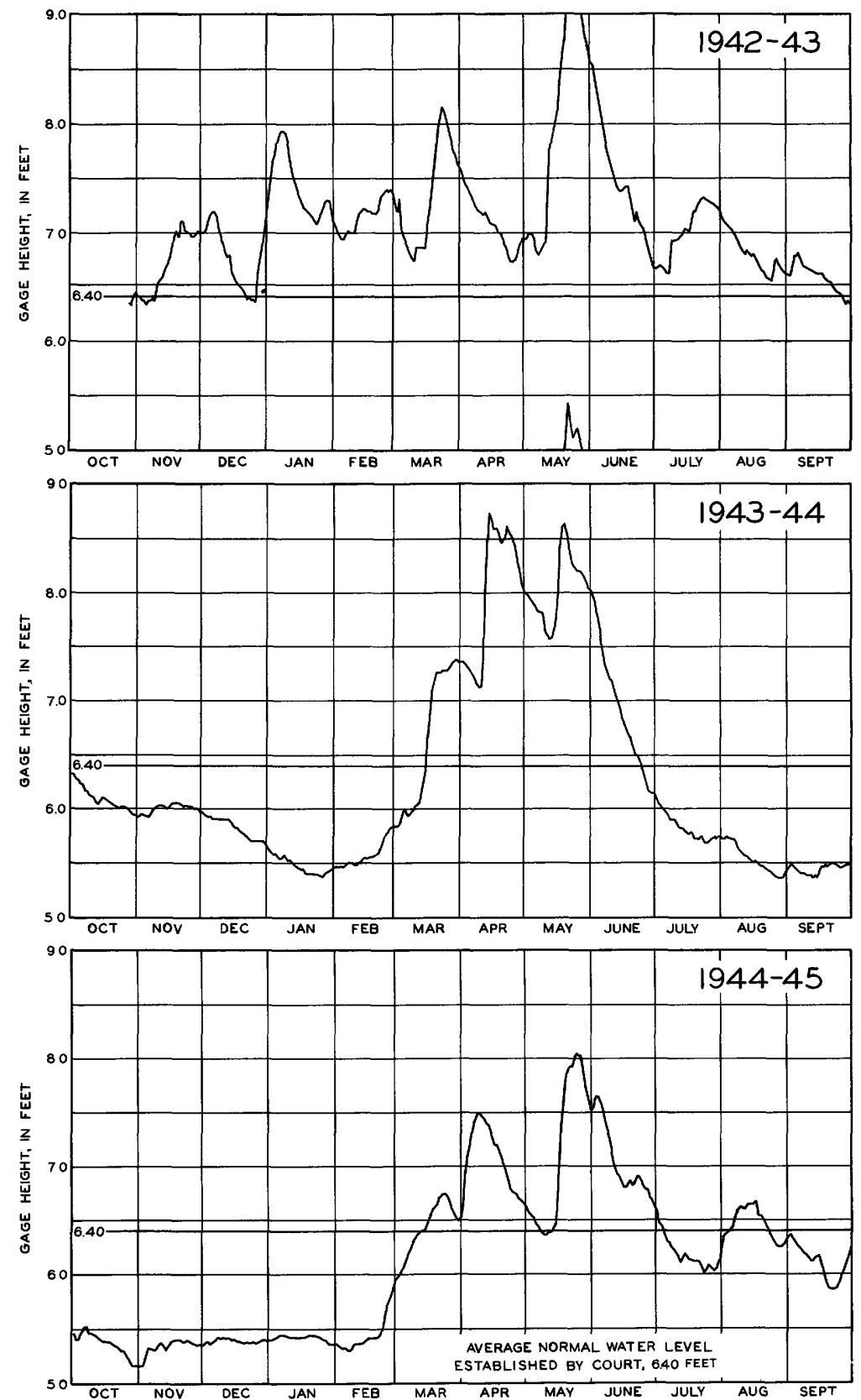

Figure 124. --Lake-level hydrographs for Tippecanoe Lake at Oswego, Ind., for water years 1943-45. 

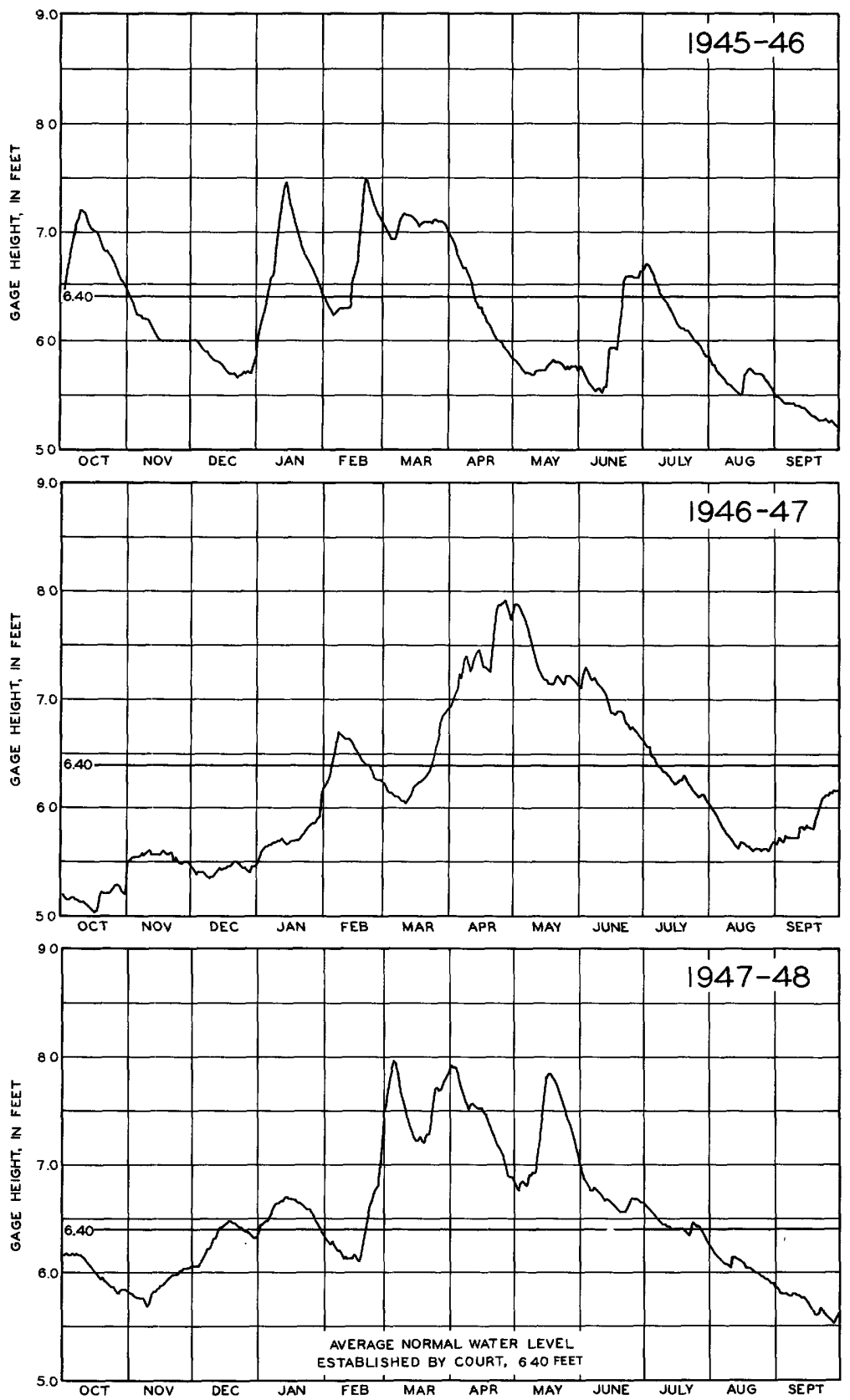

Figure 125. - -Lake-level hydrographs for Tippecanoe Lake at Oswego, Ind., for water years 1946-48. 

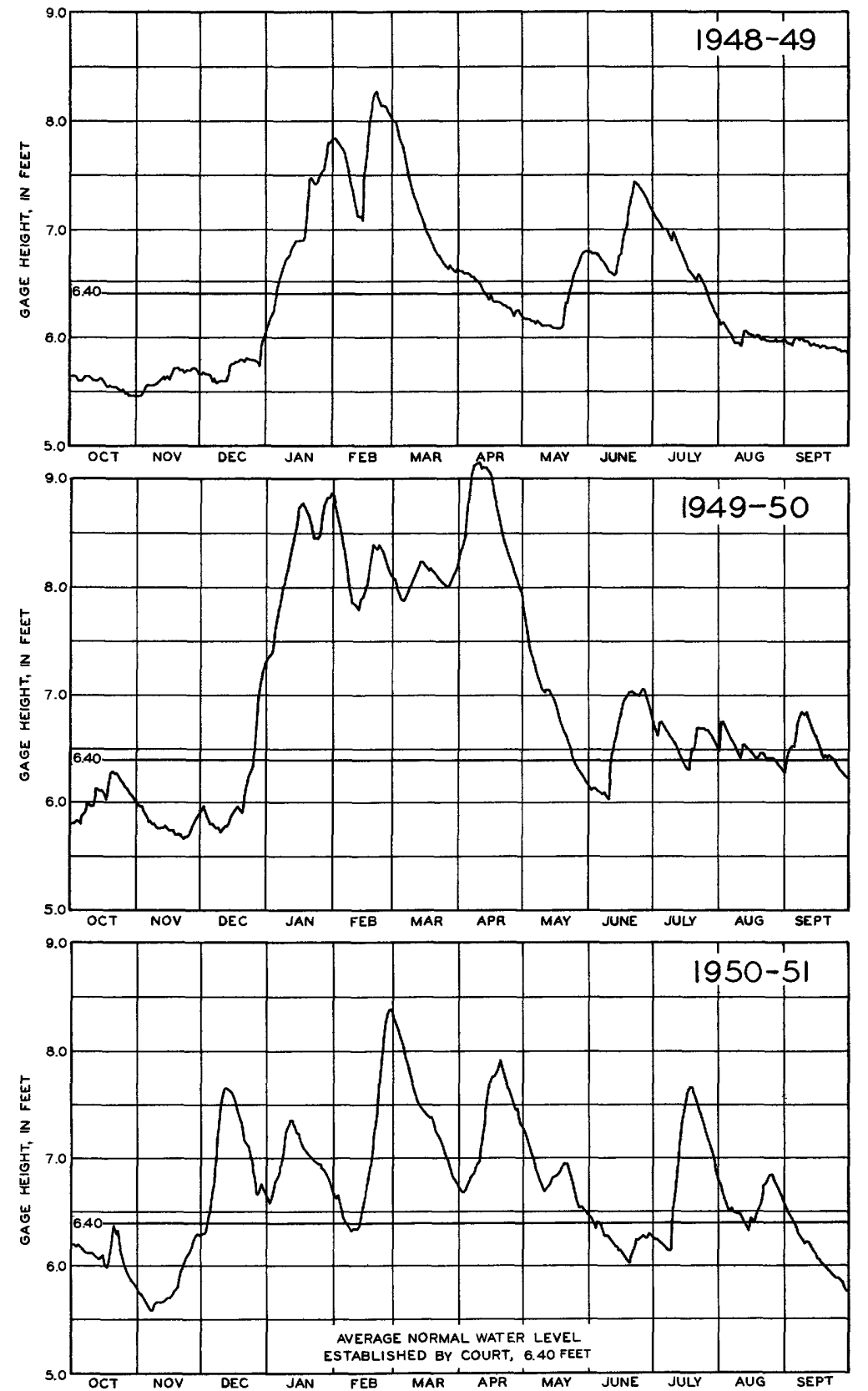

Figure 126. --Lake-level hydrographs for Tippecanoe Lake at Oswego, Ind., for water years 1949-51. 


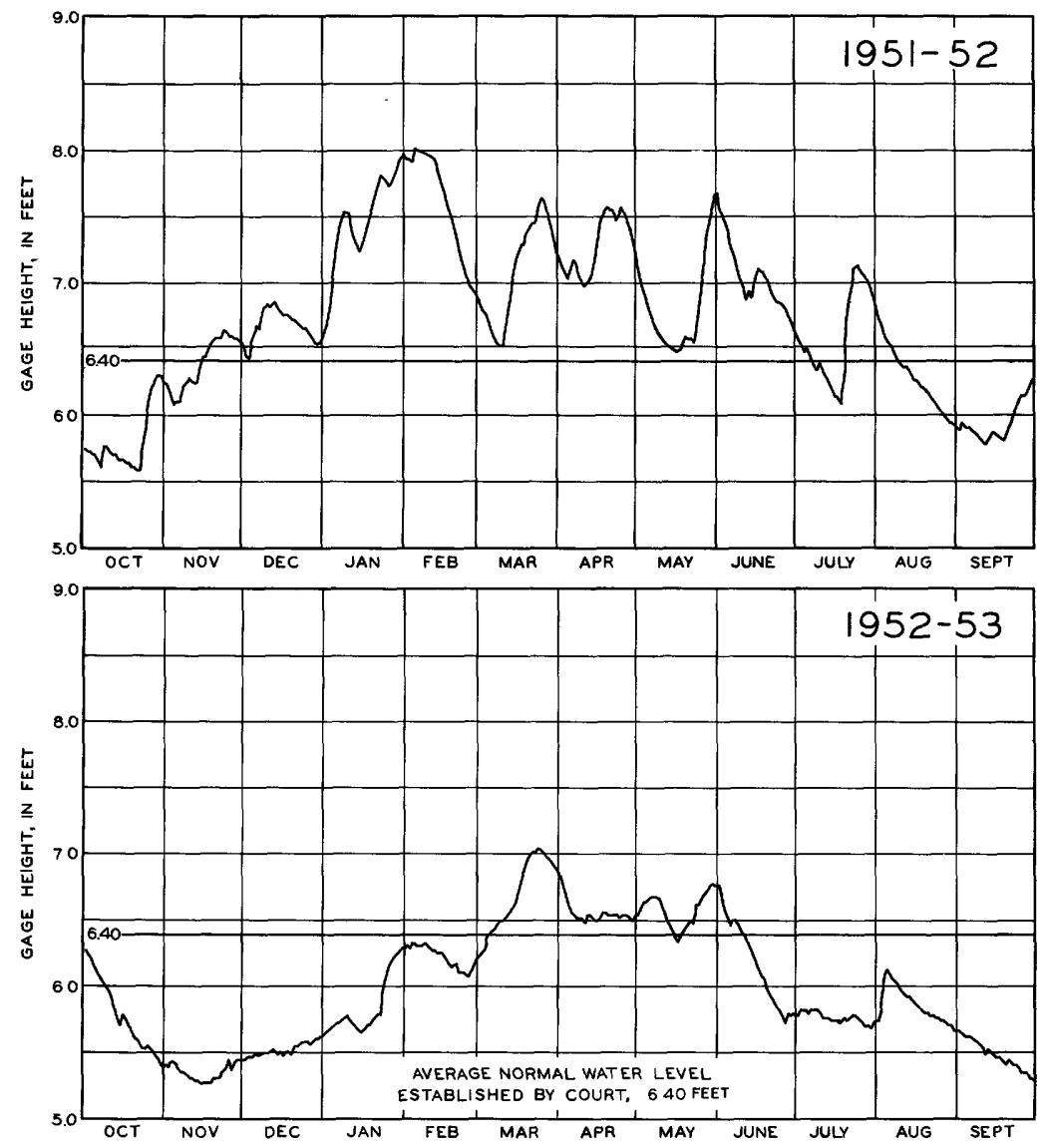

Figure 127. --Lake-level hydrographs for Tippecanoe Lake at O swego, Ind., for water years 1952-53.

Lake Wawasee near Wawasee, Ind.

Location. --Secs. 8, 9, 10, 11, 13, 14, 15, 16, 17, 22, 23, 24, 25, 26, T.

34 N., R. 7 E., Kosciusko County, 3.0 miles southeast of Wawasee. Surface area. $--2,168$ acres.

Drainage area. $--37.3 \mathrm{sq} \mathrm{mi}$.

Records available.--October 1942 to September 1953.

Gage.--Staff gage on middle pier of Wawasee Fish Hatcheryboat

house. Gage read to hundredths once daily. Datum of gage is

$850.00 \mathrm{ft}$ above mean sea level, datum of 1929 .

Average lake level. --11 years; gage height, $8.54 \mathrm{ft}$; elevation, $858.54 \mathrm{ft}$.

Established legal level. --Established September 20,1948, at gage

height $8.89 \mathrm{ft}$; elevation, $858.89 \mathrm{ft}$ above mean sea level.

Lake level control. - - Lake level cont rolled by dam at outlet of Syra-

cuse Lake. Syracuse Lake and Wawasee Lake are at same level except for slight drop through channel from Wawasee to Syracuse Lake. 
Extreme levels for Lake Wawasee, 1943-53

\begin{tabular}{|c|c|c|c|c|}
\hline \multicolumn{3}{|c|}{ Maximum } & \multicolumn{2}{|l|}{ Minimum } \\
\hline $\begin{array}{l}\text { Water } \\
\text { year }\end{array}$ & Date & $\begin{array}{c}\text { Gage } \\
\text { height } \\
\text { (feet) }\end{array}$ & Date & $\begin{array}{l}\text { Gage } \\
\text { height } \\
\text { (feet) }\end{array}$ \\
\hline 1943 & May 25, 26, 1943 $\ldots$ & 10.00 & Dec. $26,1942 \ldots$ & 8.36 \\
\hline 1944 & Mar. 17-19, $1944 \ldots$ & 9.40 & Sept. 24, 27-30, 1944 & 7.49 \\
\hline 1945 & May $18-20,1945 \ldots$ & 9.08 & Nov. $1-5,1944 \ldots$ & 7. 27 \\
\hline 1946 & July 1,1946 & 9.13 & Sept. $30,1946 \ldots$ & 7.43 \\
\hline 1947 & Apr. $22-24,1947 \ldots$ & 9.43 & Oct. $17,30,1946 \ldots$ & 7.11 \\
\hline 1948 & Mar. 7-10, $1948 \ldots$ & 9.12 & Sept. $28,29,1948 \ldots$ & 7.54 \\
\hline 1949 & July $11,1949 \ldots$ & 9.58 & Oct. $28-31,1948 \ldots$ & 7. 14 \\
\hline 1950 & Jan. 27, 28, 1950 & 10.22 & Dec. $10,11,1949 \ldots$ & 8.24 \\
\hline 1951 & Feb. 24-27, $1951 \ldots$ & 9.18 & Sept. $30,1951 \ldots$ & 8.46 \\
\hline 1952 & Feb. $10,11,1952$ & 9.31 & Sept. $30,1952 \ldots$ & 7.93 \\
\hline 1953 & May 23, 1953 $\ldots$ & 8.90 & Nov. $15-18,1952 \ldots$ & 7.28 \\
\hline
\end{tabular}

Maximum recorded thickness and periods of ice cover 1943-51

\begin{tabular}{|c|c|c|c|}
\hline $\begin{array}{l}\text { Water } \\
\text { years }\end{array}$ & $\begin{array}{l}\text { Maximum } \\
\text { thickness } \\
\text { (inches) }\end{array}$ & $\begin{array}{l}\text { Total } \\
\text { days of } \\
\text { cover }\end{array}$ & Periods \\
\hline 1943 & 14 & $-\ldots-$ & \\
\hline 1944 & 10 & $-\cdots$ & $\begin{array}{l}\text { Nov. } 29,1943 \text {, to - ?; Dec. } 14 \text {, } \\
1943 \text {, to - ? }\end{array}$ \\
\hline 1945 & 2 & ---- & Dec. 2,1944 , to - ? \\
\hline 1946 & 11 & ---- & Unknown to Mar. 6, 1946. \\
\hline 1947 & 14 & $-\cdots$ & Unknown to Mar. 29, 1947. \\
\hline 1948 & 21 &.--- & Unknown to Mar. $19,1948$. \\
\hline 1949 & 7 & 80 & Dec. 9,1948 , to Feb. 26, 1949. \\
\hline 1950 & 11 & $-\ldots$ & Dec. 10,1949 , to - ? \\
\hline 1951 & 4 & ---- & \\
\hline
\end{tabular}

Discharge measurements. --Wawasee Lake flows into Syracuse Lake. Discharge measurements are made at outlet of Syracuse Lake. 
Average lake levels for Lake Wawasee for water years 1943-53

\begin{tabular}{c|c|c|c|c|c|c|c}
\hline Year & Oct. & Nov. & Dec. & Jan. & Feb. & Mar. & Apr. \\
\hline 1943 & 8.95 & 9.16 & 8.69 & 8.67 & 8.47 & 8.84 & 8.87 \\
1944 & 8.63 & 8.67 & 8.59 & 8.54 & 8.69 & 9.10 & 9.09 \\
1945 & 7.44 & 7.35 & 7.40 & 7.47 & 7.55 & 8.14 & 8.78 \\
1946 & 8.80 & 8.66 & 8.73 & 8.75 & 8.71 & 8.64 & 8.60 \\
1947 & 7.22 & 7.35 & 7.33 & 7.46 & 7.88 & 8.08 & 9.02 \\
1948 & 8.01 & 7.90 & 8.06 & 8.39 & 8.52 & 9.05 & 9.00 \\
1949 & 7.32 & 7.37 & 7.43 & 7.98 & 8.89 & 9.13 & 8.86 \\
1950 & 8.50 & 8.37 & 8.47 & 9.73 & 9.43 & 8.91 & 9.56 \\
1951 & 8.73 & 8.68 & 9.03 & 8.90 & 8.06 & 8.94 & 8.98 \\
1952 & 8.46 & 8.77 & 9.00 & 9.03 & 9.13 & 8.96 & 9.06 \\
1953 & 7.63 & 7.35 & 7.42 & 7.47 & 7.71 & 8.28 & 8.67 \\
\hline Average & 8.15 & 8.15 & 8.20 & 8.40 & 8.53 & 8.73 & 8.95 \\
\hline \hline & & & & & & & \\
Year & May & June & July & Aug. & Sept. & Annual & \\
\hline 1943 & 9.60 & 9.21 & 9.11 & 8.99 & 8.78 & 8.95 & \\
1944 & 8.97 & 8.80 & 8.36 & 7.86 & 7.60 & 8.58 & \\
1945 & 8.88 & 8.92 & 8.75 & 8.72 & 8.68 & 8.17 & \\
1946 & 8.59 & 8.78 & 8.77 & 8.15 & 7.65 & 8.57 & \\
1947 & 9.14 & 9.00 & 8.64 & 8.23 & 8.20 & 8.13 & \\
1948 & 8.94 & 8.89 & 8.84 & 8.44 & 7.81 & 8.49 & \\
1949 & 8.99 & 9.22 & 9.42 & 9.07 & 8.54 & 8.52 & \\
1950 & 8.79 & 8.84 & 9.02 & 8.96 & 8.87 & 8.95 & \\
1951 & 8.92 & 8.81 & 8.86 & 8.77 & 8.63 & 8.84 & \\
1952 & 8.96 & 8.94 & 8.70 & 8.48 & 8.11 & 8.80 & \\
1953 & 8.84 & 8.68 & 8.21 & 7.93 & 7.50 & 7.97 & \\
\hline Average & 8.97 & 8.92 & 8.79 & 8.51 & 8.22 & 8.62 & \\
\hline
\end{tabular}




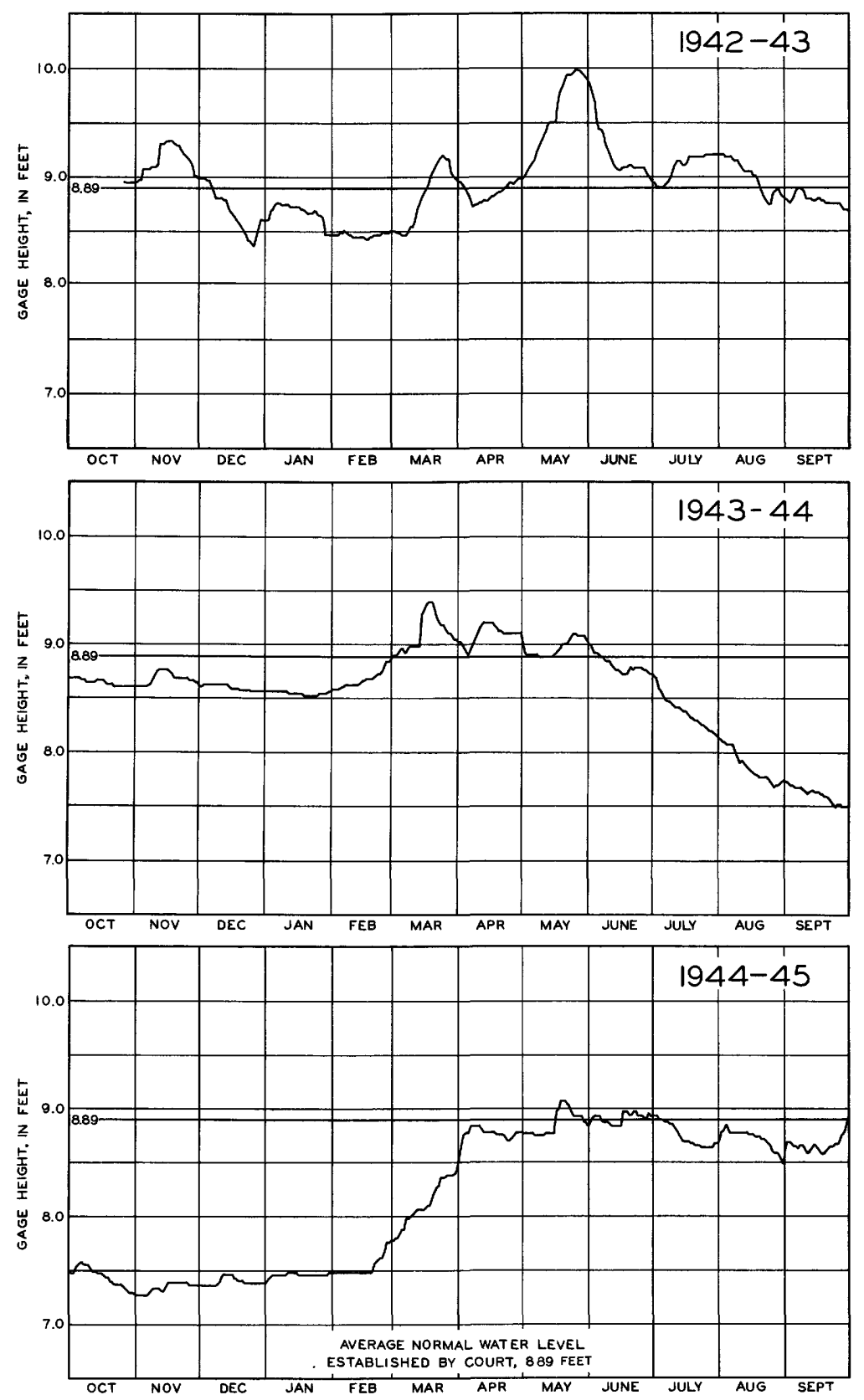

Figure 128. - Lake-level hydrographs for Lake Wawasee near Wawasee, Ind., for water years 1943-45. 

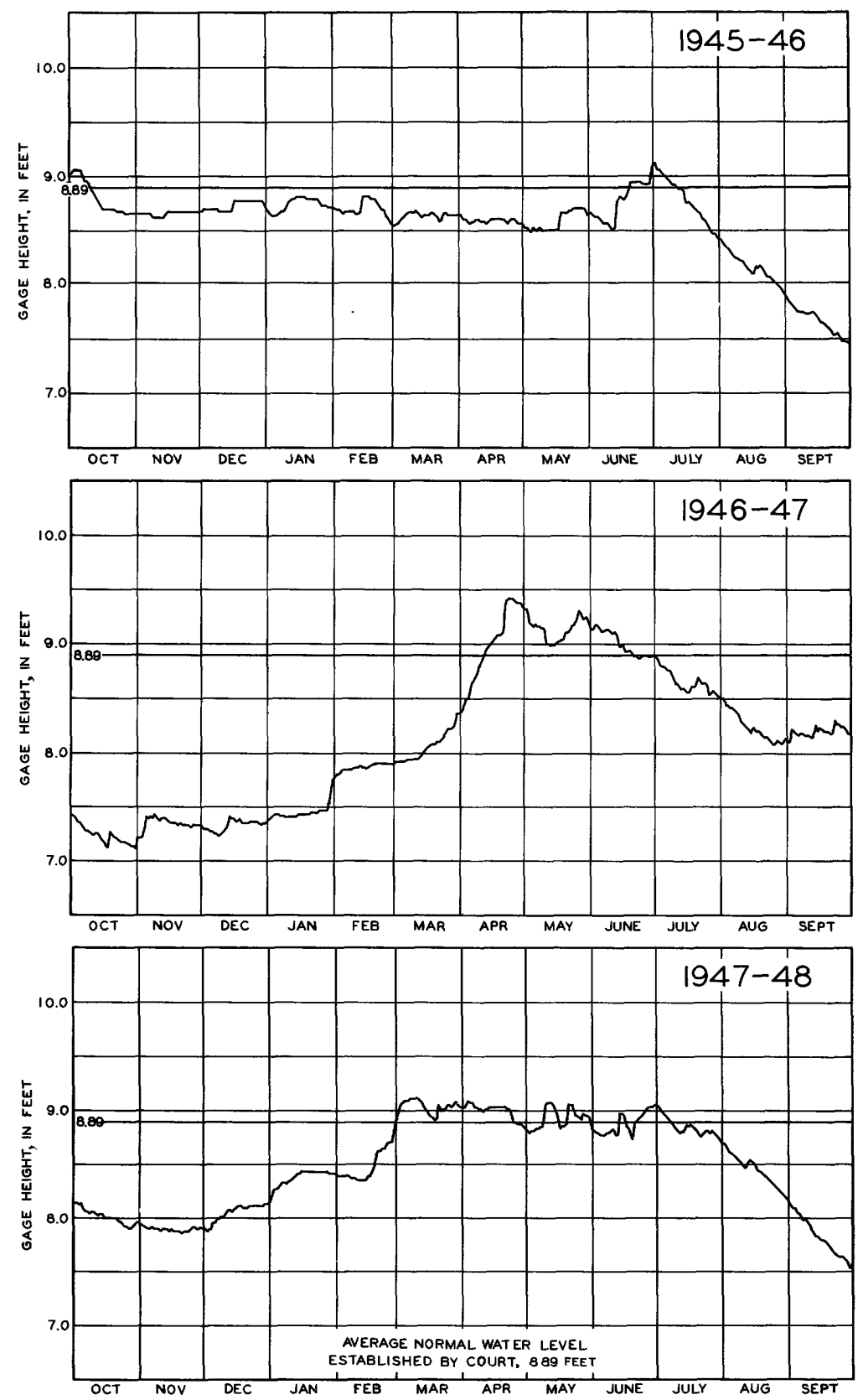

Figure 129. --Lake-level hydrographs for Lake Wawasee near Wawasee, Ind., for water years 1946-48. 

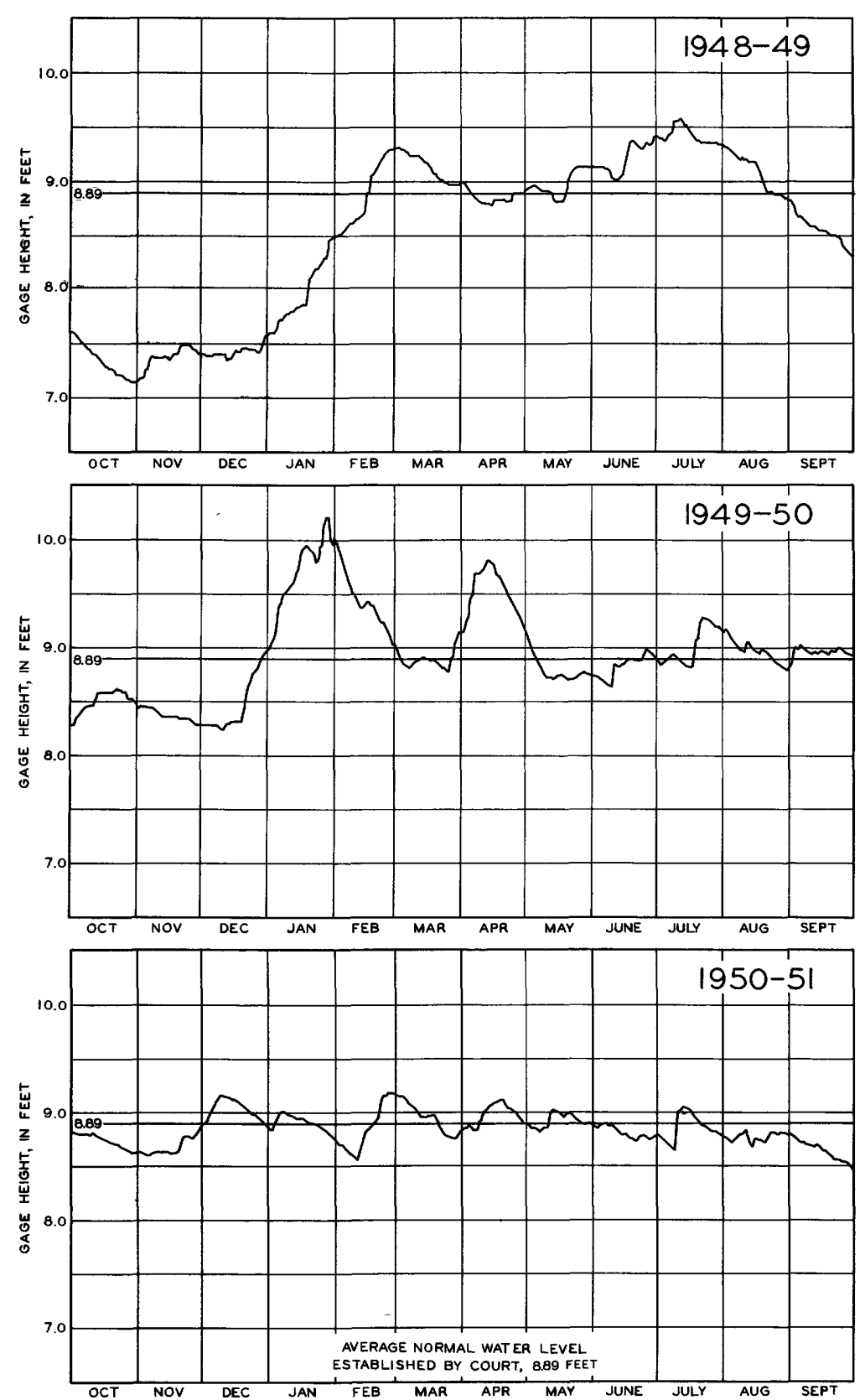

Figure 130. --Lake-level hydrographs for Lake Wawasee near Wawasee, Ind., for water years 1949-51. 
BASIC DATA ON LAKE LEVELS FOR SELECTED LAKES 251

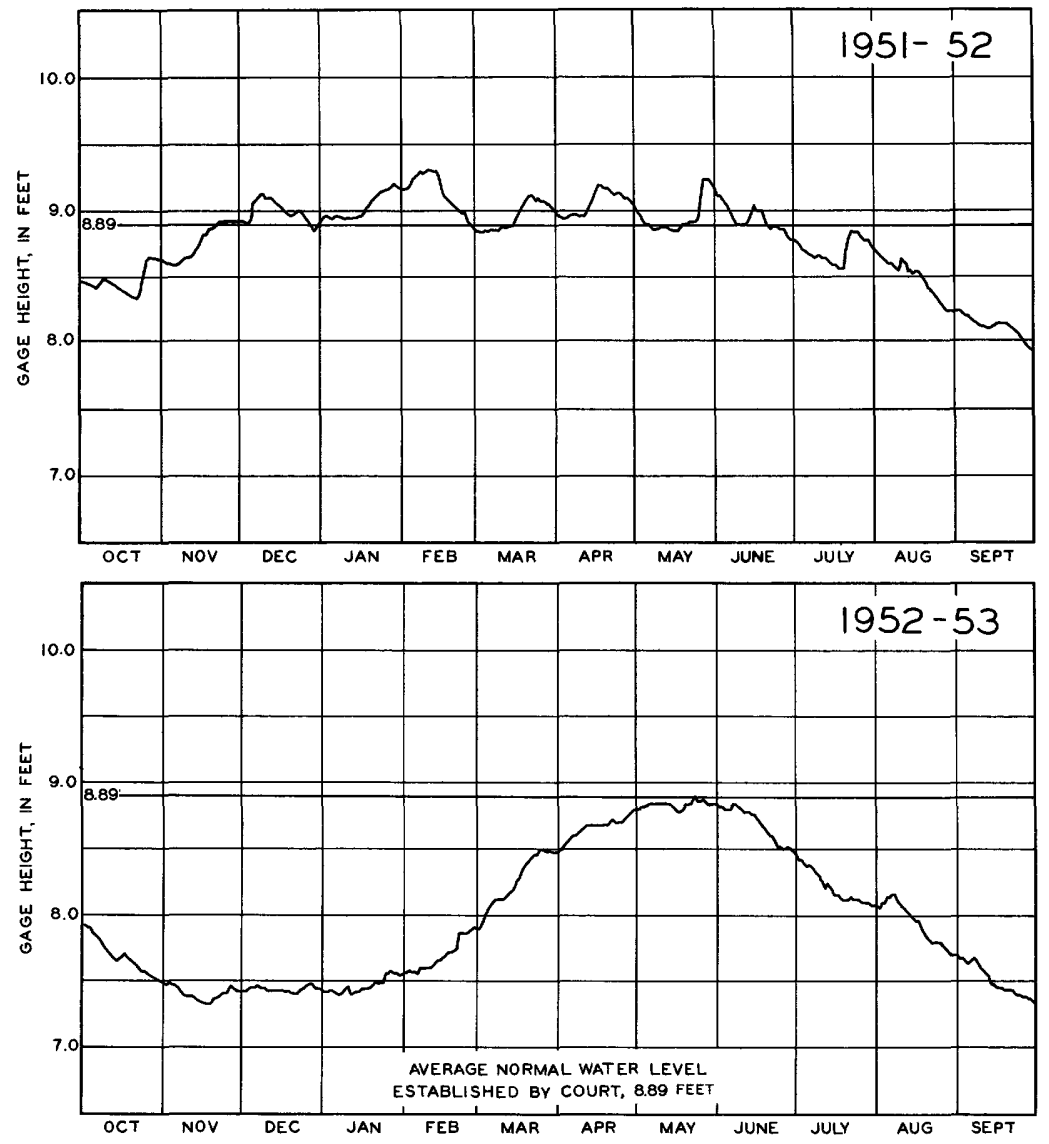

Figure 131. --Lake-level hydrographs for Lake',Wawasee near Wawasee, Ind., for water years 1952-53. 
Webster Lake at North Webster, Ind.

Location. --Secs. 10,11,12,13,14, and 15, T. 33 N., R. 7 E., Kosciusko County.

Surface area. --566 acres.

Drainage area. $--54.0 \mathrm{sq} \mathrm{mi}$.

Records available. - October 1942 to September 1953.

Gage. --Staff gage in lake bed on west shore front of Weghorst cottage. Auxiliary gage on abutment of concrete control structure at southwest corner of lake. Gage read to hundredths once daily. Datum of gage is $840.00 \mathrm{ft}$ above mean sea level, datum of 1929 .

Average lake level. --11 years; gage height,12.41 ft; elevation, $852.41 \mathrm{ft}$.

Established legal level. --None.

Lake level control. -- Lake level controlled by two outlet structures. Main structure is 35- $\mathrm{ft}$ concrete notch dam, with seven 5-ft wooden, adjustable sluice gates resting on the sill at gage height $9.5 \mathrm{ft}$. The auxiliary structure is $20-\mathrm{ft}$ concrete notch dam with five $4-\mathrm{ft}$ wooden adjustable sluice gates resting on the sill (this structure is the old mill dam) at gage height $8.2 \mathrm{ft}$.

Extreme levels for Webster Lake, 1943-53

\begin{tabular}{|c|c|c|c|c|}
\hline \multicolumn{3}{|c|}{ Maximum } & \multicolumn{2}{|l|}{ Minimum } \\
\hline $\begin{array}{c}\text { Water } \\
\text { year }\end{array}$ & Date & $\begin{array}{l}\text { Gage } \\
\text { height } \\
\text { (feet) }\end{array}$ & Date & $\begin{array}{l}\text { Gage } \\
\text { height } \\
\text { (feet) }\end{array}$ \\
\hline 1943 & Mar. 20, May 19,1943 & 13.51 & Aug. 24, $1943 \ldots$ & 12.27 \\
\hline 1944 & May $16,1944 \ldots$ & 13.51 & Feb. 1-5, $1944 \ldots$ & $* 10.75$ \\
\hline 1945 & June 18, Aug. 5-6,1945 & 13.27 & Dec. $10,1944 \ldots$ & 11.01 \\
\hline 1946 & $\begin{array}{r}\text { Oct. 11, Dec. 31, } 1945 \text {, } \\
\text { Feb. 16, } 1946\end{array}$ & 13.33 & Sept. $30,1946 \ldots$ & $* 11.55$ \\
\hline 1947 & June 1, $1947 \ldots$ & 13.11 & Oct. $17,1946 \ldots$ & 11.43 \\
\hline 1948 & May $11,12,1948 \ldots$ & 13.17 & Oct. $25,26,1947--$ & 11.17 \\
\hline 1949 & June $18,1949 \ldots$ & 13.39 & Oct. $21-24,1948--$ & 11.25 \\
\hline 1950 & Apr. $5,1950 \ldots$ & 13.26 & Feb. 5, $1950 \ldots$ & 11. 38 \\
\hline 1951 & Dec. $7,1950 \ldots$ & 13.32 & Oct. $20,1950^{-\ldots-\cdots}$ & 11.94 \\
\hline 1952 & Jan. $3,1952 \ldots$ & 13.24 & Sept. 30, 1952 $\cdots$ & 10.58 \\
\hline 1953 & May 26, $1953 \ldots$ & 13.04 & $\begin{array}{r}\text { Oct. } 4 \text { to Nov. } 14, \\
1952\end{array}$ & $* 10.16$ \\
\hline
\end{tabular}

*Estimated. 
Maximum recorded thickness and periods of ice cover 1943-53

\begin{tabular}{|c|c|c|c|}
\hline $\begin{array}{l}\text { Water } \\
\text { year }\end{array}$ & $\begin{array}{r}\text { Maximum } \\
\text { thickness } \\
\text { (inches) }\end{array}$ & $\begin{array}{c}\text { Total } \\
\text { days of } \\
\text { cover }\end{array}$ & Period \\
\hline 1943 & $\ldots--$ & $-\cdots$ & $\begin{array}{l}\text { Dec. } 6,1942 \text {, to Jan. } 1,1943 \text {; Jan. } 3 \text {, } \\
1943 \text {, to - ? }\end{array}$ \\
\hline 1944 & 2 & ---- & \\
\hline 1945 & $-\cdots$ & --- & Dec. 2,1944, to $-?$ \\
\hline 1946 & 12 & 91 & Dec. 6, 1945, to Mar. 6, 1946. \\
\hline 1947 & 14 & 107 & Dec. 17,1946, to Apr. $3,1947$. \\
\hline 1948 & 18 & 104 & $\begin{array}{l}\text { Nov. } 30 \text { to Dec. } 3 ; \text { Dec. } 12,1947 \text {, } \\
\text { to Mar. } 20,1948 .\end{array}$ \\
\hline 1949 & $6 \frac{1}{4}$ & 73 & $\begin{array}{l}\text { Dec. } 19-21 \text {; Dec. } 26,1948 \text {, to Mar. } \\
\text { 5, } 1949 .\end{array}$ \\
\hline 1950 & 5 & $\cdots--$ & $\begin{array}{l}\text { Dec. } 9-21,1949 ; \text { Jan. } 19 \text { to Mar. } 26 \text {, } \\
1950 .\end{array}$ \\
\hline 1951 & 10 & $-\cdots-$ & Dec. 11,1950, to $-?$ \\
\hline 1952 & ---- & 105 & Dec. 14, 1951, to Mar. 27, 1952. \\
\hline 1953 & $-\cdots--$ & $-\cdots--$ & Unknown to Mar. 10, 1953. \\
\hline
\end{tabular}

Discharge measurements at outlet of Webster Lake

\begin{tabular}{|c|c|c|c|c|c|c|}
\hline & Date & $\begin{array}{l}\text { Gage } \\
\text { height } \\
\text { (feet) }\end{array}$ & $\begin{array}{c}\text { Discharge } \\
\text { (cfs) }\end{array}$ & Date & $\begin{array}{l}\text { Gage } \\
\text { height } \\
\text { (feet) }\end{array}$ & $\begin{array}{c}\text { Discharge } \\
\text { (cfs) }\end{array}$ \\
\hline Aug. & 14,1943 & 12.49 & 23.4 & June $20,1946-$ & 12.96 & 94.4 \\
\hline Oct. & $7 \ldots \ldots$ & 11.99 & 54.3 & July $19 \ldots$ & 12.35 & $a_{42.8}$ \\
\hline Jan. & 8,1944 & 10.97 & 50.0 & Feb. 19, 1947- & 12.74 & 25.8 \\
\hline Apr. & $22 \ldots \ldots$ & 13.09 & 224 & Jan. 19,1950 - & 11.92 & 261 \\
\hline June & 13,1945 & 13.11 & 63.8 & Jan. $26 \ldots$ & 12.37 & 309 \\
\hline Sept. & $6 \ldots$ & 12.40 & 28.6 & bet. $24 \ldots-$ & 12.04 & b 4.19 \\
\hline May & 7,1946 & 12.56 & 18.6 & May 10,1951 - & 12.58 & 33.3 \\
\hline
\end{tabular}

a Leakage from gates.

b Combined discharge of Lake Dam, Mill Dam, and leakage. 
Average lake levels for Webster Lake for water years 1943-53

\begin{tabular}{l|c|c|c|c|c|c|c}
\hline Year & Oct. & Nov. & Dec. & Jan. & Feb. & Mar. & Apr. \\
\hline 1943 & 12.82 & 13.06 & 13.09 & 13.15 & 12.98 & 12.69 & 12.73 \\
1944 & 11.91 & 11.63 & 11.38 & 10.88 & 10.85 & 11.87 & 13.12 \\
1945 & 11.49 & 11.32 & 11.15 & 11.20 & 11.23 & 12.29 & 12.80 \\
1946 & 13.23 & 13.24 & 13.21 & 12.86 & 12.80 & 12.69 & 12.66 \\
1947 & 11.50 & 11.83 & 11.87 & 11.87 & 12.67 & 12.63 & 12.75 \\
1948 & 11.44 & 11.36 & 11.73 & 12.23 & 12.24 & 12.73 & 12.83 \\
1949 & 11.38 & 11.61 & 11.84 & 12.99 & 12.80 & 12.89 & 12.84 \\
1950 & 12.81 & 12.76 & 12.29 & 12.18 & 12.19 & 12.10 & 12.47 \\
1951 & 12.47 & 12.78 & 12.70 & 12.70 & 12.62 & 12.32 & 12.66 \\
1952 & 12.65 & 12.76 & 12.82 & 12.72 & 12.16 & 12.58 & 12.77 \\
1953 & 10.17 & 10.36 & 11.73 & 12.37 & 12.20 & 12.43 & 12.70 \\
\hline Average & 11.99 & 12.06 & 12.16 & 12.29 & 12.25 & 12.47 & 12.76 \\
\hline \hline Year & May & June & July & Aug. & Sept. & Annual & \\
\hline 1943 & 12.91 & 12.90 & 12.87 & 12.55 & 12.50 & 12.86 & \\
1944 & 13.18 & 13.11 & 12.49 & 12.04 & 11.72 & 12.02 & \\
1945 & 13.08 & 13.14 & 12.91 & 13.06 & 12.41 & 12.18 & \\
1946 & 12.69 & 12.99 & 12.45 & 11.74 & 11.62 & 12.68 & \\
1947 & 13.00 & 12.92 & 12.09 & 11.63 & 12.12 & 12.23 & \\
1948 & 12.97 & 12.78 & 12.04 & 11.87 & 11.82 & 12.17 & \\
1949 & 12.78 & 13.03 & 12.18 & 12.24 & 12.49 & 12.42 & \\
1950 & 12.74 & 12.85 & 12.86 & 12.86 & 12.74 & 12.58 & \\
1951 & 12.71 & 12.77 & 12.82 & 12.76 & 12.58 & 12.66 & \\
1952 & 12.80 & 12.78 & 12.77 & 12.73 & 12.13 & 12.64 & \\
1953 & 12.83 & 12.71 & 12.74 & 12.74 & 12.71 & 12.14 & \\
\hline Average & 12.88 & 12.91 & 12.56 & 12.38 & 12.26 & 12.41 & \\
\hline
\end{tabular}



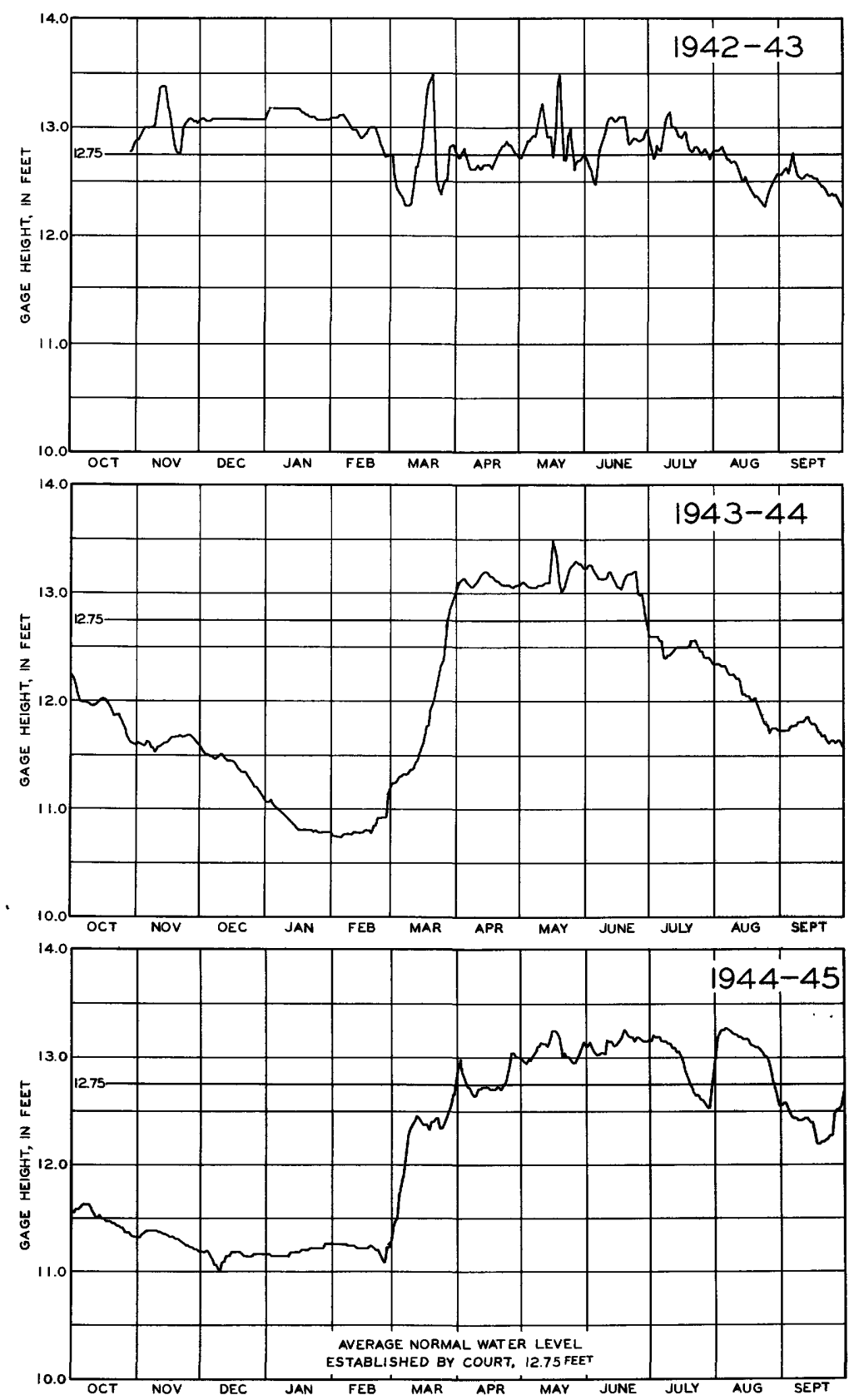

Figure 132. --Lake-level hydrographs for Webster Lake at North Webster, Ind., for water years 1943-45. 

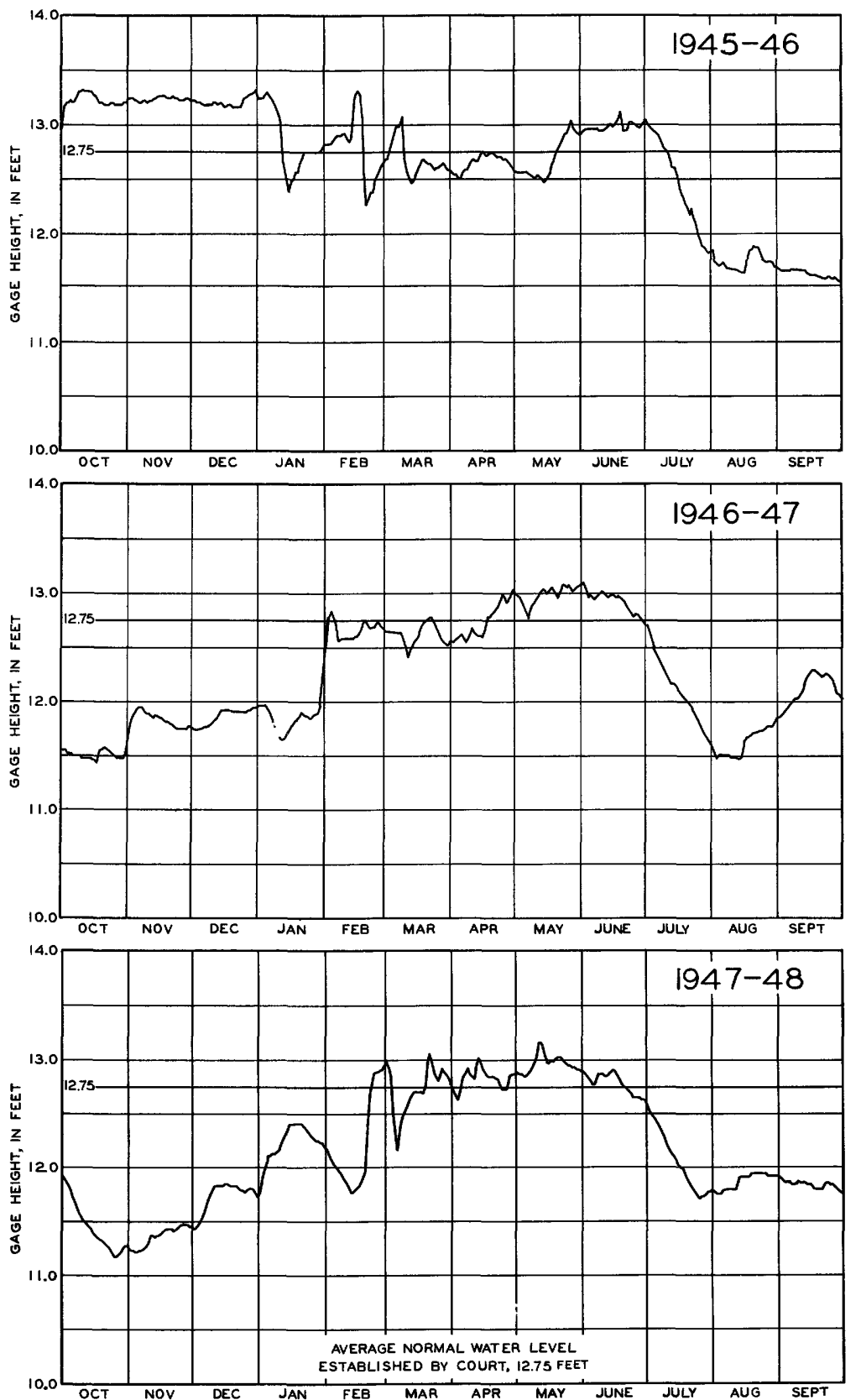

Figure 133. --Lake-level hydrographs for Webster Lake at North Webster, Ind., for water years 1946-48. 

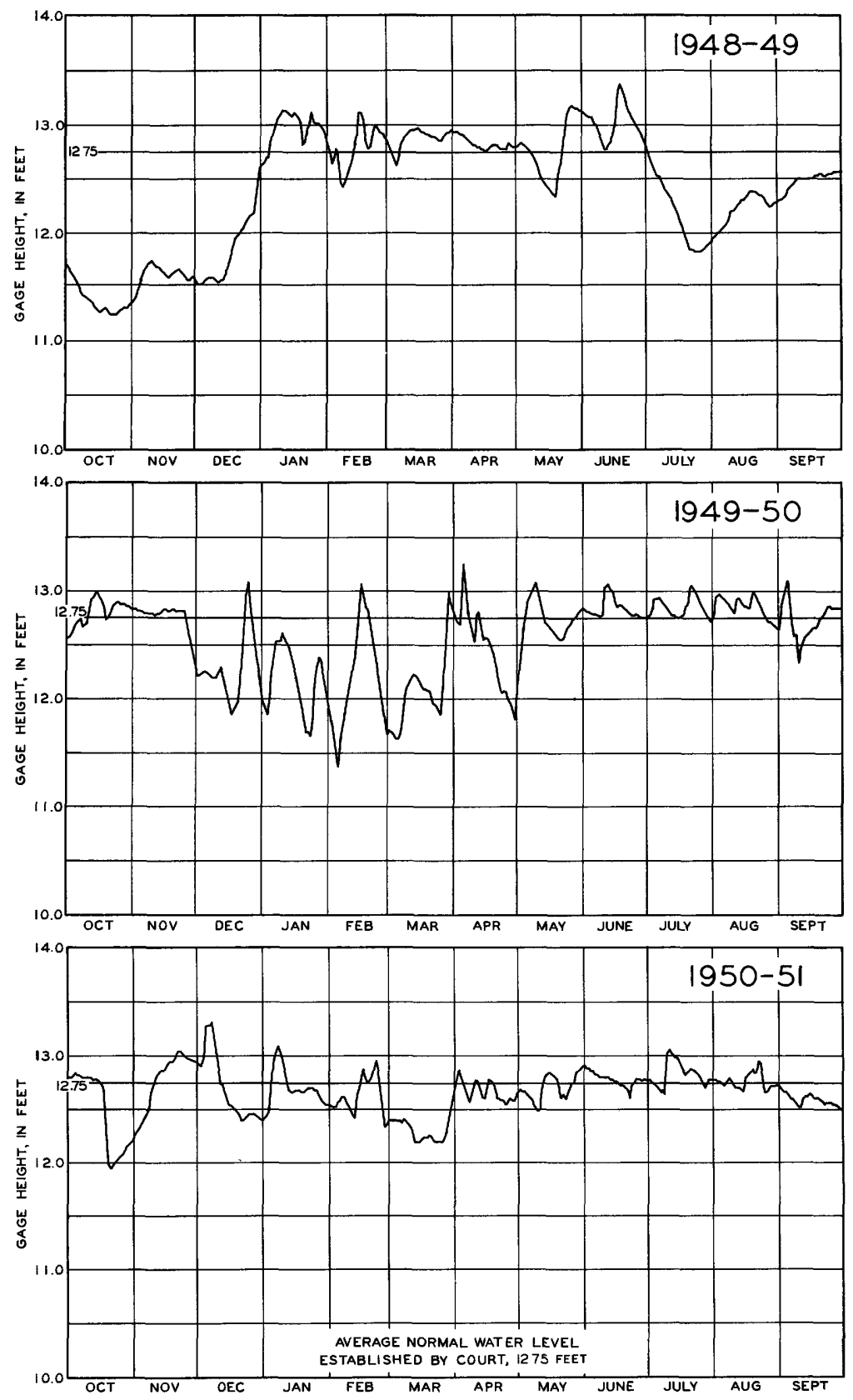

Figure 134. --Lake-level hydrographs for Webster Lake at North Webster, Ind., for water years 1949-51. 


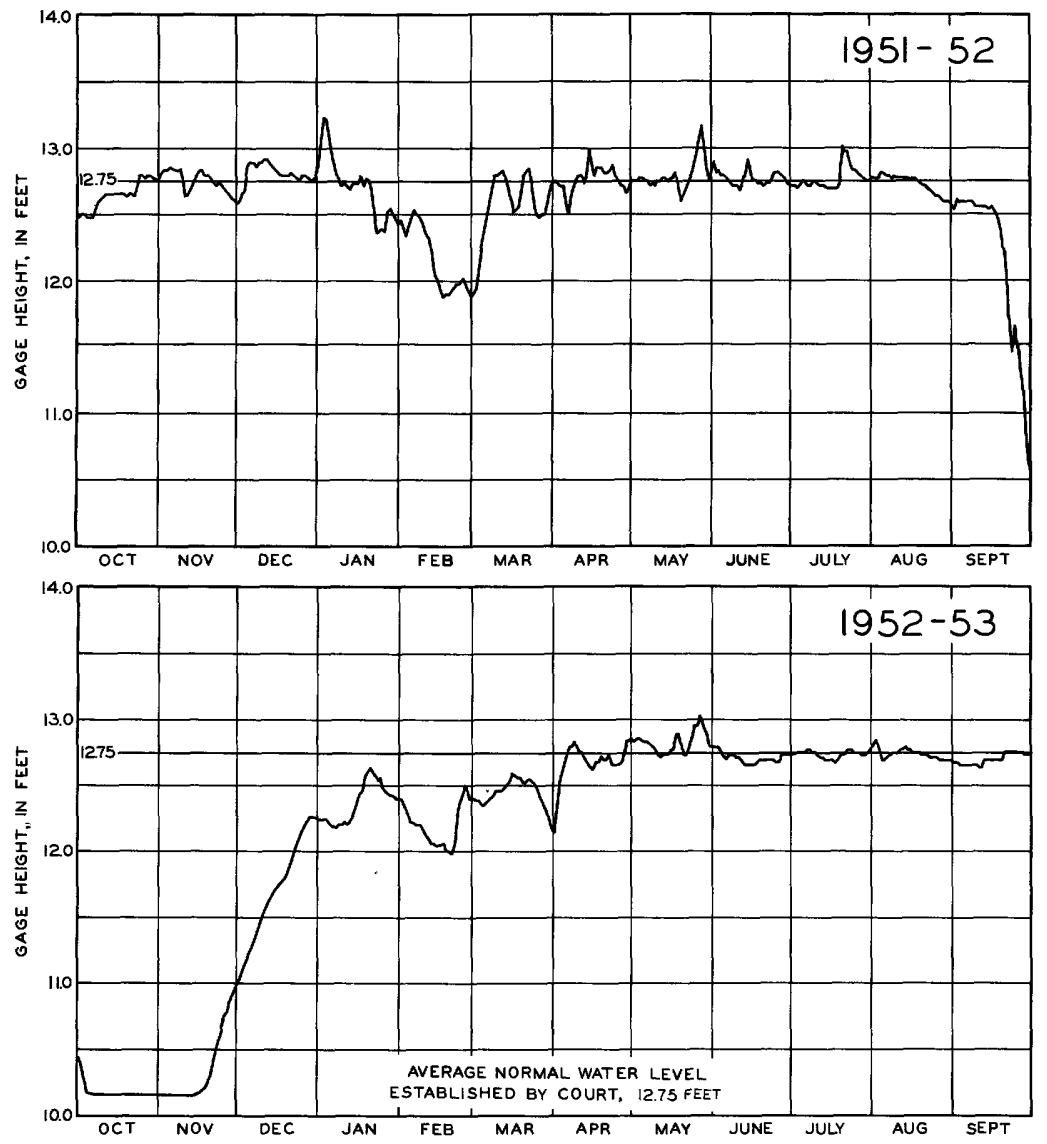

Figure 135. --Lake-level hydrographs for Webster Lake at North Webster, Ind., for water years 1952-53.

Winona Lake at Warsaw, Ind.

Location.--Secs. 15, 16, 17, 21, and 22, T. 32 N., R. 6 E., Kosciusko County.

Surface area. $-\mathbf{5 2 9}$ acres.

Drainage area. $-32.1 \mathrm{sq} \mathrm{mi}$.

Records available. --August 1943 to September 1953.

Gage.--Staff gage on outlet dam abutment at west end of lake. Gage read to hundredths once daily. Datum of gage is $810.00 \mathrm{ft}$ above mean sea level, datum of 1929 .

Average lake level. - -10 years;gage height,1.00 ft; elevation,81l.00 ft. Established legal level. --Established June 17, 1949, at gage height 1.06 ft; elevation, 811. $06 \mathrm{ft}$ above mean sea level. Lake level control. - - Lake level controlled by concrete dam built in 1937-38. When gate (12-in board) is raised sill is $20 \mathrm{ft}$ long and at gage height $0.00 \mathrm{ft}$; when gate is in place sill is $24 \mathrm{ft}$ long and at gage height $1.00 \mathrm{ft}$. 
Extreme levels for Winona Lake, 1944-53

\begin{tabular}{|c|c|c|c|c|}
\hline & \multicolumn{2}{|l|}{ Maximum } & \multicolumn{2}{|l|}{ Minimum } \\
\hline $\begin{array}{l}\text { Water } \\
\text { year }\end{array}$ & Date & $\begin{array}{c}\text { Gage } \\
\text { height } \\
\text { (feet) }\end{array}$ & Date & $\begin{array}{l}\text { Gage } \\
\text { height } \\
\text { (feet) }\end{array}$ \\
\hline $1943^{a}$ & Aug. 4, 1943 & 1.25 & Sept. $29,30,1943--$ & $a_{0.83}$ \\
\hline 1944 & Apr. 13, 1944 $\ldots$ & 2.36 & Jan. $9-20,1944 \ldots$ & .20 \\
\hline 1945 & May $18,1945 \ldots$ & 2.26 & Feb. 2-5, 1945 $\ldots$ & .16 \\
\hline 1946 & Oct. $2,1945 \ldots$ & 2. 18 & May $2,4,1946 \ldots$ & .32 \\
\hline 1947 & Feb. 1, $1947 \ldots$ & 2.05 & Oct. $2,1946 \ldots$ & .30 \\
\hline 1948 & Mar. 1, 1948...... & 1. 72 & Feb. $10-12,1948--$ & .32 \\
\hline 1949 & Feb. 17, $1949 \ldots$ & 2.32 & Mar. 20, 21, 1949-- & .54 \\
\hline 1950 & Apr. 5, $1950 \ldots$ & 2. 90 & June $6,1950 \ldots$ & .64 \\
\hline 1951 & July $12,1951 \ldots$ & 2.10 & Feb. 10, $1951 \ldots$ & .47 \\
\hline 1952 & May 26, 1952...... & 2.04 & Oct. $1,2,1951 \ldots$ & .79 \\
\hline 1953 & Mar. $16,17,1953 \ldots$ & 1.44 & Sept. 27-30, 1953-- & .42 \\
\hline
\end{tabular}

a August through September.

Maximum recorded thickness and periods of ice cover 1944-53

\begin{tabular}{|c|c|c|c|}
\hline $\begin{array}{l}\text { Water } \\
\text { year }\end{array}$ & $\begin{array}{l}\text { Maximum } \\
\text { thickness } \\
\text { (inches) }\end{array}$ & $\begin{array}{c}\text { Total } \\
\text { days of } \\
\text { cover }\end{array}$ & Periods \\
\hline 1944 & $---\cdot$ & $-\cdots$ & Dec. 15,1943 , to - ? \\
\hline 1945 & $-\cdots--$ & --- & \\
\hline 1946 & 3 & 73 & Dec. 23, 1945, to Mar. 5, 1946. \\
\hline 1947 & 12 & 110 & Dec. 10,1946 , to Mar. 29, 1947. \\
\hline 1948 & 12 & 93 & $\begin{array}{l}\text { Nov. } 30 \text { to Dec. } 3 \text {; Dec. } 12-14 \text {; } \\
\text { Dec. } 22,1947 \text {, to Mar. } 19,1948\end{array}$ \\
\hline 1949 & 5 & 70 & Dec. 19,1947, to Feb. 26,1949 \\
\hline 1950 & 8 & 64 & $\begin{array}{l}\text { Dec. } 9-11,16-24,1949 \text {; Jan. } 19 \text { to } \\
\text { Feb. } 7,1950 ; \text { Feb. } 22 \text { to Mar. } \\
\text { 25, } 1950 \text {. }\end{array}$ \\
\hline 1951 & 8 & 74 & Dec. 15,1950 , to Feb. 28,1951 \\
\hline 1952 & 6 & 89 & Dec. 14, 1951, to Mar. 11, 1952. \\
\hline 1953 & 4 & 48 & Jan. 5,1953, to Feb. $21,1953$. \\
\hline
\end{tabular}


Discharge measurements at outlet of Winona Lake

\begin{tabular}{|c|c|c|c|c|c|c|}
\hline & Date & $\begin{array}{c}\text { Gage } \\
\text { height } \\
\text { (feet) }\end{array}$ & $\begin{array}{l}\text { Discharg } \\
\text { (cfs) }\end{array}$ & Date & $\begin{array}{l}\text { Gage } \\
\text { height } \\
\text { (feet) }\end{array}$ & $\begin{array}{c}\text { Discharge } \\
\text { (cfs) }\end{array}$ \\
\hline Aug. & $4,1943-$ & .25 & 31.6 & Oct. $31,1947-$ & 1. 30 & 10.3 \\
\hline Oct. & $6--\cdots$ & .76 & 8.58 & Dec. $17 \ldots$ & .62 & 28.8 \\
\hline Jan. & $6,1944-$ & .24 & 7. 11 & Feb. 19, 1948- & 1.20 & 73.4 \\
\hline Apr. & $21 \ldots \ldots$ & 1. 49 & 122 & Mar. $2 \ldots-$ & 1. 70 & 99.6 \\
\hline June & $12,1945-$ & 1.29 & 17.7 & Mar. 25-.-- & 1. 32 & 78.9 \\
\hline pt. & $10 \ldots-\cdots$ & 26 & 23.7 & May 7--.- & 1. 11 & 36.7 \\
\hline Nov. & $26 \ldots-\cdots$ & .43 & 17.1 & May $13 \ldots \ldots$ & 1.61 & 112 \\
\hline Apr. & $19,1946-$ & .42 & & Sept. $28 \ldots$ & .64 & 1. 76 \\
\hline May & $27 \ldots-\cdots$ & .62 & 32.2 & Nov. $30 \ldots$ & .70 & $a \quad 3.52$ \\
\hline June & $20 \cdots \cdots$ & 1.28 & 94.2 & Jan. $\quad 4,1949-$ & 1. 58 & 35.9 \\
\hline June & $24 \ldots \ldots$ & .78 & 45.8 & Feb. $8 \ldots$ & .84 & 47.9 \\
\hline June & $27 \ldots \ldots$ & .99 & $\begin{array}{ll}a & 1.72\end{array}$ & Mar. 22..... & .57 & $a_{25.5}$ \\
\hline June & $29 \ldots \ldots$ & 1.30 & 12.8 & May $3 \ldots .$. & 1.08 & 5.47 \\
\hline July & $2 \ldots-\cdots$ & .48 & 27.0 & June $27 \ldots$ & 1.50 & 43.8 \\
\hline July & $19 \ldots$ & 14 & 31 & Aug. $31 \ldots$ & .99 & 6.11 \\
\hline ag. & $6 \ldots$ & .17 & 3.99 & Nov. $18 \ldots$ & 1.12 & 6.03 \\
\hline ug. & $26 \ldots$ & 1.09 & 1. 79 & Dec. 14 & .96 & 36.3 \\
\hline ct. & $23 \cdots \cdots$ & .60 & a. .42 & Jan. $14,1950-$ & 2.38 & $a_{212.0}$ \\
\hline ov. & $21 \ldots \ldots$ & 1. 22 & 5. 68 & Jan. $20 \ldots$ & 1. 68 & 115 \\
\hline Dec. & $16 \ldots$ & 1. 34 & 12.4 & Jan. $24 \ldots-$ & 1.62 & 112 \\
\hline Jan. & $8,1947-$ & 1.28 & 10.1 & Jan. $31 \ldots$ & 1.88 & 130 \\
\hline Feb. & $4 \ldots-\cdots$ & 1.86 & 67.6 & Mar. $14--\cdots$ & 1. 56 & 106 \\
\hline eb. & $18--$ & .48 & 20.4 & Apr. $14 \ldots-$ & 2.02 & 161 \\
\hline Apr. & $22 \cdots \cdots$ & 1. 58 & 123 & May $2 \ldots$ & .94 & 62.2 \\
\hline July & $30-\cdots--$ & 1. 10 & a 5.19 & $8,1951-$ & .67 & a 30.1 \\
\hline Aug. & $28 \ldots-$ & 1.06 & 4. 18 & & & \\
\hline
\end{tabular}

a Leakage. 
Average lake levels for Winona Lake for water years 1943-53

\begin{tabular}{c|r|r|r|r|r|r|r}
\hline Year & Oct. & Nov. & Dec. & Jan. & Feb. & Mar. & Apr. \\
\hline 1944 & 0.64 & 0.65 & 0.55 & 0.21 & 0.35 & 0.84 & 1.41 \\
1945 & .83 & .90 & .72 & .21 & .34 & .66 & .84 \\
1946 & .96 & .50 & .47 & .79 & .80 & .81 & .45 \\
1947 & .48 & 1.20 & 1.27 & 1.41 & .82 & .52 & 1.08 \\
1948 & 1.18 & .83 & .74 & .64 & .69 & 1.10 & 1.04 \\
1949 & .76 & .85 & 1.10 & 1.46 & 1.44 & .81 & .89 \\
1950 & 1.17 & 1.02 & 1.29 & 2.03 & 1.51 & 1.52 & 1.73 \\
1951 & .90 & .98 & 1.02 & 1.02 & 1.24 & .96 & 1.44 \\
1952 & 1.07 & 1.27 & 1.04 & 1.55 & 1.44 & 1.56 & 1.48 \\
1953 & .87 & 1.03 & .79 & .97 & 1.08 & 1.22 & 1.15 \\
\hline Average & .89 & .92 & .90 & 1.03 & .97 & 1.00 & 1.15 \\
\hline \hline & & & & & & & \\
Year & May & June & July & Aug. & Sept. & Annual & \\
\hline 1943 &..- &.--- &.--- & $* 1.08$ & 0.97 & $* 1.03$ & \\
1944 & 1.17 & 1.04 & 0.76 & .60 & .63 & .74 & \\
1945 & 1.07 & 1.33 & 1.20 & 1.36 & 1.32 & .90 & \\
1946 & .54 & .93 & 1.23 & 1.12 & .98 & .80 & \\
1947 & .81 & 1.12 & 1.14 & 1.05 & 1.20 & 1.01 & \\
1948 & 1.16 & 1.16 & 1.07 & 1.01 & .73 & .95 & \\
1949 & 1.05 & 1.30 & 1.10 & 1.02 & 1.02 & 1.06 & \\
1950 & .90 & 1.09 & .94 & 1.12 & 1.12 & 1.28 & \\
1951 & .94 & 1.11 & 1.34 & 1.06 & .89 & 1.07 & \\
1952 & 1.31 & 1.30 & 1.06 & .87 & .87 & 1.23 & \\
1953 & 1.15 & .99 & .64 & .62 & .47 & .91 & \\
\hline Average & 1.01 & 1.14 & 1.05 & .99 & .93 & 1.00 & \\
\hline
\end{tabular}

*Partial month or partial year. 

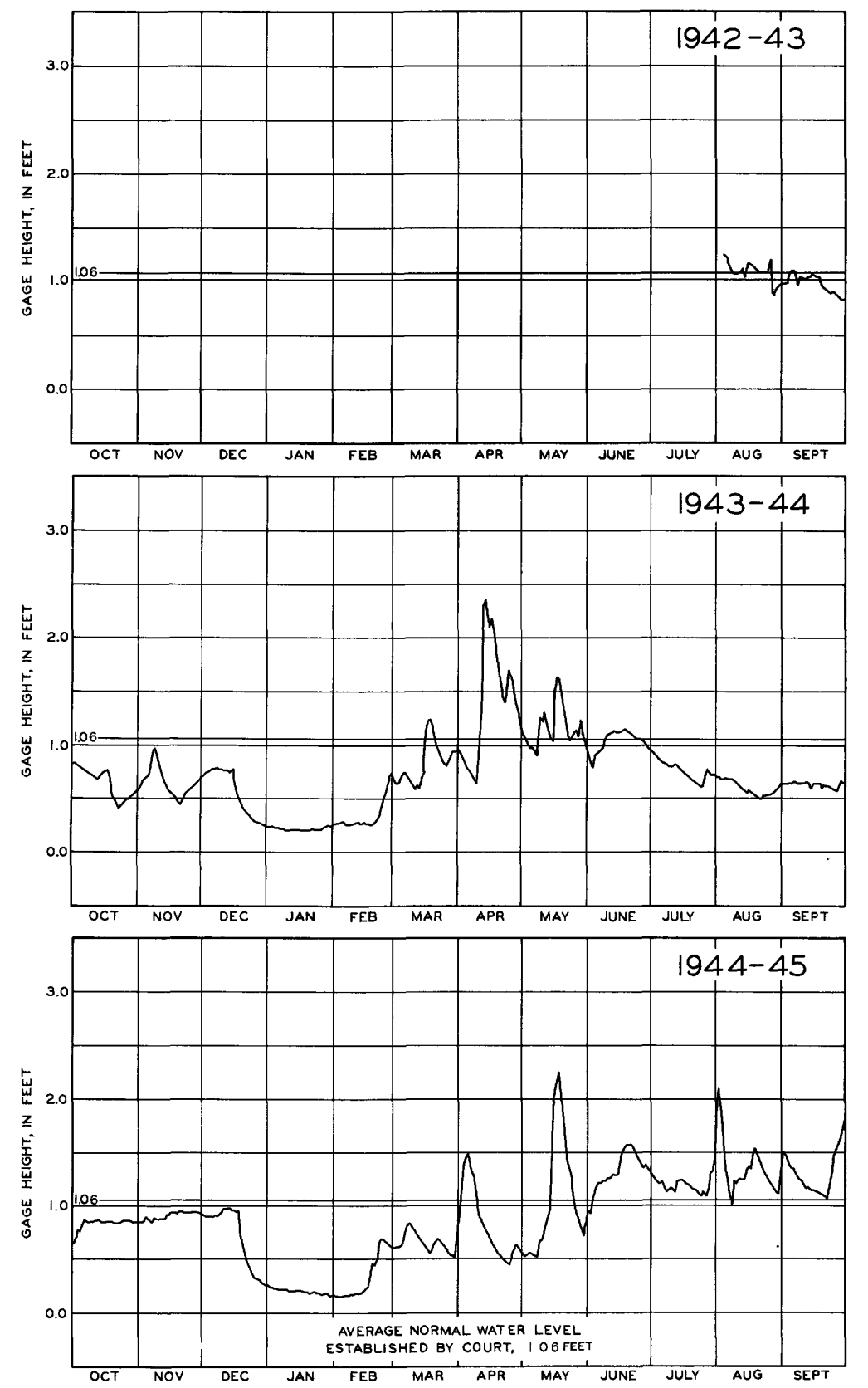

Figure 136. --Lake-level hydrographs for Winona Lake at Warsaw, Ind., for water years 1943-45. 

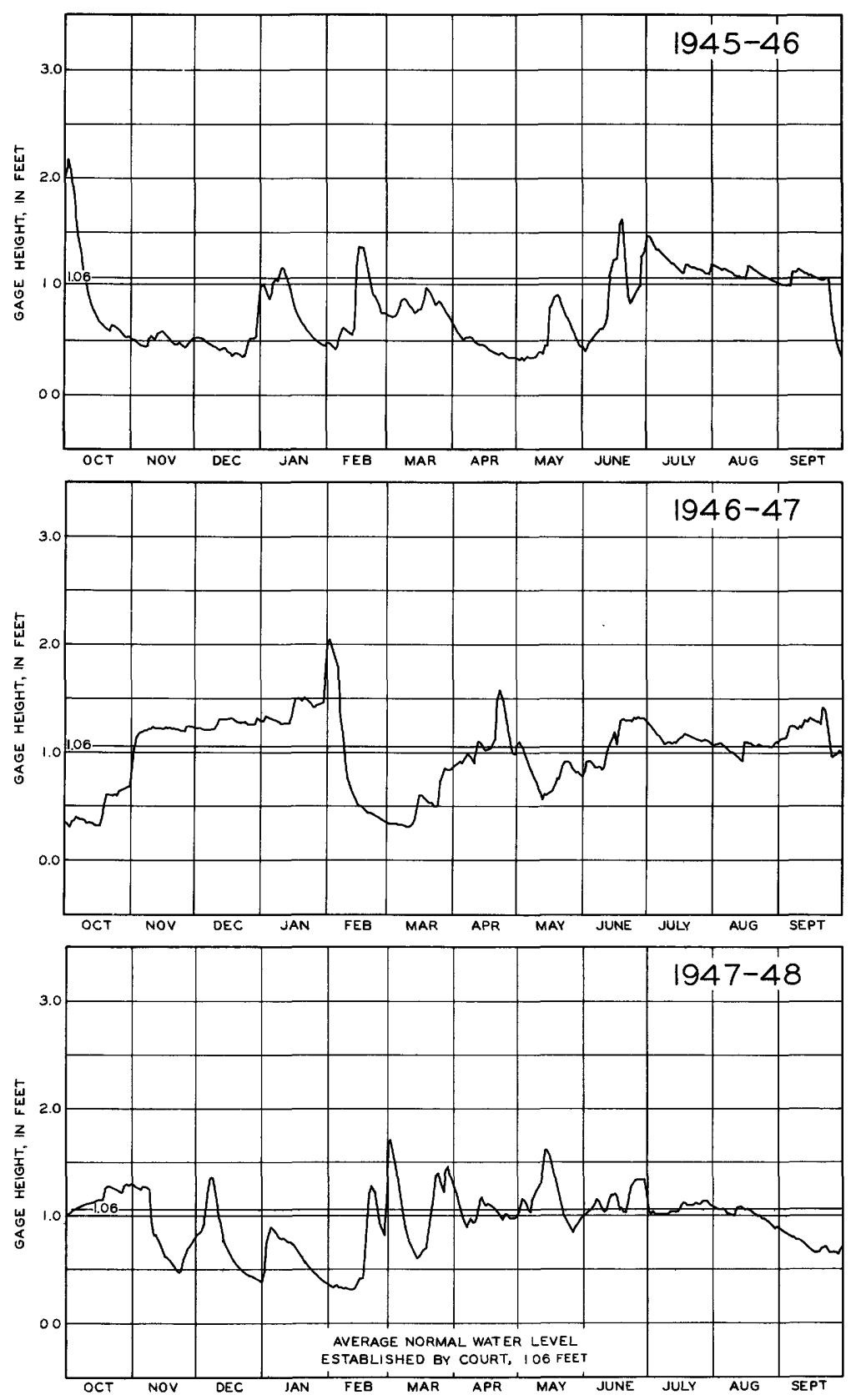

Figure 137. --Lake-level hydrographs for Winona Lake at Warsaw, Ind., for water years 1946-48. 

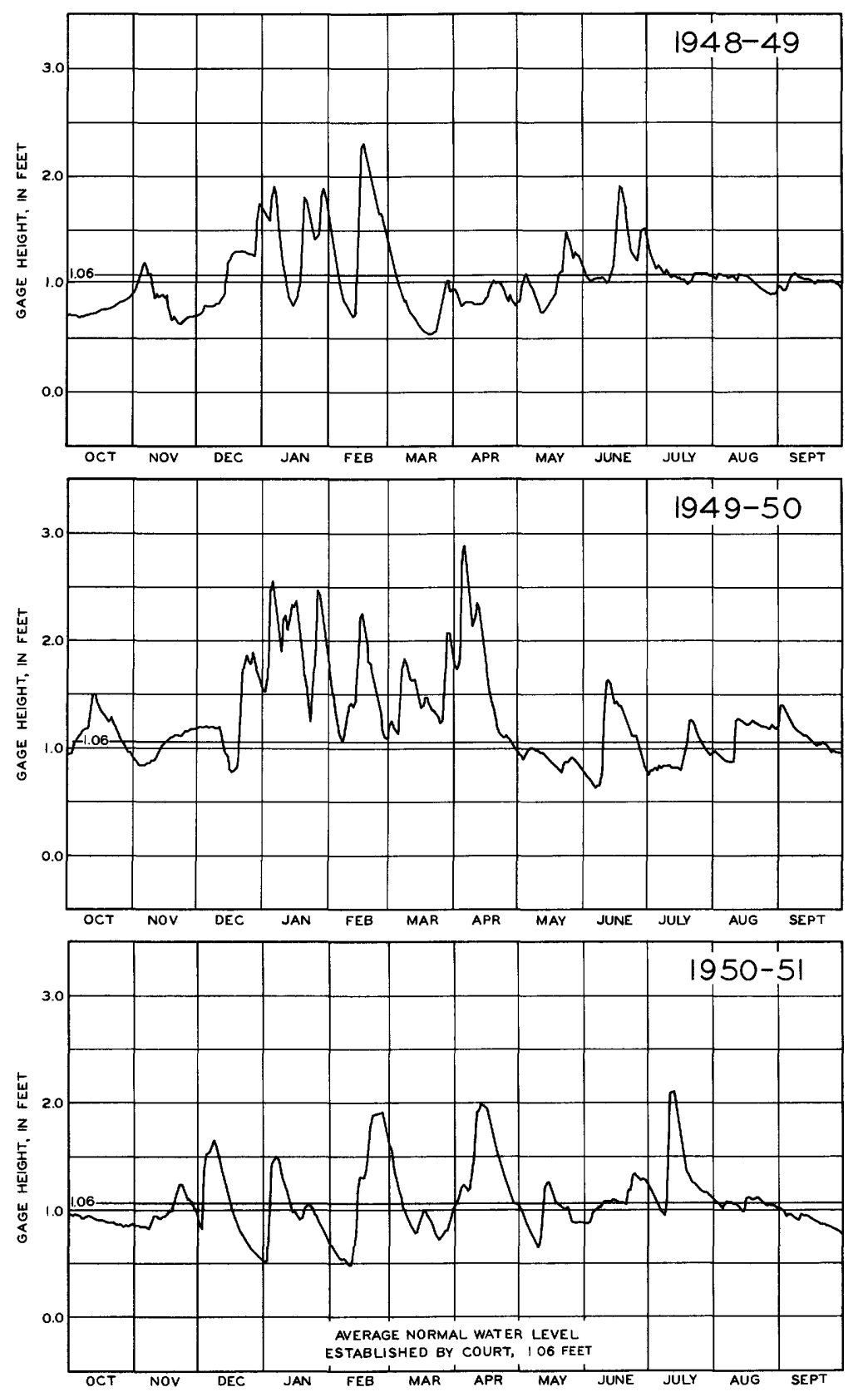

Figure 138. --Lake-level hydrographs for Winona Lake at Warsaw, Ind., for water years 1949-51. 


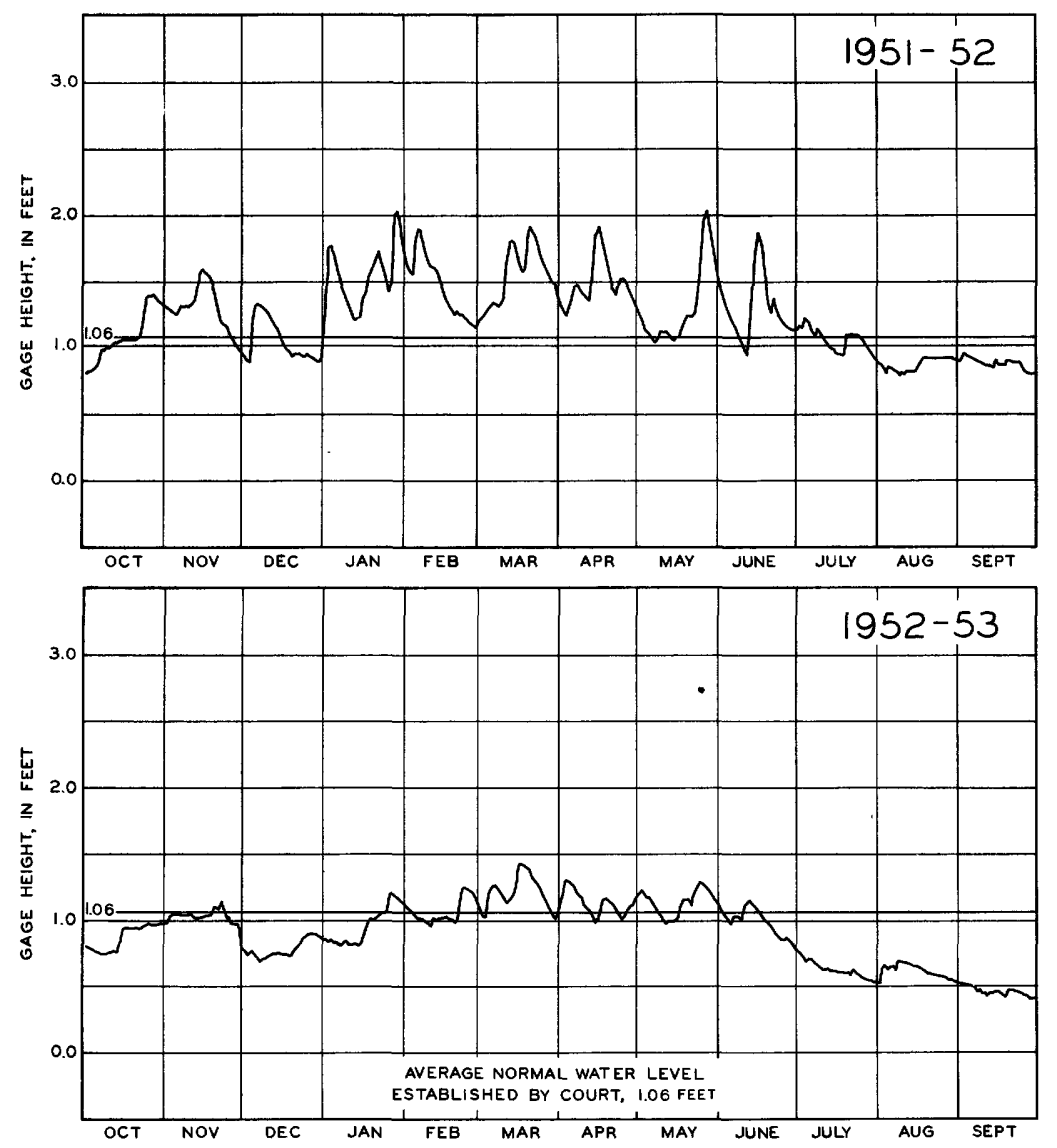

Figure 139. - -Lake-level hydrog raphs for Winona Lake at Warsaw, Ind. , for water years 1952-53. 


\section{LEGAL LAKE LEVELS}

The data contained in this section was gathered and tabulated by the Division of Water Resources, Indiana State Department of Conservation and the U. S. Geological Survey. Table 1 was furnished by C. H. Bechert, Director, Division of Water Resources, Department of Conservation and represents the status of the lakes as of September 30,1953, except for the establishment of the average normal water level which is as of December 31, 1954.

The drainage areas and surface areas of the lake were determined from aerial photographs taken in 1938 by the Agricultural Adjustment Administration except where suitable maps were available.

The lake gages were established by the U. S. Geological Survey and daily gage readings to hundredths, for the periods indicated, are available from its Indianapolis district office. The "established normal level" and "established high-water mark" shown in the table were established under the provisions of Chapter 350 of the Acts of 1947, State of Indiana.

Chapter 290 of the Acts of 1951 provides another procedure for the raising and(or)maintaining the water level of the public fresh-water lakes of Indiana. Under the provisions of this act 20 percent or more of the property owners of land abutting upon or being within 440 yards of the shore or water line of any lake containing 10 or more acres, may petition the Circuit or Superior Court of the county in which the greatest area of the lake lies, to raise or maintain the water level of the lake by constructing a control dam or other works. Although the 1951 law provides another means of establishing the water levels of the public lakes of Indiana the levels requested in the petition under this law usually conform to the average normal water level established under Chapter 350 of the acts of 1947 .

Chapter 350 of the Acts of 1947, Indiana State Legislature authorizes and empowers the Indiana Department of Conservation to establish, by appropriate monuments, the average normal water level of all of the natural and artificial lakes of the State. Such water-level monuments shall fix the average normal water levels of the lakes and shall indicate the highest point to which the water of the lake shall have arisen during the 10 years prior to the passage of the act on March 14, 1947. 
The lake-level monuments are permanent concrete markers, 18 inches square and 15 inches high. A bronze reference marker on which its elevation above sea level is stamped is set in the top of the monument and the elevation of the marker above mean sea level is shown in the column in the table headed "Elevation of lake level monument table".

Under the 1947 law the Conservation Department submits a petition containing factual data supporting the average normal water level that it proposes to establish as the legal water level of the lake to the Circuit Court having jurisdiction over the lake area. After proper public notice is given a hearing is held by the Court, and after presentation of evidence either in support of or against the recommendations of the Indiana Department of Conservation an order is issued establishing the water level of the lake.

When the normal (legal) level is established, the findings and the judgements of the court are recorded in a Lake-Level Record kept in the recorder's office of each county having lands draining into the lake. A like record is kept by the Indiana Department of Conservation and a copy is submitted to the Indiana Flood Control and Water Resources Commission.

Under the provisions of Chapter 350 of the Acts of 1947 the Indiana Department of Conservation is further authorized to construct or supervise the construction of dams or control works to maintain the average normal water level established by the Court. 


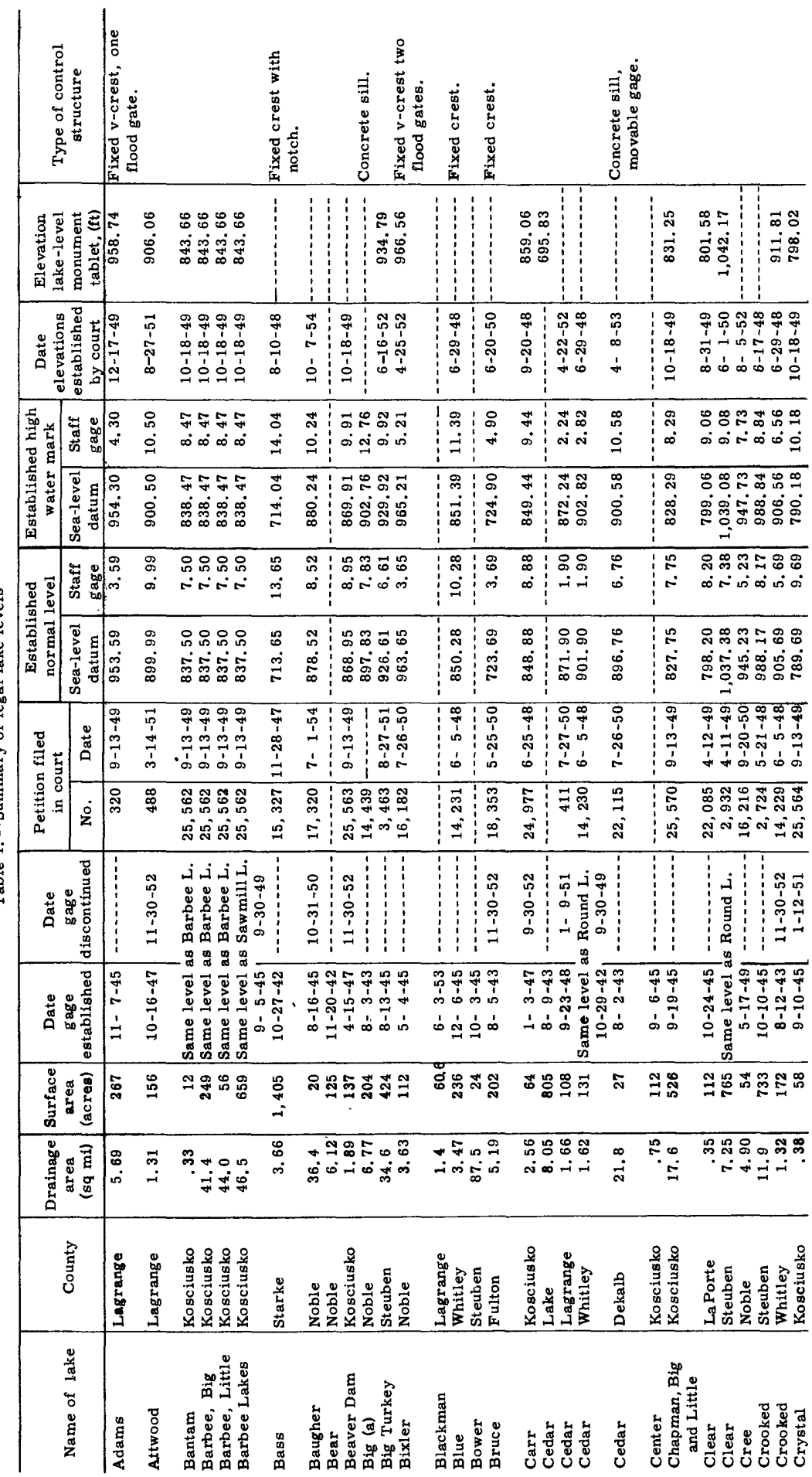




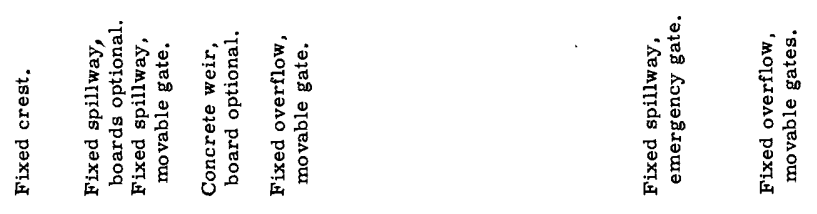

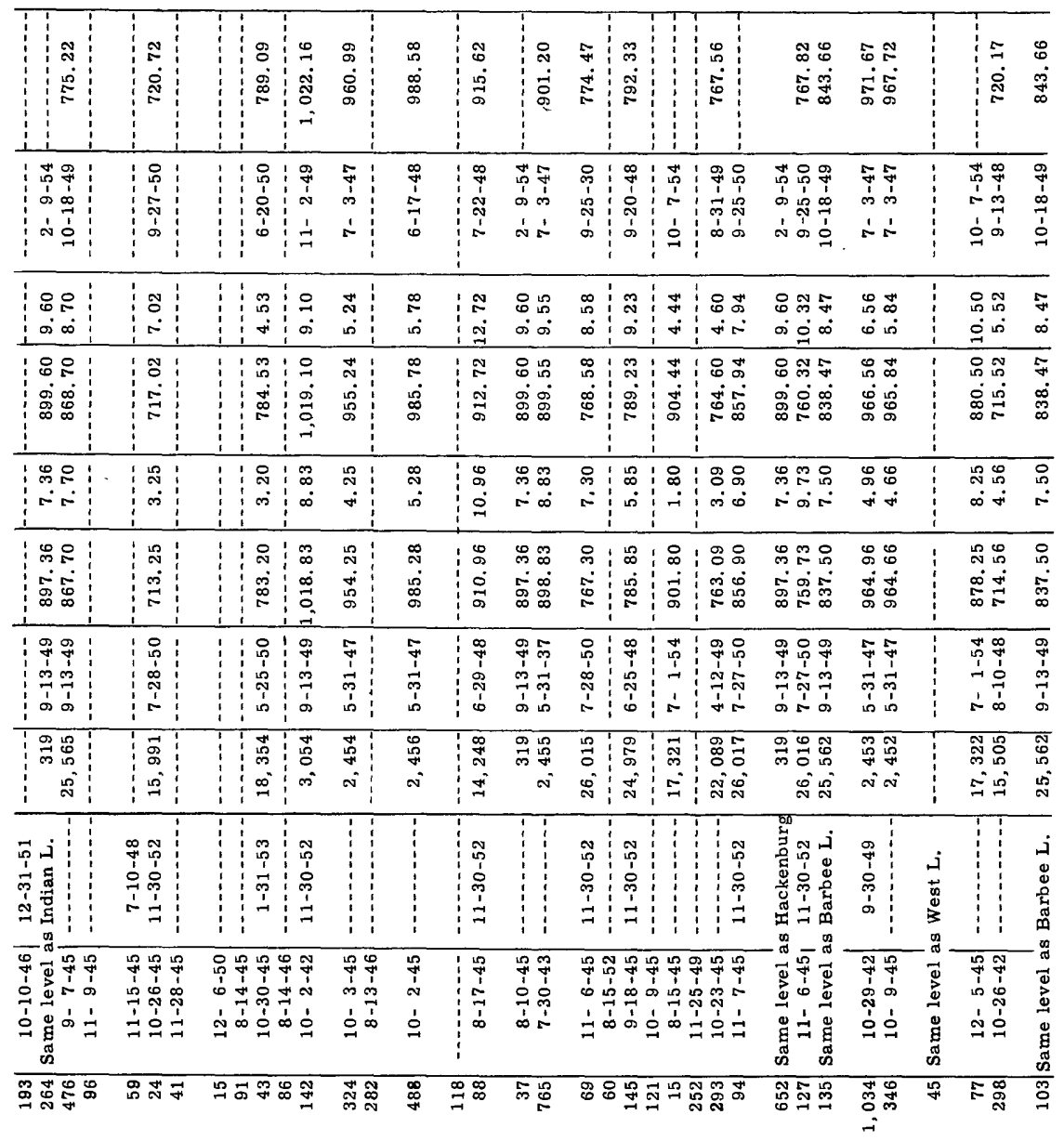

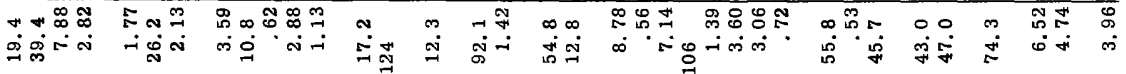

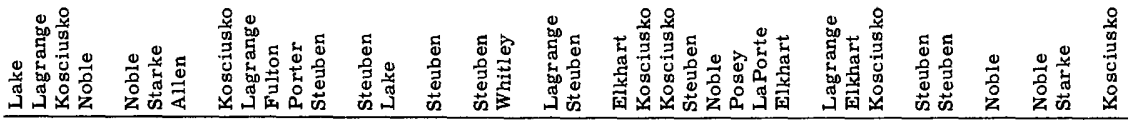

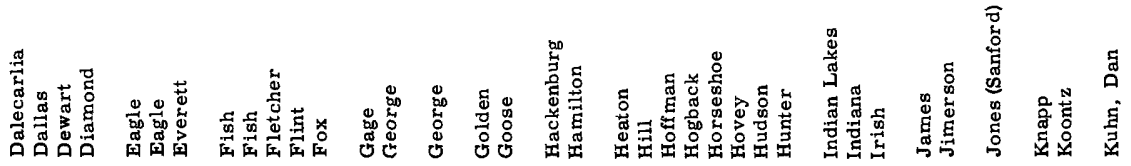




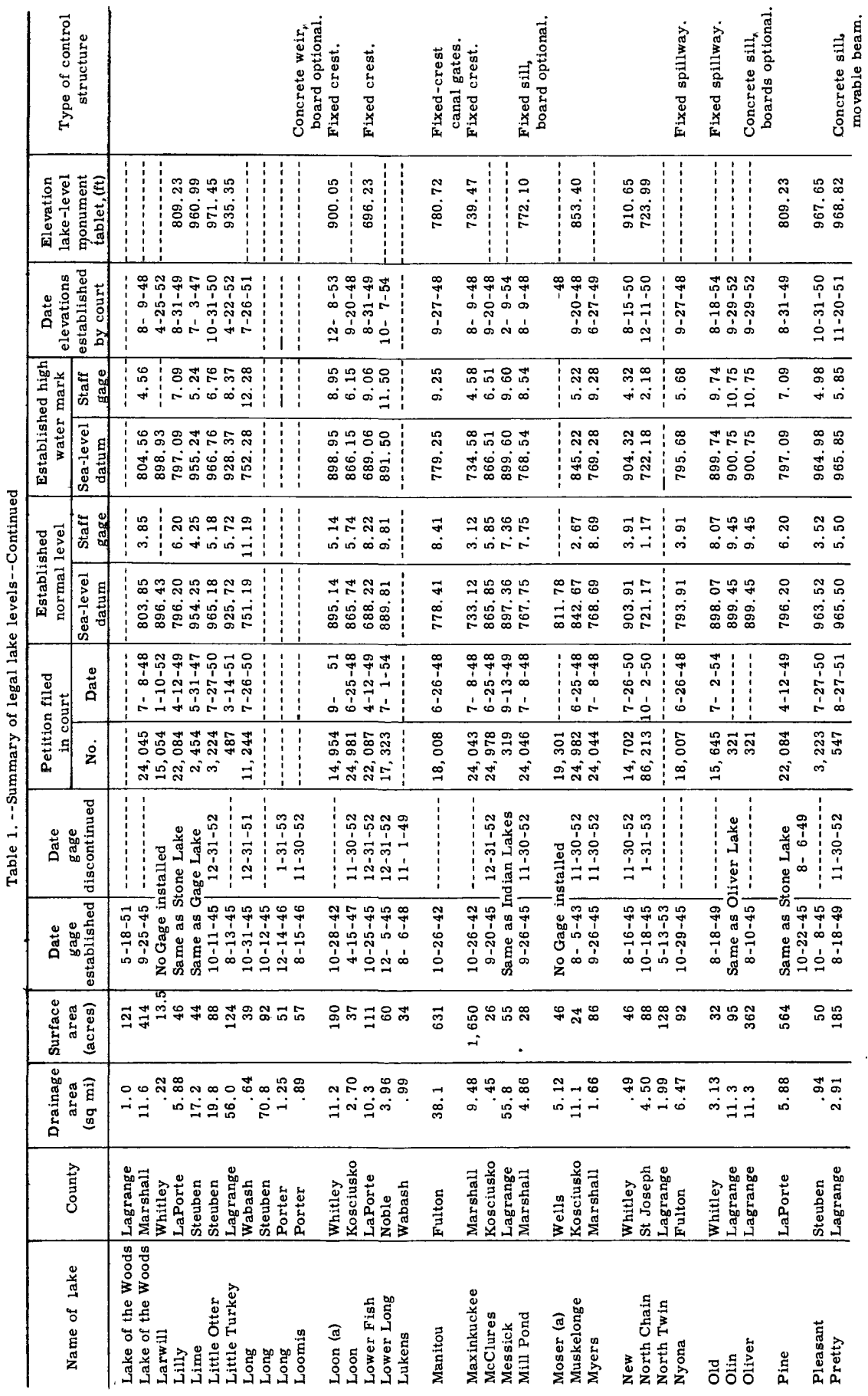




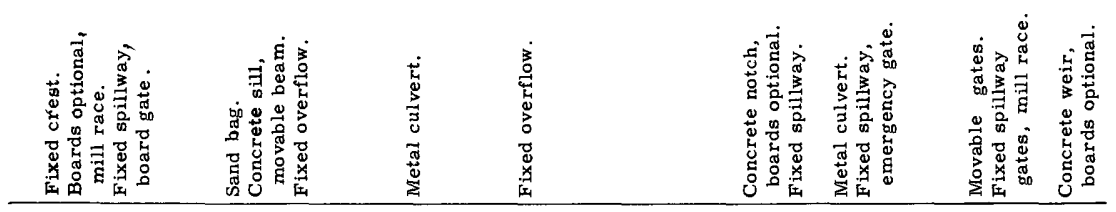

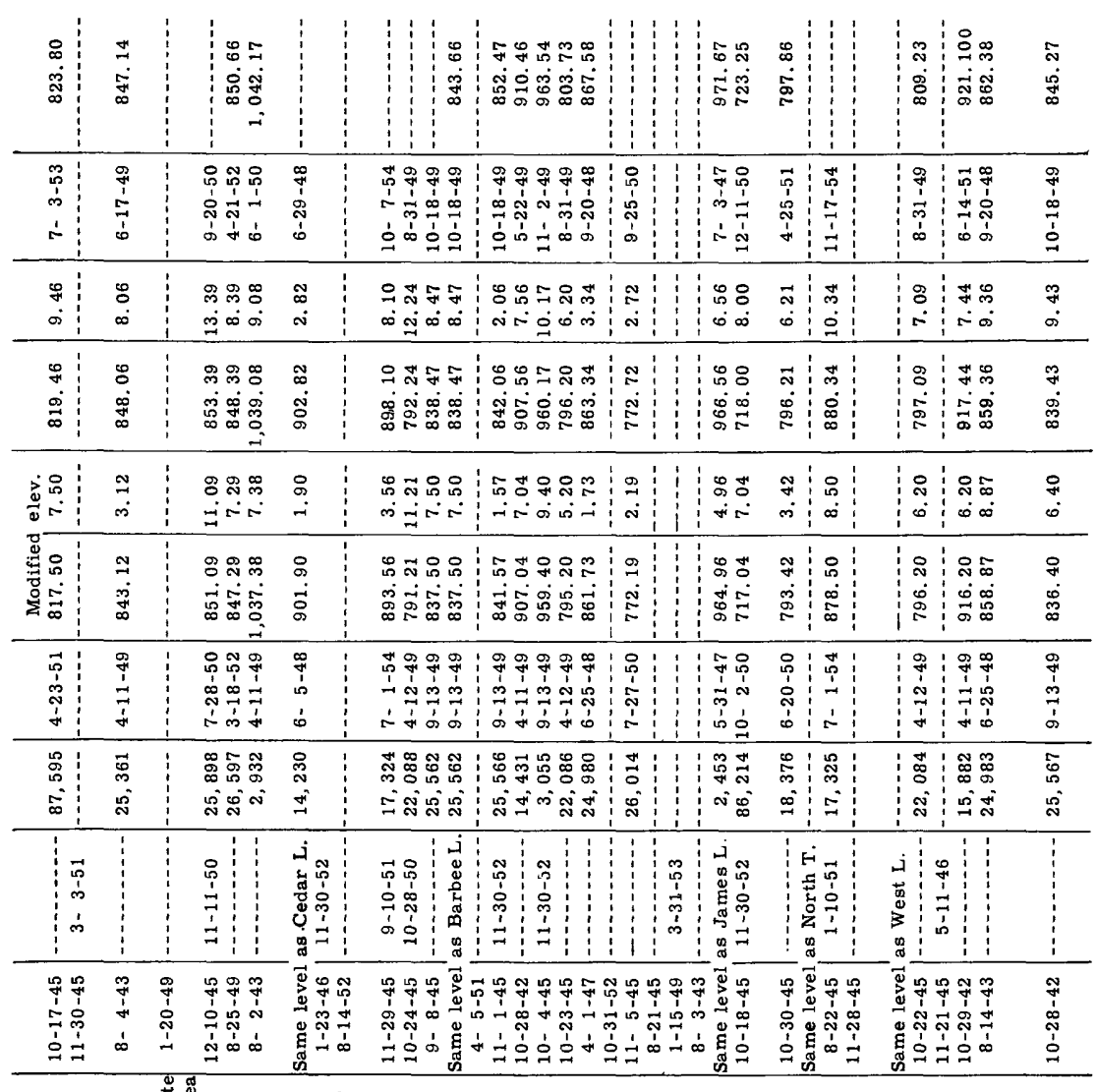

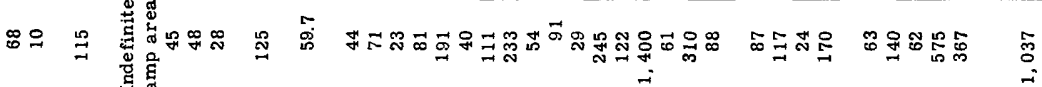
密

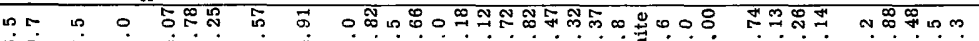

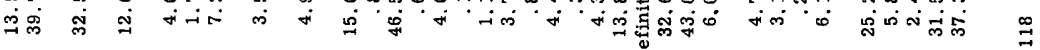
要

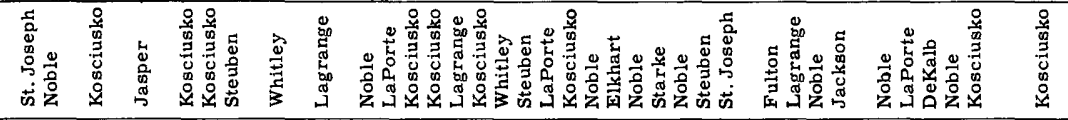

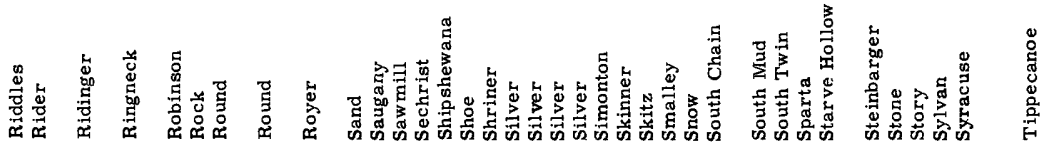




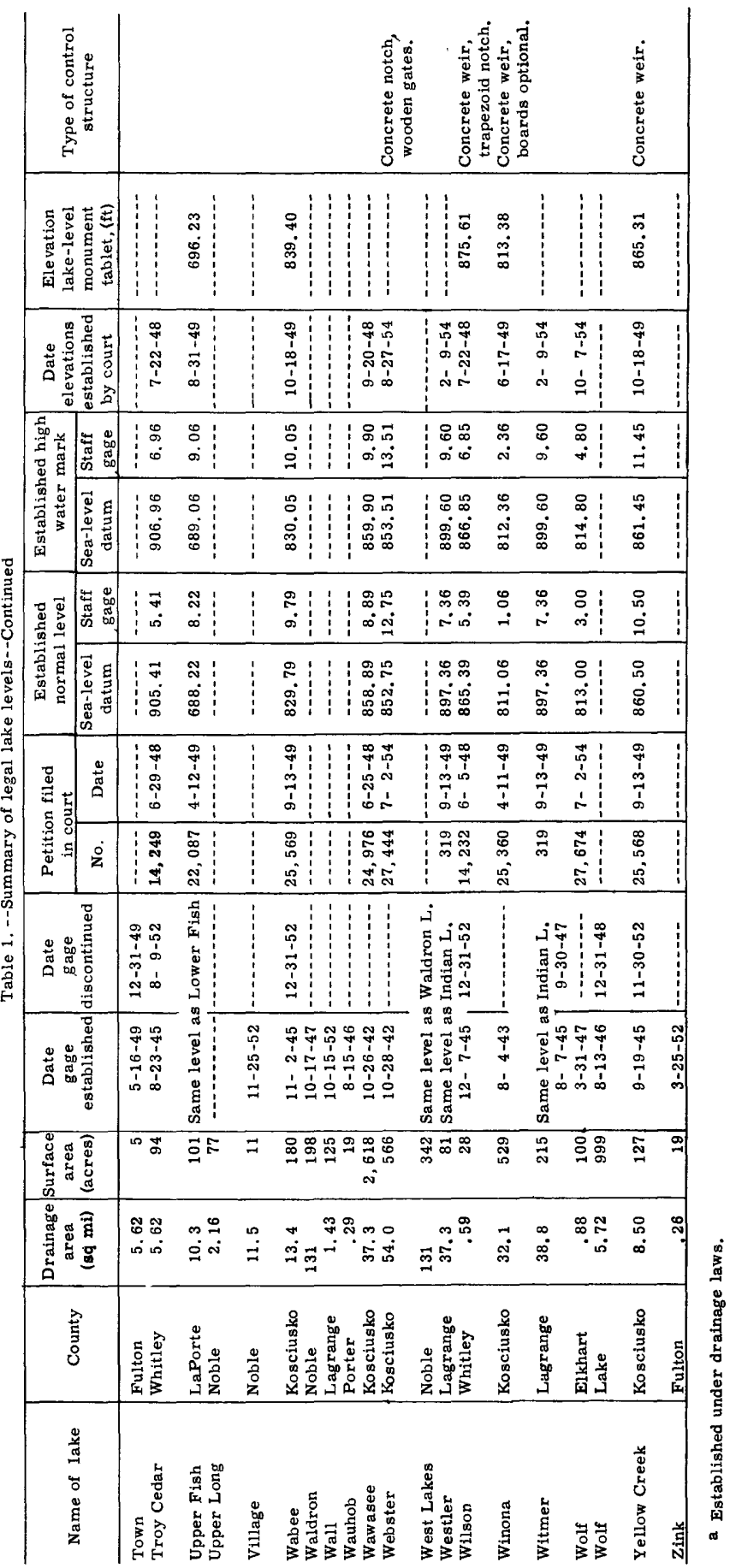




\section{TEMPERATURES FOR SELECTED LAKES}

The data contained in this section were collected by the U. S. Geological Survey except those for Maxinkuckee Lake for the period July 1899 to June 1901 which were collected by Evermann and Clark (1920).

Surface temperature readings were generally taken near the gage location by holding a mercury or liquid-filled tube thermometer about an inch below the water surface. The readings were taken by gage observers once weekly, usually on Saturday, with occasional additional observations being made by U. S. Geological Survey engineers.

The temperature profiles in Maxinkuckee Lake collected by Evermann and Clark were taken in the deepest part of the lake in what is known as the Deep Hole. A self-registering NegrettiZamba deep-sea thermometer was used except for the period July 18 to August 14,1899, when a Ritchie thermophone was used.

The temperature profiles collected by the U.S. Geological Survey were taken in the deepest parts of the lakes; a Leeds and Northrup thermohm was used. The profiles in Maxinkuckee Lake were taken in the same location in the Deep Hole used by Evermann and Clark.

In July 1946 temperature profiles were taken at three different locations in each of Koontz and Ridinger Lakes to compare temperatures in different parts of the lakes. As no significant difference was found, the practice was discontinued.

An analysis of the data contained in this section will be found on p. 14. 
Water surface temperatures for Bass Lake at Bass Lake, Ind.

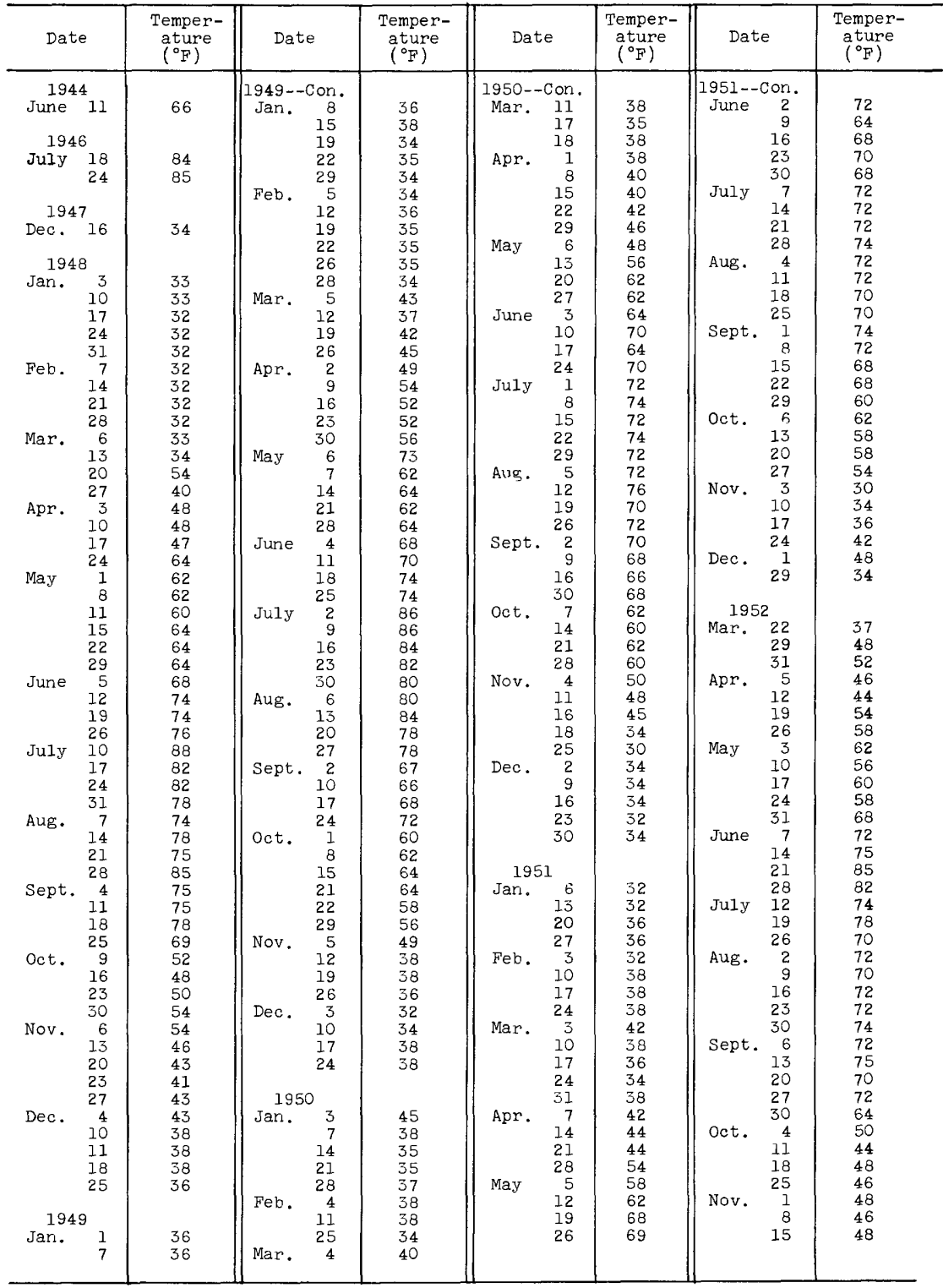


Temperature profiles $\left({ }^{\circ} \mathrm{F}\right)$ for Bass Lake, at Bass Lake, Ind.

\begin{tabular}{|c|c|c|c|c|c|c|c|c|c|}
\hline \multirow{2}{*}{$\begin{array}{l}\text { Depth } \\
\text { (feet) }\end{array}$} & \multicolumn{3}{|c|}{1946} & \multicolumn{6}{|c|}{1947} \\
\hline & July 25 & Oct. 22 & Nov. 19 & May 1 & May 27 & June 19 & July 23 & Aug. 26 & oct. 1 \\
\hline A1r & 82 & 58 & 35 & 58 & 57.5 & 78 & 78.1 & 74 & 51 \\
\hline \begin{tabular}{|c|} 
Water-surface \\
2.5 \\
5 \\
7.5 \\
10 \\
15 \\
18.5 \\
20 \\
21 \\
22 \\
22.5 \\
24 \\
25 \\
26 \\
28 \\
30 \\
32 \\
33 \\
\end{tabular} & 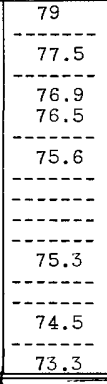 & 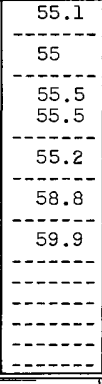 & 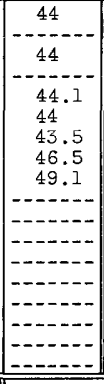 & 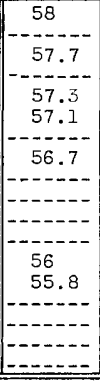 & 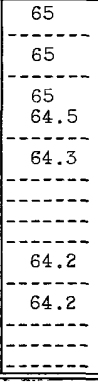 & 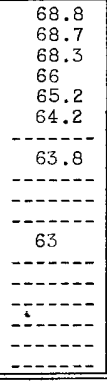 & 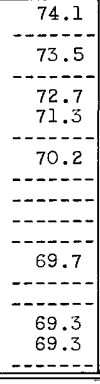 & 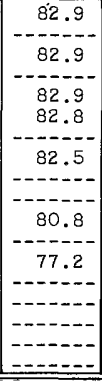 & 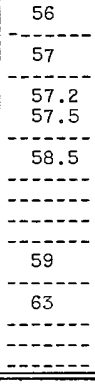 \\
\hline \multirow{2}{*}{$\begin{array}{l}\text { Depth } \\
\text { (feet) }\end{array}$} & \multicolumn{2}{|c|}{ 1947--Continued } & \multicolumn{7}{|c|}{1948} \\
\hline & oct. 31 & Nov. 18 & June 1 & July 3 & Aug. 3 & Aug. 31 & Oct. 5 & \multicolumn{2}{|l|}{ Nov. 15} \\
\hline AIr & 56 & 41 & 70 & 71 & 74 & 70 & 58 & 52 & \\
\hline $\begin{array}{c}\text { Water-surface } \\
2.5 \\
3 \\
5 \\
6 \\
9 \\
10 \\
12 \\
15 \\
18 \\
20 \\
21 \\
22.5 \\
24 \\
25 \\
26 \\
27 \\
29 \\
30 \\
32 \\
\end{array}$ & 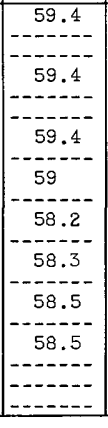 & 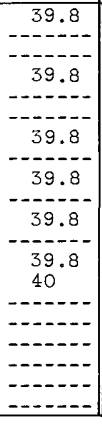 & 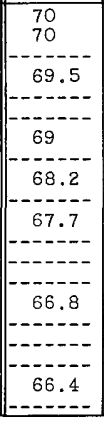 & 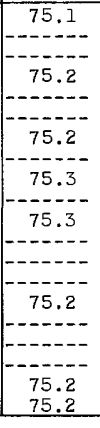 & \begin{tabular}{|c|}
78 \\
77.8 \\
77 \\
$-0-$ \\
76.7 \\
76.7 \\
76.3 \\
$-0-$ \\
76.2 \\
-0. \\
75.9 \\
75.8 \\
\end{tabular} & $\begin{array}{c}79.9 \\
79 \\
79 \\
79 \\
78.1 \\
77.4 \\
74.7 \\
74.2 \\
73.9 \\
-9-1 \\
-9 \\
\end{array}$ & 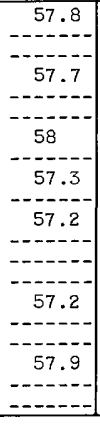 & $\begin{array}{c}44.4 \\
-44.5 \\
-0.5 \\
44.6 \\
44.6 \\
-44 \\
43.9 \\
43.7 \\
43.6 \\
43.4 \\
43.4 \\
-4 \\
\\
\end{array}$ & \\
\hline
\end{tabular}

Water surface temperatures for Bear Lake at Wolflake, Ind.

\begin{tabular}{|c|c|c|c|c|c|c|c|}
\hline Date & $\begin{array}{l}\text { Temper- } \\
\text { ature } \\
\left({ }^{\circ} \mathrm{F}\right)\end{array}$ & Date & $\begin{array}{l}\text { Temper- } \\
\text { ature } \\
\left({ }^{\circ} \mathrm{F}\right)\end{array}$ & Date & $\begin{array}{l}\text { Temper- } \\
\text { ature } \\
\left({ }^{\circ} \mathrm{F}\right)\end{array}$ & Date & $\begin{array}{c}\text { Temper- } \\
\text { ature } \\
\left({ }^{\circ} \mathrm{F}\right)\end{array}$ \\
\hline 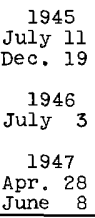 & $\begin{array}{l}75 \\
32\end{array}$ & 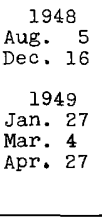 & $\begin{array}{l}34.5 \\
37 \\
61.5\end{array}$ & \begin{tabular}{l} 
1943--Con. \\
Sept. 7 \\
Dec. 1 \\
\multicolumn{1}{c}{1950} \\
Jan. 17 \\
Mar. $\quad 8$ \\
May $\quad 4$
\end{tabular} & $\begin{array}{l}35 \\
35 \\
56\end{array}$ & \begin{tabular}{lr} 
1950--Con. \\
Sept. & 13 \\
Oct. & 18 \\
Nov. & 21 \\
\multicolumn{2}{c}{1951} \\
May & 15 \\
Feb. & 2 \\
May & 6
\end{tabular} & $\begin{array}{l}70 \\
60 \\
40\end{array}$ \\
\hline
\end{tabular}

Water surface temperatures for Big Lake near Wolflake, Ind.

\begin{tabular}{|c|c|c|c|c|c|c|c|}
\hline Date & $\begin{array}{c}\text { Temper- } \\
\text { ature } \\
\left({ }^{\circ} \mathrm{F}\right)\end{array}$ & Date & $\begin{array}{l}\text { Temper- } \\
\text { ature } \\
\left({ }^{\circ} \mathrm{F}\right)\end{array}$ & Date & $\begin{array}{c}\text { Temper- } \\
\text { ature } \\
\left({ }^{\circ} \mathrm{F}\right)\end{array}$ & Date & $\begin{array}{l}\text { Temper- } \\
\text { ature } \\
\left({ }^{\circ} \mathrm{F}\right)\end{array}$ \\
\hline 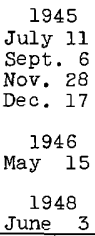 & $\begin{array}{l}67 \\
78 \\
38 \\
30\end{array}$ & $\begin{array}{c}1948-{ }_{-1} \text { Con. } \\
\text { Nov. } 4 \\
1949 \\
\text { Jan. } 21 \\
27 \\
\text { Mar. } 9 \\
\\
31 \\
\text { Apr. } 27 \\
\text { Sept. } 6 \\
\text { Dec. } 1\end{array}$ & $\begin{array}{l}34 \\
34.5 \\
37 \\
47 \\
64.5 \\
75 \\
42 \\
\end{array}$ & 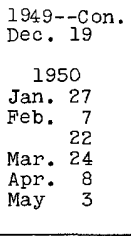 & $\begin{array}{l}35 \\
35 \\
35 \\
35 \\
40 \\
54\end{array}$ & $\begin{array}{lr}1950- & \text { Con. } \\
\text { June } & 20 \\
\text { Sept. } & 13 \\
\text { Oct. } & 17 \\
& 26 \\
\text { Nov. } & 1 \\
& 21 \\
\text { May } & 15 \\
\text { Feb. } & 2\end{array}$ & $\begin{array}{l}75 \\
72 \\
58 \\
57 \\
59 \\
40 \\
63 \\
36.5\end{array}$ \\
\hline
\end{tabular}




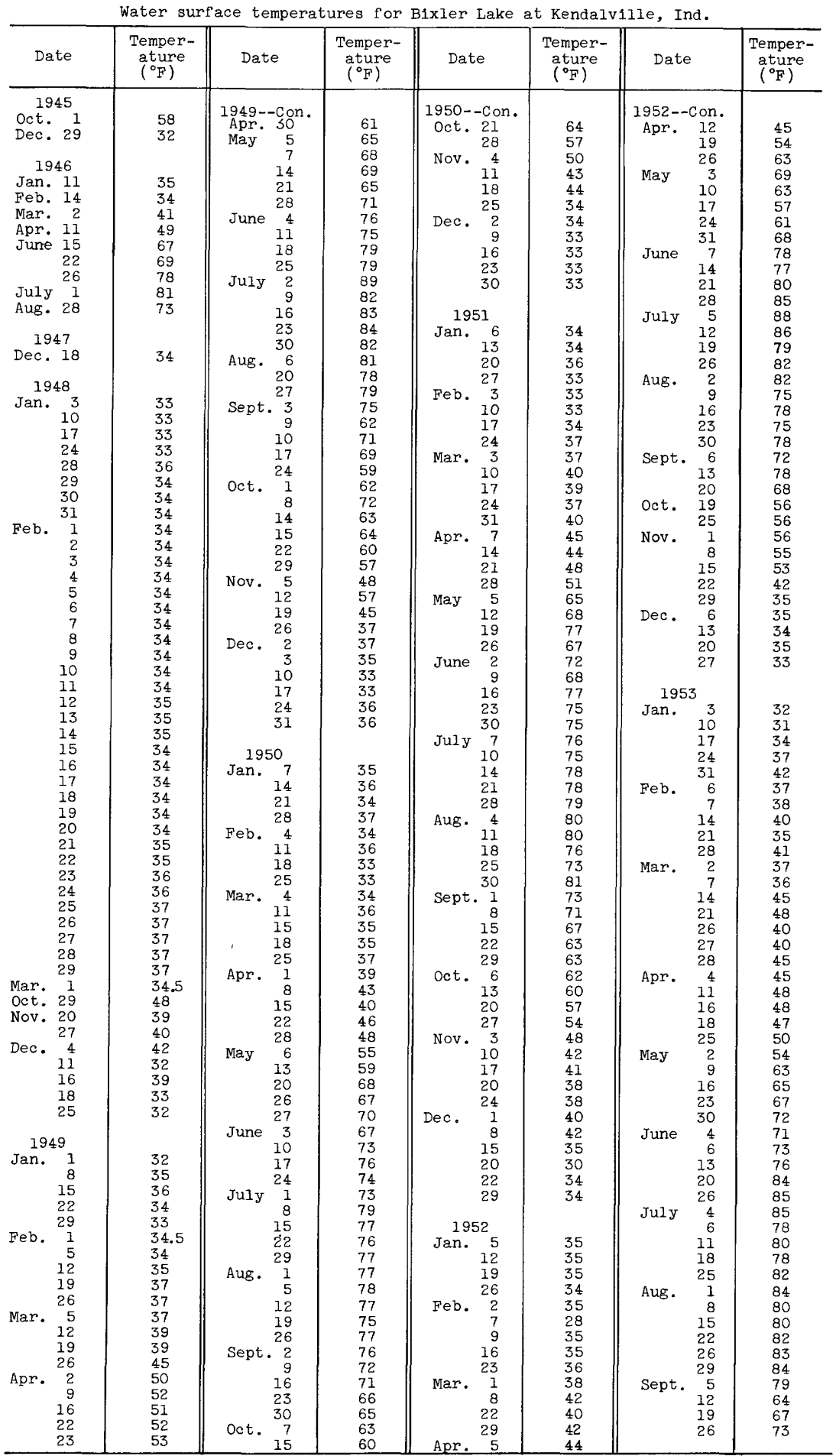


Temperature profiles ( $\left.{ }^{\circ} \mathrm{F}\right)$ for Bixler Lake, Kendallville

\begin{tabular}{|c|c|c|c|c|c|c|c|}
\hline \multirow[b]{2}{*}{$\begin{array}{l}\text { Depth } \\
\text { (feet) }\end{array}$} & \multicolumn{3}{|c|}{1946} & \multicolumn{4}{|c|}{1947} \\
\hline & Aug. 28 & oct. 16 & Nov. 21 & Jan. 8 & Mar. 27 & Apr. 28 & May 26 \\
\hline Air & $\cdots--1-\cdots$ & 64 & 60 & 32 & 47 & 59 & 61.5 \\
\hline \begin{tabular}{c|} 
Water-surface \\
2.5 \\
5 \\
7.5 \\
10 \\
12.5 \\
15 \\
17.5 \\
20 \\
25 \\
30 \\
35 \\
36 \\
36.5 \\
37 \\
37.5 \\
\end{tabular} & $\begin{array}{c}73.2 \\
73.2 \\
73.2 \\
72.9 \\
69.7 \\
62.5 \\
60.6 \\
60.1 \\
59.6 \\
- \\
\end{array}$ & \begin{tabular}{|c|}
59.5 \\
59.5 \\
59.5 \\
59.4 \\
59.5 \\
59.6 \\
59.9 \\
60.4 \\
61.9 \\
-.9 \\
\end{tabular} & $\begin{array}{c}47.2 \\
47.2 \\
47.1 \\
-47.2 \\
47.2 \\
47 \\
47 \\
47 \\
-48.5 \\
- \\
- \\
\end{array}$ & \begin{tabular}{||c|}
34.6 \\
36.1 \\
36 \\
35.9 \\
36 \\
36.1 \\
36.3 \\
37 \\
37.6 \\
38.1 \\
\\
\\
\end{tabular} & \begin{tabular}{c}
37.5 \\
$37: 3$ \\
37.1 \\
37.1 \\
37.1 \\
37.3 \\
37.5 \\
37.4 \\
37.4 \\
37.4 \\
-37.8 \\
- \\
\hdashline
\end{tabular} & $\begin{array}{l}52.1 \\
52.2 \\
51.7 \\
51.7 \\
51.7 \\
51.7 \\
50.7 \\
50.6 \\
50.3 \\
50.2 \\
50.1 \\
50 \\
50 \\
50 \\
49.6 \\
\end{array}$ & $\begin{array}{c}63.4 \\
63.4 \\
63.4 \\
62.9 \\
60.4 \\
53.3 \\
52.7 \\
52.8 \\
-9 \\
52.7 \\
-\end{array}$ \\
\hline & \multicolumn{6}{|c|}{$1947--$ Continued } & 1948 \\
\hline $\begin{array}{l}\text { Depth } \\
\text { (feet) }\end{array}$ & June 24 & July 7 & Aug. 27 & oct. 9 & Oct. 30 & Nov. 19 & June 5 \\
\hline Air & 60 & 78.8 & 104 & 59 & 53 & 43 & 82 \\
\hline \begin{tabular}{|c|} 
Water-surface \\
2.5 \\
5 \\
10 \\
12.5 \\
15 \\
17.5 \\
20 \\
22.5 \\
25 \\
30 \\
32.5 \\
35 \\
36 \\
37 \\
37.5 \\
39 \\
\end{tabular} & $\begin{array}{c}69.6 \\
69.6 \\
69.6 \\
68.8 \\
65.5 \\
62.2 \\
58.6 \\
56 \\
54.4 \\
53.5 \\
\\
\end{array}$ & 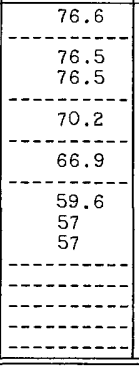 & \begin{tabular}{|c|}
83.8 \\
82.8 \\
82.5 \\
80.7 \\
73.5 \\
68.5 \\
65.2 \\
60.1 \\
58.2 \\
57 \\
56.6 \\
\\
\end{tabular} & $\begin{array}{c}64 \\
63.7 \\
63.4 \\
63.1 \\
63.1 \\
62.3 \\
61 \\
59.4 \\
-4 \\
57.7 \\
\end{array}$ & \begin{tabular}{c}
61.4 \\
61.2 \\
61.2 \\
61 \\
61 \\
-61 \\
61 \\
61 \\
61 \\
\hdashline \\
\hdashline
\end{tabular} & \begin{tabular}{|c|}
44 \\
-44 \\
43.9 \\
43.8 \\
43.8 \\
-43.8 \\
43.6 \\
43.6 \\
43.6 \\
-6 \\
\end{tabular} & $\begin{array}{c}73.8 \\
73 \\
71.7 \\
68.9 \\
66.3 \\
60.8 \\
64 \\
59.5 \\
57 \\
55.3 \\
55.1 \\
\\
\end{array}$ \\
\hline \multirow[b]{2}{*}{$\begin{array}{l}\text { Depth } \\
\text { (feet) }\end{array}$} & \multicolumn{6}{|c|}{$1948--$ Continued } & \\
\hline & July 1 & Aug. 4 & Sept. I & Oct. 8 & Nov. 18 & Dec. 9 & \\
\hline Air & 64 & 60 & 65 & 46 & 45 & 28 & \\
\hline \begin{tabular}{|c|} 
Water-surface \\
2.5 \\
5 \\
7.5 \\
10 \\
15 \\
17.5 \\
20 \\
22.5 \\
25 \\
30 \\
32.5 \\
35 \\
36 \\
37 \\
37.5
\end{tabular} & $\begin{array}{c}74.8 \\
74.6 \\
74.5 \\
74.3 \\
67.8 \\
63.4 \\
60.8 \\
59.4 \\
57.7 \\
56.7 \\
56.4\end{array}$ & \begin{tabular}{|c|}
74.5 \\
74.5 \\
-74.5 \\
74.3 \\
73.3 \\
66.1 \\
-99.5 \\
57.8 \\
56.9 \\
56.6 \\
-
\end{tabular} & $\begin{array}{r}78.5 \\
78.5 \\
.77 .5 \\
777.2 \\
76.4 \\
70 \\
66.3 \\
-.51 \\
58.5 \\
57.5 \\
57.2 \\
-7\end{array}$ & $\begin{array}{r}59.2 \\
59.2 \\
59.2 \\
59.2 \\
59.2 \\
59.2 \\
59 \\
58.8 \\
-8 \\
\\
-\end{array}$ & $\begin{array}{c}46.2 \\
45.8 \\
46.1 \\
46.3 \\
46.4 \\
46.4 \\
46.4 \\
46.4 \\
46.3 \\
46.3 \\
46.4 \\
46.4 \\
46.4 \\
- \\
- \\
- \\
-\end{array}$ & $\begin{array}{c}38.9 \\
38.5 \\
-37.3 \\
38.3 \\
39.5 \\
38.8 \\
39.3 \\
38.5 \\
38.8 \\
39.5 \\
-\end{array}$ & \\
\hline
\end{tabular}


Water surface temperatures for Bruce Lake at Lake Bruce, Ind.

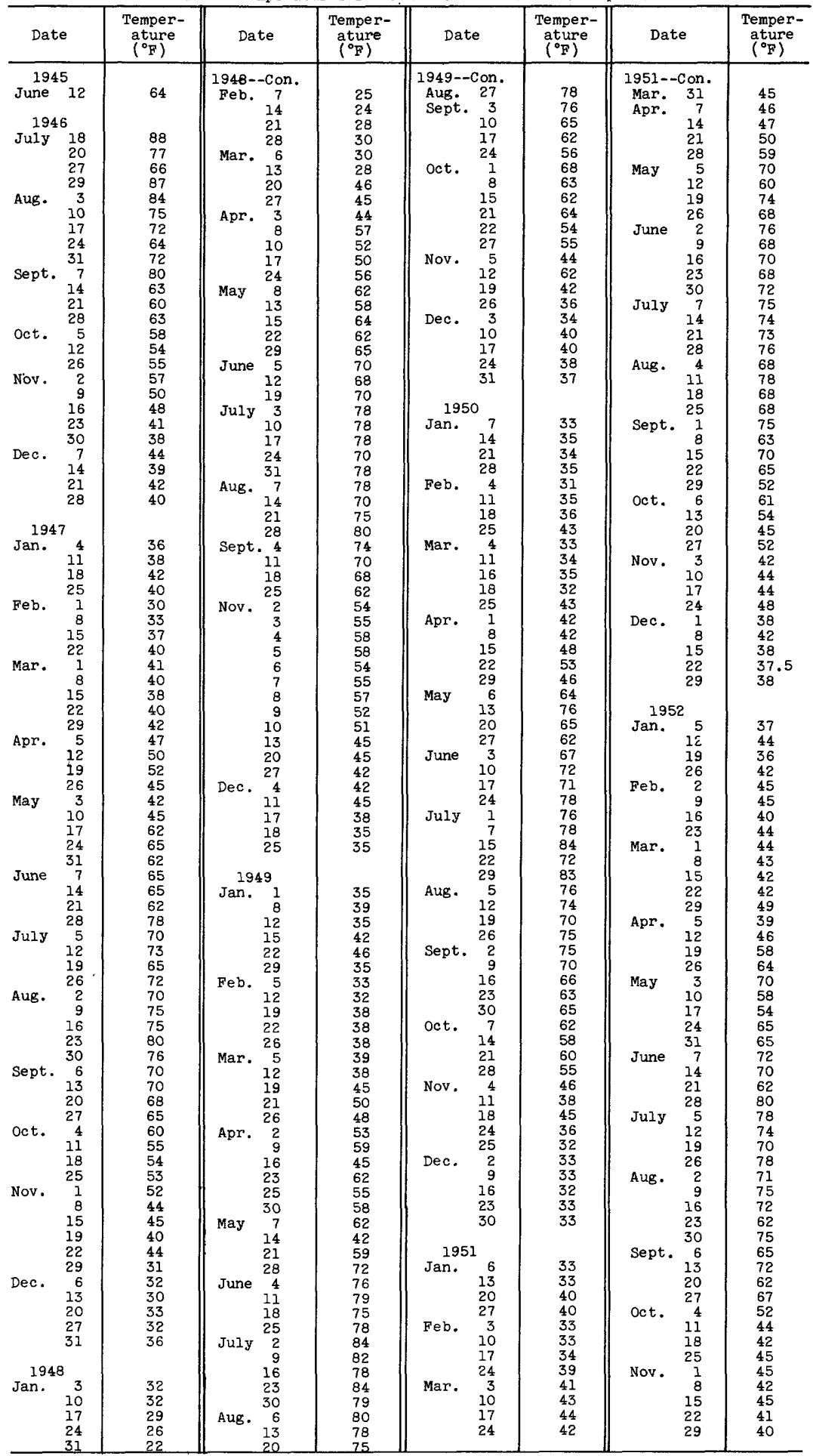


Water surface temperatures for Cedar Lake at Cedar Lake, Ind.

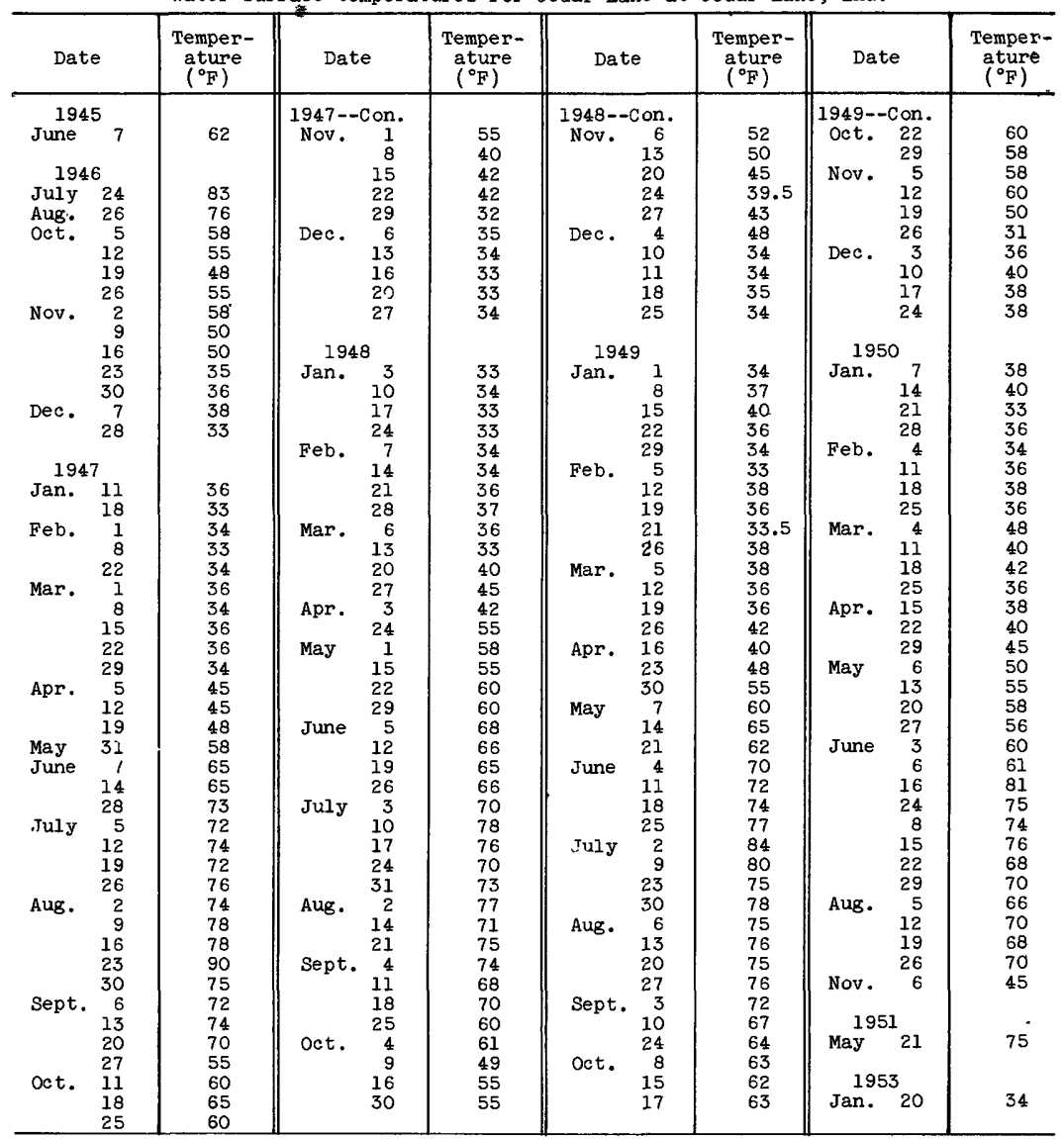

Temperature profiles ( $\left.{ }^{\circ} \mathrm{F}\right)$ for Cedar Lake, at Cedar Lake

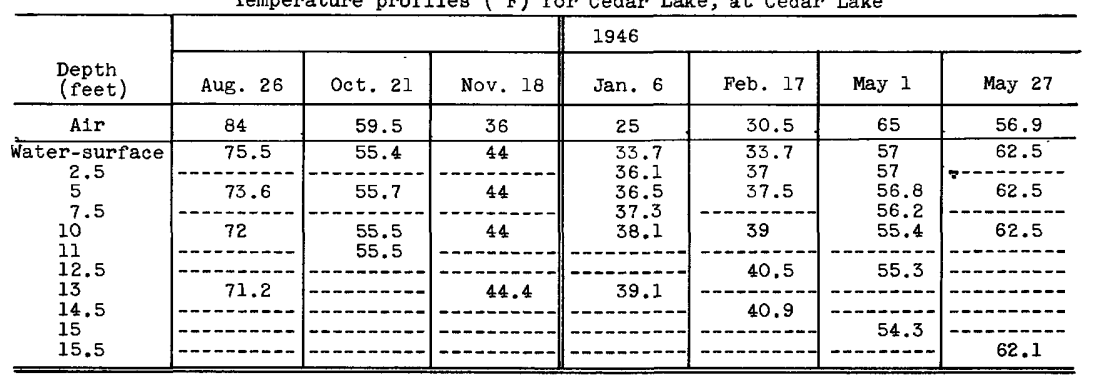


Temperature profiles $\left({ }^{\circ} \mathrm{F}\right)$ for Cedar Lake, at Cedar Lake--Continued

\begin{tabular}{|c|c|c|c|c|c|c|c|}
\hline \multirow{2}{*}{$\begin{array}{l}\text { Depth } \\
\text { (feet) }\end{array}$} & \multicolumn{6}{|c|}{1947} & \multirow{2}{*}{$\frac{1948}{\operatorname{Jan} .30}$} \\
\hline & June 16 & July 23 & Aug. 25 & oct. 1 & oct. 28 & Nov. 18 & \\
\hline Air & 87 & 75.5 & 98 & 57 & 56 & 35 & 25 \\
\hline $\begin{array}{c}\text { Water-surface } \\
2.5 \\
5 \\
7.5 \\
10 \\
12.5 \\
13.5 \\
14.5 \\
15\end{array}$ & $\begin{array}{l}68 \\
65.9 \\
63.7 \\
62.5 \\
62.3 \\
62 \\
61.4\end{array}$ & \begin{tabular}{c}
75.5 \\
73.6 \\
\hdashline 71 \\
\hdashline 71
\end{tabular} & $\begin{array}{c}85.9 \\
85.6 \\
84.8 \\
84.7 \\
82.5 \\
81 \\
79.3 \\
-0-\end{array}$ & 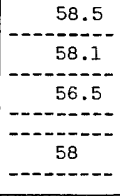 & $\begin{array}{l}62.1 \\
62.3 \\
62.3 \\
62 \\
61.8 \\
61.8 \\
61.8 \\
- \\
-\end{array}$ & $\begin{array}{c}39.2 \\
-39.2 \\
38.9 \\
39 \\
39 \\
40.5\end{array}$ & $\begin{array}{c}35.4 \\
-40 \\
40.4 \\
41.3\end{array}$ \\
\hline \multirow{2}{*}{$\begin{array}{l}\text { Depth } \\
\text { (feet) }\end{array}$} & \multicolumn{7}{|c|}{1948} \\
\hline & June 1 & July 2 & Aug. 2 & Aug. 30 & Oct. 4 & Nov. 15 & Dec. 31 \\
\hline Air & 54 & 81 & 77 & 76 & $-----n-\cdots$ & 45 & 32 \\
\hline $\begin{array}{c}\text { Water-surface } \\
2.5 \\
5 \\
7.5 \\
10 \\
12 \\
12.5 \\
13 \\
13.5 \\
14 \\
14.5\end{array}$ & $\begin{array}{c}67.4 \\
66.1 \\
65.8 \\
65.8 \\
65.8 \\
64.1 \\
63.7\end{array}$ & 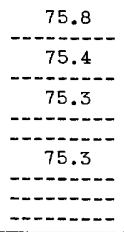 & $\begin{array}{c}79.2 \\
77.3 \\
77.2 \\
76.9 \\
76.4 \\
76.1 \\
\end{array}$ & $\begin{array}{r}82.3 \\
82.3 \\
82.4 \\
82 \\
80.5 \\
78.2 \\
77.8 \\
-0.8\end{array}$ & 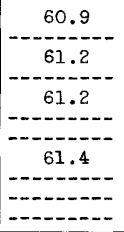 & $\begin{array}{c}42.6 \\
42.8 \\
42.2 \\
41.8 \\
41.8 \\
41.7 \\
41.6\end{array}$ & $\begin{array}{c}34.7 \\
34.1 \\
33.5 \\
34.3 \\
35.2 \\
36 \\
36 \\
-2 .\end{array}$ \\
\hline
\end{tabular}

Water surface temperatures for Cedar Lake near Waterloo, Ind.

\begin{tabular}{|c|c|c|c|c|c|c|c|}
\hline Date & $\begin{array}{l}\text { Temper- } \\
\text { ature } \\
\left({ }^{\circ} \mathrm{F}\right)\end{array}$ & Date & $\begin{array}{l}\text { Temper- } \\
\text { ature } \\
\left({ }^{\circ} \mathrm{F}\right)\end{array}$ & Date & $\begin{array}{l}\text { Temper- } \\
\text { ature } \\
\left({ }^{\circ} \mathrm{F}\right)\end{array}$ & Date & $\begin{array}{l}\text { Temper- } \\
\text { ature } \\
\left({ }^{\circ} \mathrm{F}\right)\end{array}$ \\
\hline $\begin{array}{ll}21945 \\
\text { July } & 2 \\
\text { Sept. } & 4\end{array}$ & $\begin{array}{l}72 \\
78\end{array}$ & $\begin{array}{l}\text { 1945--Con. } \\
\text { Dec. } \quad 12\end{array}$ & 36 & \begin{tabular}{l}
\multicolumn{2}{c}{1948} \\
Feb. 29 \\
Sept. 29
\end{tabular} & $\begin{array}{l}34 \\
61\end{array}$ & $\begin{array}{l}1949 \\
\operatorname{Jan} .\end{array}$ & 36 \\
\hline
\end{tabular}

Water surface temperatures for Crooked Lake near Wolflake, Ind.

\begin{tabular}{|c|c|c|c|c|c|c|c|}
\hline Date & $\begin{array}{l}\text { Temper- } \\
\text { ature } \\
\left({ }^{\circ} \mathrm{F}\right)\end{array}$ & Date & $\begin{array}{l}\text { Temper- } \\
\text { ature } \\
\left({ }^{\circ} \mathrm{F}\right)\end{array}$ & Date & $\begin{array}{l}\text { Temper- } \\
\text { ature } \\
\left({ }^{\circ} \mathrm{F}\right)\end{array}$ & Date & $\begin{array}{c}\text { Temper- } \\
\text { ature } \\
\left({ }^{\circ} \mathrm{F}\right)\end{array}$ \\
\hline 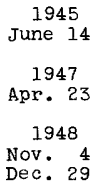 & $\begin{array}{l}57.5 \\
35\end{array}$ & \begin{tabular}{cr}
\multicolumn{2}{c}{1949} \\
Jan. & 11 \\
& 21 \\
Mar. & 9 \\
& 23 \\
& 31 \\
Sept. & 6 \\
& 14
\end{tabular} & $\begin{array}{l}34 \\
35 \\
38 \\
42 \\
45 \\
76 \\
67\end{array}$ & 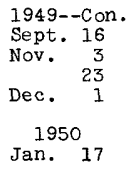 & $\begin{array}{l}64 \\
51 \\
43 \\
42 \\
\\
35\end{array}$ & $\begin{array}{c}\text { 1950--Con. } \\
\text { Mar. } 8 \\
13 \\
\text { Nov. } 7 \\
21 \\
1951 \\
\text { May } 15\end{array}$ & $\begin{array}{l}35 \\
44 \\
53 \\
40 \\
\\
64\end{array}$ \\
\hline
\end{tabular}


Water surface temperatures for Flint Lake near Valpara1so, Ind.

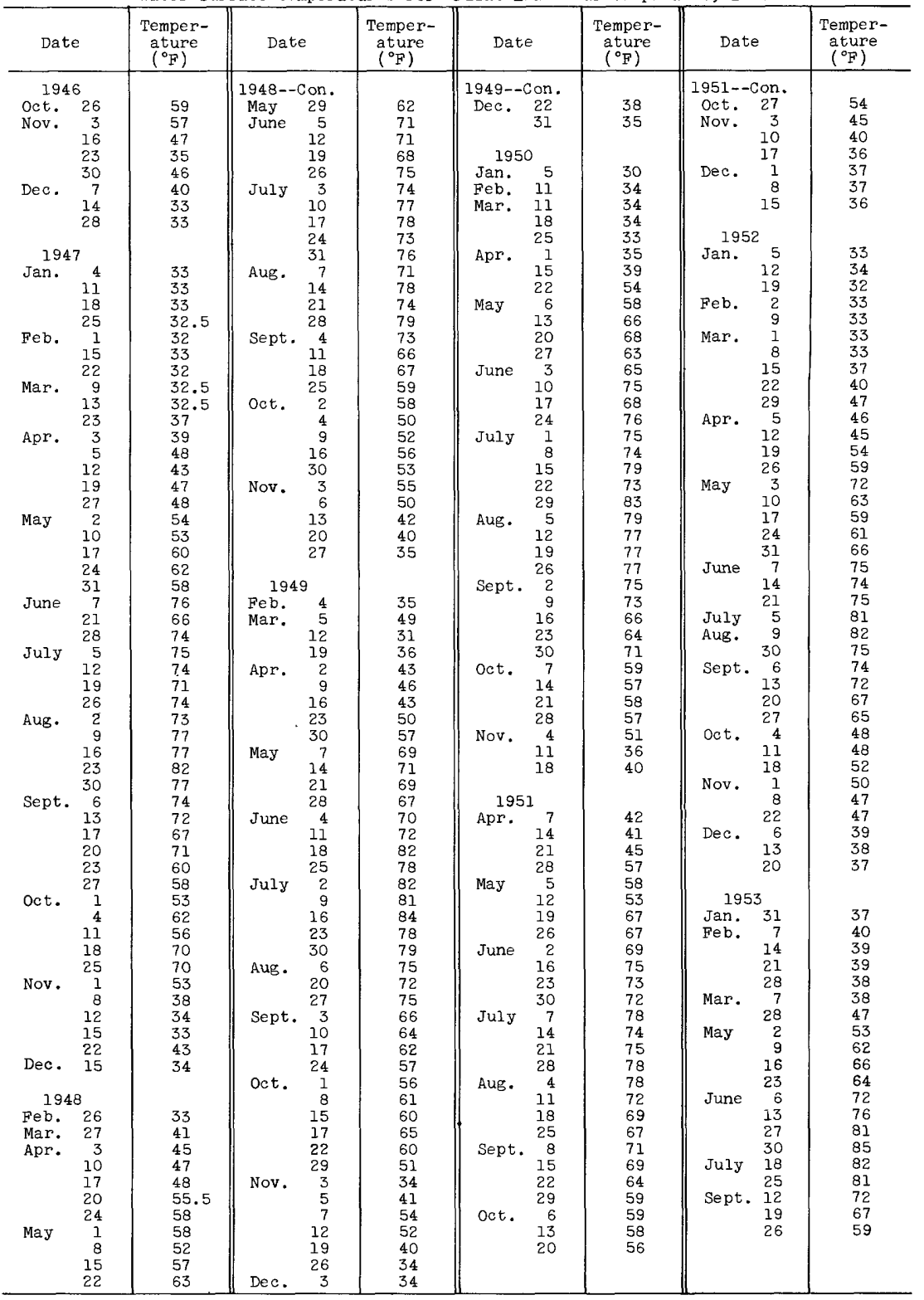


Temperature profiles $\left({ }^{\circ} \mathrm{F}\right)$ for Flint Lake, near Valparaiso

\begin{tabular}{|c|c|c|c|c|c|c|c|c|c|}
\hline \multirow{2}{*}{$\begin{array}{l}\text { Depth } \\
\text { (feet) }\end{array}$} & \multicolumn{2}{|c|}{1946} & \multicolumn{7}{|c|}{1947} \\
\hline & oct. 21 & Nov. 18 & $\operatorname{Jan} .6$ & Feb. 17 & Mar. 1 & May 27 & June 17 & July 31 & Aug. 25 \\
\hline Air & 63 & 44 & 25 & 32.5 & 53 & 58 & 72 & 75 & 95 \\
\hline $\begin{array}{c}\text { Water-surface } \\
2.5 \\
5 \\
7 \\
7.5 \\
10 \\
12.5 \\
15 \\
17.5 \\
20 \\
22.5 \\
25 \\
27.5 \\
30 \\
32.5 \\
35 \\
40 \\
45 \\
50 \\
55 \\
60 \\
64 \\
65 \\
67.5 \\
69 \\
70 \\
70.5 \\
\end{array}$ & $\begin{array}{r}57.9 \\
57.9 \\
57.8 \\
57.6 \\
57.2 \\
57 \\
56.6 \\
54.5 \\
52.3 \\
52 \\
51.7 \\
51.4 \\
51.3 \\
51.3 \\
51.3 \\
51.3 \\
-0.3 \\
-0.0 \\
-0.0 \\
\end{array}$ & 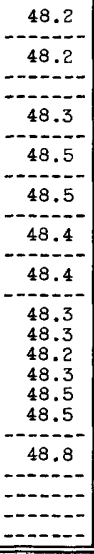 & 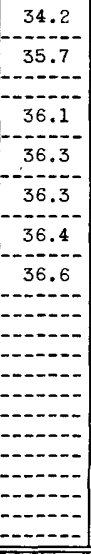 & \begin{tabular}{c}
33.6 \\
37.6 \\
39 \\
\hdashline 39.1 \\
39.3 \\
\hdashline 39.3 \\
\hdashline 39.4 \\
38.9 \\
38.8 \\
38.7 \\
38.6 \\
38.6 \\
38.8 \\
39 \\
39.1 \\
39.6 \\
-0.0 \\
\end{tabular} & $\begin{array}{c}56.1 \\
56.1 \\
56 \\
55.3 \\
-53.3 \\
52.8 \\
52.6 \\
-52 \\
-51 \\
51 \\
50.3 \\
48.9 \\
47.8 \\
47.5 \\
47.3 \\
47.1 \\
46.9 \\
46.7 \\
46.7 \\
-0.7 \\
\end{array}$ & \begin{tabular}{c}
63.7 \\
063.7 \\
\hdashline-1.7 \\
63.7 \\
62.2 \\
57.7 \\
54 \\
52 \\
50.8 \\
50 \\
49.3 \\
48.9 \\
48.8 \\
48.6 \\
48.4 \\
48.3 \\
48 \\
-4
\end{tabular} & 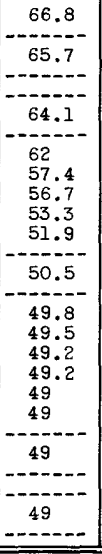 & 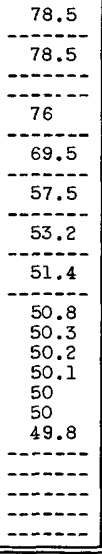 & $\begin{array}{c}85 \\
84.9 \\
84.8 \\
81.8 \\
74.2 \\
69 \\
62.3 \\
57.8 \\
52.5 \\
51.3 \\
50.6 \\
50.3 \\
50.2 \\
50.1 \\
50.1 \\
50.1 \\
50.1 \\
-1 \\
50\end{array}$ \\
\hline \multirow{2}{*}{$\begin{array}{l}\text { Depth } \\
\text { (feet) }\end{array}$} & \multicolumn{3}{|c|}{ 1947--Continued } & \multicolumn{6}{|c|}{1948} \\
\hline & oct. 2 & oct. 27 & Nov. 18 & June 1 & July 2 & Aug. 2 & Aug. 30 & oct. 4 & Nov. 19 \\
\hline Air & 57 & 57 & 39.5 & 78 & 87 & 70 & 74 & 61 & 53 \\
\hline $\begin{array}{c}\text { Water-surface } \\
2.5 \\
5 \\
10 \\
12.5 \\
15 \\
17.5 \\
20 \\
22.5 \\
25 \\
30 \\
35 \\
40 \\
45 \\
50 \\
55 \\
60 \\
64 \\
65 \\
67 \\
67.5 \\
70 \\
71.5 \\
\end{array}$ & 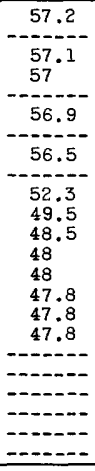 & 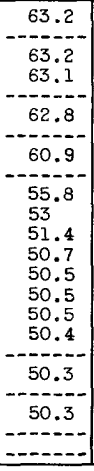 & 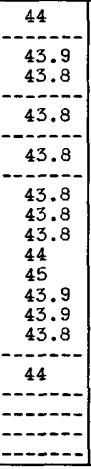 & \begin{tabular}{|l}
69.3 \\
68.8 \\
67.8 \\
65.4 \\
63.2 \\
59 \\
57.3 \\
56 \\
\hdashline 54.6 \\
53.5 \\
52 \\
50.6 \\
50 \\
49.8 \\
49.6 \\
49.4 \\
49.4 \\
-9.4 \\
49.4 \\
$-0 .-$ \\
\end{tabular} & $\begin{array}{c}74.8 \\
-74.7 \\
73.9 \\
69 \\
61.7 \\
57.3 \\
55.3 \\
-05.3 \\
53.3 \\
52.3 \\
51.5 \\
50.4 \\
50 \\
49.9 \\
49.7 \\
49.6 \\
-49.6 \\
-0.6 \\
-19.6 \\
49.6 \\
\end{array}$ & $\begin{array}{l}79.2 \\
79 \\
77.7 \\
77 \\
76 \\
68.2 \\
61.1 \\
57 \\
-0 .-- \\
53.7 \\
52.7 \\
51.7 \\
51 \\
50.7 \\
50.6 \\
50.6 \\
50.5 \\
-50.4 \\
50.3 \\
-2-- \\
-2 .- \\
\end{array}$ & 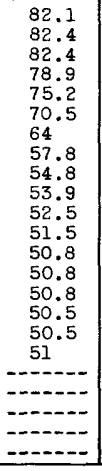 & 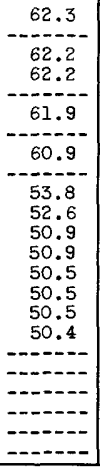 & $\begin{array}{c}46.2 \\
46.2 \\
46.4 \\
46.4 \\
46.3 \\
46.5 \\
46 \\
46.3 \\
46.6 \\
46.6 \\
46.2 \\
46.4 \\
45.9 \\
46.1 \\
46 \\
-0.9 \\
-0.9 \\
\end{array}$ \\
\hline
\end{tabular}


Water surface temperatures for Hamilton Lake at Ham1lton, Ind.

\begin{tabular}{|c|c|c|c|c|c|c|c|}
\hline Date & $\begin{array}{c}\text { Temper- } \\
\text { ature } \\
\left({ }^{\circ} \mathrm{F}\right)\end{array}$ & Date & $\begin{array}{l}\text { Temper- } \\
\text { ature } \\
\left({ }^{\circ} \mathrm{F}\right)\end{array}$ & Date & $\begin{array}{l}\text { Temper- } \\
\text { ature } \\
\left({ }^{\circ} \mathrm{F}\right)\end{array}$ & Date & $\begin{array}{c}\text { Temper- } \\
\text { ature } \\
\left({ }^{0} \mathrm{~F}\right)\end{array}$ \\
\hline 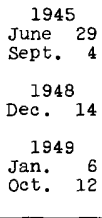 & $\begin{array}{l}84 \\
79 \\
35 \\
35.5 \\
65\end{array}$ & 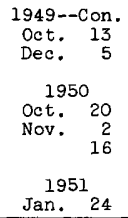 & $\begin{array}{l}64 \\
39 \\
\\
59.5 \\
57 \\
44\end{array}$ & $\begin{array}{c}1951--C o n . \\
\text { Aug. } 9 \\
\text { Oct. } 9 \\
\text { Dec. } 14 \\
1952 \\
\text { Feb. } 12 \\
\text { Dec. } 10 \\
\text { 1953 } \\
\text { Jan. } 8\end{array}$ & $\begin{array}{l}28 \\
35 \\
34\end{array}$ & $\begin{array}{cc}1953--C o n \\
\text { Jan. } & 30 \\
\text { Mar. } & 10 \\
\text { Apr. } & 2 \\
& 23 \\
\text { May } & 19 \\
\text { July } & 8 \\
\text { Aug. } & 5 \\
\text { Dec. } & 1\end{array}$ & $\begin{array}{l}35 \\
37 \\
45 \\
49 \\
60 \\
77 \\
76 \\
77\end{array}$ \\
\hline
\end{tabular}

Water surface temperatures for Jimerson Lake at Nevada M111s, Ind.

\begin{tabular}{|c|c|c|c|c|c|c|c|}
\hline Date & $\begin{array}{c}\text { Temper- } \\
\text { ature } \\
\left({ }^{\circ} \mathrm{F}\right)\end{array}$ & Date & $\begin{array}{c}\text { Temper- } \\
\text { ature } \\
\left({ }^{\circ} \mathrm{F}\right)\end{array}$ & Date & $\begin{array}{c}\text { Temper- } \\
\text { ature } \\
\left({ }^{\circ} \mathrm{F}\right)\end{array}$ & Date & $\begin{array}{c}\text { Temper- } \\
\text { ature } \\
\text { ( }{ }^{\circ} \text { il) }\end{array}$ \\
\hline 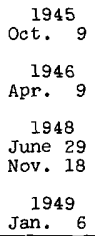 & $\begin{array}{l}51 \\
49 \\
75 \\
45 \\
35.5\end{array}$ & \begin{tabular}{cr}
$1949--C o n$. \\
Feb. & 17 \\
Sept. & 15 \\
Oct. & 12 \\
Dec. & 6 \\
& 7 \\
\multicolumn{2}{l}{} \\
1950 \\
Oct. 19 \\
Nov. 15
\end{tabular} & $\begin{array}{l}34.5 \\
65 \\
66 \\
38 \\
37 \\
\\
58 \\
48\end{array}$ & $\begin{array}{lr}1951 \\
\text { Jan. } 9 \\
\text { July } 11 \\
\text { Aug. } 9 \\
\text { Sept. } 5 \\
\text { Oct. } 9 \\
\text { Nov. } 17 \\
\text { Dec. } 21 \\
\\
\text { 1952 } \\
\text { Nov. } 14 \\
\text { Dec. } 10\end{array}$ & $\begin{array}{l}34 \\
77 \\
73 \\
68 \\
55 \\
37 \\
28 \\
\\
\\
\\
41 \\
39\end{array}$ & \begin{tabular}{lr}
\multicolumn{2}{c}{1953} \\
Jan. & 9 \\
& 30 \\
Apr. & 3 \\
May & 19 \\
June & 15 \\
July & 8 \\
Aug. & 5 \\
Sept. 29
\end{tabular} & $\begin{array}{l}34 \\
36 \\
44 \\
66 \\
73 \\
79 \\
76 \\
65\end{array}$ \\
\hline
\end{tabular}

Water surface temperatures for Koontz Lake at Koontz Lake, Ind.

\begin{tabular}{|c|c|c|c|c|c|c|c|}
\hline Date & $\begin{array}{l}\text { Temper- } \\
\text { ature } \\
\left({ }^{\circ} \mathrm{F}\right)\end{array}$ & Date & $\begin{array}{l}\text { Temper- } \\
\text { ature } \\
\left({ }^{\circ} \mathrm{F}\right)\end{array}$ & Date & $\begin{array}{l}\text { Temper- } \\
\text { ature } \\
\left({ }^{\circ} \mathrm{F}\right)\end{array}$ & Date & $\begin{array}{l}\text { Temper- } \\
\text { ature } \\
\left({ }^{\circ} \mathrm{F}\right)\end{array}$ \\
\hline $\begin{array}{cc}\text { June }^{1945} 11 \\
1946 \\
\text { Apr. } & 16\end{array}$ & 66 & $\begin{array}{c}1946--C o n \\
\text { July } 19 \\
1948 \\
\text { Feb. } 10\end{array}$ & 85 & $\begin{array}{r}1949 \\
\text { Nov. } 9 \\
28 \\
29\end{array}$ & $\begin{array}{l}49 \\
37 \\
35\end{array}$ & $\begin{array}{c}1950 \\
\text { June } 15 \\
1951 \\
\text { Jan. } 11\end{array}$ & $\begin{array}{l}79 \\
33\end{array}$ \\
\hline
\end{tabular}

Temperature prof 11 es $\left({ }^{\circ} \mathrm{F}\right)$ for Koontz Lake, at Koontz Lake July 25,1946

\begin{tabular}{c|c|c|c||c|c|c|c}
\hline $\begin{array}{c}\text { Depth } \\
\text { (feet) }\end{array}$ & Site A & Site B & Site C & $\begin{array}{c}\text { Depth } \\
\text { (feet) }\end{array}$ & Site A & Site B & Site C \\
\hline Air & 76 & 76 & 76 & 20 & 65.8 & 64.2 & 55.4 \\
\hline Water-surface & 80.3 & 79.3 & 79.8 & 23 & $* 61.8$ & -58.8 & $* 53.0$ \\
5 & 79.4 & 77.8 & 78.7 & 25 & & $* 56.5$ & \\
10 & 78.5 & 76.8 & 72.5 & & & & \\
15 & 71.2 & 70.2 & 61.5 & & & & \\
\hline
\end{tabular}

* Bottom 
Water surface temperatures for Lake James at Lake James, Ind.

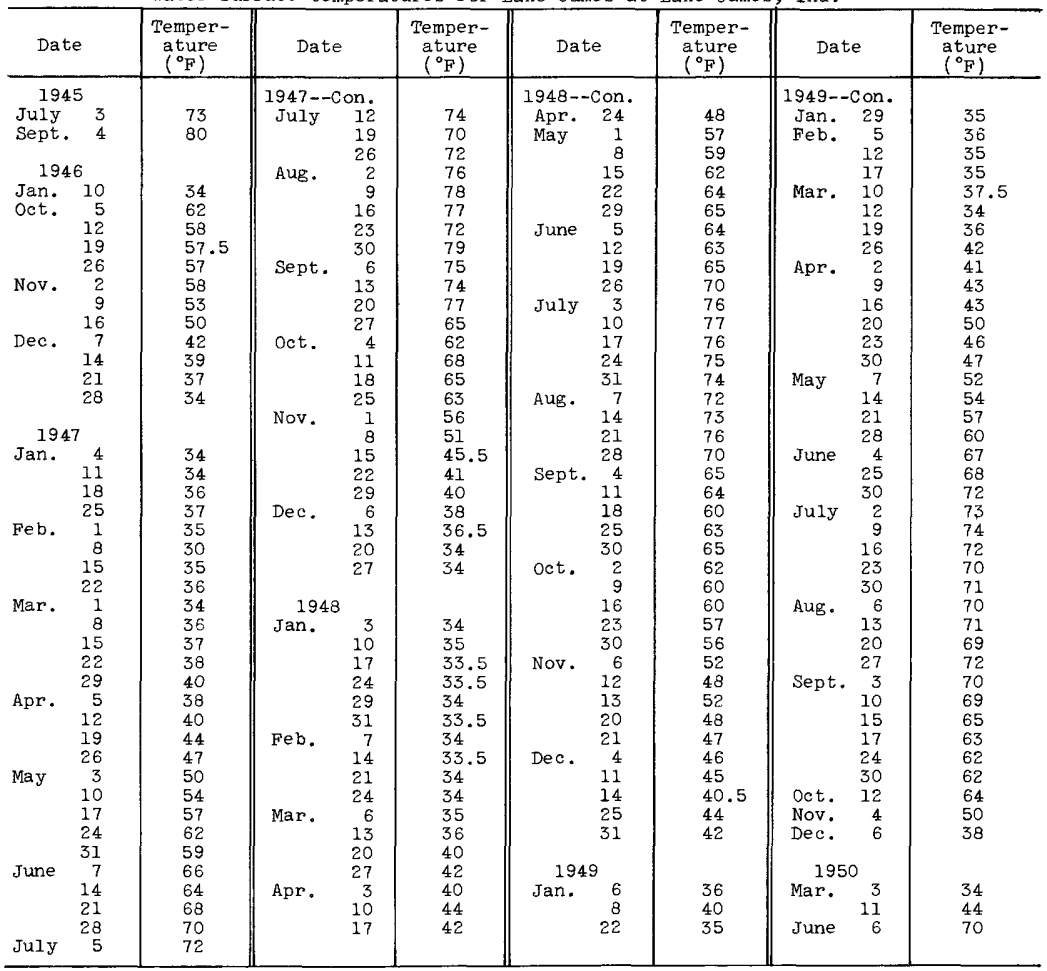

Temperature profiles $\left({ }^{\circ} \mathrm{F}\right)$ for Lake James, at Lake James

\begin{tabular}{|c|c|c|c|c|c|c|c|}
\hline \multirow{2}{*}{$\begin{array}{l}\text { Depth } \\
\text { (feet) }\end{array}$} & \multicolumn{4}{|c|}{1946} & \multicolumn{3}{|c|}{1947} \\
\hline & Sept. 29 & oct. 24 & Nov. 23 & Dec. 18 & Apr. 29 & May 25 & June 23 \\
\hline $\mathrm{A} 1 \mathrm{r}$ & 56.6 & 69.5 & 32 & 16 & 64 & 60.8 & 78 \\
\hline $\begin{array}{l}\text { Water-surface } \\
2.5 \\
5 \\
10 \\
15 \\
17.5 \\
20 \\
22 \\
24 \\
25 \\
26 \\
28 \\
30 \\
32 \\
34 \\
35 \\
36 \\
38 \\
40 \\
42.5 \\
45 \\
47.5 \\
50 \\
55 \\
60 \\
65 \\
70\end{array}$ & \begin{tabular}{|c|}
68 \\
68 \\
68 \\
68 \\
68 \\
66.8 \\
66.6 \\
66.5 \\
65.4 \\
64.8 \\
62.8 \\
60.5 \\
57.5 \\
56 \\
54.7 \\
50.9 \\
49.5 \\
49.2 \\
48.3 \\
47 \\
46.1
\end{tabular} & 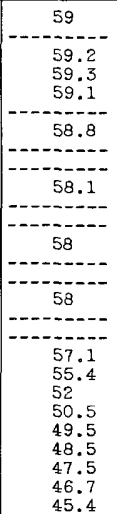 & 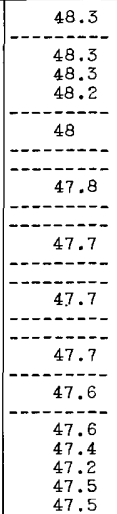 & 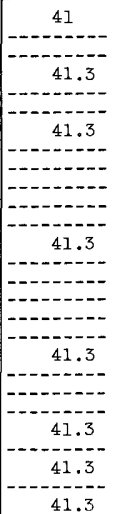 & $\begin{array}{c}48 \\
48 \\
48.4 \\
48.4 \\
48.3 \\
48.2 \\
48.1 \\
48.1 \\
47.4 \\
46.5 \\
46 \\
45.8 \\
45.5 \\
44.8 \\
44.4 \\
43.8\end{array}$ & $\begin{array}{c}62.6 \\
62.6 \\
62.6 \\
62.6 \\
61.4 \\
56.7 \\
53.1 \\
51.8 \\
51.3 \\
49.5 \\
48.8 \\
48.2 \\
47.2 \\
46.6 \\
45.7 \\
45.2 \\
44.7\end{array}$ & $\begin{array}{c}70.5 \\
70.5 \\
70.3 \\
69.7 \\
69 \\
67 \\
62 \\
56.2 \\
52.5 \\
40.3 \\
48.6 \\
47.7 \\
47.1 \\
46.2 \\
45.2 \\
45\end{array}$ \\
\hline
\end{tabular}


Temperature profiles $\left({ }^{\circ} \mathrm{F}\right)$ for Lake James, at Lake James--Cont1nued

\begin{tabular}{|c|c|c|c|c|c|c|c|}
\hline \multirow{2}{*}{$\begin{array}{l}\text { Depth } \\
\text { (feet) }\end{array}$} & \multicolumn{4}{|c|}{1946} & \multicolumn{3}{|c|}{1947} \\
\hline & Sept. 29 & Oct. 24 & Nov. 23 & Dec. 18 & Apr. 29 & May 25 & June 23 \\
\hline $\begin{array}{l}75 \\
77.5 \\
80 \\
81 \\
82 \\
82.5 \\
83 \\
85 \\
86 \\
\end{array}$ & 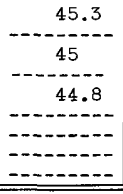 & 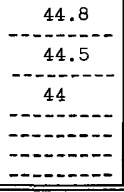 & $\begin{array}{c}47.1 \\
47 \\
46.7 \\
45.6 \\
-0 .- \\
\end{array}$ & \begin{tabular}{c}
41.3 \\
43 \\
\hdashline-10 \\
\\
\hdashline
\end{tabular} & $\begin{array}{c}43.4 \\
43.4 \\
42.8 \\
-0 . \\
\end{array}$ & $\begin{array}{c}44.4 \\
-44.2 \\
43.7 \\
-0 .- \\
\end{array}$ & $\begin{array}{l}44.7 \\
-44.5 \\
-0.5 \\
\\
\\
44.5 \\
44\end{array}$ \\
\hline \multirow{2}{*}{$\begin{array}{l}\text { Depth } \\
\text { (feet) }\end{array}$} & \multicolumn{5}{|c|}{ 1947--ContInued } & \multicolumn{2}{|c|}{1948} \\
\hline & July 30 & Aug. 27 & oct. 9 & Oct. 29 & Nov. 19 & June 3 & June 30 \\
\hline Air & 95 & 82.5 & 59 & 55 & 40 & 84 & 76 \\
\hline $\begin{array}{c}\text { Water-surface } \\
2.5 \\
5 \\
10 \\
12.5 \\
15 \\
17.5 \\
20 \\
25 \\
30 \\
35 \\
40 \\
45 \\
50 \\
55 \\
60 \\
65 \\
70 \\
75 \\
80 \\
82.5 \\
84 \\
85 \\
86 \\
87 \\
\end{array}$ & $\begin{array}{r}77.4 \\
777 \\
77 \\
76.3 \\
71.6 \\
63.7 \\
57.6 \\
53.6 \\
51.2 \\
49.3 \\
48 \\
47.7 \\
46.7 \\
46.2 \\
45.3 \\
44.8 \\
44.4 \\
4.4 \\
44.4 \\
44.2 \\
\end{array}$ & $\begin{array}{c}82.5 \\
81.9 \\
81.9 \\
79.5 \\
72.2 \\
62.7 \\
57.5 \\
54.4 \\
51.7 \\
49.8 \\
48.3 \\
47.6 \\
47 \\
46.2 \\
45.7 \\
45.2 \\
44.9 \\
-0.9 \\
44.6 \\
-0 . \\
\end{array}$ & 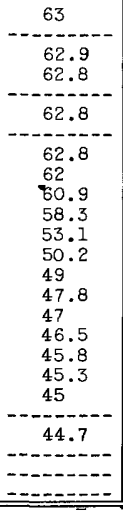 & $\begin{array}{c}61.4 \\
61.7 \\
61.7 \\
61.8 \\
61.3 \\
61.5 \\
61.5 \\
57.9 \\
53.7 \\
50.2 \\
48.8 \\
48 \\
47.2 \\
46.3 \\
45.9 \\
45.4 \\
45 \\
-0.9 \\
44.9 \\
4.9 \\
-2\end{array}$ & $\begin{array}{c}47.4 \\
47.4 \\
47.4 \\
47.4 \\
47.4 \\
47.2 \\
47.2 \\
47.2 \\
47.2 \\
47.2 \\
47.2 \\
47.2 \\
47.2 \\
47.2 \\
47.2 \\
46.6 \\
45.5 \\
45.2 \\
45.2 \\
-0.5 \\
-9.9\end{array}$ & 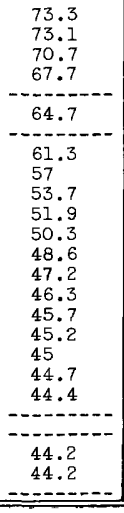 & 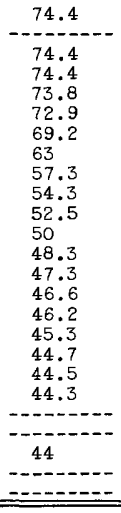 \\
\hline \multirow{2}{*}{$\begin{array}{l}\text { Depth } \\
\text { (feet) }\end{array}$} & \multicolumn{7}{|c|}{ 1948--Continued } \\
\hline & Aug. 4 & Sept. 2 & oct. 7 & Nov. 18 & Dec. 9 & \multicolumn{2}{|l|}{ Dec. 18} \\
\hline Air & 64 & 75 & 52 & 53 & $\ldots-\cdots \cdots$ & \multicolumn{2}{|l|}{16} \\
\hline $\begin{array}{c}\text { Water-surface } \\
5 \\
10 \\
15 \\
17.5 \\
20 \\
22.5 \\
25 \\
30 \\
35 \\
40 \\
45 \\
50 \\
55 \\
60 \\
65 \\
70 \\
75 \\
80 \\
81 \\
85 \\
86.5 \\
87 \\
\end{array}$ & \begin{tabular}{r}
75.1 \\
75.5 \\
75.6 \\
75.5 \\
73.6 \\
69.2 \\
\hdashline 60.8 \\
56.2 \\
53.9 \\
51.9 \\
49.7 \\
48 \\
47.3 \\
47.1 \\
46.4 \\
45.9 \\
45.6 \\
45.3 \\
45 \\
$-0-.9$ \\
.--- \\
\end{tabular} & 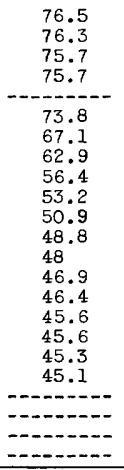 & $\begin{array}{c}60 \\
60 \\
60 \\
60 \\
-60 \\
60 \\
60 \\
54.9 \\
51.3 \\
49.1 \\
47.9 \\
47.2 \\
46.4 \\
45.7 \\
45.2 \\
44.9 \\
44.7 \\
44.5 \\
-2.7 \\
\\
\end{array}$ & $\begin{array}{c}47 \\
46.9 \\
47.3 \\
47.3 \\
-47.3 \\
47 \\
47 \\
46.6 \\
47 \\
44.5 \\
47.4 \\
47.3 \\
47.5 \\
47.5 \\
47.1 \\
47.4 \\
46 \\
44 \\
44.3 \\
-5.3 \\
44.3 \\
\end{array}$ & 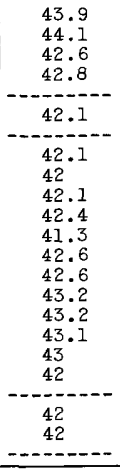 & $\begin{array}{c}41 \\
41.3 \\
41.3 \\
41.3 \\
41.3 \\
41.3 \\
41.3 \\
41.3 \\
41.3 \\
43 \\
\\
\\
\\
\end{array}$ & \\
\hline
\end{tabular}


Water surface temperatures for Lake Manttou at Rochester, Ind.

\begin{tabular}{c|c||c|c||c|c|c|c}
\hline Date & $\begin{array}{c}\text { Temper- } \\
\text { ature } \\
\left({ }^{\circ} \mathrm{F}\right)\end{array}$ & Date & $\begin{array}{c}\text { Temper- } \\
\text { ature } \\
\left({ }^{\circ} \mathrm{F}\right)\end{array}$ & Date & $\begin{array}{c}\text { Temper- } \\
\text { ature } \\
\left({ }^{\circ} \mathrm{F}\right)\end{array}$ & $\begin{array}{c}\text { Temper- } \\
\text { ature } \\
\left({ }^{\circ} \mathrm{F}\right)\end{array}$ \\
\hline $\begin{array}{c}1945 \\
\text { June } 12\end{array}$ & 67 & $\begin{array}{c}1948 \\
\text { Sept. } 14\end{array}$ & 72 & $\begin{array}{c}1949 \\
\text { Nov. } 15\end{array}$ & 47 & $\begin{array}{c}1951 \\
\text { June } 12\end{array}$ & 72 \\
\hline
\end{tabular}

Water surface temperatures for Loon Lake at ormas, Ind.

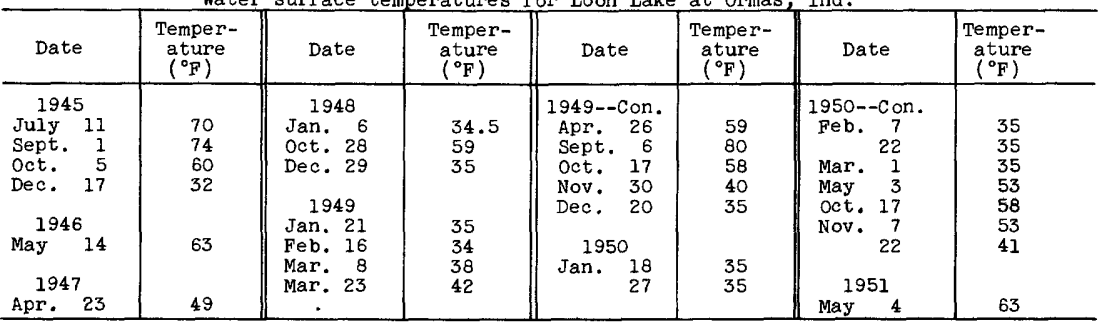

Water surface temperatures for Maxinkuckee Lake at Culver, Ind.

\begin{tabular}{|c|c|c|c|c|c|c|c|}
\hline Date & $\begin{array}{c}\text { Temper- } \\
\text { ature } \\
\left({ }^{\circ} F\right)\end{array}$ & Date & $\begin{array}{c}\text { Temper- } \\
\text { ature } \\
\left({ }^{\circ} \mathrm{F}\right)\end{array}$ & Date & $\begin{array}{c}\text { Temper- } \\
\text { ature } \\
\left({ }^{\circ} \mathrm{F}\right) \\
\end{array}$ & Date & $\begin{array}{c}\text { Temper- } \\
\text { ature } \\
\left({ }^{\circ} \mathrm{F}\right)\end{array}$ \\
\hline 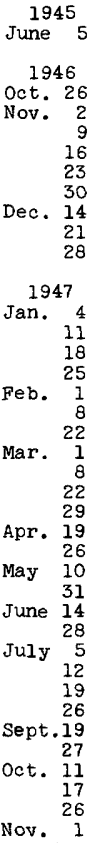 & $\begin{array}{l}33 \\
34 \\
36 \\
35 \\
33 \\
34 \\
33 \\
33 \\
34 \\
38 \\
37 \\
44 \\
50 \\
56 \\
66 \\
65 \\
78 \\
77 \\
80 \\
73 \\
78 \\
73 \\
63 \\
65 \\
68 \\
64 \\
57\end{array}$ & 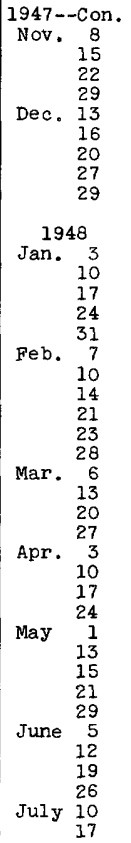 & $\begin{array}{l}43 \\
43 \\
44 \\
35 \\
33 \\
34 \\
32 \\
33 \\
33 \\
\\
33 \\
33 \\
32 \\
32 \\
32 \\
32 \\
33.5 \\
32 \\
32 \\
40.5 \\
34 \\
34 \\
38 \\
36 \\
45 \\
47 \\
52 \\
53 \\
57 \\
58 \\
58 \\
60 \\
68 \\
73 \\
72 \\
70 \\
69 \\
74 \\
85 \\
81\end{array}$ & 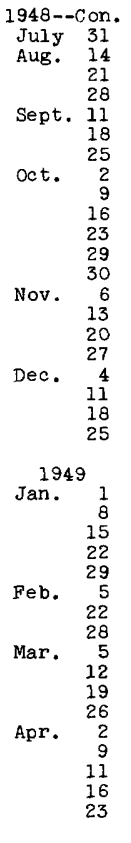 & $\begin{array}{l}33 \\
34 \\
36 \\
34 \\
33 \\
33 \\
33.5 \\
36 \\
38 \\
33 \\
38 \\
45 \\
48 \\
49 \\
56 \\
44 \\
52\end{array}$ & 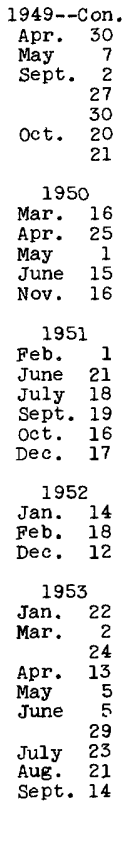 & $\begin{array}{l}59 \\
69 \\
69 \\
68.5 \\
54 \\
64 \\
42.5 \\
\\
35 \\
48 \\
54 \\
78 \\
45 \\
\\
33 \\
73 \\
83 \\
67 \\
63 \\
31\end{array}$ \\
\hline
\end{tabular}


Water temperatures $\left({ }^{\circ} \mathrm{F}\right)$ for Maxinkuckee Lake

[Data from Evermann and Clark, 1920]

\begin{tabular}{|c|c|c|c|c|c|c|c|}
\hline \multirow[t]{2}{*}{ Date } & \multirow{2}{*}{$\begin{array}{c}\text { February } \\
6 \text { a.m. }\end{array}$} & \multicolumn{4}{|c|}{ July } & \multicolumn{2}{|c|}{ August } \\
\hline & & $6 \mathrm{a} \cdot \mathrm{m}$ & Noon & 6 p.m. & Average & 6 a.m. & Noon \\
\hline 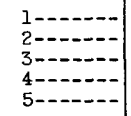 & $\begin{array}{c}32 \\
32 \\
----- \\
------\end{array}$ & $\frac{1-1 .-1}{-13.5}$ & 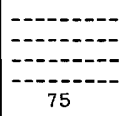 & 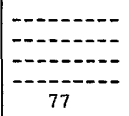 & $\frac{1}{-15.1}$ & $\begin{array}{l}74 \\
76.5 \\
76 \\
75.5 \\
77.5\end{array}$ & $\begin{array}{l}78 \\
82 \\
80 \\
84.5 \\
78.5\end{array}$ \\
\hline 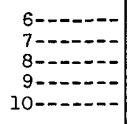 & $\frac{32}{33}$ & $\begin{array}{l}73.5 \\
72.5 \\
72 \\
70 \\
72\end{array}$ & $\begin{array}{l}80 \\
77 \\
77 \\
77 \\
76.5\end{array}$ & $\begin{array}{l}77 \\
75.5 \\
73.5 \\
77 \\
74\end{array}$ & $\begin{array}{l}76.8 \\
75 \\
74.1 \\
74.6 \\
74.1\end{array}$ & $\begin{array}{l}71.5 \\
72.5 \\
73 \\
71 \\
73\end{array}$ & $\begin{array}{l}82.5 \\
80 \\
77 \\
77.5 \\
79\end{array}$ \\
\hline 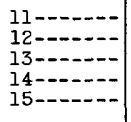 & $\frac{32}{32}$ & $\begin{array}{l}72.5 \\
74.5 \\
77 \\
75 \\
74\end{array}$ & $\begin{array}{l}77.5 \\
80.5 \\
79.5 \\
77 \\
\end{array}$ & $\begin{array}{l}77 \\
79 \\
75.5 \\
77 \\
76\end{array}$ & $\begin{array}{l}75.6 \\
78 \\
77.3 \\
76.3 \\
75\end{array}$ & $\begin{array}{l}73.5 \\
74.5 \\
70 \\
67 \\
67\end{array}$ & $\begin{array}{l}81 \cdot 5 \\
80 \\
80 \\
80 \\
80\end{array}$ \\
\hline 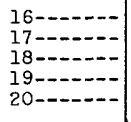 & 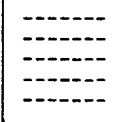 & $\begin{array}{l}75 \\
75 \\
74 \\
74 \\
76\end{array}$ & $\begin{array}{l}77.5 \\
79 \\
79 \\
79 \\
81\end{array}$ & $\begin{array}{l}76 \\
78 \\
77 \\
78 \\
78\end{array}$ & $\begin{array}{l}76.1 \\
77.3 \\
76.6 \\
77 \\
78.3\end{array}$ & $\begin{array}{l}70 \\
73 \\
73.5 \\
76 \\
76\end{array}$ & $\begin{array}{l}80 \\
81 \\
79 \\
84 \\
78.5\end{array}$ \\
\hline 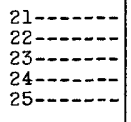 & 32 & $\begin{array}{l}75 \\
79 \\
79 \\
78 \\
79\end{array}$ & $\begin{array}{l}82 \\
86 \\
88.5 \\
86 \\
85.5\end{array}$ & $\begin{array}{l}83 \\
85 \\
85 \\
85.5 \\
82\end{array}$ & $\begin{array}{l}80 \\
83.3 \\
84.2 \\
83.2 \\
82.6\end{array}$ & $\begin{array}{l}75 \\
75 \\
75 \\
75 \\
74\end{array}$ & $\begin{array}{l}82.5 \\
82 \\
82 \\
80 \\
83.5\end{array}$ \\
\hline $\begin{array}{l}26-\cdots- \\
27-\cdots \\
28-\cdots \\
29-\cdots- \\
30-\cdots- \\
31-\cdots-\cdots \\
\end{array}$ & 32 & $\begin{array}{l}78 \\
78 \\
78.5 \\
78 \\
77 \\
75 \\
\end{array}$ & $\begin{array}{l}84 \\
82 \\
81.5 \\
82.5 \\
82.5 \\
\end{array}$ & $\begin{array}{l}80 \\
83 \\
83 \\
79.5 \\
80 \\
80 \\
\end{array}$ & $\begin{array}{l}81.6 \\
80.5 \\
81.2 \\
79.6 \\
79.8 \\
79.2 \\
\end{array}$ & $\begin{array}{l}75 \\
77 \\
70.5 \\
75.5 \\
75 \\
77 \\
\end{array}$ & $\begin{array}{l}81.5 \\
86 \\
84 \\
84 \\
84 \\
81.5 \\
\end{array}$ \\
\hline Average- & 32.1 & 75.3 & 80.5 & 78.9 & 78.25 & 73.7 & 81.1 \\
\hline \multirow{2}{*}{ Date } & \multicolumn{2}{|c|}{ August--Continued } & \multicolumn{4}{|c|}{ September } & \\
\hline & 6 p.m. & Average & $6 \mathrm{a} \cdot \mathrm{m}$. & Noon & 6 p.m. & Average & \\
\hline 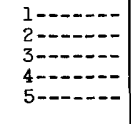 & $\begin{array}{l}78 \\
80 \\
79 \\
81 \\
78\end{array}$ & $\begin{array}{l}76.6 \\
79.5 \\
78.3 \\
80.3 \\
78\end{array}$ & $\begin{array}{l}77 \\
76.2 \\
80 \\
73.8 \\
-\end{array}$ & $\begin{array}{c}82.5 \\
82.8 \\
84.2 \\
- \\
-\end{array}$ & $\begin{array}{c}81.2 \\
80 \\
79.8 \\
-0.0-\end{array}$ & \begin{tabular}{c}
80.2 \\
79.6 \\
81.3 \\
\hdashline$-0 .-0$ \\
-0.0
\end{tabular} & \\
\hline 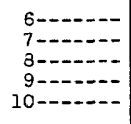 & $\begin{array}{l}80 \\
80 \\
77 \\
75 \\
76.5\end{array}$ & $\begin{array}{l}78 \\
77.5 \\
75.6 \\
74.5 \\
76.2\end{array}$ & $\begin{array}{l}72.5 \\
73.5 \\
75 \\
67 \\
67\end{array}$ & $\begin{array}{l}82 \\
79.2 \\
79 \\
68 \\
69\end{array}$ & $\begin{array}{c}79.5 \\
78.5 \\
75 \\
--29.5\end{array}$ & $\begin{array}{l}78 \\
77 \\
76.3 \\
67.5 \\
68.5\end{array}$ & \\
\hline 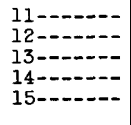 & $\begin{array}{l}80 \\
78.5 \\
75 \\
77 \\
76.5\end{array}$ & $\begin{array}{l}78.3 \\
77.6 \\
75 \\
74.6 \\
74.5\end{array}$ & $\begin{array}{l}69.5 \\
69 \\
66 \\
57 \\
64\end{array}$ & $\begin{array}{l}77 \\
77 \\
75 \\
72.5 \\
73\end{array}$ & $\begin{array}{l}74 \\
71 \\
70 \\
68.5 \\
69.5\end{array}$ & $\begin{array}{l}73.5 \\
72.3 \\
71 \\
66 \\
68.8\end{array}$ & \\
\hline 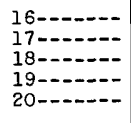 & $\begin{array}{l}80 \\
80 \\
78 \\
78 \\
-----\end{array}$ & $\begin{array}{l}76.6 \\
78 \\
76.8 \\
79.3 \\
77.2\end{array}$ & $\begin{array}{l}64 \\
66 \\
62 \\
63.1 \\
61.5\end{array}$ & $\begin{array}{l}74.5 \\
76 \\
70.5 \\
64 \\
68.5\end{array}$ & $\begin{array}{c}72 \\
70.8 \\
63.5 \\
65\end{array}$ & $\begin{array}{l}70.1 \\
70.9 \\
66.2 \\
63.5 \\
65\end{array}$ & \\
\hline 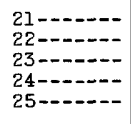 & $\begin{array}{l}77 \\
80.5 \\
82 \\
78.5 \\
80.5\end{array}$ & $\begin{array}{l}78.2 \\
79.2 \\
79.6 \\
77.8 \\
79.3\end{array}$ & $\begin{array}{l}61.5 \\
62 \\
63 \\
64 \\
61\end{array}$ & $\begin{array}{l}71 \\
69 \\
65.5 \\
66 \\
66\end{array}$ & $\begin{array}{l}67 \\
66 \\
67 \\
65 \\
63.5\end{array}$ & $\begin{array}{l}66.5 \\
65.6 \\
65.1 \\
65 \\
63.5\end{array}$ & \\
\hline 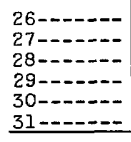 & $\begin{array}{l}82.5 \\
83 \\
82 \\
84.5 \\
82 \\
81.5 \\
\end{array}$ & $\begin{array}{l}79.3 \\
82 \\
81 \\
81.3 \\
80.5 \\
80 \\
\end{array}$ & $\begin{array}{l}57.5 \\
57 \\
55 \\
56 \\
54.8\end{array}$ & $\begin{array}{l}65.5 \\
63 \\
61.5 \\
62 \\
60\end{array}$ & $\begin{array}{l}61 \\
62 \\
60 \\
57 \\
56\end{array}$ & $\begin{array}{l}61.3 \\
60.6 \\
58.8 \\
58.3 \\
56.9\end{array}$ & \\
\hline Average- & 79.4 & 78.4 & 65.6 & 71.6. & 68.9 & 68.1 & \\
\hline
\end{tabular}


Water temperature ( F) for Maxinkuckee Lake--Continued

Year of 1899

\begin{tabular}{|c|c|c|c|c|c|c|}
\hline \multirow{2}{*}{ Date } & \multicolumn{4}{|c|}{ October } & \multicolumn{2}{|c|}{ November } \\
\hline & $6 \mathrm{a} \cdot \mathrm{m}$. & Noon & $6 \mathrm{p} . \mathrm{m}$. & Average & $6 \mathrm{a} \cdot \mathrm{m}$. & Noon \\
\hline 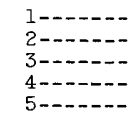 & $\begin{array}{l}48.5 \\
52.5 \\
52 \\
55 \\
55\end{array}$ & $\begin{array}{l}59.5 \\
61 \\
63.5 \\
61.5 \\
61.2\end{array}$ & $\begin{array}{l}56 \\
59 \\
60.5 \\
61 \\
60\end{array}$ & $\begin{array}{l}54.6 \\
57.5 \\
58.6 \\
59.1 \\
58.7\end{array}$ & $\begin{array}{l}54 \\
45 \\
45 \\
42 \\
44\end{array}$ & $\begin{array}{l}56 \\
48 \\
44 \\
42 \\
52.5\end{array}$ \\
\hline 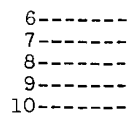 & $\begin{array}{l}54 \\
54 \\
52.5 \\
58 \\
54.5\end{array}$ & $\begin{array}{l}62 \\
63.5 \\
64 \\
64.5 \\
62\end{array}$ & $\begin{array}{l}62 \\
61 \\
61 \\
60 \\
60\end{array}$ & $\begin{array}{l}59 \\
59.5 \\
59.1 \\
60.8 \\
58.8\end{array}$ & $\begin{array}{l}47 \\
48 \\
50 \\
48 \\
48\end{array}$ & $\begin{array}{l}51 \\
50 \\
52 \\
50.5\end{array}$ \\
\hline 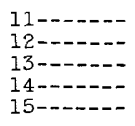 & $\begin{array}{l}58.5 \\
58.5 \\
60 \\
60 \\
60.5\end{array}$ & $\begin{array}{l}60 \\
65 \\
64 \\
68 \\
70.5\end{array}$ & $\begin{array}{l}60 \\
62 \\
64 \\
65 \\
65\end{array}$ & $\begin{array}{l}59.5 \\
61.5 \\
62.6 \\
64.3 \\
65.3\end{array}$ & $\begin{array}{l}50 \\
46 \\
42 \\
46 \\
48\end{array}$ & $\begin{array}{l}53 \\
48 \\
50 \\
49 \\
50\end{array}$ \\
\hline 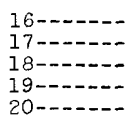 & 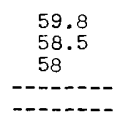 & $\begin{array}{c}67 \\
58.5 \\
-0 .- \\
-0 .-\end{array}$ & $\begin{array}{l}\quad 61 \\
57 \\
-0- \\
-0--\end{array}$ & $\begin{array}{l}62.5 \\
58 \\
-0 .- \\
-0 .\end{array}$ & $\begin{array}{l}48 \\
47 \\
50 \\
48 \\
47\end{array}$ & $\begin{array}{l}51 \\
50 \\
51 \\
51 \\
51\end{array}$ \\
\hline 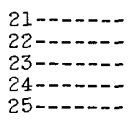 & $\begin{array}{l}50 \\
60 \\
60 \\
60\end{array}$ & $\begin{array}{l}58 \\
66 \\
66 \\
62\end{array}$ & 60 & $\begin{array}{l}54 \\
62 \\
62 \\
61\end{array}$ & $\begin{array}{l}46 \\
50 \\
47.5 \\
45 \\
43\end{array}$ & $\begin{array}{l}49 \\
51 \\
45 \\
46 \\
47\end{array}$ \\
\hline 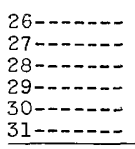 & $\begin{array}{l}55 \\
56 \\
57 \\
56 \\
53 \\
52 \\
\end{array}$ & $\begin{array}{c}64 \\
59 \\
59 \\
60 \\
\end{array}$ & $\begin{array}{l}60 \\
57 \\
57 \\
59 \\
56 \\
55 \\
\end{array}$ & $\begin{array}{l}59.6 \\
56.5 \\
57 \\
58 \\
56 \\
55.6 \\
\end{array}$ & $\begin{array}{l}42 \\
43 \\
43 \\
42 \\
42\end{array}$ & $\begin{array}{l}47 \\
46 \\
47 \\
44 \\
46\end{array}$ \\
\hline Average- & 56 & 62.8 & 59.9 & 59.3 & 46.2 & 48.9 \\
\hline \multirow[t]{2}{*}{ Date } & \multicolumn{2}{|c|}{ November--Continued } & \multicolumn{4}{|c|}{ December } \\
\hline & 6 p.m. & Average & 6 a.m. & Noon & 6 p.m. & Average \\
\hline 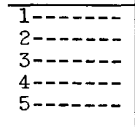 & $\begin{array}{l}------ \\
46 \\
43 \\
47 \\
50\end{array}$ & $\begin{array}{l}55 \\
46.3 \\
44 \\
43.6 \\
48.3\end{array}$ & $\begin{array}{l}44 \\
42 \\
42 \\
41 \\
35\end{array}$ & $\begin{array}{l}46 \\
46 \\
43 \\
42 \\
39\end{array}$ & $\begin{array}{l}43 \\
43 \\
42 \\
37 \\
38\end{array}$ & $\begin{array}{l}44.3 \\
43.6 \\
42.3 \\
40 \\
37.3\end{array}$ \\
\hline 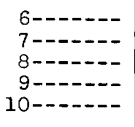 & $\begin{array}{l}48 \\
49 \\
50 \\
50 \\
50.5\end{array}$ & $\begin{array}{l}48.6 \\
49 \\
50 \\
50 \\
49.6\end{array}$ & $\begin{array}{l}38 \\
37 \\
38 \\
40 \\
44\end{array}$ & $\begin{array}{l}41 \\
40 \\
42 \\
40 \\
42\end{array}$ & $\begin{array}{l}39 \\
38.5 \\
40 \\
40 \\
42.6\end{array}$ & $\begin{array}{l}39.3 \\
38.5 \\
40 \\
40 \\
42.6\end{array}$ \\
\hline 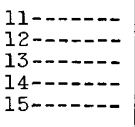 & $\begin{array}{l}50 \\
46 \\
49 \\
48 \\
49\end{array}$ & $\begin{array}{l}51 \\
46.6 \\
47 \\
47.6 \\
49\end{array}$ & $\begin{array}{l}46 \\
40 \\
40 \\
35 \\
36\end{array}$ & $\begin{array}{l}48 \\
40 \\
38 \\
36 \\
36\end{array}$ & $\begin{array}{l}47 \\
40 \\
35 \\
36 \\
36\end{array}$ & $\begin{array}{l}47 \\
40 \\
37.6 \\
35.6 \\
36\end{array}$ \\
\hline 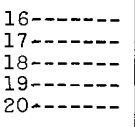 & $\begin{array}{l}50 \\
52 \\
50 \\
50 \\
47\end{array}$ & $\begin{array}{l}49.6 \\
49.6 \\
50.3 \\
49.6 \\
48.3\end{array}$ & $\begin{array}{l}32 \\
32 \\
34 \\
38 \\
36\end{array}$ & $\begin{array}{l}32 \\
35 \\
39 \\
38 \\
36\end{array}$ & $\begin{array}{l}32 \\
37 \\
38 \\
38 \\
36\end{array}$ & $\begin{array}{l}32 \\
34.6 \\
37 \\
38 \\
36\end{array}$ \\
\hline 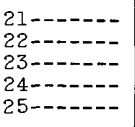 & $\begin{array}{l}50 \\
50 \\
45 \\
45 \\
45\end{array}$ & $\begin{array}{l}48.3 \\
50.3 \\
45.8 \\
45.3 \\
45\end{array}$ & $\begin{array}{l}34.5 \\
37 \\
34 \\
34 \\
32\end{array}$ & $\begin{array}{l}37 \\
38 \\
36 \\
34 \\
34\end{array}$ & $\begin{array}{l}38 \\
38 \\
34 \\
33 \\
33\end{array}$ & $\begin{array}{l}36.5 \\
37.6 \\
34.6 \\
33.6 \\
33\end{array}$ \\
\hline 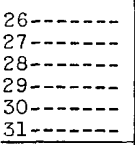 & $\begin{array}{l}44 \\
44 \\
44 \\
43 \\
47.5\end{array}$ & $\begin{array}{l}44.3 \\
44.3 \\
44.6 \\
43 \\
45.1\end{array}$ & $\begin{array}{l}33 \\
32 \\
32 \\
32 \\
32 \\
32 \\
\end{array}$ & $\begin{array}{l}34 \\
34 \\
34 \\
32 \\
32 \\
32 \\
\end{array}$ & $\begin{array}{l}34 \\
32 \\
32 \\
32 \\
32 \\
32 \\
\end{array}$ & $\begin{array}{l}33.6 \\
32.6 \\
34.6 \\
32 \\
32 \\
32 \\
\end{array}$ \\
\hline Average- & 47.6 & 47.6 & 36.6 & 37.9 & 37 & 37.2 \\
\hline
\end{tabular}


Water temperatures $\left({ }^{\circ} \mathrm{F}\right)$ for Maxinkuckee Lake--Continued

Year of 1900

\begin{tabular}{|c|c|c|c|c|c|c|}
\hline \multirow{2}{*}{ Date } & \multicolumn{4}{|c|}{ January } & \multicolumn{2}{|c|}{ February } \\
\hline & 6 a.m. & Noon & $6 \mathrm{p} . \mathrm{m}$. & Average & $6 \mathrm{a} \cdot \mathrm{m}$. & Noon \\
\hline 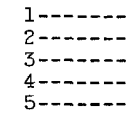 & $\begin{array}{l}32 \\
32 \\
32 \\
32 \\
32\end{array}$ & $\begin{array}{l}32 \\
32 \\
32 \\
32 \\
32\end{array}$ & $\begin{array}{l}32 \\
32 \\
32 \\
32 \\
33\end{array}$ & $\begin{array}{l}32 \\
32 \\
32 \\
32 \\
32.3\end{array}$ & $\begin{array}{l}32 \\
32 \\
32 \\
32.5 \\
32\end{array}$ & $\begin{array}{l}32 \\
32 \\
32 \\
32.5 \\
32\end{array}$ \\
\hline 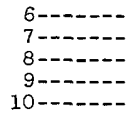 & $\begin{array}{l}33 \\
34 \\
34 \\
33 \\
33\end{array}$ & $\begin{array}{l}33 \\
34 \\
35 \\
33 \\
33\end{array}$ & $\begin{array}{l}33 \\
34 \\
35 \\
33 \\
33\end{array}$ & $\begin{array}{l}33 \\
34 \\
34.6 \\
33 \\
33\end{array}$ & $\begin{array}{l}32 \\
33 \\
33.5 \\
33 \\
33\end{array}$ & $\begin{array}{l}32.5 \\
33 \\
34 \\
33 \\
33\end{array}$ \\
\hline 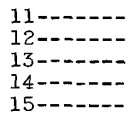 & $\begin{array}{l}33 \\
33 \\
33 \\
33 \\
33\end{array}$ & $\begin{array}{l}33 \\
34 \\
33 \\
33 \\
34\end{array}$ & $\begin{array}{l}33 \\
33 \\
33 \\
33 \\
34.5\end{array}$ & $\begin{array}{l}33 \\
33.3 \\
33 \\
33 \\
33.8\end{array}$ & $\begin{array}{l}32.5 \\
33 \\
33 \\
32 \\
32\end{array}$ & $\begin{array}{l}33 \\
33 \\
32.5 \\
32 \\
32\end{array}$ \\
\hline 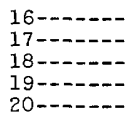 & $\begin{array}{l}35 \\
34 \\
35 \\
35 \\
35\end{array}$ & $\begin{array}{l}35 \\
34 \\
35 \\
35 \\
35\end{array}$ & $\begin{array}{l}35 \\
35 \\
35.5 \\
35 \\
35\end{array}$ & $\begin{array}{l}35 \\
34 \\
35.1 \\
35 \\
35\end{array}$ & $\begin{array}{l}32 \\
32 \\
32 \\
32 \\
32\end{array}$ & $\begin{array}{l}32 \\
32 \\
32 \\
32 \\
33\end{array}$ \\
\hline 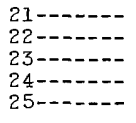 & $\begin{array}{l}35 \\
35 \\
36 \\
38 \\
37\end{array}$ & $\begin{array}{l}35 \\
35.5 \\
37 \\
40 \\
36\end{array}$ & $\begin{array}{l}35.5 \\
36.5 \\
37.5 \\
39 \\
35\end{array}$ & $\begin{array}{l}35.1 \\
35.6 \\
36.8 \\
39 \\
36\end{array}$ & $\begin{array}{l}33.5 \\
35 \\
34 \\
34 \\
32\end{array}$ & $\begin{array}{l}34 \\
34 \\
34 \\
33 \\
32\end{array}$ \\
\hline 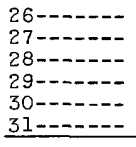 & $\begin{array}{l}34.5 \\
33.5 \\
33 \\
32 \\
32 \\
32 \\
\end{array}$ & $\begin{array}{l}34 \\
33 \\
32 \\
32 \\
32 \\
32 \\
\end{array}$ & $\begin{array}{l}34 \\
33 \\
32 \\
32 \\
32 \\
32 \\
\end{array}$ & $\begin{array}{l}34.1 \\
33.1 \\
32.3 \\
32 \\
32 \\
32 \\
\end{array}$ & $\begin{array}{l}32 \\
32 \\
32\end{array}$ & $\begin{array}{l}32 \\
32 \\
33\end{array}$ \\
\hline Average- & 33.7 & 33.8 & 33.9 & 33.8 & 32.6 & 32.6 \\
\hline \multirow[t]{2}{*}{ Date } & \multicolumn{2}{|c|}{ February--Continued } & \multicolumn{4}{|c|}{ March } \\
\hline & 6 p.m. & Average & 6 a.m. & Noon & $6 \mathrm{p.m}$ & Average \\
\hline 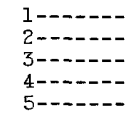 & $\begin{array}{l}32 \\
32 \\
32.5 \\
32.5 \\
32\end{array}$ & $\begin{array}{l}32 \\
32 \\
32 \cdot 1 \\
32 \cdot 5 \\
32\end{array}$ & $\begin{array}{l}33 \\
34 \\
34 \\
34 \\
34\end{array}$ & $\begin{array}{l}34.5 \\
34.5 \\
34 \\
34 \\
34\end{array}$ & $\begin{array}{l}34 \\
34 \\
34.5 \\
34 \\
34\end{array}$ & $\begin{array}{l}33.8 \\
34.1 \\
34.1 \\
34 \\
34\end{array}$ \\
\hline 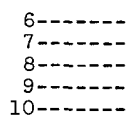 & $\begin{array}{l}33 \\
33.5 \\
34 \\
33 \\
33\end{array}$ & $\begin{array}{l}32.5 \\
33.1 \\
33.8 \\
33 \\
33\end{array}$ & $\begin{array}{l}34.5 \\
35 \\
35 \\
35.5 \\
36\end{array}$ & $\begin{array}{l}35 \\
35 \\
35.5 \\
36 \\
36\end{array}$ & $\begin{array}{l}35 \\
35 \\
35.5 \\
36 \\
36\end{array}$ & $\begin{array}{l}34.8 \\
35 \\
35.3 \\
35.8 \\
36\end{array}$ \\
\hline 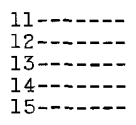 & $\begin{array}{l}33 \\
32 \\
32 \\
32\end{array}$ & $\begin{array}{l}32.8 \\
32.5 \\
32 \\
32\end{array}$ & $\begin{array}{l}36 \\
36 \\
36 \\
35\end{array}$ & $\begin{array}{l}36 \\
36 \\
36 \\
35\end{array}$ & $\begin{array}{l}36 \\
36.5 \\
36.5 \\
35\end{array}$ & $\begin{array}{l}36 \\
36.1 \\
36.1 \\
35\end{array}$ \\
\hline 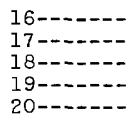 & $\begin{array}{l}32 \\
32 \\
32 \\
32 \\
33\end{array}$ & $\begin{array}{l}32 \\
32 \\
32 \\
32 \\
32.6\end{array}$ & $\begin{array}{l}35 \\
33.5 \\
33.5 \\
35 \\
36\end{array}$ & $\begin{array}{l}35 \\
33 \\
34 \\
35 \\
36\end{array}$ & $\begin{array}{l}34 \\
33 \\
34 \\
36 \\
36\end{array}$ & $\begin{array}{l}34.6 \\
33.1 \\
33.3 \\
35.3 \\
36\end{array}$ \\
\hline 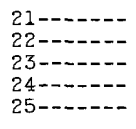 & $\begin{array}{l}34.5 \\
34.5 \\
35 \\
32 \\
32\end{array}$ & $\begin{array}{l}34 \\
34.5 \\
34.3 \\
33 \\
32\end{array}$ & $\begin{array}{l}36 \\
36 \\
36 \\
37 \\
36.5\end{array}$ & $\begin{array}{l}36 \\
36.5 \\
37 \\
37 \\
36\end{array}$ & $\begin{array}{l}36.5 \\
37 \\
37 \\
37.5 \\
36\end{array}$ & $\begin{array}{l}36.1 \\
36.5 \\
36.6 \\
37.1 \\
36.1\end{array}$ \\
\hline 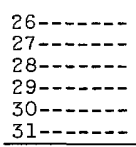 & $\begin{array}{l}32 \\
32 \\
33\end{array}$ & $\begin{array}{l}32 \\
32 \\
32.6\end{array}$ & $\begin{array}{l}36 \\
36 \\
36 \\
36 \\
37 \\
37 \\
\end{array}$ & $\begin{array}{l}36 \\
36.5 \\
36 \\
36 \\
37.5 \\
37 \\
\end{array}$ & $\begin{array}{l}36 \\
36 \\
36.5 \\
36.5 \\
36.5 \\
38.5\end{array}$ & $\begin{array}{l}36 \\
36.1 \\
36.1 \\
36.1 \\
37 \\
37.5 \\
\end{array}$ \\
\hline Average- & 32.7 & 32.7 & 35.3 & 35.5 & 35.6 & 35.5 \\
\hline
\end{tabular}


Water temperatures ( ${ }^{\circ} \mathrm{F}$ ) for Maxinkuckee Lake--Continued

Year of 1900

\begin{tabular}{|c|c|c|c|c|c|c|}
\hline \multirow{2}{*}{ Date } & \multicolumn{4}{|c|}{ April } & \multicolumn{2}{|c|}{ May } \\
\hline & $6 \mathrm{a} \cdot \mathrm{m}$. & Noon & $6 \mathrm{p} . \mathrm{m}$. & Average & $6 \mathrm{a} . \mathrm{m}$. & Noon \\
\hline 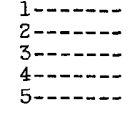 & $\begin{array}{l}39 \\
44 \\
45 \\
46 \\
47\end{array}$ & $\begin{array}{l}44 \\
44.5 \\
45.5 \\
47 \\
48\end{array}$ & $\begin{array}{l}45 \\
46 \\
45 \\
47.5 \\
48\end{array}$ & $\begin{array}{l}42.6 \\
44.8 \\
45.1 \\
46.8 \\
47.6\end{array}$ & $\begin{array}{l}60 \\
56 \\
58.5 \\
60 \\
60\end{array}$ & $\begin{array}{l}62 \\
58 \\
58 \\
60.5 \\
61\end{array}$ \\
\hline 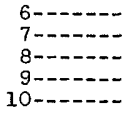 & $\begin{array}{l}48 \\
48 \\
49 \\
50 \\
48\end{array}$ & $\begin{array}{l}49 \\
49 \\
49 \\
50.5 \\
48.5\end{array}$ & $\begin{array}{l}49.5 \\
49.5 \\
50 \\
50 \\
48\end{array}$ & $\begin{array}{l}48.8 \\
48.8 \\
49.3 \\
50.1 \\
48.1\end{array}$ & $\begin{array}{l}59 \\
59 \\
59.5 \\
58 \\
57\end{array}$ & $\begin{array}{l}60.5 \\
60 \\
60 \\
58 \\
57\end{array}$ \\
\hline 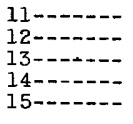 & $\begin{array}{l}48 \\
47 \\
47 \\
44 \\
45.5\end{array}$ & $\begin{array}{l}48 \\
47 \cdot 5 \\
47 \\
45 \\
46\end{array}$ & $\begin{array}{l}47.5 \\
46 \\
46 \\
46 \\
46.5\end{array}$ & $\begin{array}{l}47.8 \\
46.8 \\
46.6 \\
45 \\
46\end{array}$ & $\begin{array}{l}58 \\
62 \\
66 \\
69\end{array}$ & $\begin{array}{l}60 \\
64 \\
69 \\
71\end{array}$ \\
\hline 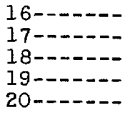 & $\begin{array}{l}46 \\
48 \\
48 \\
48 \\
48\end{array}$ & $\begin{array}{l}46.5 \\
48.5 \\
48 \\
48.5 \\
48\end{array}$ & $\begin{array}{l}48 \\
48 \\
48 \\
49 \\
49\end{array}$ & $\begin{array}{l}46.8 \\
48.1 \\
48 \\
48.5 \\
48.3\end{array}$ & $\begin{array}{l}71 \\
63 \\
62 \\
62 \\
61\end{array}$ & $\begin{array}{l}73 \\
64 \\
62 \\
62.5 \\
60\end{array}$ \\
\hline 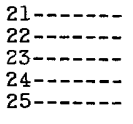 & $\begin{array}{l}50 \\
50 \\
50 \\
51 \\
50\end{array}$ & $\begin{array}{l}50 \\
51 \\
51 \cdot 5 \\
51 \cdot 5 \\
51\end{array}$ & $\begin{array}{l}51 \\
51 \\
51 \\
52 \\
51\end{array}$ & $\begin{array}{l}50.3 \\
50.6 \\
50.8 \\
51.5 \\
50.6\end{array}$ & $\begin{array}{l}59 \\
60 \\
61 \\
68 \\
70\end{array}$ & $\begin{array}{l}60 \\
61 \\
65 \\
70 \\
71\end{array}$ \\
\hline 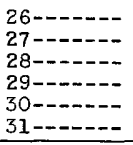 & $\begin{array}{l}50 \\
56 \\
54 \\
60 \\
67\end{array}$ & $\begin{array}{l}50 \\
54 \\
58 \\
66 \\
67\end{array}$ & $\begin{array}{l}54 \\
54 \\
64 \\
66 \\
67.5\end{array}$ & $\begin{array}{l}51.3 \\
55.3 \\
58.6 \\
64 \\
67.1\end{array}$ & $\begin{array}{l}72 \\
72 \\
72 \\
72 \\
72 \\
72+5 \\
\end{array}$ & $\begin{array}{l}73 \\
73 \\
72.5 \\
72.5 \\
74 \\
73 \\
\end{array}$ \\
\hline Average- & 49.1 & 49.9 & 50.5 & 49.8 & 63.71 & 65.41 \\
\hline \multirow{2}{*}{ Date } & \multicolumn{2}{|c|}{ May--Continued } & \multicolumn{4}{|c|}{ June } \\
\hline & $6 \mathrm{p} . \mathrm{m}$. & Average & 6 a.m. & Noon & $6 \mathrm{p} . \mathrm{m}$ & Average \\
\hline 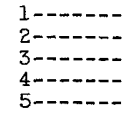 & $\begin{array}{l}61 \\
60 \\
59 \\
60 \\
60\end{array}$ & $\begin{array}{l}61 \\
58 \\
58.5 \\
60.1 \\
60.3\end{array}$ & $\begin{array}{l}73 \\
72.5 \\
71.5 \\
70 \\
71.5\end{array}$ & $\begin{array}{l}74 \\
72 \\
72 \\
71 \\
72\end{array}$ & $\begin{array}{l}73.5 \\
72 \\
21 \\
72 \\
74\end{array}$ & $\begin{array}{l}73.5 \\
72.1 \\
71.5 \\
71 \\
72.5\end{array}$ \\
\hline 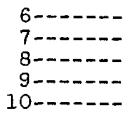 & $\begin{array}{l}60 \\
61 \\
59 \\
57.5 \\
57\end{array}$ & $\begin{array}{l}59.8 \\
60 \\
59.5 \\
57.8 \\
57\end{array}$ & $\begin{array}{l}73.5 \\
73.5 \\
76 \\
76 \\
76\end{array}$ & $\begin{array}{l}74 \\
74 \\
76.5 \\
76 \\
76.5\end{array}$ & $\begin{array}{l}73.5 \\
76 \\
77 \\
77 \\
75\end{array}$ & $\begin{array}{l}73.6 \\
74.1 \\
76.5 \\
76.3 \\
75.8\end{array}$ \\
\hline 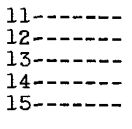 & $\begin{array}{l}60 \\
65 \\
69 \\
70\end{array}$ & $\begin{array}{l}59.3 \\
63.6 \\
68 \\
70\end{array}$ & $\begin{array}{l}74 \\
74 \\
76 \\
76 \\
77\end{array}$ & $\begin{array}{l}74.5 \\
75 \\
76.5 \\
77 \\
77\end{array}$ & $\begin{array}{l}73.5 \\
77 \\
77 \\
77.5 \\
76\end{array}$ & $\begin{array}{l}74 \\
75 \\
76.5 \\
76.8 \\
76.6\end{array}$ \\
\hline 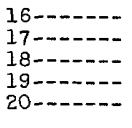 & $\begin{array}{l}70 \\
63.5 \\
62 \\
62 \\
60\end{array}$ & $\begin{array}{l}71.3 \\
63.5 \\
62 \\
62.1 \\
60.3\end{array}$ & $\begin{array}{l}75.5 \\
75.5 \\
75 \\
73 \\
70\end{array}$ & $\begin{array}{l}77 \\
76.5 \\
76.5 \\
76 \\
72\end{array}$ & $\begin{array}{l}77 \\
76 \\
77 \\
76 \\
72\end{array}$ & $\begin{array}{l}76.5 \\
76 \\
76.1 \\
75 \\
71.3\end{array}$ \\
\hline 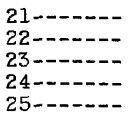 & $\begin{array}{l}60 \\
62 \\
70 \\
71 \\
73\end{array}$ & $\begin{array}{l}59.6 \\
61 \\
65.3 \\
69.6 \\
71.3\end{array}$ & $\begin{array}{l}71 \\
70 \\
71 \\
72 \\
72\end{array}$ & $\begin{array}{l}73 \cdot 2 \\
72 \\
74 \\
78 \\
78\end{array}$ & $\begin{array}{l}72 \\
70 \\
73 \\
75 \\
75\end{array}$ & $\begin{array}{l}73 \\
70.6 \\
72.6 \\
75 \\
75\end{array}$ \\
\hline 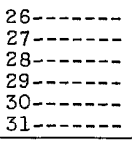 & $\begin{array}{l}74 \\
73.5 \\
73 \\
73 \\
74 \\
73 \\
\end{array}$ & $\begin{array}{l}73 \\
72.8 \\
72.5 \\
72.5 \\
73.3 \\
72.8 \\
\end{array}$ & $\begin{array}{l}78 \\
73 \\
75 \\
75 \\
68\end{array}$ & $\begin{array}{l}78 \\
77.5 \\
78 \\
78 \\
78\end{array}$ & $\begin{array}{l}75 \\
78 \\
78 \\
76 \\
77\end{array}$ & $\begin{array}{l}77 \\
76.1 \\
77 \\
76.3 \\
74.3\end{array}$ \\
\hline Average- & 65.08 & 64.74 & 73.21 & 75.32 & 74.9 & 74.6 \\
\hline
\end{tabular}


Water temperatures $\left({ }^{\circ} \mathrm{F}\right)$ for Maxinkuckee Lake--Continued

\begin{tabular}{|c|c|c|c|c|c|c|}
\hline \multirow{2}{*}{ Date } & \multicolumn{4}{|c|}{ July } & \multicolumn{2}{|c|}{ August } \\
\hline & $6 \mathrm{a} . \mathrm{m}$. & Noon & 6 p.m. & Average & $6 \mathrm{a} . \mathrm{m}$ & Noon \\
\hline 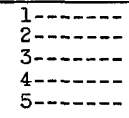 & $\begin{array}{l}71 \\
73 \\
75 \\
78 \\
78\end{array}$ & $\begin{array}{l}76 \\
80 \\
79 \\
82 \\
83\end{array}$ & $\begin{array}{l}77 \\
80 \\
78.3 \\
80 \\
80\end{array}$ & $\begin{array}{l}74.6 \\
77.6 \\
77.4 \\
80 \\
80.3\end{array}$ & $\begin{array}{l}74 \\
76.5 \\
75 \\
76 \\
77.4\end{array}$ & $\begin{array}{l}81 \\
79 \\
80.8 \\
81\end{array}$ \\
\hline 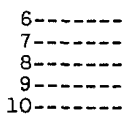 & $\begin{array}{l}78 \\
71 \\
77.5 \\
73 \\
71\end{array}$ & $\begin{array}{l}81 \\
82 \\
77 \\
78 \\
77\end{array}$ & $\begin{array}{l}80 \\
80 \\
75.5 \\
77 \\
76\end{array}$ & $\begin{array}{l}79.6 \\
77.6 \\
76.6 \\
76 \\
74.6\end{array}$ & $\begin{array}{l}79 \\
79.2 \\
78.8 \\
79.4 \\
78.9\end{array}$ & $\begin{array}{l}83.5 \\
81.8 \\
83 \\
83 \\
83.5\end{array}$ \\
\hline 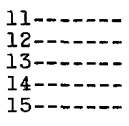 & $\begin{array}{l}73 \\
77 \\
71 \\
71 \\
75\end{array}$ & $\begin{array}{l}77.5 \\
72 \\
78 \\
79 \\
81\end{array}$ & $\begin{array}{l}77 \\
78 \\
77 \\
78 \\
79\end{array}$ & $\begin{array}{l}75.8 \\
75.6 \\
75.3 \\
76 \\
78.3\end{array}$ & $\begin{array}{l}79 \\
78.3 \\
78 \\
79 \\
76.2\end{array}$ & $\begin{array}{l}83.6 \\
81 \\
82 \\
79 \\
72.1\end{array}$ \\
\hline $\begin{array}{l}16-0-0-0 \\
17-0-0- \\
18-0-0- \\
19-0-00 \\
20-0-0-0\end{array}$ & $\begin{array}{l}75 \\
74.5 \\
72 \\
75 \\
75\end{array}$ & $\begin{array}{l}77 \\
77 \\
79 \\
79 \\
80\end{array}$ & $\begin{array}{l}77 \\
76 \\
78 \\
78 \\
75\end{array}$ & $\begin{array}{l}76.3 \\
75.5 \\
76.3 \\
77.3 \\
76.6\end{array}$ & $\begin{array}{l}75.7 \\
75.5 \\
76 \\
78.5 \\
77\end{array}$ & $\begin{array}{l}77 \\
80 \\
80.5 \\
83.5 \\
81\end{array}$ \\
\hline 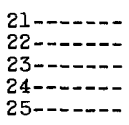 & $\begin{array}{l}73 \\
75 \\
78 \\
80 \\
75\end{array}$ & $\begin{array}{l}80 \\
80 \\
81 \\
78 \\
80\end{array}$ & $\begin{array}{l}80 \\
82 \\
80.5 \\
76 \\
79.5\end{array}$ & $\begin{array}{l}77.6 \\
79 \\
79.8 \\
78.6 \\
78.1\end{array}$ & $\begin{array}{l}76.2 \\
78.2 \\
78.9 \\
77.5 \\
77.2\end{array}$ & $\begin{array}{l}83.5 \\
84 \\
80 \\
82.5 \\
\end{array}$ \\
\hline 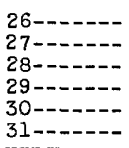 & $\begin{array}{l}75 \\
73 \\
75 \\
75.5 \\
75 \\
76\end{array}$ & $\begin{array}{l}80 \\
80 \\
80 \\
79 \\
80.5 \\
80\end{array}$ & $\begin{array}{l}79 \\
78 \\
78 \\
78 \\
79 \\
78\end{array}$ & $\begin{array}{l}78 \\
77 \\
77.6 \\
77.5 \\
78.3 \\
78\end{array}$ & $\begin{array}{l}76 \\
76.5 \\
76.5 \\
78 \\
76 \\
78\end{array}$ & $\begin{array}{l}79.5 \\
80 \\
82 \\
83.5 \\
83 \\
83.5\end{array}$ \\
\hline Average- & 74.6 & 79 & 78.3 & 78.4 & 77.2 & 81.3 \\
\hline \multirow{2}{*}{ Date } & \multicolumn{2}{|c|}{ August--Continued } & \multicolumn{4}{|c|}{ September } \\
\hline & $6 \mathrm{p} . \mathrm{m}$ & Average & 6 a.m. & Noon & $6 \mathrm{p} . \mathrm{m}$. & Average \\
\hline $\begin{array}{l}1--0-0- \\
2--0-0 \\
3--0-0- \\
4--0-0- \\
5--0-0-\end{array}$ & $\begin{array}{l}81.5 \\
78 \\
80.3 \\
78.5 \\
81\end{array}$ & $\begin{array}{l}78.8 \\
77.2 \\
78.1 \\
78.4 \\
79.8\end{array}$ & $\begin{array}{l}77.5 \\
76 \\
76 \\
75 \\
75\end{array}$ & $\begin{array}{l}83 \\
81.2 \\
80.5 \\
81 \\
80.5\end{array}$ & $\begin{array}{l}81 \\
77.2 \\
79 \\
81 \\
77.5\end{array}$ & $\begin{array}{l}80.5 \\
78.1 \\
78.5 \\
79 \\
77.3\end{array}$ \\
\hline 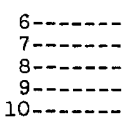 & $\begin{array}{l}79 \cdot 8 \\
81 \cdot 8 \\
81 \\
82 \\
80\end{array}$ & $\begin{array}{l}80.8 \\
81 \\
80.9 \\
81.4 \\
80.8\end{array}$ & $\begin{array}{l}76 \\
75 \\
75.5 \\
76.5 \\
75.1\end{array}$ & $\begin{array}{c}79.5 \\
78 \\
81.2 \\
80\end{array}$ & $\begin{array}{l}77.5 \\
78 \\
80.3 \\
78.6 \\
77\end{array}$ & $\begin{array}{l}77.6 \\
77 \\
77.6 \\
78.7 \\
77.3\end{array}$ \\
\hline 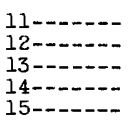 & $\begin{array}{l}80.5 \\
81.5 \\
80.5 \\
79 \\
78\end{array}$ & $\begin{array}{l}81 \\
80.2 \\
80.1 \\
79 \\
75.4\end{array}$ & $\begin{array}{l}75.8 \\
72.9 \\
72 \\
72.1 \\
68.8\end{array}$ & $\begin{array}{l}79 \\
72.5 \\
79 \\
79 \\
77\end{array}$ & $\begin{array}{l}76.3 \\
76.1 \\
78 \\
72.7 \\
74.9\end{array}$ & $\begin{array}{l}77 \\
73.8 \\
76.3 \\
74.6 \\
73.5\end{array}$ \\
\hline 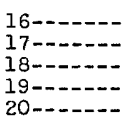 & $\begin{array}{l}80 \\
79.2 \\
79 \\
78.5 \\
79\end{array}$ & $\begin{array}{l}77.5 \\
78.2 \\
78.5 \\
80.4 \\
79\end{array}$ & $\begin{array}{l}70.1 \\
68 \\
59.3 \\
65 \\
65\end{array}$ & $\begin{array}{l}71.1 \\
73 \\
65.5 \\
71\end{array}$ & $\begin{array}{l}71.8 \\
67 \\
71.9 \\
69.9 \\
67\end{array}$ & $\begin{array}{l}71 \\
67.5 \\
67.7 \\
66.8 \\
67.6\end{array}$ \\
\hline 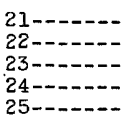 & $\begin{array}{l}83.6 \\
83 \\
82 \\
81.5 \\
79\end{array}$ & $\begin{array}{l}81.1 \\
81.7 \\
80.3 \\
80.5 \\
78.1\end{array}$ & $\begin{array}{l}63.5 \\
63.5 \\
64 \\
62.8 \\
67\end{array}$ & $\begin{array}{l}70 \\
71 \\
70.2 \\
68.5 \\
75.2\end{array}$ & $\begin{array}{l}67.5 \\
69.5 \\
67.8 \\
69 \\
71.8\end{array}$ & $\begin{array}{l}67 \\
68 \\
67.3 \\
66.7 \\
71.3\end{array}$ \\
\hline 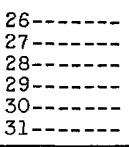 & $\begin{array}{l}81.5 \\
79 \cdot 5 \\
81.8 \\
81.5 \\
83 \\
83\end{array}$ & $\begin{array}{l}79 \\
78.6 \\
80.1 \\
81 \\
80.6 \\
81.5\end{array}$ & $\begin{array}{l}62 \\
66 \\
61.9 \\
66 \\
63.5\end{array}$ & $\begin{array}{l}69.9 \\
67.3 \\
68 \\
67.5 \\
71\end{array}$ & $\begin{array}{l}69 \\
66.5 \\
67 \\
67.5 \\
69.5\end{array}$ & $\begin{array}{l}66.9 \\
66.6 \\
65.6 \\
67 \\
68\end{array}$ \\
\hline Average- & 80.6 & 79.7 & 69.6 & 74.6 & 73.2 & 72.4 \\
\hline
\end{tabular}


Water temperatures $\left({ }^{\circ} \mathrm{F}\right)$ for Maxinkuckee Lake--Continued

Year of 1900

\begin{tabular}{|c|c|c|c|c|c|c|}
\hline \multirow{2}{*}{ Date } & \multicolumn{4}{|c|}{ October } & \multicolumn{2}{|c|}{ November } \\
\hline & $6 \mathrm{a} . \mathrm{m}$ & Noan & $6 \mathrm{p.m}$ & Average & 6 a.m. & Noon \\
\hline $\begin{array}{l}1------- \\
2------ \\
3------ \\
4--\cdots---- \\
5-------\end{array}$ & $\begin{array}{l}63.5 \\
62.5 \\
64.5 \\
65 \\
64.9\end{array}$ & $\begin{array}{l}70 \\
71 \\
72.5 \\
73.2 \\
72\end{array}$ & $\begin{array}{l}68 \\
68 \\
68.9 \\
69 \\
70.1\end{array}$ & $\begin{array}{l}67.2 \\
67.2 \\
68.6 \\
69.1 \\
69\end{array}$ & $\begin{array}{l}59.8 \\
57 \\
56.3 \\
51.1 \\
55.3\end{array}$ & $\begin{array}{l}59 \\
63.8 \\
63.5 \\
62 \\
61\end{array}$ \\
\hline 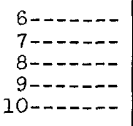 & $\begin{array}{l}67 \\
67.5 \\
65 \\
62.5 \\
69.8\end{array}$ & $\begin{array}{l}73.5 \\
68.5 \\
69.5 \\
69.5 \\
68.5\end{array}$ & $\begin{array}{l}71 \\
66.8 \\
67 \\
66.5 \\
67\end{array}$ & $\begin{array}{l}70.5 \\
67.6 \\
67.7 \\
66.2 \\
68.4\end{array}$ & $\begin{array}{l}52 \\
49.5 \\
50 \\
50 \\
45.5\end{array}$ & $\begin{array}{l}55 \\
56 \\
55 \\
51.3 \\
45.8\end{array}$ \\
\hline 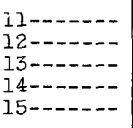 & $\begin{array}{l}62 \\
65.5 \\
60 \\
62 \\
62.3\end{array}$ & $\begin{array}{l}68.5 \\
66.5 \\
65.5 \\
68 \\
69.3\end{array}$ & $\begin{array}{l}66.9 \\
65 \\
64 \\
66.9 \\
65.5\end{array}$ & $\begin{array}{l}65.8 \\
66 \\
63.2 \\
65.6 \\
65.7\end{array}$ & $\begin{array}{l}48.9 \\
46.5 \\
44.5 \\
42 \\
42.8\end{array}$ & $\begin{array}{l}51.5 \\
49.7 \\
49.8 \\
45.8 \\
47\end{array}$ \\
\hline 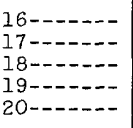 & $\begin{array}{l}61.5 \\
57.6 \\
57.2 \\
54.5 \\
54\end{array}$ & $\begin{array}{l}65 \\
64.8 \\
65.5 \\
63.6 \\
63\end{array}$ & $\begin{array}{l}60 \\
61 \\
63 \\
61.2 \\
61\end{array}$ & $\begin{array}{l}62.2 \\
61.1 \\
61.9 \\
59.8 \\
59.3\end{array}$ & $\begin{array}{l}38.9 \\
38.5 \\
44.9 \\
48.2 \\
48.9\end{array}$ & $\begin{array}{l}42.9 \\
40 \\
47 \\
49.5 \\
50\end{array}$ \\
\hline 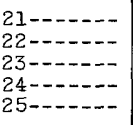 & $\begin{array}{l}61.8 \\
60.1 \\
58.9 \\
58.1 \\
58\end{array}$ & $\begin{array}{l}62 \\
62.5 \\
63.9 \\
63.5 \\
64.7\end{array}$ & $\begin{array}{l}61.9 \\
63 \\
61.2 \\
65 \\
63\end{array}$ & $\begin{array}{l}61.9 \\
61.9 \\
61.3 \\
62.2 \\
61.9\end{array}$ & $\begin{array}{l}45.8 \\
43.1 \\
43.5 \\
43 \\
39\end{array}$ & $\begin{array}{l}47.2 \\
47.5 \\
45.1 \\
43 \\
39.6\end{array}$ \\
\hline 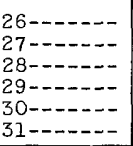 & $\begin{array}{l}58.8 \\
58.5 \\
57.5 \\
60.1 \\
61.5 \\
59.9\end{array}$ & $\begin{array}{l}64 \\
66.1 \\
64 \\
65 \\
65.2 \\
64.9\end{array}$ & $\begin{array}{l}61 \cdot 5 \\
64 \\
62 \cdot 8 \\
61 \cdot 7 \\
62.5 \\
63.9\end{array}$ & $\begin{array}{l}61.4 \\
62.9 \\
61.4 \\
62.3 \\
63.1 \\
62.9\end{array}$ & $\begin{array}{l}35.9 \\
38 \\
40.3 \\
41.1 \\
32.9\end{array}$ & $\begin{array}{l}40.5 \\
41.8 \\
41.5 \\
41.8 \\
41.2\end{array}$ \\
\hline Average- & 61.4 & 66.9 & 64.8 & 64.4 & 45.8 & 49.2 \\
\hline \multirow{2}{*}{ Date } & \multicolumn{2}{|c|}{ November--Continued } & \multicolumn{4}{|c|}{ December } \\
\hline & $6 \mathrm{p} . \mathrm{m}$ & Average & $6 \mathrm{a} \cdot \mathrm{m}$. & Noon & $6 \mathrm{p} . \mathrm{m}$. & Average \\
\hline 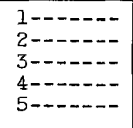 & $\begin{array}{l}58.3 \\
60.5 \\
58.1 \\
59.5 \\
57.5\end{array}$ & $\begin{array}{l}59 \\
63.8 \\
62.6 \\
57.5 \\
57.9\end{array}$ & $\begin{array}{l}39.9 \\
38.9 \\
40 \\
40.9 \\
40.9\end{array}$ & $\begin{array}{l}42 \\
40.9 \\
41.2 \\
41.3 \\
41\end{array}$ & $\begin{array}{l}42.6 \\
41.5 \\
42 \\
41 \\
40.5\end{array}$ & $\begin{array}{l}41.5 \\
40.4 \\
41.1 \\
41.1 \\
40.8\end{array}$ \\
\hline 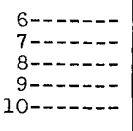 & $\begin{array}{l}54 \\
51.2 \\
52.5 \\
47.8\end{array}$ & $\begin{array}{l}53.5 \\
53.2 \\
55.1 \\
51.3 \\
46.4\end{array}$ & $\begin{array}{l}38.5 \\
40.1 \\
37.8 \\
37.5 \\
38.4\end{array}$ & $\begin{array}{l}41.1 \\
40.9 \\
38.9 \\
38.5 \\
39.2\end{array}$ & $\begin{array}{l}41 \\
39.9 \\
38.9 \\
37.7 \\
39\end{array}$ & $\begin{array}{l}40.2 \\
40.3 \\
38.5 \\
37.9 \\
38.9\end{array}$ \\
\hline $\begin{array}{l}11------- \\
12-\cdots----- \\
13------- \\
14---0--- \\
15-0-0---\end{array}$ & $\begin{array}{l}49.9 \\
50.5 \\
45.9 \\
45.5 \\
45\end{array}$ & $\begin{array}{l}50.1 \\
48.9 \\
46.7 \\
44.4 \\
44.9\end{array}$ & $\begin{array}{l}37.1 \\
35.1 \\
36.6 \\
32 \\
32\end{array}$ & $\begin{array}{l}39.5 \\
37 \\
37.3 \\
32 \\
32.9\end{array}$ & $\begin{array}{l}39 \\
36.5 \\
37.8 \\
32 \\
32\end{array}$ & $\begin{array}{l}37.5 \\
36.2 \\
37.2 \\
32 \\
32.3\end{array}$ \\
\hline 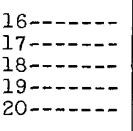 & $\begin{array}{l}41 \\
42.1 \\
47.5 \\
46.2 \\
42\end{array}$ & $\begin{array}{l}40.9 \\
40.2 \\
46.5 \\
48 \\
47\end{array}$ & $\begin{array}{l}32.1 \\
33.5 \\
33.5 \\
33.9 \\
33.8\end{array}$ & $\begin{array}{l}32.5 \\
33.8 \\
34.7 \\
36.5 \\
36\end{array}$ & $\begin{array}{l}33.2 \\
33.5 \\
34.8 \\
35 \\
36.7\end{array}$ & $\begin{array}{l}32.6 \\
33.6 \\
34.3 \\
35.1 \\
35.5\end{array}$ \\
\hline $\begin{array}{l}21------1 \\
22------1 \\
23------1 \\
24 m------ \\
25--\cdots---\end{array}$ & $\begin{array}{l}45.6 \\
46.5 \\
44.5 \\
43 \\
39.3\end{array}$ & $\begin{array}{l}46.2 \\
45.7 \\
44.4 \\
43 \\
39.3\end{array}$ & $\begin{array}{c}35 \\
34.7 \\
37.2 \\
33.9 \\
-\end{array}$ & \begin{tabular}{|}
37.8 \\
36 \\
36.7 \\
36.3 \\
\end{tabular} & $\begin{array}{c}36.4 \\
35.9 \\
34.7 \\
-0-1---\end{array}$ & $\begin{array}{r}36.4 \\
35.5 \\
36.2 \\
35.1 \\
\end{array}$ \\
\hline 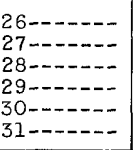 & $\begin{array}{l}40 \\
42.8 \\
41.5 \\
41.7 \\
42 \\
-\end{array}$ & $\begin{array}{c}38.8 \\
40.9 \\
41.1 \\
41.5 \\
38.7 \\
-\end{array}$ & $\begin{array}{l}33.5 \\
34 \\
33.9 \\
33.8\end{array}$ & $\begin{array}{l}35 \\
35.9 \\
35 \\
34.8\end{array}$ & $\begin{array}{l} \\
36.7 \\
35.4 \\
34 \\
33.6\end{array}$ & $\begin{array}{l}35.1 \\
35.1 \\
34.3 \\
34.1\end{array}$ \\
\hline Average- & 47.7 & 47.6 & 36 & 37.3 & 37.1 & 36.8 \\
\hline
\end{tabular}


Water temperature $\left({ }^{\circ} \mathrm{F}\right)$ for Maxinkuckee Lake--Continued

Year of 2901

\begin{tabular}{|c|c|c|c|c|c|c|}
\hline \multirow{2}{*}{ Date } & \multicolumn{4}{|c|}{ January } & \multicolumn{2}{|c|}{ February } \\
\hline & 6 a.m. & Noon & 6 p.m. & Average & $6 \mathrm{a} . \mathrm{m}$. & Noon \\
\hline 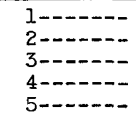 & $\begin{array}{l}33.6 \\
33.2 \\
33.5 \\
33 \\
33.5\end{array}$ & $\begin{array}{l}34.1 \\
34 \\
34.5 \\
34.7 \\
35.1\end{array}$ & $\begin{array}{l}33.5 \\
34 \\
33.9 \\
34.3 \\
34.8\end{array}$ & $\begin{array}{l}33.7 \\
33.7 \\
33.9 \\
34 \\
34.4\end{array}$ & $\begin{array}{l}34 \\
33.3 \\
35.5 \\
32.8 \\
33.8\end{array}$ & $\begin{array}{l}34.1 \\
33.5 \\
33.8 \\
34\end{array}$ \\
\hline 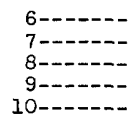 & $\begin{array}{l}34 \\
34.1 \\
34.5 \\
34 \\
34\end{array}$ & $\begin{array}{l}35 \\
35 \\
34.9 \\
35 \\
34.5\end{array}$ & $\begin{array}{l}34.8 \\
34.8 \\
34.2 \\
34 \\
35\end{array}$ & $\begin{array}{l}34.6 \\
34.6 \\
34.5 \\
34.3 \\
34.5\end{array}$ & $\begin{array}{l}33.3 \\
33.2 \\
33 \\
32.5 \\
32.5\end{array}$ & $\begin{array}{l}34.5 \\
33.8 \\
33.5 \\
32 \\
33\end{array}$ \\
\hline 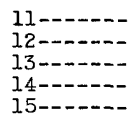 & $\begin{array}{l}35.2 \\
35.1 \\
36 \\
37 \\
37.9\end{array}$ & $\begin{array}{l}35.8 \\
36 \\
36.9 \\
38.9 \\
40.1\end{array}$ & $\begin{array}{l}35.5 \\
35.9 \\
37 \\
38.5 \\
38.2\end{array}$ & $\begin{array}{l}35.5 \\
35.5 \\
36.6 \\
38.1 \\
38.7\end{array}$ & $\begin{array}{l}33.2 \\
32.5 \\
33 \\
32.9 \\
32.9\end{array}$ & $\begin{array}{l}33.1 \\
32.9 \\
33 \\
32.9 \\
33.5\end{array}$ \\
\hline 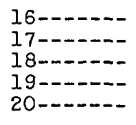 & $\begin{array}{l}36.8 \\
38.1 \\
37.9 \\
37.9 \\
37.3\end{array}$ & $\begin{array}{l}38.5 \\
38.7 \\
38.5 \\
39\end{array}$ & $\begin{array}{l}38.5 \\
38.1 \\
38.2 \\
37.3 \\
39.5\end{array}$ & $\begin{array}{l}37.1 \\
38.1 \\
38.2 \\
37.6 \\
38.6\end{array}$ & $\begin{array}{l}33 \\
33.1 \\
33 \\
33 \\
33\end{array}$ & $\begin{array}{l}33.5 \\
33.2 \\
33.2 \\
33.7 \\
33.4\end{array}$ \\
\hline 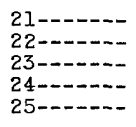 & $\begin{array}{l}37 \\
38.1 \\
39.5 \\
39.1 \\
37.6\end{array}$ & $\begin{array}{l}37.1 \\
41 \\
40 \\
38.8 \\
38.2\end{array}$ & $\begin{array}{l}37.9 \\
39 \\
40.6 \\
38.1 \\
38\end{array}$ & $\begin{array}{l}37.3 \\
39.7 \\
40 \\
38.6 \\
37.9\end{array}$ & $\begin{array}{l}32.8 \\
33.2 \\
33 \\
32.9 \\
33\end{array}$ & $\begin{array}{l}33.5 \\
33 \\
33.2 \\
33.1 \\
33\end{array}$ \\
\hline 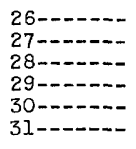 & $\begin{array}{l}37.2 \\
36.8 \\
36 \\
35.5 \\
34.9 \\
34.5\end{array}$ & $\begin{array}{l}37.3 \\
37 \\
36.9 \\
35.9 \\
35.2 \\
35\end{array}$ & $\begin{array}{l}36.1 \\
36.5 \\
37.5 \\
36.1 \\
35.7 \\
34.5\end{array}$ & $\begin{array}{l}36.8 \\
36.7 \\
36.8 \\
35.8 \\
35.2 \\
34.6\end{array}$ & $\begin{array}{l}32.5 \\
32.5 \\
33\end{array}$ & $\begin{array}{l}33 \\
33.1 \\
33\end{array}$ \\
\hline Average- & 35.4 & 36.7 & 36.8 & 36.4 & 32.7 & 33.3 \\
\hline \multirow{2}{*}{ Date } & \multicolumn{2}{|c|}{ February--Continued } & \multicolumn{4}{|c|}{ March } \\
\hline & 6 p.m. & Average & 6 a.m. & Noon & 6 p.m. & Average \\
\hline 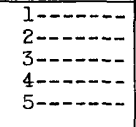 & \begin{tabular}{|l}
33.5 \\
34 \\
33.5 \\
34
\end{tabular} & $\begin{array}{l}33.8 \\
34.3 \\
33 \\
33.9\end{array}$ & $\begin{array}{l}33 \\
32.8 \\
32.6 \\
33.5 \\
33.1\end{array}$ & $\begin{array}{l}33 \\
33.3 \\
33 \\
33.8 \\
33.6\end{array}$ & $\begin{array}{l}33 \\
33 \\
33.1 \\
33.5 \\
33.6\end{array}$ & $\begin{array}{l}33 \\
33.1 \\
33 \\
33.6 \\
33.4\end{array}$ \\
\hline 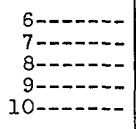 & $\begin{array}{l}33.4 \\
33.1 \\
33 \\
33 \\
33.1\end{array}$ & $\begin{array}{l}33.7 \\
33.3 \\
33.1 \\
32.5 \\
32.8\end{array}$ & $\begin{array}{l}33 \\
33 \\
32.9 \\
33.2 \\
32.3\end{array}$ & $\begin{array}{l}33.9 \\
33.9 \\
33.4 \\
33.2 \\
33.8\end{array}$ & $\begin{array}{l}33.9 \\
33.1 \\
32.9 \\
33.8 \\
33.8\end{array}$ & $\begin{array}{l}33.6 \\
33.3 \\
33 \\
33.4 \\
33.3\end{array}$ \\
\hline 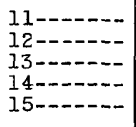 & $\begin{array}{l}33.1 \\
33 \\
33.5 \\
33.1 \\
33.3\end{array}$ & $\begin{array}{l}33.1 \\
32.8 \\
33.1 \\
32.9 \\
33.2\end{array}$ & $\begin{array}{l}33.9 \\
35.1 \\
34.3 \\
35 \\
33.8\end{array}$ & $\begin{array}{l}33 \\
34.3 \\
35.3 \\
34.9 \\
34.9\end{array}$ & $\begin{array}{l}34.8 \\
34.5 \\
34.5 \\
34.9 \\
34.6\end{array}$ & $\begin{array}{l}33.9 \\
34.6 \\
34.7 \\
34.9 \\
34.1\end{array}$ \\
\hline 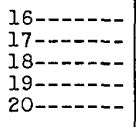 & $\begin{array}{l}33.2 \\
33 \\
33.2 \\
33.3 \\
32.9\end{array}$ & $\begin{array}{l}33.2 \\
33.1 \\
33.1 \\
33.3 \\
33.1\end{array}$ & $\begin{array}{l}35.4 \\
35.2 \\
36.9 \\
34.8 \\
37.9\end{array}$ & $\begin{array}{l}36 \\
37 \\
37.1 \\
38.5 \\
38\end{array}$ & $\begin{array}{l}34.5 \\
38 \\
37.8 \\
39.5 \\
37.5\end{array}$ & $\begin{array}{l}35.3 \\
36.7 \\
37.2 \\
37.6 \\
37.8\end{array}$ \\
\hline 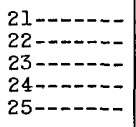 & $\begin{array}{l}33.2 \\
32.9 \\
33 \\
32.9 \\
33\end{array}$ & $\begin{array}{l}33.1 \\
33 \\
33 \\
32.9 \\
33\end{array}$ & $\begin{array}{l}37 \\
37.9 \\
38.1 \\
36.3 \\
35\end{array}$ & $\begin{array}{l}40.9 \\
44.9 \\
45.6 \\
39.5 \\
49\end{array}$ & $\begin{array}{l}38.9 \\
43.4 \\
41.5 \\
39.6 \\
42\end{array}$ & $\begin{array}{l}38.9 \\
42 \\
41.7 \\
38.4 \\
42\end{array}$ \\
\hline 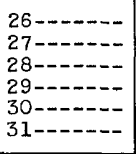 & \begin{tabular}{r}
32.9 \\
32.9 \\
32.9 \\
\hdashline$-0-$ \\
-0
\end{tabular} & \begin{tabular}{r}
32.7 \\
32.7 \\
32.9 \\
\hdashline$-1 .-$ \\
-
\end{tabular} & $\begin{array}{l}39.6 \\
38.9 \\
37.4 \\
39.8 \\
37.5 \\
37.1\end{array}$ & $\begin{array}{l}41.4 \\
41.2 \\
41 \\
44.7 \\
38.3 \\
40.1\end{array}$ & $\begin{array}{l}40.1 \\
40.3 \\
40.5 \\
42.7 \\
37.7 \\
41\end{array}$ & $\begin{array}{l}40.7 \\
40.1 \\
37.6 \\
42.4 \\
37.8 \\
39.4\end{array}$ \\
\hline Average- & 33.2 & 33.1 & 35.4 & 37.4 & 36.9 & 36.5 \\
\hline
\end{tabular}


Water temperature $\left({ }^{\circ} \mathrm{F}\right)$ for Maxinkuckee Lake--Cont1nued

\begin{tabular}{|c|c|c|c|c|c|c|}
\hline \multirow{2}{*}{ Date } & \multicolumn{4}{|c|}{ Apr11 } & \multicolumn{2}{|c|}{ May } \\
\hline & $6 \mathrm{a} . \mathrm{m}$. & Noon & 6 p.m. & Average & $6 \mathrm{a}, \mathrm{m}$. & Noon \\
\hline 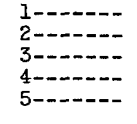 & $\begin{array}{l}36 \\
38.5 \\
38 \\
40 \\
42.5\end{array}$ & $\begin{array}{l}46.2 \\
38.5 \\
44 \\
50 \\
45.5\end{array}$ & $\begin{array}{l}45.5 \\
39 \\
43.9 \\
47.9 \\
45.5\end{array}$ & $\begin{array}{l}42.5 \\
38.6 \\
41.6 \\
45.9 \\
44.5\end{array}$ & $\begin{array}{l}57.5 \\
51.9 \\
56.9 \\
58.9\end{array}$ & $\begin{array}{l}58.3 . \\
50.5 \\
68.2 \\
67 \\
65.1\end{array}$ \\
\hline 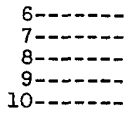 & $\begin{array}{l}44 \\
42.1 \\
40.5 \\
40.8 \\
41.9\end{array}$ & $\begin{array}{l}43.9 \\
47.6 \\
48 \\
48.9 \\
50.5\end{array}$ & $\begin{array}{l}43.5 \\
45.6 \\
45.5 \\
47.9 \\
49.9\end{array}$ & $\begin{array}{l}44.1 \\
45.1 \\
44.6 \\
45.9 \\
47.4\end{array}$ & $\begin{array}{l}60.1 \\
61 \\
60.4 \\
60.1 \\
61.9\end{array}$ & $\begin{array}{l}68 \\
63.6 \\
62.1 \\
64.5 \\
63.9\end{array}$ \\
\hline 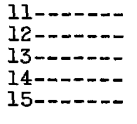 & $\begin{array}{l}42.5 \\
43.9 \\
45.3 \\
43.4 \\
44.5\end{array}$ & $\begin{array}{l}53.5 \\
46.7 \\
46.3 \\
49.3 \\
48.6\end{array}$ & $\begin{array}{l}50.9 \\
48.7 \\
47.5 \\
47.2 \\
48.5\end{array}$ & $\begin{array}{l}49.9 \\
48.4 \\
46.7 \\
46.6 \\
47.2\end{array}$ & $\begin{array}{l}60.9 \\
56 \\
55.5 \\
55 \\
57.1\end{array}$ & $\begin{array}{l}64.1 \\
59 \\
61.8 \\
61.3 \\
61.8\end{array}$ \\
\hline 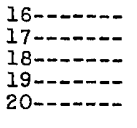 & $\begin{array}{l}44.5 \\
52.3 \\
42.5 \\
41 \\
41.5\end{array}$ & $\begin{array}{l}51.1 \\
47.5 \\
46.5 \\
48.1 \\
48.3\end{array}$ & $\begin{array}{l}49.6 \\
46.5 \\
46 \\
45 \\
46.9\end{array}$ & $\begin{array}{l}48.4 \\
48.8 \\
45 \\
44.7 \\
45.6\end{array}$ & $\begin{array}{l}61.5 \\
63.1 \\
66 \\
63 \\
58.5\end{array}$ & $\begin{array}{l}68.5 \\
70 \\
70.1 \\
65.2 \\
65\end{array}$ \\
\hline 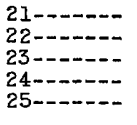 & $\begin{array}{l}42.4 \\
43.5 \\
45.5 \\
46 \\
46.8\end{array}$ & $\begin{array}{l}45.5 \\
44.5 \\
47.1 \\
52.1 \\
55.5\end{array}$ & $\begin{array}{l}43.7 \\
47.3 \\
49 \\
51.7 \\
51.5\end{array}$ & $\begin{array}{l}43.9 \\
45.1 \\
47.2 \\
49.9 \\
51.3\end{array}$ & $\begin{array}{l}58 \\
59 \\
59 \cdot 5 \\
61 \cdot 5 \\
55\end{array}$ & $\begin{array}{c}60.5 \\
62.5 \\
67.1 \\
65.4 \\
-\end{array}$ \\
\hline 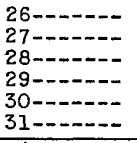 & $\begin{array}{l}47.5 \\
51.5 \\
52 \\
56.8 \\
60.1\end{array}$ & $\begin{array}{l}59 \\
57.1 \\
59 \\
65.5 \\
55.5\end{array}$ & $\begin{array}{l}57 \\
61.8 \\
64.9 \\
69.8 \\
56.7\end{array}$ & $\begin{array}{l}54.5 \\
56.8 \\
58.6 \\
64 \\
57.4\end{array}$ & $\begin{array}{l}55 \\
55 \\
55 \\
55 \\
53.1 \\
55.5 \\
\end{array}$ & $\begin{array}{l}57 \\
57.9 \\
59 \\
57.8 \\
59.5 \\
62.9 \\
\end{array}$ \\
\hline Average- & 44.6 & 49.7 & 49.5 & 47.9 & 58.23 & 62.92 \\
\hline \multirow{2}{*}{ Date } & \multicolumn{2}{|c|}{ May--Continued } & \multicolumn{4}{|c|}{ June } \\
\hline & 6 p.m. & Average & 6 a.m. & Noon & 6 p.m. & Average \\
\hline 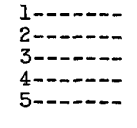 & $\begin{array}{l}54.5 \\
55 \\
65.3 \\
62.3 \\
65.3\end{array}$ & $\begin{array}{l}56.8 \\
52.8 \\
61.8 \\
62.1 \\
63.1\end{array}$ & $\begin{array}{l}58 \\
58.1 \\
60.5 \\
63.3 \\
65\end{array}$ & $\begin{array}{l}64.5 \\
65.5 \\
69 \\
71 \\
66\end{array}$ & $\begin{array}{l}63 \\
65.5 \\
68.9 \\
68.8 \\
68.9\end{array}$ & $\begin{array}{l}61.8 \\
63 \\
66.1 \\
67.7 \\
66.6\end{array}$ \\
\hline 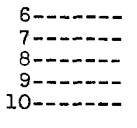 & $\begin{array}{l}63.6 \\
62 \\
62.6 \\
64.1 \\
64.9\end{array}$ & $\begin{array}{l}63.9 \\
62.2 \\
61.7 \\
62.9 \\
63.6\end{array}$ & $\begin{array}{l}64.5 \\
62.6 \\
58.9 \\
57 \\
65\end{array}$ & $\begin{array}{l}68.5 \\
64.8 \\
66.8 \\
68.5 \\
69.6\end{array}$ & $\begin{array}{l}68.8 \\
62.9 \\
63.9 \\
70 \\
70.2\end{array}$ & $\begin{array}{l}67.2 \\
63.4 \\
63.2 \\
65.2 \\
68.3\end{array}$ \\
\hline 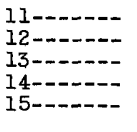 & $\begin{array}{l}61.8 \\
59.5 \\
60 \\
63.5 \\
68\end{array}$ & $\begin{array}{l}62.3 \\
58.2 \\
59.1 \\
59.9 \\
62\end{array}$ & $\begin{array}{l}66 \\
67 \\
70.9 \\
72.1 \\
75\end{array}$ & $\begin{array}{l}74.5 \\
80 \\
78 \\
80.9 \\
78.2\end{array}$ & $\begin{array}{l}70.5 \\
75 \\
74.2 \\
80 \\
78\end{array}$ & $\begin{array}{l}70.3 \\
74 \\
74.4 \\
77.7 \\
77.1\end{array}$ \\
\hline 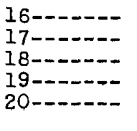 & $\begin{array}{l}66.3 \\
71 \\
69 \\
63.5\end{array}$ & $\begin{array}{l}65.4 \\
68 \\
68.4 \\
64.1 \\
62.3\end{array}$ & $\begin{array}{l}70.5 \\
74 \\
70.1 \\
72 \\
73\end{array}$ & $\begin{array}{l}80.5 \\
78.1 \\
79 \\
80 \\
77.5\end{array}$ & $\begin{array}{l}80 \\
75.5 \\
75.5 \\
78 \\
73.5\end{array}$ & $\begin{array}{l}77 \\
75.9 \\
74.9 \\
76.7 \\
74.7\end{array}$ \\
\hline 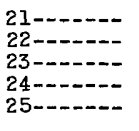 & $\begin{array}{l}61 \\
63.8 \\
65.5 \\
63.2 \\
56.9\end{array}$ & $\begin{array}{l}59.8 \\
61.8 \\
64 \\
63.4 \\
56\end{array}$ & $\begin{array}{l}74.3 \\
74.8 \\
73.9 \\
76.2 \\
77.9\end{array}$ & $\begin{array}{l}76.4 \\
82 \\
81 \\
83.5 \\
83.2\end{array}$ & $\begin{array}{l}78 \\
80.9 \\
81 \\
82 \\
81.3\end{array}$ & $\begin{array}{l}76.2 \\
79.2 \\
78.6 \\
80.6 \\
80.8\end{array}$ \\
\hline 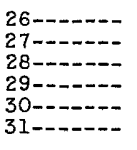 & $\begin{array}{l}51.1 \\
56.5 \\
59.5 \\
57 \\
58.9 \\
63.9\end{array}$ & $\begin{array}{l}54.4 \\
57.8 \\
61.2 \\
56.6 \\
57.2 \\
60.8\end{array}$ & $\begin{array}{l}77 \\
78.5 \\
79.2 \\
78.8 \\
78\end{array}$ & $\begin{array}{l}84.5 \\
75 \\
85.7 \\
86.2 \\
85.9\end{array}$ & $\begin{array}{l}83.9 \\
84.6 \\
84.6 \\
82.3 \\
84.3\end{array}$ & $\begin{array}{l}81.8 \\
79.4 \\
83.2 \\
82.8 \\
82.7\end{array}$ \\
\hline Average- & 61.98 & 60.93 & 73.07 & 74.13 & 75.13 & 74.11 \\
\hline
\end{tabular}


Water temperatures $\left({ }^{\circ} \mathrm{F}\right)$ for Maxinkuckee Lake--Continued

Year of 1903

\begin{tabular}{|c|c|c|c|c|c|c|}
\hline \multirow{2}{*}{ Date } & \multicolumn{4}{|c|}{ June } & \multicolumn{2}{|c|}{ July } \\
\hline & $6 \mathrm{a} . \mathrm{m}$. & Noon & $6 \mathrm{p} \cdot \mathrm{m}$ & Average & $6 \mathrm{a} \cdot \mathrm{m}$. & Noon \\
\hline 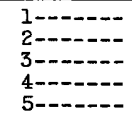 & 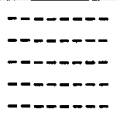 & 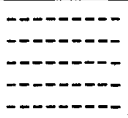 & 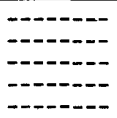 & 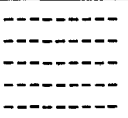 & $\begin{array}{l}71.5 \\
71 \\
74 \\
76 \\
78\end{array}$ & $\begin{array}{l}77 \\
73 \\
77 \\
79 \\
80\end{array}$ \\
\hline 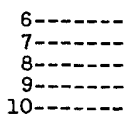 & 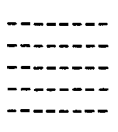 & 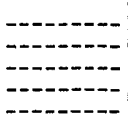 & 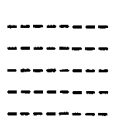 & 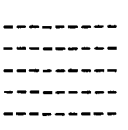 & $\begin{array}{l}77 \\
78.5 \\
79 \\
79 \\
80.5\end{array}$ & $\begin{array}{l}83.5 \\
86.5 \\
87 \\
83.5 \\
85\end{array}$ \\
\hline 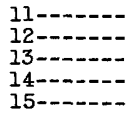 & 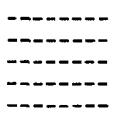 & 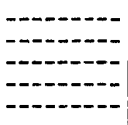 & 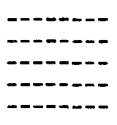 & 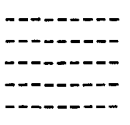 & $\begin{array}{l}81.5 \\
81 \\
78.5 \\
78 \\
74\end{array}$ & $\begin{array}{l}84.5 \\
84 \\
81.5 \\
80 \\
77.5\end{array}$ \\
\hline 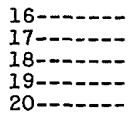 & 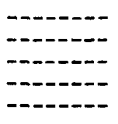 & 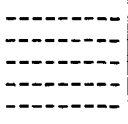 & 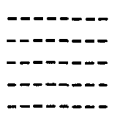 & 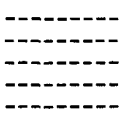 & $\begin{array}{l}75.5 \\
75 \\
73 \\
73.5 \\
72\end{array}$ & $\begin{array}{l}79 \\
78 \\
73.5 \\
74 \\
75\end{array}$ \\
\hline 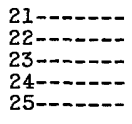 & 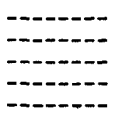 & 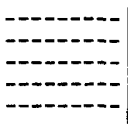 & 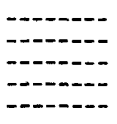 & 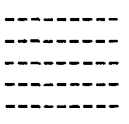 & $\begin{array}{l}72 \\
72 \\
72 \\
75 \\
78\end{array}$ & $\begin{array}{l}75 \\
75.5 \\
79 \\
81 \\
78\end{array}$ \\
\hline 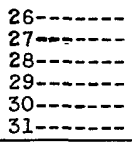 & $\begin{array}{l}65 \\
70 \\
71 \\
72\end{array}$ & $\begin{array}{l}76 \\
77 \\
73.2 \\
75.2\end{array}$ & $\begin{array}{l}75 \\
75 \\
72 \\
75\end{array}$ & $\begin{array}{r}72 \\
74 \\
72 \\
74 \\
\end{array}$ & $\begin{array}{l}76 \\
76 \\
77 \\
72 \\
76 \\
71\end{array}$ & $\begin{array}{l}79 \\
81.5 \\
80 \\
80 \\
78 \\
77 \\
\end{array}$ \\
\hline Average - & 69.5 & 75.4 & 74.3 & 73 & 75.6 & 79.4 \\
\hline \multirow{2}{*}{ Date } & \multicolumn{2}{|c|}{ July--Continued } & \multicolumn{4}{|c|}{ August } \\
\hline & $6 \mathrm{p.m}$. & Average & 6 a.m. & Noon & $6 \mathrm{p} . \mathrm{m}$. & Average \\
\hline 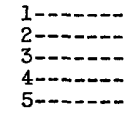 & $\begin{array}{l}71 \\
75.5 \\
78 \\
78 \\
80\end{array}$ & $\begin{array}{l}73.1 \\
73.1 \\
71.3 \\
77.6 \\
79.3\end{array}$ & $\begin{array}{l}70 \\
72 \\
77.1 \\
73.5 \\
74.5\end{array}$ & $\begin{array}{l}71 \\
77 \\
74 \\
75 \\
78.5\end{array}$ & $\begin{array}{l}71 \\
75 \\
78 \\
77 \\
75\end{array}$ & $\begin{array}{l}70.6 \\
74.6 \\
75.3 \\
75.1 \\
76\end{array}$ \\
\hline 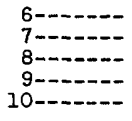 & $\begin{array}{l}84.5 \\
81.5 \\
81.5 \\
87.5 \\
85\end{array}$ & $\begin{array}{l}81.6 \\
82.1 \\
82.5 \\
83.3 \\
83\end{array}$ & $\begin{array}{l}73.5 \\
70 \\
72.5 \\
74 \\
71\end{array}$ & $\begin{array}{l}76 \\
72 \\
76.5 \\
75 \\
75\end{array}$ & $\begin{array}{l}74 \\
71 \\
74 \\
75 \\
75\end{array}$ & $\begin{array}{l}74.5 \\
71 \\
74.3 \\
74.6 \\
73.6\end{array}$ \\
\hline 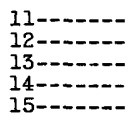 & $\begin{array}{l}84.5 \\
81 \\
78.5 \\
78.5 \\
78\end{array}$ & $\begin{array}{l}83.3 \\
82 \\
79.5 \\
78.8 \\
76.5\end{array}$ & $\begin{array}{l}72 \\
68 \\
71 \\
69 \\
68.5\end{array}$ & $\begin{array}{l}74 \\
72 \\
74 \\
76 \\
71\end{array}$ & $\begin{array}{l}74 \\
74 \\
74 \\
76 \\
70.5\end{array}$ & $\begin{array}{l}73.3 \\
71.3 \\
73 \\
73.6 \\
70\end{array}$ \\
\hline 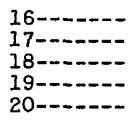 & $\begin{array}{l}79 \\
75 \\
74.5 \\
74 \\
75\end{array}$ & $\begin{array}{l}77.8 \\
76 \\
73.6 \\
77.8 \\
74\end{array}$ & $\begin{array}{l}68 \\
70 \\
73 \\
73 \\
70\end{array}$ & $\begin{array}{l}75 \\
76 \\
78 \\
74 \\
76\end{array}$ & $\begin{array}{l}74.5 \\
75 \\
76 \\
72.5 \\
75\end{array}$ & $\begin{array}{l}72.5 \\
73.6 \\
75.6 \\
73.1 \\
73.6\end{array}$ \\
\hline 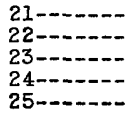 & $\begin{array}{l}73.5 \\
75.5 \\
77 \\
80 \\
77.5\end{array}$ & $\begin{array}{l}73.5 \\
74.3 \\
76 \\
78.6 \\
77.8\end{array}$ & $\begin{array}{l}71 \\
71 \\
72 \\
73 \\
73.5\end{array}$ & $\begin{array}{l}74 \\
74 \\
75.5 \\
78 \\
78\end{array}$ & $\begin{array}{l}77 \\
74 \\
73.5 \\
74.5 \\
76\end{array}$ & $\begin{array}{l}74 \\
73 \\
77 \\
75.1 \\
75.8\end{array}$ \\
\hline 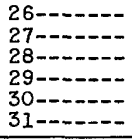 & $\begin{array}{l}78.5 \\
81.5 \\
78 \\
78.5 \\
77.5 \\
75\end{array}$ & $\begin{array}{l}76.8 \\
79.6 \\
78.3 \\
76.8 \\
77.1 \\
74.3 \\
\end{array}$ & \begin{tabular}{c}
72 \\
73 \\
73 \\
- \\
\hdashline$-0---$ \\
$-0---$ \\
\end{tabular} & $\begin{array}{c}77 \\
76 \\
76 \\
-0- \\
\end{array}$ & \begin{tabular}{l}
77.5 \\
75 \\
75 \\
\hdashline$-0-$ \\
$-0 .-0-$ \\
\end{tabular} & $\begin{array}{r}75.5 \\
74.6 \\
74.6 \\
-0 .-0 \\
\end{array}$ \\
\hline Average - & 78.5 & 77.8 & 71.7 & 75.2 & 74.6 & 73.8 \\
\hline
\end{tabular}


Water temperature $\left({ }^{\circ} \mathrm{F}\right)$ for Maxinkuckee Lake--Continued

\begin{tabular}{|c|c|c|c|c|c|c|}
\hline \multirow{2}{*}{ Date } & \multicolumn{4}{|c|}{ October } & \multicolumn{2}{|c|}{ November } \\
\hline & $6 \mathrm{a} \cdot \mathrm{m}$ & Noon & $6 \mathrm{p} . \mathrm{m}$ & Average & 6 a.m. & Noon \\
\hline 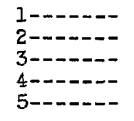 & 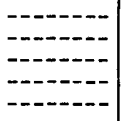 & 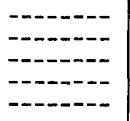 & 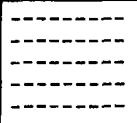 & 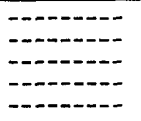 & $\begin{array}{l}50 \\
50 \\
50.5 \\
51.5 \\
49.9\end{array}$ & $\begin{array}{l}52.5 \\
53 \\
53.9 \\
53.5 \\
50.2\end{array}$ \\
\hline 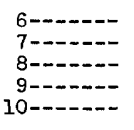 & 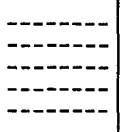 & 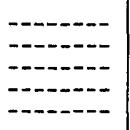 & 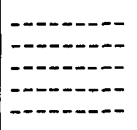 & 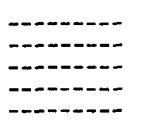 & $\begin{array}{l}42.2 \\
47.8 \\
47.9 \\
43.9 \\
47.5\end{array}$ & $\begin{array}{l}48.5 \\
48.2 \\
48.3 \\
43.9 \\
44.7\end{array}$ \\
\hline 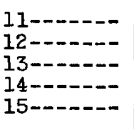 & 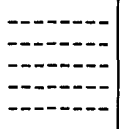 & 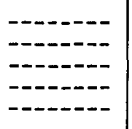 & 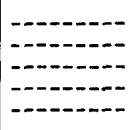 & 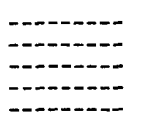 & $\begin{array}{l}42 \\
40.3 \\
42 \\
41.8 \\
41.3\end{array}$ & $\begin{array}{l}41.8 \\
43 \\
44 \\
43 \\
42\end{array}$ \\
\hline 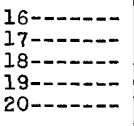 & 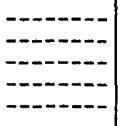 & 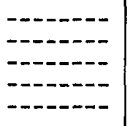 & 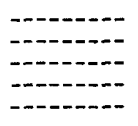 & 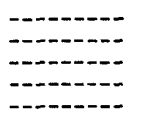 & $\begin{array}{l}42.5 \\
43.9 \\
44 \\
44.9 \\
46\end{array}$ & $\begin{array}{l}44.5 \\
45.3 \\
45.5 \\
46 \\
47\end{array}$ \\
\hline 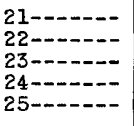 & 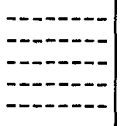 & 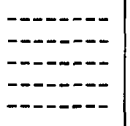 & 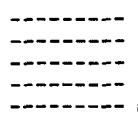 & 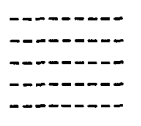 & $\begin{array}{l}43.6 \\
43 \\
43.5 \\
43.5 \\
42\end{array}$ & $\begin{array}{l}45 \cdot 5 \\
46 \\
44 \\
44.2 \\
43\end{array}$ \\
\hline 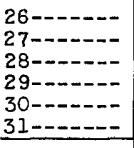 & $\begin{array}{l}47.9 \\
50.2 \\
50\end{array}$ & $\begin{array}{c}-1- \\
53 \\
54 \\
58\end{array}$ & $\begin{array}{c}52.8 \\
53.5 \\
52.9 \\
\end{array}$ & $\begin{array}{r}50.5 \\
52.6 \\
53.6\end{array}$ & $\begin{array}{l}39.8 \\
37.5 \\
43 \\
39 \\
-\end{array}$ & $\begin{array}{l}39.9 \\
38.5 \\
39 \\
-\end{array}$ \\
\hline Average- & 49.4 & 55 & 53.1 & 52.5 & 44.3 & 45.6 \\
\hline \multirow{2}{*}{ Date } & \multicolumn{2}{|c|}{ November--Continued } & \multicolumn{4}{|c|}{ December } \\
\hline & $6 \mathrm{p} \cdot \mathrm{m}$ & Average & $6 \mathrm{a} . \mathrm{m}$. & Noon & $6 \mathrm{p} . \mathrm{m}$. & Average \\
\hline 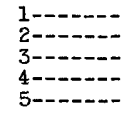 & $\begin{array}{l}53 \\
53 \\
52.9 \\
54 \\
49.2\end{array}$ & $\begin{array}{l}51.8 \\
52 \\
52.4 \\
53 \\
49.8\end{array}$ & $\begin{array}{l}35 \\
37 \\
32 \\
32 \\
33\end{array}$ & $\begin{array}{l}37.8 \\
33 \\
32.9 \\
34.5\end{array}$ & $\begin{array}{l}35 \\
33.5 \\
32.5 \\
34 \\
34.5\end{array}$ & $\begin{array}{l}35 \\
36.1 \\
32.5 \\
31.9 \\
33.5\end{array}$ \\
\hline 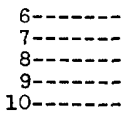 & $\begin{array}{l}47.9 \\
50.5 \\
45.1 \\
47.5 \\
44\end{array}$ & $\begin{array}{l}46.2 \\
48.8 \\
47.1 \\
45.1 \\
46.3\end{array}$ & $\begin{array}{l}34.9 \\
34.5 \\
34.5 \\
34.8 \\
32\end{array}$ & $\begin{array}{l}33 \\
36 \\
34.9 \\
35 \\
32\end{array}$ & $\begin{array}{c}35.2 \\
35.2 \\
34 \\
32\end{array}$ & $\begin{array}{l}35 \\
35.2 \\
34.6 \\
32\end{array}$ \\
\hline 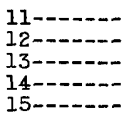 & $\begin{array}{l}43 \\
43.3 \\
42.9 \\
43.5 \\
44.8\end{array}$ & $\begin{array}{l}42.3 \\
42.2 \\
43.3 \\
42.8 \\
42.7\end{array}$ & $\begin{array}{l}34 \\
32 \\
32 \\
32 \\
32\end{array}$ & $\begin{array}{l}33.8 \\
32 \\
32 \\
32.8 \\
32\end{array}$ & $\begin{array}{l}33.8 \\
32 \\
32 \\
32 \\
32\end{array}$ & $\begin{array}{l}33.8 \\
32 \\
32 \\
32.2 \\
32\end{array}$ \\
\hline 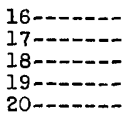 & $\begin{array}{l}47.5 \\
45 \\
46.7 \\
47 \\
45.5\end{array}$ & $\begin{array}{l}41.5 \\
44.7 \\
42.9 \\
49.9 \\
46.2\end{array}$ & $\begin{array}{l}32 \\
33 \\
33 \\
32 \\
33\end{array}$ & $\begin{array}{l}32 \\
32 \\
33 \\
33 \\
34.5\end{array}$ & $\begin{array}{l}32 \\
32 \\
33 \\
33.1 \\
35\end{array}$ & $\begin{array}{l}32 \\
32.3 \\
33 \\
32.7 \\
33.1\end{array}$ \\
\hline 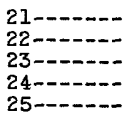 & $\begin{array}{l}45.5 \\
45.2 \\
44.8 \\
44.5 \\
42.5\end{array}$ & $\begin{array}{l}44.9 \\
44.7 \\
44.1 \\
44.1 \\
42.5\end{array}$ & $\begin{array}{l}34.9 \\
35 \\
36.4 \\
35 \\
35\end{array}$ & $\begin{array}{l}34.9 \\
37 \\
35.5 \\
34.9 \\
36.2\end{array}$ & $\begin{array}{l}35.2 \\
36.5 \\
35 \\
35 \\
35\end{array}$ & $\begin{array}{l}35 \\
36.1 \\
35.6 \\
34.9 \\
35.4\end{array}$ \\
\hline 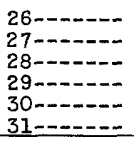 & 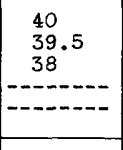 & $\begin{array}{l}39.9 \\
38.5 \\
40 \\
- \\
-\end{array}$ & \begin{tabular}{|}
35 \\
35 \\
$-2 .---$ \\
34.5 \\
34 \\
34
\end{tabular} & $\begin{array}{c}35 \\
34.5 \\
35 \\
34.8 \\
\end{array}$ & \begin{tabular}{l}
36 \\
34 \\
\hdashline-12 \\
35.1 \\
34 \\
\end{tabular} & $\begin{array}{r}35.3 \\
34.5 \\
34.2 \\
\end{array}$ \\
\hline Average - & 45.9 & 45.4 & 33.8 & 34.1 & 33.8 & 33.8 \\
\hline
\end{tabular}


Water temperatures $\left({ }^{\circ} \mathrm{F}\right)$ for Maxinkuckee Lake--Continued

Year of 1905

Year of 1906

\begin{tabular}{|c|c|c|c|c|c|c|c|c|c|}
\hline \multirow{2}{*}{ Date } & \multicolumn{4}{|c|}{ January } & \multirow{2}{*}{ Date } & \multicolumn{4}{|c|}{ Ju1y } \\
\hline & 6 a.m. & Noon & $6 \mathrm{p.m}$. & Average & & 6 a.m. & Noon & 6 p.m. & Average \\
\hline $\begin{array}{l}1 \ldots-1 \\
2 \\
3 \\
4-\cdots\end{array}$ & $\begin{array}{l}33.5 \\
34 \\
34 \\
32\end{array}$ & $\begin{array}{l}34.9 \\
34.5 \\
34.8 \\
32 \\
\end{array}$ & $\begin{array}{l}34.5 \\
32 \\
34.8 \\
32 \\
\end{array}$ & $\begin{array}{l}34.3 \\
33.5 \\
34.4 \\
32 \\
\end{array}$ & $\begin{array}{l}25-\cdots \\
26--0- \\
27 \ldots-\cdots \\
28-\cdots-\end{array}$ & $\begin{array}{l}62.5 \\
75 \\
68.5 \\
70\end{array}$ & $\begin{array}{l}72.5 \\
75.5 \\
76.8 \\
77.9\end{array}$ & $\begin{array}{l}73 \\
77.9 \\
74\end{array}$ & $\begin{array}{l}67.5 \\
74.5 \\
74.4 \\
74\end{array}$ \\
\hline Average & 33.4 & 34.1 & 33.3 & 33.6 & $\begin{array}{l}29-\ldots \\
30 \cdots \\
31 \ldots\end{array}$ & $\begin{array}{r}68.1 \\
63.5 \\
\end{array}$ & $\begin{array}{r}74 \\
82 \\
\end{array}$ & $\begin{array}{l}80 \\
82.5 \\
83.5\end{array}$ & \\
\hline & & & & & Average & 67.9 & 76.4 & 78.5 & 74.3 \\
\hline
\end{tabular}

Year of 1906

\begin{tabular}{|c|c|c|c|c|c|c|c|c|}
\hline \multirow{2}{*}{ Date } & \multicolumn{4}{|c|}{ August } & \multicolumn{4}{|c|}{ September } \\
\hline & 6 a.m. & Noon & $6 \mathrm{p}, \mathrm{m}$. & Average & $6 \mathrm{a} . \mathrm{m}$. & Noon & 6 p.m. & Average \\
\hline 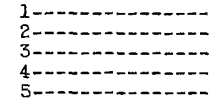 & $\begin{array}{l}71.5 \\
76.4 \\
71.9 \\
73.9 \\
73.5\end{array}$ & $\begin{array}{l}77.5 \\
81 \\
77 \\
78 \\
78.1\end{array}$ & $\begin{array}{l}84.5 \\
78.9 \\
78.9 \\
79 \\
77.5\end{array}$ & $\begin{array}{l}77.8 \\
78.8 \\
75.9 \\
77 \\
76.4\end{array}$ & $\begin{array}{l}64 \\
66 \\
67 \\
69 \\
63\end{array}$ & $\begin{array}{l}73 \\
68 \\
72.5 \\
69\end{array}$ & $\begin{array}{l}70.9 \\
69 \\
70.1 \\
69.5 \\
71\end{array}$ & $\begin{array}{l}69.3 \\
67.7 \\
69.9 \\
69.3 \\
67.7\end{array}$ \\
\hline 10-0 & $\begin{array}{l}73 \\
73.1 \\
73 \\
73.9 \\
73.9\end{array}$ & $\begin{array}{r}81.9 \\
75.4 \\
82.2 \\
75.5\end{array}$ & $\begin{array}{l}77.8 \\
77 \\
84 \\
83.5 \\
81.5\end{array}$ & $\begin{array}{l}77.6 \\
75.2 \\
79.7 \\
78.7 \\
77\end{array}$ & \begin{tabular}{l}
66.1 \\
71 \\
\hdashline 71.3 \\
76
\end{tabular} & $\begin{array}{r}71 \\
73 \\
73 \\
75 \\
-----\end{array}$ & $\begin{array}{l}73 \\
75 \\
75 \\
76 \\
74\end{array}$ & $\begin{array}{l}70 \\
73 \\
74 \\
74.1 \\
75\end{array}$ \\
\hline 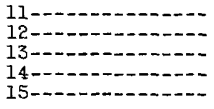 & $\begin{array}{l}72 \\
68.8 \\
69 \\
75 \\
71\end{array}$ & \begin{tabular}{c}
83 \\
75.1 \\
73.8 \\
\hdashline 76
\end{tabular} & $\begin{array}{c}79 \\
76.5 \\
75.9 \\
-72,5\end{array}$ & $\begin{array}{c}78 \\
73.5 \\
72.9 \\
73.2\end{array}$ & $\begin{array}{c}71 \\
72 \\
60 \\
-0-\end{array}$ & $\begin{array}{l}80 \\
76.8 \\
78.2 \\
67.2 \\
67\end{array}$ & $\begin{array}{l}75.2 \\
76 \\
70 \\
68 \\
67\end{array}$ & $\begin{array}{l}75.4 \\
74.9 \\
74.1 \\
65.1 \\
67\end{array}$ \\
\hline 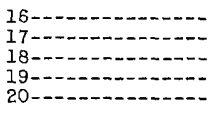 & 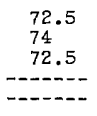 & \begin{tabular}{c}
77 \\
79 \\
77 \\
\hdashline 79.5
\end{tabular} & \begin{tabular}{l}
81 \\
77.5 \\
78 \\
\hdashline 78
\end{tabular} & $\begin{array}{c}76.8 \\
76.8 \\
75.8 \\
78.8\end{array}$ & \begin{tabular}{c}
65 \\
\hdashline 85 \\
\hdashline$-1-2-$
\end{tabular} & $\begin{array}{c}70.9 \\
74.9 \\
--0 .-\end{array}$ & $\begin{array}{c}72 \\
73 \\
80 \\
-\cdots---\end{array}$ & $\begin{array}{c}69.3 \\
-74.5 \\
-1.0-\end{array}$ \\
\hline 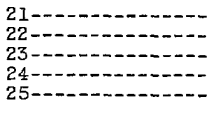 & $\begin{array}{c}74.5 \\
75.5 \\
74.8\end{array}$ & $\begin{array}{l}80 \\
80 \\
-10-\end{array}$ & $\begin{array}{c}80 \\
80.8 \\
81\end{array}$ & $\begin{array}{l}80.4 \\
77.8 \\
77.9\end{array}$ & 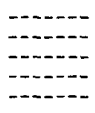 & 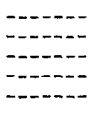 & 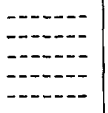 & 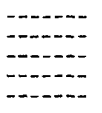 \\
\hline 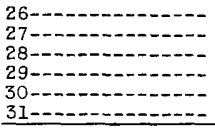 & $\begin{array}{l}70 \\
68.5 \\
70 \\
74.5 \\
67 \\
\end{array}$ & $\begin{array}{l}85 \\
73.5 \\
74.1 \\
73.5 \\
71 \\
\end{array}$ & $\begin{array}{r}77.5 \\
73.5 \\
76 \\
79 \\
72 \\
- \\
\end{array}$ & $\begin{array}{l}81.3 \\
72.3 \\
72.9 \\
74.2 \\
72.5 \\
67 \\
\end{array}$ & 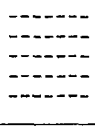 & 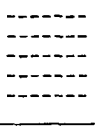 & 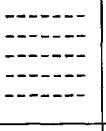 & 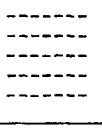 \\
\hline Average- & 72.5 & 77.7 & 78.5 & 76.2 & 67.8 & 72.6 & 72.4 & $7 I .2$ \\
\hline
\end{tabular}


Water temperatures ( $\left.{ }^{\circ} \mathrm{F}\right)$ for Maxinkuckee Lake--Continued

\begin{tabular}{|c|c|c|c|c|c|c|c|c|}
\hline \multirow[t]{2}{*}{ Date } & \multicolumn{4}{|c|}{ October } & \multicolumn{4}{|c|}{ November } \\
\hline & $6 \mathrm{a}, \mathrm{m}$. & Noon & 6 p.m. & Average & $6 \mathrm{a}, \mathrm{m}$. & Noon & $6 \mathrm{p} . \mathrm{m}$. & Average \\
\hline 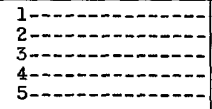 & 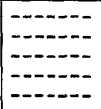 & 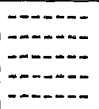 & $\frac{1}{2-\infty n+\infty}$ & 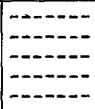 & $\begin{array}{l}45.5 \\
45 \\
48 \\
45.2 \\
47\end{array}$ & $\begin{array}{c}50.5 \\
45 \\
51 \\
-52.5\end{array}$ & $\begin{array}{l}50 \\
50 \\
45 \\
46 \\
46.5\end{array}$ & $\begin{array}{l}48.8 \\
46.6 \\
48 \\
45.6 \\
48.6\end{array}$ \\
\hline 10-0. & $\frac{-m-2}{-2-n-2}$ & 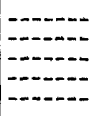 & 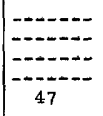 & 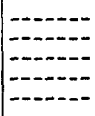 & $\begin{array}{l}43 \\
53.5 \\
45 \\
47.5 \\
41.5\end{array}$ & $\begin{array}{l}49 \\
48 \\
53.2\end{array}$ & $\begin{array}{l}48.6 \\
46 \\
44.5\end{array}$ & $\begin{array}{r}47.5 \\
47.1 \\
46.4\end{array}$ \\
\hline 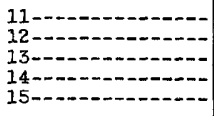 & $\begin{array}{l}44.5 \\
47.2 \\
55 \\
51 \\
51.6\end{array}$ & $\begin{array}{l}53.5 \\
52 \\
55 \\
55.8 \\
55.5\end{array}$ & \begin{tabular}{l}
49.5 \\
52 \\
\hdashline 55.5 \\
56.7
\end{tabular} & $\begin{array}{l}49.2 \\
50.4 \\
55 \\
54.1 \\
54.6\end{array}$ & $\begin{array}{l}45.2 \\
42.5 \\
41.8 \\
41 \\
43\end{array}$ & $\begin{array}{r}48.9 \\
45.1 \\
43 \\
46.6 \\
\end{array}$ & $\begin{array}{r}45 \\
42 \\
44.5 \\
-----\end{array}$ & $\begin{array}{r}44.2 \\
42.2 \\
44 \\
-\cdots\end{array}$ \\
\hline 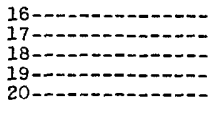 & $\begin{array}{l}51.8 \\
51.5 \\
56 \\
58.5 \\
51.9\end{array}$ & $\begin{array}{l}58 \\
58 \\
65 \\
63.3\end{array}$ & $\begin{array}{l}57 \\
58 \\
61 \\
62.5 \\
53\end{array}$ & $\begin{array}{l}54.8 \\
55.8 \\
58.3 \\
62 \\
56.1\end{array}$ & $\begin{array}{l}43.5 \\
43.5 \\
45 \\
42.5 \\
35.5\end{array}$ & $\begin{array}{r}44.5 \\
-45.5 \\
37.5 \\
37\end{array}$ & $\begin{array}{r}43.5 \\
40 \\
-37.2 \\
-\end{array}$ & $\begin{array}{l}43.8 \\
41.1 \\
45.2 \\
39 \\
36.2\end{array}$ \\
\hline 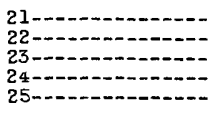 & $\begin{array}{l}56 \\
56.3 \\
59 \\
57.5 \\
-\end{array}$ & $\begin{array}{r}59 \\
57.6 \\
60.5 \\
-0.0- \\
-0 .-0\end{array}$ & $\begin{array}{l}60 \\
56 \\
58 \\
50 \\
-\end{array}$ & $\begin{array}{r}58.3 \\
56.6 \\
59.2 \\
53.8 \\
-\end{array}$ & \begin{tabular}{c}
39.5 \\
43.5 \\
\hdashline$-0 .-2$
\end{tabular} & 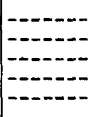 & 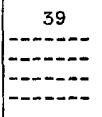 & 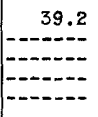 \\
\hline 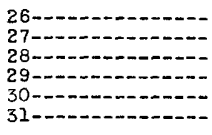 & $\begin{array}{l}49.5 \\
44 \\
41.1 \\
46 \\
42\end{array}$ & $\begin{array}{l}47 \\
43 \\
50 \\
47.5 \\
52\end{array}$ & $\begin{array}{l}45.5 \\
42.8 \\
44.5 \\
45 \\
48.5\end{array}$ & $\begin{array}{l}47.3 \\
43.3 \\
45.2 \\
46.1 \\
47.5\end{array}$ & $\frac{-2-2}{-2 m}$ & 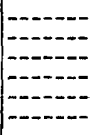 & 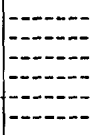 & 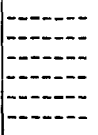 \\
\hline Average- & 51.1 & 54.9 & 53.1 & 53 & 42.9 & 46.5 & 44.5 & 44.6 \\
\hline
\end{tabular}

\begin{tabular}{|c|c|c|c|c|c|c|c|c|c|}
\hline \multirow{2}{*}{ Date } & \multicolumn{4}{|c|}{ September } & \multirow{2}{*}{ Date } & \multicolumn{4}{|c|}{ October } \\
\hline & 6 a.m. & Noon & $6 \mathrm{p} . \mathrm{m}$. & Average & & $6 \mathrm{a} . \mathrm{m}$. & Noon & $6 \mathrm{p.m}$. & Average \\
\hline 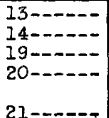 & $\begin{array}{l}67 \\
70 \\
70\end{array}$ & \begin{tabular}{c}
70 \\
\hdashline 74 \\
73
\end{tabular} & $\begin{array}{l}69 \\
73 \\
73.5\end{array}$ & $\begin{array}{c}72.2 \\
72\end{array}$ & 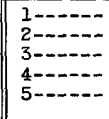 & $\begin{array}{l}57 \\
60 \\
62 \\
63 \\
59\end{array}$ & $\begin{array}{l}61 \\
65 \\
64 \\
65 \\
63\end{array}$ & $\begin{array}{l}63 \\
64 \\
63 \\
65 \\
63\end{array}$ & $\begin{array}{l}60.3 \\
63 \\
63 \\
64.3 \\
61.7\end{array}$ \\
\hline 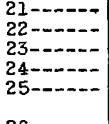 & $\begin{array}{l}67 \\
62 \\
65 \\
61 \\
58\end{array}$ & \begin{tabular}{l}
72.5 \\
70 \\
\hdashline 66 \\
62
\end{tabular} & $\begin{array}{l}70 \\
70 \\
66 \\
63 \\
62\end{array}$ & $\begin{array}{l}69.8 \\
67.3 \\
65.5 \\
63.3 \\
60.7\end{array}$ & 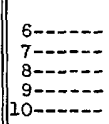 & $\begin{array}{l}59 \\
59 \\
54 \\
57 \\
54\end{array}$ & $\begin{array}{l}62 \\
62 \\
59 \\
58 \\
57\end{array}$ & $\begin{array}{l}63 \\
60 \\
61 \\
58 \\
58\end{array}$ & $\begin{array}{l}61.3 \\
60.3 \\
58 \\
57.7 \\
56.3\end{array}$ \\
\hline 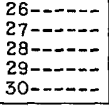 & $\begin{array}{l}60 \\
61 \\
61 \\
60 \\
57 \\
\end{array}$ & $\begin{array}{l}62 \\
61 \\
62 \\
60 \\
\end{array}$ & $\begin{array}{l}63 \\
61 \\
62 \\
59 \\
62 \\
\end{array}$ & $\begin{array}{l}61.7 \\
61 \\
61.7 \\
59.7 \\
59.5 \\
\end{array}$ & 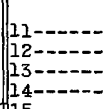 & $\begin{array}{l}54 \\
52 \\
50 \\
51\end{array}$ & $\begin{array}{l}57 \\
53 \\
52 \\
54\end{array}$ & $\begin{array}{l}55 \\
53 \\
53 \\
54\end{array}$ & $\begin{array}{l}55.3 \\
52.7 \\
51.7 \\
53\end{array}$ \\
\hline Average- & 63 & 66.6 & 65.6 & 64.5 & & 55 & 55 & 55 & 55 \\
\hline \multicolumn{5}{|c|}{ Year of 1908} & $\mid \begin{array}{l}16------ \\
17-\cdots--\end{array}$ & $\begin{array}{l}55 \\
54\end{array}$ & $\begin{array}{l}55 \\
57\end{array}$ & $\begin{array}{l}56 \\
58\end{array}$ & $\begin{array}{l}55.3 \\
56.3\end{array}$ \\
\hline \multirow[t]{2}{*}{ Date } & \multicolumn{4}{|c|}{ August } & 18 & $\begin{array}{l}54 \\
50\end{array}$ & & & \\
\hline & $6 \mathrm{a} . \mathrm{m}$. & Noon & 6 p.m. & Average & $20 \cdots$ & 53 & 54 & 53 & 53.3 \\
\hline 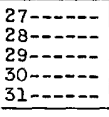 & $\begin{array}{l}65 \\
65 \\
69 \\
70 \\
72.9 \\
\end{array}$ & $\begin{array}{l}73 \\
74 \\
70.5 \\
81 \\
78.5 \\
\end{array}$ & $\begin{array}{l}74 \\
73.5 \\
80 \\
79 \\
\end{array}$ & $\begin{array}{l}69 \\
74.1 \\
71 \\
77 \\
76.8 \\
\end{array}$ & 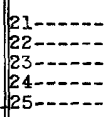 & $\begin{array}{l}51 \\
51 \\
52 \\
51 \\
50\end{array}$ & $\begin{array}{l}55 \\
55 \\
55 \\
53 \\
53\end{array}$ & $\begin{array}{l}54 \\
56 \\
53 \\
54 \\
50\end{array}$ & $\begin{array}{l}53.3 \\
54 \\
53.3 \\
52.7 \\
51\end{array}$ \\
\hline \multirow[t]{3}{*}{ Average- } & 68.4 & 75.4 & 76.6 & 73.2 & \multirow[b]{2}{*}{ 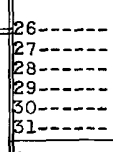 } & \multirow[b]{2}{*}{$\begin{array}{l}50 \\
49 \\
46 \\
43 \\
49 \\
48 \\
\end{array}$} & \multirow[b]{2}{*}{$\begin{array}{l}49 \\
50 \\
46 \\
45 \\
49 \\
50 \\
\end{array}$} & \multirow[b]{2}{*}{$\begin{array}{l}50 \\
48 \\
47 \\
46 \\
49 \\
51 \\
\end{array}$} & \multirow[b]{2}{*}{$\begin{array}{l}49.7 \\
49 \\
46.3 \\
44.7 \\
49 \\
49.3\end{array}$} \\
\hline & ‘ & & & & & & & & \\
\hline & & & & & Average- & 53.3 & 55.6 & 55.6 & 54.8 \\
\hline
\end{tabular}


Water temperatures $\left({ }^{\circ} \mathrm{F}\right)$ for Maxinkuckee Lake--Continued

\begin{tabular}{|c|c|c|c|c|c|c|c|c|c|}
\hline \multirow{2}{*}{ Date } & \multicolumn{4}{|c|}{ September } & \multirow{2}{*}{ Date } & \multicolumn{4}{|c|}{ October } \\
\hline & $\mathrm{t} . \mathrm{m}$. & Noon & $6 \mathrm{p.m}$. & Average & & $6 \mathrm{a} . \mathrm{m}$. & Noon & 6 p.m. & Average \\
\hline 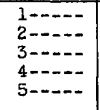 & $\begin{array}{l}75 \\
71.3 \\
61.5 \\
65 \\
67\end{array}$ & $\begin{array}{c}75 \\
73.5 \\
72 \\
72 \\
-\end{array}$ & $\begin{array}{l}70.5 \\
70 \\
70 \\
75\end{array}$ & $\begin{array}{l}75 \\
71.8 \\
67.8 \\
69 \\
71\end{array}$ & 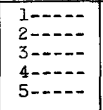 & $\begin{array}{l}61 \\
60 \\
56 \\
60 \\
60\end{array}$ & $\begin{array}{l}66 \\
65 \\
65 \\
64\end{array}$ & $\begin{array}{l}66 \\
62 \\
64 \\
65 \\
64\end{array}$ & $\begin{array}{l}64.3 \\
61 \\
61.7 \\
63.3 \\
62.7\end{array}$ \\
\hline 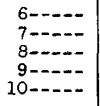 & $\begin{array}{l}71 \\
64 \\
66 \\
66.5 \\
69\end{array}$ & $\begin{array}{c}78 \\
73.5 \\
76.5\end{array}$ & $\begin{array}{c}70 \\
71.5 \\
75 \\
75.4\end{array}$ & $\begin{array}{c}73 \\
67.8 \\
71.7 \\
73.6\end{array}$ & 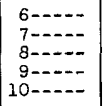 & $\begin{array}{l}62 \\
62 \\
62 \\
64 \\
65\end{array}$ & $\begin{array}{l}65 \\
67 \\
67 \\
70 \\
69\end{array}$ & $\begin{array}{l}65 \\
67 \\
67 \\
68 \\
67\end{array}$ & $\begin{array}{l}64 \\
65.3 \\
65.3 \\
67.3 \\
67\end{array}$ \\
\hline 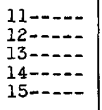 & $\begin{array}{l}71 \\
73 \\
71 \\
68.5\end{array}$ & $\begin{array}{l}77 \\
81 \\
76 \\
74 \\
72\end{array}$ & $\begin{array}{l}78 \\
73 \\
73 .\end{array}$ & $\begin{array}{l}76 \\
75.7 \\
72.7 \\
71.2\end{array}$ & 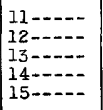 & $\begin{array}{l}61 \\
57 \\
56 \\
57 \\
56\end{array}$ & $\begin{array}{l}63 \\
58 \\
61 \\
62 \\
63\end{array}$ & $\begin{array}{l}61 \\
50 \\
62 \\
61 \\
62\end{array}$ & $\begin{array}{l}61.7 \\
55 \\
59.7 \\
60 \\
60.3\end{array}$ \\
\hline $16 \cdots$ & $\begin{array}{r}66.5 \\
-\end{array}$ & $\begin{array}{l}73.8 \\
74 \\
\end{array}$ & $\begin{array}{l}76 \\
75 \\
\end{array}$ & $\begin{array}{l}72.1 \\
74.5 \\
\end{array}$ & $16-\cdots$ & $\begin{array}{l}59 \\
60\end{array}$ & $\begin{array}{l}62 \\
62\end{array}$ & $\begin{array}{l}62 \\
52\end{array}$ & $\begin{array}{l}61 \\
58\end{array}$ \\
\hline \multirow[t]{5}{*}{ Average- } & 68 & 74.1 & 73.3 & 72.2 & $\begin{array}{l}18-\ldots-0 \\
19 \cdots \cdots\end{array}$ & $\begin{array}{l}56 \\
51\end{array}$ & $\begin{array}{l}56 \\
59\end{array}$ & $\begin{array}{l}56 \\
55\end{array}$ & $\begin{array}{l}56 \\
55\end{array}$ \\
\hline & & & & & $20 \cdots$ & 51 & 52 & 48 & 50.3 \\
\hline & & & & & $\begin{array}{l}21 \cdots \cdots \\
22-\cdots \\
23-\cdots \\
24-\cdots \\
25-\cdots\end{array}$ & $\begin{array}{l}45 \\
50 \\
48 \\
51 \\
48\end{array}$ & $\begin{array}{l}46 \\
46 \\
52 \\
54 \\
55\end{array}$ & $\begin{array}{l}46 \\
50 \\
50 \\
54 \\
54\end{array}$ & $\begin{array}{l}45.7 \\
48.7 \\
50 \\
53 \\
52.3\end{array}$ \\
\hline & & & & & 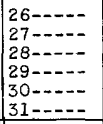 & $\begin{array}{l}50 \\
50 \\
47 \\
49 \\
45 \\
43 \\
\end{array}$ & $\begin{array}{l}54 \\
51 \\
49 \\
50 \\
45 \\
47 \\
\end{array}$ & $\begin{array}{l}55 \\
50 \\
50 \\
46 \\
45 \\
44 \\
\end{array}$ & $\begin{array}{l}53 \\
50.3 \\
48.7 \\
48.3 \\
45 \\
44.7 \\
\end{array}$ \\
\hline & & & & & Average- & 54.9 & 58.2 & 57 & 56.7 \\
\hline
\end{tabular}

Temperature proflles ( $\left.{ }^{\circ} \mathrm{F}\right)$ for Maxinkuckee Lake, at Culver

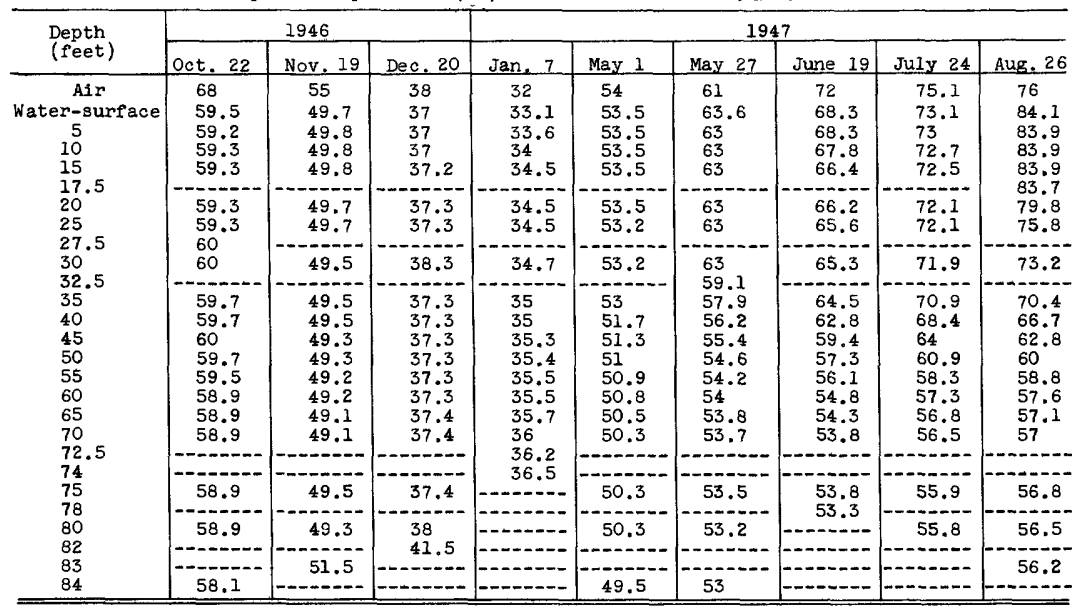


Temperature profiles $\left({ }^{\circ} \mathrm{F}\right)$ for Maxinkuckee Lake, at Culver--Continued

\begin{tabular}{|c|c|c|c|c|c|}
\hline \multirow{2}{*}{$\begin{array}{l}\text { Depth } \\
\text { (feet) }\end{array}$} & \multicolumn{3}{|c|}{ 1947--Continued } & \multicolumn{2}{|c|}{1948} \\
\hline & Oct. 3 & Oct. 31 & Nov. 20 & June 2 & July 3 \\
\hline Air & 69 & 52 & 46 & 62 & 83 \\
\hline $\begin{array}{l}\text { Water-surface } \\
5 \\
10 \\
15 \\
17.5 \\
20 \\
22.5 \\
25 \\
27.5 \\
30 \\
35 \\
40 \\
45 \\
50 \\
55 \\
60 \\
65 \\
70 \\
72.5 \\
75 \\
80 \\
85 \\
86 \\
86.5 \\
\end{array}$ & 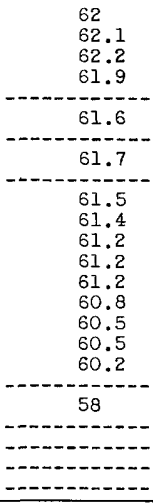 & $\begin{array}{l}61.5 \\
61.5 \\
61.5 \\
61.5 \\
61.5 \\
61.5 \\
61.5 \\
61.5 \\
61.5 \\
61.4 \\
61.3 \\
61.3 \\
61.5 \\
61.3 \\
61 \\
59.8 \\
59.3 \\
58.7 \\
57.4 \\
57.1 \\
\end{array}$ & $\begin{array}{c}46.3 \\
46.4 \\
46.4 \\
46.3 \\
46.3 \\
46.3 \\
46.2 \\
46.1 \\
46 \\
46 \\
46 \\
45.9 \\
45.9 \\
45.9 \\
45.9 \\
45.9 \\
45.7 \\
45.6 \\
-.9 \\
-9.9 \\
\end{array}$ & $\begin{array}{c}67.4 \\
67.5 \\
67.3 \\
67.1 \\
66.8 \\
65.3 \\
63.2 \\
62 \\
61.7 \\
60.7 \\
60.3 \\
60.1 \\
59.5 \\
58.7 \\
58.3 \\
58 \\
57.9 \\
57.8 \\
57.8 \\
57.6 \\
-0.0 \\
\end{array}$ & $\begin{array}{c}75 \\
75 \\
75 \\
74.8 \\
74.6 \\
74.2 \\
72 \\
68 \\
63.9 \\
62.5 \\
61.5 \\
60.3 \\
60 \\
59.7 \\
59.7 \\
59.6 \\
59.6 \\
59.4 \\
59.3 \\
59.3 \\
\end{array}$ \\
\hline Deptr & \multicolumn{5}{|c|}{ 1948--Continued } \\
\hline & Aug. 3 & Aug. 31 & Oct. 5 & Nov. 16 & Dec. 7 \\
\hline A.r & 79 & 73 & 62 & 54 & $n--n-\cdots+\cdots$ \\
\hline $\begin{array}{l}\text { Water-surface } \\
5 \\
10 \\
15 \\
17.5 \\
20 \\
22.5 \\
25 \\
27.5 \\
30 \\
32.5 \\
35 \\
40 \\
45 \\
50 \\
55 \\
60 \\
65 \\
70 \\
75 \\
78 \\
80 \\
82 \\
84 \\
85\end{array}$ & $\begin{array}{c}79.1 \\
77.6 \\
77.3 \\
77.3 \\
77 \\
76 \\
75.3 \\
72.7 \\
68 \\
66.1 \\
63.5 \\
62.2 \\
60.7 \\
59.9 \\
59.5 \\
59.2 \\
58.9 \\
58.7 \\
58.7 \\
58.7\end{array}$ & $\begin{array}{c}80.4 \\
80.4 \\
80.2 \\
80.2 \\
78.8 \\
76.4 \\
75.3 \\
74.4 \\
73.4 \\
70.3 \\
68.6 \\
63.9 \\
62.9 \\
61.2 \\
59.8 \\
59.4 \\
59.4 \\
59.2 \\
59.2 \\
59.2 \\
58.9 \\
-9.2 \\
-9\end{array}$ & $\begin{array}{c}63.2 \\
52.8 \\
62.8 \\
62.6 \\
62.7 \\
62.7 \\
62.6 \\
62.5 \\
62.3 \\
62.1 \\
62 \\
61.7 \\
59.6 \\
59.3 \\
58.9 \\
60.1 \\
59.8 \\
59.7 \\
\end{array}$ & $\begin{array}{c}48.4 \\
48.3 \\
48.3 \\
48.6 \\
48 \\
48.3 \\
48.3 \\
48.2 \\
48.6 \\
48.3 \\
49 \\
49 \\
48.8 \\
48.7 \\
48.7 \\
48.6 \\
48.7 \\
48.7 \\
-9\end{array}$ & 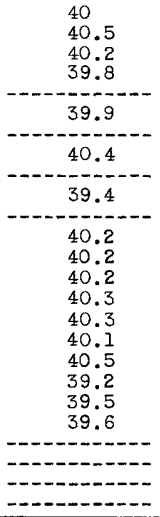 \\
\hline
\end{tabular}

Temperature profiles ( ${ }^{\circ} \mathrm{F}$ ) taken in Deep Hole, Maxinkuckee Lake

[Data from Evermann and Clark, 1920]

Temperature readings, in 1899

\begin{tabular}{|c|c|c|c|c|c|c|c|}
\hline \multirow{2}{*}{$\begin{array}{l}\text { Depth } \\
\text { (feet) }\end{array}$} & \multicolumn{7}{|c|}{ July } \\
\hline & 18 & 19 & 20 & 21 & 22 & 23 & 24 \\
\hline Air & $---\cdots---$ & $------n$ & $-\cdots-\cdots-\cdots$ & ------- & $--n---n$ & $-------n$ & \\
\hline $\begin{array}{c}\text { Water-surface } \\
5 \\
10 \\
15 \\
20 \\
25 \\
30 \\
35 \\
40 \\
50 \\
60 \\
70 \\
80 \\
85 \\
89\end{array}$ & $\begin{array}{l}77 \\
77 \\
77 \\
76.5 \\
74.7 \\
72.3 \\
69.2 \\
-23 \\
57.5 \\
52.5 \\
51.5 \\
50.3 \\
50.5\end{array}$ & $\begin{array}{l}77 \\
77 \\
77 \\
76.5 \\
74.7 \\
72.3 \\
69.2 \\
63 \\
57.5 \\
52.5 \\
51.5 \\
50.5 \\
50.3 \\
50.5 \\
\end{array}$ & $\begin{array}{l}78 \\
78 \\
78 \\
76.7 \\
75 \\
72.5 \\
69 \\
63 \\
57.3 \\
53 \\
51.7 \\
50.7 \\
50.7 \\
50.7 \\
\end{array}$ & \begin{tabular}{l}
78.2 \\
78.2 \\
78.3 \\
78.5 \\
75 \\
73.5 \\
69 \\
64.2 \\
58 \\
52.6 \\
51.2 \\
51.2 \\
50.8 \\
\hdashline 50.6
\end{tabular} & $\begin{array}{l}79.1 \\
79.1 \\
79 \\
78.1 \\
75 \\
72 \\
69.2 \\
64 \\
59.1 \\
53.1 \\
51.3 \\
51 \\
51 \\
50.5\end{array}$ & $\begin{array}{l}80.5 \\
80.5 \\
79.7 \\
78 \\
74 \\
69.5 \\
64 \\
58 \\
54 \\
50.5 \\
50.5 \\
50.5 \\
50.5 \\
- \\
\end{array}$ & $\begin{array}{l}80 \\
80.1 \\
80.1 \\
78.5 \\
75.2 \\
72.8 \\
70 \\
65 \\
58.5 \\
53.7 \\
51.5 \\
51.2 \\
50.8 \\
50.5 \\
-\end{array}$ \\
\hline
\end{tabular}


Temperature profiles $\left({ }^{\circ} \mathrm{F}\right)$ taken in Deep Hole, Maxinkuckee Lake--Continued

Temperature readings, in 1899

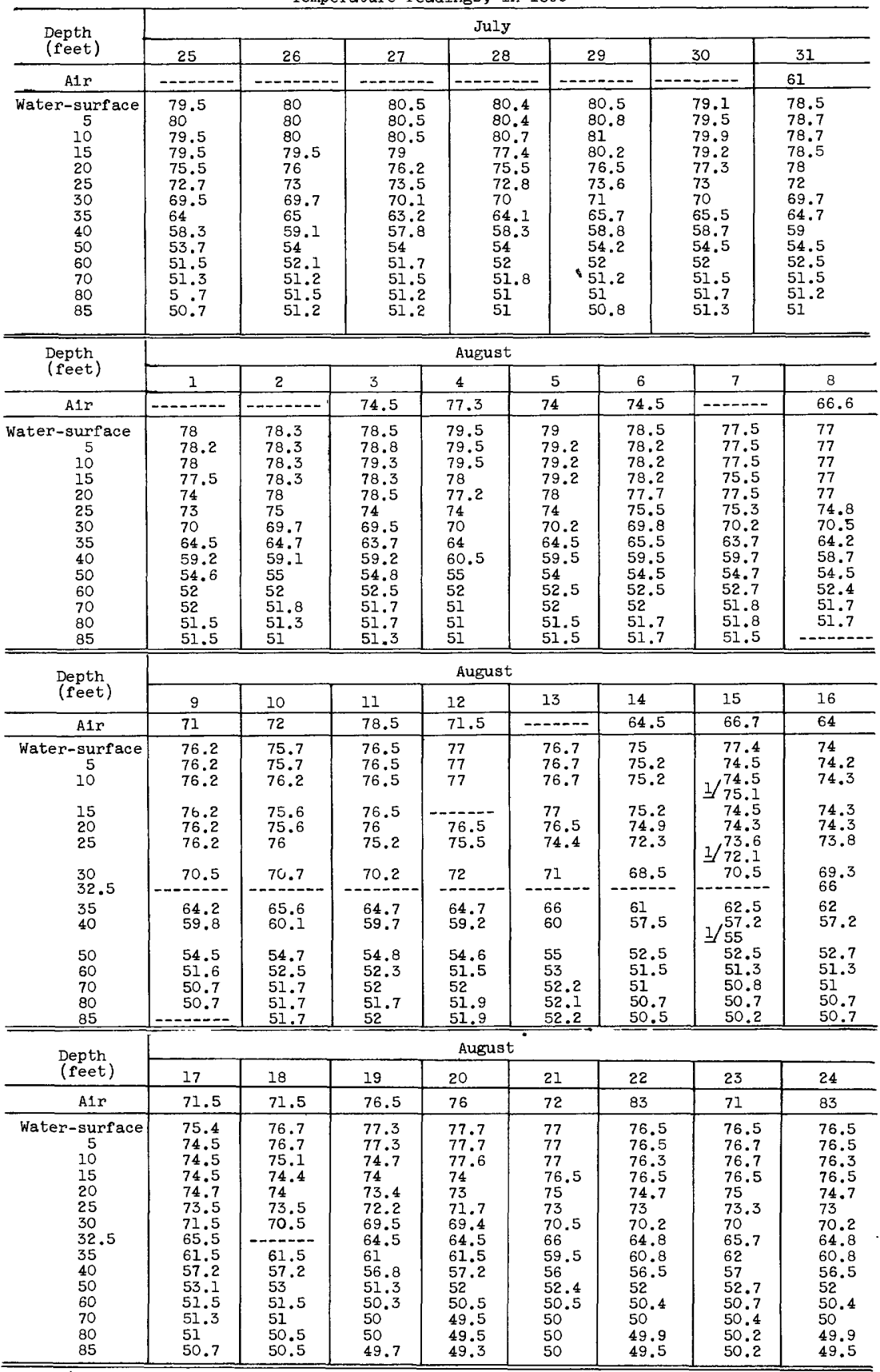

1/ Thermometer. 
Temperature profiles $\left({ }^{\circ} \mathrm{F}\right)$ taken in the Deep Hole, Maxinkuckee Lake--Continued

Temperature readings, in 1899

\begin{tabular}{|c|c|c|c|c|c|c|c|}
\hline \multirow{2}{*}{$\begin{array}{l}\text { Depth } \\
\text { (feet) }\end{array}$} & \multicolumn{7}{|c|}{ August } \\
\hline & 25 & 26 & 27 & 28 & 29 & 30 & 31 \\
\hline A1r & 72.5 & 72.3 & 71.7 & 74 & 72 & 74.2 & 73 \\
\hline \begin{tabular}{c|} 
Water-surface \\
5 \\
10 \\
15 \\
20 \\
25 \\
30 \\
32.5 \\
35 \\
40 \\
50 \\
60 \\
70 \\
80 \\
85 \\
\end{tabular} & $\begin{array}{l}76.5 \\
76.5 \\
76.5 \\
76.5 \\
75 \\
73.2 \\
70 \\
66.5 \\
62.7 \\
58 \\
52.4 \\
51 \\
50.5 \\
50 \\
50 \\
\end{array}$ & $\begin{array}{l}76.3 \\
76.3 \\
76.3 \\
76.3 \\
75.5 \\
73 \\
69.2 \\
65 \\
62 \\
57.6 \\
52.2 \\
50.8 \\
50.4 \\
50.2 \\
50 \\
\end{array}$ & $\begin{array}{l}75.5 \\
76.5 \\
76.3 \\
76.3 \\
75.1 \\
73.7 \\
69.5 \\
66.7 \\
61.2 \\
57.6 \\
52.5 \\
51 \\
50.5 \\
50.2 \\
50 \\
\end{array}$ & $\begin{array}{l}77 \\
77 \\
77 \\
76.5 \\
75 \\
73.3 \\
69.5 \\
66.3 \\
63.2 \\
57 \\
52.5 \\
50.7 \\
50.5 \\
50.5 \\
50 \\
\end{array}$ & $\begin{array}{l}76.5 \\
76.5 \\
76.7 \\
76.5 \\
76 \\
73.5 \\
70 \\
66.5 \\
63.2 \\
56.7 \\
51.7 \\
51 \\
50.7 \\
50.5 \\
50.2 \\
\end{array}$ & $\begin{array}{l}77.2 \\
77.2 \\
77 \\
77 \\
76.5 \\
73.5 \\
70.7 \\
65.5 \\
62.2 \\
57 \\
52.5 \\
51.5 \\
51 \\
51 \\
2 / 50.5 \\
\end{array}$ & $\begin{array}{l}75.7 \\
75 \\
75 \\
74.7 \\
73.5 \\
71 \\
67.3 \\
63.2 \\
60.3 \\
55.8 \\
50.7 \\
49.5 \\
49 \\
49 \\
3 / 48.7 \\
\end{array}$ \\
\hline \multirow{2}{*}{$\begin{array}{l}\text { Depth } \\
\text { (feet) }\end{array}$} & \multicolumn{7}{|c|}{ Seprember } \\
\hline & 1 & 2 & 3 & 4 & 5 & 6 & 7 \\
\hline Air & 73.5 & 77.6 & 75 & 65 & 79 & 73 & 70.5 \\
\hline $\begin{array}{l}\text { Water-surface } \\
5 \\
10 \\
15 \\
20 \\
25 \\
30 \\
32.5 \\
35 \\
40 \\
50 \\
60 \\
70 \\
80 \\
85 \\
\end{array}$ & $\begin{array}{l}75.8 \\
75.8 \\
75.8 \\
75 \\
73 \\
72 \\
68.8 \\
63.8 \\
62 \\
56.5 \\
51 \\
50 \\
49.8 \\
49.5 \\
49 \\
\end{array}$ & 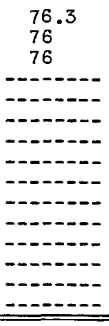 & $\begin{array}{l}77 \\
77 \\
76.5 \\
75.5 \\
73.2 \\
70.6 \\
66.2 \\
63.3 \\
59 \\
54.8 \\
50 \\
48.8 \\
48.6 \\
48.6 \\
48.4 \\
\end{array}$ & $\begin{array}{l}75.5 \\
75.5 \\
75.5 \\
74.5 \\
74 \\
72.2 \\
64.8 \\
64 \\
58.7 \\
55.9 \\
51 \\
49.9 \\
49.9 \\
49 \\
47 \\
\end{array}$ & $\begin{array}{l}76.5 \\
76 \\
75.6 \\
75 \\
74.5 \\
73.5 \\
69.5 \\
64 \\
61 \\
56 \\
51 \\
49.5 \\
49 \\
49 \\
49 \\
\end{array}$ & $\begin{array}{l}75.5 \\
75.5 \\
74.5 \\
74 \\
73 \\
72.5 \\
67 \\
63 \\
60.3 \\
57 \\
51 \\
51 \\
50.5 \\
50.5 \\
50.5 \\
\end{array}$ & $\begin{array}{l}74 \\
74 \\
74 \\
74 \\
74 \\
73 \\
68 \\
63 \\
59 \\
55 \\
50.5 \\
49 \\
48.5 \\
48.5 \\
48.5 \\
\end{array}$ \\
\hline \multirow{2}{*}{$\begin{array}{l}\text { Depth } \\
\text { (feet) }\end{array}$} & \multicolumn{7}{|c|}{ September--Cont1 nued } \\
\hline & 8 & 9 & 10 & 11 & 12 & 13 & 14 \\
\hline $\mathrm{A} 1 \mathrm{r}$ & 68.5 & 62 & 61 & 61 & 63.5 & 57 & 50.5 \\
\hline $\begin{array}{c}\text { Water-surface } \\
5 \\
10 \\
15 \\
20 \\
25 \\
30 \\
32.5 \\
35 \\
40 \\
50 \\
60 \\
70 \\
80 \\
85 \\
\end{array}$ & $\begin{array}{l}74.5 \\
74.5 \\
74.5 \\
74.5 \\
73.5 \\
72 \\
68 \\
64.5 \\
61 \\
56.5 \\
51 \\
50 \\
49.5 \\
49.5 \\
49.5 \\
\end{array}$ & $\begin{array}{l}73.5 \\
73 \\
73 \\
73 \\
73 \\
73 \\
69 \\
65.5 \\
62 \\
56 \\
51.5 \\
50 \\
49.7 \\
49.7 \\
49.7 \\
\end{array}$ & $\begin{array}{l}72.5 \\
72.5 \\
72.5 \\
72.5 \\
72.2 \\
72.2 \\
69.5 \\
65.5 \\
62.5 \\
56.5 \\
52.5 \\
50.5 \\
50.2 \\
50 \\
49.7 \\
\end{array}$ & $\begin{array}{l}72 \\
72 \\
72 \\
72 \\
72 \\
72 \\
71 \\
66.5 \\
62.5 \\
55.5 \\
51.2 \\
50 \\
49.5 \\
49.2 \\
49.2 \\
\end{array}$ & $\begin{array}{l}68.5 \\
68.5 \\
68.5 \\
68.5 \\
68.5 \\
68.5 \\
68 \\
66 \\
63 \\
55.5 \\
51 \\
50 \\
49 \\
49 \\
49 \\
\end{array}$ & $\begin{array}{l}69.5 \\
69.5 \\
69.5 \\
69.5 \\
69.5 \\
69.5 \\
68.5 \\
67 \\
64 \\
57 \\
52 \\
50 \\
50 \\
49.5 \\
49.5 \\
\end{array}$ & $\begin{array}{l}70 \\
70 \\
70 \\
70 \\
70 \\
70 \\
70 \\
69 \\
66.5 \\
61 \\
54.5 \\
53 \\
52 \\
52 \\
52 \\
\end{array}$ \\
\hline
\end{tabular}

2/ Deep sea thermometer gave surface $76^{\circ} \mathrm{F}$ and bottom $52^{\circ} \mathrm{F}$

3/ Deep sea thermometer gave surface $78.5^{\circ} \mathrm{F}$ and bottom $52^{\circ} \mathrm{F}$. 
Temperature profiles $\left({ }^{\circ} \mathrm{F}\right)$, taken in the Deep Hole, Maxinkuckee Lake--Continued

Temperature readings in 1899

\begin{tabular}{|c|c|c|c|c|c|c|c|}
\hline \multirow{2}{*}{$\begin{array}{l}\text { Depth } \\
\text { (feet) }\end{array}$} & \multicolumn{7}{|c|}{ September--Continued } \\
\hline & 15 & 16 & 17 & 19 & 20 & 21 & 22 \\
\hline Alr & 56 & 68 & 71 & 61 & 60.2 & 51.5 & 62 \\
\hline $\begin{array}{c}\text { Water-surface } \\
5 \\
10 \\
15 \\
20 \\
25 \\
30 \\
32.5 \\
35 \\
40 \\
50 \\
60 \\
70 \\
80 \\
85 \\
\end{array}$ & $\begin{array}{l}70 \\
70 \\
70 \\
70 \\
70 \\
70 \\
70 \\
68.5 \\
67 \\
60.5 \\
53.7 \\
52.7 \\
52 \\
52 \\
51.7 \\
\end{array}$ & $\begin{array}{l}68.6 \\
68.6 \\
68.6 \\
68.6 \\
68.5 \\
68.5 \\
68 \\
67 \\
66 \\
59.5 \\
53 \\
52 \\
51.4 \\
51.3 \\
51 \\
\end{array}$ & $\begin{array}{l}69 \\
69 \\
69 \\
69 \\
68.9 \\
68.5 \\
68 \\
67 \\
66 \\
58.5 \\
53 \\
51.5 \\
51.4 \\
51.3 \\
51.3 \\
\end{array}$ & $\begin{array}{l}69 \\
69 \\
69 \\
69 \\
69 \\
68.6 \\
68.6 \\
67.7 \\
66.7 \\
62.5 \\
53.5 \\
52.3 \\
52 \\
52 \\
52 \\
\end{array}$ & $\begin{array}{l}67.3 \\
67 \\
67 \\
67 \\
67 \\
67 \\
67 \\
67 \\
66.5 \\
63 \\
54.2 \\
52.1 \\
51.5 \\
51.5 \\
51.5 \\
\end{array}$ & $\begin{array}{l}66 \\
66 \\
66 \\
66 \\
66 \\
66 \\
66 \\
66 \\
66 \\
63.5 \\
53.5 \\
52.3 \\
51.8 \\
51.7 \\
51.5 \\
\end{array}$ & $\begin{array}{l}66 \\
66 \\
65.8 \\
65.8 \\
65.8 \\
65.8 \\
65 \\
64.8 \\
64.8 \\
63.5 \\
54 \\
52 \\
51.9 \\
51.8 \\
51.5 \\
\end{array}$ \\
\hline \multirow{2}{*}{$\begin{array}{l}\text { Depth } \\
\text { (feet) }\end{array}$} & \multicolumn{7}{|c|}{ September--Continued } \\
\hline & 23 & 24 & 25 & 26 & 27 & 28 & 30 \\
\hline Alr & 58.5 & 61.4 & 56 & 51.5 & 48 & 55 & 53 \\
\hline $\begin{array}{l}\text { Water-surface } \\
5 \\
10 \\
15 \\
20 \\
25 \\
30 \\
32.5 \\
35 \\
40 \\
50 \\
60 \\
70 \\
80 \\
85\end{array}$ & 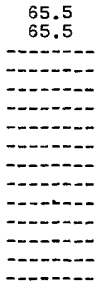 & י & $\begin{array}{l}67 \\
67 \\
66.5 \\
66.5 \\
66.5 \\
66.5 \\
67 \\
66 \\
66 \\
65.5 \\
56 \\
54 \\
52.5 \\
52 \\
53\end{array}$ & $\begin{array}{l}65.5 \\
66.5 \\
66 \\
65 \\
64.5 \\
64.5 \\
63.5 \\
63.5 \\
63.5 \\
63.5 \\
55 \\
53 \\
51.5 \\
50.5 \\
50.5\end{array}$ & $\begin{array}{l}62.5 \\
64.5 \\
64.5 \\
64.5 \\
64 \\
58 \\
58 \\
58 \\
55 \\
55 \\
52 \\
45.5 \\
45.5 \\
45 \\
42.5\end{array}$ & $\begin{array}{l}57 \\
57 \\
57 \\
57 \\
57 \\
57 \\
\\
\\
\\
\\
\end{array}$ & $\begin{array}{l}59 \\
58 \\
54 \\
54 \\
54 \\
54 \\
51 \\
51 \\
52 \\
52 \\
52 \\
51 \\
\end{array}$ \\
\hline
\end{tabular}

Monthly summary in 1899

\begin{tabular}{|c|c|c|c|c|c|c|c|c|c|}
\hline \multirow{2}{*}{$\begin{array}{l}\text { Depth } \\
\text { (feet) }\end{array}$} & \multicolumn{3}{|c|}{ July } & \multicolumn{3}{|c|}{ August } & \multicolumn{3}{|c|}{ September } \\
\hline & Maximum & Minimum & Mean & Maximum & Minimum & Mean & Maximum & Minimum & Mean \\
\hline $\begin{array}{c}\text { ater-surface } \\
5 \\
10 \\
15 \\
20 \\
25 \\
30 \\
32.5 \\
35 \\
40 \\
50 \\
60 \\
70 \\
80 \\
85\end{array}$ & $\begin{array}{l}80.5 \\
80.8 \\
81.0 \\
80.2 \\
78.0 \\
74.0 \\
71.0 \\
-1.0 \\
69.5 \\
63.0 \\
57.5 \\
52.5 \\
51.8 \\
51.7 \\
51.3\end{array}$ & \begin{tabular}{l}
77.0 \\
77.0 \\
77.0 \\
76.5 \\
74.7 \\
72.0 \\
69.0 \\
\hdashline 63.0 \\
57.3 \\
52.5 \\
50.5 \\
50.5 \\
50.3 \\
50.5
\end{tabular} & $\begin{array}{l}79.2 \\
79.3 \\
79.2 \\
78.3 \\
75.7 \\
72.8 \\
69.7 \\
-64.7 \\
58.7 \\
53.2 \\
51.7 \\
51.2 \\
50.9 \\
50.8\end{array}$ & $\begin{array}{l}79.5 \\
79.5 \\
79.5 \\
79.2 \\
78.2 \\
76.2 \\
72.0 \\
66.7 \\
66.0 \\
60.5 \\
55.5 \\
53.0 \\
52.2 \\
52.1 \\
52.2\end{array}$ & $\begin{array}{l}74.0 \\
74.2 \\
74.3 \\
74.0 \\
73.0 \\
71.0 \\
67.3 \\
63.2 \\
60.3 \\
55.8 \\
50.7 \\
49.5 \\
49.0 \\
49.0 \\
48.7\end{array}$ & $\begin{array}{l}76.6 \\
76.8 \\
76.7 \\
76.3 \\
75.6 \\
73.8 \\
70.0 \\
65.4 \\
62.9 \\
58.1 \\
53.3 \\
51.5 \\
51.0 \\
50.8 \\
50.6\end{array}$ & $\begin{array}{l}77.0 \\
77.0 \\
76.5 \\
75.5 \\
74.5 \\
73.5 \\
71.0 \\
69.0 \\
67.0 \\
63.5 \\
54.5 \\
53.0 \\
52.0 \\
52.0 \\
52.0\end{array}$ & $\begin{array}{l}66.0 \\
66.0 \\
66.0 \\
66.0 \\
66.0 \\
66.0 \\
64.8 \\
63.0 \\
58.7 \\
54.8 \\
50.0 \\
48.8 \\
48.5 \\
49.5 \\
48.4\end{array}$ & $\begin{array}{l}72.1 \\
72.0 \\
71.9 \\
71.3 \\
71.0 \\
70.5 \\
68.2 \\
65.7 \\
63.1 \\
57.9 \\
52.1 \\
50.8 \\
50.3 \\
50.2 \\
50.1\end{array}$ \\
\hline
\end{tabular}


Temperature profiles ( $\left.{ }^{\circ} \mathrm{F}\right)$ taken in Deep Hole, Maxinkuckee Lake--Continued

Temperature readings in 1900

\begin{tabular}{|c|c|c|c|c|c|c|c|c|}
\hline \multirow{2}{*}{$\begin{array}{l}\text { Depth } \\
\text { (feet) }\end{array}$} & \multicolumn{8}{|c|}{ July } \\
\hline & 17 & 18 & 19 & 20 & 21 & 22 & 23 & 24 \\
\hline Alr & 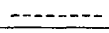 & 69.5 & 72 & 76.3 & 65 & 77 & 79.5 & 81.2 \\
\hline $\begin{array}{l}\text { Water-surface } \\
5 \\
10 \\
15 \\
20 \\
25 \\
30 \\
32.5 \\
35 \\
40 \\
45 \\
50 \\
55 \\
60 \\
65 \\
70 \\
75 \\
80 \\
85 \\
\end{array}$ & $\begin{array}{c}77.1 \\
77.1 \\
77.1 \\
72 \\
62 \\
57.3 \\
52.3 \\
50.5 \\
50 \\
50 \\
49.5 \\
\end{array}$ & $\begin{array}{l}76.3 \\
76.3 \\
76.3 \\
76.3 \\
76.3 \\
75.8 \\
74 \\
67 \\
63 \\
58.5 \\
55.5 \\
52.8 \\
51.8 \\
51 \\
50.9 \\
50 \\
49.9 \\
49.9 \\
49 \\
\end{array}$ & $\begin{array}{l}77 \\
77 \\
77 \\
77.1 \\
77 \\
76 \\
74 \\
69 \\
64.5 \\
57.7 \\
55 \\
53.8 \\
51.5 \\
50.9 \\
50.8 \\
50.3 \\
49.9 \\
49.7 \\
49.2 \\
\end{array}$ & $\begin{array}{l}78 \\
78 \\
78.5 \\
77.9 \\
77.1 \\
76 \\
73.2 \\
70 \\
63.5 \\
57.4 \\
55.5 \\
54 \\
52 \\
51 \\
50 \\
50 \\
49.9 \\
49.9 \\
49.7 \\
\end{array}$ & $\begin{array}{l}76.6 \\
77 \\
77 \\
77 \\
77 \\
75 \\
72 \\
70 \\
66.5 \\
58 \\
55.8 \\
53 \\
51.6 \\
50.7 \\
50.9 \\
50 \\
50 \\
49.9 \\
49.5 \\
\end{array}$ & $\begin{array}{l}77.5 \\
77.5 \\
78 \\
77.2 \\
76.9 \\
75.5 \\
73.1 \\
69 \\
65.5 \\
58.8 \\
55 \\
53.3 \\
52.3 \\
51.1 \\
51 \\
50 \\
50 \\
50 \\
49.2 \\
\end{array}$ & $\begin{array}{l}79 \\
79.5 \\
78.1 \\
77 \\
76.8 \\
76 \\
72 \\
68.6 \\
66 \\
58.3 \\
55 \\
53 \\
52 \\
50.9 \\
50.1 \\
50.2 \\
50.2 \\
49.8 \\
49.1 \\
\end{array}$ & $\begin{array}{l}80.3 \\
80.5 \\
80.1 \\
77.1 \\
76.6 \\
75.9 \\
73.6 \\
71.3 \\
68.9 \\
58 \\
55 \\
53 \\
52 \\
51.1 \\
50.5 \\
50 \\
50 \\
50.2 \\
49.3 \\
\end{array}$ \\
\hline \multirow{2}{*}{$\begin{array}{l}\text { Depth } \\
\text { (feet) }\end{array}$} & \multicolumn{8}{|c|}{ July--Cont Inued } \\
\hline & 25 & 26 & 27 & \multicolumn{2}{|c|}{28} & & 30 & 31 \\
\hline A I r & 78 & 68 & 69.5 & \multicolumn{2}{|l|}{69} & & 74 & 79 \\
\hline $\begin{array}{c}\text { Water-surface } \\
5 \\
10 \\
15 \\
20 \\
25 \\
30 \\
32.5 \\
35 \\
40 \\
45 \\
50 \\
55 \\
60 \\
65 \\
70 \\
75 \\
80 \\
85 \\
\end{array}$ & $\begin{array}{l}78.1 \\
78.1 \\
78.1 \\
78 \\
76.3 \\
76 \\
72.8 \\
67 \\
62.2 \\
56.6 \\
54 \\
52.1 \\
51 \\
50.5 \\
50.7 \\
50 \\
50 \\
50 \\
49.8 \\
\end{array}$ & $\begin{array}{l}77.2 \\
77.5 \\
78 \\
77.8 \\
77.5 \\
76 \\
71.5 \\
68 \\
61.5 \\
57 \\
54.6 \\
52.3 \\
51 \\
50.5 \\
50.5 \\
50.5 \\
50 \\
50 \\
50 \\
\end{array}$ & $\begin{array}{l}77 \\
77 \\
77 \\
77 \\
77 \\
76.2 \\
71 \\
68.5 \\
63 \\
57 \\
54.5 \\
52.5 \\
51 \\
50.6 \\
50.6 \\
50.4 \\
50.2 \\
50 \\
49.4 \\
\end{array}$ & \multicolumn{2}{|c|}{$\begin{array}{l}77 \\
77 \\
77 \\
77 \\
76.8 \\
75.2 \\
72.1 \\
68 \\
64 \\
56.8 \\
53.9 \\
52 \\
51 \\
50.5 \\
50.5 \\
50.4 \\
50.1 \\
50 \\
49.3 \\
\end{array}$} & & $\begin{array}{l}78 \\
78 \\
78 \\
78 \\
76.4 \\
76 \\
71 \\
67.3 \\
65 \\
57 \\
54.3 \\
52.6 \\
51 \\
50.5 \\
50 \\
50 \\
50 \\
50 \\
49.2 \\
\end{array}$ & $\begin{array}{l}78.7 \\
78.8 \\
78.5 \\
78.3 \\
77 \\
75.6 \\
72.2 \\
68.8 \\
63.2 \\
57.1 \\
54.6 \\
52.8 \\
51 \\
50.5 \\
50.3 \\
50 \\
50 \\
49.8 \\
49.5 \\
\end{array}$ \\
\hline \multirow{2}{*}{$\begin{array}{l}\text { Depth } \\
\text { (feet) }\end{array}$} & \multicolumn{8}{|c|}{ August } \\
\hline & 1 & 3 & 4 & 6 & 8 & 9 & 11 & 13 \\
\hline Alr & 73 & 73 & 73 & 83.5 & 84 & 82 & 85 & 77 \\
\hline $\begin{array}{l}\text { Water-surface } \\
5 \\
10 \\
15 \\
20 \\
25 \\
30 \\
32.5 \\
35 \\
40 \\
45 \\
50 \\
55 \\
60 \\
65 \\
70 \\
75 \\
80 \\
85\end{array}$ & $\begin{array}{l}78 \\
78.1 \\
78 \\
78 \\
76.5 \\
75.6 \\
73.2 \\
67.6 \\
64.1 \\
58 \\
54.6 \\
52.6 \\
51 \\
51 \\
50.1 \\
50.2 \\
50 \\
49.9 \\
49.5\end{array}$ & $\begin{array}{l}78 \\
78 \\
78 \\
78 \\
77 \\
76.4 \\
73 \\
68.6 \\
63.2 \\
57.2 \\
55.5 \\
52 \\
51.2 \\
51 \\
50.8 \\
50.5 \\
50.2 \\
50 \\
49.5 \\
\end{array}$ & $\begin{array}{l}78.1 \\
78.1 \\
78.1 \\
78 \\
77.6 \\
76 \\
72.5 \\
68.5 \\
64 \\
57.9 \\
55 \\
53 \\
51 \\
51 \\
50.1 \\
50.1 \\
50 \\
50 \\
49.8\end{array}$ & $\begin{array}{l}81.8 \\
81.8 \\
79.8 \\
78.2 \\
77.5 \\
76 \\
72 \\
68 \\
63.9 \\
57 \\
54.5 \\
52.5 \\
51.3 \\
50.8 \\
50.3 \\
50.1 \\
50 \\
50 \\
49.8 \\
\end{array}$ & $\begin{array}{l}82.4 \\
82.4 \\
82.2 \\
79.2 \\
77.5 \\
76 \\
70.5 \\
68.5 \\
62.5 \\
58 \\
54.5 \\
52.1 \\
51.1 \\
50.6 \\
50.5 \\
50 \\
50 \\
50 \\
49.8\end{array}$ & $\begin{array}{l}82.8 \\
82.8 \\
82.8 \\
79.5 \\
77.5 \\
76.2 \\
70.1 \\
69.2 \\
64.2 \\
58 \\
54.5 \\
52.2 \\
51.2 \\
50.7 \\
50.4 \\
50 \\
50 \\
50 \\
49.9\end{array}$ & $\begin{array}{l}83 \\
83 \\
82.5 \\
82 \\
77.6 \\
75.7 \\
70.5 \\
69.5 \\
63.5 \\
58 \\
55 \\
52.8 \\
51.2 \\
50.6 \\
50.5 \\
50.5 \\
50 \\
50 \\
49.9\end{array}$ & $\begin{array}{l}81 \\
81 \\
81 \\
80.8 \\
80.3 \\
75.7 \\
72.3 \\
68 \\
64 \\
59 \\
55 \\
52.6 \\
51.5 \\
51 \\
50.8 \\
50.3 \\
50.3 \\
50 \\
49.8\end{array}$ \\
\hline
\end{tabular}


Temperature profiles $\left({ }^{\circ} \mathrm{F}\right)$ taken in Deep Hole, Maxinkuckee Lake--Continued

Temperature readings in 1900

\begin{tabular}{|c|c|c|c|c|c|c|c|c|}
\hline \multirow{2}{*}{$\begin{array}{l}\text { Depth } \\
\text { (feet) }\end{array}$} & \multicolumn{8}{|c|}{ August--Continued } \\
\hline & 15 & 17. & 18 & 20 & 22 & 24 & 25 & 30 \\
\hline A1r & 73.2 & 78.5 & 76.2 & 80 & 80 & 76.5 & 85 & 79.8 \\
\hline $\begin{array}{c}\text { Water-surface } \\
5 \\
10 \\
15 \\
20 \\
25 \\
30 \\
32.5 \\
35 \\
40 \\
45 \\
50 \\
55 \\
60 \\
65 \\
70 \\
75 \\
80 \\
85 \\
\end{array}$ & 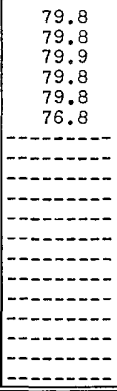 & $\begin{array}{l}78.9 \\
78.5 \\
78.5 \\
78.5 \\
78.8 \\
77 \\
72.1 \\
67.1 \\
64.6 \\
58.3 \\
55.5 \\
53 \\
52 \\
51.5 \\
51 \\
51 \\
50.5 \\
50.3 \\
49.8 \\
\end{array}$ & $\begin{array}{l}78.2 \\
78.2 \\
78.2 \\
78.2 \\
78.2 \\
76.6 \\
72.1 \\
68 \\
63.3 \\
60 \\
56 \\
54.2 \\
51.5 \\
51 \\
50.7 \\
50.6 \\
50.3 \\
50 \\
49.9 \\
\end{array}$ & $\begin{array}{l}80 \\
79.7 \\
79.5 \\
79.8 \\
79.1 \\
77.2 \\
71 \\
68.3 \\
65.7 \\
59.8 \\
56 \\
53.5 \\
52.5 \\
52 \\
51.5 \\
51.2 \\
51 \\
51 \\
50.1 \\
\end{array}$ & $\begin{array}{l}80.8 \\
80.8 \\
80.5 \\
80.3 \\
79.2 \\
76.9 \\
71.5 \\
-9.5 \\
64 \\
59.9 \\
56 \\
54 \\
52.8 \\
52.3 \\
51.8 \\
51.7 \\
51.3 \\
50.8 \\
50.2 \\
\end{array}$ & $\begin{array}{l}80.2 \\
80 \\
80 \\
80 \\
80 \\
77.5 \\
73 \\
68 \\
64 \\
60.3 \\
57 \\
54.2 \\
52.8 \\
52 \\
51.5 \\
51.2 \\
51.3 \\
51 \\
51 \\
\end{array}$ & $\begin{array}{l}80.1 \\
80.1 \\
80.1 \\
80 \\
80 \\
79.5 \\
71.5 \\
68.5 \\
66.9 \\
61 \\
57 \\
54.1 \\
52.5 \\
52.5 \\
52 \\
51.8 \\
52 \\
51.6 \\
51 \\
\end{array}$ & $\begin{array}{l}80 \\
80 \\
80 \\
79.5 \\
78.8 \\
78 \\
73 \\
70 \\
66 \\
59.8 \\
56 \\
54.3 \\
53 \\
52.2 \\
51 \\
51.8 \\
51.6 \\
51 \\
51 \\
\end{array}$ \\
\hline
\end{tabular}

\begin{tabular}{|c|c|c|c|c|c|c|c|c|c|}
\hline \multirow{2}{*}{$\begin{array}{l}\text { Depth } \\
\text { (feet) }\end{array}$} & \multicolumn{9}{|c|}{ September } \\
\hline & 1 & 3 & 5 & 12 & 14 & 22 & 24 & 26 & 28 \\
\hline A1r & 82.5 & 77.5 & 86.5 & 68 & 79 & 68 & 63 & 76 & 70.5 \\
\hline $\begin{array}{c}\text { Water-surface } \\
5 \\
10 \\
15 \\
20 \\
25 \\
30 \\
32.5 \\
35 \\
40 \\
45 \\
50 \\
52.5 \\
55 \\
60 \\
65 \\
70 \\
75 \\
80 \\
85 \\
89\end{array}$ & $\begin{array}{l}80.1 \\
80 \\
79.9 \\
79.7 \\
79.4 \\
78 \\
73 \\
69 \\
65.2 \\
60.3 \\
57 \\
54.8 \\
53 \\
52 \\
51.5 \\
51.5 \\
51.8 \\
51.6 \\
51.5 \\
50.8\end{array}$ & $\begin{array}{l}79.8 \\
79.8 \\
79.2 \\
78.5 \\
78.2 \\
78.2 \\
75.4 \\
71 \\
65.2 \\
60 \\
57 \\
54.3 \\
53.2 \\
52.5 \\
52 \\
52 \\
51.7 \\
51.7 \\
51.5 \\
50.9\end{array}$ & $\begin{array}{l}79 \\
79.1 \\
79 \\
79 \\
79 \\
79 \\
75.6 \\
70.5 \\
65 \\
60.1 \\
57 \\
54.5 \\
-53.5 \\
52.5 \\
52 \\
51.5 \\
51.3 \\
51.1 \\
51 \\
50.8\end{array}$ & $\begin{array}{c}76 \\
76 \\
76 \\
75.8 \\
75.8 \\
75.6 \\
74.5 \\
73.9 \\
73.5 \\
64.5 \\
54.6 \\
52.9 \\
51.9 \\
51.4 \\
51.1\end{array}$ & $\begin{array}{c}75 \\
75 \\
75 \\
75 \\
75 \\
74.9 \\
74.5 \\
74 \\
73 \\
64.2 \\
-54.5 \\
-95 \\
52.4 \\
52 \\
51.6 \\
51.5 \\
51.9\end{array}$ & \begin{tabular}{l}
67.4 \\
67.4 \\
67.4 \\
67.2 \\
67.2 \\
67 \\
67 \\
67 \\
66.9 \\
66.5 \\
55.1 \\
\hdashline 53.6 \\
52.9 \\
52 \\
52 \\
52 \\
51.5 \\
51.5 \\
Bottom
\end{tabular} & $\begin{array}{c}67.2 \\
67.2 \\
67.2 \\
67.2 \\
67.2 \\
67 \\
66.8 \\
66.8 \\
66.8 \\
66.6 \\
56.5 \\
53.2 \\
52.7 \\
51.9 \\
51.7 \\
51.7 \\
51.3\end{array}$ & 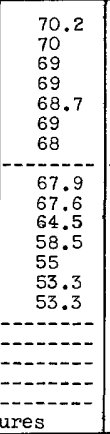 & $\begin{array}{l}68.5 \\
68.5 \\
68.2 \\
68.1 \\
68 \\
67.8 \\
67.8 \\
-0.0 \\
67 \\
67 \\
66 \\
57.9 \\
54.1 \\
53.5 \\
52.5 \\
52.4 \\
52 \\
51.6 \\
51.4 \\
51.4\end{array}$ \\
\hline
\end{tabular}

\section{October}

\begin{tabular}{c|c|c|c|c|c|c|c}
\hline $\begin{array}{c}\text { Depth } \\
\text { (feet) }\end{array}$ & 1 & 3 & 5 & 8 & 10 & 12 & 15 \\
\hline A1r & 72.5 & 72 & 75.9 & 54.1 & 54.1 & 59.5 & 63. \\
\hline Water-surface & 73 & 67.5 & 68.8 & 67.6 & 66 & 65.2 & 64.9 \\
10 & 67.9 & 67.5 & 69.5 & 68 & 66 & 65.3 & 64.9 \\
15 & 67.8 & 67.5 & 69.1 & 67.9 & 66 & 65.3 & 64.8 \\
20 & 67.5 & 67.5 & 67.8 & 67.9 & 66 & 65.3 & 64.8 \\
25 & 67.5 & 67.3 & 67.2 & 67.9 & 66 & 65.3 & 64.8 \\
30 & 67.4 & 67.1 & 66.9 & 67.8 & 66 & 65.3 & 64.6 \\
35 & 67 & 66.9 & 66.9 & 67.6 & 66 & 65.2 & 64.5 \\
40 & 67 & 66.7 & 66.8 & 67 & 66 & 65.2 & 64.5 \\
45 & 66.5 & 66.5 & 66.4 & 66.7 & 66 & 65 & 64.3 \\
50 & 66 & 66.1 & 65.5 & 66.6 & 66 & 65 & 64 \\
52.5 & 59 & 57.6 & 58 & 60.6 & 61.7 & 52 & 62.7 \\
55 & 55 & 55 & 55.3 & 57.1 & 57.5 & 56.9 & 58 \\
60 & 54.5 & 53.5 & 54 & 54.9 & 54.3 & 54.4 & 54.1 \\
65 & 52.5 & 53 & 52.3 & 52.9 & 52.9 & 52.9 & 53 \\
70 & 52.5 & 52 & 52 & 52.2 & 52.1 & 52.1 & 52.2 \\
75 & 52 & 52 & 51.9 & 52 & 52 & 52 \\
80 & 51.5 & 51.6 & 52.2 & 52 & 51.9 & 51.9 & 51.9 \\
85 & 51.5 & 51.6 & 51.3 & 51.6 & 51.9 & 51.9 & 51.9 \\
\hline
\end{tabular}


Temperature proflles ( ${ }^{\circ}$ F) taken in Deep Hole, Maxinkuckee Lake--Continued

Temperature readings in 1900

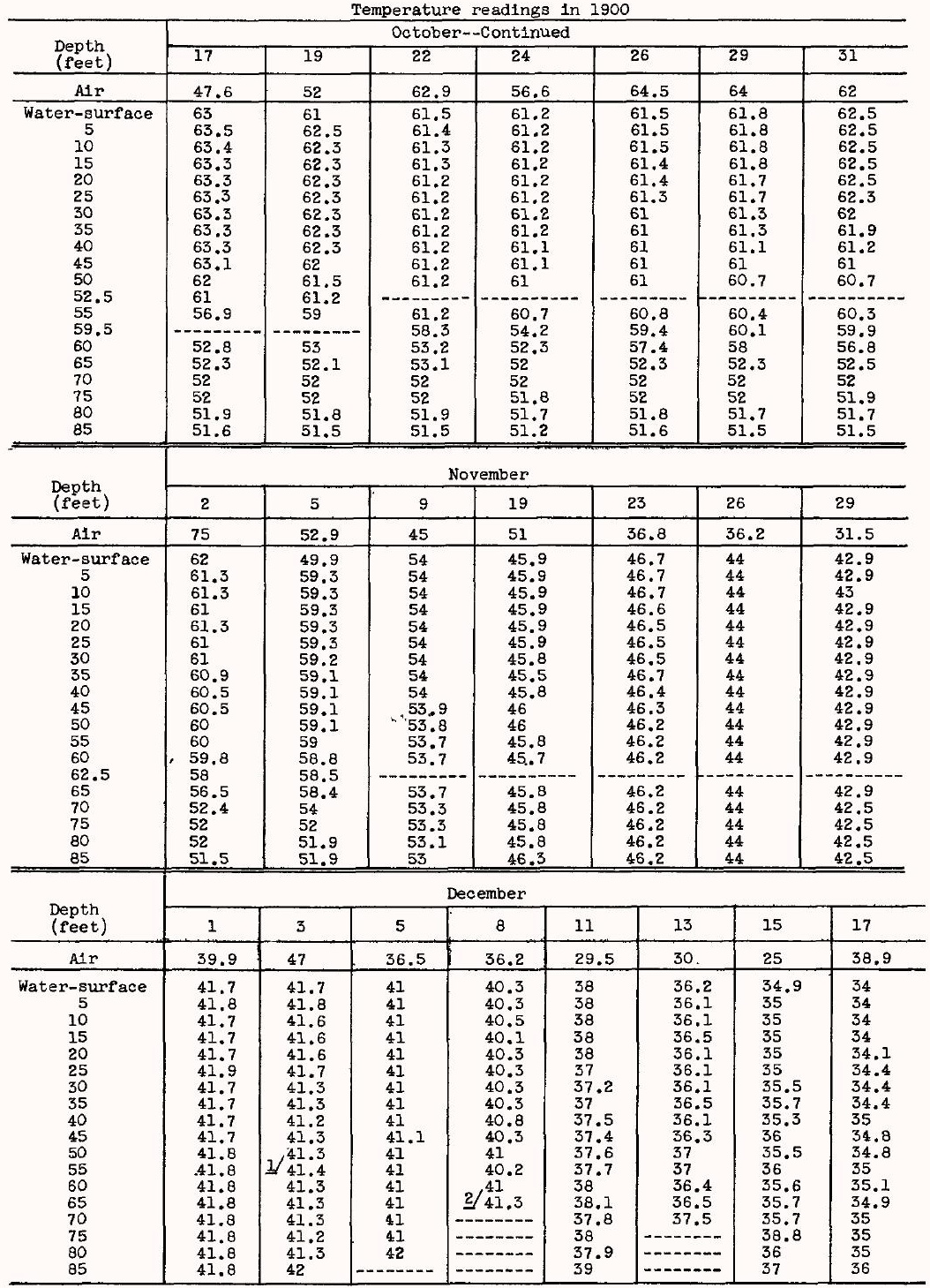

If Certain slight anomalies in the temperature readings occur in our records, but we bell the records as here given cannot be far from correct.

2/ Bottom at 62 feet. 
Temperature prof $11 \mathrm{le}\left({ }^{\circ} \mathrm{F}\right)$ at Deep Hole, Lake Maxinkuckee--ContInued

Monthly summary for 1900

\begin{tabular}{|c|c|c|c|c|c|c|c|c|c|}
\hline \multirow{2}{*}{$\begin{array}{l}\text { Depth } \\
\text { (feet) }\end{array}$} & \multicolumn{3}{|c|}{ July } & \multicolumn{3}{|c|}{ August } & \multicolumn{3}{|c|}{ September } \\
\hline & Maximum & Minimum & Mean & Maximum & Minimum & Mean & Maximum & Mintmum & Mean \\
\hline A1r & 81.2 & 65.0 & 73.9 & 85.0 & 73.0 & 78.3 & 86.5 & 70.5 & 74.6 \\
\hline $\begin{array}{c}\text { Water-surface } \\
5 \\
10 \\
15 \\
20 \\
25 \\
30 \\
32.5 \\
35 \\
40 \\
45 \\
50 \\
55 \\
60 \\
65 \\
70 \\
75 \\
80 \\
85 \\
\end{array}$ & $\begin{array}{l}80.3 \\
80.5 \\
80.1 \\
78.3 \\
77.5 \\
76.2 \\
74.0 \\
71.3 \\
68.9 \\
58.8 \\
55.8 \\
54.1 \\
52.3 \\
51.1 \\
51.0 \\
50.5 \\
50.2 \\
50.2 \\
50.0 \\
\end{array}$ & $\begin{array}{l}76.3 \\
76.3 \\
76.3 \\
76.3 \\
76.1 \\
75.0 \\
71.0 \\
67.0 \\
61.5 \\
56.6 \\
53.5 \\
.52 .0 \\
51.0 \\
50.5 \\
50.0 \\
50.0 \\
49.9 \\
49.7 \\
49.0 \\
\end{array}$ & $\begin{array}{l}77.7 \\
77.9 \\
77.8 \\
77.3 \\
76.8 \\
75.8 \\
72.5 \\
68.6 \\
64.1 \\
57.5 \\
54.7 \\
52.9 \\
51.4 \\
50.7 \\
50.5 \\
50.1 \\
50.0 \\
49.9 \\
49.5 \\
\end{array}$ & $\begin{array}{l}83.0 \\
83.0 \\
82.8 \\
82.0 \\
80.3 \\
79.5 \\
73.2 \\
70.0 \\
66.9 \\
61.0 \\
57.0 \\
54.3 \\
53.0 \\
52.5 \\
52.0 \\
51.8 \\
52.0 \\
51.6 \\
51.0 \\
\end{array}$ & $\begin{array}{l}78.0 \\
78.0 \\
78.0 \\
78.0 \\
76.5 \\
75.4 \\
70.1 \\
67.1 \\
62.5 \\
57.0 \\
54.5 \\
52.0 \\
51.0 \\
50.6 \\
50.1 \\
50.0 \\
50.0 \\
49.9 \\
49.5 \\
\end{array}$ & $\begin{array}{l}80.2 \\
80.1 \\
79.9 \\
79.3 \\
78.5 \\
76.7 \\
71.9 \\
68.4 \\
64.3 \\
58.8 \\
55.5 \\
53.1 \\
51.8 \\
51.3 \\
50.9 \\
50.7 \\
50.6 \\
50.4 \\
50.1 \\
\end{array}$ & $\begin{array}{l}80.1 \\
80.0 \\
79.9 \\
79.7 \\
79.4 \\
79.0 \\
75.6 \\
74.0 \\
73.5 \\
67.7 \\
66.0 \\
58.5 \\
53.6 \\
52.9 \\
52.4 \\
52.0 \\
52.0 \\
51.7 \\
51.9 \\
\end{array}$ & $\begin{array}{l}67.2 \\
67.2 \\
67.2 \\
67.2 \\
67.2 \\
67.0 \\
66.8 \\
66.8 \\
65.2 \\
60.0 \\
57.0 \\
54.3 \\
53.0 \\
52.0 \\
51.5 \\
51.5 \\
51.3 \\
51.1 \\
51.0 \\
\end{array}$ & $\begin{array}{l}73.7 \\
73.7 \\
73.4 \\
73.3 \\
73.2 \\
72.9 \\
71.4 \\
70.3 \\
67.8 \\
64.1 \\
61.9 \\
55.6 \\
53.3 \\
52.6 \\
52.0 \\
51.8 \\
51.7 \\
51.5 \\
51.4\end{array}$ \\
\hline \multirow{2}{*}{$\begin{array}{l}\text { Depth } \\
\text { (feet) }\end{array}$} & \multicolumn{3}{|c|}{ October } & \multicolumn{3}{|c|}{ November } & \multicolumn{3}{|c|}{ December } \\
\hline & Maximum & Minimum & Mean & Max 1 mum & Minimum & Mean & Maximum & Minimum & Mean \\
\hline Alr & 75.9 & 47.6 & 61.5 & 75.0 & 31.5 & 45.9 & 39.9 & 25.0 & 36.1 \\
\hline $\begin{array}{c}\text { Water-surface } \\
5 \\
10 \\
15 \\
20 \\
25 \\
30 \\
35 \\
40 \\
45 \\
50 \\
55 \\
60 \\
65 \\
70 \\
75 \\
80 \\
85\end{array}$ & $\begin{array}{l}73.0 \\
69.5 \\
69.1 \\
67.9 \\
67.9 \\
67.8 \\
67.6 \\
67.0 \\
66.7 \\
66.0 \\
62.7 \\
61.2 \\
58.0 \\
52.5 \\
52.0 \\
52.2 \\
51.9 \\
51.9\end{array}$ & $\begin{array}{l}61.2 \\
61.2 \\
61.2 \\
61.2 \\
61.2 \\
61.2 \\
61.0 \\
61.0 \\
61.0 \\
61.0 \\
58.0 \\
53.5 \\
52.3 \\
52.0 \\
51.9 \\
51.5 \\
51.3 \\
51.1\end{array}$ & $\begin{array}{l}64.7 \\
64.5 \\
64.4 \\
64.3 \\
64.2 \\
64.2 \\
64.0 \\
64.0 \\
63.8 \\
63.5 \\
60.7 \\
57.1 \\
53.8 \\
52.2 \\
52.0 \\
51.9 \\
51.7 \\
51.5\end{array}$ & $\begin{array}{l}62.0 \\
61.3 \\
61.3 \\
61.0 \\
61.3 \\
61.0 \\
61.0 \\
60.9 \\
60.5 \\
60.5 \\
60.0 \\
60.0 \\
59.8 \\
58.4 \\
54.0 \\
53.3 \\
53.1 \\
53.0\end{array}$ & $\begin{array}{l}42.9 \\
42.9 \\
43.0 \\
43.9 \\
42.9 \\
42.9 \\
42.9 \\
42.9 \\
42.9 \\
42.9 \\
42.9 \\
42.9 \\
42.9 \\
42.9 \\
42.5 \\
42.5 \\
42.5 \\
42.5\end{array}$ & $\begin{array}{l}49.3 \\
50.6 \\
50.6 \\
50.5 \\
50.6 \\
50.5 \\
50.5 \\
50.4 \\
50.4 \\
50.4 \\
50.3 \\
50.2 \\
50.2 \\
49.6 \\
48.3 \\
48.0 \\
47.9 \\
47.9\end{array}$ & $\begin{array}{l}41.7 \\
41.8 \\
41.7 \\
41.7 \\
41.7 \\
42.0 \\
41.7 \\
41.7 \\
41.7 \\
41.7 \\
41.8 \\
41.8 \\
41.8 \\
41.8 \\
41.8 \\
41.8 \\
41.8 \\
41.8\end{array}$ & $\begin{array}{l}34.0 \\
34.0 \\
34.0 \\
34.0 \\
34.1 \\
34.4 \\
34.4 \\
34.4 \\
35.0 \\
34.0 \\
34.0 \\
35.0 \\
35.1 \\
34.9 \\
35.0 \\
35.0 \\
35.0 \\
36.0\end{array}$ & $\begin{array}{l}38.5 \\
38.5 \\
38.5 \\
38.5 \\
38.4 \\
38.5 \\
38.4 \\
38.5 \\
38.6 \\
38.6 \\
38.7 \\
38.8 \\
38.8 \\
38.5 \\
38.6 \\
39.3 \\
39.0 \\
39.2\end{array}$ \\
\hline
\end{tabular}

Temperature readings in 1901

\begin{tabular}{|c|c|c|c|c|c|c|c|}
\hline \multirow{2}{*}{$\begin{array}{l}\text { Depth } \\
\text { (fest) }\end{array}$} & \multicolumn{7}{|c|}{ January } \\
\hline & 1 & 4 & 7 & 9 & 18 & 26 & 30 \\
\hline Air & 23.1 & 41 & 32.1 & 32 & 16.5 & 23.9 & 24.9 \\
\hline $\begin{array}{c}\text { Water-surface } \\
5 \\
10 \\
15 \\
20 \\
25 \\
30 \\
35 \\
40 \\
45 \\
50 \\
55 \\
60 \\
65 \\
70 \\
75 \\
80 \\
85 \\
\end{array}$ & $\begin{array}{l}34 \\
34.1 \\
34 \\
34.2 \\
34.2 \\
34.6 \\
34.5 \\
34.6 \\
34.8 \\
34.9 \\
34.9 \\
34.9 \\
35 \\
35 \\
35.3 \\
35.4 \\
35 \\
37 \\
\end{array}$ & $\begin{array}{l}34.5 \\
34.5 \\
34.5 \\
34.5 \\
35 \\
34.5 \\
34.5 \\
34.7 \\
34.9 \\
34.6 \\
35.2 \\
35.5 \\
35.4 \\
35.5 \\
35.8 \\
35.3 \\
35.4 \\
37 \\
\end{array}$ & $\begin{array}{l}33.7 \\
34.6 \\
34.6 \\
35 \\
35 \\
35.2 \\
35.1 \\
35.2 \\
35.7 \\
37 \\
36 \\
36 \\
36 \\
36 \\
35.3 \\
36.3 \\
37.6 \\
37.2 \\
\end{array}$ & $\begin{array}{l}34 \\
34.8 \\
35.1 \\
35.3 \\
35.4 \\
37 \\
35.5 \\
35 \\
37.5 \\
37.2 \\
37.3 \\
35.5 \\
36.1 \\
36.2 \\
36.2 \\
37.2 \\
36 \\
37.5\end{array}$ & $\begin{array}{l}33.1 \\
35.1 \\
35.7 \\
36 \\
35.5 \\
35.4 \\
35.5 \\
35.8 \\
35.9 \\
36 \\
36 \\
36 \\
36.2 \\
36.4 \\
36.5 \\
36.9 \\
36.8 \\
38.1 \\
\end{array}$ & $\begin{array}{l}34.2 \\
36.2 \\
36.3 \\
36.2 \\
36.3 \\
36.3 \\
36.5 \\
36.7 \\
36.9 \\
37 \\
37 \\
37.1 \\
37.2 \\
37.2 \\
37.3 \\
37 \\
37 \\
38.8 \\
\end{array}$ & $\begin{array}{l}33.4 \\
36.7 \\
36.2 \\
36.5 \\
36.5 \\
37 \\
37 \\
37.4 \\
37.7 \\
37.8 \\
38 \\
38 \\
37.8 \\
37.9 \\
37.3 \\
37.5 \\
37.5 \\
38.3 \\
\end{array}$ \\
\hline
\end{tabular}


Temperature profiles ( ${ }^{\circ} \mathrm{F}$ ) taken in Deep Hole, Maxinkuckee Lake--Continued

Temperature readings in 1901

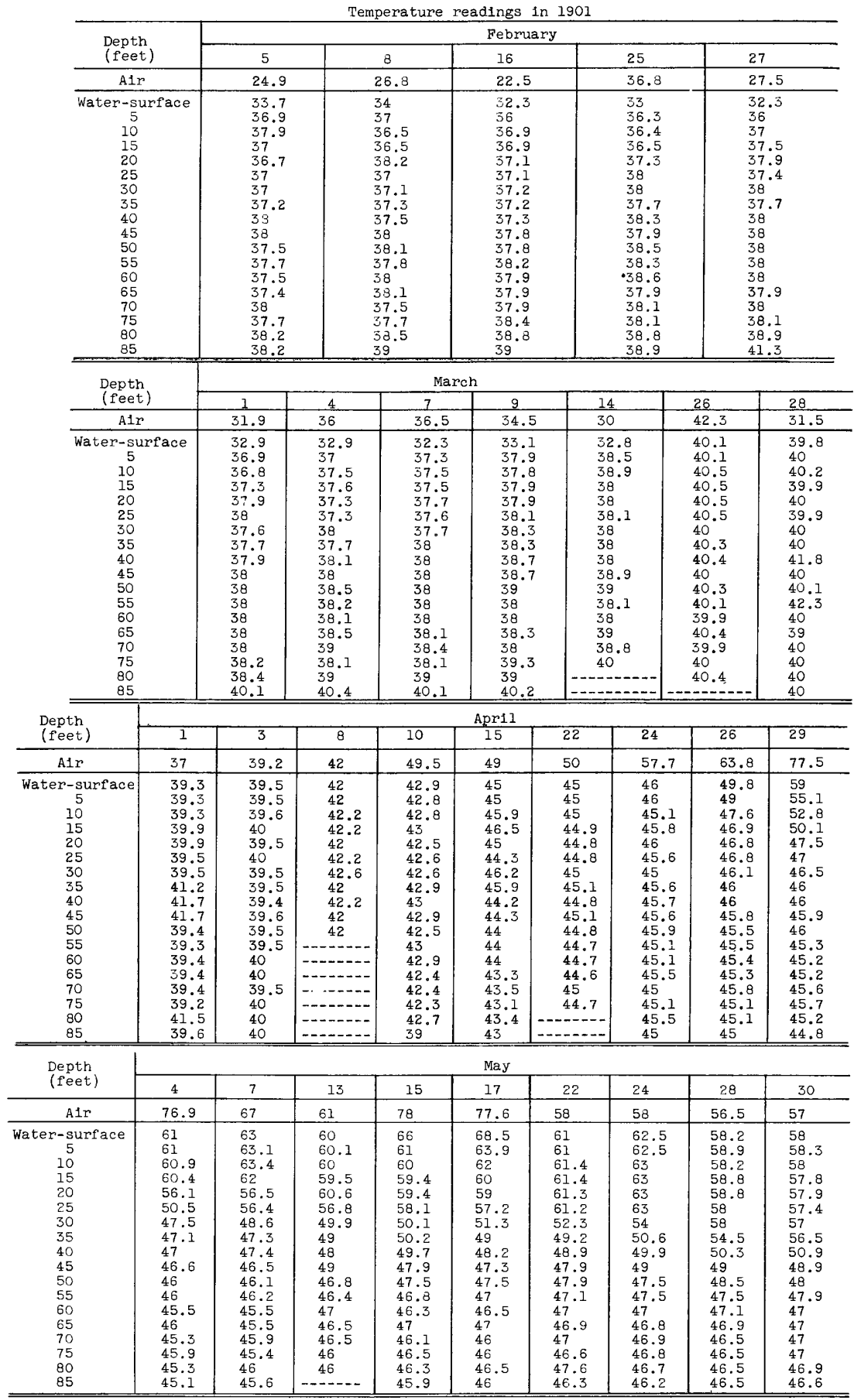


Temperature profiles ( $\left.{ }^{\circ} \mathrm{F}\right)$ taken in the Deep Hole, Maxinkuckee Lake--Continued

Temperature readings for 1901

\begin{tabular}{|c|c|c|c|c|c|c|}
\hline \multirow{2}{*}{ (fec. } & \multicolumn{6}{|c|}{ June } \\
\hline & 1 & 3 & 6 & 8 & 10 & 12 \\
\hline AIr & 66 & 65.3 & 67 & 54.5 & 66.2 & 89.5 \\
\hline $\begin{array}{r}\text { Water-surface } \\
5 \\
10 \\
15 \\
20 \\
25 \\
30 \\
35 \\
40 \\
45 \\
50 \\
55 \\
60 \\
65 \\
70 \\
75 \\
80 \\
85 \\
\end{array}$ & $\begin{array}{l}59.5 \\
59.5 \\
60 \\
59.7 \\
59 \\
58.3 \\
57.9 \\
57 \\
49.9 \\
49 \\
48.4 \\
48 \\
49 \\
47 \\
47 \\
47.5 \\
47 \\
-2 .-. \\
\end{array}$ & $\begin{array}{l}60.9 \\
61 \\
61.1 \\
61 \\
60.2 \\
58.8 \\
57.3 \\
55.9 \\
52.3 \\
50 \\
48.1 \\
47.9 \\
57.8 \\
47.1 \\
47.5 \\
47 \\
47 \\
46.9 \\
\end{array}$ & $\begin{array}{l}65.5 \\
65.5 \\
65.7 \\
64.2 \\
64 \\
60 \\
58 \\
56.2 \\
53 \\
52.1 \\
51.6 \\
51 \\
48.2 \\
47.8 \\
47.5 \\
47.5 \\
47.5 \\
47 \\
\end{array}$ & $\begin{array}{l}63.5 \\
63.5 \\
64 \\
63.4 \\
63.2 \\
61.9 \\
57.6 \\
54.8 \\
52 \\
50.9 \\
49.3 \\
52.8 \\
48.2 \\
48 \\
48.2 \\
48 \\
47.8 \\
47.3 \\
\end{array}$ & $\begin{array}{l}65 \\
64.8 \\
65.1 \\
64.9 \\
64 \\
62.8 \\
59.4 \\
54.1 \\
52 \\
50.2 \\
49.8 \\
48.6 \\
48.3 \\
48.2 \\
48 \\
47.9 \\
47.9 \\
47.3 \\
\end{array}$ & $\begin{array}{l}79.6 \\
72.5 \\
69 \\
66.5 \\
64.5 \\
62 \\
58.7 \\
54.8 \\
52.3 \\
51 \\
50 \\
49 \\
48.3 \\
48.5 \\
48 \\
48.3 \\
48 \\
48.1 \\
\end{array}$ \\
\hline \multirow{2}{*}{$\begin{array}{l}\text { Depth } \\
\text { (feet) }\end{array}$} & \multicolumn{6}{|c|}{ June --Cont Inued } \\
\hline & 17 & 19 & 21 & 24 & 26 & 28 \\
\hline Alr & 74 & 83 & 77.9 & 83.5 & 83 & 88.2 \\
\hline $\begin{array}{c}\text { Water-surface } \\
5 \\
10 \\
15 \\
20 \\
25 \\
30 \\
35 \\
40 \\
45 \\
50 \\
55 \\
60 \\
65 \\
70 \\
75 \\
80 \\
85 \\
\end{array}$ & $\begin{array}{l}76 \\
76.1 \\
76 \\
68.6 \\
66.1 \\
62.8 \\
59 \\
54.9 \\
53.5 \\
50.9 \\
50 \\
50 \\
49 \\
48.8 \\
49 \\
48.2 \\
48.1 \\
48.5\end{array}$ & $\begin{array}{l}80 \\
75.5 \\
74.9 \\
71.5 \\
66 \\
62 \\
58.1 \\
54.5 \\
52 \\
51 \\
51 \\
49.5 \\
49 \\
48.6 \\
48.4 \\
48.2 \\
48.1 \\
48\end{array}$ & $\begin{array}{l}76.9 \\
77 \\
77 \\
75.9 \\
66.5 \\
62.3 \\
58.1 \\
55.6 \\
53.2 \\
50.9 \\
50 \\
49.3 \\
49.9 \\
49 \\
48.8 \\
49 \\
49 \\
48 \\
\end{array}$ & $\begin{array}{l}78.8 \\
78.3 \\
78.2 \\
78 \\
67.1 \\
61.6 \\
58 \\
55.6 \\
53 \\
52 \\
50.2 \\
49.9 \\
49.2 \\
49 \\
48.9 \\
48.8 \\
48.5 \\
48.4\end{array}$ & $\begin{array}{l}82 \\
82.5 \\
80.8 \\
77.4 \\
68 \\
63 \\
58 \\
55.5 \\
54 \\
51.9 \\
50.1 \\
50.5 \\
49.5 \\
49.1 \\
49 \\
49.1 \\
49 \\
48.8\end{array}$ & $\begin{array}{l}83 \\
83 \\
83 \\
82.9 \\
67.2 \\
62 \\
58.9 \\
55.6 \\
53.5 \\
52 \\
51 \\
50.4 \\
49.7 \\
49.2 \\
49 \\
49 \\
49 \\
49 \\
\end{array}$ \\
\hline
\end{tabular}

\begin{tabular}{|c|c|c|c|c|c|c|c|c|c|}
\hline \multirow{3}{*}{$\begin{array}{l}\text { Depth } \\
\text { (feet) }\end{array}$} & \multirow{2}{*}{\multicolumn{3}{|c|}{ January }} & \multirow{2}{*}{\multicolumn{3}{|c|}{ February }} & \multirow{2}{*}{\multicolumn{3}{|c|}{ March }} \\
\hline & & & & & & & & & \\
\hline & Maximum & Minimim & Mean & Maximum & Minimum & Mean & Maximum & Mensmum & Mean \\
\hline Alr & 41.0 & 16.5 & 27.6 & 36.8 & 22.5 & 27.7 & 42.3 & 26.5 & 33.2 \\
\hline $\begin{array}{c}\text { Water-surface } \\
5 \\
10 \\
15 \\
20 \\
25 \\
30 \\
35 \\
40 \\
45 \\
50 \\
55 \\
60 \\
65 \\
70 \\
75 \\
80 \\
85 \\
\end{array}$ & $\begin{array}{l}34.5 \\
36.7 \\
36.3 \\
36.5 \\
36.5 \\
37.0 \\
37.0 \\
37.4 \\
37.7 \\
37.8 \\
38.0 \\
38.0 \\
37.8 \\
37.9 \\
37.3 \\
37.5 \\
37.6 \\
38.8 \\
\end{array}$ & $\begin{array}{l}33.1 \\
34.1 \\
34.0 \\
34.2 \\
34.2 \\
34.5 \\
34.5 \\
34.6 \\
34.8 \\
34.6 \\
34.9 \\
34.9 \\
35.0 \\
35.0 \\
35.3 \\
35.3 \\
35.0 \\
37.0 \\
\end{array}$ & $\begin{array}{l}33.8 \\
35.1 \\
35.0 \\
35.7 \\
35.6 \\
35.7 \\
35.5 \\
35.6 \\
36.2 \\
36.4 \\
36.3 \\
36.2 \\
36.2 \\
36.3 \\
36.2 \\
36.5 \\
36.5 \\
37.7 \\
\end{array}$ & $\begin{array}{l}34.0 \\
37.0 \\
37.9 \\
37.5 \\
38.2 \\
38.0 \\
38.0 \\
37.7 \\
38.0 \\
38.0 \\
38.5 \\
38.3 \\
38.6 \\
38.1 \\
38.1 \\
38.4 \\
38.9 \\
41.3 \\
\end{array}$ & $\begin{array}{l}32.3 \\
36.0 \\
36.4 \\
36.5 \\
36.7 \\
37.0 \\
37.0 \\
37.2 \\
37.3 \\
37.8 \\
37.5 \\
37.7 \\
37.5 \\
37.4 \\
37.5 \\
37.7 \\
38.2 \\
38.2 \\
\end{array}$ & $\begin{array}{l}33.1 \\
36.4 \\
36.9 \\
36.9 \\
37.4 \\
37.5 \\
37.5 \\
37.4 \\
37.8 \\
37.9 \\
38.0 \\
38.0 \\
38.0 \\
37.8 \\
37.9 \\
38.0 \\
38.6 \\
39.3 \\
\end{array}$ & $\begin{array}{l}40.1 \\
40.1 \\
40.5 \\
40.5 \\
40.5 \\
40.5 \\
40.0 \\
40.3 \\
41.8 \\
40.0 \\
40.3 \\
42.3 \\
40.0 \\
40.4 \\
40.0 \\
40.0 \\
40.4 \\
40.4 \\
\end{array}$ & $\begin{array}{l}32.3 \\
36.9 \\
36.8 \\
37.3 \\
37.3 \\
37.3 \\
37.6 \\
37.7 \\
37.9 \\
38.0 \\
38.0 \\
38.0 \\
38.0 \\
38.0 \\
38.0 \\
38.1 \\
38.4 \\
40.0 \\
\end{array}$ & $\begin{array}{l}34.8 \\
38.2 \\
38.5 \\
38.4 \\
38.5 \\
38.5 \\
38.5 \\
38.6 \\
39.0 \\
38.8 \\
39.0 \\
38.9 \\
38.6 \\
38.8 \\
38.9 \\
39.1 \\
39.3 \\
40.2 \\
\end{array}$ \\
\hline \multirow{2}{*}{$\begin{array}{l}\text { Depth } \\
\text { (feet) }\end{array}$} & \multicolumn{3}{|c|}{ April } & \multicolumn{3}{|c|}{ May } & \multicolumn{3}{|c|}{ June } \\
\hline & Max1mum & Minimum & Mean & Maximum & Minimum & Mean & Maximum & Minimum & Mean \\
\hline A1r & 77.5 & 37.0 & 53.0 & 78.0 & 56.5 & 59.0 & 89.0 & 54.5 & 74.0 \\
\hline $\begin{array}{c}\text { Water-surface } \\
5 \\
10 \\
15 \\
20 \\
25 \\
30 \\
35 \\
40 \\
45 \\
50 \\
55 \\
60 \\
65 \\
70 \\
75 \\
80 \\
85 \\
\end{array}$ & $\begin{array}{l}59.0 \\
55.1 \\
52.8 \\
50.1 \\
47.5 \\
47.0 \\
46.5 \\
46.0 \\
46.0 \\
45.9 \\
46.0 \\
46.3 \\
45.4 \\
45.5 \\
45.8 \\
45.7 \\
45.5 \\
45.0\end{array}$ & $\begin{array}{l}39.0 \\
39.3 \\
39.3 \\
39.9 \\
39.5 \\
39.5 \\
39.5 \\
39.5 \\
39.4 \\
39.6 \\
39.4 \\
39.3 \\
39.4 \\
39.4 \\
39.4 \\
39.2 \\
40.0 \\
39.6 \\
\end{array}$ & $\begin{array}{l}45.8 \\
45.2 \\
44.8 \\
44.6 \\
44.0 \\
43.8 \\
43.8 \\
44.0 \\
43.9 \\
43.9 \\
43.4 \\
43.4 \\
43.3 \\
43.2 \\
43.3 \\
43.2 \\
43.3 \\
42.3 \\
\end{array}$ & $\begin{array}{l}68.5 \\
63.9 \\
63.0 \\
63.0 \\
63.0 \\
63.0 \\
58.0 \\
56.5 \\
50.9 \\
49.0 \\
48.5 \\
47.9 \\
47.1 \\
47.0 \\
47.0 \\
47.0 \\
47.6 \\
46.6 \\
\end{array}$ & $\begin{array}{l}58.0 \\
58.3 \\
58.0 \\
57.8 \\
56.1 \\
50.5 \\
47.5 \\
47.1 \\
47.0 \\
46.5 \\
46.0 \\
46.0 \\
45.5 \\
45.5 \\
45.3 \\
45.4 \\
45.3 \\
45.1\end{array}$ & $\begin{array}{l}62.0 \\
61.1 \\
60.7 \\
60.3 \\
59.2 \\
57.6 \\
52.2 \\
50.4 \\
48.9 \\
48.0 \\
47.3 \\
46.9 \\
46.5 \\
46.5 \\
46.3 \\
46.3 \\
46.4 \\
46.0 \\
\end{array}$ & $\begin{array}{l}83.0 \\
83.0 \\
83.0 \\
82.9 \\
68.0 \\
63.0 \\
59.0 \\
54.0 \\
54.0 \\
52.1 \\
51.6 \\
52.8 \\
49.9 \\
49.2 \\
49.0 \\
49.4 \\
49.0 \\
49.0\end{array}$ & $\begin{array}{l}59.5 \\
59.5 \\
60.0 \\
59.7 \\
59.0 \\
58.3 \\
57.3 \\
54.1 \\
49.9 \\
49.0 \\
48.1 \\
47.9 \\
47.8 \\
47.0 \\
47.0 \\
47.0 \\
47.0 \\
46.9\end{array}$ & $\begin{array}{l}72.6 \\
71.6 \\
71.2 \\
69.5 \\
64.6 \\
61.5 \\
58.3 \\
55.4 \\
52.6 \\
51.0 \\
50.0 \\
49.7 \\
48.8 \\
48.4 \\
48.3 \\
48.2 \\
48.1 \\
47.9\end{array}$ \\
\hline
\end{tabular}


Water surface temperatures for Muskelonge Lake near Warsaw

\begin{tabular}{|c|c|c|c|c|c|c|c|}
\hline Date & $\begin{array}{c}\text { Temper- } \\
\text { ature } \\
\left({ }^{\circ} \mathrm{F}\right)\end{array}$ & Date & $\begin{array}{l}\text { Temper- } \\
\text { ature } \\
\left({ }^{\circ} \mathrm{F}\right)\end{array}$ & Date & $\begin{array}{l}\text { Temper- } \\
\text { ature } \\
(\mathrm{o} \text { F })\end{array}$ & Date & $\begin{array}{l}\text { Temper- } \\
\text { ature } \\
\left({ }^{\circ} \mathrm{F}\right)\end{array}$ \\
\hline 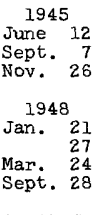 & $\begin{array}{l}35 \\
36 \\
51.5 \\
54\end{array}$ & \begin{tabular}{lr}
$1948--C o n$. \\
Oct. & 14 \\
Nov. & 30 \\
\multicolumn{2}{c}{1949} \\
Jan. & 4 \\
Feb. & 15 \\
Mar. & 2 \\
& 22 \\
May & 3
\end{tabular} & $\begin{array}{l}37 \\
33.5 \\
35 \\
48 \\
59\end{array}$ & \begin{tabular}{cc}
$1949--C o n$. \\
July & 13 \\
Aug. & 30 \\
Nov. & 1 \\
& 17 \\
Dec. & 14 \\
\multicolumn{3}{c}{1950} \\
Jan. & 12 \\
& 19
\end{tabular} & $\begin{array}{l}79 \\
79.5 \\
48 \\
46 \\
35 \\
\\
36 \\
35\end{array}$ & 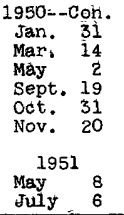 & $\begin{array}{l}35 \\
35 \\
55 \\
69 \\
57 \\
40 \\
\\
69 \\
77\end{array}$ \\
\hline
\end{tabular}

Water surface temperatures for Rldinger Lake near Plereeton

\begin{tabular}{|c|c|c|c|c|c|c|c|}
\hline Date & $\begin{array}{c}\text { Temper- } \\
\text { ature } \\
\left({ }^{\circ} \mathrm{F}\right)\end{array}$ & Date & $\begin{array}{l}\text { Temper- } \\
\text { ature } \\
\left({ }^{\circ} \mathrm{F}\right)\end{array}$ & Date & $\begin{array}{l}\text { Temper- } \\
\text { ature } \\
(0 \mathrm{~F})\end{array}$ & Date & $\begin{array}{l}\text { Temper- } \\
\text { ature } \\
\left({ }^{\circ} \mathrm{F}\right)\end{array}$ \\
\hline \begin{tabular}{lr}
\multicolumn{2}{c}{1945} \\
Mar. & 2 \\
June & 14 \\
Sept. & 7 \\
Nov. & 27 \\
\multicolumn{2}{c}{1947} \\
Apr. $\quad 22$
\end{tabular} & $\begin{array}{l}39 \\
73 \\
79 \\
38 \\
\\
46\end{array}$ & $\begin{array}{l}\text { 1947--Con. } \\
\text { Aug. } 28 \\
\text { Sept. } 4 \\
\begin{array}{ll}1948 \\
\text { Feb. } & 18 \\
\text { Aug. } & 24 \\
& 27\end{array}\end{array}$ & $\begin{array}{l}81 \\
75.5 \\
\\
34.5 \\
83 \\
84\end{array}$ & 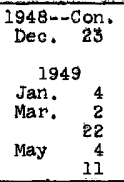 & $\begin{array}{l}36 \\
36 \\
40 \\
68 \\
57\end{array}$ & $\begin{array}{l}\text { 1949--Con: } \\
\text { Aug: } 31 \\
\text { Nov. } 29 \\
1950 \\
\text { Nov. } 22 \\
\text { 1951 } \\
\text { June } 14\end{array}$ & $\begin{array}{l}74.5 \\
45\end{array}$ \\
\hline
\end{tabular}

Temperature profiles ( $\left.{ }^{\circ} \mathrm{F}\right)$ for Ridinger Lake, Kosclusko County, July 26, 1946

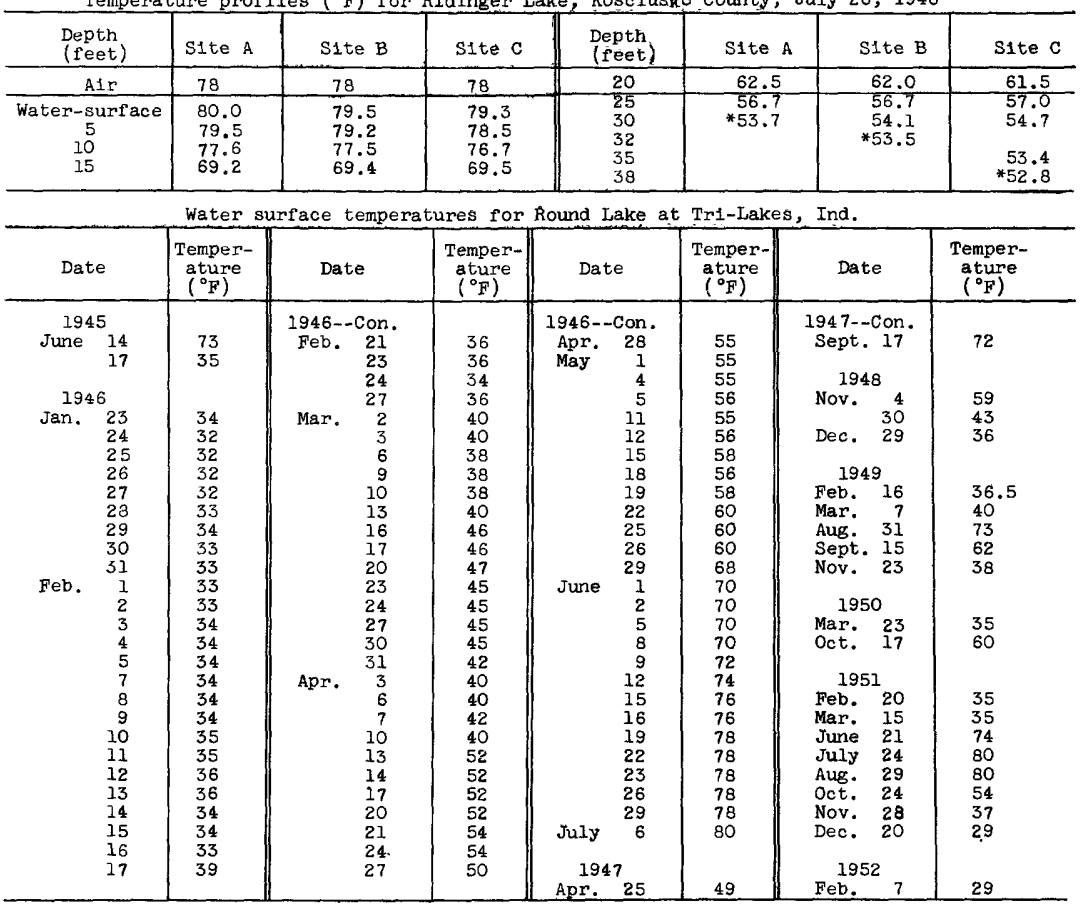


Water surface temperatures for Round Lake at Clear Lake, Ind.

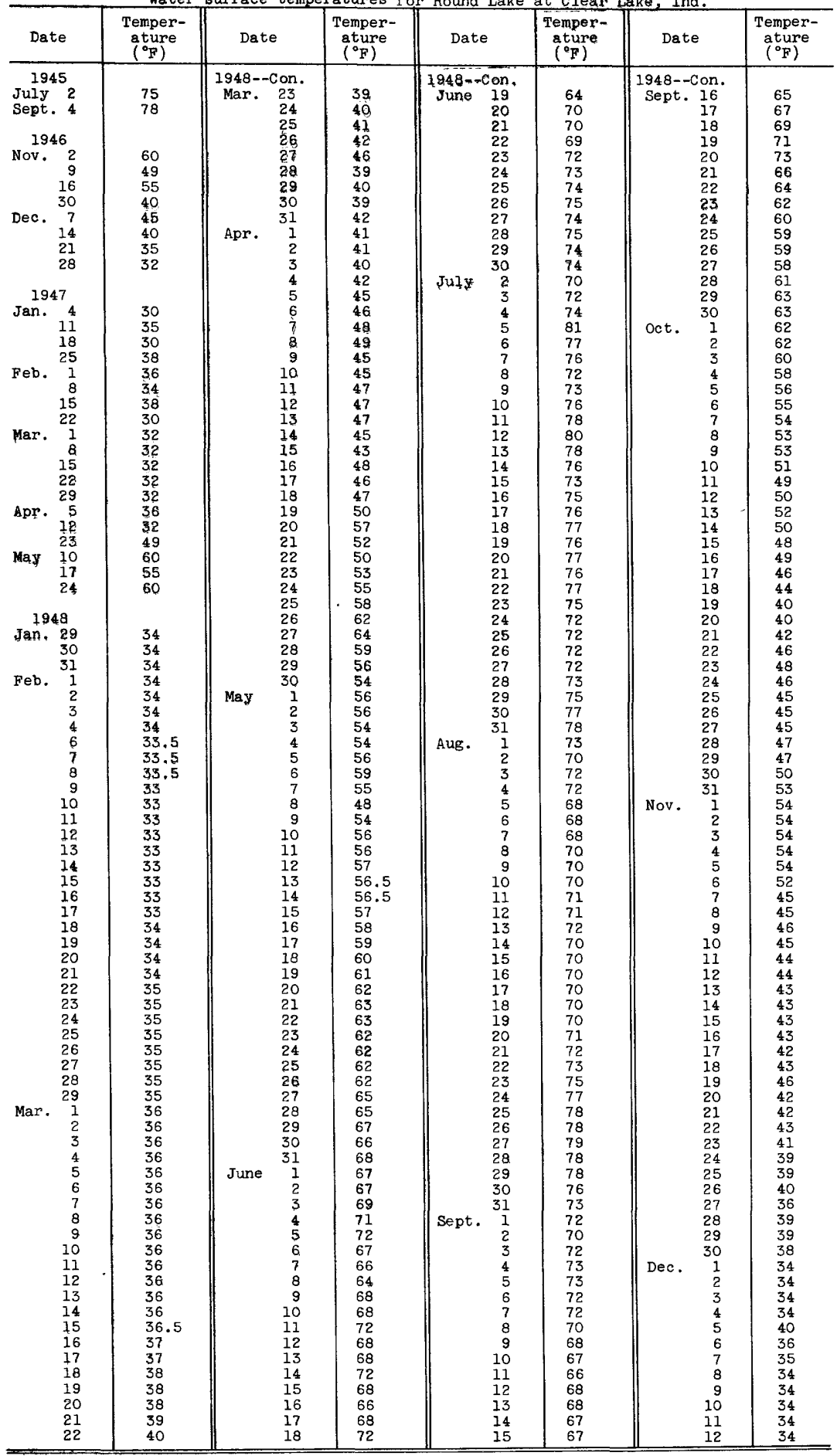


Water surface temperatures for Round Lake at Clear Lake, Ind.--Cont1nued

\begin{tabular}{|c|c|c|c|c|c|c|c|}
\hline Date & $\begin{array}{l}\text { Temper- } \\
\text { ature } \\
\left({ }^{\circ} \mathrm{F}\right)\end{array}$ & Date & $\begin{array}{l}\text { Temper- } \\
\text { ature } \\
\left({ }^{\circ} \mathrm{F}\right)\end{array}$ & Date & $\begin{array}{l}\text { Temper- } \\
\text { ature } \\
\left({ }^{\circ} \mathrm{s}\right)\end{array}$ & Date & $\begin{array}{l}\text { Temper- } \\
\text { ature } \\
\left({ }^{\circ} \mathrm{F}\right)\end{array}$ \\
\hline 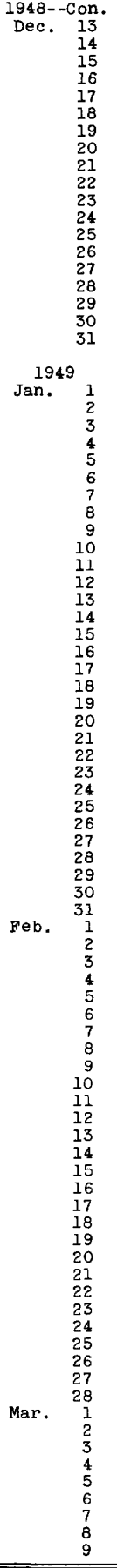 & 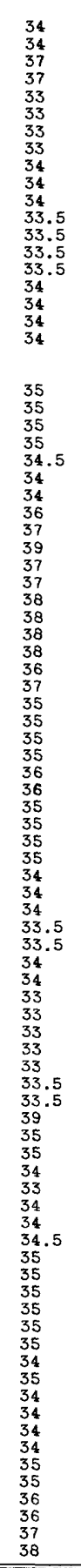 & 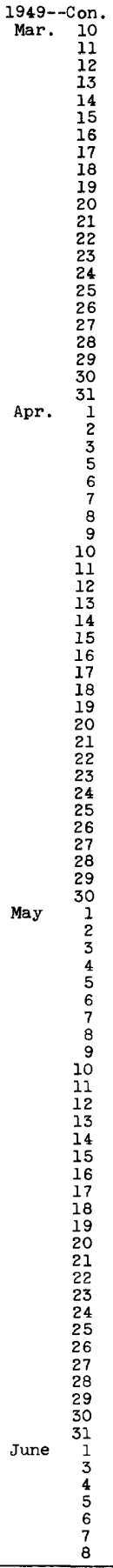 & 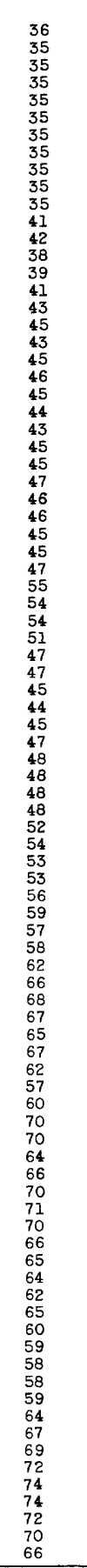 & 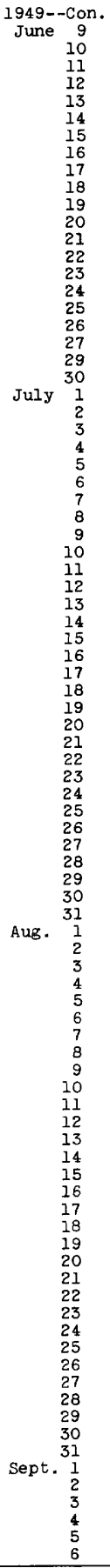 & 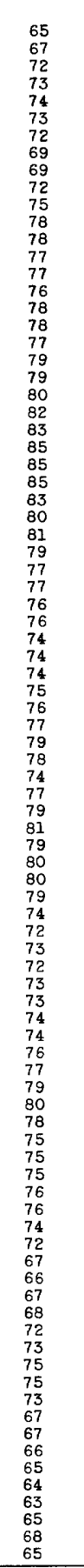 & 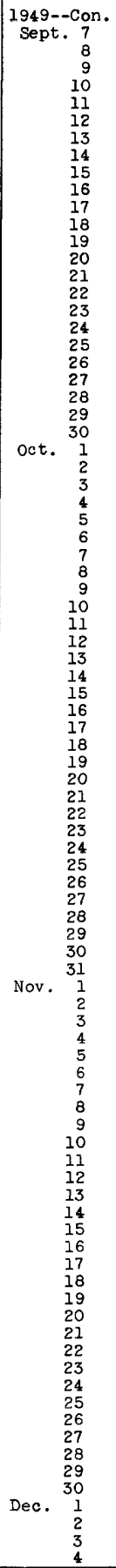 & 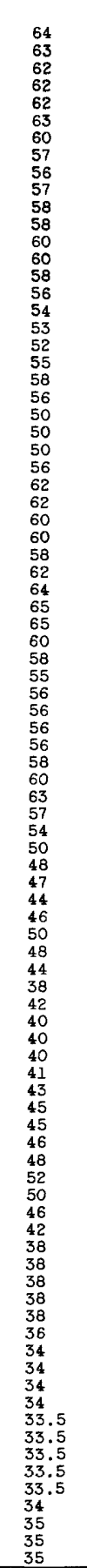 \\
\hline
\end{tabular}




\begin{tabular}{|c|c|c|c|c|c|c|c|}
\hline Date & $\begin{array}{l}\text { Temper- } \\
\text { ature } \\
\left({ }^{\circ} \mathrm{F}\right)\end{array}$ & Date & $\begin{array}{l}\text { Temper- } \\
\text { ature } \\
\left({ }^{\circ} \mathrm{F}\right)\end{array}$ & Date & $\begin{array}{l}\text { Temper- } \\
\text { ature } \\
\left({ }^{\circ} \mathrm{F}\right)\end{array}$ & Date & $\begin{array}{l}\text { Temper- } \\
\text { ature } \\
\left({ }^{\circ} \mathrm{F}\right)\end{array}$ \\
\hline 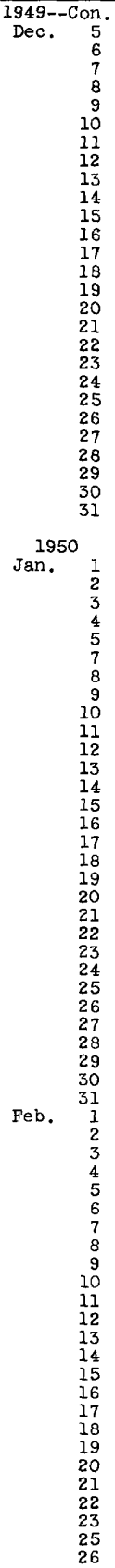 & 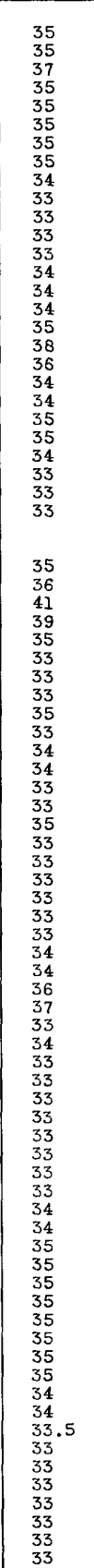 & 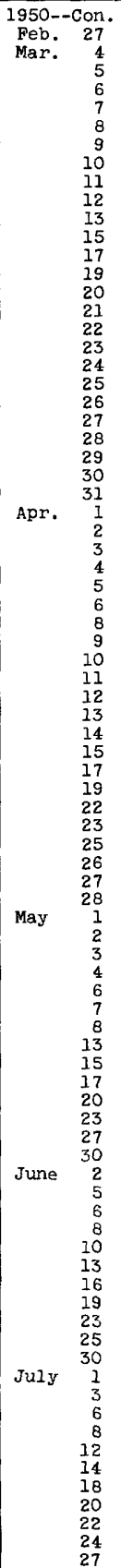 & 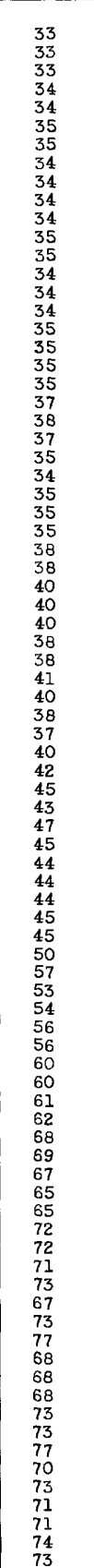 & 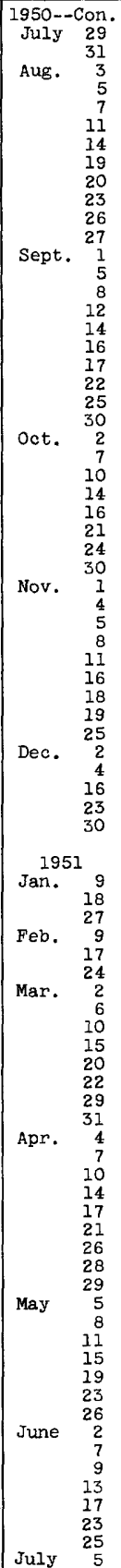 & $\begin{array}{l}33 \\
36 \\
34.5 \\
33 \\
34 \\
37 \\
36 \\
38 \\
37 \\
37 \\
34 \\
33 \\
39 \\
38 \\
38 \\
39 \\
42 \\
42 \\
41 \\
44 \\
47 \\
54 \\
56 \\
60 \\
56.5 \\
54 \\
60 \\
67 \\
65 \\
67 \\
68 \\
67 \\
66 \\
69 \\
72 \\
70 \\
70 \\
72\end{array}$ & 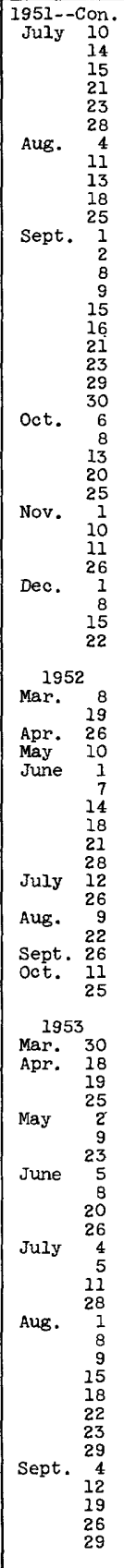 & $\begin{array}{l}73 \\
75 \\
75 \\
74 \\
74 \\
76 \\
74 \\
74 \\
74 \\
73 \\
68 \\
72 \\
72 \\
65 \\
65 \\
64 \\
63 \\
65 \\
64 \\
57 \\
57 \\
59 \\
52 \\
55 \\
53 \\
51 \\
45 \\
36 \\
36 \\
35 \\
36 \\
37 \\
33.5 \\
33 \\
\end{array}$ \\
\hline
\end{tabular}




\begin{tabular}{|c|c|c|c|c|c|c|c|c|c|}
\hline & \multicolumn{4}{|c|}{1946} & \multicolumn{5}{|c|}{1947} \\
\hline & Sept. 26 & Oct. 24 & Nov. 23 & Dec. 19 & Jan. 8 & Feb. 19 & Apr. 29 & May 25 & June 23 \\
\hline $\mathrm{A} \perp \mathrm{r}$ & & 60 & 24 & 10 & 31 & 23 & 51 & 54.8 & 69 \\
\hline $\begin{array}{c}\text { Water-surface } \\
2.5 \\
5 \\
10 \\
15 \\
20 \\
22.5 \\
25 \\
30 \\
32 \\
34 \\
35 \\
36 \\
38 \\
40 \\
45 \\
47.5 \\
50 \\
52.5 \\
55 \\
60 \\
65 \\
70 \\
75 \\
80 \\
85 \\
87.5 \\
90 \\
92.5 \\
95 \\
100 \\
101 \\
102 \\
102.5 \\
103.5 \\
105 \\
107 \\
\end{array}$ & \begin{tabular}{c}
66.6 \\
66.4 \\
66.4 \\
66.4 \\
66.2 \\
\hdashline 66.3 \\
66 \\
66.5 \\
63.1 \\
61.2 \\
60 \\
57 \\
55.3 \\
52.3 \\
51 \\
50.8 \\
50.6 \\
50.3 \\
50.1 \\
50.1 \\
50.1 \\
50 \\
50 \\
49.8 \\
49.4 \\
$-2-1$ \\
49.5 \\
-2 \\
\end{tabular} & 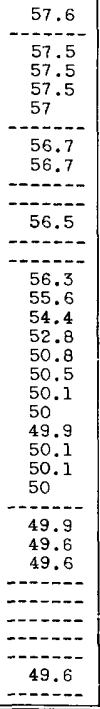 & 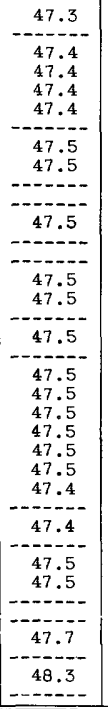 & 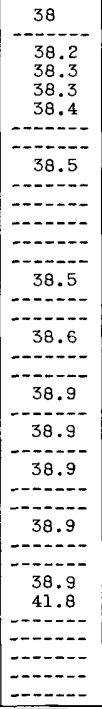 & $\begin{array}{c}33 \\
35 \\
35.4 \\
35.4 \\
35.4 \\
35.3 \\
35.4 \\
35.4 \\
35.5 \\
35.7 \\
36 \\
36.1 \\
36.1 \\
36.1 \\
36.6 \\
38 \\
37 \\
36 \\
36\end{array}$ & 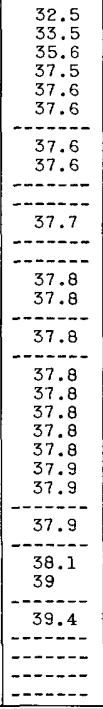 & 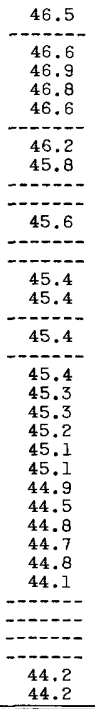 & 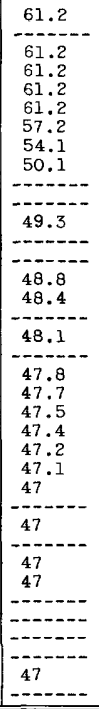 & 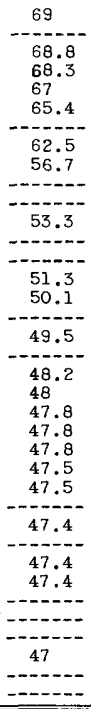 \\
\hline \multirow{2}{*}{$\begin{array}{l}\text { Depth } \\
\text { (feet) }\end{array}$} & \multicolumn{6}{|c|}{ 1947--Continued } & \multicolumn{3}{|c|}{1948} \\
\hline & July 30 & Aug. 27 & oct. 10 & oct. 30 & Nov. 19 & Dec. 19 & Jan. 29 & June 3 & July 1 \\
\hline A1r & 91.4 & 70 & 57 & 51 & 34 & 30.5 & 21 & 81 & 58 \\
\hline $\begin{array}{c}\text { Water-surface } \\
2.5 \\
5 \\
10 \\
15 \\
16 \\
17.5 \\
20 \\
22.5 \\
25 \\
30 \\
35 \\
40 \\
45 \\
50 \\
55 \\
60 \\
65 \\
70 \\
75 \\
80 \\
85 \\
90 \\
95 \\
97 \\
100 \\
101 \\
102 \\
103 \\
104 \\
105 \\
106\end{array}$ & \begin{tabular}{c}
77.1 \\
\hdashline 77.1 \\
76.4 \\
76.4 \\
\hdashline-1.4 \\
76.2 \\
67.4 \\
59.5 \\
53.4 \\
50.6 \\
49.3 \\
48.4 \\
48.4 \\
48.4 \\
48.4 \\
48.4 \\
48 \\
48 \\
48 \\
48 \\
47.5 \\
47.5 \\
-2 \\
-9
\end{tabular} & $\begin{array}{c}81.2 \\
81.2 \\
81.2 \\
81.2 \\
79.9 \\
76.8 \\
75.3 \\
-66.8 \\
58.8 \\
55 \\
51.8 \\
50.3 \\
49.2 \\
48.9 \\
48.7 \\
48.5 \\
48.5 \\
48.5 \\
48.5 \\
48.5 \\
48.4 \\
48.4 \\
48.3 \\
-2.5 \\
-2 \\
-2- \\
48.2 \\
48\end{array}$ & 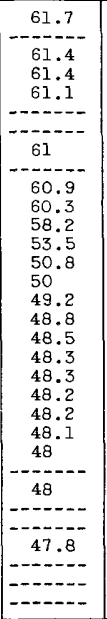 & \begin{tabular}{c}
59.6 \\
59.6 \\
59.6 \\
59.5 \\
\hdashline-1.5 \\
59.5 \\
59.5 \\
59.5 \\
58.3 \\
54.3 \\
50.8 \\
49.7 \\
49.2 \\
48.5 \\
48.5 \\
48.5 \\
48.5 \\
48.5 \\
48.5 \\
48.3 \\
48.3
\end{tabular} & 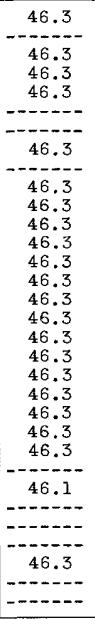 & 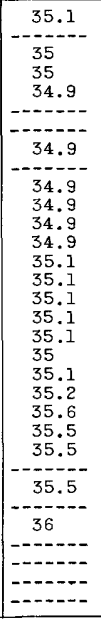 & $\begin{array}{c}33.3 \\
36 \\
36.4 \\
36.5 \\
36.6 \\
36.5 \\
36.5 \\
36.5 \\
36.5 \\
36.6 \\
36.6 \\
36.6 \\
36.6 \\
36.6 \\
36.6 \\
36.6 \\
36.6 \\
36.8 \\
36.8 \\
37.2 \\
37.6 \\
38.1 \\
-2\end{array}$ & 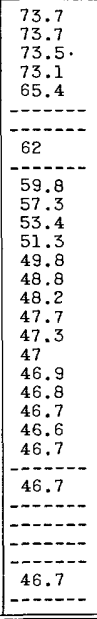 & 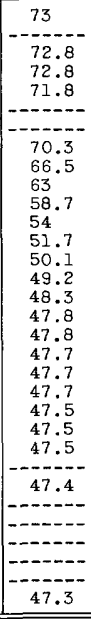 \\
\hline
\end{tabular}


Temperature profiles $\left({ }^{\circ} \mathrm{F}\right)$ for Clear lake, at Clear Lake--Continued

\begin{tabular}{|c|c|c|c|c|c|}
\hline \multirow{2}{*}{$\begin{array}{l}\text { Depth } \\
\text { (feet) }\end{array}$} & \multicolumn{5}{|c|}{ 1948--Continued } \\
\hline & Aug. 4 & Sept. 2 & Oct. 7 & Nov. 18 & Dec. 9 \\
\hline $\mathrm{A} 1 \mathrm{r}$ & 62 & 74 & 53 & 51.5 & $--0-1-1-0=-1$ \\
\hline $\begin{array}{l}\text { Water-surface } \\
5 \\
10 \\
15 \\
20 \\
25 \\
27.5 \\
30 \\
32.5 \\
35 \\
40 \\
45 \\
50 \\
55 \\
60 \\
65 \\
70 \\
75 \\
80 \\
85 \\
90 \\
95 \\
100 \\
102 \\
103 \\
105 \\
106 \\
\end{array}$ & $\begin{array}{l}74.3 \\
74.3 \\
74.4 \\
74.4 \\
74.2 \\
73.2 \\
69.5 \\
60 \\
53.7 \\
51.5 \\
50.2 \\
49.1 \\
48.3 \\
48 \\
47.7 \\
47.6 \\
47.5 \\
47.4 \\
47.3 \\
47.2 \\
47.3 \\
47.3 \\
-.-.-2 \\
47.2 \\
47.2 \\
\end{array}$ & $\begin{array}{l}76.2 \\
76.2 \\
76 \\
76 \\
74.2 \\
69.7 \\
66.3 \\
60.8 \\
57.5 \\
54.8 \\
51.7 \\
49.8 \\
48.8 \\
48.2 \\
48.1 \\
47.9 \\
47.9 \\
47.7 \\
47.6 \\
47.6 \\
47.6 \\
47.4 \\
47.4 \\
-. .5 \\
47.4 \\
47.4 \\
-1.5\end{array}$ & $\begin{array}{r}58.8 \\
58.8 \\
58.8 \\
58.8 \\
58.8 \\
58.8 \\
58.6 \\
57.4 \\
54.5 \\
49.6 \\
47.5 \\
47.6 \\
47.5 \\
\\
\\
\end{array}$ & 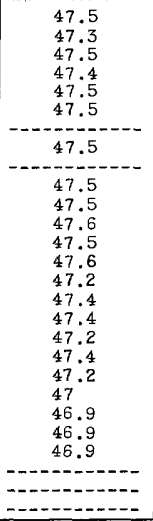 & \begin{tabular}{|l}
40.4 \\
40.5 \\
40.5 \\
40.6 \\
40.1 \\
40.2 \\
39.1 \\
38.6 \\
38.6 \\
38 \\
39.8 \\
37.5 \\
40 \\
40 \\
40.4 \\
39.7 \\
39.8 \\
39.6 \\
39.8 \\
39.8 \\
40 \\
40.2 \\
\\
\\
\end{tabular} \\
\hline
\end{tabular}

Water surface temperature for Shriner Lake at Tr1-Lakes, Ind.

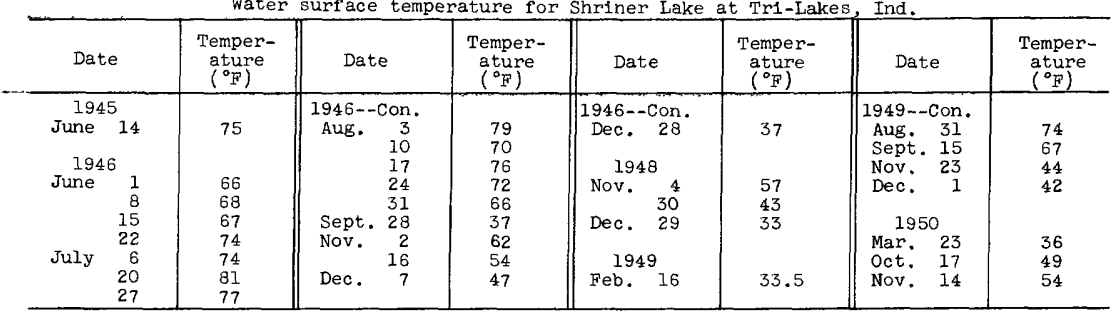

Water surface temperature for Smalley Lake near Washington Center

\begin{tabular}{|c|c|c|c|c|c|c|c|}
\hline Date & $\begin{array}{l}\text { Temper- } \\
\text { ature } \\
\left({ }^{\circ} \mathrm{F}\right)\end{array}$ & Date & $\begin{array}{c}\text { Temper- } \\
\text { ature } \\
\left({ }^{\circ} \mathrm{F}\right)\end{array}$ & Date & $\begin{array}{l}\text { Temper- } \\
\text { ature } \\
\left({ }^{\circ} \mathrm{F}\right)\end{array}$ & Date & $\begin{array}{l}\text { Temper- } \\
\text { ature } \\
\left({ }^{\circ} \mathrm{F}\right)\end{array}$ \\
\hline 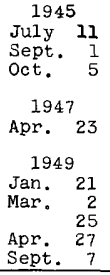 & $\begin{array}{l}47 \\
\\
\\
35 \\
34 \\
44 \\
58 \\
69 \\
\end{array}$ & 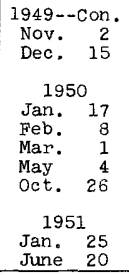 & $\begin{array}{l}51 \\
34 \\
\\
35 \\
34 \\
35 \\
55 \\
57 \\
\\
\\
36 \\
74 \\
\end{array}$ & 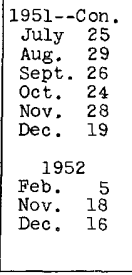 & $\begin{array}{l}80 \\
80 \\
62 \\
55 \\
37 \\
29 \\
\\
\\
26 \\
48 \\
37\end{array}$ & \begin{tabular}{lr}
\multicolumn{2}{c}{1953} \\
Jan. & 15 \\
Feb. & 3 \\
Mar. & 25 \\
Apr. & 17 \\
May & 13 \\
June & 8 \\
July & 2 \\
& 27 \\
Aug. & 25 \\
Sept. & 18
\end{tabular} & $\begin{array}{l}36 \\
36 \\
40 \\
47 \\
62 \\
75 \\
75 \\
78 \\
77 \\
73\end{array}$ \\
\hline \multicolumn{8}{|c|}{ Water surface temperature for Sylvan Lake at Rome City } \\
\hline Date & $\begin{array}{l}\text { Temper- } \\
\text { ature } \\
\left({ }^{\circ} \mathrm{F}\right)\end{array}$ & Date & $\begin{array}{l}\text { Temper- } \\
\text { ature } \\
\left({ }^{\circ} \mathrm{F}\right)\end{array}$ & Date & $\begin{array}{l}\text { Temper- } \\
\text { ature } \\
\left({ }^{\circ} \mathrm{F}\right)\end{array}$ & Date & $\begin{array}{l}\text { Temper- } \\
\text { ature } \\
\left({ }^{\circ} \mathrm{F}\right)\end{array}$ \\
\hline \begin{tabular}{lr}
\multicolumn{2}{c}{1945} \\
Mar. & 2 \\
July & 2 \\
Sept. & 5 \\
Nov. & 29 \\
\multicolumn{2}{c}{1947} \\
May & \\
& 12
\end{tabular} & $\begin{array}{l}39 \\
75 \\
78 \\
35\end{array}$ & \begin{tabular}{c}
1947 - \\
June \\
\multicolumn{1}{c}{8} \\
1948 \\
Jan. 21 \\
Sept. \\
Nov. $\begin{array}{r}5 \\
26\end{array}$
\end{tabular} & $\begin{array}{l}33 \\
75 \\
55 \\
43\end{array}$ & $\begin{array}{c}1948--C o n . \\
\text { Dec. }\end{array}$ & $\begin{array}{l}36 \\
35 \\
38.5 \\
66\end{array}$ & $\begin{array}{l}1949-- \text { Con. } \\
\text { Oct. } 14 \\
\text { Dec. }\end{array}$ & $\begin{array}{l}64 \\
40\end{array}$ \\
\hline
\end{tabular}


Water surface temperatures for Syracuse Lake at Syracuse, Ind.

\begin{tabular}{|c|c|c|c|c|c|c|c|}
\hline Date & $\begin{array}{c}\text { Temper- } \\
\text { ature } \\
\left({ }^{\circ} \mathrm{F}\right)\end{array}$ & Date & $\begin{array}{c}\text { Temper- } \\
\text { ature } \\
\left({ }^{\circ} \mathrm{F}\right)\end{array}$ & Date & $\begin{array}{c}\text { Temper- } \\
\text { ature } \\
\left({ }^{\circ} \mathrm{F}\right)\end{array}$ & Date & $\begin{array}{c}\text { Temper- } \\
\text { ature } \\
\left({ }^{\circ} \mathrm{F}\right)\end{array}$ \\
\hline \begin{tabular}{lr}
\multicolumn{2}{c}{1945} \\
June & 13 \\
Sept. & 6 \\
& 1948 \\
Aug. & 6
\end{tabular} & $\begin{array}{l}70 \\
70\end{array}$ & $\begin{array}{c}1948-- \text { Con. } \\
\text { oct. } 6 \\
28 \\
1949 \\
\operatorname{Jan} .5\end{array}$ & $\begin{array}{l}62 \\
51 \\
35\end{array}$ & $\begin{array}{c}1949--C o n . \\
\text { Feb. } 2 \\
\text { Mar. } 25 \\
\text { Apr. } 22 \\
\text { July } 29 \\
\text { Nov. } 22\end{array}$ & $\begin{array}{l}33 \\
46 \\
57.5 \\
81.5 \\
40\end{array}$ & \begin{tabular}{lr}
\multicolumn{2}{c}{1950} \\
Feb. & 1 \\
& 10 \\
Mar. & 7 \\
& 14 \\
May & 9 \\
Oct. & 25
\end{tabular} & $\begin{array}{l}35 \\
35 \\
35 \\
35 \\
63 \\
57\end{array}$ \\
\hline
\end{tabular}

Water surface temperatures for Tippecanoe Lake at oswego, Ind.

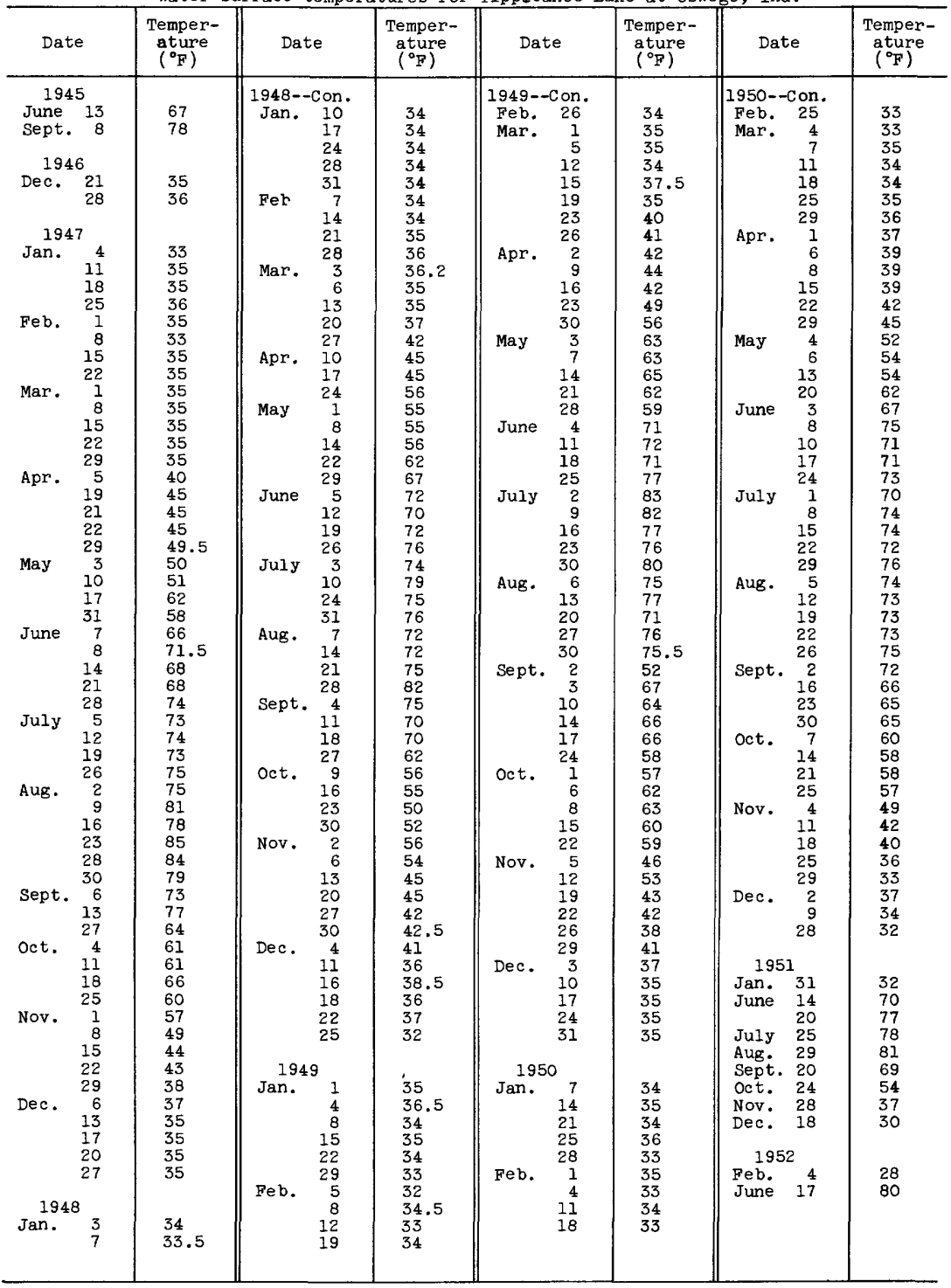


Temperature profiles ( ${ }^{\circ} \mathrm{F}$ ) for Tippecanoe Lake, at Oswego

\begin{tabular}{|c|c|c|c|c|c|c|c|}
\hline \multirow{2}{*}{$\begin{array}{l}\text { Depth } \\
\text { (feet) }\end{array}$} & \multicolumn{4}{|c|}{1946} & \multicolumn{3}{|c|}{1947} \\
\hline & Aug. 27 & Oct. 23 & Nov. 20 & Dec. 19 & $\operatorname{Jan} .9$ & Apr. 30 & May 24 \\
\hline Alr & 74 & 74 & 56 & 32 & 26 & 63 & 74 \\
\hline $\begin{array}{c}\text { Water-surface } \\
2.5 \\
5 \\
10 \\
15 \\
20 \\
25 \\
30 \\
35 \\
40 \\
42 \\
44 \\
45 \\
46 \\
48 \\
50 \\
55 \\
60 \\
65 \\
70 \\
75 \\
77.5 \\
80 \\
85 \\
90 \\
95 \\
100 \\
105 \\
110 \\
115 \\
116 \\
117 \\
117.5 \\
118.5 \\
119 \\
\end{array}$ & 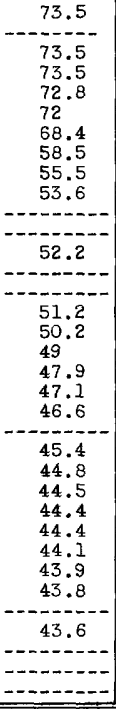 & 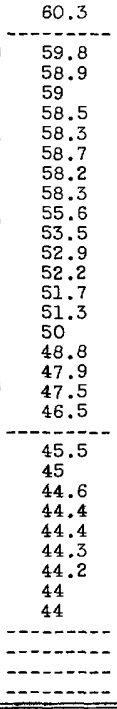 & 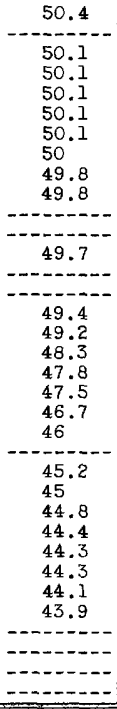 & \begin{tabular}{|c}
41.2 \\
41.1 \\
41.1 \\
41 \\
41 \\
41 \\
40.9 \\
40.8 \\
40.8 \\
40.8 \\
-10.8 \\
40.8 \\
40.8 \\
40.6 \\
40.7 \\
40.5 \\
40.5 \\
40.5 \\
40.3 \\
40.2 \\
40.2 \\
40.1 \\
40.1 \\
-9
\end{tabular} & $\begin{array}{c}34 \\
36.5 \\
36.6 \\
36.6 \\
36.6 \\
36.7 \\
36.7 \\
37 \\
37.3 \\
37.6 \\
37.9 \\
38 \\
38 \\
38.1 \\
38.2 \\
38.3 \\
38.3 \\
38.4 \\
38.5 \\
38.5 \\
38.4 \\
38.3 \\
38.4 \\
38.6 \\
39.2 \\
-2 \\
-2\end{array}$ & $\begin{array}{c}52.1 \\
52.1 \\
51.9 \\
51.6 \\
51 \\
49.6 \\
48.8 \\
48.3 \\
48.1 \\
47.7 \\
46.8 \\
46.5 \\
46.5 \\
46.5 \\
46.1 \\
45.6 \\
45.2 \\
44.9 \\
44.8 \\
44.6 \\
43.5 \\
43.5 \\
43.5 \\
43.8 \\
43.7 \\
43.4 \\
\end{array}$ & \begin{tabular}{|l}
66.7 \\
65.3 \\
64.4 \\
63.6 \\
62.6 \\
59 \\
55.2 \\
51 \\
50 \\
49.6 \\
49.2 \\
48.4 \\
47.4 \\
47 \\
46.8 \\
46.4. \\
45.8 \\
45.4 \\
45.1 \\
44.9 \\
44.9 \\
44.9 \\
44.8 \\
44.7 \\
44.6 \\
-2 \\
44.4 \\
44.3 \\
$-9-1$ \\
\end{tabular} \\
\hline \multirow{2}{*}{$\begin{array}{l}\text { Depth } \\
\text { (feet) }\end{array}$} & \multicolumn{5}{|c|}{ 1947--Cont1nued } & \multicolumn{2}{|c|}{1948} \\
\hline & June 20 & Jul. 25 & Aug. 26 & Oct. 30 & Nov. 20 & Jan. 28 & June 3 \\
\hline A1r & 74 & 78.6 & 84.5 & 60 & 43 & 12 & 72 \\
\hline $\begin{array}{c}\text { Water-surface } \\
2.5 \\
5 \\
10 \\
12.5 \\
15 \\
20 \\
25 \\
30 \\
32.5 \\
35 \\
40 \\
45 \\
50 \\
55 \\
60 \\
65 \\
70 \\
75 \\
80 \\
85 \\
90 \\
95 \\
100 \\
105 \\
110 \\
115 \\
116 \\
117 \\
118 \\
\end{array}$ & $\begin{array}{c}67.5 \\
67.5 \\
67.5 \\
-66.8 \\
-64.5 \\
60.8 \\
58 \\
53.5 \\
51.7 \\
51.2 \\
49.6 \\
48.8 \\
48.2 \\
47.7 \\
47.1 \\
46.8 \\
46.3 \\
45.9 \\
45.8 \\
45.6 \\
45.5 \\
45.2 \\
45.2 \\
45.2 \\
45.1 \\
-0-1 \\
45.1 \\
4\end{array}$ & 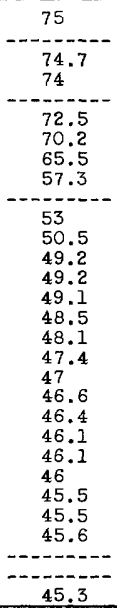 & $\begin{array}{l}85 \\
-85 \\
84.4 \\
83.4 \\
80 \\
72.3 \\
64.4 \\
56.8 \\
-53.4 \\
51.2 \\
50.1 \\
49.2 \\
48.5 \\
47.8 \\
47.3 \\
46.7 \\
46.5 \\
46.3 \\
45.9 \\
45.9 \\
45.8 \\
45.5 \\
45.4 \\
45.3 \\
45.2 \\
45.1 \\
-1 . .--\end{array}$ & 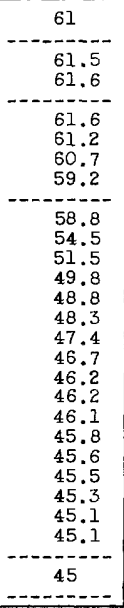 & $\begin{array}{c}46.8 \\
46.8 \\
47 \\
47 \\
47 \\
47 \\
46.8 \\
46.8 \\
46.8 \\
46.7 \\
46.5 \\
46.5 \\
46.5 \\
46.5 \\
46.5 \\
46.4 \\
46.3 \\
46.2 \\
46.1 \\
46 \\
45.9 \\
45.8 \\
45.8 \\
45.7 \\
45.5 \\
-9\end{array}$ & $\begin{array}{c}34.4 \\
33.7 \\
38 \\
38.3 \\
38.4 \\
38.5 \\
38.5 \\
38.5 \\
38.5 \\
38.5 \\
38.3 \\
38.2 \\
38.2 \\
38.1 \\
38.1 \\
38.1 \\
38 \\
38 \\
38 \\
37.9 \\
37.7 \\
37.7 \\
38.8 \\
38.6 \\
39 \\
-2.0 \\
-2\end{array}$ & $\begin{array}{r}71.5 \\
69.9 \\
70.7 \\
67.8 \\
64.8 \\
60.8 \\
58.3 \\
56.2 \\
54.4 \\
53.1 \\
52 \\
50.7 \\
49.1 \\
48.4 \\
47 \\
46.3 \\
45.8 \\
45.4 \\
45.3 \\
45.2 \\
44.9 \\
44.8 \\
44.7 \\
44.6 \\
44.5 \\
-0 .- \\
44.5 \\
\end{array}$ \\
\hline
\end{tabular}


Temperature profiles $\left({ }^{\circ} \mathrm{F}\right)$ for Tippecanoe Lake, at Oswego--Continued

\begin{tabular}{|c|c|c|c|c|c|c|}
\hline \multirow{2}{*}{$\begin{array}{l}\text { Depth } \\
\text { (feet) }\end{array}$} & \multicolumn{6}{|c|}{ 1948--Continued } \\
\hline & July 1 & Aug. 6 & Sept. I & oct. 6 & Nov. 17 & Dec. 7 \\
\hline Air & 76 & 68 & 65 & 51.5 & 56 & $-\ldots-\cdots$ \\
\hline $\begin{array}{l}\text { Water-surface } \\
2.5 \\
5 \\
10 \\
15 \\
17.5 \\
20 \\
22.5 \\
25 \\
27.5 \\
30 \\
32.5 \\
35 \\
40 \\
45 \\
50 \\
55 \\
60 \\
65 \\
70 \\
75 \\
80 \\
85 \\
90 \\
95 \\
100 \\
105 \\
110 \\
115 \\
116 \\
117 \\
118\end{array}$ & 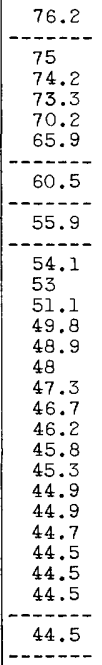 & 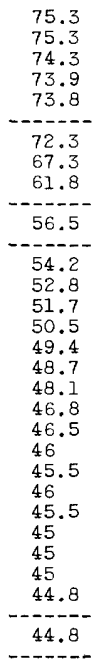 & $\begin{array}{l}78.1 \\
78.1 \\
78.1 \\
78.1 \\
78.1 \\
75.3 \\
72.3 \\
69.7 \\
65.4 \\
60.7 \\
58.2 \\
56.2 \\
54.9 \\
53.4 \\
52.1 \\
50.8 \\
49.4 \\
48.7 \\
48 \\
47.3 \\
46.6 \\
46 \\
45.5 \\
45.5 \\
45.3 \\
45.1 \\
45 \\
44.9 \\
44.9 \\
-24.5 \\
44 \\
---1--\end{array}$ & 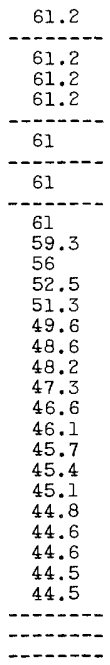 & $\begin{array}{c}48.7 \\
48.6 \\
48.6 \\
48.5 \\
48.2 \\
48.2 \\
48.1 \\
48.4 \\
48.4 \\
48.5 \\
48.5 \\
48.5 \\
48.5 \\
48.5 \\
48.4 \\
48.2 \\
48 \\
47.7 \\
46.5 \\
45.6 \\
45.6 \\
45.6 \\
45.2 \\
45.2 \\
45.2 \\
--2.5 \\
-1-1\end{array}$ & $\begin{array}{c}43.3 \\
43.4 \\
43.3 \\
43.2 \\
43.3 \\
43.5 \\
43.5 \\
43.6 \\
43.6 \\
43.6 \\
43.6 \\
43.8 \\
43.6 \\
43.3 \\
43 \\
42.7 \\
42.4 \\
43.7 \\
42.5 \\
43.8 \\
43.7 \\
43.3 \\
43.3 \\
42.5 \\
---2 \\
43\end{array}$ \\
\hline
\end{tabular}

Water surface temperatures for Lake Wawasee near Wawasee, Ind.

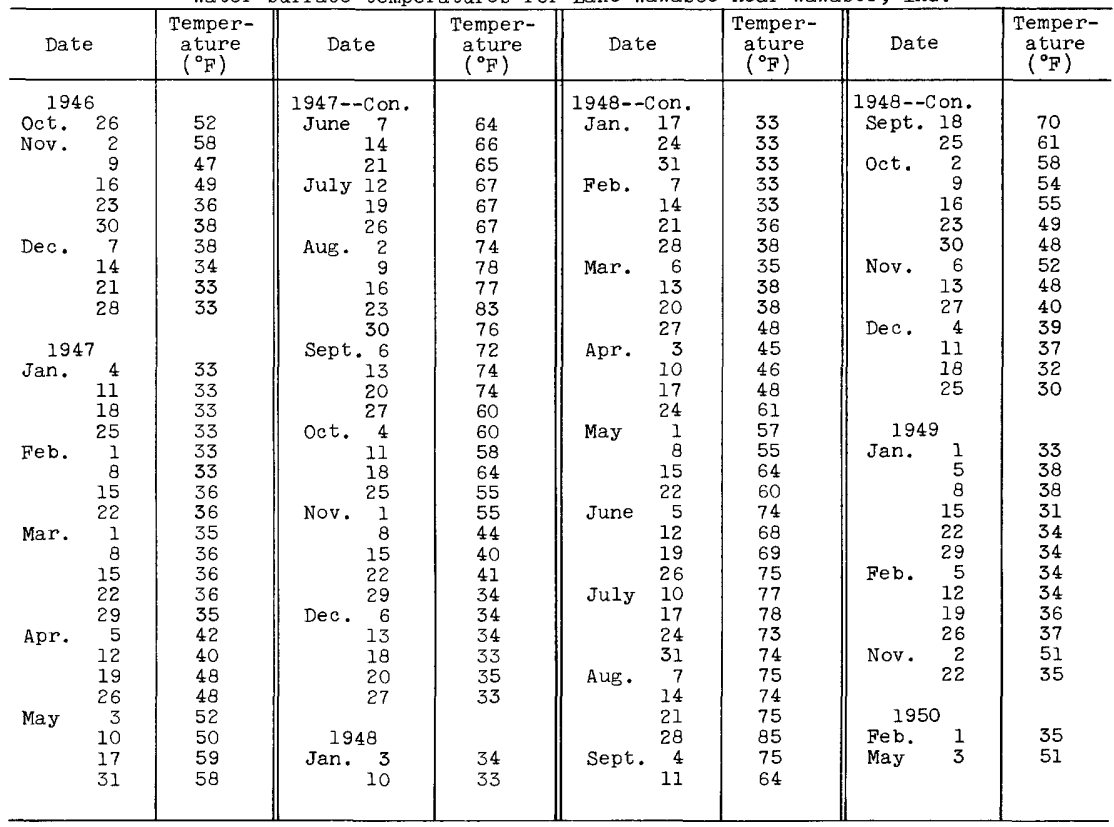


TEMPERATURE FOR SELECTED LAKES

Temperature profiles $\left({ }^{\circ} \mathrm{F}\right)$ for Lake Wawasee near 1 Wawasee

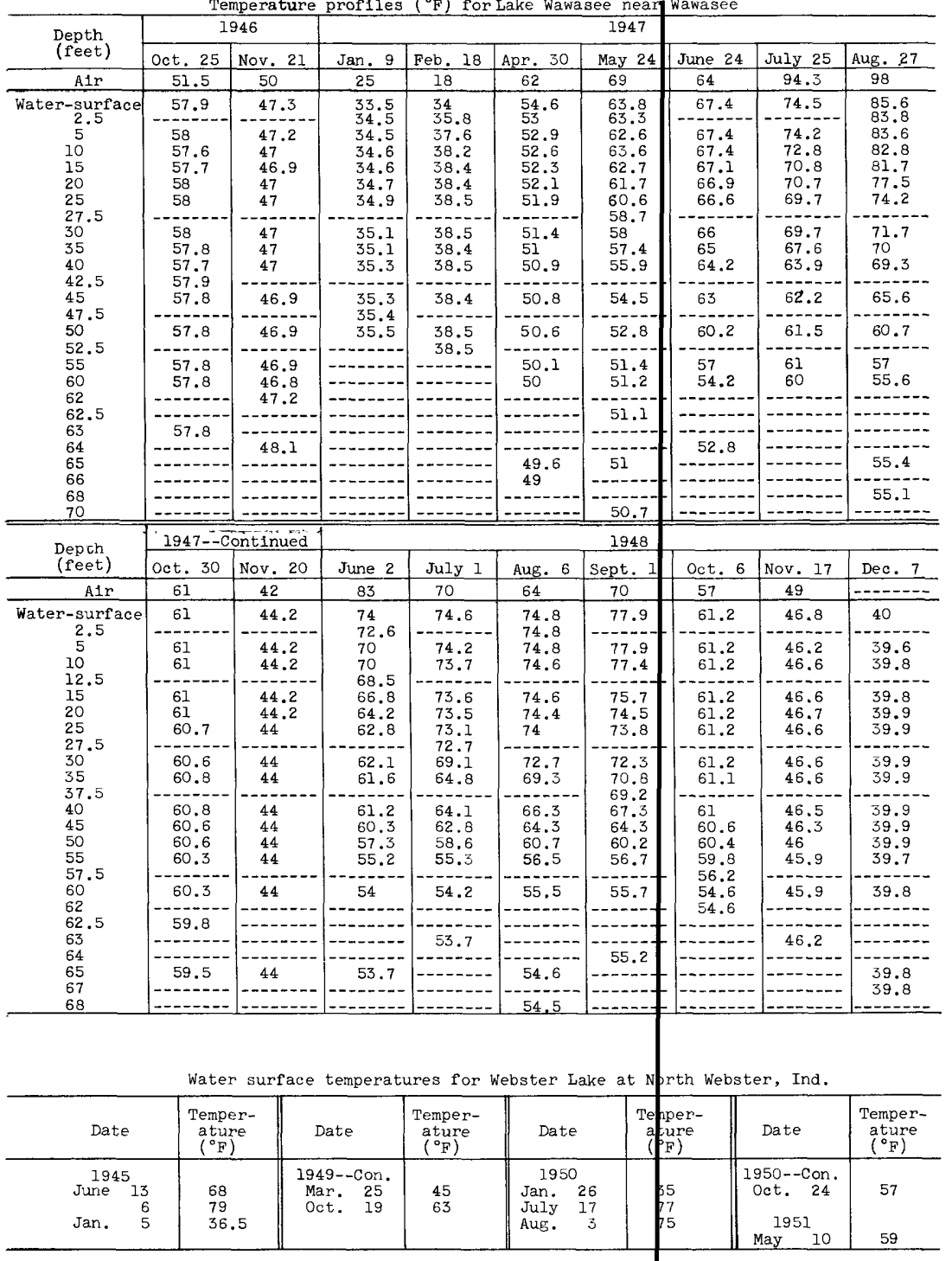


Water surface temperatures for Winona Lake at Warsaw, Ind.

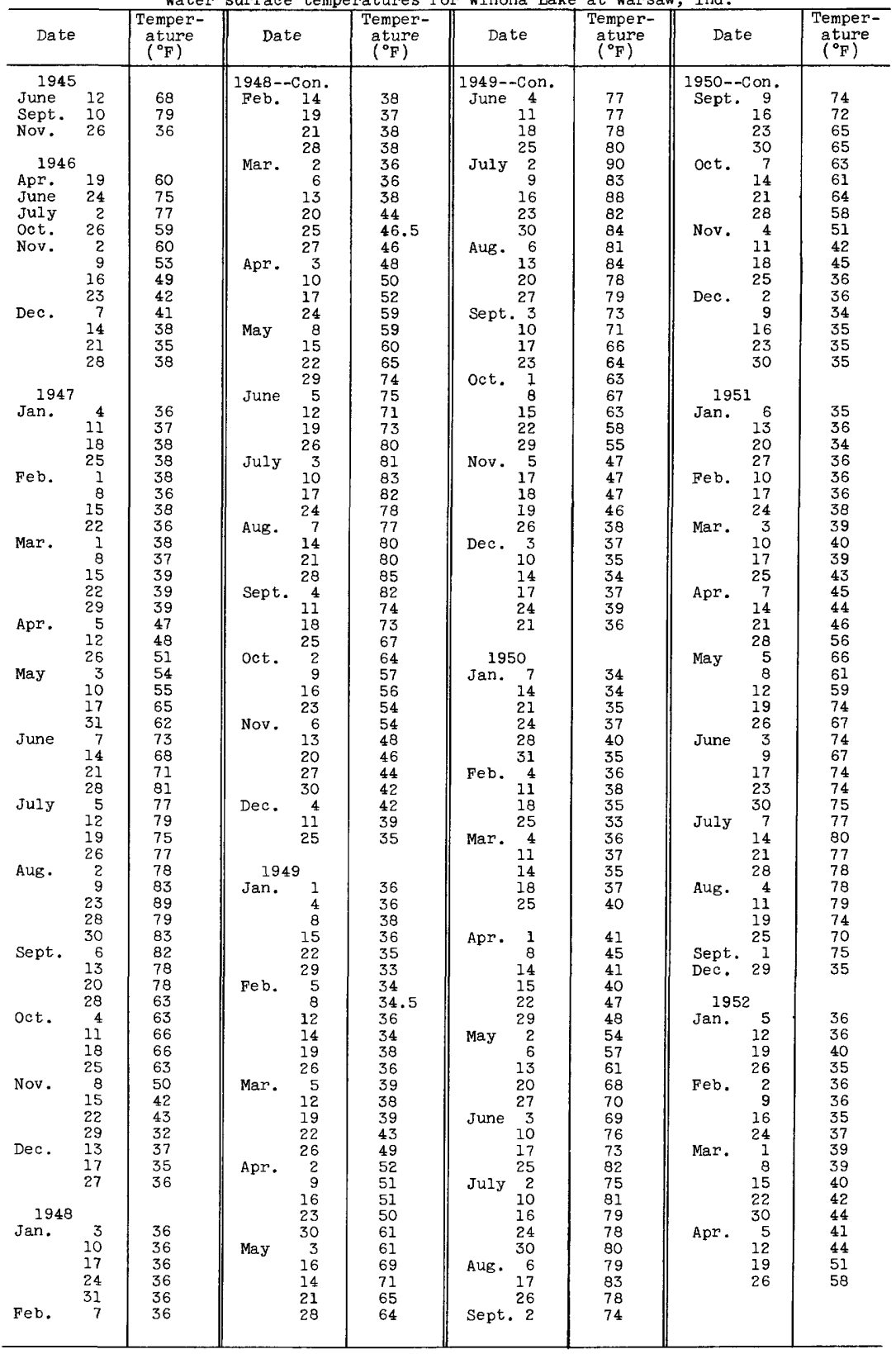


Temperature profiles $\left({ }^{\circ} \mathrm{F}\right)$ for Winona Lake, a Warsaw

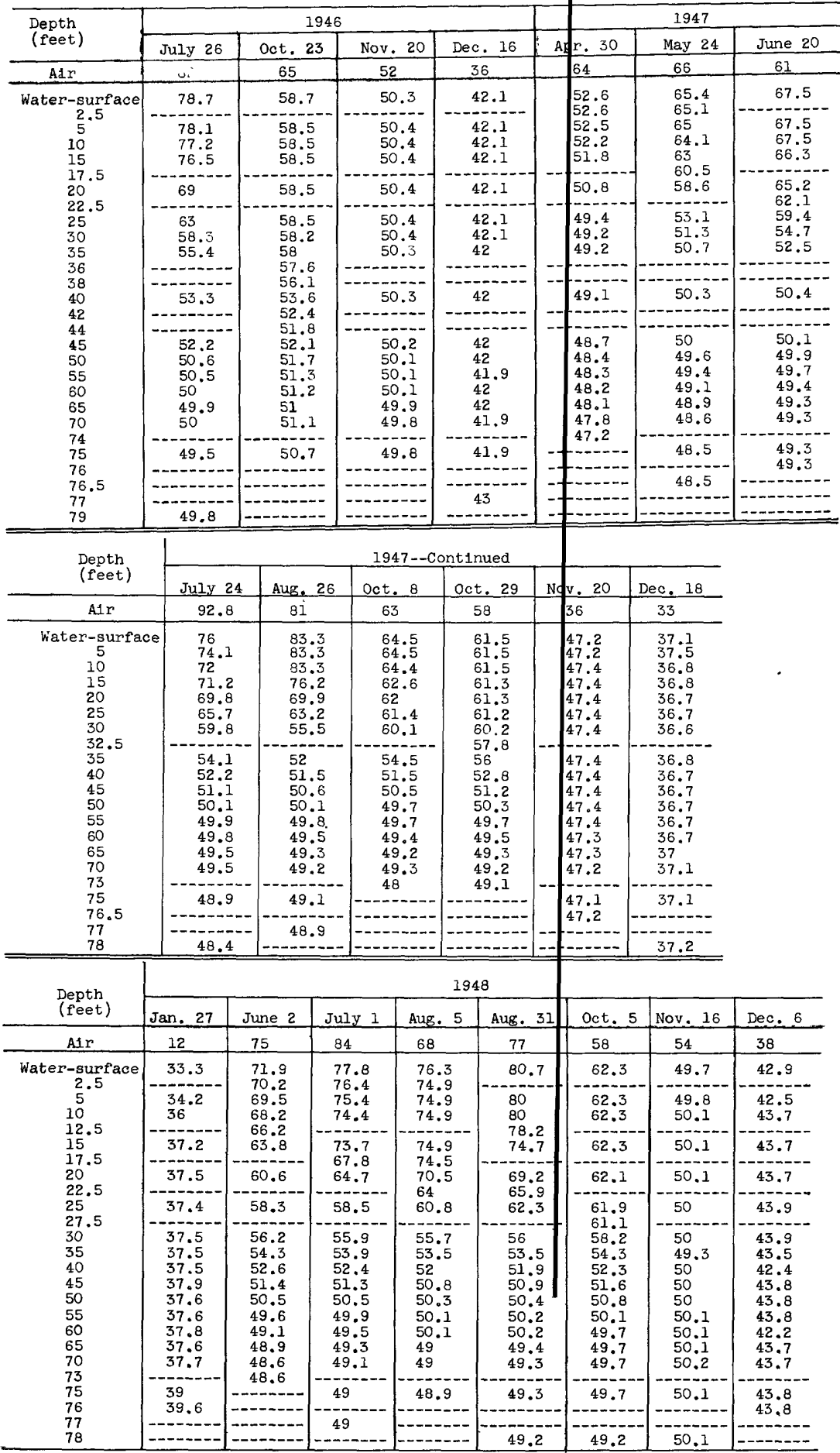




\section{EVAPORATION DATA}

The data in this section were collected at class A evaporation stations in Evansville, Indianapolis, Kendallville, and Valparaiso and compiled from data in the files of the U. S. Weather Bureau Office at Indianapolis. Some previously unpublished data, corrections of errors in previously published data, and estimates to supply missing data for completing monthly totals are included in this tabulation. These estimated were made by the author in collaboration with the members of the U. S. Weather Bureau at the Indianapolis office. Estimates were based on air temperatures, wind movement, and relative humidity. Water temperatures were not available for use in making estimates in most instances.

In studying the records it was found that in nearly all instances rain occurred on the days on which negative evaporation was observed. The exceptions to this occurred on November 23 and 24 , 1948, at Evansville and on December 3 and 4, 1948, and November 4, 1950, at Kendallville. On the latter 3 days the observer reported slushice in the pan which may have caused errors in the observations. It is believed that errors in determining the amount of rain that fell into the evaporation pan at times of low evaporation rates may account for negative evaporation being observed when it was not actually negative.

It was also noticed that heavy rain fell on days on which unusually high evaporation was observed. In some instances the record indicated that possibly the pan was filled to overflowing and some of the water lost and not reported by the observer. Where the observed amounts were obviously in error, estimations of the evaporation have been made by the author.

Records have been kept at most of the stations until freezing weather in the fall months prevented further readings. Where observations were made through the major part of a month, the remainder of the month was estimated in order to obtain a monthly total and also to fill out daily amounts for use in computing daily averages for the period of record. In using these figures, it should be kept in mind that observations could be made in winter months only when temperatures were above freezing. Consequently the evaporation amounts tabulated here are likely to be higher than a long time average would be that would include evaporation rates at both above and below freezing temperatures.

The daily observations in the tables are given in inches and represent evaporation from the class A type pan described on p. 50. 
Evansville. - The Evansville evaporation station was established on May 1, 1946 at the Evansville Airport, lat $38^{\circ} 01^{\prime} 55^{\prime \prime}$, long $87^{\circ} 32^{\prime} 19^{\prime \prime}$. Evaporation readings were made at 7 a.m. central standard time, from May 1946 to December 1946 and at 6 a. $\mathrm{m}$. since that time. Maximum and minimum pan water temperatures have been observed since October 1, 1952, and are available through the U. S. Weather Bureau.

Indianapolis. - - The Indianapolis evaporation station was established May 1, 1937, at the Riverside pumping staticn of the Indianapolis Water Company, lat $39^{\circ} 46^{\prime} 57^{\prime \prime}$, long. $86^{\circ} 11^{\prime} 07^{\prime \prime}$. The sta tion was moved to Geist Reservoir, lat $39^{\circ} 54^{\prime} 40^{\prime \prime}$, long. $85^{\circ} 59^{\prime} 14^{\prime \prime}$, on July 7, 1944. Evaporation observations were made at 7 a. $\mathrm{m}$. central standard time from May 1937 to July 6, 1944; at 5:30 a. m. from July 7, 1944, to October 31, 1944; and at noon either central standard time or central daylight saving time since April 1, 1945. Pan water temperatures have been observed since April 1, 1948, and are available through the U. S. Weather Bureau.

Kendallville. - - The Kendallville evaporation station was established on April 1, 1947, at the Kendallville water works, lat $41^{\circ}$ $26^{\prime} 26^{\prime \prime}$, long. $85^{\circ} 15^{\prime} 07^{\prime \prime}$. The station is located on top of a buried reservoir and is about 6 or 7 feet higher then the surrounding ground. Evaporation readings were generally made at about 9 a. $m$. central standard time during the months of October to April and at 8 a.m. from May to September until June 1948. From July 1948 to December 1953 observations were made at about $4 \mathrm{p}$. $\mathrm{m}$. The observations at Kendallville from April through October average about 6 percent higher than those at Indianapolis and 14 percent higher than those at Valparaiso. These higher values seem abnormal and may be due to the exposure at Kendallville. The reservoir on which the evaporation pan is located, although covered with earth, may produce different ground temperature characteristics than normal ground because of the water being continually changed in the reservoir. The elevated position of the pan may also cause higher evaporation rates.

Valparaiso. - The Valparaiso evaporation station was established on April 1, 1947, at the Valparaiso water plant on the shore of Flint Lake, lat $41^{\circ} 30^{\prime} 44^{\prime \prime}$, long. 87 $02^{\prime} 24^{\prime \prime}$. Observations were generally made between 7 and 8 a. m. central standard time, with the readings being nearer $7 \mathrm{a} . \mathrm{m}$. in the months of May to September and near 8 a.m. in the months October to April, during the period April 1947 to September 1951. Observations were changed to 5 p. m. on October 1, 1951, and have been made at about that time since then. 
MEASUREMENTS OF EVAPORATION AT 4 STATIONS IN INDIANA

Evaporation in inches, at Evansville, Ind.

[Observations were not made before May 1946 and during November 1951, 1952, and 1953]

\begin{tabular}{|c|c|c|c|c|c|c|c|c|c|}
\hline \multirow{2}{*}{\multicolumn{2}{|c|}{ 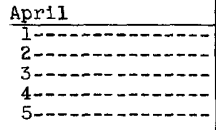 }} & 1947 & 1948 & 1949 & 1950 & 1951 & 1952 & 1953 & Average \\
\hline & & $\begin{array}{r}0.160 \\
.062 \\
.126 \\
.131 \\
.039\end{array}$ & $\begin{array}{r}0.158 \\
.163 \\
.087 \\
.188 \\
.223\end{array}$ & $\begin{array}{r}0.301 \\
.053 \\
.105 \\
.091 \\
.161\end{array}$ & $\begin{array}{l}0.23 \\
.15 \\
.15 \\
.00 \\
.17\end{array}$ & $\begin{array}{l}0.06 \\
.12 \\
.08 \\
.07 \\
.16\end{array}$ & $\begin{array}{l}0.13 \\
.24 \\
.19 \\
.09 \\
.06\end{array}$ & $\begin{array}{l}0.16 \\
.10 \\
.03 \\
.08 \\
.22\end{array}$ & $\begin{array}{l}0.171 \\
.127 \\
.110 \\
.093 \\
.148\end{array}$ \\
\hline \multicolumn{2}{|c|}{ 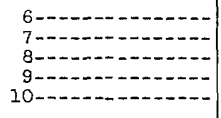 } & $\begin{array}{l}.241 \\
.334 \\
.149 \\
.037 \\
.163\end{array}$ & $\begin{array}{l}.126 \\
.198 \\
.080 \\
.323 \\
.183\end{array}$ & $\begin{array}{l}.035 \\
.173 \\
.184 \\
.196 \\
.214\end{array}$ & $\begin{array}{l}a \\
a \\
.05 \\
.07 \\
.23 \\
.00\end{array}$ & $\begin{array}{l}.12 \\
.07 \\
.08 \\
.10 \\
.10\end{array}$ & $\begin{array}{l}.09 \\
.10 \\
.15 \\
.23 \\
.30\end{array}$ & $\begin{array}{l}.10 \\
a .05 \\
a .03 \\
.09 \\
.33\end{array}$ & $\begin{array}{l}.112 \\
.139 \\
.120 \\
.172 \\
.184\end{array}$ \\
\hline \multicolumn{2}{|c|}{ 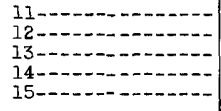 } & $\begin{array}{l}.241 \\
.113 \\
.188 \\
.129 \\
.172\end{array}$ & $\begin{array}{l}.137 \\
.277 \\
.282 \\
.026 \\
.045\end{array}$ & $\begin{array}{l}.150 \\
.035 \\
.107 \\
.081 \\
.347\end{array}$ & $\begin{array}{l}.25 \\
.15 \\
.04 \\
.16 \\
.14\end{array}$ & $\begin{array}{l}.14 \\
.02 \\
.06 \\
.00 \\
.14\end{array}$ & $\begin{array}{l}.08 \\
.08 \\
.18 \\
.09 \\
.03\end{array}$ & $\begin{array}{l}.21 \\
.06 \\
.03 \\
.18 \\
.22\end{array}$ & $\begin{array}{l}.173 \\
.112 \\
.127 \\
.095 \\
.156\end{array}$ \\
\hline \multicolumn{2}{|c|}{ 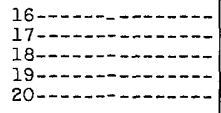 } & $\begin{array}{l}.045 \\
.086 \\
.230 \\
.298 \\
.220\end{array}$ & $\begin{array}{l}.085 \\
.269 \\
.181 \\
.226 \\
.312\end{array}$ & $\begin{array}{l}.094 \\
.129 \\
.186 \\
.098 \\
.132\end{array}$ & $\begin{array}{l}.13 \\
.17 \\
.09 \\
.20 \\
.15\end{array}$ & $\begin{array}{l}.17 \\
.11 \\
.17 \\
.22 \\
.33\end{array}$ & $\begin{array}{l}.10 \\
.18 \\
.15 \\
.17 \\
.24\end{array}$ & $\begin{array}{r}.28 \\
.22 \\
.11 \\
a .04 \\
. .03 \\
\end{array}$ & $\begin{array}{l}.129 \\
.166 \\
.160 \\
.179 \\
.202\end{array}$ \\
\hline \multicolumn{2}{|c|}{ 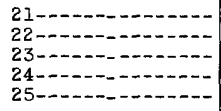 } & $\begin{array}{l}.143 \\
.042 \\
.075 \\
.296 \\
.010\end{array}$ & $\begin{array}{l}.211 \\
.161 \\
.141 \\
.255 \\
.293\end{array}$ & $\begin{array}{l}.129 \\
.036 \\
.156 \\
.377 \\
.214\end{array}$ & $\begin{array}{l}.20 \\
.25 \\
.24 \\
.26 \\
.25\end{array}$ & $\begin{array}{l}.11 \\
.02 \\
.10 \\
.16 \\
.27\end{array}$ & $\begin{array}{l}.24 \\
.07 \\
.13 \\
.01 \\
.06\end{array}$ & $\begin{array}{l}.27 \\
.14 \\
.33 \\
.40 \\
.12\end{array}$ & $\begin{array}{l}.186 \\
.103 \\
.167 \\
.251 \\
.174\end{array}$ \\
\hline \multicolumn{2}{|c|}{ 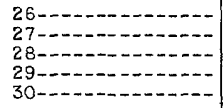 } & $\begin{array}{l}.052 \\
.194 \\
.247 \\
.133 \\
.126\end{array}$ & $\begin{array}{l}.270 \\
.205 \\
.374 \\
.156 \\
.209\end{array}$ & $\begin{array}{l}.274 \\
.073 \\
.157 \\
.305 \\
.187\end{array}$ & $\begin{array}{l}.21 \\
.19 \\
.12 \\
.18 \\
.09\end{array}$ & $\begin{array}{l}.33 \\
.12 \\
.18 \\
.34 \\
.28\end{array}$ & $\begin{array}{l}.15 \\
.33 \\
.28 \\
.42 \\
.30\end{array}$ & $\begin{array}{l}.33 \\
.06 \\
.12 \\
.31 \\
.12 \\
\end{array}$ & $\begin{array}{l}.231 \\
.167 \\
.211 \\
.263 \\
.187 \\
\end{array}$ \\
\hline \multicolumn{2}{|c|}{ TotaI--.-- } & 4.482 & 5.844 & 4.830 & 4.69 & 4,23 & 4.87 & 4.77 & 4.815 \\
\hline May & 1946 & 1947 & 1948 & 1949 & 1950 & 1951 & 1952 & 1953 & Average \\
\hline 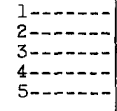 & $\begin{array}{r}0.043 \\
.109 \\
.102 \\
.085 \\
.117\end{array}$ & $\begin{array}{r}0.178 \\
.422 \\
.109 \\
.080 \\
.238\end{array}$ & $\begin{array}{r}0.158 \\
.139 \\
.293 \\
.134 \\
.076\end{array}$ & $\begin{array}{l}0.03 \\
.09 \\
.27 \\
.29 \\
.31\end{array}$ & $\begin{array}{r}0.08 \\
.02 \\
.01 \\
.12 \\
.27\end{array}$ & $\begin{array}{r}0.32 \\
.32 \\
.14 \\
.26 \\
.36\end{array}$ & $\begin{array}{r}0.26 \\
.23 \\
.25 \\
.14 \\
.32\end{array}$ & $\begin{array}{l}0.01 \\
.36 \\
.09 \\
.21 \\
.03\end{array}$ & $\begin{array}{r}0.135 \\
.211 \\
.158 \\
.165 \\
.215\end{array}$ \\
\hline 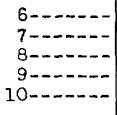 & $\begin{array}{l}.162 \\
.204 \\
.185 \\
.139 \\
.226\end{array}$ & $\begin{array}{l}.197 \\
.168 \\
.174 \\
.180 \\
.210\end{array}$ & $\begin{array}{l}.161 \\
.031 \\
.216 \\
.251 \\
.296\end{array}$ & $\begin{array}{l}.29 \\
.28 \\
.29 \\
.16 \\
.42\end{array}$ & $\begin{array}{r}.21 \\
.13 \\
.10 \\
.27 \\
. .15\end{array}$ & $\begin{array}{l}.18 \\
.07 \\
.19 \\
.27 \\
.19\end{array}$ & $\begin{array}{l}.42 \\
.31 \\
.32 \\
.21 \\
.21\end{array}$ & $\begin{array}{l}.16 \\
.17 \\
.08 \\
.12 \\
.22\end{array}$ & $\begin{array}{l}.222 \\
.170 \\
.194 \\
.200 \\
.240\end{array}$ \\
\hline 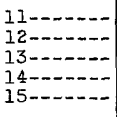 & $\begin{array}{l}.059 \\
.037 \\
.235 \\
.119 \\
.168\end{array}$ & $\begin{array}{l}.222 \\
.235 \\
.264 \\
.242 \\
.232\end{array}$ & $\begin{array}{l}.250 \\
.171 \\
.154 \\
.109 \\
.163\end{array}$ & $\begin{array}{l}.14 \\
.18 \\
.21 \\
.26 \\
.27\end{array}$ & $\begin{array}{r}c . .01 \\
.02 \\
.13 \\
.20 \\
.17\end{array}$ & $\begin{array}{l}.16 \\
.06 \\
.24 \\
.26 \\
.33\end{array}$ & $\begin{array}{l}.02 \\
.12 \\
.05 \\
.24 \\
.38\end{array}$ & $\begin{array}{r}.35 \\
.08 \\
.01 \\
.01 \\
a .06\end{array}$ & $\begin{array}{l}.149 \\
.113 \\
.162 \\
.180 \\
.222\end{array}$ \\
\hline 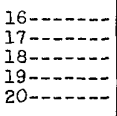 & $\begin{array}{l}.120 \\
.061 \\
.090 \\
.152 \\
.111\end{array}$ & $\begin{array}{l}.268 \\
.261 \\
.169 \\
.224 \\
.102\end{array}$ & $\begin{array}{l}.034 \\
.214 \\
.247 \\
.251 \\
.226\end{array}$ & $\begin{array}{l}.27 \\
.19 \\
.31 \\
.26 \\
.26\end{array}$ & $\begin{array}{l}.27 \\
.24 \\
.27 \\
.25 \\
.13\end{array}$ & $\begin{array}{l}.33 \\
.31 \\
.33 \\
.33 \\
.32\end{array}$ & $\begin{array}{l}.15 \\
.42 \\
.17 \\
.03 \\
.10\end{array}$ & $\begin{array}{r}a .08 \\
a .10 \\
a .10 \\
.10 \\
.18\end{array}$ & $\begin{array}{l}.190 \\
.224 \\
.211 \\
.200 \\
.179\end{array}$ \\
\hline 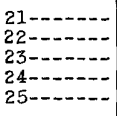 & $\begin{array}{l}.057 \\
.194 \\
.230 \\
.137 \\
.335\end{array}$ & $\begin{array}{l}.114 \\
.168 \\
.226 \\
.238 \\
.029\end{array}$ & $\begin{array}{l}.274 \\
.172 \\
.248 \\
.215 \\
.290\end{array}$ & $\begin{array}{l}.23 \\
.20 \\
.16 \\
.26 \\
.08\end{array}$ & $\begin{array}{l}.31 \\
.23 \\
.25 \\
.34 \\
.11\end{array}$ & $\begin{array}{l}.16 \\
.28 \\
.26 \\
.27 \\
.23\end{array}$ & $\begin{array}{l}.09 \\
.06 \\
.24 \\
.20 \\
.15\end{array}$ & $\begin{array}{l}.22 \\
.33 \\
.24 \\
.12 \\
.31\end{array}$ & $\begin{array}{l}.186 \\
.204 \\
.232 \\
.222 \\
.192\end{array}$ \\
\hline 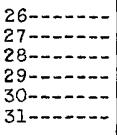 & $\begin{array}{l}.105 \\
.037 \\
.055 \\
.245 \\
.196 \\
.228\end{array}$ & $\begin{array}{l}.108 \\
.219 \\
.257 \\
.338 \\
.161 \\
.141 \\
\end{array}$ & $\begin{array}{l}.227 \\
.300 \\
.201 \\
.174 \\
.308 \\
.348 \\
\end{array}$ & $\begin{array}{l}.23 \\
.25 \\
.21 \\
.19 \\
.34 \\
.09 \\
\end{array}$ & $\begin{array}{l}.48 \\
.29 \\
.14 \\
.25 \\
.24 \\
.05 \\
\end{array}$ & $\begin{array}{l}.32 \\
.33 \\
.54 \\
.13 \\
.26 \\
.35 \\
\end{array}$ & $\begin{array}{l}.08 \\
.20 \\
.25 \\
.24 \\
.28 \\
.23 \\
\end{array}$ & $\begin{array}{l}.36 \\
.37 \\
.31 \\
.31 \\
.29 \\
.44\end{array}$ & $\begin{array}{r}.239 \\
.250 \\
.245 \\
.235 \\
.259 \\
.235 \\
\end{array}$ \\
\hline Tota1- & 4.343 & 6.204 & 6.331 & 7.02 & 5.72 & 8.10 & 6.37 & 5.82 & 6.239 \\
\hline
\end{tabular}

see footnotes at end of table. 
Evaporation in Inches at Evansville, Ind.--Continued

\begin{tabular}{|c|c|c|c|c|c|c|c|c|c|}
\hline June & 1946 & 1947 & 1948 & 1949 & 1950 & 1951 & 1952 & 1953 & Average \\
\hline 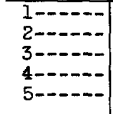 & $\begin{array}{r}0.155 \\
.216 \\
.071 \\
.201 \\
.228\end{array}$ & $\begin{array}{r}0.278 \\
.384 \\
.184 \\
.189 \\
.203\end{array}$ & $\begin{array}{r}0.382 \\
.337 \\
.274 \\
.295 \\
.378\end{array}$ & $\begin{array}{r}0.28 \\
.25 \\
.31 \\
.25 \\
.23\end{array}$ & $\begin{array}{r}0.27 \\
.19 \\
.17 \\
.05 \\
.22\end{array}$ & $\begin{array}{r}0.20 \\
.39 \\
.30 \\
.13 \\
.00\end{array}$ & $\begin{array}{r}0.35 \\
.32 \\
.24 \\
.24 \\
.32\end{array}$ & $\begin{array}{l}0.36 \\
.40 \\
.28 \\
.31 \\
.43\end{array}$ & $\begin{array}{r}0.284 \\
.311 \\
.229 \\
.208 \\
.251\end{array}$ \\
\hline 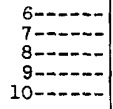 & $\begin{array}{l}.255 \\
.341 \\
.359 \\
.181 \\
.468\end{array}$ & $\begin{array}{l}.243 \\
.232 \\
.363 \\
.278 \\
.328\end{array}$ & $\begin{array}{l}.321 \\
.122 \\
.264 \\
.378 \\
.122\end{array}$ & $\begin{array}{l}.34 \\
.30 \\
.41 \\
.39 \\
.20\end{array}$ & $\begin{array}{l}.25 \\
.27 \\
.31 \\
.21 \\
.21\end{array}$ & $\begin{array}{l}.20 \\
.15 \\
.11 \\
.23 \\
.06\end{array}$ & $\begin{array}{l}.24 \\
.33 \\
.42 \\
.42 \\
.13\end{array}$ & $\begin{array}{l}.65 \\
.28 \\
.28 \\
.46 \\
.32\end{array}$ & $\begin{array}{l}.312 \\
.253 \\
.314 \\
.318 \\
.230\end{array}$ \\
\hline 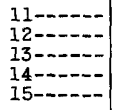 & $\begin{array}{l}.305 \\
.367 \\
.359 \\
.335 \\
.277\end{array}$ & $\begin{array}{l}.368 \\
.277 \\
.241 \\
.316 \\
.270\end{array}$ & $\begin{array}{l}.324 \\
.209 \\
.336 \\
.287 \\
.193\end{array}$ & $\begin{array}{l}.12 \\
.13 \\
.11 \\
.08 \\
.07\end{array}$ & $\begin{array}{l}.26 \\
.31 \\
.26 \\
.16 \\
.18\end{array}$ & $\begin{array}{l}.29 \\
.32 \\
.00 \\
.15 \\
.29\end{array}$ & $\begin{array}{l}.12 \\
.28 \\
.20 \\
.32 \\
.26\end{array}$ & $\begin{array}{l}.50 \\
.17 \\
.34 \\
.10 \\
.31\end{array}$ & $\begin{array}{l}.286 \\
.258 \\
.231 \\
.218 \\
.231\end{array}$ \\
\hline 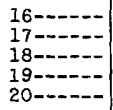 & $\begin{array}{l}.083 \\
.371 \\
.385 \\
.421 \\
.296\end{array}$ & $\begin{array}{l}.328 \\
.239 \\
.177 \\
.167 \\
.210\end{array}$ & $\begin{array}{l}.299 \\
.259 \\
.163 \\
.241 \\
.228\end{array}$ & $\begin{array}{l}.08 \\
.06 \\
.05 \\
.14 \\
.24\end{array}$ & $\begin{array}{l}.29 \\
.38 \\
.29 \\
.22 \\
.25\end{array}$ & $\begin{array}{l}.26 \\
.16 \\
.08 \\
.24 \\
.18\end{array}$ & $\begin{array}{l}.36 \\
.49 \\
.27 \\
.36 \\
.38\end{array}$ & $\begin{array}{l}.29 \\
.42 \\
.38 \\
.30 \\
.41\end{array}$ & $\begin{array}{l}.249 \\
.297 \\
.224 \\
.261 \\
.274\end{array}$ \\
\hline 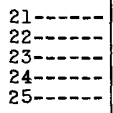 & $\begin{array}{l}.263 \\
.332 \\
.270 \\
.342 \\
.320\end{array}$ & $\begin{array}{l}.313 \\
.103 \\
.116 \\
.207 \\
.036\end{array}$ & $\begin{array}{l}.253 \\
.326 \\
.349 \\
.394 \\
.321\end{array}$ & $\begin{array}{l}.27 \\
.17 \\
.31 \\
.28 \\
.30\end{array}$ & $\begin{array}{l}.14 \\
.20 \\
.21 \\
.26 \\
.29\end{array}$ & $\begin{array}{l}.30 \\
.21 \\
.20 \\
.04 \\
.40\end{array}$ & $\begin{array}{l}.38 \\
.37 \\
.25 \\
.35 \\
.46\end{array}$ & $\begin{array}{l}.50 \\
.48 \\
.37 \\
.37 \\
.43\end{array}$ & $\begin{array}{l}.302 \\
.274 \\
.259 \\
.280 \\
.320\end{array}$ \\
\hline 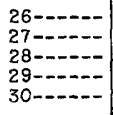 & $\begin{array}{r}.171 \\
.258 \\
.253 \\
.313 \\
.362\end{array}$ & $\begin{array}{l}.040 \\
.167 \\
.090 \\
.231 \\
.202\end{array}$ & $\begin{array}{l}.379 \\
.237 \\
.175 \\
.221 \\
.172\end{array}$ & $\begin{array}{l}.24 \\
.24 \\
.18 \\
.27 \\
.18\end{array}$ & $\begin{array}{l}.30 \\
.36 \\
.34 \\
.19 \\
.33\end{array}$ & $\begin{array}{l}.37 \\
.11 \\
.32 \\
.18 \\
.11\end{array}$ & $\begin{array}{l}.45 \\
.34 \\
.33 \\
.34 \\
.35\end{array}$ & $\begin{array}{l}.42 \\
.15 \\
.34 \\
.24 \\
.29\end{array}$ & $\begin{array}{l}.296 \\
.233 \\
.254 \\
.248 \\
.250\end{array}$ \\
\hline Total- & 8.558 & 6.780 & 8.239 & 6.48 & 7.47 & 5.98 & 9.67 & 10.58 & 7.955 \\
\hline July & 1946 & 1947 & 1948 & 1949 & 1950 & 1951 & 1952 & 1953 & Average \\
\hline 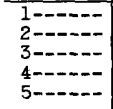 & $\begin{array}{r}0.360 \\
.209 \\
.328 \\
.330 \\
.198\end{array}$ & $\begin{array}{r}0.376 \\
.211 \\
.306 \\
.258 \\
.272\end{array}$ & $\begin{array}{r}0.283 \\
.294 \\
.288 \\
.316 \\
.251\end{array}$ & $\begin{array}{l}0.29 \\
.31 \\
.30 \\
.32 \\
.18\end{array}$ & $\begin{array}{l}0.28 \\
.30 \\
.20 \\
.33 \\
.11\end{array}$ & $\begin{array}{r}0.00 \\
.21 \\
.19 \\
.23 \\
.28\end{array}$ & $\begin{array}{r}0.47 \\
.33 \\
.35 \\
.14 \\
.35\end{array}$ & $\begin{array}{l}0.28 \\
.33 \\
.45 \\
.27 \\
.21\end{array}$ & $\begin{array}{r}0.292 \\
.274 \\
.302 \\
.274 \\
.231\end{array}$ \\
\hline 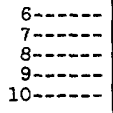 & $\begin{array}{l}.123 \\
.188 \\
.342 \\
.282 \\
.274\end{array}$ & $\begin{array}{l}.165 \\
.121 \\
.210 \\
.304 \\
.158\end{array}$ & $\begin{array}{l}.278 \\
.352 \\
.422 \\
.368 \\
.288\end{array}$ & $\begin{array}{l}.34 \\
.24 \\
.24 \\
.20 \\
.24\end{array}$ & $\begin{array}{l}.24 \\
.29 \\
.28 \\
.31 \\
.34\end{array}$ & $\begin{array}{l}.17 \\
.29 \\
.33 \\
.41 \\
.15\end{array}$ & $\begin{array}{l}.31 \\
.31 \\
.32 \\
.11 \\
.19\end{array}$ & $\begin{array}{l}.40 \\
.41 \\
.31 \\
.46 \\
.34\end{array}$ & $\begin{array}{l}.253 \\
.275 \\
.307 \\
.306 \\
.248\end{array}$ \\
\hline 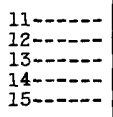 & $\begin{array}{l}.089 \\
.273 \\
.306 \\
.308 \\
.274\end{array}$ & $\begin{array}{l}.218 \\
.273 \\
.153 \\
.440 \\
.231\end{array}$ & $\begin{array}{l}.326 \\
.379 \\
.094 \\
.204 \\
.138\end{array}$ & $\begin{array}{l}.31 \\
.27 \\
.27 \\
.16 \\
.27\end{array}$ & $\begin{array}{l}.32 \\
.30 \\
.28 \\
.26 \\
.35\end{array}$ & $\begin{array}{l}.21 \\
.17 \\
.16 \\
.07 \\
.22\end{array}$ & $\begin{array}{l}.26 \\
.26 \\
.32 \\
.29 \\
.22\end{array}$ & $\begin{array}{l}.34 \\
.37 \\
.30 \\
.30 \\
.29\end{array}$ & $\begin{array}{l}.259 \\
.287 \\
.235 \\
.254 \\
.249\end{array}$ \\
\hline 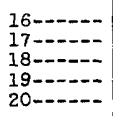 & $\begin{array}{l}.267 \\
.285 \\
.137 \\
.274 \\
.331\end{array}$ & $\begin{array}{l}.282 \\
.208 \\
.242 \\
.324 \\
.206\end{array}$ & $\begin{array}{l}.182 \\
.237 \\
.209 \\
.216 \\
.197\end{array}$ & $\begin{array}{l}.13 \\
.14 \\
.11 \\
.17 \\
.16\end{array}$ & $\begin{array}{l}.28 \\
.25 \\
.25 \\
.10 \\
.32\end{array}$ & $\begin{array}{l}.41 \\
.19 \\
.20 \\
.26 \\
.37\end{array}$ & $\begin{array}{l}.09 \\
.22 \\
.19 \\
.30 \\
.33\end{array}$ & $\begin{array}{r}.25 \\
.25 \\
a .09 \\
a .10 \\
.25\end{array}$ & $\begin{array}{l}.236 \\
.222 \\
.178 \\
.218 \\
.270\end{array}$ \\
\hline 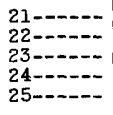 & $\begin{array}{l}.124 \\
.165 \\
.258 \\
.304 \\
.353\end{array}$ & $\begin{array}{l}.242 \\
.271 \\
.257 \\
.224 \\
.253\end{array}$ & $\begin{array}{l}.135 \\
.306 \\
.130 \\
.228 \\
.210\end{array}$ & $\begin{array}{l}.08 \\
.20 \\
.25 \\
.26 \\
.32\end{array}$ & $\begin{array}{l}.25 \\
.12 \\
.07 \\
.14 \\
.19\end{array}$ & $\begin{array}{l}.29 \\
.27 \\
.20 \\
.35 \\
.17\end{array}$ & $\begin{array}{l}.38 \\
.41 \\
.50 \\
.46 \\
.37\end{array}$ & $\begin{array}{l}.30 \\
.06 \\
.22 \\
.30 \\
.29\end{array}$ & $\begin{array}{l}.225 \\
.225 \\
.236 \\
.283 \\
.270\end{array}$ \\
\hline 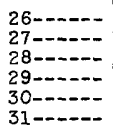 & $\begin{array}{l}.280 \\
.347 \\
.293 \\
.238 \\
.200 \\
.336\end{array}$ & $\begin{array}{l}.171 \\
.120 \\
.475 \\
.174 \\
.262 \\
.285\end{array}$ & $\begin{array}{l}.278 \\
.116 \\
.200 \\
.256 \\
.196 \\
.274\end{array}$ & $\begin{array}{l}.24 \\
.24 \\
.29 \\
.16 \\
.27 \\
.22\end{array}$ & $\begin{array}{l}.26 \\
.14 \\
.18 \\
.21 \\
.21 \\
.19\end{array}$ & $\begin{array}{l}.20 \\
.34 \\
.18 \\
.36 \\
.23 \\
.29\end{array}$ & $\begin{array}{l}.37 \\
.35 \\
.36 \\
.50 \\
.43 \\
.12\end{array}$ & $\begin{array}{l}.34 \\
.29 \\
.30 \\
.10 \\
.33 \\
.42\end{array}$ & $\begin{array}{l}.267 \\
.243 \\
.285 \\
.250 \\
.266 \\
.267\end{array}$ \\
\hline Total- & 8.076 & 7.692 & 7.651 & 7.18 & 7.35 & 7.40 & 9.61 & 8.95 & 7.987 \\
\hline
\end{tabular}

see footnotes at end of table. 
Evaporation in inches at Evansville, Ind.--Continued

\begin{tabular}{|c|c|c|c|c|c|c|c|c|c|}
\hline August & 1946 & 1947 & 1948 & 1949 & 1950 & 1951 & 1952 & 1953 & Average \\
\hline 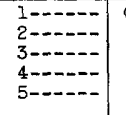 & $\begin{array}{r}0.256 \\
.243 \\
.026 \\
.175 \\
.181\end{array}$ & $\begin{array}{r}0.335 \\
.235 \\
.269 \\
.320 \\
.310\end{array}$ & $\begin{array}{r}0.330 \\
.261 \\
.242 \\
.282 \\
.100\end{array}$ & $\begin{array}{l}0.30 \\
.24 \\
.22 \\
.21 \\
.24\end{array}$ & $\begin{array}{l}0.15 \\
.23 \\
.19 \\
.32 \\
.21\end{array}$ & $\begin{array}{l}0.21 \\
.36 \\
.30 \\
.30 \\
.31\end{array}$ & $\begin{array}{l}0.31 \\
.27 \\
.26 \\
.30 \\
.24\end{array}$ & $\begin{array}{l}0.35 \\
.20 \\
.34 \\
.39 \\
.14\end{array}$ & $\begin{array}{r}0.280 \\
.255 \\
.231 \\
.287 \\
.216\end{array}$ \\
\hline 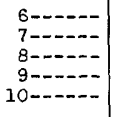 & $\begin{array}{l}.282 \\
.245 \\
.233 \\
.101 \\
.253\end{array}$ & $\begin{array}{l}.291 \\
.387 \\
.408 \\
.216 \\
.264\end{array}$ & $\begin{array}{l}.212 \\
.192 \\
.150 \\
.264 \\
.235\end{array}$ & $\begin{array}{r}.18 \\
.22 \\
.28 \\
.24 \\
6.20\end{array}$ & $\begin{array}{l}.25 \\
.26 \\
.24 \\
.08 \\
.06\end{array}$ & $\begin{array}{l}.36 \\
.22 \\
.31 \\
.17 \\
.23\end{array}$ & $\begin{array}{l}.26 \\
.21 \\
.28 \\
.24 \\
.24\end{array}$ & $\begin{array}{l}.23 \\
.22 \\
.27 \\
.27 \\
.20\end{array}$ & $\begin{array}{l}.258 \\
.244 \\
.271 \\
.198 \\
.210\end{array}$ \\
\hline 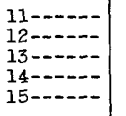 & $\begin{array}{l}.332 \\
.226 \\
.159 \\
.057 \\
.050\end{array}$ & $\begin{array}{l}.281 \\
.268 \\
.192 \\
.242 \\
.295\end{array}$ & $\begin{array}{l}.202 \\
.173 \\
.210 \\
.273 \\
.232\end{array}$ & $\begin{array}{l}.19 \\
.29 \\
.14 \\
.16 \\
.13\end{array}$ & $\begin{array}{l}.13 \\
.30 \\
.17 \\
.09 \\
.08\end{array}$ & $\begin{array}{l}.23 \\
.16 \\
.28 \\
.31 \\
.25\end{array}$ & $\begin{array}{l}.30 \\
.20 \\
.12 \\
.21 \\
.16\end{array}$ & $\begin{array}{l}.24 \\
.24 \\
.18 \\
.27 \\
.29\end{array}$ & $\begin{array}{l}.238 \\
.232 \\
.181 \\
.202 \\
.186\end{array}$ \\
\hline 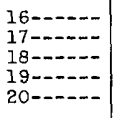 & $\begin{array}{l}.318 \\
.054 \\
.508 \\
.123 \\
.274\end{array}$ & $\begin{array}{l}.164 \\
.138 \\
.225 \\
.184 \\
.210\end{array}$ & $\begin{array}{l}.228 \\
.240 \\
.257 \\
.188 \\
.274\end{array}$ & $\begin{array}{l}.16 \\
.13 \\
.20 \\
.24 \\
.16\end{array}$ & $\begin{array}{l}.05 \\
.25 \\
.22 \\
.13 \\
.19\end{array}$ & $\begin{array}{l}.29 \\
.30 \\
.23 \\
.24 \\
.28\end{array}$ & $\begin{array}{l}.24 \\
.34 \\
.19 \\
.25 \\
.32\end{array}$ & $\begin{array}{l}.33 \\
.34 \\
.28 \\
.36 \\
.33\end{array}$ & $\begin{array}{l}.222 \\
.224 \\
.264 \\
.214 \\
.255\end{array}$ \\
\hline 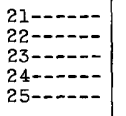 & $\begin{array}{l}.231 \\
.139 \\
.137 \\
.242 \\
.160\end{array}$ & $\begin{array}{l}.181 \\
.231 \\
.259 \\
.225 \\
.272\end{array}$ & $\begin{array}{l}.267 \\
.224 \\
.272 \\
.259 \\
.258\end{array}$ & $\begin{array}{l}.25 \\
.29 \\
.08 \\
.14 \\
.23\end{array}$ & $\begin{array}{l}.24 \\
.10 \\
.30 \\
.21 \\
.21\end{array}$ & $\begin{array}{l}.23 \\
.29 \\
.32 \\
.34 \\
.28\end{array}$ & $\begin{array}{l}.21 \\
.17 \\
.32 \\
.29 \\
.28\end{array}$ & $\begin{array}{l}.22 \\
.24 \\
.21 \\
.21 \\
.24\end{array}$ & $\begin{array}{l}.229 \\
.210 \\
.237 \\
.240 \\
.241\end{array}$ \\
\hline 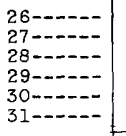 & $\begin{array}{l}.196 \\
.242 \\
.195 \\
.159 \\
.152 \\
.160 \\
\end{array}$ & $\begin{array}{l}.253 \\
.145 \\
.288 \\
.138 \\
.250 \\
.252 \\
\end{array}$ & $\begin{array}{l}.238 \\
.200 \\
.237 \\
.272 \\
.189 \\
.229 \\
\end{array}$ & $\begin{array}{l}.23 \\
.12 \\
.22 \\
.10 \\
.20 \\
.22 \\
\end{array}$ & $\begin{array}{l}.22 \\
.16 \\
.15 \\
.16 \\
.05 \\
.07 \\
\end{array}$ & $\begin{array}{l}.32 \\
.21 \\
.01 \\
.06 \\
.05 \\
.27 \\
\end{array}$ & $\begin{array}{l}.19 \\
.24 \\
.27 \\
.26 \\
.17 \\
.06\end{array}$ & $\begin{array}{l}.27 \\
.31 \\
.29 \\
.30 \\
.17 \\
.32 \\
\end{array}$ & $\begin{array}{l}.240 \\
.203 \\
.208 \\
.181 \\
.154 \\
.128 \\
\end{array}$ \\
\hline Total- & 6.109 & 7.728 & 7.190 & 6.21 & 5.47 & 7.72 & 7.40 & 8.25 & 7.009 \\
\hline September & 1946 & 1947 & 1948 & 1949 & 1950 & 1951 & 1952 & 1953 & Average \\
\hline 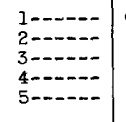 & $\begin{array}{r}0.147 \\
.035 \\
.163 \\
.182 \\
.177\end{array}$ & $\begin{array}{r}0.064 \\
.224 \\
.243 \\
.264 \\
.257\end{array}$ & $\begin{array}{r}0.341 \\
.282 \\
.240 \\
.199 \\
.220\end{array}$ & $\begin{array}{r}0.27 \\
.21 \\
.21 \\
.18 \\
.20\end{array}$ & $\begin{array}{l}0.26 \\
.03 \\
.01 \\
.12 \\
.28\end{array}$ & $\begin{array}{r}0.29 \\
.28 \\
.28 \\
.25 \\
.40\end{array}$ & $\begin{array}{r}0.22 \\
.34 \\
.21 \\
.19 \\
.18\end{array}$ & $\begin{array}{l}0.31 \\
.28 \\
.25 \\
.34 \\
.10\end{array}$ & $\begin{array}{r}0.238 \\
.210 \\
.201 \\
.216 \\
.227\end{array}$ \\
\hline $\begin{array}{r}6 \ldots-\ldots \\
7 \ldots-\cdots \\
9-\cdots-\cdots-\end{array}$ & $\begin{array}{l}.158 \\
.193 \\
.164 \\
.189 \\
.096\end{array}$ & $\begin{array}{l}.294 \\
.206 \\
.242 \\
.280 \\
.114\end{array}$ & $\begin{array}{l}.091 \\
.076 \\
.089 \\
.197 \\
.134\end{array}$ & $\begin{array}{l}.21 \\
.23 \\
.18 \\
.25 \\
.19\end{array}$ & $\begin{array}{l}.25 \\
.23 \\
.10 \\
.05 \\
.05\end{array}$ & $\begin{array}{l}.15 \\
.20 \\
.28 \\
.20 \\
.27\end{array}$ & $\begin{array}{r}.17 \\
.20 \\
6.20 \\
.18 \\
.19\end{array}$ & $\begin{array}{l}.26 \\
.27 \\
.28 \\
.25 \\
.23\end{array}$ & $\begin{array}{l}.198 \\
.201 \\
.192 \\
.200 \\
.159\end{array}$ \\
\hline 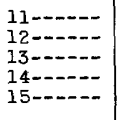 & $\begin{array}{l}.236 \\
.194 \\
.088 \\
.238 \\
.083\end{array}$ & $\begin{array}{l}.162 \\
.286 \\
.061 \\
.267 \\
.344\end{array}$ & $\begin{array}{l}.203 \\
.252 \\
.214 \\
.170 \\
.247\end{array}$ & $\begin{array}{l}.20 \\
.28 \\
.06 \\
.14 \\
.14\end{array}$ & $\begin{array}{l}.03 \\
.08 \\
.12 \\
.18 \\
.11\end{array}$ & $\begin{array}{l}.07 \\
.32 \\
.29 \\
.00 \\
.20\end{array}$ & $\begin{array}{l}.26 \\
.26 \\
.23 \\
.19 \\
.11\end{array}$ & $\begin{array}{l}.22 \\
.43 \\
.26 \\
.23 \\
.29\end{array}$ & $\begin{array}{l}.173 \\
.263 \\
.165 \\
.177 \\
.190\end{array}$ \\
\hline 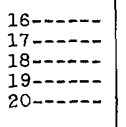 & $\begin{array}{l}.172 \\
.196 \\
.139 \\
.111 \\
.034\end{array}$ & $\begin{array}{l}.156 \\
.196 \\
.164 \\
.207 \\
.233\end{array}$ & $\begin{array}{l}.216 \\
.176 \\
.198 \\
.225 \\
.363\end{array}$ & $\begin{array}{l}.05 \\
.04 \\
.08 \\
.10 \\
.27\end{array}$ & $\begin{array}{l}.09 \\
.15 \\
.19 \\
.17 \\
.20\end{array}$ & $\begin{array}{l}.24 \\
.20 \\
.20 \\
.19 \\
.19\end{array}$ & $\begin{array}{l}.25 \\
.27 \\
.12 \\
.19 \\
.17\end{array}$ & $\begin{array}{l}.21 \\
.30 \\
.24 \\
.26 \\
.07\end{array}$ & $\begin{array}{l}.173 \\
.191 \\
.166 \\
.182 \\
.191\end{array}$ \\
\hline $\begin{array}{l}21 \ldots \ldots \\
22 \ldots \ldots \ldots- \\
23 \ldots \ldots \\
24 \ldots \ldots- \\
25 \ldots \ldots-\end{array}$ & $\begin{array}{l}.201 \\
.145 \\
.046 \\
.170 \\
.161\end{array}$ & $\begin{array}{l}.296 \\
.384 \\
.238 \\
.158 \\
.186\end{array}$ & $\begin{array}{l}.280 \\
.169 \\
.099 \\
.259 \\
.178\end{array}$ & $\begin{array}{l}.09 \\
.20 \\
.26 \\
.19 \\
.14\end{array}$ & $\begin{array}{l}.18 \\
.19 \\
.08 \\
.21 \\
.15\end{array}$ & $\begin{array}{l}.29 \\
.12 \\
.13 \\
.22 \\
.01\end{array}$ & $\begin{array}{l}.17 \\
.20 \\
.18 \\
.18 \\
.18\end{array}$ & $\begin{array}{l}.26 \\
.25 \\
.11 \\
.22 \\
.30\end{array}$ & $\begin{array}{l}.221 \\
.207 \\
.143 \\
.201 \\
.163\end{array}$ \\
\hline 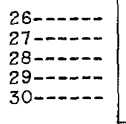 & $\begin{array}{l}.148 \\
.144 \\
.163 \\
.094 \\
.160\end{array}$ & $\begin{array}{l}.152 \\
.228 \\
.200 \\
.170 \\
.165\end{array}$ & $\begin{array}{l}.276 \\
.225 \\
.326 \\
.011 \\
.035\end{array}$ & $\begin{array}{l}.16 \\
.16 \\
.17 \\
.25 \\
.16\end{array}$ & $\begin{array}{l}.14 \\
.04 \\
.03 \\
.09 \\
.10\end{array}$ & $\begin{array}{l}.10 \\
.17 \\
.29 \\
.18 \\
.14\end{array}$ & $\begin{array}{l}.15 \\
.22 \\
.17 \\
.20 \\
.22\end{array}$ & $\begin{array}{l}.26 \\
.21 \\
.29 \\
.25 \\
.37\end{array}$ & $\begin{array}{l}.173 \\
.175 \\
.205 \\
.156 \\
.169\end{array}$ \\
\hline Total- & 4.427 & 6.441 & 5.991 & 5.27 & 3.91 & 6.15 & 6.00 & 7.60 & 5.726 \\
\hline
\end{tabular}

See footnotes at end of table. 
Evaporation, in inches, at Evansville, Ind.--Continued

\begin{tabular}{|c|c|c|c|c|c|c|c|c|c|}
\hline October & 1946 & 1947 & 1948 & 1949 & 1950 & 1951 & 1952 & 1953 & Average \\
\hline 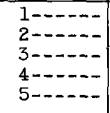 & $\begin{array}{r}0.180 \\
.133 \\
.141 \\
.147 \\
.166\end{array}$ & $\begin{array}{r}0.187 \\
.124 \\
.321 \\
.157 \\
.150\end{array}$ & $\begin{array}{r}0.107 \\
.120 \\
.198 \\
.139 \\
.090\end{array}$ & $\begin{array}{r}0.15 \\
.16 \\
.03 \\
.08 \\
.01\end{array}$ & $\begin{array}{l}0.10 \\
.08 \\
.03 \\
.07 \\
.20\end{array}$ & $\begin{array}{r}0.19 \\
.11 \\
.27 \\
.24 \\
.24\end{array}$ & $\begin{array}{r}0.23 \\
.31 \\
.28 \\
.12 \\
.23\end{array}$ & $\begin{array}{r}0.35 \\
.27 \\
.22 \\
.24 \\
.15\end{array}$ & $\begin{array}{r}0.187 \\
.163 \\
.186 \\
.149 \\
.154\end{array}$ \\
\hline $\begin{array}{r}6 \cdots-\cdots- \\
7-\cdots-\cdots- \\
8-\cdots-\cdots- \\
9-\cdots-\cdots- \\
10-\cdots-\cdots-\end{array}$ & $\begin{array}{l}.149 \\
.160 \\
.171 \\
.138 \\
.130\end{array}$ & $\begin{array}{l}.124 \\
.174 \\
.219 \\
.181 \\
.156\end{array}$ & $\begin{array}{l}.108 \\
.159 \\
.227 \\
.091 \\
.152\end{array}$ & $\begin{array}{l}.01 \\
.57 \\
.14 \\
.05 \\
.11\end{array}$ & $\begin{array}{l}.10 \\
.14 \\
.12 \\
.10 \\
.04\end{array}$ & $\begin{array}{l}.22 \\
.17 \\
.08 \\
.09 \\
.11\end{array}$ & $\begin{array}{l}.03 \\
.06 \\
.13 \\
.16 \\
.17\end{array}$ & $\begin{array}{l}.03 \\
.08 \\
.14 \\
.08 \\
.12\end{array}$ & $\begin{array}{l}.096 \\
.189 \\
.153 \\
.111 \\
.124\end{array}$ \\
\hline 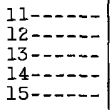 & $\begin{array}{l}.125 \\
.101 \\
.150 \\
.117 \\
.005\end{array}$ & $\begin{array}{l}.111 \\
.110 \\
.120 \\
.159 \\
.110\end{array}$ & $\begin{array}{l}.101 \\
.159 \\
.096 \\
.146 \\
.134\end{array}$ & $\begin{array}{l}.24 \\
.19 \\
.19 \\
.14 \\
.13\end{array}$ & $\begin{array}{l}.13 \\
.22 \\
.23 \\
.12 \\
.21\end{array}$ & $\begin{array}{l}.12 \\
.11 \\
.12 \\
.16 \\
.17\end{array}$ & $\begin{array}{l}.13 \\
.09 \\
.18 \\
.23 \\
.16\end{array}$ & $\begin{array}{l}.14 \\
.26 \\
.19 \\
.19 \\
.12\end{array}$ & $\begin{array}{l}.137 \\
.155 \\
.160 \\
.158 \\
.130\end{array}$ \\
\hline 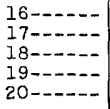 & $\begin{array}{l}.132 \\
.108 \\
.062 \\
.056 \\
.039\end{array}$ & $\begin{array}{l}.392 \\
.134 \\
.031 \\
.133 \\
.110\end{array}$ & $\begin{array}{l}.211 \\
.039 \\
.046 \\
.092 \\
.131\end{array}$ & $\begin{array}{l}.18 \\
.13 \\
.08 \\
.12 \\
.11\end{array}$ & $\begin{array}{l}.12 \\
.14 \\
.12 \\
.14 \\
.17\end{array}$ & $\begin{array}{l}.15 \\
.17 \\
.14 \\
.19 \\
.20\end{array}$ & $\begin{array}{l}.11 \\
.10 \\
.18 \\
.11 \\
.17\end{array}$ & $\begin{array}{l}.16 \\
.21 \\
.18 \\
.17 \\
.12\end{array}$ & $\begin{array}{l}.182 \\
.129 \\
.105 \\
.126 \\
.131\end{array}$ \\
\hline $\begin{array}{l}21-\cdots---- \\
22-\cdots--- \\
23-\cdots---- \\
24-\cdots-\cdots- \\
25-\cdots-\cdots\end{array}$ & $\begin{array}{l}.100 \\
.048 \\
.099 \\
.095 \\
.127\end{array}$ & $\begin{array}{l}.130 \\
.088 \\
.166 \\
.151 \\
.078\end{array}$ & $\begin{array}{l}.110 \\
.010 \\
.086 \\
.087 \\
.048\end{array}$ & $\begin{array}{l}.05 \\
.05 \\
.15 \\
.15 \\
.08\end{array}$ & $\begin{array}{l}.26 \\
.11 \\
.05 \\
.09 \\
.05\end{array}$ & $\begin{array}{l}.09 \\
.12 \\
.10 \\
.12 \\
.12\end{array}$ & $\begin{array}{l}a .14 \\
\alpha .12 \\
a .10 \\
.11 \\
.11\end{array}$ & $\begin{array}{l}.14 \\
.16 \\
.15 \\
.15 \\
.11\end{array}$ & $\begin{array}{l}.128 \\
.088 \\
.113 \\
.119 \\
.090\end{array}$ \\
\hline 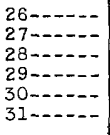 & $\begin{array}{l}.094 \\
.120 \\
.060 \\
.082 \\
.164 \\
.138\end{array}$ & $\begin{array}{l}.129 \\
.194 \\
.181 \\
.091 \\
.027 \\
.022\end{array}$ & $\begin{array}{l}.066 \\
.047 \\
.060 \\
.063 \\
.091 \\
.062\end{array}$ & $\begin{array}{l}.11 \\
.11 \\
.07 \\
.08 \\
.06 \\
.04\end{array}$ & $\begin{array}{l}.11 \\
.10 \\
.09 \\
.12 \\
.10 \\
.09\end{array}$ & $\begin{array}{l}.10 \\
.11 \\
.01 \\
.07 \\
.02 \\
.16 \\
\end{array}$ & $\begin{array}{l}.10 \\
.21 \\
.31 \\
.16 \\
.07 \\
.22 \\
\end{array}$ & $\begin{array}{r}.10 \\
a .02 \\
a .03 \\
. .03 \\
.10 \\
.12 \\
\end{array}$ & $\begin{array}{l}.101 \\
.114 \\
.101 \\
.087 \\
.079 \\
.106 \\
\end{array}$ \\
\hline Total - & 3.537 & 4.460 & 3.276 & 3.77 & 3.75 & 4.27 & 4.83 & 4.53 & 4.051 \\
\hline November & 1946 & 1947 & 1948 & 1949 & 1950 & Average & December & 1946 & \\
\hline 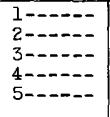 & $\begin{array}{r}0.059 \\
.068 \\
.001 \\
.049 \\
.053\end{array}$ & $\begin{array}{r}-0.007 \\
-.013 \\
.002 \\
.010 \\
.016\end{array}$ & $\begin{array}{r}-0.013 \\
.051 \\
-.024 \\
.011 \\
.265\end{array}$ & $\begin{array}{r}0.07 \\
.11 \\
.12 \\
.07 \\
.12\end{array}$ & $\begin{array}{r}0.17 \\
.24 \\
.13 \\
.01 \\
. .05\end{array}$ & $\begin{array}{r}0.056 \\
.091 \\
.046 \\
.030 \\
.101\end{array}$ & 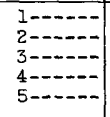 & $\begin{array}{l}0.058 \\
a .040 \\
.040 \\
a .040 \\
a .040 \\
\end{array}$ & \\
\hline 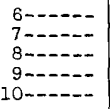 & $\begin{array}{l}.100 \\
.050 \\
.182 \\
.046 \\
.037\end{array}$ & $\begin{array}{l}.056 \\
.084 \\
.066 \\
.103 \\
.102\end{array}$ & $\begin{array}{l}.037 \\
.057 \\
.069 \\
.082 \\
.047\end{array}$ & $\begin{array}{l}.07 \\
.09 \\
.12 \\
.11 \\
.13\end{array}$ & $\begin{array}{l}.09 \\
.13 \\
.04 \\
.05 \\
.11\end{array}$ & $\begin{array}{l}.071 \\
.082 \\
.095 \\
.078 \\
.085\end{array}$ & $\begin{array}{r}6-\cdots-- \\
7 \cdots-\cdots \\
8-\cdots-\cdots \\
9-\cdots \cdots \\
10-\cdots-\cdots\end{array}$ & $\begin{array}{r}.033 \\
.046 \\
.080 \\
-.016 \\
.073\end{array}$ & \\
\hline 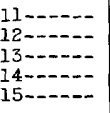 & $\begin{array}{l}.012 \\
.059 \\
.038 \\
.056 \\
.056\end{array}$ & $\begin{array}{r}.077 \\
.010 \\
.009 \\
.084 \\
. .012\end{array}$ & $\begin{array}{l}.071 \\
.063 \\
.050 \\
.081 \\
.111\end{array}$ & $\begin{array}{l}.16 \\
.11 \\
.11 \\
.04 \\
.10\end{array}$ & $\begin{array}{l}a .01 \\
\alpha .02 \\
\alpha .03 \\
.09 \\
.11\end{array}$ & $\begin{array}{l}.066 \\
.052 \\
.047 \\
.070 \\
.073\end{array}$ & 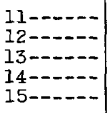 & $\begin{array}{r}.095 \\
-.045 \\
.059 \\
.032 \\
.076\end{array}$ & \\
\hline 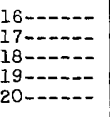 & $\begin{array}{r}.087 \\
.005 \\
a .061 \\
a .062 \\
.021\end{array}$ & $\begin{array}{r}.032 \\
.128 \\
.048 \\
-.024 \\
.065\end{array}$ & $\begin{array}{r}.070 \\
-.018 \\
.072 \\
.039 \\
.155\end{array}$ & $\begin{array}{l}.11 \\
.14 \\
.03 \\
.07 \\
.11\end{array}$ & $\begin{array}{r}c .00 \\
.19 \\
.07 \\
.10 \\
.08\end{array}$ & $\begin{array}{l}.060 \\
.089 \\
.056 \\
.049 \\
.086\end{array}$ & 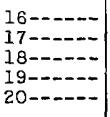 & $\begin{array}{r}.037 \\
.076 \\
\hdashline-0-0 \\
\hdashline-0-0\end{array}$ & \\
\hline 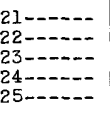 & $\begin{array}{r}.023 \\
.063 \\
a .080 \\
a .108 \\
.156\end{array}$ & $\begin{array}{r}.048 \\
-.001 \\
.047 \\
-.006 \\
b .040\end{array}$ & $\begin{array}{r}.054 \\
.045 \\
-.015 \\
-.022 \\
.0121\end{array}$ & $\begin{array}{r}.14 \\
a .03 \\
\alpha .02 \\
.08 \\
.02\end{array}$ & $\begin{array}{l}b .06 \\
b .05 \\
b .04 \\
b .01 \\
b .01\end{array}$ & $\begin{array}{l}.065 \\
.037 \\
.034 \\
.034 \\
.069\end{array}$ & $\begin{array}{l}2.1-\cdots---- \\
22------ \\
23------ \\
24-\cdots---- \\
25-\cdots----\end{array}$ & 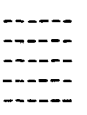 & \\
\hline 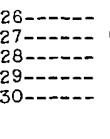 & $\begin{array}{l}.176 \\
.008 \\
.007 \\
.072 \\
.044\end{array}$ & $\begin{array}{l}b .040 \\
b .030 \\
b .040 \\
b .030 \\
b .020\end{array}$ & $\begin{array}{l}.090 \\
.002 \\
.120 \\
.056 \\
.038\end{array}$ & $\begin{array}{l}.02 \\
.09 \\
.04 \\
.00 \\
.05\end{array}$ & $\begin{array}{l}b .02 \\
.6 .02 \\
b .02 \\
b .03 \\
b .03\end{array}$ & $\begin{array}{l}.069 \\
.030 \\
.045 \\
.038 \\
.036\end{array}$ & $\begin{array}{l}26-\cdots--- \\
27-\cdots--- \\
28 \cdots---- \\
29-\cdots--- \\
30-\cdots--- \\
31-\cdots----\end{array}$ & 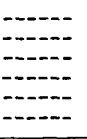 & \\
\hline Total- & 1.839 & 1.124 & 1.765 & 2.48 & 2.01 & 1.840 & & & \\
\hline
\end{tabular}

astimated from total observed on underlined day.

- Estimated because of missing or erroneous data.

- Corrected since orlginally published by U. S. Weather Bureau.

$d$ Not previously published. 
MEASUREMENTS OF EVAPORATION AT 4 STATIONS IN INDIANA

Evaporation in inches, in Indianapolis, Ind.

[No observations were made during November 1951].

\begin{tabular}{|c|c|c|c|c|c|c|c|c|c|}
\hline \multirow{2}{*}{\multicolumn{2}{|c|}{ April }} & & & & & & & & \\
\hline & & 1938 & 1939 & 1940 & 1941 & 1942 & 1943 & 1944 & 1945 \\
\hline \multicolumn{2}{|c|}{ 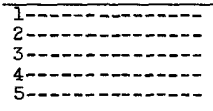 } & $\begin{array}{r}0.182 \\
.162 \\
.072 \\
.113 \\
.152\end{array}$ & $\begin{array}{l}0.104 \\
6.164 \\
6.130 \\
6.138 \\
. .118\end{array}$ & $\begin{array}{r}0.388 \\
.177 \\
.196 \\
.236 \\
.070\end{array}$ & $\begin{array}{r}0.144 \\
.182 \\
.164 \\
.073 \\
.013\end{array}$ & $\begin{array}{r}6.159 \\
. .247 \\
b .159 \\
b .191 \\
b .243\end{array}$ & $\begin{array}{r}0.266 \\
.218 \\
.084 \\
.132 \\
.131\end{array}$ & $\begin{array}{r}0.116 \\
.083 \\
.079 \\
.190 \\
.047\end{array}$ & $\begin{array}{r}0.052 \\
.060 \\
.070 \\
.040 \\
.044\end{array}$ \\
\hline \multicolumn{2}{|c|}{ 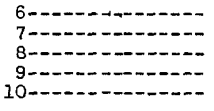 } & $\begin{array}{l}.153 \\
.162 \\
.015 \\
.089 \\
.103\end{array}$ & $\begin{array}{r}.150 \\
.138 \\
.084 \\
.098 \\
.066\end{array}$ & $\begin{array}{l}.241 \\
.166 \\
.008 \\
.057 \\
.040\end{array}$ & $\begin{array}{l}.036 \\
.064 \\
.189 \\
.077 \\
.019\end{array}$ & $\begin{array}{l}b .207 \\
6.130 \\
6.071 \\
b .111 \\
b .159\end{array}$ & $\begin{array}{l}.102 \\
.201 \\
.192 \\
.190 \\
.030\end{array}$ & $\begin{array}{r}.061 \\
.109 \\
.084 \\
.018 \\
.091\end{array}$ & $\begin{array}{r}.178 \\
.172 \\
.104 \\
.232 \\
.195\end{array}$ \\
\hline \multicolumn{2}{|c|}{ 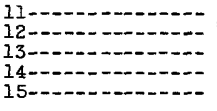 } & $\begin{array}{l}.108 \\
.149 \\
.136 \\
.218 \\
.260\end{array}$ & $\begin{array}{l}.212 \\
.072 \\
.162 \\
.111 \\
.120\end{array}$ & $\begin{array}{l}.072 \\
.060 \\
.010 \\
.010 \\
.125\end{array}$ & $\begin{array}{l}.095 \\
.222 \\
.172 \\
.292 \\
.296\end{array}$ & $\begin{array}{r}.075 \\
.071 \\
.110 \\
.111 \\
.123\end{array}$ & $\begin{array}{l}.201 \\
.049 \\
.214 \\
.045 \\
.062\end{array}$ & $\begin{array}{r}.140 \\
.195 \\
.021 \\
.246 \\
.101\end{array}$ & $\begin{array}{l}.117 \\
.333 \\
.094 \\
.070 \\
.048\end{array}$ \\
\hline \multicolumn{2}{|c|}{ 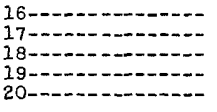 } & $\begin{array}{l}.179 \\
.148 \\
.223 \\
.130 \\
.269\end{array}$ & $\begin{array}{l}. .100 \\
.100 \\
.116 \\
.030 \\
.097\end{array}$ & $\begin{array}{l}.033 \\
.122 \\
.191 \\
.071 \\
.031\end{array}$ & $\begin{array}{l}.076 \\
.170 \\
.164 \\
.062 \\
.332\end{array}$ & $\begin{array}{l}.209 \\
.214 \\
.258 \\
.166 \\
.156\end{array}$ & $\begin{array}{r}.141 \\
.086 \\
.150 \\
.126 \\
. .034\end{array}$ & $\begin{array}{l}.019 \\
.015 \\
.094 \\
.120 \\
.184\end{array}$ & $\begin{array}{l}.011 \\
.154 \\
.128 \\
.120 \\
.142\end{array}$ \\
\hline \multicolumn{2}{|c|}{ 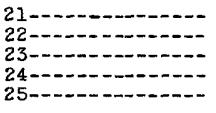 } & $\begin{array}{l}.100 \\
.202 \\
.223 \\
.139 \\
.265\end{array}$ & $\begin{array}{l}.130 \\
.003 \\
.092 \\
.048 \\
.449\end{array}$ & $\begin{array}{l}.027 \\
.142 \\
.203 \\
.011 \\
.104\end{array}$ & $\begin{array}{l}.223 \\
.183 \\
.154 \\
.118 \\
.196\end{array}$ & $\begin{array}{l}.174 \\
.157 \\
.149 \\
.189 \\
.141\end{array}$ & $\begin{array}{r}.020 \\
.100 \\
.247 \\
-.050 \\
.103\end{array}$ & $\begin{array}{l}.032 \\
.162 \\
.043 \\
.085 \\
.118\end{array}$ & $\begin{array}{l}.156 \\
.150 \\
.117 \\
.010 \\
.031\end{array}$ \\
\hline \multicolumn{2}{|c|}{ 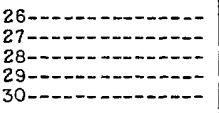 } & $\begin{array}{l}.229 \\
.297 \\
.399 \\
.230 \\
.140\end{array}$ & $\begin{array}{l}.058 \\
.141 \\
.177 \\
.140 \\
.218\end{array}$ & $\begin{array}{l}.148 \\
.130 \\
.222 \\
.288 \\
.104\end{array}$ & $\begin{array}{l}.150 \\
.138 \\
.130 \\
.200 \\
.214\end{array}$ & $\begin{array}{l}.178 \\
.074 \\
.188 \\
.142 \\
.216\end{array}$ & $\begin{array}{l}.052 \\
.390 \\
.184 \\
.148 \\
.120\end{array}$ & $\begin{array}{l}.284 \\
.054 \\
.162 \\
.188 \\
.224\end{array}$ & $\begin{array}{l}.093 \\
.043 \\
.128 \\
.128 \\
.105\end{array}$ \\
\hline \multicolumn{2}{|c|}{ Total $\ldots$} & 5.247 & 3.766 & 3.683 & 4.548 & 4.778 & 3.900 & 3.365 & 3.325 \\
\hline Apr11 & 1946 & 1947 & 1948 & 1949 & 1950 & 1951 & 1952 & 2953 & Average \\
\hline 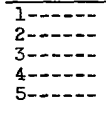 & $\begin{array}{r}0.102 \\
.189 \\
.175 \\
.260 \\
.014\end{array}$ & $\begin{array}{r}0.038 \\
.054 \\
.072 \\
.144 \\
.004\end{array}$ & $\begin{array}{r}0.193 \\
.008 \\
.091 \\
.225 \\
.189\end{array}$ & $\begin{array}{r}0.046 \\
.028 \\
.111 \\
.089 \\
.111\end{array}$ & $\begin{array}{l}0.09 \\
.10 \\
.00 \\
.05 \\
.07\end{array}$ & $\begin{array}{l}0.17 \\
.02 \\
.06 \\
.05 \\
.06\end{array}$ & $\begin{array}{l}0.05 \\
.14 \\
.12 \\
.08 \\
.04\end{array}$ & $\begin{array}{l}0.26 \\
.02 \\
.06 \\
.09 \\
.14\end{array}$ & $\begin{array}{r}0.148 \\
.116 \\
.103 \\
.131 \\
.090\end{array}$ \\
\hline 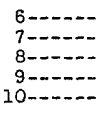 & $\begin{array}{l}.100 \\
.176 \\
.102 \\
.010 \\
.128\end{array}$ & $\begin{array}{l}.286 \\
.164 \\
.112 \\
.004 \\
.158\end{array}$ & $\begin{array}{r}.136 \\
.016 \\
.155 \\
.201 \\
.155\end{array}$ & $\begin{array}{l}.047 \\
.112 \\
.136 \\
.102 \\
.134\end{array}$ & $\begin{array}{l}.06 \\
.05 \\
.12 \\
.09 \\
.05\end{array}$ & $\begin{array}{l}.11 \\
.02 \\
.02 \\
.08 \\
.04\end{array}$ & $\begin{array}{l}.02 \\
.05 \\
.09 \\
.17 \\
.18\end{array}$ & $\begin{array}{l}.07 \\
.06 \\
.10 \\
.07 \\
.27\end{array}$ & $\begin{array}{l}.122 \\
.112 \\
.099 \\
.100 \\
.114\end{array}$ \\
\hline 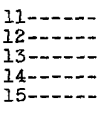 & $\begin{array}{l}.130 \\
.010 \\
.231 \\
.133 \\
.180\end{array}$ & $\begin{array}{l}.178 \\
.116 \\
.112 \\
.089 \\
.053\end{array}$ & $\begin{array}{l}.052 \\
.075 \\
.008 \\
.000 \\
.028\end{array}$ & $\begin{array}{l}.070 \\
.129 \\
.104 \\
.124 \\
.105\end{array}$ & $\begin{array}{l}.30 \\
.05 \\
.02 \\
.00 \\
.01\end{array}$ & $\begin{array}{l}.03 \\
.03 \\
.02 \\
.04 \\
.16\end{array}$ & $\begin{array}{l}.06 \\
.06 \\
.07 \\
.06 \\
.00\end{array}$ & $\begin{array}{l}.07 \\
.07 \\
.03 \\
.06 \\
.13\end{array}$ & $\begin{array}{l}.119 \\
.105 \\
.095 \\
.100 \\
.113\end{array}$ \\
\hline 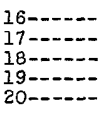 & $\begin{array}{l}.092 \\
.060 \\
.166 \\
.116 \\
.284\end{array}$ & $\begin{array}{l}.192 \\
.046 \\
.148 \\
.190 \\
.124\end{array}$ & $\begin{array}{l}.144 \\
.208 \\
.148 \\
.202 \\
.290\end{array}$ & $\begin{array}{l}.020 \\
.071 \\
.007 \\
.000 \\
.108\end{array}$ & $\begin{array}{l}.10 \\
.19 \\
.16 \\
.14 \\
.14\end{array}$ & $\begin{array}{l}.06 \\
.07 \\
.14 \\
.19 \\
.16\end{array}$ & $\begin{array}{l}.05 \\
.15 \\
.19 \\
.18 \\
.21\end{array}$ & $\begin{array}{l}.17 \\
.14 \\
.02 \\
.05 \\
.05\end{array}$ & $\begin{array}{l}.100 \\
.122 \\
.144 \\
.118 \\
.159\end{array}$ \\
\hline 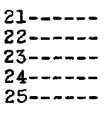 & $\begin{array}{l}.182 \\
.179 \\
.133 \\
.178 \\
.110\end{array}$ & $\begin{array}{l}.038 \\
.054 \\
.050 \\
.236 \\
.126\end{array}$ & $\begin{array}{l}.182 \\
.102 \\
.118 \\
.198 \\
.158\end{array}$ & $\begin{array}{l}.131 \\
.117 \\
.207 \\
.194 \\
.119\end{array}$ & $\begin{array}{l}.13 \\
.15 \\
.19 \\
.09 \\
.08\end{array}$ & $\begin{array}{l}.24 \\
.02 \\
.12 \\
.13 \\
.19\end{array}$ & $\begin{array}{l}.23 \\
.17 \\
.09 \\
.03 \\
.06\end{array}$ & $\begin{array}{l}.12 \\
.22 \\
.19 \\
.24 \\
.04\end{array}$ & $\begin{array}{r}.132 \\
.132 \\
.145 \\
.115 \\
.143\end{array}$ \\
\hline $26 \cdots-\ldots$ & .248 & .044 & .202 & .212 & .21 & .19 & .20 & .23 & .170 \\
\hline $\begin{array}{l}27 \cdots-\cdots \\
28 \cdots \cdots\end{array}$ & $\begin{array}{l}.146 \\
.130\end{array}$ & $\begin{array}{l}.182 \\
.126\end{array}$ & $\begin{array}{l}.139 \\
.202\end{array}$ & $\begin{array}{l}.065 \\
.207\end{array}$ & .14 & $\begin{array}{l}.08 \\
.11\end{array}$ & .19 & .01 & $\begin{array}{l}.139 \\
.369\end{array}$ \\
\hline $29-\ldots$ & .046 & .180 & .072 & .212 & .09 & .20 & .23 & .04 & .158 \\
\hline $30-\cdots---$ & .070 & .006 & .069 & .135 & .01 & .12 & .19 & .11 & .128 \\
\hline Total- & 4.080 & 3.326 & 3.964 & 3.243 & 2.95 & 2.93 & 3.58 & 3.18 & 3.741 \\
\hline
\end{tabular}

See footnotes at end of table. 
Evaporation, in 1nches, in Indianapolis, Ind.--Continued

\begin{tabular}{|c|c|c|c|c|c|c|c|c|c|}
\hline May & 1937 & 1938 & 1939 & 1940 & 1941 & 1942 & 1943 & 1944 & 1945 \\
\hline 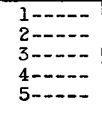 & $\begin{array}{r}0.112 \\
.051 \\
.208 \\
.095 \\
.087\end{array}$ & $\begin{array}{r}0.351 \\
.401 \\
.256 \\
.280 \\
.292\end{array}$ & $\begin{array}{r}0.260 \\
.166 \\
.192 \\
.152 \\
.072\end{array}$ & $\begin{array}{r}0.092 \\
.036 \\
.032 \\
.020 \\
.124\end{array}$ & $\begin{array}{r}0.163 \\
.091 \\
.260 \\
.280 \\
.161\end{array}$ & $\begin{array}{r}0.238 \\
.306 \\
.307 \\
.206 \\
.057\end{array}$ & $\begin{array}{r}0.146 \\
.165 \\
.103 \\
.116 \\
.180\end{array}$ & $\begin{array}{r}0.139 \\
.081 \\
.186 \\
.260 \\
.110\end{array}$ & $\begin{array}{r}0.187 \\
.154 \\
.004 \\
.109 \\
.073\end{array}$ \\
\hline 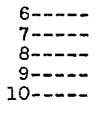 & $\begin{array}{l}.055 \\
.077 \\
.242 \\
.204 \\
.095\end{array}$ & $\begin{array}{l}.290 \\
.270 \\
.120 \\
.150 \\
.169\end{array}$ & $\begin{array}{l}.282 \\
.302 \\
.236 \\
.138 \\
.251\end{array}$ & $\begin{array}{l}.261 \\
.261 \\
.164 \\
.199 \\
.227\end{array}$ & $\begin{array}{l}.220 \\
.029 \\
.132 \\
.205 \\
.066\end{array}$ & $\begin{array}{l}.164 \\
.115 \\
.055 \\
.108 \\
.156\end{array}$ & $\begin{array}{l}.320 \\
.175 \\
.086 \\
.079 \\
.006\end{array}$ & $\begin{array}{l}.110 \\
.130 \\
.078 \\
.014 \\
.022\end{array}$ & $\begin{array}{l}.138 \\
.151 \\
.100 \\
.152 \\
.043\end{array}$ \\
\hline 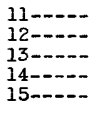 & $\begin{array}{l}.217 \\
.262 \\
.032 \\
.136 \\
.033\end{array}$ & $\begin{array}{l}.140 \\
.048 \\
.128 \\
.240 \\
.038\end{array}$ & $\begin{array}{l}.205 \\
.297 \\
.201 \\
.202 \\
.177\end{array}$ & $\begin{array}{l}.124 \\
.215 \\
.275 \\
.260 \\
.310\end{array}$ & $\begin{array}{l}.045 \\
.184 \\
.144 \\
.104 \\
.142\end{array}$ & $\begin{array}{l}.082 \\
.154 \\
.215 \\
.283 \\
.120\end{array}$ & $\begin{array}{r}.141 \\
.074 \\
.178 \\
.079 \\
.238\end{array}$ & $\begin{array}{l}.108 \\
.146 \\
.178 \\
.216 \\
.120\end{array}$ & $\begin{array}{l}.062 \\
.143 \\
.070 \\
.157 \\
.270\end{array}$ \\
\hline 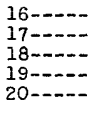 & $\begin{array}{l}.093 \\
.284 \\
.204 \\
.042 \\
.194\end{array}$ & $\begin{array}{l}.102 \\
.173 \\
.029 \\
.016 \\
.217\end{array}$ & $\begin{array}{l}.123 \\
.179 \\
.287 \\
.217 \\
.194\end{array}$ & $\begin{array}{l}.072 \\
.041 \\
.122 \\
.148 \\
.159\end{array}$ & $\begin{array}{l}.200 \\
.098 \\
.348 \\
.190 \\
.224\end{array}$ & $\begin{array}{l}.196 \\
.088 \\
.128 \\
.050 \\
.184\end{array}$ & $\begin{array}{l}.037 \\
.228 \\
.248 \\
.048 \\
.049\end{array}$ & $\begin{array}{l}.320 \\
.185 \\
.137 \\
.252 \\
.214\end{array}$ & $\begin{array}{l}.010 \\
.036 \\
.076 \\
.064 \\
.178\end{array}$ \\
\hline 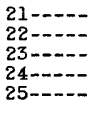 & $\begin{array}{l}.135 \\
.187 \\
.102 \\
.186 \\
.186\end{array}$ & $\begin{array}{l}.220 \\
.150 \\
.120 \\
.139 \\
.288\end{array}$ & $\begin{array}{l}.160 \\
.126 \\
.137 \\
.295 \\
.292\end{array}$ & $\begin{array}{l}.111 \\
.210 \\
.293 \\
.214 \\
.048\end{array}$ & $\begin{array}{l}.216 \\
.286 \\
.326 \\
.280 \\
.290\end{array}$ & $\begin{array}{l}.052 \\
.122 \\
.141 \\
.073 \\
.225\end{array}$ & $\begin{array}{l}.014 \\
.234 \\
.184 \\
.076 \\
.068\end{array}$ & $\begin{array}{l}.183 \\
.226 \\
.135 \\
.120 \\
.158\end{array}$ & $\begin{array}{r}.154 \\
.340 \\
.116 \\
.160 \\
.148\end{array}$ \\
\hline 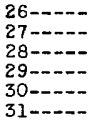 & $\begin{array}{l}.188 \\
.265 \\
.178 \\
.275 \\
.225 \\
.237\end{array}$ & $\begin{array}{l}.086 \\
.174 \\
.152 \\
.175 \\
.059 \\
.154 \\
\end{array}$ & $\begin{array}{l}.235 \\
.290 \\
.158 \\
.333 \\
.246 \\
.264 \\
\end{array}$ & $\begin{array}{l}.008 \\
.212 \\
.328 \\
.156 \\
.110 \\
.038 \\
\end{array}$ & $\begin{array}{l}.205 \\
.238 \\
.342 \\
.328 \\
.262 \\
.361 \\
\end{array}$ & $\begin{array}{l}.221 \\
.036 \\
.092 \\
.200 \\
.270 \\
.183 \\
\end{array}$ & $\begin{array}{l}.192 \\
.186 \\
.174 \\
.180 \\
.256 \\
.108 \\
\end{array}$ & $\begin{array}{r}.191 \\
.186 \\
.210 \\
.189 \\
.184 \\
.184 \\
\end{array}$ & $\begin{array}{r}.062 \\
.146 \\
.214 \\
.208 \\
.196 \\
.124 \\
\end{array}$ \\
\hline Total- & 4.887 & 5.427 & 6.669 & 4.970 & 6.465 & 4.827 & 4.368 & 4.972 & 4.045 \\
\hline May & 1946 & 1947 & 1948 & 1949 & 1950 & 1951 & 1952 & 1953 & Average \\
\hline 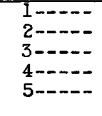 & $\begin{array}{r}0.037 \\
.009 \\
.155 \\
.064 \\
.078\end{array}$ & $\begin{array}{r}0.123 \\
.043 \\
.097 \\
.070 \\
.187\end{array}$ & $\begin{array}{r}0.142 \\
.112 \\
.100 \\
.124 \\
.032\end{array}$ & $\begin{array}{l}0.03 \\
.10 \\
.21 \\
.20 \\
.25\end{array}$ & $\begin{array}{r}0.04 \\
.14 \\
.10 \\
.15 \\
.16\end{array}$ & $\begin{array}{r}0.19 \\
.23 \\
.19 \\
.20 \\
.13\end{array}$ & $\begin{array}{r}0.20 \\
.21 \\
.25 \\
.18 \\
.32\end{array}$ & $\begin{array}{l}0.20 \\
.27 \\
.24 \\
.21 \\
.04\end{array}$ & $\begin{array}{r}0.156 \\
.151 \\
.170 \\
.166 \\
.138\end{array}$ \\
\hline 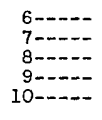 & $\begin{array}{l}.118 \\
.088 \\
.110 \\
.100 \\
.122\end{array}$ & $\begin{array}{l}.065 \\
.121 \\
.122 \\
.156 \\
.094\end{array}$ & $\begin{array}{l}.083 \\
.085 \\
.129 \\
.105 \\
.150\end{array}$ & $\begin{array}{r}.23 \\
.27 \\
. .07 \\
.13 \\
.14\end{array}$ & $\begin{array}{l}.37 \\
.11 \\
.25 \\
.21 \\
.14\end{array}$ & $\begin{array}{l}.11 \\
.09 \\
.15 \\
.20 \\
.18\end{array}$ & $\begin{array}{l}.36 \\
.27 \\
.10 \\
.08 \\
.03\end{array}$ & $\begin{array}{l}.09 \\
.11 \\
.08 \\
.10 \\
.20\end{array}$ & $\begin{array}{r}.192 \\
.156 \\
.131 \\
.137 \\
.123\end{array}$ \\
\hline 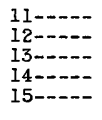 & $\begin{array}{l}.121 \\
.091 \\
.136 \\
.099 \\
.062\end{array}$ & $\begin{array}{l}.160 \\
.189 \\
.254 \\
.158 \\
.071\end{array}$ & $\begin{array}{l}.368 \\
.147 \\
.035 \\
.038 \\
.057\end{array}$ & $\begin{array}{l}.17 \\
.15 \\
.17 \\
.14 \\
.31\end{array}$ & $\begin{array}{l}.13 \\
.05 \\
.13 \\
.19 \\
.19\end{array}$ & $\begin{array}{l}.06 \\
.03 \\
.18 \\
.20 \\
.22\end{array}$ & $\begin{array}{l}.05 \\
.08 \\
.00 \\
.25 \\
.16\end{array}$ & $\begin{array}{l}.26 \\
.07 \\
.08 \\
.02 \\
.00\end{array}$ & $\begin{array}{r}.144 \\
.137 \\
.142 \\
.163 \\
.148\end{array}$ \\
\hline $\begin{array}{l}16 \ldots \\
17-\ldots \\
18 \ldots- \\
19-\ldots \\
20 \ldots\end{array}$ & $\begin{array}{l}.145 \\
.075 \\
.025 \\
.024 \\
.102\end{array}$ & $\begin{array}{l}.230 \\
.072 \\
.422 \\
.083 \\
.138\end{array}$ & $\begin{array}{l}.067 \\
.214 \\
.182 \\
.189 \\
.144\end{array}$ & $\begin{array}{l}.18 \\
.09 \\
.23 \\
.22 \\
.16\end{array}$ & $\begin{array}{l}.15 \\
.19 \\
.13 \\
.23 \\
.17\end{array}$ & $\begin{array}{l}.23 \\
.22 \\
.19 \\
.18 \\
.20\end{array}$ & $\begin{array}{l}.21 \\
.03 \\
.09 \\
.04 \\
.00\end{array}$ & $\begin{array}{l}.04 \\
.01 \\
.11 \\
.15 \\
.15\end{array}$ & $\begin{array}{l}.141 \\
.130 \\
.174 \\
.126 \\
.157\end{array}$ \\
\hline 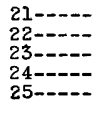 & $\begin{array}{l}.120 \\
.132 \\
.175 \\
.089 \\
.060\end{array}$ & $\begin{array}{l}.264 \\
.099 \\
.188 \\
.215 \\
.100\end{array}$ & $\begin{array}{l}.213 \\
.287 \\
.148 \\
.240 \\
.210\end{array}$ & $\begin{array}{r}.04 \\
.17 \\
.20 \\
.09 \\
.14\end{array}$ & $\begin{array}{l}.23 \\
.21 \\
.08 \\
.36 \\
.19\end{array}$ & $\begin{array}{l}.22 \\
.23 \\
.18 \\
.20 \\
.20\end{array}$ & $\begin{array}{l}.01 \\
.14 \\
.06 \\
.25 \\
.11\end{array}$ & $\begin{array}{l}.23 \\
.13 \\
.13 \\
.22 \\
.04\end{array}$ & $\begin{array}{l}.151 \\
.193 \\
.160 \\
.189 \\
.162\end{array}$ \\
\hline 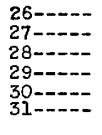 & $\begin{array}{l}.160 \\
.038 \\
.158 \\
.180 \\
.230 \\
.150\end{array}$ & $\begin{array}{l}.067 \\
.154 \\
.020 \\
.214 \\
.100 \\
.116\end{array}$ & $\begin{array}{l}.154 \\
.208 \\
.176 \\
.175 \\
.255 \\
.219\end{array}$ & $\begin{array}{l}.14 \\
.17 \\
.11 \\
.18 \\
.19 \\
.16 \\
\end{array}$ & $\begin{array}{l}.27 \\
.21 \\
.02 \\
.03 \\
.08 \\
.16 \\
\end{array}$ & $\begin{array}{l}.16 \\
.20 \\
.01 \\
.08 \\
.13 \\
.20 \\
\end{array}$ & $\begin{array}{l}.05 \\
.18 \\
.17 \\
.21 \\
.15 \\
.20 \\
\end{array}$ & $\begin{array}{l}.22 \\
.34 \\
.14 \\
.21 \\
.24 \\
.27 \\
\end{array}$ & $\begin{array}{r}.156 \\
.190 \\
.156 \\
.195 \\
.187 \\
.184 \\
\end{array}$ \\
\hline Total- & 3.253 & 4.392 & 4.788 & $\div 5.04$ & 5.07 & 5.19 & 4.44 & 4.60 & 4.905 \\
\hline
\end{tabular}

See footnotes at end of table. 
Evaporation, in inches, in Indianpolis, Ind.--Continued

\begin{tabular}{|c|c|c|c|c|c|c|c|c|c|}
\hline June & 1937 & 1938 & 1939 & 1940 & 1941 & 1942 & 1943 & 1944 & 1945 \\
\hline 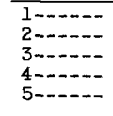 & $\begin{array}{r}0.312 \\
.267 \\
.220 \\
.190 \\
.163\end{array}$ & $\begin{array}{r}0.152 \\
.040 \\
.239 \\
.252 \\
.223\end{array}$ & $\begin{array}{r}0.230 \\
.091 \\
.089 \\
.171 \\
.260\end{array}$ & $\begin{array}{r}0.002 \\
.112 \\
.168 \\
.218 \\
.206\end{array}$ & $\begin{array}{r}0.269 \\
.127 \\
\text { to. } .150 \\
.054 \\
.222\end{array}$ & $\begin{array}{r}0.087 \\
.368 \\
.238 \\
.200 \\
.225\end{array}$ & $\begin{array}{r}0.130 \\
.220 \\
.232 \\
.252 \\
.144\end{array}$ & $\begin{array}{r}0.204 \\
.236 \\
.200 \\
.254 \\
.258\end{array}$ & $\begin{array}{l}0.104 \\
.274 \\
.118 \\
.072 \\
.156\end{array}$ \\
\hline 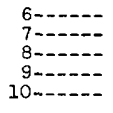 & $\begin{array}{l}.119 \\
.260 \\
.231 \\
.272 \\
.046\end{array}$ & $\begin{array}{l}.256 \\
.295 \\
.175 \\
.096 \\
.250\end{array}$ & $\begin{array}{l}.246 \\
.198 \\
.322 \\
.228 \\
.240\end{array}$ & $\begin{array}{l}.256 \\
.250 \\
.214 \\
.250 \\
.182\end{array}$ & $\begin{array}{l}.190 \\
.260 \\
.182 \\
.283 \\
.087\end{array}$ & $\begin{array}{l}.267 \\
.089 \\
.022 \\
.016 \\
.112\end{array}$ & $\begin{array}{l}.266 \\
.136 \\
.141 \\
.136 \\
.180\end{array}$ & $\begin{array}{l}.274 \\
.312 \\
.174 \\
.280 \\
.086\end{array}$ & $\begin{array}{l}.164 \\
.144 \\
.130 \\
.190 \\
.072\end{array}$ \\
\hline 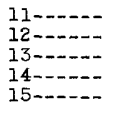 & $\begin{array}{l}.030 \\
.167 \\
.200 \\
.125 \\
.119\end{array}$ & $\begin{array}{l}.062 \\
.306 \\
.253 \\
.197 \\
.224\end{array}$ & $\begin{array}{l}.256 \\
.146 \\
.066 \\
.018 \\
.159\end{array}$ & $\begin{array}{l}.247 \\
.112 \\
.241 \\
.170 \\
.246\end{array}$ & $\begin{array}{r}.100 \\
. .120 \\
6.237 \\
.184 \\
.128\end{array}$ & $\begin{array}{l}.110 \\
.201 \\
.129 \\
.286 \\
.190\end{array}$ & $\begin{array}{l}.110 \\
.137 \\
.256 \\
.166 \\
.343\end{array}$ & $\begin{array}{l}.185 \\
.228 \\
.250 \\
.372 \\
.192\end{array}$ & $\begin{array}{r}.114 \\
.049 \\
6.150 \\
.128 \\
.212\end{array}$ \\
\hline 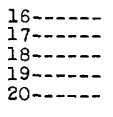 & $\begin{array}{l}.230 \\
.190 \\
.189 \\
.090 \\
.160\end{array}$ & $\begin{array}{l}.232 \\
.274 \\
.104 \\
.185 \\
.239\end{array}$ & $\begin{array}{l}.230 \\
.283 \\
.268 \\
.234 \\
.363\end{array}$ & $\begin{array}{l}.228 \\
.186 \\
.132 \\
.222 \\
.328\end{array}$ & $\begin{array}{l}.090 \\
.104 \\
.206 \\
.266 \\
.244\end{array}$ & $\begin{array}{l}.108 \\
.091 \\
.120 \\
.107 \\
.138\end{array}$ & $\begin{array}{l}.225 \\
.184 \\
.366 \\
.242 \\
.250\end{array}$ & $\begin{array}{l}.161 \\
.204 \\
.288 \\
.294 \\
.262\end{array}$ & $\begin{array}{l}.157 \\
.221 \\
.057 \\
.136 \\
.096\end{array}$ \\
\hline 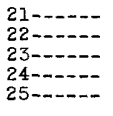 & $\begin{array}{l}.229 \\
.218 \\
.279 \\
.176 \\
.177\end{array}$ & $\begin{array}{l}.233 \\
.230 \\
.210 \\
.139 \\
.087\end{array}$ & $\begin{array}{r}. .210 \\
.211 \\
.301 \\
.200 \\
.200\end{array}$ & $\begin{array}{l}.278 \\
.248 \\
.186 \\
.250 \\
.218\end{array}$ & $\begin{array}{l}.220 \\
.166 \\
.238 \\
.216 \\
.304\end{array}$ & $\begin{array}{l}.164 \\
.182 \\
.203 \\
.259 \\
.175\end{array}$ & $\begin{array}{l}.225 \\
.289 \\
.196 \\
.278 \\
.206\end{array}$ & $\begin{array}{l}.220 \\
.232 \\
.077 \\
.279 \\
.272\end{array}$ & $\begin{array}{l}.173 \\
.157 \\
.190 \\
.197 \\
.162\end{array}$ \\
\hline 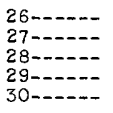 & $\begin{array}{l}.109 \\
.210 \\
.274 \\
.289 \\
.258\end{array}$ & $\begin{array}{l}.020 \\
.185 \\
.161 \\
.258 \\
.218\end{array}$ & $\begin{array}{l}.158 \\
.332 \\
.145 \\
.233 \\
.202\end{array}$ & $\begin{array}{l}.250 \\
.130 \\
.159 \\
.340 \\
.236\end{array}$ & $\begin{array}{l}.256 \\
.246 \\
.244 \\
.190 \\
.228\end{array}$ & $\begin{array}{l}.135 \\
.073 \\
.223 \\
.210 \\
.214\end{array}$ & $\begin{array}{r}.231 \\
.123 \\
.388 \\
.191 \\
.308\end{array}$ & $\begin{array}{l}.248 \\
.201 \\
.291 \\
.259 \\
.376\end{array}$ & $\begin{array}{l}.087 \\
.221 \\
.192 \\
.223 \\
.167\end{array}$ \\
\hline Total- & 5.799 & 5.795 & 6.290 & 6.265 & 5.811 & 4.942 & 6.551 & 7.169 & 4.513 \\
\hline June & 1946 & 1947 & 1948 & d 1949 & t 1950 & 1951 & 1952 & 1953 & Average \\
\hline 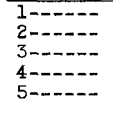 & $\begin{array}{r}0.042 \\
.062 \\
.118 \\
.190 \\
.194\end{array}$ & $\begin{array}{r}0.211 \\
.003 \\
.068 \\
.180 \\
.070\end{array}$ & $\begin{array}{r}0.217 \\
.227 \\
.167 \\
.228 \\
.261\end{array}$ & $\begin{array}{l}0.18 \\
.20 \\
.22 \\
.20 \\
.25\end{array}$ & $\begin{array}{r}0.20 \\
.22 \\
.21 \\
.09 \\
.19\end{array}$ & $\begin{array}{r}0.11 \\
.26 \\
.23 \\
.12 \\
.09\end{array}$ & $\begin{array}{r}0.24 \\
.24 \\
.20 \\
.19 \\
.20\end{array}$ & $\begin{array}{r}0.37 \\
.12 \\
.21 \\
.25 \\
.30\end{array}$ & $\begin{array}{r}0.185 \\
.180 \\
.181 \\
.183 \\
.201\end{array}$ \\
\hline 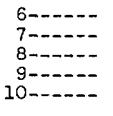 & $\begin{array}{l}.170 \\
.075 \\
.219 \\
.230 \\
.220\end{array}$ & $\begin{array}{l}.248 \\
.035 \\
.168 \\
.167 \\
.232\end{array}$ & $\begin{array}{l}.174 \\
.073 \\
.134 \\
.174 \\
.180\end{array}$ & $\begin{array}{l}.29 \\
.22 \\
.14 \\
.20 \\
.42\end{array}$ & $\begin{array}{l}.23 \\
.21 \\
.25 \\
.26 \\
.14\end{array}$ & $\begin{array}{l}.15 \\
.16 \\
.07 \\
.15 \\
.09\end{array}$ & $\begin{array}{l}.17 \\
.15 \\
.20 \\
.30 \\
.16\end{array}$ & $\begin{array}{l}.25 \\
.14 \\
.25 \\
.18 \\
.21\end{array}$ & $\begin{array}{l}.219 \\
.177 \\
.178 \\
.201 \\
.171\end{array}$ \\
\hline 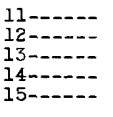 & $\begin{array}{l}.240 \\
.175 \\
.207 \\
.152 \\
.197\end{array}$ & $\begin{array}{l}.253 \\
.217 \\
.188 \\
.218 \\
.102\end{array}$ & $\begin{array}{l}.170 \\
.260 \\
.127 \\
.211 \\
.182\end{array}$ & $\begin{array}{l}.02 \\
.08 \\
.13 \\
.10 \\
.15\end{array}$ & $\begin{array}{l}.27 \\
.19 \\
.25 \\
.03 \\
.10\end{array}$ & $\begin{array}{r}6.16 \\
.18 \\
.08 \\
.15 \\
.16\end{array}$ & $\begin{array}{l}.23 \\
.41 \\
.11 \\
.17 \\
.28\end{array}$ & $\begin{array}{l}.11 \\
.19 \\
.19 \\
.15 \\
.19\end{array}$ & $\begin{array}{l}.157 \\
.186 \\
.180 \\
.166 \\
.187\end{array}$ \\
\hline 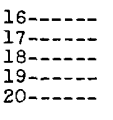 & $\begin{array}{l}.061 \\
.194 \\
.182 \\
.184 \\
.120\end{array}$ & $\begin{array}{l}.114 \\
.174 \\
.045 \\
.025 \\
.080\end{array}$ & $\begin{array}{l}.118 \\
.192 \\
.218 \\
.185 \\
.275\end{array}$ & $\begin{array}{l}.01 \\
.02 \\
.11 \\
.15 \\
.26\end{array}$ & $\begin{array}{l}.28 \\
.37 \\
.10 \\
.02 \\
.07\end{array}$ & $\begin{array}{l}.20 \\
.14 \\
.20 \\
.1 \\
.2\end{array}$ & $\begin{array}{r}.22 \\
.29 \\
.31 \\
.19 \\
. .22\end{array}$ & $\begin{array}{l}.19 \\
.24 \\
.15 \\
.27 \\
.27\end{array}$ & $\begin{array}{l}.168 \\
.197 \\
.179 \\
.177 \\
.211\end{array}$ \\
\hline 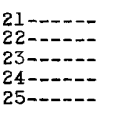 & $\begin{array}{l}.150 \\
.177 \\
.171 \\
.180 \\
.254\end{array}$ & $\begin{array}{l}.240 \\
.277 \\
.260 \\
.161 \\
.038\end{array}$ & $\begin{array}{l}.110 \\
.176 \\
.182 \\
.272 \\
.142\end{array}$ & $\begin{array}{l}.25 \\
.12 \\
.22 \\
.21 \\
.27\end{array}$ & $\begin{array}{l}.07 \\
.16 \\
.18 \\
.24 \\
.18\end{array}$ & $\begin{array}{l}.28 \\
.21 \\
.19 \\
.11 \\
.23\end{array}$ & $\begin{array}{r}.15 \\
.23 \\
.07 \\
.26 \\
.16\end{array}$ & $\begin{array}{l}.39 \\
.30 \\
.25 \\
.28 \\
.36\end{array}$ & $\begin{array}{l}.211 \\
.211 \\
.200 \\
.218 \\
.202\end{array}$ \\
\hline 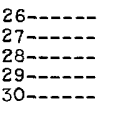 & $\begin{array}{l}.150 \\
.090 \\
.100 \\
.194 \\
.234\end{array}$ & $\begin{array}{l}.057 \\
.164 \\
.190 \\
.206 \\
.118\end{array}$ & $\begin{array}{l}.184 \\
.162 \\
.156 \\
.278 \\
.106\end{array}$ & $\begin{array}{l}.20 \\
.18 \\
.16 \\
.17 \\
.11\end{array}$ & $\begin{array}{l}.17 \\
.30 \\
.21 \\
.10 \\
.21\end{array}$ & $\begin{array}{l}.19 \\
.16 \\
.17 \\
.14 \\
.11\end{array}$ & $\begin{array}{l}.39 \\
.37 \\
.14 \\
.25 \\
.23\end{array}$ & $\begin{array}{r}.20 \\
.14 \\
.26 \\
6.23 \\
.19\end{array}$ & $\begin{array}{l}.179 \\
.193 \\
.204 \\
.221 \\
.207\end{array}$ \\
\hline Total- & 4.932 & 4.509 & 5.541 & 5.24 & 5.50 & 4.92 & 6.73 & 6.83 & 5.725 \\
\hline
\end{tabular}

See footnotes at end of table. 
EVAPORATION DATA

Evaporation, in inches, in Indianapolis, Ind.--Continued

\begin{tabular}{|c|c|c|c|c|c|c|c|c|c|}
\hline July & 1937 & 1938 & 1939 & 1940 & 1941 & 1942 & 1943 & 1944 & 1945 \\
\hline 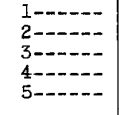 & $\begin{array}{r}0.241 \\
.132 \\
.254 \\
.226 \\
\text { b. } 221\end{array}$ & $\begin{array}{r}0.240 \\
.108 \\
.200 \\
.182 \\
.344\end{array}$ & $\begin{array}{r}0.302 \\
.212 \\
.238 \\
.160 \\
.110\end{array}$ & $\begin{array}{r}0.195 \\
.313 \\
.282 \\
.148 \\
.376\end{array}$ & $\begin{array}{r}0.268 \\
.276 \\
.326 \\
.148 \\
.198\end{array}$ & $\begin{array}{r}0.272 \\
.268 \\
.204 \\
.251 \\
.180\end{array}$ & $\begin{array}{r}0.232 \\
.171 \\
.216 \\
.192 \\
.211\end{array}$ & $\begin{array}{r}0.310 \\
.335 \\
.326 \\
.363 \\
.274\end{array}$ & $\begin{array}{r}0.176 \\
.178 \\
.146 \\
.144 \\
.171\end{array}$ \\
\hline 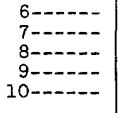 & $\begin{array}{l}.225 \\
.245 \\
.126 \\
.154 \\
.282\end{array}$ & $\begin{array}{l}.220 \\
.232 \\
.336 \\
.098 \\
.187\end{array}$ & $\begin{array}{l}.220 \\
.325 \\
.005 \\
.268 \\
.257\end{array}$ & $\begin{array}{l}.140 \\
.404 \\
.382 \\
.214 \\
.182\end{array}$ & $\begin{array}{l}.284 \\
.200 \\
.244 \\
.304 \\
.228\end{array}$ & $\begin{array}{l}.151 \\
.300 \\
.373 \\
.140 \\
.137\end{array}$ & $\begin{array}{l}.189 \\
.175 \\
.211 \\
.152 \\
.254\end{array}$ & $\begin{array}{r}.206 \\
.246 \\
.192 \\
.220 \\
.127\end{array}$ & $\begin{array}{l}.213 \\
.228 \\
.213 \\
.158 \\
.189\end{array}$ \\
\hline 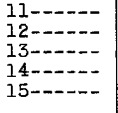 & $\begin{array}{l}.302 \\
.214 \\
.206 \\
.048 \\
.274\end{array}$ & $\begin{array}{l}.224 \\
.262 \\
.226 \\
.200 \\
.199\end{array}$ & $\begin{array}{r}.316 \\
.190 \\
.330 \\
. .416 \\
.272\end{array}$ & $\begin{array}{l}.284 \\
.260 \\
.088 \\
.266 \\
.276\end{array}$ & $\begin{array}{l}.110 \\
.213 \\
.165 \\
.330 \\
.198\end{array}$ & $\begin{array}{l}.222 \\
.138 \\
.221 \\
.318 \\
.296\end{array}$ & $\begin{array}{l}.132 \\
.152 \\
.195 \\
.237 \\
.286\end{array}$ & $\begin{array}{l}.350 \\
.186 \\
.171 \\
.261 \\
.264\end{array}$ & $\begin{array}{l}.231 \\
.201 \\
.172 \\
.198 \\
.135\end{array}$ \\
\hline 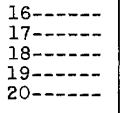 & $\begin{array}{l}.304 \\
.306 \\
.290 \\
.215 \\
.132\end{array}$ & $\begin{array}{l}.257 \\
.235 \\
.056 \\
.182 \\
.125\end{array}$ & $\begin{array}{r}.378 \\
.261 \\
.300 \\
.261 \\
.130\end{array}$ & $\begin{array}{l}.246 \\
.114 \\
.250 \\
.196 \\
.317\end{array}$ & $\begin{array}{l}.262 \\
.279 \\
.266 \\
.150 \\
.284\end{array}$ & $\begin{array}{l}.124 \\
.226 \\
.290 \\
.222 \\
.294\end{array}$ & $\begin{array}{l}.261 \\
.288 \\
.141 \\
.249 \\
.120\end{array}$ & $\begin{array}{l}.244 \\
.268 \\
.160 \\
.207 \\
.295\end{array}$ & $\begin{array}{l}.211 \\
.199 \\
.167 \\
.147 \\
.188\end{array}$ \\
\hline 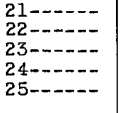 & $\begin{array}{l}.115 \\
.313 \\
.145 \\
.217 \\
.237\end{array}$ & $\begin{array}{l}.348 \\
.200 \\
.278 \\
.178 \\
.345\end{array}$ & $\begin{array}{l}.114 \\
.258 \\
.166 \\
.148 \\
.200\end{array}$ & $\begin{array}{l}.319 \\
.309 \\
.239 \\
.290 \\
.435\end{array}$ & $\begin{array}{l}.286 \\
.270 \\
.270 \\
.252 \\
.248\end{array}$ & $\begin{array}{l}.100 \\
.226 \\
.274 \\
.152 \\
.210\end{array}$ & $\begin{array}{l}.266 \\
.200 \\
.242 \\
.218 \\
.171\end{array}$ & $\begin{array}{l}.287 \\
.212 \\
.145 \\
.393 \\
.241\end{array}$ & $\begin{array}{l}.216 \\
.108 \\
.217 \\
.195 \\
.244\end{array}$ \\
\hline 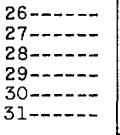 & $\begin{array}{l}.141 \\
.218 \\
.211 \\
.212 \\
.114 \\
.244\end{array}$ & $\begin{array}{l}.151 \\
.348 \\
.250 \\
.212 \\
.234 \\
.160\end{array}$ & $\begin{array}{r}.260 \\
.190 \\
.216 \\
b .210 \\
.014 \\
.124 \\
\end{array}$ & $\begin{array}{l}.350 \\
.454 \\
.180 \\
.424 \\
.374 \\
.312 \\
\end{array}$ & $\begin{array}{l}.252 \\
.240 \\
.300 \\
.354 \\
.362 \\
.338 \\
\end{array}$ & $\begin{array}{l}.166 \\
.252 \\
.116 \\
.177 \\
.366 \\
.240 \\
\end{array}$ & $\begin{array}{r}.169 \\
.248 \\
.238 \\
.273 \\
.153 \\
.211 \\
\end{array}$ & $\begin{array}{r}.250 \\
.164 \\
.299 \\
.295 \\
.115 \\
.231 \\
\end{array}$ & $\begin{array}{l}.149 \\
.218 \\
.080 \\
.041 \\
.139 \\
.150 \\
\end{array}$ \\
\hline Total- & 6.564 & 6.817 & 6.851 & 8.619 & 7.905 & 6.906 & 6.453 & 7.637 & 5.422 \\
\hline July & 1946 & 1947 & 1948 & 1949 & 1950 & 1951 & 1952 & 1953 & Average \\
\hline 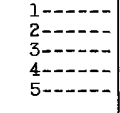 & $\begin{array}{r}0.194 \\
.204 \\
.230 \\
.206 \\
.194\end{array}$ & $\begin{array}{r}0.244 \\
.296 \\
.229 \\
.186 \\
.211\end{array}$ & $\begin{array}{r}0.212 \\
.194 \\
.222 \\
.218 \\
.168\end{array}$ & $\begin{array}{l}0.21 \\
.19 \\
.24 \\
.24 \\
.20\end{array}$ & $\begin{array}{r}0.16 \\
.20 \\
.07 \\
.11 \\
.28\end{array}$ & $\begin{array}{r}0.08 \\
.18 \\
.21 \\
.24 \\
.28\end{array}$ & $\begin{array}{l}0.34 \\
.18 \\
.38 \\
.04 \\
.24\end{array}$ & $\begin{array}{l}0.27 \\
.11 \\
.20 \\
.18 \\
.35\end{array}$ & $\begin{array}{r}0.232 \\
.209 \\
.234 \\
.190 \\
.236\end{array}$ \\
\hline 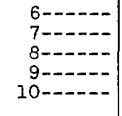 & $\begin{array}{l}.202 \\
.202 \\
.207 \\
.084 \\
.199\end{array}$ & $\begin{array}{l}.125 \\
.058 \\
.252 \\
.265 \\
.006\end{array}$ & $\begin{array}{l}.180 \\
.274 \\
.200 \\
.206 \\
.194\end{array}$ & $\begin{array}{l}.16 \\
.20 \\
.15 \\
.23 \\
.12\end{array}$ & $\begin{array}{l}.18 \\
.20 \\
.18 \\
.23 \\
.23\end{array}$ & $\begin{array}{l}.19 \\
.23 \\
.15 \\
.34 \\
.10\end{array}$ & $\begin{array}{l}.21 \\
.25 \\
.25 \\
.09 \\
.19\end{array}$ & $\begin{array}{l}.41 \\
.13 \\
.25 \\
.23 \\
.18\end{array}$ & $\begin{array}{r}.206 \\
.229 \\
.219 \\
.199 \\
.180\end{array}$ \\
\hline 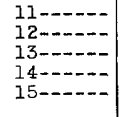 & $\begin{array}{l}.191 \\
.140 \\
.207 \\
.196 \\
.212\end{array}$ & $\begin{array}{l}.153 \\
.152 \\
.246 \\
.112 \\
.240\end{array}$ & $\begin{array}{l}.207 \\
.175 \\
.084 \\
.070 \\
.227\end{array}$ & $\begin{array}{l}.22 \\
.22 \\
.23 \\
.22 \\
.23\end{array}$ & $\begin{array}{l}.19 \\
.18 \\
.19 \\
.15 \\
.17\end{array}$ & $\begin{array}{l}.10 \\
.16 \\
.16 \\
.07 \\
.21\end{array}$ & $\begin{array}{l}.20 \\
.23 \\
.23 \\
.21 \\
.17\end{array}$ & $\begin{array}{l}.19 \\
.20 \\
.19 \\
.24 \\
.20\end{array}$ & $\begin{array}{l}.213 \\
.193 \\
.195 \\
.208 \\
.227\end{array}$ \\
\hline 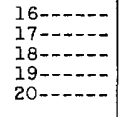 & $\begin{array}{l}.125 \\
.189 \\
.209 \\
.191 \\
.246\end{array}$ & $\begin{array}{l}.173 \\
.037 \\
.145 \\
.067 \\
.180\end{array}$ & $\begin{array}{l}.135 \\
.153 \\
.186 \\
.249 \\
.109\end{array}$ & $\begin{array}{l}.19 \\
.10 \\
.08 \\
.23 \\
.21\end{array}$ & $\begin{array}{l}.23 \\
.21 \\
.23 \\
.12 \\
.16\end{array}$ & $\begin{array}{l}.21 \\
.18 \\
.22 \\
.18 \\
.29\end{array}$ & $\begin{array}{l}.18 \\
.08 \\
.12 \\
.21 \\
.19\end{array}$ & $\begin{array}{l}.20 \\
.20 \\
.01 \\
.22 \\
.19\end{array}$ & $\begin{array}{l}.219 \\
.196 \\
.184 \\
.194 \\
.204\end{array}$ \\
\hline 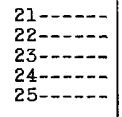 & $\begin{array}{l}.062 \\
.208 \\
.222 \\
.232 \\
.255\end{array}$ & $\begin{array}{l}.092 \\
.090 \\
.274 \\
.176 \\
.200\end{array}$ & $\begin{array}{l}.122 \\
.074 \\
.102 \\
.158 \\
.168\end{array}$ & $\begin{array}{l}.16 \\
.10 \\
.17 \\
.13 \\
.21\end{array}$ & $\begin{array}{l}.12 \\
.21 \\
.08 \\
.27 \\
.22\end{array}$ & $\begin{array}{l}.22 \\
.12 \\
.17 \\
.13 \\
.16\end{array}$ & $\begin{array}{l}.30 \\
.25 \\
.30 \\
.31 \\
.13\end{array}$ & $\begin{array}{l}.21 \\
.17 \\
.09 \\
.24 \\
.21\end{array}$ & $\begin{array}{l}.196 \\
.195 \\
.199 \\
.217 \\
.228\end{array}$ \\
\hline 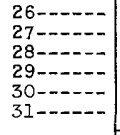 & $\begin{array}{l}.181 \\
.110 \\
.218 \\
.214 \\
.214 \\
.208\end{array}$ & $\begin{array}{l}.192 \\
.170 \\
.158 \\
.168 \\
.226 \\
.428\end{array}$ & $\begin{array}{l}.194 \\
.086 \\
.109 \\
.311 \\
.192 \\
.219 \\
\end{array}$ & $\begin{array}{l}.19 \\
.13 \\
.20 \\
.23 \\
.14 \\
.10 \\
\end{array}$ & $\begin{array}{l}.16 \\
.12 \\
.19 \\
.20 \\
.23 \\
.20 \\
\end{array}$ & $\begin{array}{l}.22 \\
.21 \\
.11 \\
.20 \\
.19 \\
.21 \\
\end{array}$ & $\begin{array}{l}.24 \\
.26 \\
.26 \\
.36 \\
.13 \\
.24 \\
\end{array}$ & $\begin{array}{l}.20 \\
.21 \\
.21 \\
.22 \\
.22 \\
.14 \\
\end{array}$ & $\begin{array}{l}.204 \\
.213 \\
.197 \\
.241 \\
.201 \\
.221 \\
\end{array}$ \\
\hline Total- & 5.952 & 5.551 & 5.398 & 5.63 & 5.67 & 5.72 & 6.77 & 6.27 & 6.479 \\
\hline
\end{tabular}

\footnotetext{
See footnotes at end of table.
} 
Evaporation, in 1nches, in Indianapolis, Ind.--Continued

\begin{tabular}{|c|c|c|c|c|c|c|c|c|c|}
\hline August & 1937 & 1938 & 1939 & 1940 & 1941 & 1942 & 1943 & 1944 & 1945 \\
\hline 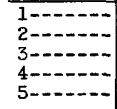 & $\begin{array}{r}0.150 \\
.355 \\
.225 \\
.225 \\
.092\end{array}$ & $\begin{array}{r}0.076 \\
.169 \\
.215 \\
.242 \\
.340\end{array}$ & $\begin{array}{r}0.222 \\
.210 \\
.100 \\
.203 \\
.239\end{array}$ & $\begin{array}{r}0.334 \\
.378 \\
.348 \\
.292 \\
.308\end{array}$ & $\begin{array}{r}0.210 \\
.330 \\
.355 \\
.324 \\
.296\end{array}$ & $\begin{array}{r}0.212 \\
.146 \\
.264 \\
.217 \\
.145\end{array}$ & $\begin{array}{r}0.204 \\
.256 \\
.165 \\
.071 \\
.150\end{array}$ & $\begin{array}{r}0.242 \\
.269 \\
.276 \\
.212 \\
.128\end{array}$ & $\begin{array}{r}0.167 \\
.229 \\
.206 \\
.238 \\
.211\end{array}$ \\
\hline 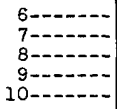 & $\begin{array}{l}.042 \\
.236 \\
.230 \\
.297 \\
.197\end{array}$ & $\begin{array}{l}.158 \\
.165 \\
.222 \\
.137 \\
.190\end{array}$ & $\begin{array}{l}.154 \\
.285 \\
.059 \\
.244 \\
.226\end{array}$ & $\begin{array}{l}.328 \\
.312 \\
.256 \\
.168 \\
.365\end{array}$ & $\begin{array}{l}.205 \\
.354 \\
.239 \\
.296 \\
.168\end{array}$ & $\begin{array}{l}.161 \\
.111 \\
.088 \\
.108 \\
.178\end{array}$ & $\begin{array}{l}.188 \\
.172 \\
.143 \\
.181 \\
.108\end{array}$ & $\begin{array}{l}.188 \\
.279 \\
.283 \\
.249 \\
.270\end{array}$ & $\begin{array}{l}.091 \\
.168 \\
.177 \\
.206 \\
.177\end{array}$ \\
\hline 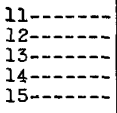 & $\begin{array}{l}.176 \\
.158 \\
.196 \\
.190 \\
.222\end{array}$ & $\begin{array}{l}.160 \\
.356 \\
.220 \\
.254 \\
.296\end{array}$ & $\begin{array}{l}.222 \\
.202 \\
.218 \\
.170 \\
.142\end{array}$ & $\begin{array}{l}.450 \\
.346 \\
.391 \\
.291 \\
.296\end{array}$ & $\begin{array}{l}.301 \\
.378 \\
.228 \\
.248 \\
.178\end{array}$ & $\begin{array}{l}.152 \\
.254 \\
.208 \\
.211 \\
.218\end{array}$ & $\begin{array}{l}.290 \\
.262 \\
.248 \\
.182 \\
.188\end{array}$ & $\begin{array}{l}.246 \\
.352 \\
.140 \\
.200 \\
.205\end{array}$ & $\begin{array}{l}.186 \\
.093 \\
.103 \\
.195 \\
.051\end{array}$ \\
\hline 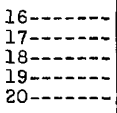 & $\begin{array}{l}.234 \\
.199 \\
.234 \\
.184 \\
.287\end{array}$ & $\begin{array}{l}.026 \\
.308 \\
.166 \\
.202 \\
.169\end{array}$ & $\begin{array}{l}.100 \\
.188 \\
.220 \\
.122 \\
.056\end{array}$ & $\begin{array}{r}.244 \\
.230 \\
.362 \\
6.192 \\
.194\end{array}$ & $\begin{array}{l}.186 \\
.288 \\
.110 \\
.020 \\
.192\end{array}$ & $\begin{array}{l}.076 \\
.148 \\
.181 \\
.150 \\
.143\end{array}$ & $\begin{array}{l}.276 \\
.230 \\
.140 \\
.224 \\
.172\end{array}$ & $\begin{array}{l}.249 \\
.322 \\
.030 \\
.240 \\
.235\end{array}$ & $\begin{array}{l}.310 \\
.152 \\
.162 \\
.125 \\
.166\end{array}$ \\
\hline 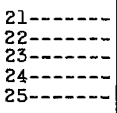 & $\begin{array}{l}.254 \\
.172 \\
.016 \\
.266 \\
.162\end{array}$ & $\begin{array}{l}.413 \\
.137 \\
.197 \\
.156 \\
.132\end{array}$ & $\begin{array}{l}.263 \\
.156 \\
.201 \\
.143 \\
.205\end{array}$ & $\begin{array}{l}.168 \\
.172 \\
.158 \\
.202 \\
.146\end{array}$ & $\begin{array}{l}.154 \\
.167 \\
.170 \\
.199 \\
.268\end{array}$ & $\begin{array}{l}.196 \\
.174 \\
.328 \\
.218 \\
.187\end{array}$ & $\begin{array}{l}.066 \\
.254 \\
.124 \\
.156 \\
.190\end{array}$ & $\begin{array}{l}.234 \\
.047 \\
.256 \\
.196 \\
.208\end{array}$ & $\begin{array}{l}.192 \\
.203 \\
.106 \\
.042 \\
.175\end{array}$ \\
\hline 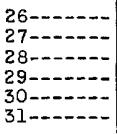 & $\begin{array}{l}.184 \\
.147 \\
.207 \\
.288 \\
.216 \\
.186\end{array}$ & $\begin{array}{l}.180 \\
.202 \\
.241 \\
.183 \\
.247 \\
.152\end{array}$ & $\begin{array}{l}.226 \\
.272 \\
.154 \\
.180 \\
.157 \\
.183\end{array}$ & $\begin{array}{l}.148 \\
.250 \\
.032 \\
.132 \\
.123 \\
.111\end{array}$ & $\begin{array}{l}.176 \\
.213 \\
.194 \\
.264 \\
.196 \\
.200\end{array}$ & $\begin{array}{l}.199 \\
.116 \\
.070 \\
.123 \\
.139 \\
.146 \\
\end{array}$ & $\begin{array}{l}.070 \\
.146 \\
.190 \\
.140 \\
.138 \\
.194 \\
\end{array}$ & $\begin{array}{l}.265 \\
.059 \\
.014 \\
.051 \\
.161 \\
.259\end{array}$ & $\begin{array}{l}.192 \\
.137 \\
.170 \\
.182 \\
.189 \\
.202\end{array}$ \\
\hline Total - & 6.227 & 6.311 & 5.722 & 7.727 & 7.107 & 5.267 & 5.518 & 6.365 & 5.203 \\
\hline August & 1946 & 1947 & 1948 & 1949 & 1950 & 1951 & 1952 & 1953 & Average \\
\hline 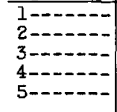 & $\begin{array}{r}0.205 \\
.245 \\
.248 \\
.192 \\
.228\end{array}$ & $\begin{array}{r}0.296 \\
.204 \\
.056 \\
.170 \\
.232\end{array}$ & $\begin{array}{r}0.213 \\
.252 \\
.216 \\
.218 \\
.048\end{array}$ & $\begin{array}{l}0.16 \\
.17 \\
.12 \\
.20 \\
.19\end{array}$ & $\begin{array}{l}0.09 \\
.20 \\
.26 \\
.18 \\
.13\end{array}$ & $\begin{array}{l}0.16 \\
.27 \\
.14 \\
.27 \\
.26\end{array}$ & $\begin{array}{l}0.25 \\
.21 \\
.23 \\
.18 \\
.22\end{array}$ & $\begin{array}{r}0.17 \\
6.18 \\
.17 \\
.24 \\
.13\end{array}$ & $\begin{array}{r}0.198 \\
.245 \\
.211 \\
.216 \\
.197\end{array}$ \\
\hline 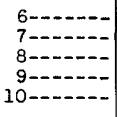 & $\begin{array}{l}.138 \\
.119 \\
.231 \\
.108 \\
.211\end{array}$ & $\begin{array}{r}.252 \\
.254 \\
.118 \\
.220 \\
.226\end{array}$ & $\begin{array}{l}.184 \\
.153 \\
.203 \\
.179 \\
.203\end{array}$ & $\begin{array}{l}.18 \\
.17 \\
.19 \\
.16 \\
.21\end{array}$ & $\begin{array}{l}.20 \\
.20 \\
.25 \\
.13 \\
.04\end{array}$ & $\begin{array}{l}.19 \\
.03 \\
.14 \\
.15 \\
.09\end{array}$ & $\begin{array}{l}.21 \\
.17 \\
.22 \\
.20 \\
.09\end{array}$ & $\begin{array}{l}.08 \\
.14 \\
.18 \\
.10 \\
.13\end{array}$ & $\begin{array}{l}.173 \\
.195 \\
.190 \\
.184 \\
.181\end{array}$ \\
\hline 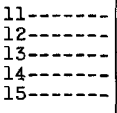 & $\begin{array}{l}.201 \\
.114 \\
.154 \\
.130 \\
.134\end{array}$ & $\begin{array}{l}.112 \\
.334 \\
.105 \\
.185 \\
.246\end{array}$ & $\begin{array}{l}.178 \\
.128 \\
.194 \\
.204 \\
.184\end{array}$ & $\begin{array}{l}.22 \\
.18 \\
.06 \\
.10 \\
.08\end{array}$ & $\begin{array}{l}.21 \\
.11 \\
.12 \\
.21 \\
.17\end{array}$ & $\begin{array}{l}.21 \\
.16 \\
.21 \\
.20 \\
.21\end{array}$ & $\begin{array}{l}.17 \\
.02 \\
.11 \\
.22 \\
.09\end{array}$ & $\begin{array}{l}.16 \\
.11 \\
.22 \\
.18 \\
.27\end{array}$ & $\begin{array}{l}.214 \\
.209 \\
.184 \\
.200 \\
.187\end{array}$ \\
\hline $\begin{array}{l}16------- \\
17------ \\
18------ \\
19------- \\
20-------\end{array}$ & $\begin{array}{l}.130 \\
.104 \\
.068 \\
.150 \\
.180\end{array}$ & $\begin{array}{r}.183 \\
.011 \\
.159 \\
.154 \\
.122\end{array}$ & $\begin{array}{l}.171 \\
.171 \\
.180 \\
.164 \\
.178\end{array}$ & $\begin{array}{l}.05 \\
.03 \\
.14 \\
.20 \\
.18\end{array}$ & $\begin{array}{l}.16 \\
.20 \\
.20 \\
.01 \\
.22\end{array}$ & $\begin{array}{r}.22 \\
.17 \\
b .17 \\
.20 \\
.18\end{array}$ & $\begin{array}{l}.11 \\
.18 \\
.20 \\
.09 \\
.16\end{array}$ & $\begin{array}{l}.20 \\
.13 \\
.21 \\
.14 \\
.20\end{array}$ & $\begin{array}{l}.172 \\
.180 \\
.172 \\
.151 \\
.178\end{array}$ \\
\hline $\begin{array}{l}21------- \\
22------- \\
23------- \\
24------- \\
25-m-----\end{array}$ & $\begin{array}{l}.146 \\
.125 \\
.155 \\
.134 \\
.142\end{array}$ & $\begin{array}{l}.132 \\
.166 \\
.174 \\
.118 \\
.234\end{array}$ & $\begin{array}{l}.155 \\
.137 \\
.266 \\
.192 \\
.230\end{array}$ & $\begin{array}{l}.20 \\
.18 \\
.16 \\
.18 \\
.17\end{array}$ & $\begin{array}{l}.18 \\
.20 \\
.19 \\
.19 \\
.19\end{array}$ & $\begin{array}{l}.15 \\
.19 \\
.19 \\
.21 \\
.21\end{array}$ & $\begin{array}{l}.09 \\
.07 \\
.19 \\
.20 \\
.19\end{array}$ & $\begin{array}{l}.19 \\
.17 \\
.18 \\
.18 \\
.18\end{array}$ & $\begin{array}{l}.187 \\
.160 \\
.180 \\
.175 \\
.189\end{array}$ \\
\hline 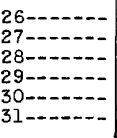 & $\begin{array}{l}.162 \\
.188 \\
.136 \\
.174 \\
.100 \\
.100\end{array}$ & $\begin{array}{l}.084 \\
.070 \\
.114 \\
.142 \\
.090 \\
.146\end{array}$ & $\begin{array}{l}.212 \\
.194 \\
.212 \\
.226 \\
.276 \\
.202\end{array}$ & $\begin{array}{l}.20 \\
.18 \\
.05 \\
.07 \\
.1 .5 \\
.07\end{array}$ & $\begin{array}{l}.17 \\
.22 \\
.18 \\
.07 \\
.11 \\
.04\end{array}$ & $\begin{array}{l}.20 \\
.05 \\
.01 \\
.12 \\
.05 \\
.17 \\
\end{array}$ & $\begin{array}{l}.18 \\
.20 \\
.16 \\
.25 \\
.20 \\
.12 \\
\end{array}$ & $\begin{array}{l}.20 \\
.21 \\
.19 \\
.22 \\
.21 \\
.24 \\
\end{array}$ & $\begin{array}{l}.179 \\
.168 \\
.137 \\
.166 \\
.162 \\
.160 \\
\end{array}$ \\
\hline Total- & 4.852 & 5.109 & 5,923 & 4.63 & 5.03 & 5.18 & 5.18 & 5.51 & 5.695 \\
\hline
\end{tabular}

see footnotes at end of table. 
EVAPORATION DATA

Evaporation, in Inches, in Indianapolis, Ind.--Continued

\begin{tabular}{|c|c|c|c|c|c|c|c|c|c|}
\hline September & 1937 & 1938 & 1939 & 1940 & 1941 & 1942 & 1943 & 1944 & 1945 \\
\hline 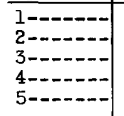 & $\begin{array}{r}0.085 \\
.129 \\
.194 \\
.167 \\
.199\end{array}$ & $\begin{array}{r}0.161 \\
.222 \\
.096 \\
.200 \\
.243\end{array}$ & $\begin{array}{r}0.048 \\
.262 \\
.148 \\
.224 \\
.266\end{array}$ & $\begin{array}{r}0.200 \\
.155 \\
.113 \\
.086 \\
.190\end{array}$ & $\begin{array}{r}0.322 \\
.092 \\
.102 \\
.126 \\
.100\end{array}$ & $\begin{array}{r}0.190 \\
.166 \\
.138 \\
.120 \\
.164\end{array}$ & $\begin{array}{r}0.282 \\
.242 \\
.170 \\
.150 \\
.096\end{array}$ & $\begin{array}{r}0.108 \\
.210 \\
.187 \\
.216 \\
.174\end{array}$ & $\begin{array}{r}0.218 \\
.230 \\
.192 \\
.155 \\
.182\end{array}$ \\
\hline 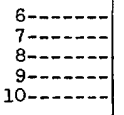 & $\begin{array}{l}.125 \\
.070 \\
.281 \\
.197 \\
.200\end{array}$ & $\begin{array}{l}.193 \\
.208 \\
.238 \\
.222 \\
.180\end{array}$ & $\begin{array}{l}.212 \\
.218 \\
.296 \\
.310 \\
.298\end{array}$ & $\begin{array}{l}.055 \\
.189 \\
.136 \\
.256 \\
.204\end{array}$ & $\begin{array}{l}.174 \\
.190 \\
.110 \\
.236 \\
.256\end{array}$ & $\begin{array}{l}.152 \\
.126 \\
.082 \\
.090 \\
.105\end{array}$ & $\begin{array}{l}.156 \\
.084 \\
.174 \\
.232 \\
.118\end{array}$ & $\begin{array}{l}.155 \\
.114 \\
.127 \\
.141 \\
.152\end{array}$ & $\begin{array}{l}.186 \\
.170 \\
.143 \\
.140 \\
.165\end{array}$ \\
\hline 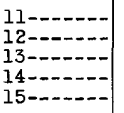 & $\begin{array}{l}.113 \\
.156 \\
.126 \\
.148 \\
.178\end{array}$ & $\begin{array}{l}.086 \\
.116 \\
.172 \\
.054 \\
.166\end{array}$ & $\begin{array}{l}.256 \\
.174 \\
.236 \\
.235 \\
.125\end{array}$ & $\begin{array}{l}.174 \\
.090 \\
.108 \\
.226 \\
.198\end{array}$ & $\begin{array}{l}.194 \\
.178 \\
.222 \\
.153 \\
.246\end{array}$ & $\begin{array}{l}.149 \\
.158 \\
.151 \\
.153 \\
.182\end{array}$ & $\begin{array}{l}.158 \\
.104 \\
.034 \\
.056 \\
.104\end{array}$ & $\begin{array}{l}.177 \\
.104 \\
.047 \\
.095 \\
.116\end{array}$ & $\begin{array}{l}.156 \\
.119 \\
.030 \\
.096 \\
.072\end{array}$ \\
\hline 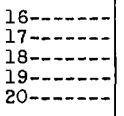 & $\begin{array}{l}.187 \\
.168 \\
.102 \\
.173 \\
.174\end{array}$ & $\begin{array}{l}.198 \\
.102 \\
.122 \\
.137 \\
.159\end{array}$ & $\begin{array}{l}.224 \\
.372 \\
.368 \\
.100 \\
.146\end{array}$ & $\begin{array}{l}.095 \\
.123 \\
.126 \\
.132 \\
.188\end{array}$ & $\begin{array}{l}.217 \\
.222 \\
.167 \\
.187 \\
.210\end{array}$ & $\begin{array}{l}.177 \\
.130 \\
.053 \\
.128 \\
.112\end{array}$ & $\begin{array}{l}.112 \\
.155 \\
.095 \\
.126 \\
.080\end{array}$ & $\begin{array}{l}.096 \\
.130 \\
.159 \\
.141 \\
.144\end{array}$ & $\begin{array}{l}.089 \\
.127 \\
.121 \\
.033 \\
.117\end{array}$ \\
\hline 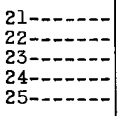 & $\begin{array}{l}.128 \\
.124 \\
.118 \\
.121 \\
.164\end{array}$ & $\begin{array}{l}.060 \\
.094 \\
.015 \\
.131 \\
.164\end{array}$ & $\begin{array}{l}.162 \\
.186 \\
.154 \\
.204 \\
.250\end{array}$ & $\begin{array}{l}.114 \\
.198 \\
.199 \\
.110 \\
.088\end{array}$ & $\begin{array}{l}.196 \\
.186 \\
.144 \\
.159 \\
.322\end{array}$ & $\begin{array}{l}.178 \\
.096 \\
.112 \\
.188 \\
.141\end{array}$ & $\begin{array}{l}.054 \\
.046 \\
.118 \\
.102 \\
.064\end{array}$ & $\begin{array}{l}.048 \\
.190 \\
.170 \\
.081 \\
.103\end{array}$ & $\begin{array}{l}.100 \\
.093 \\
.046 \\
.161 \\
.141\end{array}$ \\
\hline 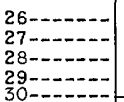 & $\begin{array}{l}.059 \\
.122 \\
.067 \\
.060 \\
.120\end{array}$ & $\begin{array}{l}.240 \\
.182 \\
.180 \\
.138 \\
.100 \\
\end{array}$ & $\begin{array}{l}.258 \\
.132 \\
.100 \\
.160 \\
.136 \\
\end{array}$ & $\begin{array}{r}.182 \\
.112 \\
.098 \\
.076 \\
.137 \\
\end{array}$ & $\begin{array}{r}.110 \\
.114 \\
.187 \\
.088 \\
.070 \\
\end{array}$ & $\begin{array}{l}.104 \\
.070 \\
.094 \\
.086 \\
.037\end{array}$ & $\begin{array}{r}.046 \\
.110 \\
.096 \\
.089 \\
.106 \\
\end{array}$ & $\begin{array}{l}.100 \\
.123 \\
.120 \\
.042 \\
.091\end{array}$ & $\begin{array}{l}.052 \\
.051 \\
.144 \\
.158 \\
.046 \\
\end{array}$ \\
\hline Total- & 4.255 & 4.579 & 6.260 & 4.358 & 5.280 & 3.832 & 3.559 & 3.861 & 3.733 \\
\hline September & 1946 & 1947 & 1948 & 1949 & 1950 & 1951 & 1952 & 1953 & Average \\
\hline 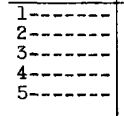 & $\begin{array}{r}0.170 \\
.076 \\
.138 \\
.153 \\
.169\end{array}$ & $\begin{array}{r}0.090 \\
.134 \\
.188 \\
.232 \\
.068\end{array}$ & $\begin{array}{r}0.214 \\
.157 \\
.157 \\
.012 \\
.186\end{array}$ & $\begin{array}{l}0.28 \\
.15 \\
.13 \\
.18 \\
.07\end{array}$ & $\begin{array}{l}0.04 \\
.16 \\
.13 \\
.07 \\
.19\end{array}$ & $\begin{array}{l}0.30 \\
.04 \\
.19 \\
.10 \\
.11\end{array}$ & $\begin{array}{r}0.20 \\
.27 \\
.11 \\
.11 \\
.14\end{array}$ & $\begin{array}{l}0.24 \\
.25 \\
.26 \\
.04 \\
.08\end{array}$ & $\begin{array}{r}0.185 \\
.173 \\
.155 \\
.138 \\
.154\end{array}$ \\
\hline 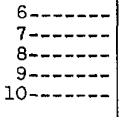 & $\begin{array}{l}.156 \\
.144 \\
.096 \\
.125 \\
.113\end{array}$ & $\begin{array}{l}.090 \\
.102 \\
.119 \\
.141 \\
.102\end{array}$ & $\begin{array}{l}.130 \\
.022 \\
.144 \\
.049 \\
.191\end{array}$ & $\begin{array}{l}.17 \\
.18 \\
.13 \\
.09 \\
.14\end{array}$ & $\begin{array}{l}.19 \\
.15 \\
.17 \\
.00 \\
.04\end{array}$ & $\begin{array}{l}.09 \\
.19 \\
.17 \\
.14 \\
.13\end{array}$ & $\begin{array}{l}.15 \\
.20 \\
.15 \\
.16 \\
.15\end{array}$ & $\begin{array}{l}.15 \\
.16 \\
.22 \\
.17 \\
.14\end{array}$ & $\begin{array}{l}.149 \\
.148 \\
.164 \\
.159 \\
.158\end{array}$ \\
\hline 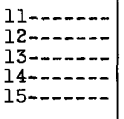 & $\begin{array}{l}.205 \\
.154 \\
.089 \\
.108 \\
.097\end{array}$ & $\begin{array}{l}.128 \\
.186 \\
.024 \\
.171 \\
.221\end{array}$ & $\begin{array}{l}.124 \\
.182 \\
.186 \\
.209 \\
.167\end{array}$ & $\begin{array}{l}.15 \\
.14 \\
.02 \\
.09 \\
.07\end{array}$ & $\begin{array}{l}.05 \\
.07 \\
.05 \\
.15 \\
.09\end{array}$ & $\begin{array}{l}.06 \\
.22 \\
.20 \\
.07 \\
.21\end{array}$ & $\begin{array}{r}.18 \\
.21 \\
.18 \\
.20 \\
b .15\end{array}$ & $\begin{array}{l}.20 \\
.20 \\
.21 \\
.13 \\
.05\end{array}$ & $\begin{array}{l}.151 \\
.151 \\
.123 \\
.138 \\
.144\end{array}$ \\
\hline 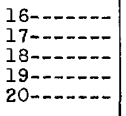 & $\begin{array}{l}.150 \\
.174 \\
.063 \\
.178 \\
.150\end{array}$ & $\begin{array}{l}.098 \\
.124 \\
.104 \\
.144 \\
.135\end{array}$ & $\begin{array}{l}.162 \\
.156 \\
.100 \\
.100 \\
.272\end{array}$ & $\begin{array}{l}.05 \\
.05 \\
.08 \\
.10 \\
.19\end{array}$ & $\begin{array}{l}.12 \\
.13 \\
.13 \\
.13 \\
.12\end{array}$ & $\begin{array}{l}.14 \\
.13 \\
.15 \\
.17 \\
.15\end{array}$ & $\begin{array}{l}.17 \\
.17 \\
.14 \\
.17 \\
.12\end{array}$ & $\begin{array}{l}.14 \\
.19 \\
.18 \\
.16 \\
.03\end{array}$ & $\begin{array}{l}.143 \\
.156 \\
.133 \\
.136 \\
.147\end{array}$ \\
\hline 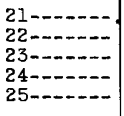 & $\begin{array}{l}.088 \\
.096 \\
.037 \\
.109 \\
.188\end{array}$ & $\begin{array}{l}.168 \\
.175 \\
.129 \\
.083 \\
.108\end{array}$ & $\begin{array}{l}.092 \\
.152 \\
.096 \\
.094 \\
.110\end{array}$ & $\begin{array}{l}.10 \\
.06 \\
.12 \\
.10 \\
.10\end{array}$ & $\begin{array}{r}.10 \\
.13 \\
.09 \\
.17 \\
.06\end{array}$ & $\begin{array}{l}.22 \\
.10 \\
.09 \\
.13 \\
.02\end{array}$ & $\begin{array}{l}.11 \\
.15 \\
.10 \\
.09 \\
.14\end{array}$ & $\begin{array}{l}.21 \\
.15 \\
.15 \\
.11 \\
.16\end{array}$ & $\begin{array}{l}.125 \\
.131 \\
.111 \\
.126 \\
.137\end{array}$ \\
\hline 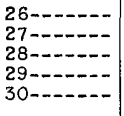 & $\begin{array}{l}.117 \\
.113 \\
.180 \\
.080 \\
.092\end{array}$ & $\begin{array}{l}.102 \\
.141 \\
.117 \\
.052 \\
.081\end{array}$ & $\begin{array}{l}.102 \\
.136 \\
.136 \\
.084 \\
.056\end{array}$ & $\begin{array}{l}.11 \\
.12 \\
.11 \\
.13 \\
.11\end{array}$ & $\begin{array}{l}.07 \\
.08 \\
.05 \\
.04 \\
.11\end{array}$ & $\begin{array}{l}.07 \\
.11 \\
.20 \\
.11 \\
.11\end{array}$ & $\begin{array}{l}.08 \\
.18 \\
.12 \\
.13 \\
.09\end{array}$ & $\begin{array}{l}.15 \\
.17 \\
.21 \\
.19 \\
.29\end{array}$ & $\begin{array}{l}.115 \\
.122 \\
.130 \\
.101 \\
.105\end{array}$ \\
\hline Total- & 3.808 & 3.757 & 3.978 & 3.52 & 3.08 & 4.12 & 4.52 & 4.99 & 4.208 \\
\hline
\end{tabular}

See footnotes at end of table. 
Evaporation, in inches, in Indianapolis, Ind.--Continued

\begin{tabular}{|c|c|c|c|c|c|c|c|c|}
\hline October & 1944 & 1945 & 1946 & 1947 & 1948 & 1949 & 1950 & 1951 \\
\hline 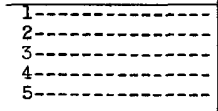 & $\begin{array}{r}0.071 \\
.070 \\
.021 \\
.023 \\
.060\end{array}$ & $\begin{array}{r}0.025 \\
.107 \\
.069 \\
.086 \\
.072\end{array}$ & $\begin{array}{r}0.148 \\
.112 \\
.094 \\
.126 \\
.116\end{array}$ & $\begin{array}{r}0.091 \\
.076 \\
.092 \\
.114 \\
.090\end{array}$ & $\begin{array}{r}0.045 \\
.123 \\
.122 \\
.096 \\
.077\end{array}$ & $\begin{array}{r}0.08 \\
.13 \\
.11 \\
.00 \\
.01\end{array}$ & $\begin{array}{l}0.07 \\
.08 \\
.08 \\
.08 \\
.10\end{array}$ & $\begin{array}{l}0.05 \\
.11 \\
.15 \\
.20 \\
.27\end{array}$ \\
\hline 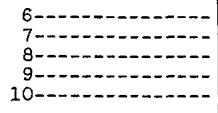 & $\begin{array}{r}b .071 \\
b .049 \\
b .089 \\
b .068 \\
.088\end{array}$ & $\begin{array}{l}.021 \\
.054 \\
.007 \\
.002 \\
.012\end{array}$ & $\begin{array}{l}.126 \\
.112 \\
.126 \\
.091 \\
.087\end{array}$ & $\begin{array}{l}.100 \\
.114 \\
.118 \\
.096 \\
.085\end{array}$ & $\begin{array}{l}.088 \\
.099 \\
.056 \\
.029 \\
.108\end{array}$ & $\begin{array}{l}.06 \\
.01 \\
.12 \\
.11 \\
.15\end{array}$ & $\begin{array}{l}.09 \\
.12 \\
.10 \\
.05 \\
.05\end{array}$ & $\begin{array}{l}.18 \\
.05 \\
.07 \\
.09 \\
.08\end{array}$ \\
\hline 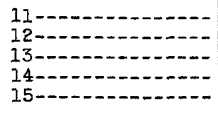 & $\begin{array}{l}.040 \\
.026 \\
.049 \\
.100 \\
.112\end{array}$ & $\begin{array}{l}.163 \\
.114 \\
.125 \\
.060 \\
.038\end{array}$ & $\begin{array}{l}.124 \\
.102 \\
.056 \\
.116 \\
.114\end{array}$ & $\begin{array}{l}.055 \\
.052 \\
.072 \\
.101 \\
.129\end{array}$ & $\begin{array}{l}.043 \\
.030 \\
.060 \\
.100 \\
.070\end{array}$ & $\begin{array}{l}.21 \\
.11 \\
.14 \\
.10 \\
.07\end{array}$ & $\begin{array}{l}.11 \\
.10 \\
.15 \\
.08 \\
.10\end{array}$ & $\begin{array}{l}.07 \\
.07 \\
.09 \\
.11 \\
.14\end{array}$ \\
\hline 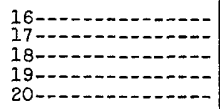 & $\begin{array}{l}.075 \\
.065 \\
.070 \\
.060 \\
.094\end{array}$ & $\begin{array}{l}.062 \\
.068 \\
.094 \\
.138 \\
.052\end{array}$ & $\begin{array}{l}.116 \\
.054 \\
.032 \\
.058 \\
.091\end{array}$ & $\begin{array}{l}.076 \\
.095 \\
.019 \\
.034 \\
.055\end{array}$ & $\begin{array}{l}.089 \\
.047 \\
.052 \\
.070 \\
.081\end{array}$ & $\begin{array}{l}.07 \\
.05 \\
.03 \\
.05 \\
.08\end{array}$ & $\begin{array}{l}.12 \\
.08 \\
.09 \\
.11 \\
.04\end{array}$ & $\begin{array}{l}.11 \\
.14 \\
.12 \\
.15 \\
.08\end{array}$ \\
\hline 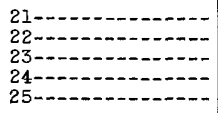 & $\begin{array}{l}.041 \\
.092 \\
.072 \\
.080 \\
.094\end{array}$ & $\begin{array}{l}.060 \\
.016 \\
.026 \\
.066 \\
.044\end{array}$ & $\begin{array}{l}.041 \\
.060 \\
.072 \\
.098 \\
.132\end{array}$ & $\begin{array}{l}.065 \\
.064 \\
.097 \\
.117 \\
.049\end{array}$ & $\begin{array}{l}.061 \\
.004 \\
.043 \\
.054 \\
.027\end{array}$ & $\begin{array}{l}.06 \\
.07 \\
.04 \\
.19 \\
.06\end{array}$ & $\begin{array}{l}.17 \\
.07 \\
.04 \\
.02 \\
.05\end{array}$ & $\begin{array}{l}.08 \\
.12 \\
.10 \\
.07 \\
.02\end{array}$ \\
\hline 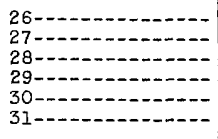 & $\begin{array}{l}.107 \\
.099 \\
.048 \\
.100 \\
.052 \\
.058\end{array}$ & $\begin{array}{l}.078 \\
.096 \\
.080 \\
.022 \\
.100 \\
.148\end{array}$ & $\begin{array}{l}.066 \\
.092 \\
.082 \\
.108 \\
.114 \\
.102\end{array}$ & $\begin{array}{l}.057 \\
.084 \\
.092 \\
.011 \\
.032 \\
.035\end{array}$ & $\begin{array}{l}c .000 \\
.036 \\
.054 \\
.043 \\
.073 \\
.033\end{array}$ & $\begin{array}{l}.06 \\
.10 \\
.02 \\
.08 \\
.05 \\
.10\end{array}$ & $\begin{array}{l}.06 \\
.04 \\
.06 \\
.04 \\
.04 \\
.07\end{array}$ & $\begin{array}{l}.08 \\
.07 \\
.03 \\
.10 \\
.05 \\
.11\end{array}$ \\
\hline Total- & 2.144 & 2.105 & 2. 968 & 2.367 & 1.913 & 2.52 & 2.46 & 3.16 \\
\hline October & 1952 & 1953 & Average & \multicolumn{2}{|c|}{ November } & 1950 & 1952 & 1953 \\
\hline 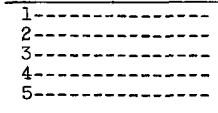 & $\begin{array}{l}0.20 \\
.23 \\
.09 \\
.10 \\
.14\end{array}$ & $\begin{array}{r}0.20 \\
.18 \\
.14 \\
.14 \\
.07\end{array}$ & $\begin{array}{l}0.098 \\
.122 \\
.097 \\
.096 \\
.100\end{array}$ & \multicolumn{2}{|c|}{ 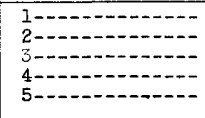 } & $\begin{array}{l}0.19 \\
.08 \\
.05 \\
.08 \\
.02\end{array}$ & $\begin{array}{l}0.07 \\
.08 \\
.15 \\
.09 \\
.12\end{array}$ & $\begin{array}{l}0.08 \\
.07 \\
.12 \\
.11 \\
.09\end{array}$ \\
\hline 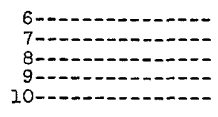 & $\begin{array}{l}.03 \\
.09 \\
.07 \\
.09 \\
.11\end{array}$ & $\begin{array}{l}.04 \\
.08 \\
.09 \\
.09 \\
.09\end{array}$ & $\begin{array}{l}.081 \\
.078 \\
.085 \\
.072 \\
.086\end{array}$ & \multicolumn{2}{|c|}{ 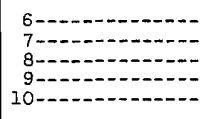 } & $\begin{array}{l}.10 \\
.07 \\
.05 \\
.03 \\
.03\end{array}$ & $\begin{array}{l}.17 \\
.06 \\
.05 \\
.02 \\
.06\end{array}$ & $\begin{array}{l}.06 \\
a .03 \\
.05 \\
.09 \\
.08\end{array}$ \\
\hline 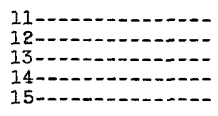 & $\begin{array}{l}.07 \\
.08 \\
.16 \\
.10 \\
.06\end{array}$ & $\begin{array}{l}.12 \\
.16 \\
.13 \\
.20 \\
.00\end{array}$ & $\begin{array}{l}.100 \\
.084 \\
.103 \\
.107 \\
.083\end{array}$ & \multicolumn{2}{|c|}{ 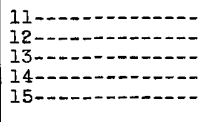 } & $\begin{array}{r}.04 \\
.05 \\
.06 \\
.01 \\
.03\end{array}$ & $\begin{array}{l}.04 \\
.04 \\
.04 \\
.10 \\
.02\end{array}$ & $\begin{array}{l}.06 \\
.07 \\
.03 \\
.04 \\
.13\end{array}$ \\
\hline 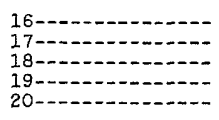 & $\begin{array}{l}.05 \\
.09 \\
.09 \\
.11 \\
.13\end{array}$ & $\begin{array}{l}.11 \\
.12 \\
.12 \\
.13 \\
.14\end{array}$ & $\begin{array}{l}.088 \\
.081 \\
.072 \\
.091 \\
.084\end{array}$ & \multicolumn{2}{|c|}{ 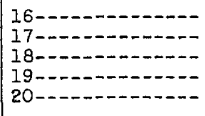 } & $\begin{array}{l}.07 \\
.10 \\
.04 \\
.05 \\
\text { b.04 }\end{array}$ & $\begin{array}{l}.05 \\
.09 \\
.10 \\
.03 \\
.05\end{array}$ & $\begin{array}{l}.09 \\
.10 \\
.11 \\
.05 \\
.09\end{array}$ \\
\hline 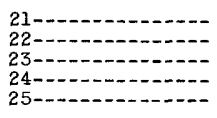 & $\begin{array}{l}.07 \\
.05 \\
.10 \\
.10 \\
.09\end{array}$ & $\begin{array}{l}.10 \\
.12 \\
.10 \\
.13 \\
.05\end{array}$ & $\begin{array}{l}.075 \\
.067 \\
.069 \\
.092 \\
.062\end{array}$ & \multicolumn{2}{|c|}{ 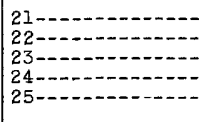 } & $\begin{array}{l}b .03 \\
b .03 \\
b .01 \\
b .00 \\
b .01\end{array}$ & $\begin{array}{l}.03 \\
.04 \\
.05 \\
.02 \\
.00\end{array}$ & $\begin{array}{l}.06 \\
.08 \\
.02 \\
.04 \\
.03\end{array}$ \\
\hline 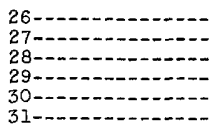 & $\begin{array}{l}.11 \\
.23 \\
.05 \\
.18 \\
.10 \\
.21\end{array}$ & $\begin{array}{l}.05 \\
.02 \\
.01 \\
.06 \\
.09 \\
.09\end{array}$ & $\begin{array}{l}.067 \\
.087 \\
.053 \\
.074 \\
.070 \\
.096\end{array}$ & \multirow{2}{*}{\multicolumn{2}{|c|}{ 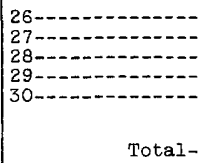 }} & $\begin{array}{l}b .02 \\
b .01 \\
b .02 \\
b .02 \\
b .02\end{array}$ & $\begin{array}{l}.02 \\
b .02 \\
b .01 \\
b .02 \\
b .02\end{array}$ & $\begin{array}{l}b .03 \\
b .02 \\
b .01 \\
b .02 \\
b .02\end{array}$ \\
\hline Total- & 3.38 & 3.17 & 2.620 & & & 1.36 & 1.66 & 1.88 \\
\hline
\end{tabular}

a Estimated from total observed on underlined day.

$b$ Estimated because of missing or erroneous data.

- Corrected since originaliy published by U. S. Weather Bureau. dNot previously published. 
Evaporation, in Inches, at Kendallville, Ind.

[No record for Apr11 1950; pan was leaking]

\begin{tabular}{|c|c|c|c|c|c|c|c|c|c|}
\hline March & 1948 & Apr11 & 1947 & 1948 & 1949 & 1951. & 1952 & 1953 & Average \\
\hline 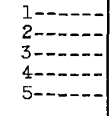 & $\begin{array}{r}b 0.050 \\
.075 \\
.050 \\
.102 \\
.134\end{array}$ & 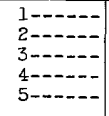 & $\begin{array}{r}0.109 \\
.036 \\
.088 \\
. .140 \\
-.010\end{array}$ & $\begin{array}{r}0.018 \\
.048 \\
.109 \\
.175 \\
.150\end{array}$ & $\begin{array}{r}0.059 \\
.118 \\
.155 \\
.120 \\
.102\end{array}$ & $\begin{array}{l}0.00 \\
.06 \\
.05 \\
.08 \\
.15\end{array}$ & $\begin{array}{l}0.09 \\
.13 \\
.12 \\
.05 \\
.02\end{array}$ & $\begin{array}{r}0.06 \\
.12 \\
.12 \\
.07 \\
.11\end{array}$ & $\begin{array}{r}0.056 \\
.085 \\
.107 \\
.106 \\
.087\end{array}$ \\
\hline 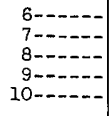 & $\begin{array}{l}b .080 \\
b .050 \\
b .110 \\
b .090 \\
b .070\end{array}$ & $\begin{array}{r}6-\cdots-\cdots \\
7-\cdots-\cdots \\
8 \cdots-\cdots- \\
9 \cdots-\cdots- \\
10-\cdots=-\cdots\end{array}$ & $\begin{array}{r}.217 \\
.228 \\
.059 \\
.054 \\
.132\end{array}$ & $\begin{array}{l}.209 \\
.078 \\
.105 \\
.380 \\
.171\end{array}$ & $\begin{array}{l}.100 \\
.146 \\
.137 \\
.156 \\
.167\end{array}$ & $\begin{array}{l}.11 \\
.09 \\
.07 \\
.03 \\
.05\end{array}$ & $\begin{array}{l}.02 \\
.07 \\
.13 \\
.18 \\
.17\end{array}$ & $\begin{array}{l}.11 \\
.16 \\
.12 \\
.20 \\
.21\end{array}$ & $\begin{array}{l}.128 \\
.129 \\
.104 \\
.167 \\
.150\end{array}$ \\
\hline 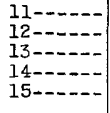 & $\begin{array}{l}b .050 \\
b .100 \\
b .080 \\
b .090 \\
b .070\end{array}$ & 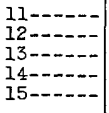 & $\begin{array}{l}.144 \\
.104 \\
.094 \\
.101 \\
.120\end{array}$ & $\begin{array}{l}.147 \\
.043 \\
.039 \\
.035 \\
.077\end{array}$ & $\begin{array}{l}.172 \\
.194 \\
.162 \\
.209 \\
.094\end{array}$ & $\begin{array}{r}. .06 \\
c . .15 \\
-.01 \\
.02 \\
.10\end{array}$ & $\begin{array}{r}.07 \\
.10 \\
6.04 \\
.04 \\
.08\end{array}$ & $\begin{array}{l}.08 \\
.08 \\
.09 \\
.14 \\
.15\end{array}$ & $\begin{array}{l}.112 \\
.112 \\
.069 \\
.091 \\
.104\end{array}$ \\
\hline 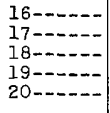 & $\begin{array}{r}b .140 \\
6.070 \\
.021 \\
.050 \\
.133\end{array}$ & 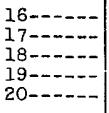 & $\begin{array}{l}.078 \\
.046 \\
.191 \\
.078 \\
.076\end{array}$ & $\begin{array}{l}.309 \\
.187 \\
.152 \\
.198 \\
.255\end{array}$ & $\begin{array}{l}.112 \\
.004 \\
.001 \\
.118 \\
.170\end{array}$ & $\begin{array}{l}.07 \\
.08 \\
.15 \\
.20 \\
.14\end{array}$ & $\begin{array}{l}.13 \\
.34 \\
.19 \\
.30 \\
.24\end{array}$ & $\begin{array}{l}.01 \\
.10 \\
.05 \\
.10 \\
.05\end{array}$ & $\begin{array}{l}.118 \\
.126 \\
.122 \\
.166 \\
.155\end{array}$ \\
\hline $\begin{array}{l}21-\cdots--- \\
22-\cdots--- \\
23-\cdots--- \\
24-\cdots---- \\
25-\cdots----\end{array}$ & $\begin{array}{l}.076 \\
.100 \\
.081 \\
.070 \\
.135\end{array}$ & 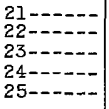 & $\begin{array}{r}c .007 \\
.096 \\
c .039 \\
c .198 \\
c .167\end{array}$ & $\begin{array}{l}.258 \\
.211 \\
.124 \\
.080 \\
.060\end{array}$ & $\begin{array}{r}.199 \\
.255 \\
b .233 \\
b .233 \\
.106\end{array}$ & $\begin{array}{l}.18 \\
.09 \\
.13 \\
.13 \\
.10\end{array}$ & $\begin{array}{l}.21 \\
.12 \\
.09 \\
.09 \\
.11\end{array}$ & $\begin{array}{l}.16 \\
.17 \\
.27 \\
.18 \\
.18\end{array}$ & $\begin{array}{l}.169 \\
.157 \\
.148 \\
.152 \\
.120\end{array}$ \\
\hline 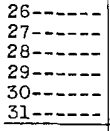 & $\begin{array}{l}.199 \\
.126 \\
.095 \\
.117 \\
.071 \\
.127 \\
\end{array}$ & 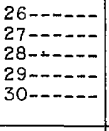 & $\begin{array}{l}.057 \\
.202 \\
.175 \\
.222 \\
.018\end{array}$ & $\begin{array}{l}.302 \\
.089 \\
.289 \\
.090 \\
.207\end{array}$ & $\begin{array}{l}.136 \\
.144 \\
.275 \\
.279 \\
.159\end{array}$ & $\begin{array}{l}.11 \\
.19 \\
.16 \\
.28 \\
.16\end{array}$ & $\begin{array}{l}.23 \\
.26 \\
.25 \\
.14 \\
.23\end{array}$ & $\begin{array}{l}.08 \\
.04 \\
.03 \\
.15 \\
.18\end{array}$ & $\begin{array}{l}.152 \\
.154 \\
.196 \\
.194 \\
.159\end{array}$ \\
\hline Total- & 2.812 & Total- & $c 3.266$ & 4.595 & 4.515 & 3.18 & 4.24 & 3.57 & 3.895 \\
\hline May & & 1947 & 1948 & 1949 & 1950 & 1951 & 1952 & 1953 & Average \\
\hline 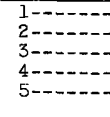 & & $\begin{array}{r}-0.021 \\
.177 \\
.042 \\
.070 \\
.155\end{array}$ & $\begin{array}{r}0.091 \\
.050 \\
.236 \\
.100 \\
.032\end{array}$ & $\begin{array}{r}0.05 \\
.18 \\
.25 \\
.26 \\
.28\end{array}$ & $\begin{array}{r}60.05 \\
b .10 \\
b . .25 \\
b .15 \\
6.18\end{array}$ & $\begin{array}{l}0.27 \\
.22 \\
.24 \\
.20 \\
.10\end{array}$ & $\begin{array}{l}0.27 \\
.23 \\
.24 \\
.31 \\
.27\end{array}$ & $\begin{array}{r}0.16 \\
.22 \\
.16 \\
.15 \\
.16\end{array}$ & $\begin{array}{r}0.124 \\
.168 \\
.203 \\
.177 \\
.168\end{array}$ \\
\hline & 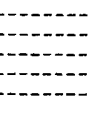 & $\begin{array}{l}.152 \\
.118 \\
.135 \\
.169 \\
.142\end{array}$ & $\begin{array}{l}.190 \\
.168 \\
.168 \\
.122 \\
.059\end{array}$ & $\begin{array}{l}.29 \\
.26 \\
.20 \\
.13 \\
.20\end{array}$ & $\begin{array}{l}b .33 \\
b .28 \\
b .18 \\
b .23 \\
b .15\end{array}$ & $\begin{array}{r}.22 \\
.19 \\
.27 \\
.22 \\
6.19\end{array}$ & $\begin{array}{l}.30 \\
.18 \\
.07 \\
.08 \\
.07\end{array}$ & $\begin{array}{l}.09 \\
.09 \\
.08 \\
.18 \\
.27\end{array}$ & $\begin{array}{l}.225 \\
.184 \\
.158 \\
.162 \\
.154\end{array}$ \\
\hline $\begin{array}{l}11= \\
12 \\
13 \\
14 \\
15\end{array}$ & 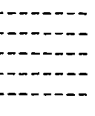 & $\begin{array}{l}.152 \\
.202 \\
.155 \\
.133 \\
.112\end{array}$ & $\begin{array}{l}.343 \\
.076 \\
.036 \\
.083 \\
.083\end{array}$ & $\begin{array}{r}c .21 \\
.20 \\
.26 \\
.24 \\
.18\end{array}$ & $\begin{array}{l}b .10 \\
b .15 \\
.18 \\
.26 \\
.22\end{array}$ & $\begin{array}{l}.00 \\
.11 \\
.21 \\
.20 \\
.33\end{array}$ & $\begin{array}{l}.17 \\
.10 \\
.18 \\
.19 \\
.12\end{array}$ & $\begin{array}{l}.23 \\
.10 \\
.14 \\
.07 \\
.04\end{array}$ & $\begin{array}{l}.172 \\
.134 \\
.166 \\
.168 \\
.155\end{array}$ \\
\hline $\begin{array}{l}16- \\
17-0 \\
18 \\
19- \\
20-0\end{array}$ & 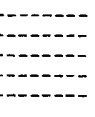 & $\begin{array}{l}.080 \\
.148 \\
.075 \\
.084 \\
.170\end{array}$ & $\begin{array}{l}.351 \\
a .148 \\
a .147 \\
a .148 \\
a .147 \\
\end{array}$ & $\begin{array}{l}.11 \\
.21 \\
.25 \\
.15 \\
.13\end{array}$ & $\begin{array}{l}.20 \\
.28 \\
.17 \\
.27 \\
.28\end{array}$ & $\begin{array}{l}.27 \\
.20 \\
.21 \\
.19 \\
.24\end{array}$ & $\begin{array}{l}.06 \\
.04 \\
.10 \\
.10 \\
.02\end{array}$ & $\begin{array}{l}.06 \\
.05 \\
.12 \\
.25 \\
.19\end{array}$ & $\begin{array}{l}.162 \\
.154 \\
.153 \\
.170 \\
.168\end{array}$ \\
\hline $\begin{array}{l}21-\cdots \\
22-\cdots \\
23-\cdots \\
24 \ldots \\
25 \ldots\end{array}$ & 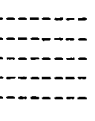 & $\begin{array}{r}.252 \\
.087 \\
.302 \\
.340 \\
.6-.010\end{array}$ & $\begin{array}{l}.268 \\
.184 \\
.184 \\
.261 \\
.207\end{array}$ & $\begin{array}{r}.05 \\
.10 \\
.17 \\
.14 \\
.16\end{array}$ & $\begin{array}{l}.34 \\
.17 \\
.15 \\
.23 \\
.26\end{array}$ & $\begin{array}{l}.25 \\
.07 \\
.11 \\
.25 \\
.29\end{array}$ & $\begin{array}{l}.12 \\
.13 \\
.09 \\
.16 \\
.04\end{array}$ & $\begin{array}{l}.24 \\
.27 \\
.05 \\
.14 \\
.10\end{array}$ & $\begin{array}{l}.217 \\
.144 \\
.151 \\
.217 \\
.150\end{array}$ \\
\hline $\begin{array}{l}26 \cdots \\
27-\cdots \\
28 \\
29 \\
30 \cdots \\
31 \ldots\end{array}$ & 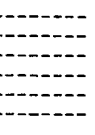 & $\begin{array}{r}.106 \\
.192 \\
.008 \\
.124 \\
.127 \\
.163 \\
\end{array}$ & $\begin{array}{r}.179 \\
a .179 \\
.227 \\
.216 \\
.319 \\
.142 \\
\end{array}$ & $\begin{array}{l}.14 \\
.10 \\
.23 \\
.20 \\
.19 \\
.18 \\
\end{array}$ & $\begin{array}{l}.19 \\
.15 \\
.18 \\
.12 \\
.24 \\
.18 \\
\end{array}$ & $\begin{array}{l}.18 \\
.08 \\
.02 \\
.04 \\
.21 \\
.17\end{array}$ & $\begin{array}{l}.11 \\
.19 \\
.26 \\
.23 \\
.22 \\
.18 \\
\end{array}$ & $\begin{array}{l}.25 \\
.26 \\
.19 \\
.23 \\
.28 \\
.36 \\
\end{array}$ & $\begin{array}{l}.165 \\
.164 \\
.159 \\
.166 \\
.227 \\
.196 \\
\end{array}$ \\
\hline \multicolumn{2}{|c|}{ Total-_-_- } & 4.141 & 5.144 & 5.70 & 6.22 & 5.75 & 4.83 & 5.18 & 5.281 \\
\hline
\end{tabular}

See footnotes at end of table. 
Evaporation in inches at Kendallville, Ind.--Continued

\begin{tabular}{|c|c|c|c|c|c|c|c|c|}
\hline June & 1947 & 1948 & 1949 & 1950 & 1951 & 1952 & 1953 & Average \\
\hline 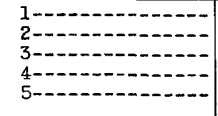 & $\begin{array}{r}0.145 \\
.002 \\
.163 \\
.161 \\
.127\end{array}$ & $\begin{array}{r}0.272 \\
.133 \\
.221 \\
.071 \\
.070\end{array}$ & $\begin{array}{l}0.22 \\
.24 \\
.25 \\
.25 \\
.28\end{array}$ & $\begin{array}{r}0.34 \\
.15 \\
.11 \\
.23 \\
.32\end{array}$ & $\begin{array}{l}0.18 \\
.31 \\
.25 \\
.11 \\
.10\end{array}$ & $\begin{array}{l}0.23 \\
.27 \\
.19 \\
.22 \\
.15\end{array}$ & $\begin{array}{l}0.17 \\
.22 \\
.21 \\
.28 \\
.28\end{array}$ & $\begin{array}{r}0.222 \\
.189 \\
.199 \\
.189 \\
.190\end{array}$ \\
\hline 10-10.0. & $\begin{array}{l}.220 \\
.100 \\
.138 \\
.232 \\
.271\end{array}$ & $\begin{array}{l}.000 \\
.216 \\
.138 \\
.142 \\
.138\end{array}$ & $\begin{array}{l}.28 \\
.26 \\
.29 \\
.30 \\
.18\end{array}$ & $\begin{array}{l}.38 \\
.30 \\
.38 \\
.17 \\
.39\end{array}$ & $\begin{array}{l}.11 \\
.22 \\
.18 \\
.03 \\
.13\end{array}$ & $\begin{array}{l}.22 \\
.28 \\
.29 \\
.26 \\
.33\end{array}$ & $\begin{array}{l}.19 \\
.12 \\
.17 \\
.20 \\
.16\end{array}$ & $\begin{array}{l}.200 \\
.214 \\
.227 \\
.191 \\
.228\end{array}$ \\
\hline 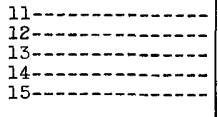 & $\begin{array}{r}.359 \\
.270 \\
.100 \\
. .199 \\
.049\end{array}$ & $\begin{array}{l}.109 \\
.095 \\
.172 \\
.234 \\
.114\end{array}$ & $\begin{array}{l}.17 \\
.22 \\
.18 \\
.20 \\
.10\end{array}$ & $\begin{array}{l}.27 \\
.22 \\
.12 \\
.16 \\
.15\end{array}$ & $\begin{array}{l}.22 \\
.19 \\
.12 \\
.19 \\
.30\end{array}$ & $\begin{array}{l}.23 \\
.13 \\
.12 \\
.21 \\
.18\end{array}$ & $\begin{array}{l}.16 \\
.26 \\
.24 \\
.24 \\
.18\end{array}$ & $\begin{array}{l}.217 \\
.198 \\
.150 \\
.205 \\
.153\end{array}$ \\
\hline 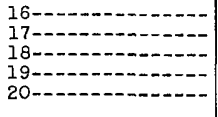 & $\begin{array}{r}.052 \\
.210 \\
c .022 \\
.118 \\
.290\end{array}$ & $\begin{array}{l}.259 \\
.184 \\
.227 \\
.158 \\
.120\end{array}$ & $\begin{array}{l}.02 \\
.03 \\
.13 \\
.22 \\
.28\end{array}$ & $\begin{array}{l}.18 \\
.34 \\
.13 \\
.04 \\
.18\end{array}$ & $\begin{array}{l}.16 \\
.25 \\
.17 \\
.23 \\
.23\end{array}$ & $\begin{array}{l}.32 \\
.27 \\
.32 \\
.30 \\
.18\end{array}$ & $\begin{array}{l}.18 \\
.15 \\
.08 \\
.21 \\
.39\end{array}$ & $\begin{array}{l}.167 \\
.205 \\
.154 \\
.182 \\
.239\end{array}$ \\
\hline 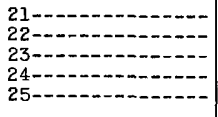 & $\begin{array}{l}.268 \\
.282 \\
.320 \\
.208 \\
.052\end{array}$ & $\begin{array}{l}.094 \\
.129 \\
.091 \\
.114 \\
.410\end{array}$ & $\begin{array}{l}.22 \\
.19 \\
.22 \\
.20 \\
.24\end{array}$ & $\begin{array}{l}.16 \\
.21 \\
.23 \\
.36 \\
.18\end{array}$ & $\begin{array}{l}.21 \\
.23 \\
.05 \\
.27 \\
.17\end{array}$ & $\begin{array}{l}.07 \\
.13 \\
.10 \\
.20 \\
.27\end{array}$ & $\begin{array}{l}.38 \\
.22 \\
.30 \\
.30 \\
.32\end{array}$ & $\begin{array}{l}.200 \\
.199 \\
.187 \\
.236 \\
.235\end{array}$ \\
\hline 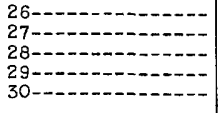 & $\begin{array}{l}.218 \\
.032 \\
.370 \\
.180 \\
.230 \\
\end{array}$ & $\begin{array}{l}.154 \\
.130 \\
.162 \\
.102 \\
.178 \\
\end{array}$ & $\begin{array}{l}.20 \\
.08 \\
.30 \\
.08 \\
.14 \\
\end{array}$ & $\begin{array}{l}.34 \\
.36 \\
.25 \\
.26 \\
.26 \\
\end{array}$ & $\begin{array}{l}.17 \\
.22 \\
.15 \\
.14 \\
.16 \\
\end{array}$ & $\begin{array}{l}.30 \\
.25 \\
.21 \\
.38 \\
.12 \\
\end{array}$ & $\begin{array}{l}.16 \\
.26 \\
.35 \\
.28 \\
.35 \\
\end{array}$ & $\begin{array}{r}.220 \\
.190 \\
.256 \\
.203 \\
.205 \\
\end{array}$ \\
\hline Total-n--2t-m- & 5.388 & 4.637 & 5.97 & 7.17 & 5.45 & 6.73 & 7.01 & 6.050 \\
\hline July & 1947 & 1948 & 1949 & 1950 & 1951 & 1952 & 1953 & Average \\
\hline 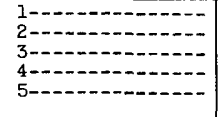 & $\begin{array}{r}0.188 \\
.282 \\
.184 \\
.190 \\
.182\end{array}$ & $\begin{array}{r}0.223 \\
.182 \\
.241 \\
.287 \\
.244\end{array}$ & $\begin{array}{r}0.25 \\
.31 \\
.24 \\
.32 \\
.24\end{array}$ & $\begin{array}{l}0.20 \\
.25 \\
.09 \\
.19 \\
.20\end{array}$ & $\begin{array}{l}0.12 \\
.23 \\
.20 \\
.29 \\
.19\end{array}$ & $\begin{array}{l}0.30 \\
.29 \\
.23 \\
.29 \\
.16\end{array}$ & $\begin{array}{l}0.28 \\
.19 \\
.26 \\
.27 \\
.31\end{array}$ & $\begin{array}{r}0.223 \\
.248 \\
.206 \\
.262 \\
.218\end{array}$ \\
\hline 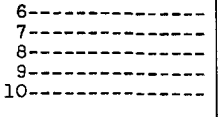 & $\begin{array}{l}.118 \\
.234 \\
.293 \\
.083 \\
.057\end{array}$ & $\begin{array}{l}.268 \\
.202 \\
.207 \\
.225 \\
.244\end{array}$ & $\begin{array}{l}.16 \\
.11 \\
.23 \\
.19 \\
.24\end{array}$ & $\begin{array}{l}.22 \\
.25 \\
.22 \\
.35 \\
.17\end{array}$ & $\begin{array}{r}.22 \\
.29 \\
.27 \\
. .19 \\
.15\end{array}$ & $\begin{array}{l}.31 \\
.28 \\
.19 \\
.13 \\
.24\end{array}$ & $\begin{array}{l}.24 \\
.29 \\
.28 \\
.27 \\
.22\end{array}$ & $\begin{array}{l}.219 \\
.237 \\
.241 \\
.205 \\
.189\end{array}$ \\
\hline 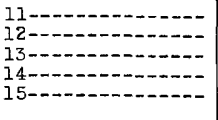 & $\begin{array}{l}.177 \\
.232 \\
.194 \\
.100 \\
.138\end{array}$ & $\begin{array}{l}.211 \\
.140 \\
.184 \\
.150 \\
.151\end{array}$ & $\begin{array}{l}.20 \\
.24 \\
.18 \\
.26 \\
.24\end{array}$ & $\begin{array}{l}.24 \\
.29 \\
.23 \\
.22 \\
.26\end{array}$ & $\begin{array}{l}.17 \\
.12 \\
.10 \\
.19 \\
.17\end{array}$ & $\begin{array}{l}.28 \\
.26 \\
.33 \\
.22 \\
.15\end{array}$ & $\begin{array}{l}.24 \\
.26 \\
.21 \\
.25 \\
.27\end{array}$ & $\begin{array}{l}.217 \\
.220 \\
.204 \\
.199 \\
.197\end{array}$ \\
\hline 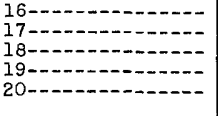 & $\begin{array}{l}.124 \\
.054 \\
.154 \\
.182 \\
.108\end{array}$ & $\begin{array}{l}.216 \\
.235 \\
.270 \\
.239 \\
.291\end{array}$ & $\begin{array}{l}.26 \\
.20 \\
.20 \\
.23 \\
.19\end{array}$ & $\begin{array}{r}.24 \\
.18 \\
.33 \\
.20 \\
.09\end{array}$ & $\begin{array}{l}.25 \\
.15 \\
.14 \\
.29 \\
.23\end{array}$ & $\begin{array}{l}.08 \\
.12 \\
.22 \\
.22 \\
.19\end{array}$ & $\begin{array}{l}.25 \\
.20 \\
.14 \\
.23 \\
.21\end{array}$ & $\begin{array}{l}.203 \\
.163 \\
.208 \\
.227 \\
.187\end{array}$ \\
\hline 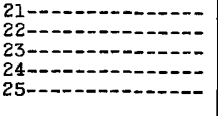 & $\begin{array}{l}.066 \\
.332 \\
.190 \\
.173 \\
.191\end{array}$ & $\begin{array}{l}.083 \\
.107 \\
.158 \\
.158 \\
.281\end{array}$ & $\begin{array}{l}.08 \\
.36 \\
.25 \\
.25 \\
.27\end{array}$ & $\begin{array}{l}.14 \\
.21 \\
.21 \\
.23 \\
.23\end{array}$ & $\begin{array}{l}.18 \\
.05 \\
.15 \\
.18 \\
.19\end{array}$ & $\begin{array}{l}.32 \\
.25 \\
.35 \\
.23 \\
.26\end{array}$ & $\begin{array}{l}.22 \\
.17 \\
.31 \\
.25 \\
.27\end{array}$ & $\begin{array}{l}.156 \\
.211 \\
.231 \\
.210 \\
.242\end{array}$ \\
\hline 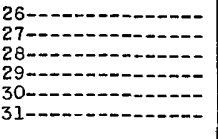 & $\begin{array}{l}.219 \\
.251 \\
.183 \\
.207 \\
.307 \\
.359 \\
\end{array}$ & $\begin{array}{r}.066 \\
.206 \\
.190 \\
.214 \\
. .252 \\
.295 \\
\end{array}$ & $\begin{array}{l}.25 \\
.25 \\
.34 \\
.24 \\
.19 \\
.22 \\
\end{array}$ & $\begin{array}{l}.16 \\
.18 \\
.19 \\
.22 \\
.22 \\
.24 \\
\end{array}$ & $\begin{array}{l}.23 \\
.20 \\
.04 \\
.15 \\
.23 \\
.20 \\
\end{array}$ & $\begin{array}{l}.27 \\
.16 \\
.19 \\
.24 \\
.19 \\
.25 \\
\end{array}$ & $\begin{array}{l}.32 \\
.20 \\
.28 \\
.22 \\
.20 \\
.25 \\
\end{array}$ & $\begin{array}{l}.216 \\
.207 \\
.202 \\
.213 \\
.227 \\
.259 \\
\end{array}$ \\
\hline Total-n-n- & 5.752 & 6.420 & 7.19 & 6.65 & 5.76 & 7.20 & 7.56 & 6.647 \\
\hline
\end{tabular}

See footnotes at end of table. 
Evaporation in Inches at Kendallville, Ind.--Continued

\begin{tabular}{|c|c|c|c|c|c|c|c|c|}
\hline August & 1947 & 1948 & 1949 & 1950 & 1951 & 1952 & 1953 & Average \\
\hline 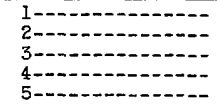 & $\begin{array}{r}0.290 \\
.200 \\
.196 \\
.297 \\
.180\end{array}$ & $\begin{array}{r}0.230 \\
.223 \\
.212 \\
.123 \\
.107\end{array}$ & $\begin{array}{r}0.20 \\
.16 \\
.21 \\
.20 \\
.23\end{array}$ & $\begin{array}{l}0.19 \\
.21 \\
.21 \\
.19 \\
.21\end{array}$ & $\begin{array}{l}0.14 \\
.24 \\
.24 \\
.28 \\
.18\end{array}$ & $\begin{array}{l}0.22 \\
.23 \\
.22 \\
.23 \\
.14\end{array}$ & $\begin{array}{r}0.29 \\
.21 \\
.22 \\
.25 \\
.18\end{array}$ & $\begin{array}{r}0.223 \\
.210 \\
.201 \\
.224 \\
.175\end{array}$ \\
\hline 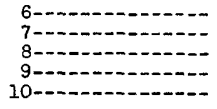 & $\begin{array}{l}.346 \\
.354 \\
.290 \\
.293 \\
.210\end{array}$ & $\begin{array}{l}.178 \\
.216 \\
.213 \\
.127 \\
.270\end{array}$ & $\begin{array}{l}.20 \\
.26 \\
.20 \\
.32 \\
.26\end{array}$ & $\begin{array}{l}.24 \\
.20 \\
.20 \\
.12 \\
.18\end{array}$ & $\begin{array}{l}.14 \\
.03 \\
.14 \\
.15 \\
.16\end{array}$ & $\begin{array}{r}.10 \\
.20 \\
.24 \\
b .17 \\
.28\end{array}$ & $\begin{array}{l}.14 \\
.23 \\
.21 \\
.16 \\
.17\end{array}$ & $\begin{array}{l}.192 \\
.213 \\
.213 \\
.191 \\
.219\end{array}$ \\
\hline 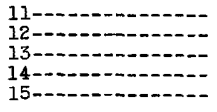 & $\begin{array}{l}.289 \\
.243 \\
.260 \\
.080 \\
.335\end{array}$ & $\begin{array}{l}.156 \\
.184 \\
.177 \\
.191 \\
.188\end{array}$ & $\begin{array}{l}.23 \\
.08 \\
.18 \\
.39 \\
.12\end{array}$ & $\begin{array}{l}.19 \\
.14 \\
.21 \\
.19 \\
.21\end{array}$ & $\begin{array}{r}.21 \\
.22 \\
.23 \\
.21 \\
6.17\end{array}$ & $\begin{array}{l}.28 \\
.04 \\
.18 \\
.16 \\
.12\end{array}$ & $\begin{array}{l}.22 \\
.17 \\
.13 \\
.25 \\
.29\end{array}$ & $\begin{array}{l}.225 \\
.154 \\
.195 \\
.210 \\
.205\end{array}$ \\
\hline 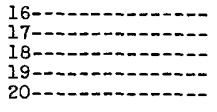 & $\begin{array}{l}.086 \\
.257 \\
.195 \\
.297 \\
.264\end{array}$ & $\begin{array}{l}.176 \\
.212 \\
.140 \\
.205 \\
.211\end{array}$ & $\begin{array}{l}.23 \\
.15 \\
.16 \\
.26 \\
.21\end{array}$ & $\begin{array}{l}.23 \\
.25 \\
.13 \\
.15 \\
.21\end{array}$ & $\begin{array}{l}.14 \\
.14 \\
.13 \\
.16 \\
.14\end{array}$ & $\begin{array}{l}.18 \\
.16 \\
.24 \\
.18 \\
.20\end{array}$ & $\begin{array}{l}.17 \\
.20 \\
.24 \\
.18 \\
.25\end{array}$ & $\begin{array}{l}.173 \\
.196 \\
.176 \\
.205 \\
.212\end{array}$ \\
\hline 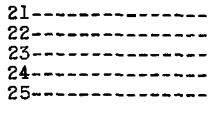 & $\begin{array}{l}.299 \\
.102 \\
.284 \\
.236 \\
.151\end{array}$ & $\begin{array}{l}.236 \\
.179 \\
.269 \\
.231 \\
.289\end{array}$ & $\begin{array}{l}.27 \\
.20 \\
.22 \\
.20 \\
.20\end{array}$ & $\begin{array}{l}.21 \\
.19 \\
.26 \\
.19 \\
.20\end{array}$ & $\begin{array}{r}.16 \\
.16 \\
.19 \\
.23 \\
.17\end{array}$ & $\begin{array}{r}.13 \\
.23 \\
.22 \\
.17 \\
.25\end{array}$ & $\begin{array}{l}.20 \\
.22 \\
.20 \\
.26 \\
.23\end{array}$ & $\begin{array}{l}.215 \\
.183 \\
.235 \\
.217 \\
.213\end{array}$ \\
\hline 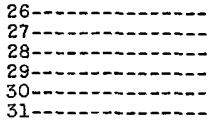 & $\begin{array}{l}.091 \\
.145 \\
.164 \\
.224 \\
.136 \\
.161 \\
\end{array}$ & $\begin{array}{l}.195 \\
.249 \\
.263 \\
.217 \\
.265 \\
.235 \\
\end{array}$ & $\begin{array}{l}.25 \\
.21 \\
.10 \\
.18 \\
.17 \\
.18\end{array}$ & $\begin{array}{l}.18 \\
.21 \\
.15 \\
.28 \\
.13 \\
.09 \\
\end{array}$ & $\begin{array}{l}.11 \\
.02 \\
.12 \\
.17 \\
.15 \\
.19\end{array}$ & $\begin{array}{l}.20 \\
.27 \\
.22 \\
.25 \\
.21 \\
.18 \\
\end{array}$ & $\begin{array}{l}.22 \\
.26 \\
.24 \\
.27 \\
.35 \\
.32 \\
\end{array}$ & $\begin{array}{l}.178 \\
.195 \\
.180 \\
.227 \\
.202 \\
.194 \\
\end{array}$ \\
\hline Total - & 6.955 & 6.367 & 6.33 & 5.95 & 5.12 & 6.10 & 6.93 & 6.251 \\
\hline September & 1947 & 1948 & 1949 & 1950 & 1951 & 1952 & 1953 & Average \\
\hline 14-20 & $\begin{array}{r}0.298 \\
.231 \\
.225 \\
.101 \\
.191\end{array}$ & $\begin{array}{r}0.186 \\
.153 \\
.193 \\
.179 \\
.201\end{array}$ & $\begin{array}{r}0.20 \\
.17 \\
.20 \\
.11 \\
.19\end{array}$ & $\begin{array}{r}0.11 \\
.14 \\
.02 \\
.23 \\
.10\end{array}$ & $\begin{array}{r}0.23 \\
.04 \\
.09 \\
.14 \\
.14\end{array}$ & $\begin{array}{r}0.21 \\
.16 \\
.12 \\
.12 \\
.20\end{array}$ & $\begin{array}{l}0.33 \\
.38 \\
.15 \\
.10 \\
.13\end{array}$ & $\begin{array}{r}0.223 \\
.182 \\
.168 \\
.140 \\
.165\end{array}$ \\
\hline 10-10. & $\begin{array}{l}.055 \\
.113 \\
.141 \\
.166 \\
.180\end{array}$ & $\begin{array}{r}.136 \\
. .149 \\
. .114 \\
.181 \\
.180\end{array}$ & $\begin{array}{l}.17 \\
.11 \\
.13 \\
.15 \\
.17\end{array}$ & $\begin{array}{l}.18 \\
.19 \\
.14 \\
.16 \\
.09\end{array}$ & $\begin{array}{l}.15 \\
.17 \\
.12 \\
.13 \\
.11\end{array}$ & $\begin{array}{l}.17 \\
.13 \\
.12 \\
.16 \\
.19\end{array}$ & $\begin{array}{l}.22 \\
.20 \\
.19 \\
.16 \\
.18\end{array}$ & $\begin{array}{l}.154 \\
.152 \\
.136 \\
.158 \\
.157\end{array}$ \\
\hline 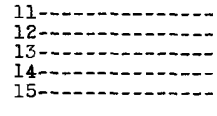 & $\begin{array}{r}.024 \\
.178 \\
.081 \\
.214 \\
.355\end{array}$ & $\begin{array}{l}.187 \\
.207 \\
.192 \\
.246 \\
.128\end{array}$ & $\begin{array}{l}.11 \\
.12 \\
.08 \\
.11 \\
.10\end{array}$ & $\begin{array}{l}.05 \\
.10 \\
.08 \\
.07 \\
.09\end{array}$ & $\begin{array}{l}.17 \\
.20 \\
.13 \\
.12 \\
.12\end{array}$ & $\begin{array}{l}.22 \\
.22 \\
.23 \\
.20 \\
.12\end{array}$ & $\begin{array}{l}.24 \\
.22 \\
.13 \\
.08 \\
.09\end{array}$ & $\begin{array}{l}.143 \\
.178 \\
.132 \\
.149 \\
.143\end{array}$ \\
\hline 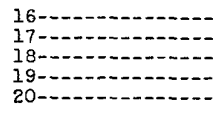 & $\begin{array}{l}.153 \\
.133 \\
.135 \\
.116 \\
.171\end{array}$ & $\begin{array}{l}.168 \\
.170 \\
.149 \\
.174 \\
.110\end{array}$ & $\begin{array}{l}.13 \\
.11 \\
.11 \\
.21 \\
.15\end{array}$ & $\begin{array}{l}.12 \\
.13 \\
.14 \\
.18 \\
.06\end{array}$ & $\begin{array}{l}.10 \\
.15 \\
.10 \\
.19 \\
.18\end{array}$ & $\begin{array}{l}.25 \\
.15 \\
.10 \\
.13 \\
.09\end{array}$ & $\begin{array}{l}.15 \\
.19 \\
.20 \\
.11 \\
.10\end{array}$ & $\begin{array}{l}.153 \\
.148 \\
.133 \\
.159 \\
.123\end{array}$ \\
\hline 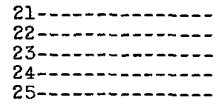 & $\begin{array}{l}.033 \\
.213 \\
.118 \\
.025 \\
.215\end{array}$ & $\begin{array}{l}.090 \\
.131 \\
.126 \\
.093 \\
.101\end{array}$ & $\begin{array}{l}.12 \\
.07 \\
.04 \\
.09 \\
.10\end{array}$ & $\begin{array}{l}.11 \\
.09 \\
.18 \\
.11 \\
.08\end{array}$ & $\begin{array}{l}.20 \\
.12 \\
.10 \\
.10 \\
.04\end{array}$ & $\begin{array}{l}.13 \\
.10 \\
.12 \\
.13 \\
.16\end{array}$ & $\begin{array}{l}.20 \\
.11 \\
.13 \\
.19 \\
.17\end{array}$ & $\begin{array}{l}.126 \\
.119 \\
.116 \\
.105 \\
.124\end{array}$ \\
\hline 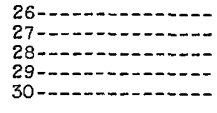 & $\begin{array}{l}.193 \\
.035 \\
.143 \\
.028 \\
.077\end{array}$ & $\begin{array}{l}.151 \\
.122 \\
.160 \\
.090 \\
.030 \\
\end{array}$ & $\begin{array}{l}.16 \\
.10 \\
.09 \\
.10 \\
.17 \\
\end{array}$ & $\begin{array}{l}.10 \\
.08 \\
.07 \\
.11 \\
.07 \\
\end{array}$ & $\begin{array}{l}.06 \\
.19 \\
.12 \\
.09 \\
.08\end{array}$ & $\begin{array}{l}.17 \\
.14 \\
.17 \\
.17 \\
.15 \\
\end{array}$ & $\begin{array}{l}.15 \\
.22 \\
.19 \\
.24 \\
.31 \\
\end{array}$ & $\begin{array}{l}.141 \\
.127 \\
.135 \\
.118 \\
.127 \\
\end{array}$ \\
\hline Total & 4.341 & 4.497 & 3.87 & 3.56 & 3.88 & 4.73 & 5.46 & 4.334 \\
\hline
\end{tabular}

See footnotes at end of table. 
Evaporation, in Inches, at Kendallville, Ind.--Continued

\begin{tabular}{|c|c|c|c|c|c|c|c|c|c|c|}
\hline \multicolumn{2}{|l|}{ October } & 1947 & c 1948 & 1949 & & 1950 & 1951 & 1952 & 1953 & Average \\
\hline \multicolumn{2}{|c|}{ 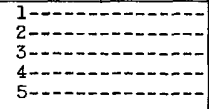 } & $\begin{array}{r}0.073 \\
.102 \\
.052 \\
.109 \\
.098\end{array}$ & $\begin{array}{r}.104 \\
.110 \\
.106 \\
.152 \\
.132\end{array}$ & $\begin{array}{l}0.12 \\
.12 \\
.07 \\
.01 \\
.13\end{array}$ & & $\begin{array}{r}0.06 \\
.08 \\
.15 \\
.09 \\
.06\end{array}$ & $\begin{array}{l}0.05 \\
.14 \\
.17 \\
.20 \\
.15\end{array}$ & $\begin{array}{l}0.19 \\
.12 \\
.09 \\
.18 \\
.15\end{array}$ & $\begin{array}{l}0.22 \\
.23 \\
.21 \\
.14 \\
.09\end{array}$ & $\begin{array}{r}0.117 \\
.129 \\
.121 \\
.126 \\
.116\end{array}$ \\
\hline \multicolumn{2}{|c|}{ 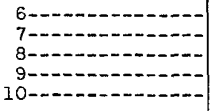 } & $\begin{array}{l}.010 \\
.235 \\
.135 \\
.053 \\
.096\end{array}$ & $\begin{array}{l}.118 \\
.056 \\
.042 \\
.166 \\
.102\end{array}$ & $\begin{array}{l}.22 \\
.06 \\
.11 \\
.17 \\
.20\end{array}$ & & $\begin{array}{l}.09 \\
.12 \\
.07 \\
.01 \\
.05\end{array}$ & $\begin{array}{l}.09 \\
.11 \\
.06 \\
.06 \\
.09\end{array}$ & $\begin{array}{l}.10 \\
.05 \\
.08 \\
.10 \\
.14\end{array}$ & $\begin{array}{l}.10 \\
.10 \\
.09 \\
.09 \\
.13\end{array}$ & $\begin{array}{l}.104 \\
.104 \\
.084 \\
.093 \\
.115\end{array}$ \\
\hline \multicolumn{2}{|c|}{ 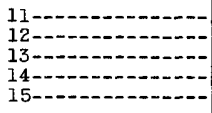 } & $\begin{array}{l}.117 \\
.050 \\
.089 \\
.089 \\
.088\end{array}$ & $\begin{array}{l}.010 \\
.030 \\
.064 \\
.061 \\
.087\end{array}$ & $\begin{array}{l}.12 \\
.16 \\
.15 \\
.08 \\
.11\end{array}$ & & $\begin{array}{l}.05 \\
.12 \\
.08 \\
.08 \\
.09\end{array}$ & $\begin{array}{l}.09 \\
.07 \\
.08 \\
.12 \\
.10\end{array}$ & $\begin{array}{l}.10 \\
.10 \\
.18 \\
.08 \\
.10\end{array}$ & $\begin{array}{l}.12 \\
.18 \\
.12 \\
.12 \\
.18\end{array}$ & $\begin{array}{l}.087 \\
.101 \\
.100 \\
.090 \\
.108\end{array}$ \\
\hline \multicolumn{2}{|c|}{ 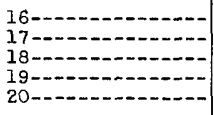 } & $\begin{array}{l}.145 \\
.214 \\
.068 \\
.025 \\
.130\end{array}$ & $\begin{array}{l}.025 \\
.059 \\
.064 \\
.054 \\
.050\end{array}$ & $\begin{array}{l}.10 \\
.12 \\
.03 \\
.08 \\
.08\end{array}$ & & $\begin{array}{l}.10 \\
.09 \\
.11 \\
.13 \\
.16\end{array}$ & $\begin{array}{l}.12 \\
.10 \\
.11 \\
.14 \\
.06\end{array}$ & $\begin{array}{l}.11 \\
.09 \\
.09 \\
.14 \\
.07\end{array}$ & $\begin{array}{l}.12 \\
.13 \\
.15 \\
.19 \\
.16\end{array}$ & $\begin{array}{l}.103 \\
.115 \\
.089 \\
.108 \\
.101\end{array}$ \\
\hline \multicolumn{2}{|c|}{ 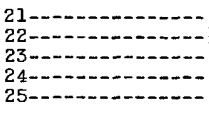 } & $\begin{array}{l}.114 \\
.275 \\
.026 \\
.067 \\
.081\end{array}$ & $\begin{array}{l}.040 \\
.067 \\
.021 \\
.028 \\
.040\end{array}$ & $\begin{array}{l}.04 \\
.21 \\
.10 \\
.09 \\
.07\end{array}$ & & $\begin{array}{l}.07 \\
.07 \\
.04 \\
.04 \\
.06\end{array}$ & $\begin{array}{l}.08 \\
.07 \\
.09 \\
.07 \\
.01\end{array}$ & $\begin{array}{l}.06 \\
.12 \\
.15 \\
.14 \\
.10\end{array}$ & $\begin{array}{l}.11 \\
.15 \\
.22 \\
.05 \\
.04\end{array}$ & $\begin{array}{l}.073 \\
.137 \\
.092 \\
.069 \\
.057\end{array}$ \\
\hline \multicolumn{2}{|c|}{ 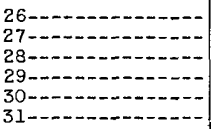 } & $\begin{array}{r}.038 \\
.080 \\
.039 \\
.102 \\
.094 \\
.037 \\
\end{array}$ & $\begin{array}{l}.052 \\
.064 \\
.048 \\
.056 \\
.078 \\
.026 \\
\end{array}$ & $\begin{array}{l}.14 \\
.08 \\
.07 \\
.10 \\
.07 \\
.07\end{array}$ & & $\begin{array}{l}.07 \\
.06 \\
.02 \\
.06 \\
.08 \\
.12 \\
\end{array}$ & $\begin{array}{l}.05 \\
.08 \\
.02 \\
.05 \\
.05 \\
.13 \\
\end{array}$ & $\begin{array}{l}.14 \\
.17 \\
.08 \\
.03 \\
.10 \\
.17 \\
\end{array}$ & $\begin{array}{r}.05 \\
.03 \\
.08 \\
.08 \\
.09 \\
.10 \\
\end{array}$ & $\begin{array}{r}.077 \\
.081 \\
.051 \\
.068 \\
.080 \\
.093 \\
\end{array}$ \\
\hline \multicolumn{2}{|c|}{ Total- } & 2.931 & 2.112 & 3.28 & & 2.48 & 2.81 & 3.52 & 3.87 & 2.989 \\
\hline November & d 1947 & 1948 & 1949 & $d 1950$ & di95] & 1952 & d 1953 & Average & December & \& 1948 \\
\hline 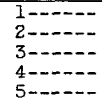 & $\begin{array}{r}0.015 \\
.048 \\
.039 \\
.001 \\
.034\end{array}$ & $\begin{array}{r}0.022 \\
.028 \\
.006 \\
.031 \\
.105\end{array}$ & $\begin{array}{r}0.07 \\
.05 \\
.04 \\
.03 \\
.04\end{array}$ & $\begin{array}{l}0.16 \\
.17 \\
.04 \\
.03 \\
.03\end{array}$ & $\begin{array}{l}0.05 \\
---- \\
---- \\
---\end{array}$ & $\begin{array}{r}0.08 \\
.08 \\
.10 \\
.09 \\
.11\end{array}$ & $\begin{array}{l}0.07 \\
a .10 \\
a .13 \\
.10 \\
.06\end{array}$ & $\begin{array}{r}0.067 \\
.079 \\
.059 \\
.037 \\
.063\end{array}$ & $\begin{array}{l}1-\cdots \\
2-\ldots- \\
3---- \\
4-\cdots- \\
5-\cdots\end{array}$ & $\begin{array}{r}0.044 \\
.018 \\
-.009 \\
-.029 \\
.018\end{array}$ \\
\hline 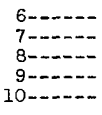 & $\begin{array}{l}.019 \\
.067 \\
.076 \\
.083 \\
.020\end{array}$ & $\begin{array}{l}.077 \\
.104 \\
.050 \\
.046 \\
.058\end{array}$ & $\begin{array}{l}.07 \\
.07 \\
.12 \\
.08 \\
.08\end{array}$ & $\begin{array}{r}.08 \\
.05 \\
.05 \\
.12 \\
-.--\end{array}$ & $\begin{array}{l}--\cdots \\
--- \\
--- \\
--- \\
---\end{array}$ & $\begin{array}{l}.11 \\
.11 \\
.02 \\
.09 \\
.06\end{array}$ & 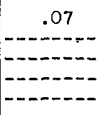 & $\begin{array}{l}.071 \\
.080 \\
.063 \\
.084 \\
.054\end{array}$ & $\begin{array}{r}6-\cdots- \\
7-\cdots- \\
8-\cdots- \\
9-\cdots- \\
10-\cdots-\end{array}$ & $\begin{array}{r}.101 \\
.045 \\
- \\
-\end{array}$ \\
\hline $\begin{array}{l}11 \ldots \ldots \\
12-\ldots \\
13 \\
14 \ldots \\
15 \ldots\end{array}$ & $\begin{array}{r}6.030 \\
.031 \\
.037 \\
.043 \\
.019\end{array}$ & $\begin{array}{l}.074 \\
.027 \\
.021 \\
.040 \\
.080\end{array}$ & $\begin{array}{l}.06 \\
.06 \\
.08 \\
.06 \\
.06\end{array}$ & $\begin{array}{l}---- \\
---- \\
---- \\
-\cdots- \\
-\cdots-\end{array}$ & $\begin{array}{l}--- \\
--- \\
-\cdots- \\
--- \\
---\end{array}$ & $\begin{array}{l}.06 \\
.03 \\
.06 \\
.07 \\
.01\end{array}$ & 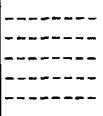 & $\begin{array}{l}.056 \\
.037 \\
.050 \\
.053 \\
.042\end{array}$ & 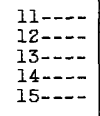 & $\overline{-}$ \\
\hline 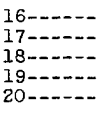 & $\begin{array}{l}.076 \\
.020 \\
.027 \\
.013 \\
.030\end{array}$ & $\begin{array}{l}.061 \\
.129 \\
.066 \\
.122 \\
.066\end{array}$ & $\begin{array}{l}.03 \\
.00 \\
.04 \\
.06 \\
.07\end{array}$ & $\begin{array}{c}---- \\
---- \\
---- \\
---- \\
----\end{array}$ & $\begin{array}{l}---- \\
---- \\
--- \\
---- \\
----\end{array}$ & $\begin{array}{l}.03 \\
.04 \\
.07 \\
.07 \\
.05\end{array}$ & 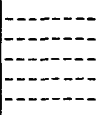 & $\begin{array}{l}.049 \\
.047 \\
.051 \\
.066 \\
.054\end{array}$ & $\begin{array}{l}16-\cdots- \\
17-\cdots- \\
18-\cdots-- \\
19--n- \\
20-\cdots\end{array}$ & 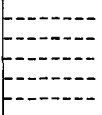 \\
\hline 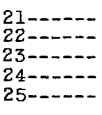 & $\begin{array}{r}.036 \\
.007 \\
.026 \\
.071 \\
.045\end{array}$ & $\begin{array}{l}.082 \\
.016 \\
.029 \\
.016 \\
.023\end{array}$ & $\begin{array}{l}.01 \\
.001 \\
.01 \\
\text { b.02 } \\
\text { b.03 }\end{array}$ & $\begin{array}{l}---- \\
---- \\
---- \\
---- \\
----\end{array}$ & $\begin{array}{l}---- \\
---- \\
---- \\
---- \\
----\end{array}$ & $\begin{array}{l}.02 \\
.12 \\
.00 \\
.01 \\
.07\end{array}$ & 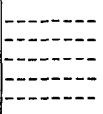 & $\begin{array}{l}.037 \\
.038 \\
.016 \\
.029 \\
.042\end{array}$ & 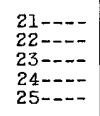 & 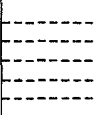 \\
\hline 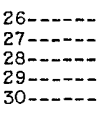 & $\begin{array}{l}b .030 \\
b .020 \\
b .030 \\
b .010 \\
b .010\end{array}$ & $\begin{array}{l}.010 \\
.032 \\
.034 \\
.008 \\
.020\end{array}$ & $\begin{array}{l}b .03 \\
b .02 \\
b .02 \\
b .03 \\
b .02\end{array}$ & $\begin{array}{l}---- \\
---- \\
--- \\
---- \\
----\end{array}$ & $\begin{array}{l}---- \\
---- \\
---- \\
---- \\
----\end{array}$ & $\begin{array}{l}.09 \\
.05 \\
6.02 \\
.02 \\
6.03\end{array}$ & 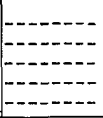 & $\begin{array}{l}.040 \\
.030 \\
.026 \\
.017 \\
.020\end{array}$ & 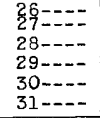 & 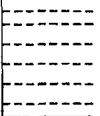 \\
\hline Total- & 1.013 & 1.483 & 1.37 & $\ldots$ & --- & 1.77 & - & 1.457 & ----- & $---\infty$ \\
\hline
\end{tabular}

a Estimated from total observed on underlined day.

$b$ Estimated because of missing or erroneous data.

- Dorrected since orlginally published by U. S. Weather Bureau.

Not previously published. 
Evaporation, in inches, at Valparaiso, Ind.

[No observations were made during part of October 1952, and during November 1951 and 1952 ]

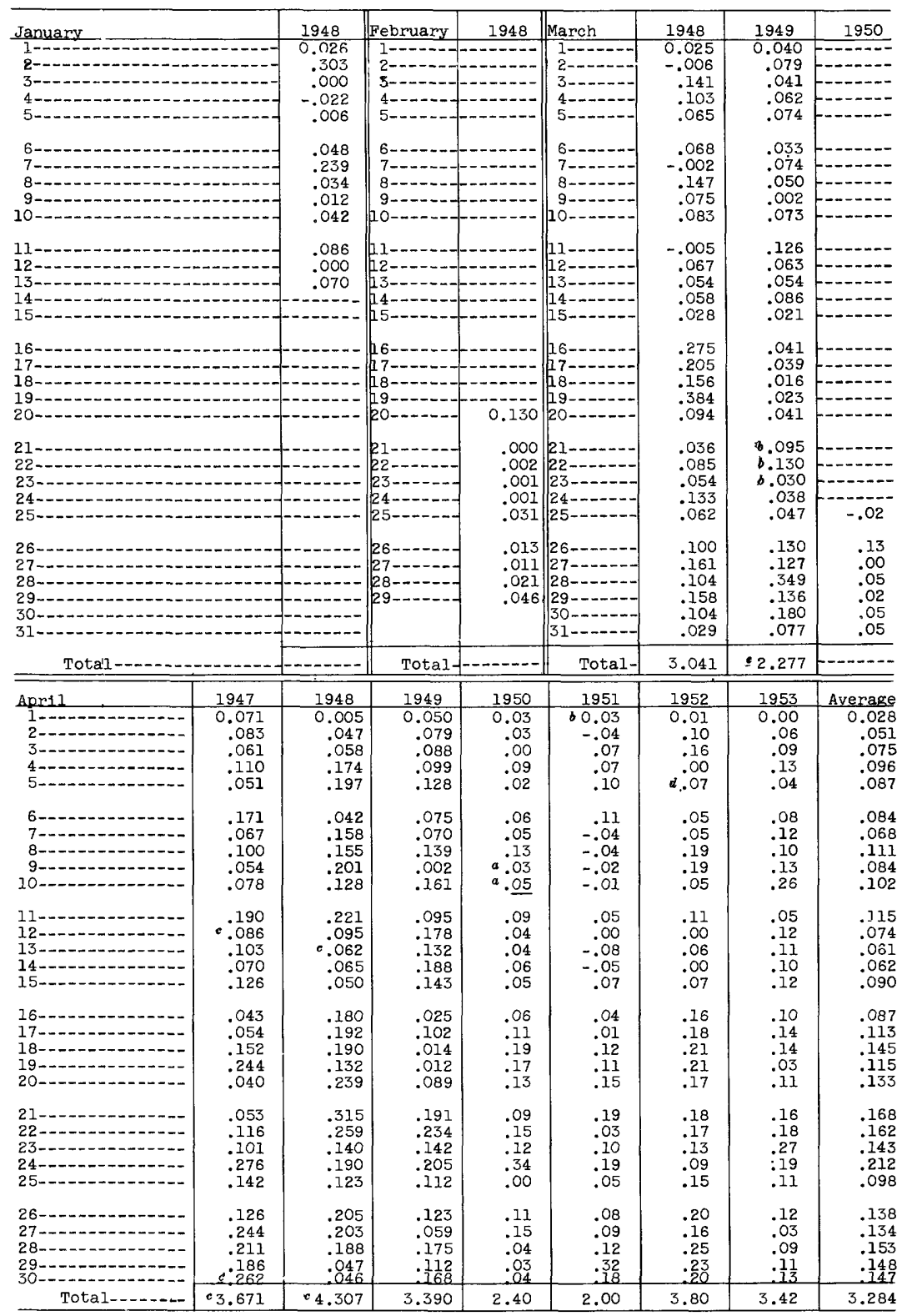

See footnotes at end of table. 
Evaporation, in inches, at Valpara1so, Ind.--Continued

\begin{tabular}{|c|c|c|c|c|c|c|c|c|}
\hline May & 1947 & 1948 & 1949 & 1950 & 1951 & 1952 & 1953 & Average \\
\hline 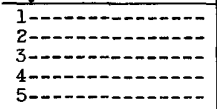 & $\begin{array}{r}0.125 \\
.113 \\
.053 \\
.043 \\
.148\end{array}$ & $\begin{array}{r}0.137 \\
.177 \\
.163 \\
.155 \\
.020\end{array}$ & $\begin{array}{l}0.08 \\
. .08 \\
.23 \\
.29 \\
.26\end{array}$ & $\begin{array}{l}0.05 \\
.12 \\
.22 \\
.05 \\
.09\end{array}$ & $\begin{array}{r}0.22 \\
.24 \\
.17 \\
.18 \\
.08\end{array}$ & $\begin{array}{l}0.21 \\
.26 \\
.16 \\
.28 \\
.25\end{array}$ & $\begin{array}{r}0.14 \\
.31 \\
.10 \\
.00 \\
.16\end{array}$ & $\begin{array}{r}0.137 \\
.186 \\
.157 \\
.143 \\
.144\end{array}$ \\
\hline 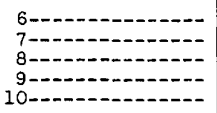 & $\begin{array}{l}.101 \\
.115 \\
.235 \\
.169 \\
.183\end{array}$ & $\begin{array}{l}.348 \\
.045 \\
.119 \\
.141 \\
.125\end{array}$ & $\begin{array}{l}.28 \\
.27 \\
.12 \\
.14 \\
.11\end{array}$ & $\begin{array}{l}.36 \\
.34 \\
.09 \\
.34 \\
.05\end{array}$ & $\begin{array}{l}.09 \\
.04 \\
.05 \\
.29 \\
.18\end{array}$ & $\begin{array}{l}.18 \\
.21 \\
.11 \\
.04 \\
.09\end{array}$ & $\begin{array}{l}.14 \\
.15 \\
.11 \\
.24 \\
.31\end{array}$ & $\begin{array}{l}.214 \\
.167 \\
.119 \\
.194 \\
.150\end{array}$ \\
\hline 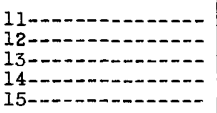 & $\begin{array}{l}.160 \\
.254 \\
.239 \\
.135 \\
.147\end{array}$ & $\begin{array}{l}.208 \\
.049 \\
.012 \\
.034 \\
.166\end{array}$ & $\begin{array}{l}.21 \\
.17 \\
.19 \\
.14 \\
.26\end{array}$ & $\begin{array}{l}.12 \\
.12 \\
.22 \\
.18 \\
.22\end{array}$ & $\begin{array}{l}.20 \\
.00 \\
.19 \\
.20 \\
.25\end{array}$ & $\begin{array}{l}.14 \\
.00 \\
.16 \\
.17 \\
.06\end{array}$ & $\begin{array}{l}.13 \\
.06 \\
.16 \\
.09 \\
.11\end{array}$ & $\begin{array}{l}.167 \\
.093 \\
.167 \\
.136 \\
.173\end{array}$ \\
\hline 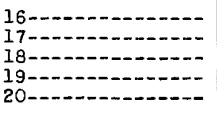 & $\begin{array}{r}.170 \\
.163 \\
.070 \\
.137 \\
.168\end{array}$ & $\begin{array}{r}. .089 \\
. .253 \\
.200 \\
.116 \\
.162\end{array}$ & $\begin{array}{l}.10 \\
.03 \\
.20 \\
.19 \\
.12\end{array}$ & $\begin{array}{r}.23 \\
.19 \\
.18 \\
.22 \\
.14\end{array}$ & $\begin{array}{l}.32 \\
.26 \\
.07 \\
.13 \\
.30\end{array}$ & $\begin{array}{l}.04 \\
.02 \\
.10 \\
.05 \\
.02\end{array}$ & $\begin{array}{l}.05 \\
.06 \\
.11 \\
.23 \\
.21\end{array}$ & $\begin{array}{l}.143 \\
.139 \\
.133 \\
.153 \\
.160\end{array}$ \\
\hline 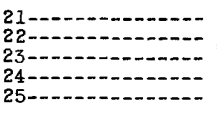 & $\begin{array}{r}.067 \\
.125 \\
.278 \\
.129 \\
.128\end{array}$ & $\begin{array}{l}.216 \\
.250 \\
.225 \\
.204 \\
.153\end{array}$ & $\begin{array}{l}.07 \\
.03 \\
.03 \\
.09 \\
.21\end{array}$ & $\begin{array}{l}.15 \\
.40 \\
.08 \\
.21 \\
.20\end{array}$ & $\begin{array}{l}.25 \\
.21 \\
.06 \\
.18 \\
.26\end{array}$ & $\begin{array}{l}.07 \\
.09 \\
.13 \\
.09 \\
.16\end{array}$ & $\begin{array}{r}.11 \\
.19 \\
.30 \\
.05 \\
.06\end{array}$ & $\begin{array}{l}.133 \\
.185 \\
.158 \\
.122 \\
.167\end{array}$ \\
\hline 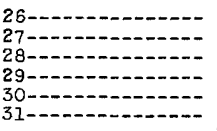 & $\begin{array}{l}.134 \\
.205 \\
.001 \\
.035 \\
.073 \\
.162\end{array}$ & $\begin{array}{l}.184 \\
.217 \\
.207 \\
.223 \\
.243 \\
.197\end{array}$ & $\begin{array}{l}.06 \\
.16 \\
.14 \\
.16 \\
.16 \\
.21\end{array}$ & $\begin{array}{l}.35 \\
.08 \\
.10 \\
.13 \\
.17 \\
.20\end{array}$ & $\begin{array}{l}.29 \\
.08 \\
.00 \\
.14 \\
.02 \\
.22\end{array}$ & $\begin{array}{l}.16 \\
.28 \\
.27 \\
.16 \\
.23 \\
.13\end{array}$ & $\begin{array}{l}.23 \\
.18 \\
.20 \\
.30 \\
.28 \\
.35\end{array}$ & $\begin{array}{l}.201 \\
.172 \\
.131 \\
.164 \\
.168 \\
.210\end{array}$ \\
\hline Total-....... & 04.265 & 5.038 & $c_{4.79}$ & 5.60 & 5.17 & 4.32 & $\div 5.02$ & 4.886 \\
\hline June & 1947 & 1948 & 1949 & 1950 & 1951 & 1952 & 1953 & Average \\
\hline 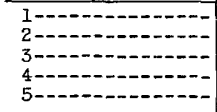 & $\begin{array}{r}0.305 \\
.101 \\
.070 \\
.131 \\
.193\end{array}$ & $\begin{array}{r}0.200 \\
.204 \\
.137 \\
.145 \\
.254\end{array}$ & $\begin{array}{r}0.07 \\
.15 \\
.18 \\
.14 \\
.20\end{array}$ & $\begin{array}{r}0.30 \\
.30 \\
.40 \\
.15 \\
.16\end{array}$ & $\begin{array}{r}0.09 \\
.31 \\
.14 \\
.10 \\
.10\end{array}$ & $\begin{array}{l}0.16 \\
.15 \\
.23 \\
.26 \\
.18\end{array}$ & $\begin{array}{r}0.12 \\
.24 \\
.22 \\
.28 \\
.31\end{array}$ & $\begin{array}{r}0.178 \\
.208 \\
.197 \\
.172 \\
.200\end{array}$ \\
\hline 610-0. & $\begin{array}{l}.174 \\
.059 \\
.142 \\
.175 \\
.243\end{array}$ & $\begin{array}{r}.151 \\
.156 \\
.132 \\
.136 \\
.140\end{array}$ & $\begin{array}{r}.21 \\
.33 \\
.25 \\
.19 \\
.20\end{array}$ & $\begin{array}{l}.34 \\
.26 \\
.24 \\
.25 \\
.21\end{array}$ & $\begin{array}{l}.14 \\
.12 \\
.17 \\
.04 \\
.15\end{array}$ & $\begin{array}{l}.41 \\
.26 \\
.37 \\
.51 \\
.31\end{array}$ & $\begin{array}{l}.12 \\
.16 \\
.26 \\
.20 \\
.26\end{array}$ & $\begin{array}{l}.221 \\
.192 \\
.223 \\
.214 \\
.216\end{array}$ \\
\hline 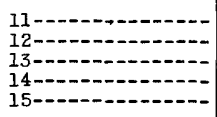 & $\begin{array}{l}.410 \\
.218 \\
.170 \\
.149 \\
.052\end{array}$ & $\begin{array}{r}.148 \\
.131 \\
. .057 \\
. .189 \\
. .103\end{array}$ & $\begin{array}{l}.15 \\
.20 \\
.20 \\
.15 \\
.19\end{array}$ & $\begin{array}{l}.24 \\
.24 \\
.20 \\
.15 \\
.20\end{array}$ & $\begin{array}{l}.15 \\
.17 \\
.08 \\
.14 \\
.22\end{array}$ & $\begin{array}{l}.12 \\
.23 \\
.15 \\
.12 \\
.19\end{array}$ & $\begin{array}{l}.20 \\
.15 \\
.18 \\
.23 \\
.19\end{array}$ & $\begin{array}{l}.203 \\
.191 \\
.148 \\
.161 \\
.164\end{array}$ \\
\hline 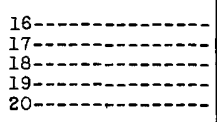 & $\begin{array}{l}.040 \\
.160 \\
.008 \\
.148 \\
.135\end{array}$ & $\begin{array}{r}.123 \\
.214 \\
.191 \\
.141 \\
. .091\end{array}$ & $\begin{array}{l}.06 \\
.05 \\
.04 \\
.17 \\
.20\end{array}$ & $\begin{array}{r}.11 \\
.29 \\
a .09 \\
a .16 \\
.08\end{array}$ & $\begin{array}{l}.21 \\
.10 \\
.10 \\
.20 \\
.15\end{array}$ & $\begin{array}{l}.29 \\
.33 \\
.32 \\
.36 \\
.00\end{array}$ & $\begin{array}{r}.18 \\
.16 \\
.26 \\
. .15 \\
.32\end{array}$ & $\begin{array}{l}.145 \\
.186 \\
.144 \\
.190 \\
.139\end{array}$ \\
\hline 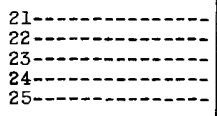 & $\begin{array}{l}.283 \\
.215 \\
.257 \\
.203 \\
.200\end{array}$ & $\begin{array}{r}c .153 \\
.163 \\
. .201 \\
.172 \\
.147\end{array}$ & $\begin{array}{l}.24 \\
.08 \\
.09 \\
.15 \\
.32\end{array}$ & $\begin{array}{l}a .08 \\
.18 \\
.20 \\
.28 \\
.20\end{array}$ & $\begin{array}{l}.23 \\
.07 \\
.11 \\
.10 \\
.21\end{array}$ & $\begin{array}{l}.10 \\
.06 \\
.23 \\
.16 \\
.26\end{array}$ & $\begin{array}{l}.20 \\
.12 \\
.14 \\
.15 \\
.23\end{array}$ & $\begin{array}{l}.184 \\
.127 \\
.175 \\
.174 \\
.224\end{array}$ \\
\hline 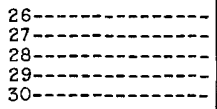 & $\begin{array}{l}.163 \\
.192 \\
.171 \\
.263 \\
.102 \\
\end{array}$ & $\begin{array}{r}.215 \\
.093 \\
. .066 \\
.184 \\
.081 \\
\end{array}$ & $\begin{array}{l}.19 \\
.10 \\
.21 \\
.22 \\
.14 \\
\end{array}$ & $\begin{array}{l}.18 \\
.30 \\
.19 \\
.17 \\
.22 \\
\end{array}$ & $\begin{array}{l}.15 \\
.17 \\
.10 \\
.08 \\
.06 \\
\end{array}$ & $\begin{array}{l}.14 \\
.29 \\
.26 \\
.27 \\
.13 \\
\end{array}$ & $\begin{array}{l}.24 \\
.22 \\
.17 \\
.24 \\
.29 \\
\end{array}$ & $\begin{array}{l}.183 \\
.195 \\
.167 \\
.204 \\
.146 \\
\end{array}$ \\
\hline Total-....... & 5.132 & ${ }^{c} 4.518$ & 5.07 & 6.37 & 4.16 & 6.85 & 6.19 & 5.471 \\
\hline
\end{tabular}

See footnotes at end of table. 
EVAPORATION DATA

Evaporation, in inches, at Valparaiso, Ind.--Continued

\begin{tabular}{|c|c|c|c|c|c|c|c|c|}
\hline July & 1947 & 1948 & 1949 & 1950 & 1951 & 1952 & 1953 & Average \\
\hline 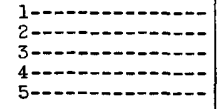 & $\begin{array}{r}0.178 \\
.216 \\
.203 \\
.212 \\
.217\end{array}$ & $\begin{array}{r}0.215 \\
.122 \\
.256 \\
.242 \\
.262\end{array}$ & $\begin{array}{r}0.22 \\
.11 \\
.27 \\
.22 \\
.23\end{array}$ & $\begin{array}{r}0.16 \\
.19 \\
.19 \\
. .00 \\
.15\end{array}$ & $\begin{array}{r}0.12 \\
.24 \\
.11 \\
.06 \\
.02\end{array}$ & $\begin{array}{r}0.29 \\
.12 \\
.23 \\
.14 \\
.25\end{array}$ & $\begin{array}{l}0.26 \\
.23 \\
.21 \\
.26 \\
.15\end{array}$ & $\begin{array}{r}0.206 \\
.175 \\
.210 \\
.162 \\
.183\end{array}$ \\
\hline 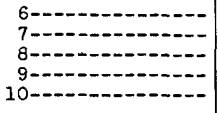 & $\begin{array}{l}.136 \\
.140 \\
.185 \\
.249 \\
.139\end{array}$ & $\begin{array}{l}.197 \\
.227 \\
.200 \\
.218 \\
.218\end{array}$ & $\begin{array}{l}.22 \\
.14 \\
.15 \\
.19 \\
.15\end{array}$ & $\begin{array}{l}.06 \\
.32 \\
.25 \\
.17 \\
.20\end{array}$ & $\begin{array}{r}.09 \\
.21 \\
c .28 \\
. .16 \\
.13\end{array}$ & $\begin{array}{l}.21 \\
.21 \\
.27 \\
.15 \\
.25\end{array}$ & $\begin{array}{l}.30 \\
.28 \\
.23 \\
.23 \\
.12\end{array}$ & $\begin{array}{l}.173 \\
.218 \\
.224 \\
.195 \\
.172\end{array}$ \\
\hline 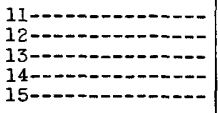 & $\begin{array}{l}.116 \\
.186 \\
.243 \\
.137 \\
.168\end{array}$ & $\begin{array}{l}.157 \\
.151 \\
.137 \\
.189 \\
.155\end{array}$ & $\begin{array}{l}.28 \\
.22 \\
.08 \\
.16 \\
.29\end{array}$ & $\begin{array}{l}.20 \\
.10 \\
.09 \\
.28 \\
.18\end{array}$ & $\begin{array}{l}.11 \\
.05 \\
.13 \\
.12 \\
.13\end{array}$ & $\begin{array}{l}.17 \\
.21 \\
.24 \\
.18 \\
.05\end{array}$ & $\begin{array}{l}.20 \\
.26 \\
.20 \\
.21 \\
.23\end{array}$ & $\begin{array}{l}.176 \\
.168 \\
.160 \\
.182 \\
.172\end{array}$ \\
\hline 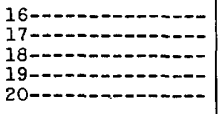 & $\begin{array}{l}.099 \\
.093 \\
.195 \\
.209 \\
.243\end{array}$ & $\begin{array}{r}.110 \\
.195 \\
. .279 \\
.157 \\
.221\end{array}$ & $\begin{array}{l}.11 \\
.35 \\
.26 \\
.21 \\
.25\end{array}$ & $\begin{array}{l}.21 \\
.04 \\
.07 \\
.17 \\
.00\end{array}$ & $\begin{array}{l}.31 \\
.20 \\
.18 \\
.23 \\
.16\end{array}$ & $\begin{array}{l}.13 \\
.07 \\
.23 \\
.07 \\
.19\end{array}$ & $\begin{array}{l}.20 \\
.01 \\
.20 \\
.25 \\
.18\end{array}$ & $\begin{array}{l}.167 \\
.137 \\
.202 \\
.185 \\
.178\end{array}$ \\
\hline 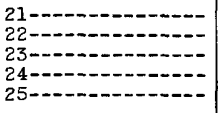 & $\begin{array}{l}.113 \\
.198 \\
.186 \\
.159 \\
.195\end{array}$ & $\begin{array}{r}c .233 \\
.162 \\
. .137 \\
.085 \\
.158\end{array}$ & $\begin{array}{l}.09 \\
.09 \\
.20 \\
.21 \\
.26\end{array}$ & $\begin{array}{l}.01 \\
.20 \\
.18 \\
.14 \\
.19\end{array}$ & $\begin{array}{l}.27 \\
.15 \\
.07 \\
.06 \\
.10\end{array}$ & $\begin{array}{l}.22 \\
.26 \\
.32 \\
.19 \\
.17\end{array}$ & $\begin{array}{l}.21 \\
.17 \\
.27 \\
.22 \\
.22\end{array}$ & $\begin{array}{l}.164 \\
.176 \\
.195 \\
.152 \\
.185\end{array}$ \\
\hline 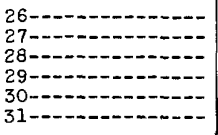 & $\begin{array}{l}.252 \\
.242 \\
.112 \\
.169 \\
.209 \\
.360\end{array}$ & $\begin{array}{l}.193 \\
.211 \\
.199 \\
.211 \\
.245 \\
.182\end{array}$ & $\begin{array}{l}.12 \\
.24 \\
.26 \\
.27 \\
.16 \\
.20\end{array}$ & $\begin{array}{l}.14 \\
.04 \\
.22 \\
.20 \\
.16 \\
.28\end{array}$ & $\begin{array}{l}.25 \\
.10 \\
.25 \\
.07 \\
.24 \\
.11\end{array}$ & $\begin{array}{l}.18 \\
.22 \\
.12 \\
.20 \\
.16 \\
.21\end{array}$ & $\begin{array}{l}.23 \\
.25 \\
.24 \\
.21 \\
.23 \\
.14 \\
\end{array}$ & $\begin{array}{l}.195 \\
.186 \\
.200 \\
.190 \\
.201 \\
.212 \\
\end{array}$ \\
\hline Total_...... & 5.759 & c 5.924 & 6.21 & 4.79 & c4.71 & 5.91 & 6.60 & 5.701 \\
\hline August & 1947 & 1948 & 1949 & 1950 & 1951 & 1952 & 1953 & Average \\
\hline 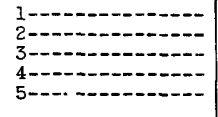 & $\begin{array}{r}0.246 \\
.186 \\
.125 \\
.198 \\
.237\end{array}$ & $\begin{array}{r}0.151 \\
.199 \\
.213 \\
.146 \\
.139\end{array}$ & $\begin{array}{l}0.18 \\
.21 \\
.18 \\
.23 \\
.24\end{array}$ & $\begin{array}{l}0.14 \\
.23 \\
.08 \\
.18 \\
.19\end{array}$ & $\begin{array}{r}0.10 \\
.19 \\
.28 \\
.15 \\
.10\end{array}$ & $\begin{array}{r}0.22 \\
.18 \\
.19 \\
.16 \\
.12\end{array}$ & $\begin{array}{r}0.23 \\
.31 \\
.12 \\
.27 \\
.16\end{array}$ & $\begin{array}{r}0.181 \\
.215 \\
.170 \\
.191 \\
.169\end{array}$ \\
\hline 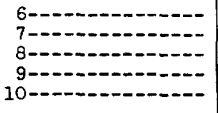 & $\begin{array}{l}.300 \\
.280 \\
.150 \\
.250 \\
.200\end{array}$ & $\begin{array}{l}.069 \\
.161 \\
.130 \\
.052 \\
.044\end{array}$ & $\begin{array}{l}.20 \\
.20 \\
.20 \\
.16 \\
.23\end{array}$ & $\begin{array}{l}.20 \\
.20 \\
.17 \\
.12 \\
.14\end{array}$ & $\begin{array}{r}.10 \\
.20 \\
. .25 \\
.05 \\
.05\end{array}$ & $\begin{array}{l}.15 \\
.17 \\
.18 \\
.39 \\
.14\end{array}$ & $\begin{array}{l}.12 \\
.15 \\
.13 \\
.17 \\
.18\end{array}$ & $\begin{array}{l}.163 \\
.194 \\
.173 \\
.170 \\
.141\end{array}$ \\
\hline 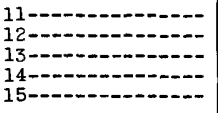 & $\begin{array}{l}.230 \\
.200 \\
.230 \\
.280 \\
.180\end{array}$ & $\begin{array}{l}.204 \\
.102 \\
.187 \\
.200 \\
.161\end{array}$ & $\begin{array}{l}.21 \\
.09 \\
.08 \\
.14 \\
.22\end{array}$ & $\begin{array}{l}.17 \\
.18 \\
.17 \\
.18 \\
.13\end{array}$ & $\begin{array}{r}.10 \\
.10 \\
.10 \\
.42 \\
. .00\end{array}$ & $\begin{array}{r}c .02 \\
.12 \\
.29 \\
.06 \\
.25\end{array}$ & $\begin{array}{r}.11 \\
. .11 \\
. .25 \\
.20 \\
.20\end{array}$ & $\begin{array}{l}.143 \\
.129 \\
.187 \\
.211 \\
.163\end{array}$ \\
\hline 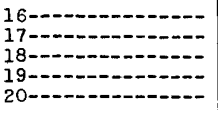 & $\begin{array}{l}.200 \\
.238 \\
.191 \\
.245 \\
.212\end{array}$ & $\begin{array}{r}. .161 \\
.164 \\
.170 \\
.200 \\
.150\end{array}$ & $\begin{array}{l}.18 \\
.11 \\
.14 \\
.20 \\
.22\end{array}$ & $\begin{array}{l}.11 \\
.20 \\
.19 \\
.14 \\
.05\end{array}$ & $\begin{array}{l}.14 \\
.27 \\
.20 \\
.09 \\
.09\end{array}$ & $\begin{array}{l}.03 \\
.11 \\
.20 \\
.18 \\
.12\end{array}$ & $\begin{array}{l}.17 \\
.14 \\
.18 \\
.16 \\
.24\end{array}$ & $\begin{array}{l}.142 \\
.176 \\
.182 \\
.174 \\
.155\end{array}$ \\
\hline 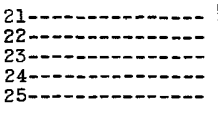 & $\begin{array}{l}.223 \\
.190 \\
.127 \\
.149 \\
.183\end{array}$ & $\begin{array}{l}.418 \\
.221 \\
.210 \\
.310 \\
.240\end{array}$ & $\begin{array}{l}.21 \\
.20 \\
.19 \\
.19 \\
.17\end{array}$ & $\begin{array}{l}.23 \\
.20 \\
.20 \\
.24 \\
.11\end{array}$ & $\begin{array}{l}.14 \\
.20 \\
.30 \\
.12 \\
.18\end{array}$ & $\begin{array}{l}.03 \\
.15 \\
.10 \\
.28 \\
.10\end{array}$ & $\begin{array}{l}.17 \\
.17 \\
.20 \\
.19 \\
.21\end{array}$ & $\begin{array}{l}.203 \\
.190 \\
.190 \\
.211 \\
.170\end{array}$ \\
\hline 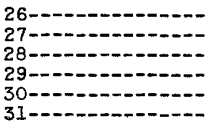 & $\begin{array}{l}.096 \\
.095 \\
.199 \\
.180 \\
.122 \\
.252 \\
\end{array}$ & $\begin{array}{l}.256 \\
.206 \\
.190 \\
.187 \\
.275 \\
.179 \\
\end{array}$ & $\begin{array}{l}.09 \\
.32 \\
.13 \\
.10 \\
.17 \\
.17 \\
\end{array}$ & $\begin{array}{l}.13 \\
.12 \\
.09 \\
.08 \\
.12 \\
.11 \\
\end{array}$ & $\begin{array}{l}.12 \\
.04 \\
.12 \\
.12 \\
.07 \\
.10\end{array}$ & $\begin{array}{l}.19 \\
.22 \\
.18 \\
.20 \\
.30 \\
.01 \\
\end{array}$ & $\begin{array}{l}.23 \\
.22 \\
.24 \\
.22 \\
.24 \\
.29 \\
\end{array}$ & $\begin{array}{l}.159 \\
.174 \\
.164 \\
.155 \\
.185 \\
.159 \\
\end{array}$ \\
\hline Total $=\ldots$ & 6.194 & c 5.695 & 5.56 & 4.80 & 4.49 & 5.00 & 5.98 & 5.389 \\
\hline
\end{tabular}

See footnotes at end of table. 
Evaporation, in inches, at Valpara1so, Ind.--Continued

\begin{tabular}{|c|c|c|c|c|c|c|c|c|}
\hline September & 1947 & 1948 & 1949 & 1950 & 1951 & 1952 & 1953 & Average \\
\hline 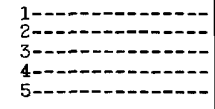 & $\begin{array}{r}0.152 \\
.156 \\
.193 \\
.234 \\
.111\end{array}$ & $\begin{array}{r}0.279 \\
.081 \\
.114 \\
.177 \\
.199\end{array}$ & $\begin{array}{r}0.20 \\
.19 \\
.18 \\
.20 \\
.20\end{array}$ & $\begin{array}{r}0.03 \\
.10 \\
.11 \\
.15 \\
.16\end{array}$ & $\begin{array}{l}0.24 \\
.02 \\
.04 \\
.08 \\
.13\end{array}$ & $\begin{array}{r}0.14 \\
.09 \\
.11 \\
.14 \\
.11\end{array}$ & $\begin{array}{l}0.31 \\
.28 \\
.16 \\
.20 \\
.18\end{array}$ & $\begin{array}{r}0.193 \\
.131 \\
.130 \\
.169 \\
.156\end{array}$ \\
\hline 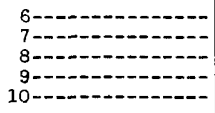 & $\begin{array}{l}.067 \\
.109 \\
.121 \\
.136 \\
.139\end{array}$ & $\begin{array}{l}.128 \\
.065 \\
.113 \\
.071 \\
.125\end{array}$ & $\begin{array}{r}.09 \\
.08 \\
.09 \\
.08 \\
.06\end{array}$ & $\begin{array}{l}.14 \\
.13 \\
.13 \\
.08 \\
.10\end{array}$ & $\begin{array}{l}.08 \\
.12 \\
.08 \\
.07 \\
.14\end{array}$ & $\begin{array}{l}.20 \\
.11 \\
.10 \\
.16 \\
.20\end{array}$ & $\begin{array}{l}.16 \\
.21 \\
.16 \\
.12 \\
.14\end{array}$ & $\begin{array}{l}.124 \\
.118 \\
.113 \\
.102 \\
.129\end{array}$ \\
\hline 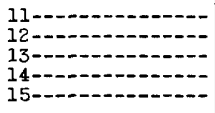 & $\begin{array}{l}.136 \\
.203 \\
.125 \\
.199 \\
.226\end{array}$ & $\begin{array}{l}.121 \\
.202 \\
.253 \\
.155 \\
.156\end{array}$ & $\begin{array}{l}\text { b. } .11 \\
b .04 \\
\text { b. } 14 \\
\text { b.12 } \\
\text { b. } 05\end{array}$ & $\begin{array}{l}.07 \\
.05 \\
.03 \\
.02 \\
.12\end{array}$ & $\begin{array}{l}.14 \\
.17 \\
.26 \\
.14 \\
.17\end{array}$ & $\begin{array}{r}.17 \\
.20 \\
.10 \\
c .10 \\
c .15\end{array}$ & $\begin{array}{l}.07 \\
.31 \\
.12 \\
.08 \\
.12\end{array}$ & $\begin{array}{l}.117 \\
.168 \\
.147 \\
.116 \\
.142\end{array}$ \\
\hline 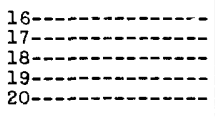 & $\begin{array}{l}.149 \\
.146 \\
.109 \\
.129 \\
.118\end{array}$ & $\begin{array}{l}.066 \\
.187 \\
.224 \\
.199 \\
.167\end{array}$ & $\begin{array}{l}\text { b. } 13 \\
b .20 \\
b .19 \\
b .13 \\
b .16\end{array}$ & $\begin{array}{l}.03 \\
.06 \\
.09 \\
.14 \\
.04\end{array}$ & $\begin{array}{l}.07 \\
.16 \\
.12 \\
.09 \\
.16\end{array}$ & $\begin{array}{l}.31 \\
.17 \\
.07 \\
.09 \\
.07\end{array}$ & $\begin{array}{l}.14 \\
.18 \\
.20 \\
.06 \\
.15\end{array}$ & $\begin{array}{l}.128 \\
.158 \\
.143 \\
.120 \\
.124\end{array}$ \\
\hline 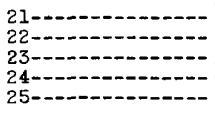 & $\begin{array}{l}.144 \\
.166 \\
.104 \\
.121 \\
.120\end{array}$ & $\begin{array}{l}.060 \\
.044 \\
.098 \\
.085 \\
.115\end{array}$ & $\begin{array}{l}6.17 \\
.10 \\
.06 \\
.06 \\
.11\end{array}$ & $\begin{array}{l}.03 \\
.04 \\
.11 \\
.14 \\
.04\end{array}$ & $\begin{array}{l}.17 \\
.14 \\
.07 \\
.11 \\
.06\end{array}$ & $\begin{array}{l}.14 \\
.09 \\
.10 \\
.10 \\
.20\end{array}$ & $\begin{array}{l}.15 \\
.07 \\
.16 \\
.18 \\
.16\end{array}$ & $\begin{array}{l}.123 \\
.093 \\
.100 \\
.114 \\
.115\end{array}$ \\
\hline 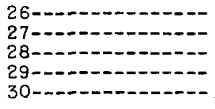 & $\begin{array}{l}.101 \\
.101 \\
.083 \\
.078 \\
.081 \\
\end{array}$ & $\begin{array}{l}.158 \\
.113 \\
.121 \\
.168 \\
.040 \\
\end{array}$ & $\begin{array}{l}.12 \\
.18 \\
.12 \\
.03 \\
.06\end{array}$ & $\begin{array}{l}.09 \\
.07 \\
.08 \\
.10 \\
.06 \\
\end{array}$ & $\begin{array}{l}.05 \\
.26 \\
.17 \\
.07 \\
.07 \\
\end{array}$ & $\begin{array}{l}.20 \\
.14 \\
.21 \\
.19 \\
.12 \\
\end{array}$ & $\begin{array}{l}.16 \\
.20 \\
.12 \\
.26 \\
.27 \\
\end{array}$ & $\begin{array}{l}.126 \\
.152 \\
.129 \\
.128 \\
.100 \\
\end{array}$ \\
\hline Total -...- & 4.057 & 4.084 & 3.65 & 2.54 & 3.65 & 4.28 & 5.08 & 3.908 \\
\hline October & 1947 & 1948 & 1949 & 1950 & 1951 & 1952 & 1953 & Average \\
\hline 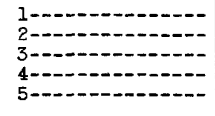 & $\begin{array}{r}0.085 \\
.073 \\
.093 \\
.133 \\
.083\end{array}$ & $\begin{array}{r}0.008 \\
.120 \\
.126 \\
.110 \\
.121\end{array}$ & $\begin{array}{l}0.13 \\
.11 \\
.08 \\
.02 \\
.02\end{array}$ & $\begin{array}{l}0.05 \\
.05 \\
.13 \\
.07 \\
.06\end{array}$ & $\begin{array}{r}0.06 \\
.17 \\
.10 \\
.17 \\
.17\end{array}$ & $\begin{array}{r}0.17 \\
.14 \\
.05 \\
.11 \\
.13\end{array}$ & $\begin{array}{l}0.19 \\
.16 \\
.18 \\
.12 \\
.08\end{array}$ & $\begin{array}{r}0.099 \\
.118 \\
.108 \\
.105 \\
.095\end{array}$ \\
\hline 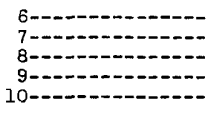 & $\begin{array}{l}.090 \\
.182 \\
.105 \\
.104 \\
.096\end{array}$ & $\begin{array}{l}.123 \\
.100 \\
.046 \\
.069 \\
.133\end{array}$ & $\begin{array}{l}.03 \\
.04 \\
.14 \\
.12 \\
.14\end{array}$ & $\begin{array}{l}.07 \\
.12 \\
.02 \\
.07 \\
.02\end{array}$ & $\begin{array}{l}.06 \\
.04 \\
.07 \\
.06 \\
.05\end{array}$ & & $\begin{array}{l}.11 \\
.09 \\
.09 \\
.09 \\
.12\end{array}$ & $\begin{array}{l}.080 \\
.095 \\
.078 \\
.086 \\
.093\end{array}$ \\
\hline 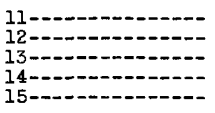 & $\begin{array}{l}.065 \\
.051 \\
.128 \\
.104 \\
.098\end{array}$ & $\begin{array}{l}.082 \\
.026 \\
.042 \\
.045 \\
.059\end{array}$ & $\begin{array}{l}.21 \\
.03 \\
.11 \\
.08 \\
.09\end{array}$ & $\begin{array}{l}.02 \\
.05 \\
.10 \\
.03 \\
.09\end{array}$ & $\begin{array}{l}.05 \\
.07 \\
.07 \\
.12 \\
.10\end{array}$ & & $\begin{array}{l}.10 \\
.08 \\
.11 \\
.09 \\
.13\end{array}$ & $\begin{array}{l}.088 \\
.051 \\
.093 \\
.078 \\
.094\end{array}$ \\
\hline 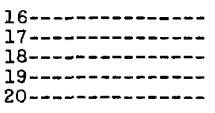 & $\begin{array}{l}.110 \\
.112 \\
.010 \\
.100 \\
.076\end{array}$ & $\begin{array}{l}.081 \\
.068 \\
.086 \\
.079 \\
.050\end{array}$ & $\begin{array}{l}.09 \\
.09 \\
.06 \\
.07 \\
.05\end{array}$ & $\begin{array}{l}.08 \\
.07 \\
.11 \\
.10 \\
.02\end{array}$ & $\begin{array}{l}.11 \\
.09 \\
.05 \\
.10 \\
.04\end{array}$ & -- & $\begin{array}{l}.09 \\
.13 \\
.17 \\
.17 \\
.10\end{array}$ & $\begin{array}{l}.094 \\
.093 \\
.081 \\
.103 \\
.056\end{array}$ \\
\hline 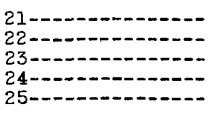 & $\begin{array}{l}.120 \\
.145 \\
.139 \\
.126 \\
.118\end{array}$ & $\begin{array}{l}.076 \\
.010 \\
.063 \\
.039 \\
.017\end{array}$ & $\begin{array}{l}.15 \\
.08 \\
.09 \\
.09 \\
.06\end{array}$ & $\begin{array}{l}.12 \\
.11 \\
.05 \\
.03 \\
.03\end{array}$ & $\begin{array}{r}.08 \\
.05 \\
.10 \\
.01 \\
. .02\end{array}$ & & $\begin{array}{l}.22 \\
.11 \\
.09 \\
.07 \\
.08\end{array}$ & $\begin{array}{l}.128 \\
.084 \\
.089 \\
.061 \\
.054\end{array}$ \\
\hline 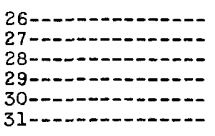 & $\begin{array}{l}.058 \\
.018 \\
.052 \\
.017 \\
.003 \\
.071 \\
\end{array}$ & $\begin{array}{l}.048 \\
.064 \\
.057 \\
.059 \\
.060 \\
.083 \\
\end{array}$ & $\begin{array}{l}.08 \\
.07 \\
.06 \\
.08 \\
.13 \\
.07 \\
\end{array}$ & $\begin{array}{l}.08 \\
.05 \\
.04 \\
.05 \\
.09 \\
.11\end{array}$ & $\begin{array}{l}.10 \\
.07 \\
.01 \\
.09 \\
.05 \\
.07\end{array}$ & 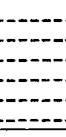 & $\begin{array}{l}.02 \\
.07 \\
.05 \\
.05 \\
.09 \\
.09\end{array}$ & $\begin{array}{l}.064 \\
.057 \\
.045 \\
.058 \\
.070 \\
.082 \\
\end{array}$ \\
\hline Total-_..... & 2.765 & $c_{2} .150$ & 2.67 & 2.09 & e 2.40 & 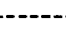 & 3.34 & 2.580 \\
\hline
\end{tabular}

See footnotes at end of table. 
Evaporation, In inches, at Valparaiso, Ind.--Continued

\begin{tabular}{|c|c|c|c|c|c|c|c|c|c|}
\hline November & $1947 d$ & 1948 & 1949 & 1950 & 1953 & Average & December & $1948 d$ & 1949 \\
\hline 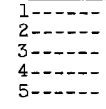 & $\begin{array}{r}0.029 \\
.069 \\
.069 \\
.035 \\
.000\end{array}$ & $\begin{array}{r}0.011 \\
.056 \\
.041 \\
.000 \\
.058\end{array}$ & $\begin{array}{l}0.08 \\
.05 \\
.03 \\
.04 \\
.02\end{array}$ & $\begin{array}{l}0.17 \\
.20 \\
.05 \\
.08 \\
.01\end{array}$ & $\begin{array}{l}0.06 \\
.06 \\
.15 \\
.14 \\
.08\end{array}$ & $\begin{array}{l}0.070 \\
.087 \\
.068 \\
.059 \\
.034\end{array}$ & 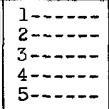 & $\begin{array}{r}0.040 \\
.050 \\
.006 \\
.009 \\
.002\end{array}$ & $\begin{array}{l}0.01 \\
a .00 \\
a .00 \\
a .00 \\
.02\end{array}$ \\
\hline 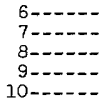 & $\begin{array}{l}.033 \\
.090 \\
.009 \\
.005 \\
.098\end{array}$ & $\begin{array}{l}.080 \\
.054 \\
.055 \\
.043 \\
.064\end{array}$ & $\begin{array}{l}.04 \\
.05 \\
.13 \\
.07 \\
.10\end{array}$ & $\begin{array}{l}.08 \\
.13 \\
.00 \\
.06 \\
.01\end{array}$ & $\begin{array}{l}.02 \\
.01 \\
.06 \\
.08 \\
.11\end{array}$ & $\begin{array}{l}.051 \\
.067 \\
.051 \\
.052 \\
.076\end{array}$ & 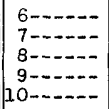 & $\begin{array}{l}.086 \\
.043 \\
.055 \\
.055 \\
.006\end{array}$ & $\begin{array}{l}a .02 \\
9.03 \\
.03 \\
.01 \\
.01\end{array}$ \\
\hline 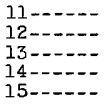 & $\begin{array}{l}.160 \\
.060 \\
.055 \\
.024 \\
.007\end{array}$ & $\begin{array}{l}.038 \\
.057 \\
.016 \\
.018 \\
.044\end{array}$ & $\begin{array}{l}.05 \\
.05 \\
.01 \\
.15 \\
.10\end{array}$ & $\begin{array}{r}a .03 \\
a .02 \\
a .02 \\
a .03 \\
-.02\end{array}$ & $\begin{array}{l}.03 \\
.05 \\
.03 \\
.04 \\
.13\end{array}$ & $\begin{array}{l}.062 \\
.047 \\
.026 \\
.052 \\
.052\end{array}$ & 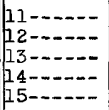 & $\begin{array}{l}.002 \\
.029 \\
.010 \\
.019 \\
.010\end{array}$ & $\begin{array}{r}a .03 \\
0.07 \\
-0 .---\end{array}$ \\
\hline 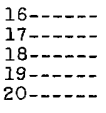 & $\begin{array}{l}.084 \\
.035 \\
.030 \\
.027 \\
.017\end{array}$ & $\begin{array}{r}.066 \\
.080 \\
.053 \\
.073 \\
. .014\end{array}$ & $\begin{array}{r}.11 \\
.03 \\
-.01 \\
.05 \\
.06\end{array}$ & $\begin{array}{l}.07 \\
.01 \\
.05 \\
.06 \\
.00\end{array}$ & $\begin{array}{l}.11 \\
.10 \\
.09 \\
.08 \\
.06\end{array}$ & $\begin{array}{l}.088 \\
.051 \\
.043 \\
.058 \\
.030\end{array}$ & 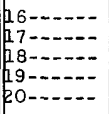 & $\begin{array}{r}.017 \\
.037 \\
.022 \\
c .0112 \\
\text { c. } 002\end{array}$ & 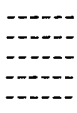 \\
\hline 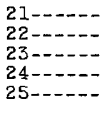 & $\begin{array}{r}.037 \\
.011 \\
.051 \\
.008 \\
.030\end{array}$ & $\begin{array}{l}.009 \\
.007 \\
.026 \\
.030 \\
.026\end{array}$ & $\begin{array}{l}.05 \\
a-.02 \\
a-.06 \\
a-.02 \\
a-.03 \\
\end{array}$ & $\begin{array}{l}a .03 \\
a .05 \\
a .02 \\
a .01 \\
a .00\end{array}$ & $\begin{array}{l}.02 \\
.00 \\
.01 \\
.00 \\
.05\end{array}$ & $\begin{array}{l}.029 \\
.010 \\
.009 \\
.006 \\
.015\end{array}$ & $\begin{array}{l}21-\ldots \ldots \\
22-\ldots \ldots \\
23-\ldots \ldots \\
24-\ldots \ldots- \\
25-\ldots \ldots-\end{array}$ & $\begin{array}{l}c .307 \\
a .100 \\
a .035 \\
a .021 \\
a .035\end{array}$ & 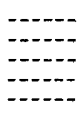 \\
\hline 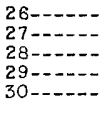 & $\begin{array}{l}b .030 \\
b .020 \\
b .030 \\
b .010 \\
b .010\end{array}$ & $\begin{array}{l}.010 \\
.049 \\
.019 \\
.024 \\
.024\end{array}$ & $\begin{array}{l}a .04 \\
a \\
a \\
.12 \\
.06 \\
.05 \\
.01\end{array}$ & $\begin{array}{l}a .01 \\
a .02 \\
a .04 \\
a .02 \\
a .03\end{array}$ & $\begin{array}{l}.06 \\
b .02 \\
b .01 \\
b .00 \\
b .02\end{array}$ & $\begin{array}{l}.030 \\
.046 \\
.032 \\
.021 \\
.019\end{array}$ & 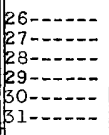 & $\begin{array}{l}a .020 \\
a .060 \\
a .065 \\
a .095 \\
a . \overline{230} \\
. .034 \\
\end{array}$ & - \\
\hline Total- & 1.173 & $c_{1.145}$ & 1.41 & 1.29 & 1.68 & 1.341 & Total- & 1.614 & $----\cdots$ \\
\hline
\end{tabular}

a Estimated from total observed on underlined day.

$b$ Estimated because of missing or erroneous data.

c Corrected since originally published by U. S. Weather Bureau.

d Not previously published. 


\section{LITERATURE CITED}

Blatchley, W. S., and Ashley, G. H., 1900, The lakes of northern Indiana and their associated marl deposits: Ind. Dept. Geol. and Nat. Res., 25th Ann. Rept., p. 31-321.

Birge, E. A., and Juday C., 1911, The inland lakes of Wisconsin, the dissolved gases of the water and their biological significance: Wis. Geol. and Nat. History Survey Bull. 22.

Dolan, J. P., 1896, Temperature of Lake Wawasee: Ind. Acad. Sci. Proc., p. 279-285.

Evermann, B. W., and Clark, H. W., 1920, Lake Maxinkuckee, a physical and biological survey: Ind. Dept. Conserv. Pub. 7 , v. 1 , p. $152-216$.

Indiana State Legislature, 1947, Acts of 1947: chap. 350.

Indiana State Legislature, 1951, Acts of 1951: chap. 290.

Levette, G. M., 1876, Observations on the depth and temperature of some lakes in northern Indiana: Ind. Geol. Survey, 7th Ann. Rept., p. 469-503.

Miner, D. H., October 1949, Fourteen years under water: Outdoor Indiana, p. 8.

Ricker, W. E., 1937, Physical and chemical characteristics of Cultis Lake, British Columbia: Jour. Biol. Board of Canada 3 (4).

Scott, Will, 1915, Report on the Lakes of Tippecanoe basin, Ind. Acad, Sci. Pro., p. 377-378.

Scott, Will and Miner, D. H., 1935, Sedimentation in Winona Lake and Tippecanoe Lake, Koscuisko County, Ind., July 31, 1930. to July 30, 1935: Ind. Acad. Sci. Proc., v. 45, p. 275-286.

Trewartha, G. T., 1943, An introduction to weather and climate, 2d ed.: New York, McGraw-Hill Book Co., Inc.

Wilson, Ira T., 1935, A study of sedimentation of Winona Lake: Ind. Acad. Sci. Proc., v. 45, p. 295-304.

Wilson, Ira T., 1937, The accumulated sediment in Tippecanoe Lake and a comparison with Winona Lake: Ind. Acad. Sci. Proc., v. 47, p. 234-253.

Anonymous, 1952, Water-loss investigations: v. 1, Lake Hefner studies, Technical Report: U. S. Geol. Survey Circ. 229; also 1954, U. S. Geol. Survey Prof. Paper 269 and 270. 
Abstract . . . . . . . . . . . . . 1

Analyses of evaporation records ....52-59

Artificial lakes, construction of .4-5, 13-14

Bass Lake at Bass Lake, Ind., control of lake level of . . . . . . . . 68

lake levels for. ...........78-84, 268 surface temperatures for . . . . . . 274 temperature profile for. . . . . .33, 38, 275

Bear Lake at Wolflake, lake levels for . . . . . . . . . 84-90, 268

surface temperatures for . . . . . 275

Big Lake near Wolflake, lake levels for. ........... 91-97, 268

surface temperatures for....... 275

Bixler Lake at Kendallville, inflow and outflow studies ........ 70

lake levels for. ......... 98-103, 268 surface temperatures for . . . . . 276 temperature profiles for. . . . .39, 42, 277 water loss by evaporation. .... 55-56, 57

Bruce Lake at Lake Bruce, Ind., lake levels for. ........104-110, 268

surface temperatures for .......278

Cedar Lake at Cedar Lake, inflow and outflow studies.......... 70

lake levels for.........110-116, 268

surface temperatures for .......279

temperature profiles for. $33,36,279-280$

Cedar Lake at Tri-Lakes, Ind, , lake levels for. ........ 197-204, 268

Cedar Lake in DeKalb County. See Cedar Lake near Water $\overline{l o o}$, Ind.

Cedar Lake in Lake County. See Cedar Lake at Cedar Lake, Ind.

Cedar Lake in Whitley County. See Cedar Lake at Tri-Lakes, Ind.

Cedar Lake near Waterloo, Ind., lake levels for..........117-123, 268

surface temperatures for ........ 280

Clear Lake at Clear Lake, Ind., lake levels for. .......205-211, 268

temperature profiles for. $39,44,314-315$

Clear Lake at LaPorte, Ind., legal lake levels for ..........268

water loss from ..........57,58

Clear Lake in LaPorte County. See Clear Lake at LaPorte.

Clear Lake in Steuben County. See Clear Lake at Clear Lake, Ind.

Creation of new lakes........... 13-14

Crooked Lake near Wolflake, lake levels for......... 124-130, 268

surface temperatures for . . . . . . 280

Culver, Maxinkuckee Lake at .20, 25, 27,29 , $30,32,33,35,176-183,270,286-309$

Dams, for controlling lake levels .. 69-70 Deep Hole, Maxinkuckee Lake, temperature profiles for...... 300-309

Depth of water, effect on temperature of lakes ............45-46

Distribution of Indiana lakes ........ 8 Drainage area, effect of size of, on temperature of lakes.........45

Drainage of lakes.............11-13
Epilimnion ................ 22-31

Evansville, Ind., evaporation station at ................. 323

measurement of evaporation at ............. 324-328

Evaporation, measurement of, from water surfaces. . . . . . . 50-51

variations in Indiana .......... 52

Evaporation data, collection of $51-52,322$

tables................ 324-343

Evaporation from lakes, analyses of records ........... 52-59

effect on lake levels .......... 50 evaporation stations ... 51-52, 323-324 factors influencing rate .......49-50

Evermann, B. W, and Clark, H. W. , quoted .. 46, 60, 287-299, 300-309

Extinction of Indiana lakes, cause of 8-13

Flint Lake near Valparaiso, lake levels for...........130-135, 268

surface temperatures for . . . . . 281

temperature profiles for. . . 33, 37, 282 Flood control, relation to storage capacity of lakes ....... 70-72

Geist Reservoir, average daily evaporation at.

Hamilton Lake at Hamilton, lake levels for. ...........136-142, 268 surface temperatures for . . . . . 283 Hydrology of lakes. . . . . . . . . 3-5 Hydrologic data, basic types of .... 3-4 Hypolimnion ..............22-23

Ice, coefficient of linear expansion of 63 damage by .............. 65 formation of, on lakes. ....... 59-60 thickness of ..............61-64 See also lake levels for individual lakes.

lce conditions, effect of temperature on ..............61-64 Ice cover, duration of. . . . . . 60-61 See also lake levels for individual lakes.

Indianapolis, evaporation station at. . 323 measurement of evaporation at $328-334$ Inflow of lakes, studies of . . . . . 69-70 Introduction $\ldots \ldots \ldots \ldots \ldots \ldots \ldots .2-3$

James, Lake, at Lake James, Ind.,

lake levels for .....159-163, 269 surface temperatures for . . . . . 284 temperature profiles for. . $39,43,284$ -

Jimerson Lake at Nevada Mills, lake levels for........ 143-152, 269 surface temperatures for . . . . . 283

Kendalville, Bixler Lake at 39, 42, 55-56, $57,70,98-103,268,276,277$ evaporation station at ......... 323 measured evaporation at $\ldots 55,56,335-$ 
Koontz Lake at Koontz Lake, Ind., lake levels for .........152-158, 269 surface temperatures for. . . . . . . 283

Lake levels, basic data for selected lakes.............,77-266 control dams for maintaining ....69-70 effect of evaporation on ......... 50 legal, establishment of. . . . 266-267 table of. .............268-272 problems of control ..........68-69 requirenients for maintaining. . . 67-68 Laws, for preservation of lakes . . . . 5-6 Legal lake levels, table of ...... 268-272

Literature cited ... . . . . . . . . . . . . 344 Loomis Lake, legal lake levels for. . . . 270 water loss by evaporation ......57, 58

Loon Lake at Ormas, lake levels

for ...........170-176, 270

surface temperatures for........286

Manitou, Lake, at Rochester, lake levels for .........163-169, 270

at Rochester, surface temperature for $\ldots \ldots \ldots \ldots \ldots \ldots \ldots \ldots \ldots$

Marl deposits in lakes............ 9

Maxinkuckee Lake at Culver, lake levels for . . ..........176-183, 270 surface temperatures for. . . 20, 286-299 temperature profiles for . .25, 27, 29, 30, $32,33,35,299-309$

Muskelonge Lake near Warsaw, lake levels for ........183-189, 270 surface temperatures for. ........310

Nevada Mills, Jimerson Lake at. 143-152, 269,283

North Webster, Webster Lake at . .68-69, $252-258,272,319$

Origin of Indiana lakes $\ldots \ldots \ldots \ldots \ldots$ 7-8

Organic matter, lake filling by ...... 9 Ormas, Loon lake at .... 170-176, 270, 286 Oswego, Tippecanoe Lake at . . . 33, 34, 69, 238-245, 271, 316-318

Outflow of lakes, studies of ......69-70

Pierceton, Ridinger Lake at. . 68-69, 70, 72 $76,189-196,271,310$

Pleasant Lake, legal lake levels... . . .270

water loss by evaporation..... 56-57

Present investigation...........6-7

Preservation of lakes, Indiana laws

for ............... 5-6

Profiles, of lake temperatures....17-49

Purpose of the report $\ldots \ldots \ldots \ldots \ldots \ldots 7$

Records of evaporation, analyses of .52-59 Ridinger Lake near Pierceton, control of level of.......68-69, 72-76

inflow and outflow studies....... 70 lake levels for.........189-196, 271

surface temperatures for . . . . . 310

temperature profiles for ........... 310

Rochester, Lake Manitou at. . 163-169, 270,

286

Rome City, Sulvan Lake at 68, 69, 224-230,
Round Lake at Clear Lake, Ind., lake levels for. ......,203 211, 271

surface temperatures for . . . 311-313

Round Lake at Tri-Lakes, Ind, lake levels for. . 197-200, 203-204, 271

surface temperatures for , .......310

Round Lake in Steuben County. See Round Lake at Clear Lake, Ind.

Round Lake in Whitley County, See Round Lake at Tri-Lakes, Ind.

Sedimentation of lakes, methods of measuring ............10-11

Shriner Lake at Tri-Lakes, Ind., lake levels for. ......212-217, 271

surface temperatures for . . . . . . 315

Smalley Lake near Washington Center, lake levels for. ....218-224,271

surface temperatures for . . . . . 315

Stabilization of lakes..........65-77

Storage capacity of lakes, relation to flood control......... 70-72

Streamflow, effect of storage in lakes on .............. 70-72

Surface area of lakes, effect of size of, on temperature .........4 45

Surface temperatures of lakes .... 15-17?

See also surface temperatures for individual lakes.

Sylvan Lake at Rome City, control of level of... . . . . . . 68,69

lake levels for. . : : ., . 224-230,271

surface temperatures for . . ...... 315

Syracuse Lake at Syracuse, lake levels for. ..........231 =237,271

surface temperatures for . . . . . 316

Temperature, effects of, on ice conditions of lakes.........61-64 Temperature of lakes, density relation to $\ldots \ldots \ldots \ldots \ldots \ldots \ldots 18=19$

effect of air temperatures ....... effect of solar insolation ......., 21 effect of wind action .....,..., 21 implications of information about 47-49 methods of measuring ........273 profiles. ......, .,........ 17-49 scope of studies ........... 14-15 surface................ 15-17 Temperature profiles, comparison

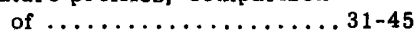

effect of depth of water on .... 45-46 effect of size of drainage area on. 45 effect of size of surface area on... 45 theory of changes below the surface ............19-31

Temperature zones in lakes . . . . 22-23

Thermocline................22-31

Tippecanoe Lake at Oswego, capacity of. ............... 48

control of level of . . . . . . . 69 lake levels for ........ 238-245, 271 surface temperatures for . . . . . 316 temperature profiles for. . $33,34,0,7=$

Tippecanoe River drainage basin, effect of lakes on discharge in... 71-72 
Round Lake at 197-200, 203-204, 271, 310

Shriner Lake at . . . . . 212-217, 271, 315

Turnover of lakes ............46-47

Use of lakes, agricultural.

66

effect of imprc* ed transportation on ................65-66

recreational ............66-67

Valparaiso, evaporation station at . . . 323 Flint Lake at. . .33, 37, 130-135, 268, 281-

measured evaporation at . . . 58, 339-343

Warsaw, Muskelonge Lake near. ..183-189,

Winona Lake at $39,40,258-265,272,320-$ near.........218-224, 271, 315

Waterloo, Cedar Lake near. ..117-123, 268 ,

Water loss from lakes . . . . . . . . 54-59

Wáwásé, Lakè, neả Wawasee, lake levels for. .......245-251,272

surface temperatures for........ 318 temperature profiles for. .....39, 41, 319 Webster Lake at North Webster, control of level of ...........68-69 lake levels for. . . . . . . . 252-258, 272 surface temperatures for ....... . : \$19 Wind action, affect on lake temperatures 21 Winona Lake at Warsaw, lake levels for ............258-265, 272 surface temperatures for ....... 320 temperature profiles for . . . .39, 40, 321 Wolflake, Bear Lake at . . . .84-90, 268, 275 Big Lake near.........91-97, 268, 275 Crooked Lake near. . . . 124-130, 268, 280

\ U. 8. GOVERNMEHT PRINTHNG OFFICE : 1956 O - 384117 
